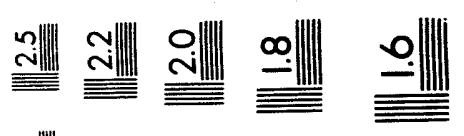
竞: 

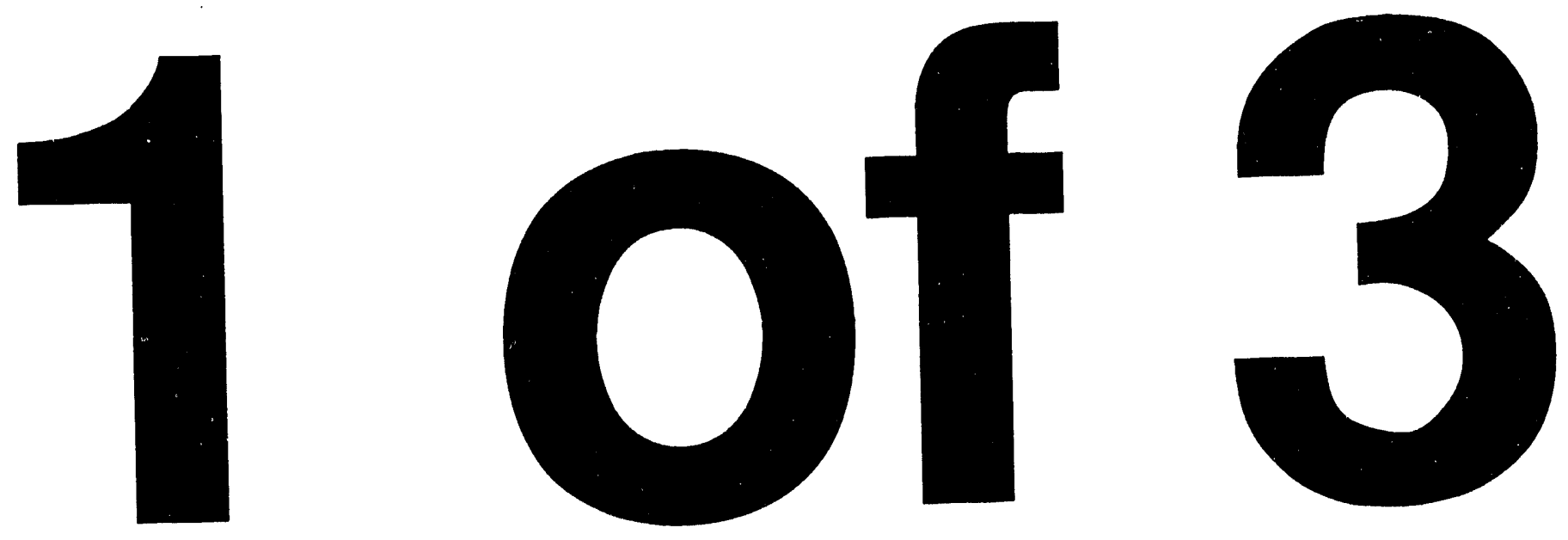
NUREG/CR-5843

SAND92-0167

\section{CORCON-MOD3: An Integrated Computer Model for Analysis of Molten Core-Concrete Interactions}

\section{User's Manual}

Manuscript Completed: August 1993

Date Published: October 1993

Prepared by

D. R. Bradley, D. R. Gardner, J. E. Brockmann, R. O. Griffith

Sandia National Laboratories

Albuquerque, NM 87185

Prepared for

Division of Systems Research

Office of Nuclear Regulatory Research

U.S. Nuclear Regulatory Commission

Washington, DC 20555-0001

NRC FIN L1434 


\begin{abstract}
The CORCON-Mod3 computer code was developed to mechanistically model the important core-concrete interaction phenomena, including those phenomena relevant to the assessment of containment failure and radionuclide release. The code can be applied to a wide range of severe accident scenarios and reactor plants. The code represents the current state of the art for simulating core debris interactions with concrete. This document comprises the user's manual and gives a brief description of the models and the assumptions and limitations in the code. Also discussed are the input parameters and the code output. Two sample problems are also given.
\end{abstract}




\section{Contents}

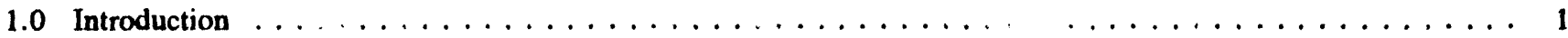

1.1 Historical Background $\ldots \ldots \ldots \ldots \ldots \ldots \ldots \ldots \ldots \ldots \ldots \ldots \ldots \ldots \ldots \ldots$

1.2 Broad Capabilities of CORCON-Mod3 $\ldots \ldots \ldots \ldots \ldots \ldots \ldots \ldots \ldots \ldots \ldots \ldots \ldots \ldots$

1.3 Improvements in CORCON-Mod3 $\ldots \ldots \ldots \ldots \ldots \ldots \ldots \ldots \ldots \ldots \ldots \ldots \ldots \ldots$

2.0 Brief Descriptions of the Phenomenological Models $\ldots \ldots \ldots \ldots \ldots \ldots \ldots \ldots$

2.1 Overview of CORCON Phenomenology $\ldots \ldots \ldots \ldots \ldots \ldots \ldots \ldots \ldots \ldots \ldots \ldots \ldots \ldots$

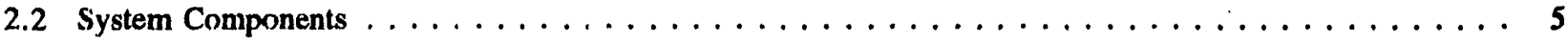

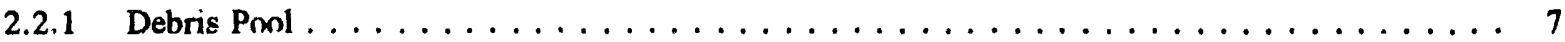

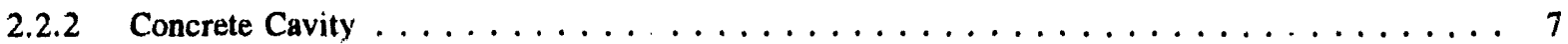

2.2 .3 Aimosphere and Surroundings $\ldots \ldots \ldots \ldots \ldots \ldots \ldots \ldots \ldots \ldots \ldots \ldots$

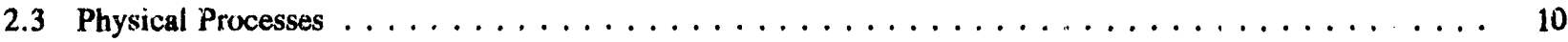

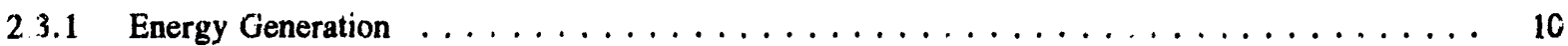

2.3.2 Melt/Concrete Heat Transfer $\ldots \ldots \ldots \ldots \ldots \ldots \ldots \ldots \ldots \ldots \ldots \ldots \ldots$

2.3 .3 Coolant Heat Transfer . . . . . . . . . . . . . . . . . . . . . 19

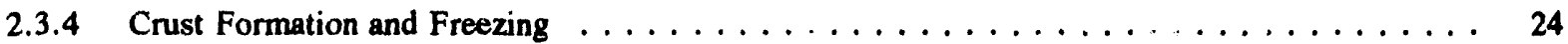

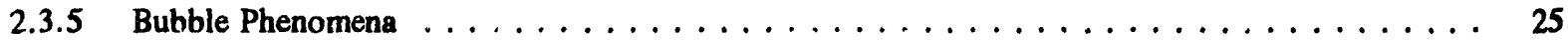

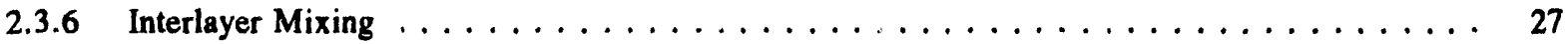

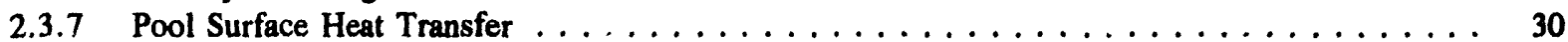

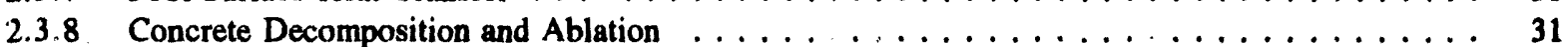

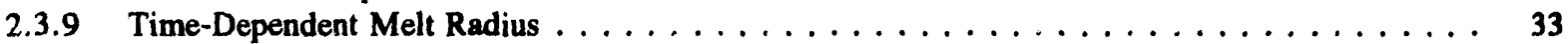

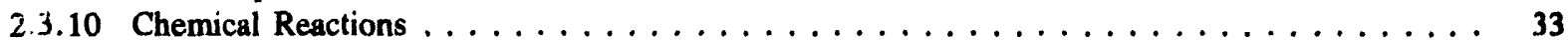

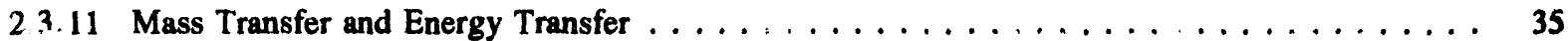

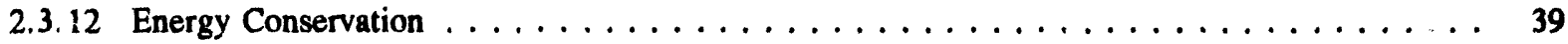

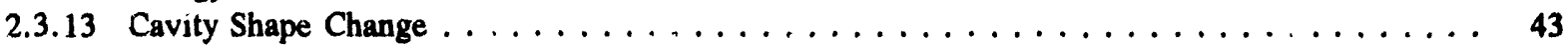

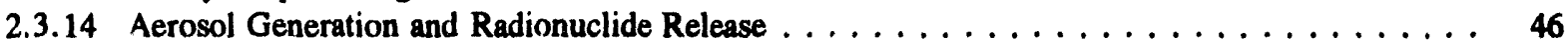

2.3.15 Aerosol Removal By Overlying Water Pools $\ldots \ldots \ldots \ldots \ldots \ldots \ldots \ldots \ldots \ldots$

2.4 Material Properties $\ldots \ldots \ldots \ldots \ldots \ldots \ldots \ldots \ldots \ldots \ldots \ldots \ldots \ldots \ldots \ldots$

2.4.1 Thermodynamic Properties $\ldots \ldots \ldots \ldots \ldots \ldots \ldots \ldots \ldots \ldots \ldots \ldots \ldots \ldots \ldots \ldots$

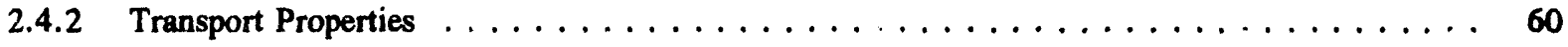

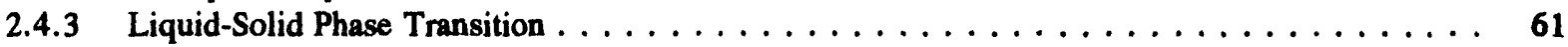

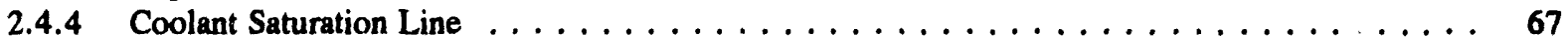

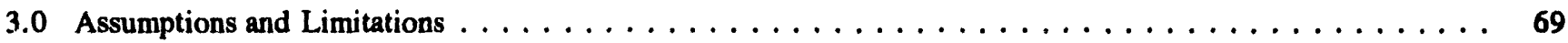

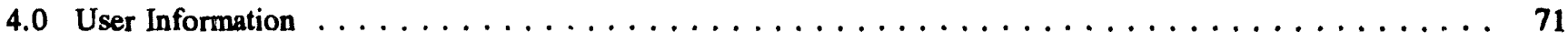

4.1 A Typical Calculational Cycle in CORCON-Mod3 $\ldots \ldots \ldots \ldots \ldots \ldots \ldots \ldots \ldots$

4.2 Description of the Input Parameters $\ldots \ldots \ldots \ldots \ldots \ldots \ldots \ldots \ldots \ldots \ldots \ldots \ldots$

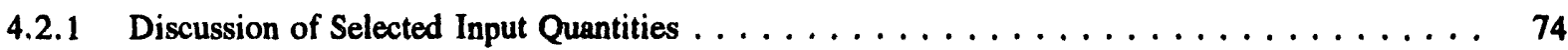

4.2.2 Recornmended Values and Default Values for Input Quantities $\ldots \ldots \ldots \ldots \ldots \ldots \ldots$ 
4.3 Description of the Output $\ldots \ldots \ldots \ldots \ldots \ldots \ldots \ldots \ldots \ldots \ldots \ldots \ldots \ldots \ldots$

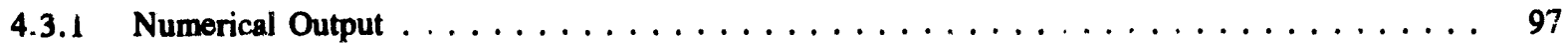

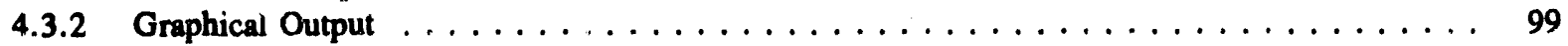

4.4 Description of the Warning and Error Messages $\ldots \ldots \ldots \ldots \ldots \ldots \ldots \ldots \ldots \ldots$

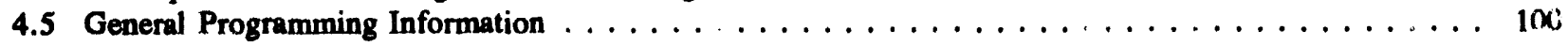

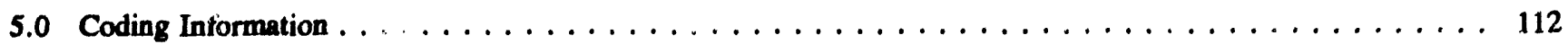

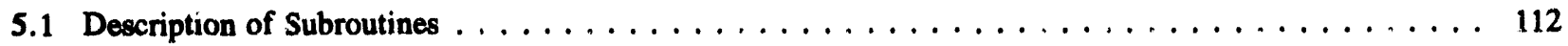

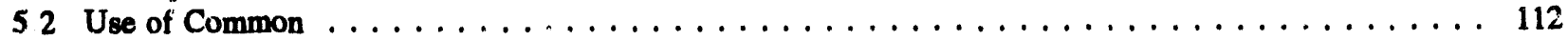

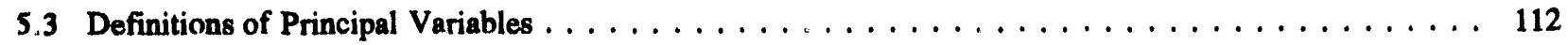

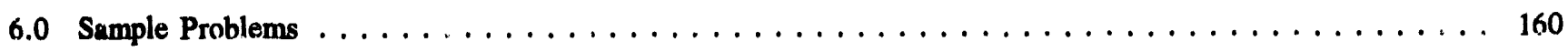

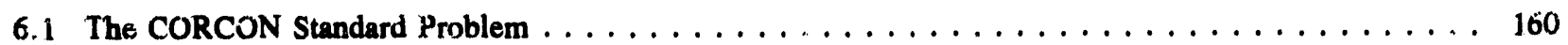

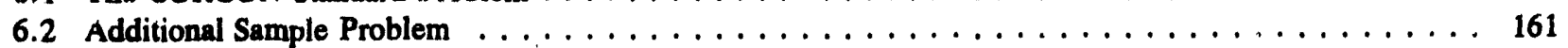

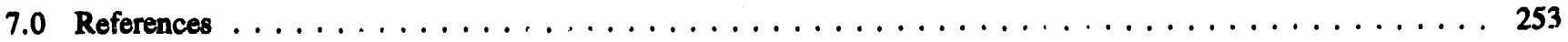




\section{Figures}

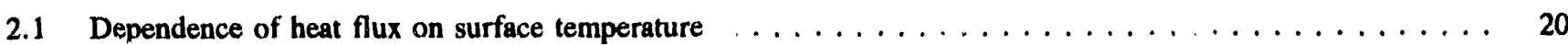

2.2 Approximation to heat flux as a function of interface temperate $\ldots \ldots \ldots \ldots \ldots \ldots \ldots \ldots \ldots$

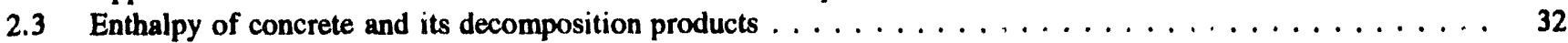

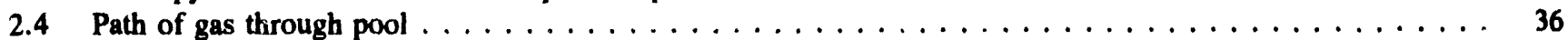

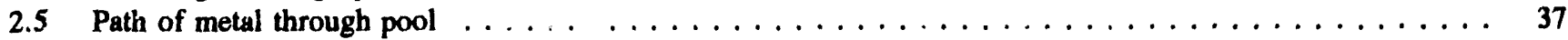

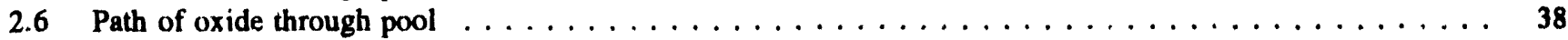

2.7 Normal surface recession $\ldots \ldots \ldots \ldots \ldots \ldots \ldots \ldots \ldots \ldots \ldots \ldots \ldots$

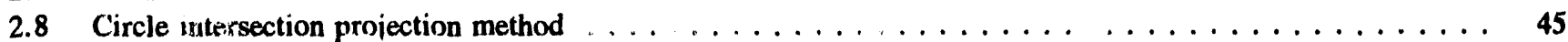

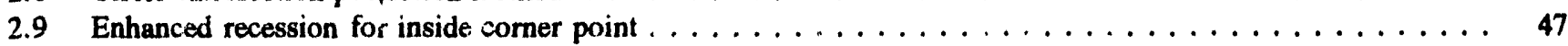

2.10 Sensitivity of the decontamination factor to bubble size (pool depth is $3 \mathrm{~m}$,

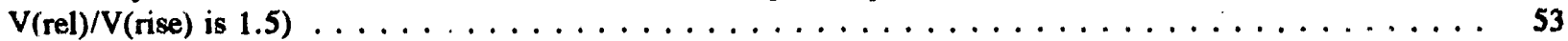

2.11 Sensitivity of the decontamination factor to pool depth (bubble diameter is $1 \mathrm{~cm}$.

$\mathrm{V}($ rel $) / \mathrm{V}$ (rise) is 1.5$) \ldots \ldots \ldots \ldots \ldots \ldots \ldots \ldots \ldots \ldots \ldots \ldots \ldots \ldots$

2.12 Sensitivity of the decontamination factor of the velocity ratio (ponl depth is $2 \mathrm{~m}$,

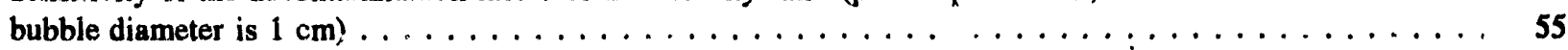

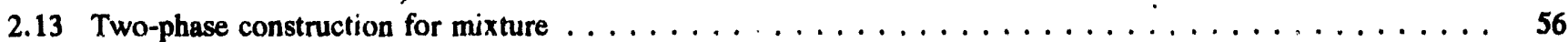

2.14 Laquidus and solidus temperature fits for $\mathrm{Cr}-\mathrm{Fe}-\mathrm{Ni}$ system $\ldots \ldots \ldots \ldots \ldots \ldots \ldots \ldots \ldots \ldots \ldots$

2.15 Example liquidus and solidus temperature for oxidic mixtures $\ldots \ldots \ldots \ldots \ldots \ldots \ldots$

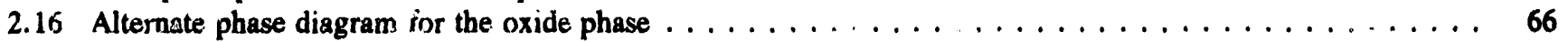

4.1 Flow diagram for CORCON-Mod3 $\ldots \ldots \ldots \ldots \ldots \ldots \ldots \ldots \ldots \ldots \ldots \ldots \ldots \ldots$

4.2 Initial cavity geometry - cylinder with hemispherical buse $\ldots \ldots \ldots \ldots \ldots \ldots \ldots \ldots \ldots$

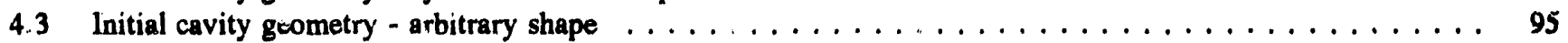

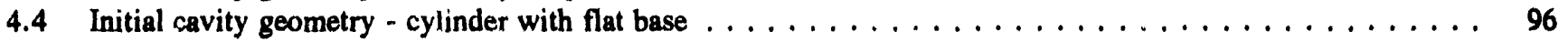

6.1 Axial ablation rate culculated for the CORCON standard problem $\ldots \ldots \ldots \ldots \ldots \ldots \ldots$

6.2 Axial and radial ablation distance calculated for the CORCON standard problem . . . . . . . . . , 165

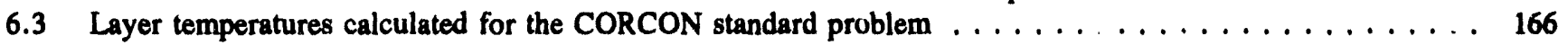

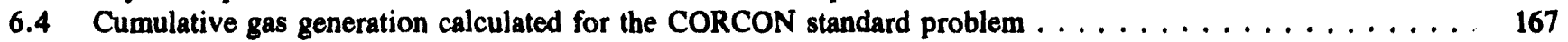

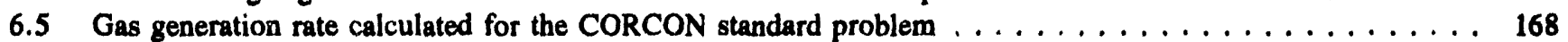

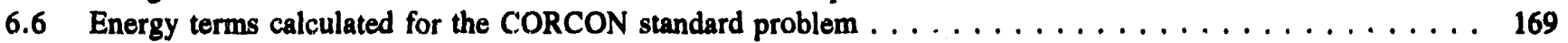

6.7 Aerosol concentration (ambient conditions) calculated for the CORCON standard problem . . . . . . . 170

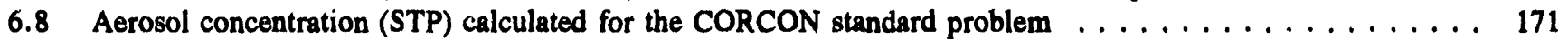

6.9 Aerosol generation rate calculated for the CORCON standard problem $\ldots \ldots \ldots \ldots \ldots \ldots \ldots \ldots \ldots$

6.10 Axial ablation rate calculated for the BWR sample problem $\ldots \ldots \ldots \ldots \ldots \ldots \ldots \ldots \ldots \ldots$

6.11 Axial and radial ablation distances calculated for the BWR sample problem . . . . . . . . . . . . 174

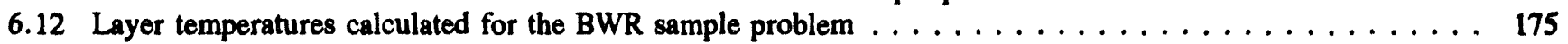

6.13 Cumulative gas generation calculated for the BWR sample problem $\ldots \ldots \ldots \ldots \ldots \ldots \ldots \ldots$

6.14 Gas generation rate calculated for the BWR sample problem $\ldots \ldots \ldots \ldots \ldots \ldots \ldots \ldots \ldots \ldots \ldots$

6.15 Energy terms calculated for the BWR sample problem $\ldots \ldots \ldots \ldots \ldots \ldots \ldots \ldots \ldots \ldots \ldots$

6.16 Aerosol concentration (ambient conditions) calculated for the BWR sample problem . . . . . . . . 179

6.17 Aerosol concentration (STP) calculated for the BWR sample problem $\ldots \ldots \ldots \ldots \ldots \ldots \ldots \ldots$

6.18 Aerosol generation rate calculated for the BWR sample problem $\ldots \ldots \ldots \ldots \ldots \ldots \ldots \ldots \ldots$ 


\section{Tables}

Table

2.1 Chemical species included in CORCON master species list $\ldots \ldots \ldots \ldots \ldots \ldots \ldots \ldots \ldots$

2.2 Chemical species included in the VANESA species list $\ldots \ldots \ldots \ldots \ldots \ldots \ldots \ldots \ldots \ldots$

2.3 Chemical compositions of default concretes (Values in w/o) $\ldots \ldots \ldots \ldots \ldots \ldots \ldots \ldots \ldots \ldots$

2.4 Melting ranges of default concretes $\ldots \ldots \ldots \ldots \ldots \ldots \ldots \ldots \ldots \ldots \ldots \ldots \ldots \ldots$

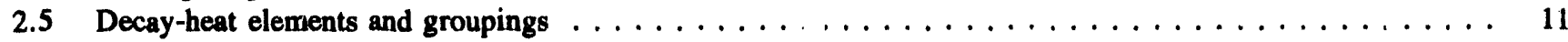

2.6 Chemical species included in the CORCON chemical equilibrium solution $\ldots \ldots \ldots \ldots \ldots \ldots \ldots$

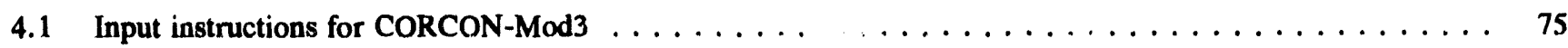

4.2 CORCON-generated warning and error messages for each subroutine $\ldots \ldots \ldots \ldots \ldots \ldots \ldots$

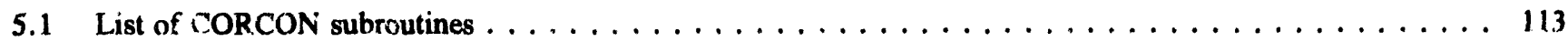

5.2 Subroutines called by each program routine $\ldots \ldots \ldots \ldots \ldots \ldots \ldots \ldots \ldots \ldots \ldots \ldots \ldots \ldots$

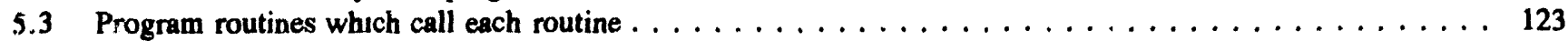

5.4 COMMON blocks contained by each program routine $\ldots \ldots \ldots \ldots \ldots \ldots \ldots \ldots \ldots \ldots \ldots \ldots \ldots$

5.5 Program routines sontaining each COMMON block $\ldots \ldots \ldots \ldots \ldots \ldots \ldots \ldots \ldots \ldots \ldots \ldots$

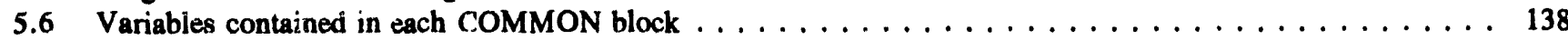

5.7 Dictionary of principai variables in CORCON $\ldots \ldots \ldots \ldots \ldots \ldots \ldots \ldots \ldots \ldots \ldots \ldots \ldots \ldots$

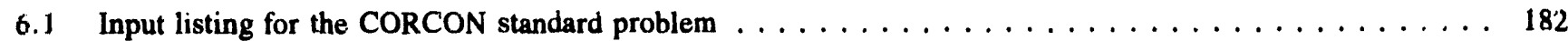

6.2 Output listing for the CORCON standard problem $\ldots \ldots \ldots \ldots \ldots \ldots \ldots \ldots \ldots \ldots \ldots \ldots$

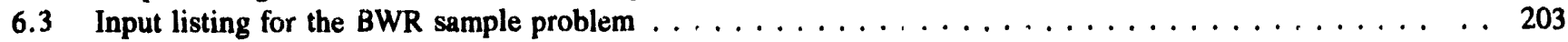

6.4 Output listing for the BWR sample problem $\ldots \ldots \ldots \ldots \ldots \ldots \ldots \ldots \ldots \ldots \ldots \ldots \ldots \ldots \ldots \ldots$. 206 


\section{Nomenclature}

a Laplace constant $\left(=\left[\sigma / g\left(\rho_{1}-\rho_{2}\right)\right]^{1 / 2}\right)$

A Area; initial mass concentration of condensing vapor

[A] Aerosol concentration

$A_{i j} \quad$ Oxide phase interaction parameters

c Speed of sound in the gas

c Specific heat

C Cunningham slip correction factor

$C_{1} \quad$ Heat capacity of layer $i$ for the new mass at the old temperature

$\mathrm{C}_{\text {lvoy }}$ Coefficient used in the boiling heat transfer model

$\mathrm{C}_{\text {of }} \quad$ Coefficient used in the boiling heat transfer model

$C_{d} \quad$ Drag coefficient

d Diameter

$D_{1} \quad$ Diffusion coefficient for species i

E Dimensionless entrainment volume due to bubble bursting

E* Dimensionless droplet entrainment flux

f Fraction of evolved gas entering bubbles

fef $_{i} \quad$ Free energy function for species $i$

g Gravitational acceleration $\left(=9.806 \mathrm{~m} / \mathrm{s}^{2}\right)$

$g_{i} \quad$ Gibbs free energy of species $i$

G Gibbs free energy

$\Delta G \quad$ Free energy of formation

h Convective heat transfer coefficient; specific enthalpy

$h_{f_{g}} \quad$ Enthalpy of vaporization

$h_{i j} \quad$ Metal phase interaction parameter
H Total enthalpy

$\Delta \mathrm{H} \quad$ Change in enthalpy; latent heat of fusion

$\Delta \mathrm{H}_{\mathrm{abl}} \quad$ Heat of ablation

j Superficial velocity (volumetric flux)

$\mathrm{Ja}^{*} \quad$ Dimensionless variable in Equation (64)

k Thermal conductivity; Boltzmann's constant

K Extinction coefficient

$\mathrm{K}_{\text {off,i }}$ Effective rate constant for formation of vapor species i

$\mathrm{K}_{\mathrm{a}, 1} \quad$ Gas phase mass transport coefficient

$\mathrm{K}_{\mathrm{m}, \mathrm{i}} \quad$ Rate constant for condensed phase mass transport

$\mathrm{K}_{\mathrm{v}, \mathrm{l}} \quad$ Vaporization rate constant

$\mathrm{Ku}$ Dimensionless number in the Kutateladze correlation

l Liquid layer thickness

L Characteristic length; layer thickness; radiation path length

m Mass

M Morton number $\left(=g \mu^{4} \Delta \rho / \rho^{2} \sigma^{2}\right)$

$\mathrm{M}_{\mathrm{i}} \quad$ Molecular weight of species i

n Number concentration of aerosol particles

N Number of size segments used to describe the aerosol particle size distribution

$\mathrm{N}_{\mathrm{A}} \quad$ Avogadro's number $\left(6.023 \times 10^{23}\right.$ atoms/mole)

$N_{k} \quad$ Number of moles of species $k$

$\mathrm{N}\left(\mu_{v}\right)$ Dimensionless gas viscosity

$\mathrm{Nu} \quad$ Nusselt number $(=\mathrm{hL} / \mathrm{k})$

p Pressure 


\begin{tabular}{|c|c|c|c|}
\hline $\mathbf{P}$ & Power & $\mathbf{w}_{\mathrm{ij}}$ & Metal phase interaction parameter \\
\hline $\mathbf{P}_{\mathbf{i}}$ & Partial pressure of species $i$ & $\mathbf{W}_{\mathbf{j}}$ & Weight fraction of species $\mathbf{j}$ \\
\hline $\mathbf{P}^{*}$ & $\begin{array}{l}\text { Product of partial pressure times fugacity } \\
\text { coefficient }\end{array}$ & We & Weber number $\left(=\mathrm{U}^{2} \mathrm{~d} \rho_{\ell} / \sigma_{\ell}\right)$ \\
\hline & & $\mathbf{x}$ & Spatial location \\
\hline Pr & Prandtl number $\left(=\mu \mathrm{c}_{\mathrm{p}} / \mathrm{k}\right)$ & $\mathbf{x}_{\mathbf{a}}$ & Position of concrete surface \\
\hline $\mathbf{q}$ & Heat flux & $\mathbf{x}_{\mathbf{i}}$ & Mole fraction of species $i$ \\
\hline $\mathbf{Q}$ & Heat transfer enhancement factor & $\mathbf{X}$ & $\begin{array}{l}\text { Position of solidification and melting fronts; } \\
\text { enthalpy change in temperature units }\end{array}$ \\
\hline $\mathbf{r}$ & Radius; radial coordinate & $\mathbf{z}$ & Axial (vertical) coordinate \\
\hline $\mathbf{R}$ & Radial melt dimension & Greek & \\
\hline $\mathbf{R}_{\mathbf{0}}$ & Universal gas constant & $\alpha$ & $\begin{array}{l}\text { Thermal diffusivity; gas volume fraction; } \\
\text { parameter in melt radius solution }\end{array}$ \\
\hline $\mathbf{R a}$ & Rayleigh number $\left(=g \beta \Delta \mathrm{TL}^{3} / \nu \alpha\right)$ & & \\
\hline $\mathbf{R e}$ & Reynolds number $(=\rho \mathrm{uL} / \mu)$ & $\boldsymbol{\alpha}_{\boldsymbol{d}}$ & Droplet volume traction \\
\hline & & $\alpha_{D}$ & Coefficient for aerosol removal by diffusion \\
\hline & Specinc Entropy or species I & $\alpha_{1}$ & Coefficient for aerosol removal by impaction \\
\hline $\mathbf{S}$ & $\begin{array}{l}\text { Surface recession rate (ablation rate) } \\
\text { Volumetric.heat source }\end{array}$ & $\alpha_{s}$ & $\begin{array}{l}\text { Coefficient for aerosol removal by } \\
\text { sedimentation }\end{array}$ \\
\hline Sh & Sherwood number $\left(=K_{m, l} d / D_{1}\right)$ & $\beta$ & Thermal expansivity \\
\hline $\mathbf{t}$ & Time & $\gamma$ & Temperature difference ratio \\
\hline :t & Timestep & $\boldsymbol{\gamma}_{\mathbf{i}}$ & Activity coefficient for species $\mathrm{i}$ \\
\hline $\mathbf{T}$ & Temperature & $\delta$ & Film thickness \\
\hline$\delta \mathrm{T}$ & Temperature correction & $\epsilon$ & Emissivity \\
\hline$\Delta \mathbf{T}$ & Temperature difference; temperature change & $\eta$ & Dimensionless gas velocity \\
\hline $\mathbf{u}$ & Flow velocity in the film & $\theta$ & Wall inclination \\
\hline U & Velocity of rising bubbles & $\lambda$ & Melting and solidification constants \\
\hline $\mathbf{v}_{\mathbf{d}}$ & Droplet settling velocity & $\mu$ & Dynamic viscosity; mean particle size \\
\hline $\mathbf{v}_{\mathbf{i}}$ & Molar volume of species i & $\mu_{k}$ & Chemical potential of species $k$ \\
\hline V & Volume & $\nu$ & Kinematic viscosity \\
\hline $\mathbf{V}_{\mathbf{t s}}$ & Transition gas velocity & $\boldsymbol{\xi}$ & Normalized excess bubble volume \\
\hline
\end{tabular}




\begin{tabular}{|c|c|c|c|}
\hline$\rho$ & Density & $\mathbf{F}$ & Film \\
\hline$\rho_{\text {molur }}$ & Molar density of the condensed phase & fg & Vaporization \\
\hline $\boldsymbol{\sigma}$ & $\begin{array}{l}\text { Surface tension; geometric standard deviation } \\
\text { of aerosol particle size distribution }\end{array}$ & g & Gas phase \\
\hline \multirow[t]{2}{*}{$\sigma_{\mathbf{B}}$} & Stefan-Boltzmann constant $\left(=5.668 \times 10^{-8}\right.$ & gas & Gas sparged \\
\hline & $\left.\mathbf{W} / \mathbf{m}^{2} \mathbf{K}^{4}\right)$ & $\mathbf{i}$ & ith species or layer \\
\hline$\phi$ & Volume fraction solids in a slurry & in & Incoming \\
\hline$\Phi$ & Flux & I & Interface \\
\hline$\psi_{k}$ & Volume fraction of species $k$ & ? & Liquid phase \\
\hline $\boldsymbol{\omega}$ & Dimensionless bubble volume & leave & Leaving \\
\hline $\mathbf{\Omega}$ & $\begin{array}{l}\text { Implicitness factor (fully explicit = 0; fully } \\
\text { implicit }=1 \text { ) }\end{array}$ & liq & Liquidus \\
\hline \multicolumn{4}{|r|}{ 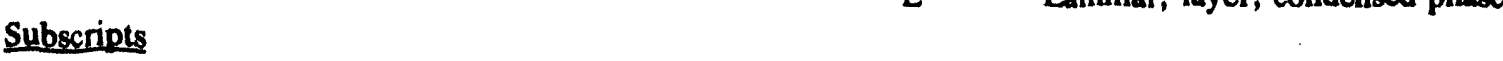 } \\
\hline $\mathbf{a}$ & Based on the Laplace constant; atmosphere & Leid & Leidenfrost (film collapse) point \\
\hline abl & Ablation & $\mathbf{m}$ & Melting; mixture \\
\hline A & Atmosphere & $\max$ & Maximum \\
\hline \multirow[t]{2}{*}{ b } & Bubble & $\mathrm{mp}$ & Melting point \\
\hline & & 0 & Overall \\
\hline B & Bubbling film; bottom & & \\
\hline \multirow[t]{2}{*}{ c } & Concrete; convective & out & Outgoing \\
\hline & & $\mathbf{p}$ & Pool; Aerosol particle \\
\hline $\operatorname{cln}$ & Coolant & pen & Penetration \\
\hline conv & Convective & & \\
\hline \multirow[t]{2}{*}{ crit } & Critical & $\mathbf{r}$ & Radiative; radial \\
\hline & & react & Chemical reactions \\
\hline CHF & Critical Heat Flux & & \\
\hline \multirow[t]{2}{*}{ d } & Droplet & rel & Relative \\
\hline & & rise & Rise \\
\hline e & Elemental; entrainment; equivalent & & \\
\hline \multirow[t]{2}{*}{ eff } & Effective & $\mathbf{R}$ & Radial \\
\hline & Entering & $\mathrm{rad}_{\mathrm{raw}}$ & Net radiation \\
\hline enter & & $\mathbf{s}$ & Surface; solidification \\
\hline eq & Equilibrium & & \\
\hline f & Fluid & sat & Saturation \\
\hline
\end{tabular}


Nomenclature

\begin{tabular}{ll} 
sol & Solidus \\
source & Decay heat source \\
stable & Stable thermal gradient \\
sub & Subcooled \\
sur & Surroundings \\
S & Stokes \\
tot & Total \\
T & Turbulent; top \\
tr & Transition \\
unstable & Unstable thermal gradient \\
w & Ablating concrete surface (wall) \\
z & Axial \\
& \\
\hline
\end{tabular}

1 Initially molten phase; component 1 of binary mixture

2 Substrate; component 2 of binary mixture

\section{Superscripts}

k kth temperature range in thermal equation of state

Liquid; liquidus

m Melting state

nth time level

- Undisturbed or unperturbed state

s Solid; solidus; slag

xs Excess

$\sim \quad$ Projected

A Property for new composition at old temperature 


\subsection{Introduction}

A proper assessment of the risks to the public associated with the operation of nuclear power plants requires a realistic evaluation of the important accident sequences. The Reactor Safety Study' demonstrated that the risks associated with light water reactors (LWRs) are dominated by core meltdown sequences. In these hypothetical accident sequences, loss of normal and emergency cooling systems leads to melting and slumping of the core. If uninterrupted, this is followed by failure of the pressure vessel and deposition of molten core and associated structural materials onto the concrete floor of the reactor cavity.

The interaction of the resulting pool of debris with the concrete has been identified as an important part of the accident sequence. The debris is maintained at elevated temperatures by decay heat from non-volatile fission products retained in the melt. The temperatures and heat fluxes involved are sufficient to decompose and ablate concrete; such attack could fail containment by basemat penetration. This would result in a release of radioactive materials to the soil underneath the reactor building.

Potentially more important are the various mechanisms which can lead to above-ground failure of containment and release of radioactive materials to the atmosphere. The most obvious of these involve the large amounts of water vapor and carbon dioxide which are produced by the decomposition of concrete. These gases can then be reduced to hydrogen and carbon monoxide in chemical reactions with the core debris. (Small quantities of hydrocarbons and other species are also formed.) All four major gases contribute to the risk of eventual overpressurization of containment. Hydrogen and carbon monioxide are also combustible, presenting an additional risk of sudden overpressurization if they are ignited. Also, ablative attack may cause the failure of internal structures in the containment building such as the reactor pedestal in a boiling water reactor (BWR); the resulting mechanical disruption could fail the containment itself. Finally, heat from the debris may be sufficient by itself to cause the failure of containment. This might happen, for example, by degradation of the containment penetration seals in a BWR or by overheating of the drywell liner in a BWR Mark I containment.

The CORCON-Mod3 computer code was developed to mechanistically model the important core-concrete interaction phenomena, including those phenomena relevant to the assessment of coitainment failure and radionuclide release. The code is sufficiently flexible that it can be applied to a wide range of severe accident scenarios, and reactor plants. As such, the code represents the current state of the art for simulating core debris interactions with concrete.

\subsection{Historical Background}

A research program to investigate molten core/concrete interactions was initiated at Sandia National Laboratories in july 1975, under the sponsorship of the Reactor Safety Research Division of the U.S. Nuclear Regulatory Commission. This program was initially experimental, but its scope was soon broadened to include the development of computer models to describe the interactions. A preliminary model, based on limited data and using untested assumptions, was quickly developed by W. B. Murfin in $1977 .^{2}$ This model, INTER1, was intended as a qualitative tool, suitable for sensitivity studies; its use for quantitative prediction was specifically discouraged by its author. ${ }^{2}$

Following the release of INTER1, work was begun on development of an improved computer code, CORCON. This program was intended to provide a more detailed--and more mechanistic--description of the physical processes involved in molten core/concrete interactions. The first version of the code, CORCON-Mod1 ${ }^{3}$ was released in 1981. It lacked models for the freezing of core debris and for interactions between debris and a coolant (water), which limited its applicability to the early stages of accidents involving dry reactor cavities. Later development work has concentrated on removing these limitations. Also, as experience with the code accumulated, several deficiencies in the existing modeling became apparent and improvements were made to correct them.

It was decided that CORCON-Mod1 would be supported as originally issued. That is, errors which prevented the code from performing as intended (as indicated by the users manual) would be corrected, but improved models would not be issued until an entirely new version of the code was completed. In the second version of the code, CORCON-Mod $2,{ }^{4}$ the restrictions mentioned above were removed by the inclusion of freezing and coolant models. Heat transfer and viscosity models were improved, and the code was brought into conformity with the 1977 ANSI standard for FORTRAN programming. ${ }^{5}$ CORCON-Mod2 was released in 1984.

Since its release, CORCON-Mod2 has been used extensively to analyze core-concrete interaction experiments, such as SURC $-4,{ }^{6}$ and to analyze core-concrete interactions in various severe accident scenarios. It is incorporated in the systems-level codes CONTAIN ${ }^{\prime}$ and MELCOR, ${ }^{8}$ which are used to analyze containment response and overall accident progression, respectively. 
This experience with CORCON-Mod2 and the improved knowledge of core-concrete interactions gained since the release of the code, have revealed some deficiencies in CORCON-Mod2. For example, the difficulties encountered by core-concrete interaction codes in simulating the results of International Standard Problem 24 (ISP-24), ${ }^{9}$ which is based on the SURC-4 experiment," revealed the need to include condensed-phase chemical reactions and improved heat transfer models.

In CORCON-Mod3, many of the deficiencies previously identified have been removed, and several significant new features have been added. A summary of model improvements and additions is provided in Section 1.3 of this report.

\subsection{Broad Capabilities of CORCON-Mod3}

CORCON is a general computational model describing the interactions between molten core materials and concrete in LWRs. The molten core debris is assumed to lie in an axisymmetric concrete cavity, with gravity acting parallel to the axis of symmetry. Several standard concretes may be used, or the user may specify a non-standard concrete. Coolant (water) may be present. The user also may specify addition of core material and/or coolant as a function of time.

The model includes heat transfer between the corium and the concrete and between the corium and the atmosphere. If coolant is present, then the heat transfer from the corium to the coolant, and from the coolant to the atmosphere, is modeled.

For heat transfer between the corium and the concrete, the user may select to model the interfacial region as a gas film or a slag film. Heat transfer across the gas film is by combined radiation and convection (the models in the code are identical to those used in CORCON-Mod2). The model for heat transfer across the slag film is based on analysis of transient slag and crust growth at the interface during intermittent contact between the corium and the concrete. Convective heat transfer between the bulk melt and the film (gas or slag) is modeled using heat transfer correlations derived for boiling and gas barbotage.

The heat transfer model for the coolant includes a representation of the full pool boiling curve. The effect of ambient pressure is included in the models for film, nucleate, and transition boiling. The effect of coolant subcooling is included in the nucleate boiling model, while the effects of subcooling and gas barbotage are included in the film boiling models. The transition boiling regime is treated using a log-linear interpolation between the critical heat flux and the Leidenfrost points.

Models have been added to simulate the mixing between corium layers which occurs via droplet entrainment.

Melt stratification via de-entrainment has been added as well. The user may choose to disable the mixing calculation, forcing the corium to remain stratified into distinct oxidic and metallic layers. Here, as in CORCON-Mod2, the layering configuration is determined by the relative densities of the layers.

Both gas-phase and condensed-phase chemical reacticas are modeled. The model assumes chemical equilibrium between the oxides, metals, and gases in each layer containing metals. Chemical reactions between gases and oxides in a purely oxidic layer are not treated. The user may disable the oxide-metal condensed phase reactions if desired.

CORCON-Mod3 models the generation of aerosols and the release of radionuclides using the VANESA model, ${ }^{10}$ which has been fully integrated into the code. The VANESA model, which was developed originally as a stand-alone code, treats aerosol generation by vaporization and by mechanical processes (e.g., bubble bursting). Kinetic limitations to the vaporization process are considered. VANESA also models aerosol removal by an overlying water pool.

CORCON-Mod3 includes a much broader range of user options than were available in CORCON-Mod2. Through input the user can modify many of the more important models and parameters in the code. This capability allows the code to be applied to a broader range of accident conditions. It also allows the user greater flexibility in modeling uncertain phenomena.

The features described above allow CORCON-Mod3 to model a wide range of core-concrete interaction phenomena and allow the code to be used to simulate the effects of core-concrete interactions in a wide range of severe accident scenarios. CORCON-Mod3 is a state-of-the-art computer code for simulating the interaction of molten core debris with concrete in LWRs.

\subsection{Improvements in CORCON-Mod3}

Many improvements have been made to CORCON-Mod2 during the development of CORCON-Mod3. Several of 
the phenomenological models in CORCON-Mod2 were improved, and several new models were added.

The model improvements include:

- the debris-concrete heat transfer models now allow either a stable gas film or an unstable gas film with intermittent melt-concrete contact,

- the coolant heat transfer model now includes the enhancement of film boiling heat transfer by gas barbotage and coolant subcooling, and

- the models for bubble phenomena (bubble size, rise velocity, and void fraction) have been upgraded to reflect our improved understanding of bubble behavior.

The new models include:

- an integrated version of the VANESA model ${ }^{10}$ that includes models for aerosol generation and radionuclide release from the melt, and scrubbing in overlying water pools.
- the models for condensed phase chemical reactions between oxide and metals,

- activity coefficient models for the condensed phases (used in the aerosol generation and radionuclide release calculation),

- an aerosol scrubbing model for subcooled pools, and

- a parametric treatment of core debris spreading across the concrete floor of the reactor cavity.

In addition to these model changes, CORCON-Mod3 provides the user with the capability of modifying a wide range of models and model parameters. This additional flexibility allows the code to be used in a broader range of applications.

The new and improved models are described briefly in Section 2. A more detailed description of the current phenomenological models will be provided in the forthcoming CORCON-Mod3 models and correlations report. 


\subsection{Brief Descriptions of the Phenomenological Models}

\subsection{Overview of CORCON Phenomenology}

CORCON-Mod3 is a mechanistic computer model that describes the core-concrete interaction phenomena relevant to the assessment of containment failure and radionuclide release. In this section, we present a brief description of the principal interaction phenomena modeled in the code.

A great deal may be understood about core/concrete interactions from a very simple picture. The attack of core debris on concrete is largely thermal in a light-water reactor. Energy is generated in the core debris from radionuclide decay and from chemical reactions, and may be lost either through its top surface or to the adjacent concrete. (In many experimental studies, externally supplied induction or joule heating is substituted for the reactor decay heat.) In either case, so long as the heat source is sufficiently large, the situation rapidly approaches a quasi-steady state where the losses from the core debris balance the internal sources. The partition of internally generated heat between concrete and surface is determined by the ratio of the thermal resistances of the corresponding paths. In this simple view, pool behavior is dominated by conservation of energy, with heat-transfer relations providing the most important constitutive relations.

Under most circumstances, the heat flux to the concrete is sufficient to decompose it, releasing water vapor (adsorbed and from hydroxides) and carbon dioxide (from carbonates), and to melt the residual oxides. The surface of the concrete is ablated at a rate which is typically several centimeters per hour. The molten oxides and molten steel from reinforcing bars in the concrete are added to the pool. The gases are strongly oxidizing at pool temperatures and will be reduced, primarily to hydrogen and carbon monoxide, on contact with metals in the pool. Ultimately the reacted and unreacted gases enter the atmosphere above the pool. These gases may or may not burn immediately, depending on their temperature and on the relative concentrations of oxygen, steam, and combustible gases. Combustion of these flammable gases are an important consideration in the assessment of containment failure during a severe reactor accident. Modeling of these above-pool phenomena is not included in CORCONMod3.
Gas released at the bottom of the melt pool rises through it in the form of bubbles. Gas released at the sides of the melt also form bubbles and rise to the surface. At high gas release rates a stable gas film may form at either the bottom or side surfaces. The presence of gas bubbles in the pool increases the volume of the pool, and increases the interfacial area in contact with concrete.

Vaporization of melt constituents into the rising bubbles leads to aerosol generation when these vapors condense in the cooler atmosphere above the melt. Aerosol particles are generated also when the bubbles rupture at the surface of the melt and are entrained by the rising gases. The aerosols that are produced by these processes include both radioactive and non-radioactive species. Radionuclide release during core-concrete interactions can be an important contributor to the radionuclide source term arising from the accident.

Because the concrete thermally decomposes at depths well below the surface, the thermal response of the concrete is complex. The released gases produce internal pressures which drive flows of carbon dioxide, steam, and liquid water through the pores of the concrete. Experiments have shown that at low incident heat fluxes, the concrete may be heated to substantial depths prior to the onset of ablation. At higher heat fluxes, however, penetration of the thermal front will be minimal prior to the onset of ablation. In either case, a steady temperature profile is eventually attained and ablation assumes a pseudo-steady character. Given the high heat fluxes expected uuring core-concrete interactions, we have always assumed steady state ablation in CORCON. This is true also of CORCON-Mod3.

Experimental evidence ${ }^{11 a, 12}$ shows that the various oxidic species in the melt are highly miscible, as are the metallic species, but that the two groups are mutually immiscible. In the absence of gas bubbling, the core debris will stratify into distinct layers based on their relative densities. A stratified layer configuration has been assumed in previous versions of CORCON.

Mixing of the immiscible layers can occur at high gas fluxes or when the densities of the layers are close. ${ }^{13}$ Mixing occurs when droplets of the lower (denser) layer are entrained by bubbles passing through the interface between the layers. Once entrained into the mixture, the droplets will settle out. Therefore, during a core- 
concrete interaction, there may be times in which the molten core debris is mixed and other times in which the debris is stratified. CORCON-Mod3 models both entrainment and droplet settling (deentrainment). The user may disable the mixing calculation and force the core debris to remain fully mixed or fully stratified.

If water is present, it will form an additional layer at the top of the pool. Though explosive interactions have been observed when molten material has been poured through water, ${ }^{14}$ recent experiments have shown that explosive interactions are unlikely when water is poured onto melts composed of prototypic LWR core materials. ${ }^{15,16}$ (The reader should note that there have been experiments in which energetic events have been observed in this configuration. ${ }^{17,18}$ However, these were tests using highly superheated metallic melts under vigorous gas sparging. We do not expect these conditions to exist in LWR accidents.) If the water does not react violently with the molten material underneath it, it will provide an enhanced heat sink. This is likely to cool the top of the melt below the solidification temperature, resulting in a solid crust on the surface. It has been suggested that these crusts will progressively fragment and allow water ingression until the core debris is completely quenched. ${ }^{19}$ This progressive quenching of the melt has not been observed in any experiments to date. CORCON-Mod3 treats heat transfer to the coolant using pool boiling correlations. It does not allow for either steam explosions or the progressive quenching of a molten pool to a coolable debris bed.

An overlying coolant pool will also trap aerosols generated during the core-concrete interaction. Depending on the depth of the pool and its degree of subcooling, there may be a factor of ten or more reduction in the amount of aerosol released into the containment atmosphere. ${ }^{15}$

As time progresses, the debris pool grows; its surface area increases, and decay heating decreases. Therefore, pool temperatures and heat fluxes decrease, and the possibility of refreezing arises. Substantial freezing of the metallic phase may occur. However, the large internal heating and small thermal conductivity of the oxidic phase prevent the existence of steady crusts more than a few centimeters thick. The bulk of this phase will remain liquid, probably for weeks. ${ }^{11 f}$

Coupling between the molten pool and the rest of containment is rather one-sided; the pool serves as a source of mass and energy while being only weakly influenced by conditions in the containment. Containment pressure affects the properties of gases in the pool and of any water over the molten debris.
However, the effects on gas-related heat transfer coefficients, on equilibrium gas compositions, and on the temperature and latent heat of the water are relatively small. Heat loss from the top of the molten debris is dominated by radiation to containment structures or to the overlying water. Because of the fourth-power temperature dependence of the radiative flux, this loss is rather insensitive to containment temperatures (unless they are very high). In the absence of a water layer, the optical properties of the atmosphere may become significant. Molecular absorption by atmospheric gases is a relatively small effect, ${ }^{11 f}$ but aerosol concentrations may be great enough that the atmosphere is optically thick. ${ }^{118}$

Because CORCON-Mod3 is not intended to serve as a full containment code, no attempt is made to model above-pool structures. The surroundings, temperature, and atmospheric pressure are user-specified and may be given as functions of time. The decrease in radiative heat transfer from the pool surface to the surroundings, associated with atmospheric attenuation by aerosols, is approximately accounted for. The calculation is based on diffusion theory for gray, infinite parallel plates, and an aerosol concentration is calculated internally for the purpose of determining the atmospheric extinction coefficient during the interaction.

\subsection{System Components}

The principal components of the CORCON system are the core debris, the concrete, and the atmosphere and surroundings above the debris. The code will also treat an overlying coolant pool if one is present. The composition of each component is specified through user input in terms of a "master list" of chemical species. The list, presented in Table 2.1, is divided into four groups: oxidic compounds, metals and other elements, gases, and miscellaneous compounds. As noted in the table, not all of the species in the master list are available to the user for specification of initial compositions. The aluminates (species 12 to 17) are a hold-over from the viscosity modeling of CORCON-Modl and are not used in CORCON-Mod3. The fission-product pseudo species (oxides 24 to 28, metal 47 and gases 82 to 83 ) are used in the decay-heat generation model. The initial fission product composition is determined within the code from the concentration of fission products in the fuel. The fission product composition is then updated during the calculation to account for addition of core material into the reactor cavity and release in the form of aerosols.

Although we believe that the list of available species is more than ample for describing the physical and chemical processes pertinent to the interaction of molten LWR 
Table 2.1 Chemical species included in the COR ${ }^{\circ}$ ON master species list ${ }^{t}$

\begin{tabular}{|c|c|c|c|c|c|c|c|}
\hline & Oxides & & $\begin{array}{l}\text { tals and } \\
\text { elements }\end{array}$ & & Gases & & Miscellaneous \\
\hline 1 & $\mathrm{SiO}_{2}$ & 43 & $\mathrm{Fe}$ & 63 & $C(g)$ & 100 & $\mathrm{H}_{2} \mathrm{O}$ evap \\
\hline 2 & $\mathrm{TiO}_{2}$ & 44 & $\mathrm{Cr}$ & 64 & $\mathrm{CH}_{4}$ & 101 & $\mathrm{H}_{2} \mathrm{O}$ chem \\
\hline 3 & $\mathrm{FeO}^{2}$ & 45 & $\mathrm{Ni}$ & 65 & $\mathrm{CO}$ & 102 & $\mathrm{CaCO}_{3}$ \\
\hline 4 & $\mathrm{MnO}$ & 46 & $\mathrm{Zr}_{\mathbf{r}}$ & 66 & $\mathrm{CO}_{2}$ & 103 & $\mathrm{Ca}(\mathrm{OH})_{2}$ \\
\hline 5 & $\mathrm{MgO}$ & 47 & "FpM & 67 & $\mathrm{C}_{2} \mathrm{H}_{2}$ & 104 & $\mathrm{H}_{2} \mathrm{O} \mathrm{cln}$ \\
\hline 6 & $\mathrm{CaO}$ & 48 & $\mathrm{Mn}$ & 68 & $\mathrm{C}_{2} \mathrm{H}_{4}$ & $105-109$ & Blank \\
\hline 7 & Sro & 49 & $C(c)$ & 69 & $\mathrm{C}_{2} \mathrm{H}_{6}$ & & \\
\hline 8 & $\mathrm{BaO}$ & 50 & $\mathrm{Al}$ & 70 & $\mathrm{H}^{*}$ & & \\
\hline 9 & $\mathrm{Li}_{2} \mathrm{O}$ & 51 & $\mathbf{U}$ & 71 & $\mathrm{H}_{2}$ & & \\
\hline 10 & $\mathrm{Na}_{2} \mathrm{O}$ & 52 & $\mathrm{Si}$ & 72 & $\mathrm{H}_{2} \mathrm{O}$ & & \\
\hline 11 & $\mathrm{~K}_{2} \mathrm{O}$ & 53 & $\mathrm{UAl}_{3}$ & 73 & $\mathbf{N}$ & & \\
\hline 12 & ${\mathrm{KA} 1 \mathrm{O}_{2}}_{2}$ & 54 & $\mathrm{UAl}_{2}$ & 74 & $\mathrm{NH}_{3}$ & & \\
\hline 13 & $\mathrm{NaAlO}_{2}$ & 55 & $\mathrm{Na}$ & 75 & $\mathrm{~N}_{2}$ & & \\
\hline 14 & $\mathrm{BaAl}_{2} \mathrm{O}_{4}$ & 56 & $\mathrm{Ca}$ & 76 & 0 & & \\
\hline 15 & $\mathrm{CaAl}_{2} \mathrm{O}_{4}$ & 57 & $-\mathrm{x}$ & 77 & $\mathrm{O}_{2}$ & & \\
\hline 16 & $\mathrm{MgAl}_{2} \mathrm{O}_{4}$ & $58-62$ & Blank & 78 & $\mathrm{OH}$ & & \\
\hline 17 & $\mathrm{MnAl}_{2} \mathrm{O}_{4}$ & & & 79 & $\mathrm{CHO}$ & & \\
\hline 18 & $\mathrm{Fe}_{2} \mathrm{O}_{3}$ & & & 80 & $\mathrm{CH}_{2} \mathrm{O}$ & & \\
\hline 19 & $\mathrm{Al}_{2} \mathrm{O}$ & & & 81 & $\mathrm{CrO}_{3}(\mathrm{~g})$ & & \\
\hline 20 & $\mathrm{UO}_{2}$ & & & 82 & ${ }^{*} \mathrm{ppMO}_{2}(\mathrm{~g})$ & & \\
\hline 21 & $\mathrm{ZrO}_{2}$ & & & 83 & ${ }^{-} \mathrm{FpMO}_{3}(\mathrm{~g})$ & & \\
\hline 22 & $\mathrm{Cr}_{2} \mathrm{O}_{3}$ & & & 84 & $\mathrm{Al}_{2} \mathrm{O}_{2}(\mathrm{~g})$ & & \\
\hline 23 & $\mathrm{NiO}$ & & & 85 & $\mathrm{Al}_{2} \mathrm{O}(\mathrm{g})$ & & \\
\hline 24 & $" \mathrm{FpMO}_{2}(\mathrm{c})$ & & & 86 & $\mathrm{AlO}(\mathrm{g})$ & & \\
\hline 25 & $\mathrm{FpMO}_{3}$ & & & 87 & OAlH $(\mathrm{g})$ & & \\
\hline 26 & FpOx & & & 88 & $\mathrm{AlOH}(\mathrm{g})$ & & \\
\hline 27 & FpAlkMet(c) & & & 89 & $\mathrm{OAIOH}(\mathrm{g})$ & & \\
\hline 28 & FpHalogn(c) & & & 90 & $\mathrm{AlO}_{2}(\mathrm{~g})$ & & \\
\hline 29 & $\mathrm{Fe}_{2} \mathrm{O}$ & & & $91-99$ & Blank & & \\
\hline 30 & $\mathrm{Mn}_{3} \mathrm{O}_{4}$ & & & & & & \\
\hline 31 & $\mathrm{PuO}_{2}$ & & & & & & \\
\hline 32 & $\mathrm{UO}_{3}$ & & & & & & \\
\hline 33 & $\mathrm{U}_{3} \mathrm{O}_{8}$ & & & & & & \\
\hline $34-42$ & Blank & & & & & & \\
\hline \multicolumn{8}{|c|}{ Pseudo species representing four condensed phase fission product groups. } \\
\hline \multicolumn{8}{|c|}{ "Inert oxidic apecies treated as an element " $\mathrm{X}$ " in chemical equilibrium calculation. } \\
\hline Alumin & species 12-17, not & $\mathrm{d}$ in $\mathrm{COR}$ & -Mod3. & & & & \\
\hline
\end{tabular}


core materials (and coolant) with concrete, blank spaces have been left in the list for future additions. Such additions to the list might be required, for example, for treatment of the sodium chemistry associated with core/concrete interactions in Liquid Metal Fast Breeder Reactor (LMFBR) accidents.

The VANESA model was developed using a somewhat simpler description of the melt, and considers only the major condensed phase species. VANESA focussed primarily on gas phase chemistry and it therefore includes a much more extensive species list for the gas phase. The VANESA species list is shown in Table 2.2.

\subsubsection{Debris Pool}

CORCON models the debris pool as consisting of a number of layers contained in a concrete cavity. These layers are, from bottom up, a heavy (i.e., dense) oxide phase (HOX), a heterogeneous mixture of heavy oxides and metals (HMX), a metallic phase (MET), a heterogeneous mixture of metals and light oxides (LMX), a light oxide phase (LOX), a coolant (CLN), and the atmosphere (ATM). The three-letter mnemonics are useful in describing the pool structure, and have been used in appropriate variable names throughout the code. Layer volumes, including the swelling effects of gas bubbles, determine the elevations of layer interfaces and of the pool surface.

These seven layers are always present in the data structure but may be "empty" in the sense of containing no material. Note that the coolant (CLN) is treated in the same manner as the debris-containing layers.

Many different layer configurations are possible now that CORCON-Mod3 allows for the existence of mixture layers. Mixing, stratification, and density changes resulting from material addition can lead to changes in the layer orientation during a calculation.

If an overlying coolant layer is present, it will form a layer overlying the core debris. The user may specify the presence of a coolant layer at the start of the calculation or may specify addition of coolant at some later time. Currently, water is the only acceptable coolant.

\subsubsection{Concrete Cavity}

The concrete cavity containing the core debris is assumed to be axisymmetric. Several simple geometries are available to describe its initial shape. The user may specify a cylinder with either a flat base or a hemispherical base. A general (axisymmetric) initial shape may also be defined by specifying the initial position of each body point. The shape of the cavity is represented and tracked by the position of a number of points, termed "body points," on its surface.

The composition of the concrete must also be specified, and may be chosen as one of the three built-in default concretes. These are referred to as "basaltic aggregate concrete," "limestone aggregate common sand concrete," and "limestone aggregate concrete." The compositions of these concretes are given in Table 2.3; they are based on measured compositions reported by Powers. ${ }^{1 / b}$ The solidus and liquidus temperatures for these concretes, also included in internal data, are given in Table 2.4; the user must specify an ablation temperature somewhere between these limits.

Alternatively, provision is made for the user to define a "non-standard" concrete. In this case, the composition and melting range of the concrete must be user-specified, in addition to the ablation temperature. The concrete composition may be specified either in terms of $\mathrm{CaCO}_{3}$ and $\mathrm{Ca}(\mathrm{OH})_{2}$, or in terms of their decomposition products, $\mathrm{CaO}, \mathrm{CO}_{2}$, and $\mathrm{H}_{2} \mathrm{OCHEM}$ (chemically bound water). The latter form is used internally, with the code performing the conversion if necessary.

Steel reinforcing bar in the concrete is also permitted; if present, it is assumed to be pure iron (Fe). Alternatively, CORCON-Mod3 allows the user to specify the composition of the rebar to be something other than iron. This option was included to enable the user to simulate experiments with zirconium rods embedded in the concrete. ${ }^{20}$ Concrete, with or without reinforcing rods, is treated as a homogeneous material.

\subsubsection{Atmosphere and Surroundings}

The atmosphere above the pool and its surroundings serve as sinks for mass (evolved gases) and energy (convection and radiation from the pool surface). CORCON-Mod3 contains very simple models for these components. The atmosphere is described by a specified (constant) temperature and (time-dependent) pressure. The surroundings are described by a specified (time-dependent) temperature and (time- or temperature-dependent) emissivity. Within the code, the pool surface (bottom of the atmosphere) is treated as a major computational interface. Very limited information, restricted to mass flows and (linearized) heat transfer relations, is passed across the interface. Thus, because the interface is well-defined and actively used in the stand-alone code, a more detailed above-pool model--or a full containment response code--could be easily 


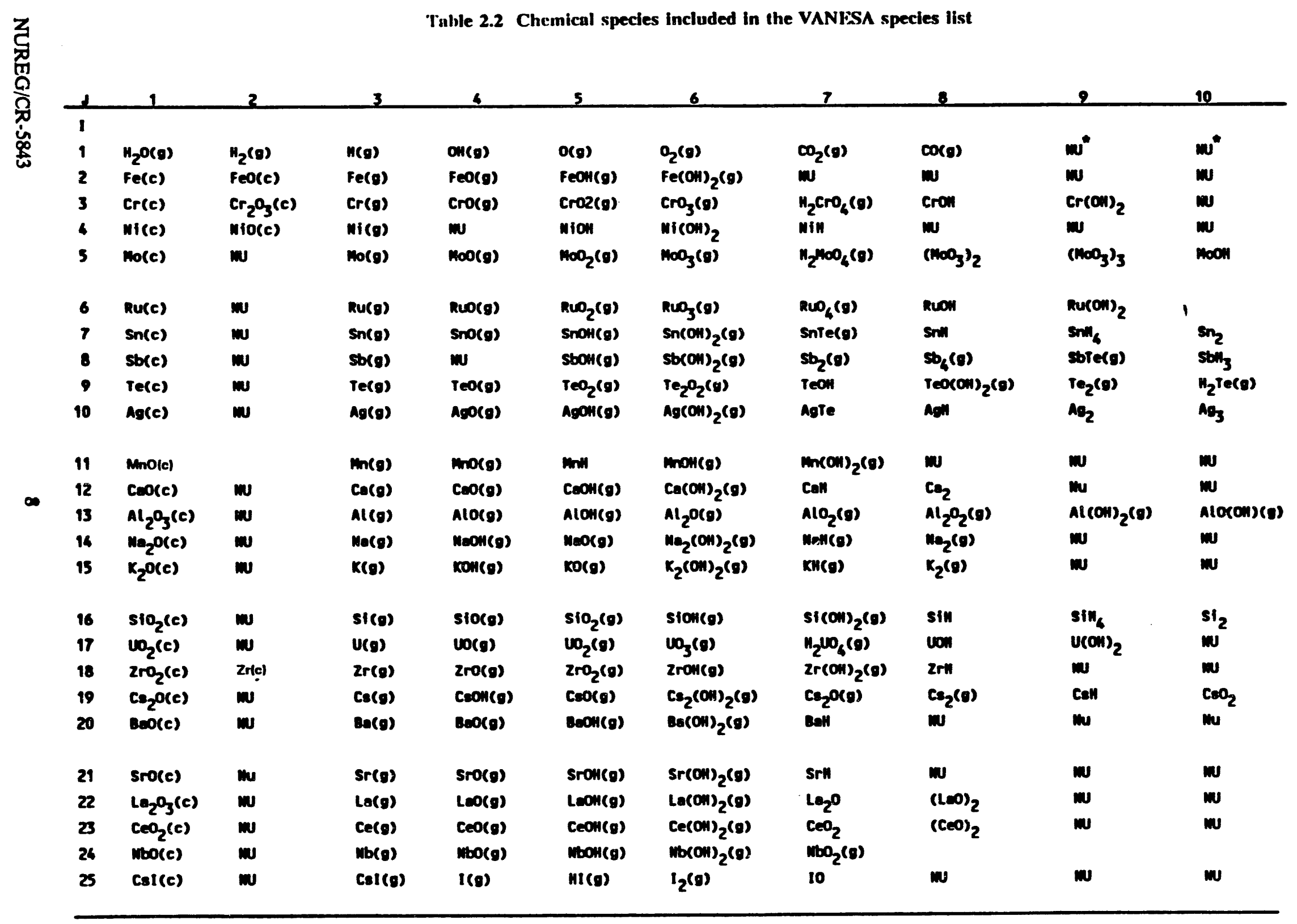


Table 2.3 Chemical compositions of default concretes (Values in $w / 0$ )

\begin{tabular}{|c|c|c|c|c|}
\hline $\begin{array}{l}\text { specie } \\
\text { s }\end{array}$ & $\begin{array}{l}\text { speci } \\
\text { es } \\
\text { no. }\end{array}$ & $\begin{array}{l}\text { Basalti } \\
\text { c } \\
\text { aggrega } \\
\text { te } \\
\text { concret } \\
\text { e }\end{array}$ & $\begin{array}{l}\text { Limestone } \\
\text { aggregate } \\
- \\
\text { common } \\
\text { sand } \\
\text { concrete }\end{array}$ & $\begin{array}{l}\text { Limest } \\
\text { one } \\
\text { aggreg } \\
\text { ate } \\
\text { concre } \\
\text { te }\end{array}$ \\
\hline $\mathrm{SiO}_{2}$ & 1 & 54.84 & 35.80 & 3.60 \\
\hline $\mathrm{TiO}_{2}$ & 2 & 1.05 & 0.18 & 0.12 \\
\hline Mno & 4 & 0.00 & 0.03 & 0.01 \\
\hline Mgo & 5 & 6.16 & 0.48 & 5.67 \\
\hline $\mathrm{CaO}$ & 6 & 8.82 & 31.30 & 45.40 \\
\hline $\mathrm{Na}_{2} \mathrm{O}$ & 10 & 1.80 & 0.082 & 0.078 \\
\hline $\mathrm{K}_{2} \mathrm{O}$ & 11 & 5.39 & 1.22 & 0.68 \\
\hline $\mathrm{Fe}_{2} \mathrm{O}_{3}$ & 18 & 6.26 & 1.44 & 1.20 \\
\hline $\mathrm{Al}_{2} \mathrm{O}_{3}$ & 19 & 8.32 & 3.60 & 1.60 \\
\hline $\mathrm{Cr}_{2} \mathrm{O}_{3}$ & 22 & 0.00 & 0.014 & 0.004 \\
\hline $\mathrm{CO}_{2}$ & 66 & 1.50 & 21.154 & 35.698 \\
\hline $\begin{array}{l}\mathrm{H}_{2} \mathrm{O} \\
\text { evap }\end{array}$ & 100 & 3.86 & 2.70 & 3.94 \\
\hline $\begin{array}{l}\mathrm{H}_{2} \mathrm{O} \\
\text { chem }\end{array}$ & 101 & 2.00 & 2.00 & 2.00 \\
\hline
\end{tabular}

Table 2.4 Melting ranges of default concretes

\begin{tabular}{lcc}
\hline & \multicolumn{2}{c}{ Temperature (K) } \\
Concrete & solidus & $\begin{array}{c}\text { Liquidu } \\
\mathbf{8}\end{array}$ \\
\hline $\begin{array}{l}\text { Basaltic } \\
\text { Aggregate }\end{array}$ & 1350 & 1650 \\
$\begin{array}{l}\text { Limestone } \\
\text { Aggregate- } \\
\text { Common Sand }\end{array}$ & 1420 & 1670 \\
$\begin{array}{l}\text { Limestone } \\
\text { Aggregate }\end{array}$ & 1690 & 1875 \\
\hline
\end{tabular}


substituted. This is typically what is done when integrating CORCON into integral accident analysis codes such as CONTAIN ${ }^{7}$ and MELCOR. ${ }^{8}$

Heat transfer from the pool surface is by convection and radiation with the latter mode ordinarily dominant. Convective heat transfer is calculated using the temperature of the atmosphere while radiative heat transfer uses the temperature of surrounding surfaces. If desired, CORCON-Mod3 computes atmospheric aerosol concentration for the purpose of estimating the optical thickness of the cavity atmosphere above the pool.

\subsection{Physical Processes}

A number of physical processes are included in the modeling of molten-fuel/concrete interactions; these include internal energy generation, mass and heat transfer, chemical reactions, concrete response, and bubble phenomena. The models are described in the following subsections. The level of detail is intended to be sufficient for understanding of the basic concepts and the code implementation; for details, the reader should consult the references.

In several cases, one or more phenomena are tightly coupled and must be considered simultaneously. For example, concrete response determines gas generation, which affects heat transfer and the heat flux to concrete, which in turn feeds back to determine concrete response. We will note such coupled interactions in the discussion which follows.

\subsubsection{Energy Generation}

The entire fuel/concrete interaction process is driven by decay heat generated in the pool, including actinides, decay products and irradiated structural materials. Because of the loss of some of the more volatile fission products before the pool is formed (i.e., during the in-vessel core melt progression), use of the ANS Standard decay curve is not appropriate. While decay heating could be calculated using detailed decay chains, as described in the Users' Manual for CONTAIN, ${ }^{7}$ the isotopic information is not needed for fission-product tracking in CORCON-Mod3. Therefore, we have included a much simpler decay heat model in the code.
Bennett has shown that the decay power for typical reactor cores in the 1-hour to 10-day time-frame is nearly proportional to operating power and relatively insensitive to burnup. Therefore, a SANDIA-ORIGEN ${ }^{21}$ calculation was performed for a reference core representative of a large PWR core at equilibrium burnup (3320 MWt and $33000 \mathrm{MWD} / \mathrm{MTU}$ ). From the results of this calculation, we identified 27 elements (excluding noble gases) which account for essentially all the heat production in the reference core in the time-frame of interest. We assume that the initial intact-core inventory is proportional to core operating power, as specified by the user. The initial pool inventory of fission products is then determined by the fraction of the core contained in the melt, as given by the mass of $\mathrm{UO}_{2}$ and the user-specified core size, multiplied by a "retention fraction" for each element. (Note that as a result of this scheme, $\mathrm{UO}_{2}$ must be present for decay heat to be represented.) These retention fractions account for partial loss of the more volatile species during the invessel melt progression phase. The elements, their assumed chemical forms ${ }^{b}$ and concentrations in the core, and the default retention fraction for each species' are given in Table 2.5. The retention fractions may be overridden by the user if desired.

The radionuclide release model in the code requires the initial fission product composition of the core debris to be specified. The user has two options for specifying the fission product composition. The default is to use the fission product composition determined for the decay power calculation. As another option, the user may specify the fission product composition. This option is useful when performing experiment simulations since the melt has a given composition of fission product simulants.

The decay power is calculated using the further assumption that the specific decay power (W/g-atom) associated with each element is a simple function of time after SCRAM. The values of the decay power associated with each element (also taken from the reference SANDIA-ORIGEN calculation) are tabulated at eight time points: $0.5,1.0,2.0,4.0,10.0,24.0,72.0$, and 240 hours. A power-law interpolation (i.e., log-power is assumed to be linear in log-time) is then used to determine the decay power at intermediate times. If needed, the decay power may be extrapolated to earlier or later times.

\footnotetext{
"Bennett, D. E., "Power Level-Burnup Parametric Study," memorandum to M. Berman, Sandia National Laboratories, Albuquerque, NM, September 10, 1979.

bowers, D. A., private communication, 1980.
} 
Table 2.5 Decay-heat elements and groupings

\begin{tabular}{|c|c|c|c|}
\hline $\begin{array}{l}\text { Pseudo-ele } \\
\text { ment }\end{array}$ & Element & $\begin{array}{c}\text { Mass } \\
\text { concentra } \\
\text { tion } \\
{[g \text {-atom/M }} \\
\text { (thermal) } \\
1\end{array}$ & $\begin{array}{l}\text { Retent } \\
\text { Ion } \\
\text { fracti } \\
\text { on }\end{array}$ \\
\hline \multicolumn{4}{|l|}{ FpM } \\
\hline \multirow[t]{6}{*}{ Metals } & Mo & .6053 & .97 \\
\hline & TC & .1545 & .97 \\
\hline & $\mathrm{Ru}$ & .3885 & .97 \\
\hline & $\mathbf{R h}$ & .0690 & .97 \\
\hline & $\mathbf{S b}$ & .00244 & .85 \\
\hline & $\mathrm{Te}$ & .0627 & .85 \\
\hline \multicolumn{4}{|l|}{ Fpox } \\
\hline \multirow[t]{2}{*}{ Monoxides } & sr & .2155 & .90 \\
\hline & $\mathrm{Ba}$ & .1915 & .90 \\
\hline \multirow[t]{7}{*}{ Dioxides } & $\mathrm{Zr}$ & .7352 & .99 \\
\hline & $\mathrm{Ce}$ & .3870 & .99 \\
\hline & $\mathrm{Np}$ & .0422 & .99 \\
\hline & $\mathrm{cm}$ & .00204 & .99 \\
\hline & $\mathrm{Nb}$ & .01139 & .99 \\
\hline & $\mathrm{Pu}$ & .7921 & .99 \\
\hline & Am & .00593 & .99 \\
\hline \multirow{6}{*}{ deşquoioxi } & $Y$ & .1099 & .99 \\
\hline & La & .1662 & .99 \\
\hline & Pr & .1446 & .99 \\
\hline & $\mathrm{Nd}$ & .4638 & .99 \\
\hline & $\mathrm{Sm}$ & .0539 & .99 \\
\hline & $\mathrm{Eu}$ & .01705 & .99 \\
\hline \multicolumn{4}{|l|}{ FpAlkMet } \\
\hline \multirow[t]{2}{*}{ Me elatgli } & $\mathrm{Rb}$ & .0819 & .19 \\
\hline & Cs & .3776 & .19 \\
\hline FpHalogn & $\mathrm{Br}$ & .00530 & .10 \\
\hline Halogens & $I$ & .0320 & .10 \\
\hline $\mathrm{UO}_{2}$ & U & insert & \\
\hline $\mathrm{Zr}, \mathrm{ZrO}_{2}$ & $\begin{array}{l}\text { Zr } \\
\text { (struct } \\
\text { ural) }\end{array}$ & itsput & \\
\hline
\end{tabular}


There may be further losses from the melt during the interaction due to further vaporization and to mechanical sparging and aerosol generation driven by concrete decomposition gases. The user has two options for determining release from the melt: a complete radionuclide release and aerosol generation calculation using the VANESA model, or the simple release model employed in CORCON-Mod2 and retained in CORCONMod3. The detailed treatment in the VANESA model is described in Section 2.3.14 and will not be discussed here. The simple CORCON-Mod2 model is discussed below.

CORCON-Mod2 considered only vaporization of alkali metals and halogens. It is extremely unlikely that either group will be present in elemental form because the alkali metals boil (at one atmosphere) slightly below 1000 $\mathrm{K}$ and the halogens boil below $500 \mathrm{~K}$. The halogens will probably be present as alkali halides (e.g., Csl) with boiling points of 1500 to $1600 \mathrm{~K}$. Under ordinary conditions, the melt will contain more alkali metal than halogen; the excess will most probably occur as hydroxides with boiling points comparable to the halides. However, the retention fractions may be specified such that the amount of halogen present exceeds the amount of alkali metal. If this occurs, the model in CORCON-Mod3 will eliminate the excess halogen during initialization; an appropriate message is written to the output file. The remaining halogens and alkali metals are then removed exponentially in time with an arbitrary but reasonable half-life of 10 minutes. Except in the decay power model, the fission products and actinides are grouped as four pseudo elements, as follows:

FpM $\quad \begin{aligned} & \text { - metals which may oxidize, and whose } \\ & \text { oxides may volatilize }\end{aligned}$
FpOx - chemically inert oxides
FpAlkMet - alkali metals
FpHalogn - halogens.

Thus, the composition of the melt is represented in terms of these four pseudo elements which are resolved into actual elements only for the calculation of decay power.

\subsubsection{Melt/Concrete Heat Transfer}

Heat transfer between molten core debris and reactor cavity concrete is controlled by the bubbling of concrete decomposition gases through the melt. This process is similar to nucleate boiling or gas barbotage except that at the interface between the core debris and the concrete, gas is being released coincident with melting of the concrete surface. The molten concrete is miscible in molten oxidic core debris, but is immiscible in metallic core debris. At bubble departure, drops of concrete slag are displaced from the surface of the concrete by the buoyancy of the concrete slag in the denser molten core material and the suction caused by the low pressure region in the wake of the rising bubbles.

Coincident with gas bubbling and concrete melting at the interface, the molten core debris may begin to solidify as a crust adjacent to the melting concrete surface. This crust may be stable or unstable depending on its growth rate, its strength properties, and the disruptive forces acting to destabilize it. If the crust is unstable, it will fragment and be carried away by the rising bubbles. If the crust is stable, it will continue to grow at a rate determined by the local energy balance. Eventually, it may provide a barrier to the flow of gases and concrete slag. If this occurs, the flow of gas and slag will then be parallel to the concrete surface.

At extremely high gas generation rates, it may be possible to form a stable gas film at the melt-concrete interface. This is the description assumed in previous versions of CORCON. When a stable film is present, heat transfer across the film is by combined radiation and convection.

In order to adequately represent melt/concrete heat transfer in the cases described above, models are required for bubble-driven heat transfer, slag layer growth and removal, crust growth and stability, and heat transfer across a stable film. Models for bubble-driven convection heat transfer are described in Section 2.3.2.1. The models for interfacial heat transfer phenomena are discussed in Section 2.3.2.2.

\subsubsection{Bulk Pool Heat Transfer}

Heat is removed at the boundaries of the pool, which are its top surface and its interface with concrete.

As discussed in Section 2, the internal temperature of the pool adjusts quickly so that these heat losses balance the internal heat generation, and the heat transfer approaches a steady state. Therefore, we use quasi-steady models for heat transfer in CORCON-Mod3. The principal advantage of quasi-steady models is that heat fluxes at any time depend on the current state of the pool and not on its history. For example, fully developed flow is assumed in convective correlations and the temporal development of boundary layers is not considered.

In CORCON-Mod3 we employ a multi-layered pool model (Section 2.2.1) for which it is convenient to consider heat transfer one layer at a time. Given a trial 
set of interfacial temperatures, a solution is found (independently) for each layer. Newton's iteration is then used to revise the interfacial temperatures to satisfy the requirement that the heat flux must be continuous at all interfaces between layers. The solutions for the individual layers are repeated at each step. The heat-transfer model allows for several possible configurations in each layer: the layer may be completely molten, it may have a solid crust on one or more surfaces, or it may be completely solid. In this section, we will address heat transfer in a liquid layer or the liquid portion of a partially-solidified layer. The modifications necessary to account for crusting or freezing will be described in the next section.

Heat transfer coefficients are required from the interior of a liquid layer to its surfaces. If the layer were a right circular cylinder, there would be three such coefficients: to the upper, lower, and radial surfaces. In CORCON-Mod3, these three heat transfer coefficients are evaluated for a cylinder whose thickness and volume match those of the layer. Boundaries with other layers are assumed to be horizontal, and the corresponding heat transfer coefficients employed directly. For the boundary with concrete, an appropriate combination is constructed, as described in Section 2.3.2.2, to account for the actual inclination of the surface.

The analysis includes the effects of the passage of concrete decomposition gases through the liquid. Models are included for gas injection at the bottom surface of the melt, and gas agitation along the sides of the melt. The bulk pool heat transfer model in CORCON-Mod3 has evolved from those used in CORCON-Mod1, and CORCON-Mod2. The model in CORCON-Mod1 was based on the correlation recommended by Blottner ${ }^{22}$ which was a modification of a correlation by Konsetov. ${ }^{23}$ CORCON-Mod2 retained this model for the radial (vertical) surface, but utilized the empirical correlation developed by Ginsberg and Green $e^{24}$ for axial (horizontal) interfaces.

Early comparisons between CORCON-Mod2 predictions and the results from experiments demonstrated very clearly that the heat transfer models in the code were not accurate. Because of this, Bradley 25 initiated a reassessment of the heat transfer models in the code. His recommendations formed the basis for selection of the models implemented into CORCON-Mod3.

For the bottom interface of the melt pool, where gas bubbles may be injected from the incoming concrete, the heat transfer coefficient for a liquid layer is calculated using the correlation devised by Kutateladze. The Kutateladze $^{26}$ correlation is given by:

$$
\mathrm{Nu}=1.5 \times 10^{-3} \mathrm{Ku}^{23} \mathrm{f}(\eta)
$$

where $\mathrm{Nu}_{\mathrm{a}}$ is the Nusselt number based on a characteristic length equal to the Laplace constant a,

$$
a=\left\{\sigma_{\ell} /\left[g\left(\rho_{\ell}-\rho_{g}\right)\right]\right\}^{1 / 2},
$$

$\mathrm{Ku}$ is a dimensionless number defined as

$$
\mathrm{Ku}=\frac{\operatorname{Pr} \mathrm{p} \mathrm{j}_{\mathrm{g}}}{\mathrm{g} \mu}
$$

$\eta$ is a dimensionless gas velocity defined by

$$
\eta=\mathrm{j}_{\mathrm{z}} / \mathrm{V}_{\mathrm{t}}
$$

and $f(\eta)$ is given by

$$
f(\eta)=\left\{\begin{array}{l}
1, \text { if } \eta \leq 1 \\
\eta^{-1 / 2} \text { if } \eta>1
\end{array} .\right.
$$

In these equations, $j_{k}$ is the superficial velocity for the gas entering the melt, $V_{t s}$ is a transition velocity correlated by

$$
V_{t r}=4.0 \times 10^{-4} \sigma_{l} / \mu_{l}
$$

$\sigma_{t}$ is the surface tension of the liquid, $\rho_{\ell}$ is the density of the liquid, $\rho_{B}$ is the density of the gas, $p$ is the pressure at the interface, and Pr is the Prandtl number for the liquid. For many fluids, the transition velocity calculated using the above equation is comparable to the velocity for transition from bubbly to churn-turbulent flow. The heat transfer correlations have been formulated such that alternate equations for the transition velocity can be easily substituted into the model.

In CORCON-Mod3, a smooth transition for $\mathrm{Nu}_{\mathrm{a}}$ through the value $\eta=1$ is effected by using

$$
N u_{a}=\frac{N u_{a 1} / \eta+N u_{a 2} \eta}{1 / \eta+\eta}
$$

where $\mathrm{Nu}_{\mathrm{a} 1}$ and $\mathrm{Nu}_{\mathrm{a} 2}$ are defined as follows: 


$$
\begin{aligned}
& N u_{a_{1}}=1.5 \times 10^{-3} \mathrm{Ku}^{2 / 3} \\
& N u_{a^{2}}=1.5 \times 10^{-3} \mathrm{Ku}^{23} \eta^{-1 / 2}
\end{aligned}
$$

Results from experiments with gas agitation of heated surfaces suggest that heat transfer along vertical walls is less sensitive to the superficial gas velocity than is indicated by the Kutateladze correlation. ${ }^{27, c}$ The experiments show also that at high gas velocities convective heat transfer at horizontal and vertical surfaces is nearly the same. These results suggest that a modified form of the Kutateladze correlation should be used for calculating the heat transfer coefficient to the side of a liquid layer. We have chosen, therefore, to represent convective heat transfer along the vertical sidewalls using only the churn-turbulent (i.e., $\eta>1$ ) form of the Kutateladze correlation. This form of the correlation depends on the superficial gas velocity raised to the one-sixth power. This dependence is in the range of literature values which indicate a 0.14 to 0.33 dependence on gas velocity. ${ }^{2,27}$

For liquid layers within the melt pool, a correlation devised by Greene ${ }^{28}$ is used to calculate the heat transfer coefficient in each layer, except for the uppermost melt layer. Greene's correlation is

$$
\mathrm{h}=1.95 \mathrm{k}(\operatorname{Re} \operatorname{Pr})^{0.72 / \mathrm{r}_{\mathrm{b}}}
$$

where $k$ is the thermal conductivity, $R e$ is the Reynolds number for the liquid based on the characteristic length $r_{b}$ and the superficial gas velocity $j_{k}, \operatorname{Pr}$ is the Prandtl number for the liquid, and $r_{b}$ is the average bubble radius in the layer.

For the uppermost melt layer (adjacent to the atmosphere or coolant), the heat transfer coefficient is calculated using a modified form of the Kutateladze correlation (Equation (1)), which accounts for the greater surface area of the unstable surface. For the upper melt surface, the Kutateladze correlation is simply multiplied by an area enhancement derived by Farmer: ${ }^{29}$

$$
A^{*}=1+4.5 \frac{j_{g}}{U_{b}}
$$

At sufficiently low gas velocities, heat transfer in molten debris is dominated by natural convection. This process is modeled in CORCON-Mod3 by conventional Nusselt-Rayleigh correlations in the form: ${ }^{30}$

$$
\mathrm{Nu}=\max \left(0.54 \mathrm{Ra}^{1 / 4}, 0.14 \mathrm{Ra}^{1 / 3}\right),
$$

for axial heat transfer in an unstable thermal gradient, and

$$
\mathrm{Nu}=\max \left(0.59 \mathrm{Ra}^{1 / 4}, 0.10 \mathrm{Ra}^{1 / 3}\right)
$$

for radial heat transfer. Here

$$
\mathrm{Nu}=\mathrm{h} \ell / \mathrm{k}
$$

is the Nusselt number, based on the layer thickness, $\ell$, and

$$
\mathbf{R a}=\mathbf{g} \beta \Delta \mathrm{T} \ell^{3} / \nu \alpha
$$

is the Rayleigh number. $\Delta \mathrm{T}$ is the temperature difference, fluid to boundary. In Equations (11) and (12), the first expression in parentheses corresponds to laminar (low Rayleigh number) convection and the second to turbulent (high Rayleigh number) convection.

The heat transfer coefficient at a surface where the temperature gradient is stable is calculated directly from Equation (12). If the temperature gradient is stable at one interface but unstable at the other, convective flows driven by the unstable gradient steepen the stable gradient. To account for the resulting increase in heat transfer, the heat transfer coefficient for the stable gradient is then calculated from

$$
N u_{\text {stable }}=1+\left(1+2 N u_{\text {unstable }} \frac{\left|\Delta \mathrm{T}_{\text {umatuble }}\right|}{\left|\Delta \mathrm{T}_{\text {otuble }}\right|}\right)^{1 / 2}
$$

Kulacki and co-workers ${ }^{31.33}$ have developed correlations for convective heat transfer in internally heated fluid layers. The model in CORCON-Mod3 reproduces the various correlations with a maximum error of 30 percent and an average error closer to 10 percent. $1 \mathrm{~d}$

The natural convection limit is imposed in CORCON-Mod3 by choosing the greater of the Nusselt numbers calculated for bubble-enhanced and for natural convection. The actual implementation assumes that

'Greene, G. A., private communication, March, 1991. 
Equation (15) may be applied even when $\mathrm{Nu}_{\text {monuble }}$ is evaluated for bubble-enhanced convection.

For very thin or very viscous layers, the natural convection correlations above can yield smaller heat fluxes than would result from simple conduction. Therefore, an approximate conduction limit is imposed in CORCON-Mod3, in the form of a lower limit in the Nusselt number. The formulation is based on the average temperature of the liquid layer, $T$, which is consistent with CORCON usage and is normal practice for convective heat transfer. For convection, there is an (usually unstated) assumption that the boundary layers are thin and that the local temperature is essentially equal to the average temperature. The assumption fails at or near the conduction limit, where the temperature profile is quadratic (for a uniform volumetric source). This is the reason that we have chosen an "approximate" limit rather than an "exact" one. A full discussion is contained in Reference $11 \mathrm{e}$.

In the radial direction, the exact conduction result for a quadratic temperature profile is

$$
N u_{R}+\frac{q_{R} \ell}{k\left(T_{\ell}-T_{R}\right)}=\frac{4 \ell}{R}
$$

where $\mathrm{Nu}_{\mathrm{R}}$ is the Nusselt number based on layer thickness, and $\mathbf{R}$ is the average radius of the layer. This provides the desired lower bound on radial heat transfer.

The exact axial conduction result is

$$
\begin{aligned}
& q_{T}=2 k\left[T_{\ell}-T_{T}+\left(-T_{B}+2 T_{\ell}-T_{T}\right)\right] / \ell \\
& q_{B}=-2 k\left[T_{\ell}-T_{B}+\left(-T_{B}+2 T_{\ell}-T_{T}\right)\right] / \ell
\end{aligned}
$$

where the fluxes are positive up, and subscripts "B" and " $T$ " refer to the bottom and top surfaces, respectively. Note that $q_{B}$ in Equation (17) does not necessarily have the same sign as $\left(T_{B}-T_{\ell}\right)$ and similarly for $q_{T}$ and $\left(T_{\ell}-\right.$ $T_{T}$ ) in Equation (18), which would greatly complicate an attempt to impose the "exact" limit. In order to avoid this, we have employed the approximations

$$
\begin{aligned}
\mathrm{q}_{\mathrm{T}} \approx & 2 \mathrm{k}\left\{\mathrm{T}_{\mathrm{l}}-\mathrm{T}_{\mathrm{T}}\right. \\
& \left.+2\left[\max \left(\left(\mathrm{T}_{\ell}-\mathrm{T}_{\mathrm{T}}\right)\left(\mathrm{T}_{\ell}-\mathrm{T}_{\mathrm{B}}\right), 0\right)\right]^{1 / 2}\right\} / \ell
\end{aligned}
$$

and

$$
\begin{aligned}
\mathrm{q}_{\mathrm{B}} \approx & -2 \mathrm{k}\left\{\mathrm{T}_{\ell}-\mathrm{T}_{\mathrm{B}}\right. \\
& \left.+2\left[\max \left(\left(\mathrm{T}_{\ell}-\mathrm{T}_{\mathrm{T}}\right)\left(\mathrm{T}_{\ell}-\mathrm{T}_{\mathrm{B}}\right), 0\right)\right]^{1 / 2}\right\} / \ell
\end{aligned}
$$

which have the desired properties, and the same values as Equations (17) and (18) for the limiting cases of no internal heating $\left(T=\left(T_{B}+T_{T}\right) / 2\right)$ and of large internal heating $\left(T_{B}-T_{l}=T_{T}-T_{\ell}\right)$. In fact, as discussed in Reference $11 \mathrm{e}$, the approximation errs only in the temperature at which steady state is achieved for given boundary temperatures and volumetric heating. This error is unavoidable if we wish the heat fluxes to have the same sign as the temperature differences. In any case, if the conduction limit in a liquid is reached, the layer must be relatively thin, and the maximum error in the average temperature, one third of the temperature difference across the layer, ${ }^{110}$ must be relatively small. It represents only a minor error in the sensible heat content of the layer and has no other consequences. Equations (19) and (20), rewritten in the form of Nusselt numbers based on layer thickness, are employed in CORCONMod3 as lower bounds in the Nusselt numbers in a liquid layer.

\subsubsection{Interfacial Heat Transfer}

At the time CORCON-Mod2 was released, it was assumed that gas release from the decoinposing concrete was sufficient to form a stable gas film between the concrete and the debris pool. There $w$ as no convincing experimental evidence to support or disprove this assumption. Consequently, CORCON-Mod2 employed a model for melt/concrete heat transfer that assumed the boundary region was dominated by a stable gas film and that on horizontal and nearly horizontal surfaces the Taylor instability leads to formation of bubbles which enter the melt, while on more steeply inclined surfaces the gas forms a flowing film.

Since that time, the assumption of an initially stable gas film has been shown to be incorrect under most realistic accident conditions. ${ }^{25}$ Instead, gas release is usually far less than that required to form a stable gas film, and intermittent debris-concrete contact occurs. This intermittent contact results in periodic growth and removal of slag from the interface, and, depending on the temperature of the molten debris, may also lead to periodic growth and removal of debris crusts. Simplified models for these processes have been developed and implemented in CORCON-Mod3. The stable gas film model implemented in CORCON-Mod2 has been retained, and the user can select either the slag or gas film model at the start of a calculation. No transition from one model to the other is included. 


\section{Model Descriptions}

The following is a brief discussion of the slag film and gas film models. For further discussion of the slag film model the reader is referred to Reference 25 .

\section{Slag Film Model}

Simultaneous concrete melting and molten core debris solidification can be modeled using an analysis similar to that used by Epstein ${ }^{34}$ to model freezing of molten reactor fuel on the surface of stainless steel cladding. Epstein's model considers four regions: the initially molten phase, the solid substrate, the solidified molten phase crust, and the layer of melting substrate. The energy equation in each of these regions has the form:

$$
\frac{\partial^{2} T_{i}}{\partial x^{2}}=\frac{1}{\alpha_{i}} \frac{\partial T_{i}}{\partial t}
$$

where $T_{i}$ is the temperature in region $i, \alpha_{i}$ is the thermal diffusivity of region $i, x$ is the spatial location, and $t$ is time. (Note that internal heating of the molten phase due to decay heat has been neglected. It can be easily shown that during a bubble cycle, internal energy generation can be neglected.)

The energy equation is solved subject to the following boundary conditions: continuity of temperature at each interface, continuity of heat flux (including effects of phase change) at each interface, and the temperature of the melt and substrate at "infinity" is fixed at the initial temperature of the region.

Given these boundary conditions, the temperature of each of the four regions has the general form:

$$
T_{i}=A_{i}+B_{i} \operatorname{erf}\left(\frac{x}{2\left(\alpha_{i}\right)^{1 / 2}}\right)
$$

with the positions of the solidification and melting fronts given by equations of the following form:

$$
X_{i}(t)=2 \lambda_{i}\left(\alpha_{i} t\right)^{1 / 2}
$$

In these equations, $A_{i}, B_{i}$, and $\lambda_{i}$ are constants determined by applying the boundary conditions.

Application of the boundary conditions results in a set of algebraic equations for the constants. By algebraic manipulation, this set of equations can be reduced to two simultaneous transcendental equations for $\lambda_{1}$ and $\lambda_{2}$. After $\lambda_{1}$ and $\lambda_{2}$ have been determined, the temperature at the interface between the core debris and concrete can be determined from the following equation:

$$
\frac{T_{1}-T_{2 m p}}{T_{\text {Imp }}-T_{2 \operatorname{mp}}}=\frac{\operatorname{erf}\left(\lambda_{2}\right)}{\operatorname{erf}\left(\lambda_{2}\right)-\sigma \operatorname{erf}\left(\lambda_{1}\right)}
$$

where $T_{i}$ is the interface temperature, $T_{1 m p}$ and $T_{2 m p}$ are the melting points of the initially molten phase and solid substrate, and $\sigma$ is the following function of material properties:

$$
\sigma=\frac{k_{2 m}}{k_{1 s}}\left(\frac{\alpha_{1 s}}{\alpha_{2 m}}\right)^{1 / 2}
$$

where $\mathrm{k}$ and $\alpha$ are the thermal conductivity and thermal diffusivity of the respective regions, and the subscripts $1 \mathrm{~s}$ and $2 \mathrm{~m}$ refer to the solidifying molten phase and melting substrate, respectively.

Under some conditions, solidification of the molten phase does not occur. The equation for the interface temperature is then derived following the same procedure but with three regions rather than four. The resulting equation for the interface temperature is

$$
\frac{T_{10}-T_{i}}{T_{10}-T_{2 m p}}=\frac{\sigma}{\sigma+\operatorname{erf}\left(\lambda_{2}\right)}
$$

where $T_{10}$ is the initial temperature of the molten phase, and $\sigma$ is now based on the thermal conductivity and thermal diffusivity of the molten phase rather than the crust.

The preceding analysis is implemented in CORCON-Mod3 by treating the transient slag layer growth and removal process as a heat transfer resistance in series with convective heat transfer within the molten pool. At the interface between the slag layer and the molten pool

$$
h_{p}\left(T_{p}-T_{i}\right)=h_{s}\left(T_{i}-T_{d}\right)
$$

where $h$ is the convective heat transfer coefficient, and the subscripts $p, i, c$, and $s$ refer to the debris pool, the interface, the concrete surface, and slag layer. The pool heat transfer coefficient is determined using the equations discussed in the preceding section. The concrete surface temperature is simply the user-specified ablation temperature. The interface temperature is determined using the analysis described above. Solving this equation for the slag heat transfer coefficient yields 


$$
\begin{aligned}
h_{1} & =h_{p} \frac{T_{p}-T_{1}}{T_{i}-T_{c}} \\
& =h_{p} \gamma
\end{aligned}
$$

The overall heat transfer coefficient (between the molten debris and the concrete surface) is given by

$$
h_{0}=\frac{h_{1} h_{p}}{h_{c}+h_{p}}
$$

Substituting for $h_{\mathrm{f}}$ into this equation results in

$$
h_{0}=h_{p}\left(\frac{\gamma}{\gamma+1}\right)
$$

Bradley25 found that the value of $\gamma /(\gamma+1)$ is relatively insensitive to the material properties of the core debris. Whether the core debris is metallic or oxidic, $\gamma /(\gamma+1)$ was found to fall within a narrow range of $0.29 \pm 0.07$. In light of the uncertainty in the heat transfer models, we decided to implement the model assuming a constant value of $\gamma$. The value of $\gamma$ corresponding to a value of $\gamma /(\gamma+1)$ equal to 0.29 is 0.41 . Hence, the slag heat transfer coefficient is assumed to be given by

$$
h_{\text {s }}=0.41 h_{p}
$$

Currently, the code provides a lower limit to the slag heat transfer coefficient of $1000 \mathrm{~W} / \mathrm{m}^{2} \mathrm{~K}$. This value simulates conduction-limited heat transfer across a thin (1 $\mathrm{mm}$ ) slag layer. Calculated results should be relatively insensitive to this value since it is likely to be invoked only when a debris crust exists at the interface with the concrete. Therefore, heat transfer would be controlled by conduction through the crust, and the contribution from the slag thermal resistance would be small.

\section{Stable Gas Film Model}

For a stable gas film, two models for heat transfer are used, one for nearly horizontal films in which there is essentially no gas flow parallel to the concrete surface, and one for inclined films in which there is gas flow parallel to the concrete surface. A film is classified as "nearly horizontal" if its inclination is less than 15 degrees.

For a stable gas film on a nearly horizontal surface, heat transfer is computed from a mechanistic model based on momentum balance in a Taylor-instability bubbling cell. ${ }^{35}$
The result may be cast in the form of a Nusselt number based on film thickness as

$$
\mathrm{Nu}_{\mathrm{B}}=\frac{\mathrm{h}_{\mathrm{B}} \delta_{\mathrm{B}}}{\mathrm{k}_{\mathrm{s}}}=0.804
$$

where $h_{B}$ is the heat-transfer coefficient, $\delta_{B}$ is the film thickness, and $k$ is the thermal conductivity of the gas. The factor $\mathbf{0 . 8 0 4}$ is the fraction of the surface not occupied by bubble sites and, therefore, available for heat transfer. The film thickness satisfies

$$
\delta_{B}^{3}=15.05 \operatorname{Re}_{B} L^{3}
$$

Here $\mathrm{L}$ is a material property

$$
L=\left(\frac{\mu_{8}^{2}}{g \rho_{2}\left(\rho_{l}-\rho_{8}\right)}\right)^{1 / 3}
$$

where $\mathbf{g}$ is the acceleration of gravity, subscripts $\ell$ and $g$ refer to pool material and film properties, respectively, and

$$
\operatorname{Re}_{B}=\frac{\rho_{8} j_{8} a}{\mu_{3}}
$$

is the Reynolds number based on the Laplace constant (defined in Equation (2)), and the superficial velocity $j_{k}$ with which gas enters the film. Note that if the process modeled were really film boiling, the equations would be closed through the relations

$$
q=h_{B} \Delta T=\rho_{8} j_{g} h_{f_{8}}^{*}
$$

where $h_{f_{g}}^{*}$ is the effective heat of vaporization. Relatively simple manipulation can then be used to reduce the present model to a form similar to Berenson' $\mathrm{s}^{36}$ correlation for boiling on a flat plate,

$$
h=0.68\left[\frac{k_{g}^{3} h_{f_{k}}^{*} g_{\ell}\left(\rho_{\ell}-\rho_{k}\right)}{\mu_{k} \Delta T(2 \pi a)}\right]^{1 / 4} \text {. }
$$

This bubble model is used for inclinations less than 15 degrees in CORCON-Mod3. Above 30 degrees we use a flowing-film model, and consider both laminar and turbulent films. The transition model used between 15 degrees and 30 degrees will be described later. The film models are mechanistic, based on momentum balances in 
an inclined flowing film, with the Reynolds analogy used for heat transfer in the turbulent case. The results, expressed as Nusselt numbers, are

$$
N u_{L F}=h_{L F} \delta_{L F} / k_{2}=1
$$

for the laminar case and

$$
N u_{T F}=h_{T F} \delta_{T F} / k_{z}=0.0325 \operatorname{Pr}^{1 / 3} \operatorname{Re}_{F}^{3 / 4}
$$

for the turbulent case. In these equations, $\operatorname{Pr}$ is the Prandtl number for the film, and $\mathrm{Re}_{\mathrm{F}}$ is the Reynolds number based on film thickness:

$$
\operatorname{Re}_{\mathrm{F}}=\rho_{\mathrm{B}} \overline{\mathrm{u}} \delta / \mu_{\mathrm{z}}
$$

with a the average flow velocity in the film. The film thicknesses satisfy

$$
\begin{gathered}
\delta_{L F}^{3}=5.61 \operatorname{Re}_{F} L^{3} / \sin \theta \\
\delta_{T F}^{3}=0.0469 \operatorname{Re}_{F}^{7 / 4} L^{3} / \sin \theta
\end{gathered}
$$

where $\theta$ is the inclination of the film from the horizontal.

In CORCON-Mod3, we employ a simple transition between the laminar and turbulent flow regimes which will ensure continuity of film thickness and heat-transfer coefficient with the appropriate limits:

$$
\begin{gathered}
\delta_{F}=\max \left(\delta_{L F}, \delta_{T F}\right), \\
N u_{F}=\max \left(N u_{L F}, N u_{T F}\right), \\
h_{F}=N u_{F} k_{b} / \delta_{F},
\end{gathered}
$$

where the various film thicknesses and Nusselt numbers have been defined above.

A transition between the bubbling model and the film-flow model is also required. We have incorporated a mechanistic model ${ }^{1 / 8}$ based on a momentum balance with a fraction, $f$, of injected gas going into bubbles and the rest into establishing the film. The results have the form

$$
\begin{aligned}
& \delta^{3}=\delta_{F}^{3}+f\left(\sin 15^{\circ} / \sin \theta\right) \delta_{B}^{3} \\
& h=\left[f N u_{B}+(1-f) N u_{F}\right] k_{8} / \delta .
\end{aligned}
$$

A transition is achieved by decreasing $f$ linearly with sin $\theta$ from 1 at 15 degrees to 0 at 30 degrees.

Because of the high temperatures involved, heat transfer by radiation across the gas film must also be included. The radiative component accounts for about one half of the total heat flux in many CORCON calculations. We use the form for a transparent gas between infinite parallel gray walls

$$
q_{n d-}=\frac{\sigma_{B}\left(T_{A}^{4}-T_{w}^{4}\right)}{\left(1 / \varepsilon_{p}+1 / \varepsilon_{w}-1\right)} .
$$

Here $\sigma_{B}$ is the Stefan-Boltzmann constant, $T_{A}$ and $T_{w}$ are the temperatures of the pool side of the gas film and of the ablating concrete surface, respectively, and $\epsilon_{\mathrm{p}}$ and $\epsilon_{\mathrm{w}}$ are the corresponding emissivities.

The convective heat-transfer coefficients above involve the superficial velocity of gas entering the interface region and, in film flow regions, the film-wise mass flow. The latter is determined by the entering gas flow at all upstream points. The superficial gas velocity is determined by the concrete response, which is determined by the total heat flux. This, in turn, involves the heat-transfer coefficients themselves. A self-consistent solution has been found advisable for numerical stability; this is obtained through a simple iteration.

Note that $\rho_{g} u \delta$ is the mass flow per unit width of film, which satisfies

$$
\frac{d}{d s}\left(r \rho_{z} \bar{u} \delta\right)=r(1-f) \rho_{z} j_{z}
$$

where $r$ is the local radius of the cavity, $s$ is the path length measured along the film, and $f$ is the interpolation factor which imposes the transition from bubbling to film flow.

We solve this equation by use of a simple predictor-corrector method. An inner iteration, as described above, is required to solve the non-linear (because of radiation) energy balance at each point. In cases where the spacing between body points is too great to resolve the development of the gas film, 
intermediate points are considered by the integration routine.

\section{Numerical Solution Technique}

Heat transfer through the (slag or gas) film is formulated in terms of the temperatures of its surfaces. On the melt pool side, this temperature, $T_{A}$, is determined implicitly by the requirement that the heat flux be continuous. The subroutine SURFEB is used to perform a surface energy balance at the interface between the melt and the film and to evaluate its temperature at each spatial point. The nonlinear equation

$$
q_{p}=q_{n d}+h_{1}\left(T_{A}-T_{w}\right)
$$

is solved for $T_{A}$ using Newton's iteration. (Note that the radiation term disappears if the slag film model is selected by the user. This greatly simplifies the solution for $T_{A}$.) A complete solution, including the full evaluation of pool-side heat transfer within the iteration loop, would be extremely expensive in computer time because the energy balance is typically performed at hundreds of points along the pool/concrete interface.

The crust formation model in CORCON-Mod3 was developed originally for CORCON-Mod2. This model introduces a discontinuity in behavior when $T_{A}$ passes through the solidification temperature, $T_{\text {. }}$. The qualitative dependence of $q_{\phi}$ on $T_{A}$ is shown in Figure 2.1. When $T_{A}$ is slightly greater than $T_{\text {, }}$ no crust exists and the derivative $d q_{p} / d T_{A}$ is given by a convective heat-transfer correlation. When $T_{A}$ is slightly less than $T_{\text {, a }}$ a crust must be present. When a crust is present, the derivative is small because a small change in surface temperature merely changes the (steady) crust thickness with very little change in the heat flux. The discontinuity in slope must be accounted for to prevent unphysical results or failure of the iteration which determines $T_{\Lambda}$.

As in CORCON-Mod2, CORCON-Mod3 uses a piecewise linear approximation to $q_{p}$ as a function of $T_{A}$. There are two cases, as shown in Figure 2.2, depending on whether or not a crust was present $\left(T_{A}\right.$ was less than $T_{.}$) when within-pool heat transfer was last evaluated. If so, $q_{\phi}$ is evaluated as

$$
q_{p}=q_{p}^{\text {old }}+\frac{d q_{p}}{d T_{A}}\left(T_{A}-T_{A}^{\text {old }}\right) \text {, }
$$

for $T_{A} \leq T_{*}$. For $T_{A}>T_{,}, q_{p}$ is extrapolated linearly to zero at $T_{A}=T_{M}$, where $T_{M}$ is currently taken as the temperature of the liquid center of the layer. This isillustrated in Figure 2.2A. If, on the other hand, no crust was previously present ( $T_{A}$ was greater than $\left.T_{N}\right)$, Equation (51) is used to evaluate of for $T_{A}>T_{\text {. }}$. For $T_{A}<T_{\text {, }}$, the heat flux $q_{0}$ is assumed to be constant as shown in Figure 2.2B.

\subsubsection{Coolant Heat Transfer}

If a coolant layer is present, CORCON-Mod3 calculates boiling heat transfer. The boiling heat transfer model in the code includes the full boiling curve, based on standard pool boiling correlations as summarized by Bergles. ${ }^{37}$ Corrections are made for the effects of gas injection at the melt/coolant interface and coolant subcooling. The various correlations involved are not used directly in CORCON-Mod3. This is possible because the boiling heat transfer coefficient for a given fluid, water in this case, is a function of pressure and temperature only, with all of the detailed dependence of material properties on temperature and pressure contained in the one function. A series of calculations was performed outside the code, using thermal and transport properties from the Steam Tables, ${ }^{38}$ to generate tables of values. These were then fit by simple analytic forms which reproduce the tables within 3 percent over the pressure range of $10 \mathrm{kPa}$ to $10 \mathrm{MPa}$ (saturation temperatures from $320 \mathrm{~K}$ to $580 \mathrm{~K}$ ). The principal advantage of using these fits is that extensive libraries of water properties need not be included in the code.

Nucleate boiling is treated by using the Rohsenow ${ }^{39}$ correlation for the temperature rise and the Zuber ${ }^{0,41}$ correlation (with Rohsenow's coefficient) ${ }^{12}$ for the critical heat flux to calculate the values of $q_{c h F}$ and of $T_{w}-T_{m a}$ at the point of critical heat flux (where $T_{w}$, as used here, is the temperature of the debris surface). These are represented as

$$
\begin{gathered}
q_{C H F}=\frac{1.50 \times 10^{6}\left(10^{-5} \mathrm{p}\right)^{0.415}}{1.0+5.97 \times 10^{-3}\left(10^{-5} \mathrm{p}\right)^{1.117}} \\
\left(T_{w}-T_{\text {mat }}\right)_{C H F}=\frac{1.71 \times 10^{3} \mathrm{C}_{\mathrm{dF}}\left(10^{-5} \mathrm{p}\right)^{-0.112}}{1.0+7.02 \times 10^{-8}\left(10^{-5} \mathrm{p}\right)^{3.181}},
\end{gathered}
$$

where all values are in S.I. units (i.e., $q$ is in $W / \mathrm{m}^{2}, p$ is in $\mathrm{Pa}$, and $\mathrm{T}$ is in $\mathrm{K}$ ) and the surface coefficient, $\mathrm{C}_{a b}$, is taken as 0.01 . The nucleate-boiling portion of the boiling curve is then represented as 


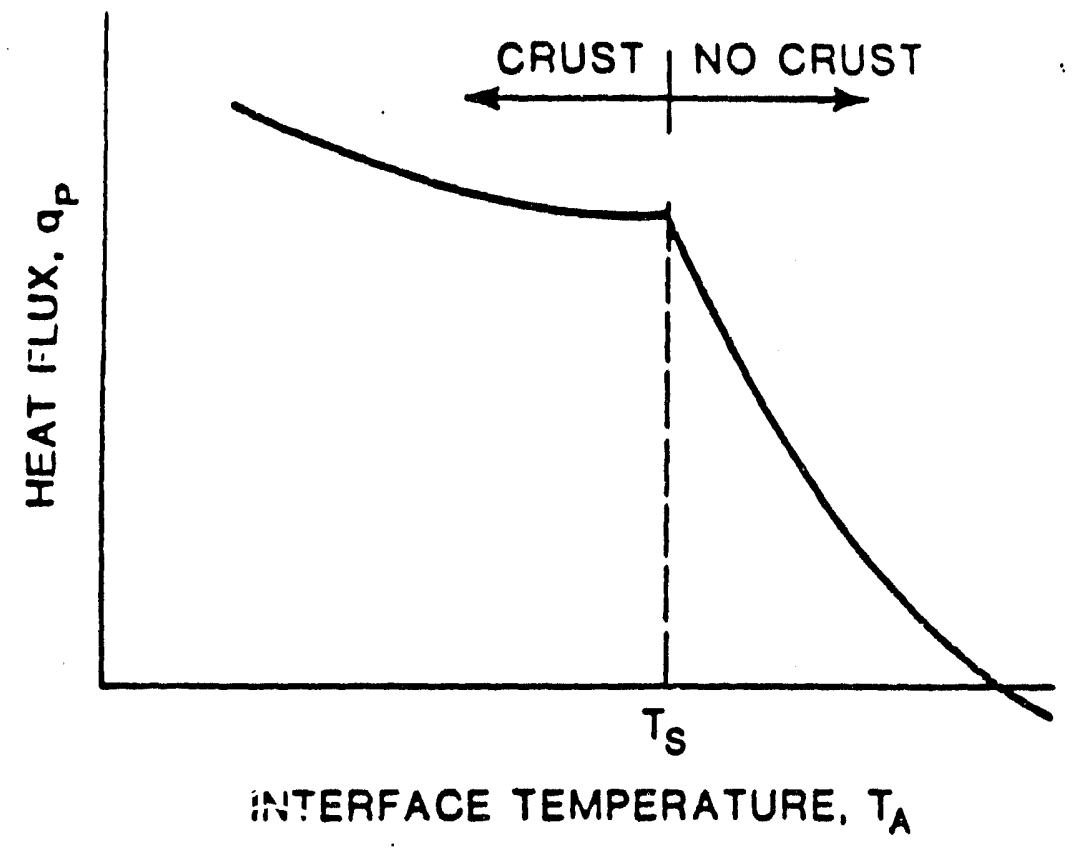

Figure 2.1 Dependence of heat fux on surface temperature 


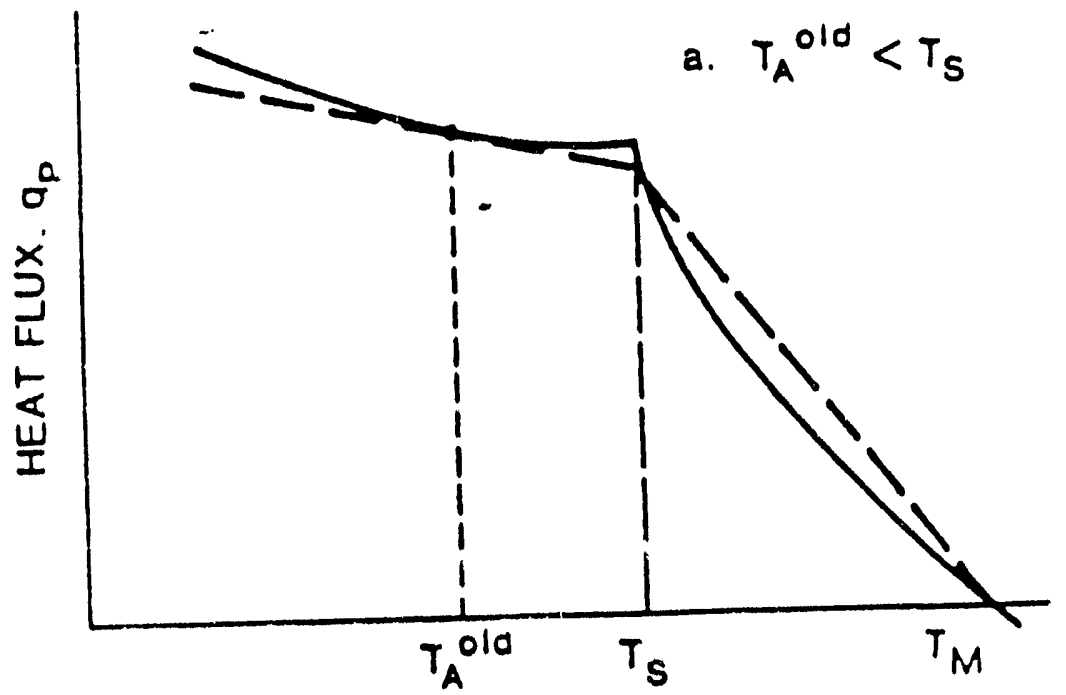

INTERFACE TEMPERATURE, $T_{A}$

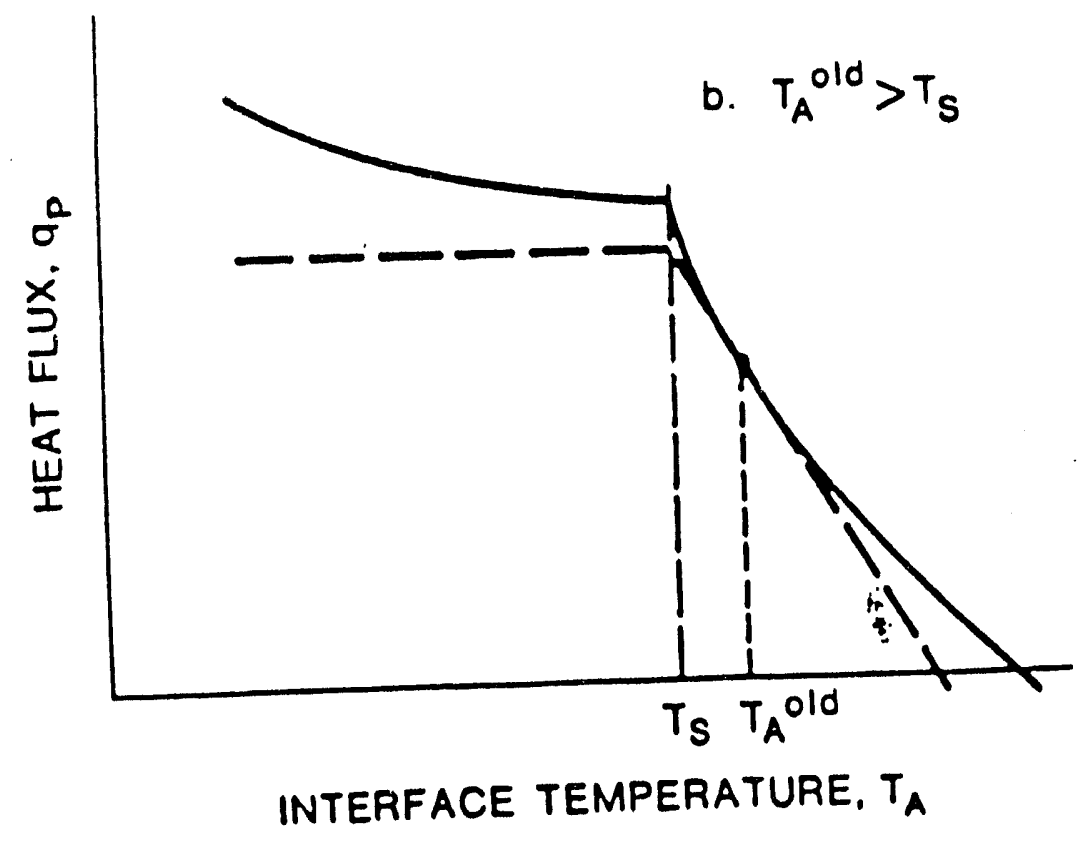

Figure 2.2 Approximation to heat hux as a function of interface temperature 


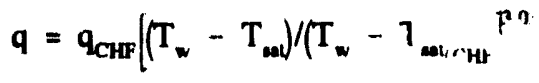

where the exponent is that attributed w Rnhsenou in Reference 37.

The effect of subcooling on nucleate builing is included, using the expression recommended by Ivey: ${ }^{43}$

$$
\mathrm{q}_{\mathrm{CHF}, \text { aw }} / \mathbf{q}_{\mathrm{CHY}, \text { ant }}=1+\mathrm{C}_{\text {hoy }}\left(\mathrm{T}_{\text {wat }}-\mathrm{T}_{.} !\right. \text {. }
$$

where $T_{b}$ is the bulk temperature ot the fluid and the coefficient $C_{\text {wey }}$ is given by

$$
C_{\text {two }}=0.1\left(\frac{\rho_{i}}{\rho_{\mathrm{v}}}\right)^{3 / 4} \frac{\rho_{\mathrm{f}}}{\mathrm{h}_{\mathrm{fi}}} .
$$

This coefficient is calculated as a function of pressure from the fit

$$
C_{\text {hoy }}=\frac{4.77 \times 10^{-2}\left(10^{-5} p\right)^{-0.68 .3}}{1.0 \cdot 6.29 \times 10^{-3}\left(10^{-5} p\right)^{0.862}}
$$

The film boiling regime is based on the Berenson correlations $^{36}$ for the heat-transfer coefficient in film boiling and for the temperature difference at the Leidenfrost temperature (minimum film-boiling point). These have been fit for use in CORCON-Mod3 as

$$
q_{\text {Lod }}=\frac{1.88 \times 10^{4}\left(10^{-5} p\right)^{0.894}}{1.0+7.58 \times 10^{-3}\left(10^{-5} p\right)^{0.986}}
$$

and

$$
\left(T_{w}-T_{\text {mat }}\right)_{\text {Lotd }}=\frac{8.56 \times 10^{1}\left(10^{-5} p\right)^{0.848}}{1.0+1.38 \times 10^{-1}\left(10^{-5} p\right)^{0.750}}
$$

Above the Leidenfrost point, the total heat flux including radiation is represented, in accordance with Reference 37 , as

$$
q=q_{c}\left(q_{c} / q\right)^{1 / 3}+q_{r}
$$

Here $q_{c}$ is the convective heat flux in the absence of radiation, and the factor $\left(q_{c} / q\right)^{1 / 3}$ accounts for the fact that the total heat flux contributes to the vaporization rate, which determines the thickness and thermal resistance of vapor film. The heat flux $q_{c}$ has an explicit variation with temperature as the $3 / 4$ power of $\left(T_{w}-T_{n a t}\right)$. We assume that this dominates the implicit temperature dependence through material properties, so that $\mathrm{q}_{c}$ may be calculated as

$$
q_{c}=q_{c, \text { Let }}\left[\left(T_{w}-T_{\text {cat }}\right) /\left(T_{w}-T_{\text {eat }}\right)_{\text {Lots }}\right]^{0.75} \text {. }
$$

The radiative contribution, $q_{r}$, is given for infinite parallel gray walls by

$$
q_{f}=\frac{\sigma_{B}\left(T_{w}^{4}-T_{m a 1}^{4}\right)}{1 / \varepsilon_{w}+1 / \varepsilon_{f}-1}
$$

where $\epsilon_{w}$ is the emissivity of the wall and $\epsilon_{\mathrm{f}}$ that of the coolant.

CORCON-Mod3 includes the effects of gas barbotage and coolant subccoling on film boiling heat transfer. Both gas barbotage (i.e., noncondensible gas injection at the interface) and coolant subcooling can greatly increase the film boiling heat flux, while also increasing the temperature at which the vapor film collapses (the Leidenfrost point).

Gas barbotage increases film boiling heat transfer by increasing agitation of the coolant, and by increasing agitation of the melt surface. In CORCON-Mod3, the enhancement to the film boiling heat flux due to gas barbotage is included as a multiplicative factor. The factor used depends on whether the surface underlying the coolant is solid or liquid.

If the surface underlying the coolant is liquid, then the enhancement factor is calculated using a correlation of experimental results advanced by Greene. The experimental results were for freon and water on three different molten metals, bismuth, lead, and Wood's metal. The expression proposed by Greene is

$$
Q_{\ell}^{*}=\min \left\{1+11.85\left(\frac{\mathrm{j}_{z}^{*}}{\mathrm{Ja}^{*}}\right)^{0.91}\left(1+2 \mathrm{j}_{\mathrm{z}}^{*}\right), 5\right\}
$$

where $Q^{*}$ is the ratio of the measured heat flux to the heat flux calculated using the Berenson correlation, $j_{b}{ }^{*}=$ $j_{g} / U_{\infty}, j_{z}$ is the superficial gas velocity, $U_{\infty}$ is the terminal rise velocity of the noncondensible gas bubbles in the liquid metal, and $\mathrm{Ja}^{*}$ is defined by

\footnotetext{
'Greene, G. A., private communication, February, 1990.
} 


$$
J^{*}=\frac{c_{p, v} \Delta T_{a a l}}{h_{f_{g}}+0.5 C_{p, v} \Delta T_{\text {aat }}}
$$

where $\Delta T_{\text {cas }}$ is the wall superheat $T_{T}-T_{\text {aul }}$, and $c_{p, v}$ is the specific heat of the vapor at constant pressure.

If the surface underlying the coolant is solid, then the enhancement factor is calculated using a correlation advanced by Duignan. ${ }^{4}$ This correlation is based on experiments in which gas was injected through heated, drilled plates in contact with an overlying water pool.

The correlation used in CORCON-Mod3 is

$$
Q_{1}^{*}=1+0.99\left(j_{b^{*}}^{*} / \mathrm{Ja}^{*}\right)^{0.92}
$$

where all variables have been previously defined.

When the temperature of the core debris is calculated to lie between the solidus and liquidus temperatures of the debris mixture, the two enhancement factors shown above are weighted by the surface solids fraction. The solids fraction is estimated using

$$
\phi=\left(\mathrm{T}_{1 \mathrm{lq}}-\mathrm{T}_{\mathrm{T}}\right) /\left(\mathrm{T}_{\mathrm{lqq}}-\mathrm{T}_{\text {sol }}\right)
$$

where $T_{\| q}$ is the liquidus temperature, $T_{\text {col }}$ is the solidus temperature, and $T_{1}$ is the surface temperature. The gas barbotage enhancement factor is then calculated from

$$
Q^{*}=Q_{i}^{*}(1-\phi)+Q_{0}^{*} \phi
$$

The increased agitation of the melt-coolant interface caused by gas barbotage destabilizes the vapor film, thereby increasing the temperature at which the film collapses. The effect of gas barbotage on the minimum film boiling temperature is accounted for by the equation

$$
\Delta T_{\text {Lot, en }}=\Delta T_{\text {Loti, } 0}+463.1 j_{8}^{0.39 s 3}
$$

where $\Delta T_{\text {Lotd,ee }}$ is the minimum film boiling superheat in the presence of gas barbotage, $\Delta T_{L e t}, 0$ is the minimum film boiling superheat in the absence of gas barbotage, and $j_{3}$ is the superficial gas velocity.

Subcooling of the overlying coolant pool can also enhance heat transfer in the film boiling regime. When the overlying coolant pool is subcooled, energy is removed from the gas film by the overlying subcooled coolant. The net effect of this cooling is a reduction in the thickness of the vapor film. The reduced film thickness permits greater heat transfer by conduction.

The enhancement to heat transfer owing to coolant subcooling in the film boiling regime is included as a multiplicative factor. The factor is calculated using an equation of the form proposed by Siviour and Ede ${ }^{45}$ and Dhir and Purohit: ${ }^{46}$

$$
Q_{\text {oub }}^{*}=1+C^{\prime}\left(\Delta T_{\text {sub }}\right)^{5 / 4}\left(\Delta T_{\text {sat }}\right)^{3 / 4}
$$

where $Q_{n u b}^{*}$ is the coolant subcooling factor, $\Delta T_{\text {sut }}$ is the coolant subcouling (i.e., $T_{\text {att }}-T_{\text {oub }}$ ), and $C$ is chosen to be 0.98 , based on companson to experimental results in References 46 and 47

By reducing the thickness of the vapor film, the subs:ooling of the coolant reduces the stability of the film, and increases the minimum film boiling temperature. The effect of coolant subcnoling on the minimum film boiling temperature is calculated using a simple linear correlation of experinental data: $46,47,48$

$$
\Delta T_{\text {Lodid, oub }}=\Delta T_{\text {Lold, }, 0}+8.0 \Delta T_{\text {oub }}
$$

where $\Delta T_{\text {Lob,oub }}=T_{T}-T_{\text {oub }}$ is the minimum film boiling superheat in the presence of coolant subcooling, and $\Delta T_{\text {Lot }, 0}=T_{T}-T_{\text {aul }}$ is the minimum film boiling superheat in the absence of coolant subcooling.

In the absence of experimental data for the combined effects of coolant subcooling and gas barbotage, we have implemented the following simple equations to describe the combined effect of these phenomena on the film boiling heat flux and minimum film boiling temperature:

$$
Q_{10}^{*}=1+\left[\left(Q_{n u b}^{*}-1\right)^{2}+\left(Q_{m}^{*}-1\right)^{2}\right]^{1 / 2} \text {, }
$$

and

$$
\begin{aligned}
& \Delta T_{\text {Lold }}=\Delta T_{\text {Lot }, 0}+\left[\left(\Delta T_{\text {Lot, oub }}-\Delta T_{\text {Lot }, 0}\right)^{2}\right.
\end{aligned}
$$

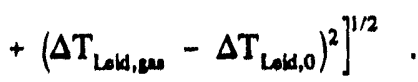

The transition-boiling regime is represented by a simple linear interpolation in $\ell \mathrm{n} q \mathrm{vs}$. $\mathrm{ln} \Delta \mathrm{T}_{\boldsymbol{m} 1}$ between the critical heat flux and the Leidenfrost point. The latter is adjusted to account for the effects of coolant subcooling and gas barbotage. 


\subsubsection{Crust Formation and Freezing}

After sume penod of interaction, core debris temperatures will fall to the point where solidification hegins In the early stages we assume that crusts will form at one or more interfices with the interior of the layer remaning liquid (This is not the only possibility: the crusts may th unstable. or the entire melt may form a slurry.) At latei times, considerable freezing may occur. If part or all of a layer becomes frozen, heal can be removed from it by conduction only, which is ordinarily far less effective than convectiun. Because of internal heating and the fact that cooling cannot continue unless heat iosses exiced sources. freezing is largely self-lımıting.

For some accident scenarios, the core debris may inttially be solid or partially solid. If the degree of solidification is such thai internally-generated heat sannot be removed. the debris temperature will rise and material will melt until convective heat transter is sufficient to allow a balance to be achieved. In general, melting will proceed outward from the center of the debris.

A complete formulation of the problem involves transient, two-dimensional heat transfer with conduction, convection, and change of phase. The spatial resolution must be sufficient to resolve centimeter-thick crusts on layers with dimensions of meters. A numerical solution of the full problem would be very difficult, if not impossible.

A major effect of the presence of solid crusts on heat transfer is the limitation of convective losses because the boundary temperature of the liquid cannot fall below the solidification temperature. Also, a crust provides an additional thermal resistance between the interior of the pool and its surroundings. Both effects tend to reduce heat losses and slow internal cooling rates (or force reheating) so that a steady state is approached. We have retained in CORCON-Mod3 the relatively simple quasi-steady-state model developed for inclusion in CORCON-Mod2. ${ }^{49}$ The model is described in the following paragraphs.

The model is formulated in terms of the average temperature of the layer, which is known from its mass and energy content, although it assunies the existence of a temperature profile within the layer. The basic approach is to construct a steady-state solution to the heat-transfer equations in a right circular cylinder whose average temperature, boundary temperatures, thickness, and volume all match those of the actual layer. The resulting heat fluxes at the boundaries are then used at the corresponding boundaries of the actual layer. As described above, the state of a layer will evolve toward a situation where heat losses balance internal heat generation. The average temperature and the boundary heat fluxes at this steady state are determined by the internal heating and the houndary temperatures for the layer.

As a further simplification, the problem is teduced to two independent one-dimensional protilems, one axial and one radial, by performing radial and axial averages, respectively, of the fuil two-dimensional problems. This is a familiar approximation for conve:tive heat transfer in an almost isothermal liquid layer with thin thermal houndary layers, and its accuracy is seldom yuestioned. It might be expected to he less accurate in the opposite limit of conduction in a solid. Therefore, the model was tested hy comparing its predicuons with the exact solution for steady-state conduction in a right circular cylinder with uniform volumetric heating and specified surface temperatures. ${ }^{1 / \mathrm{h}}$ The agreement between the two calculations was found to be gond: differences in the partition of heat and in the effect of boundary temperatures in the average temperature were less than 10 perceni. and the calculated temperature rises due to internal heating differed by less than 20 percent. Therefore, we believe that the one-dimensional simplification is sufficiently accurate for use in CORCON-Mod3.

Within a one-dimensional calculation, a layer may be entirely liquid, entırely solid, or liquid with a solid crust. For the axial case, a crust may exist on the top, on the bottom, or both. In liquid regions, heat transfer is by convection (natural or bubble-enhanced) with a conduction limit as described in Section 2.3.2.1. In solid regions, it is by conduction. The all-liquid case employs the results of Section 2.3.2.1 directly, while the all-solid case uses the analytic results for steady-state conduction with a constant volumetric source which follows from

$$
\begin{gathered}
\mathrm{q}_{\mathrm{z}}=-\mathrm{kdT} / \mathrm{dz} \\
\mathrm{dq}_{\mathrm{z}} / \mathrm{dz}=\mathrm{S}_{2} \\
\mathrm{q}_{\mathrm{r}}=-\mathrm{kdT} / \mathrm{dr} \\
\mathrm{d}\left(\mathrm{rq}_{\mathrm{t}}\right) / \mathrm{dr}=\mathrm{rS},
\end{gathered}
$$

Here the heat flux, $q$, is positive upward or outward, and $S$ is the volumetric heat source. These relations lead to 
familiar quadratıc temperature profiles. In terms of boundary and average temperatures, the heat fluxes are

$$
\begin{gathered}
q_{B}=k\left(-4 T_{B}+6 \bar{T}-2 T_{T}\right) / L, \\
q_{T}=k\left(-2 T_{B}+6 \bar{T}-2 T_{T}\right) / L, \\
q_{R}=4 k(\bar{T}-R) / R,
\end{gathered}
$$

with subscripts $B, T$, and $\mathbf{R}$ referring to the hottom, top, and radial surfaces. Here $T$ is the average temperature of the layer, and $L$ and $R$ are its thickness and radius, respectively. Note that the volumetric source does not appear in these results; the implications of this are further discussed in Reference $11 \mathrm{~h}$.

In the case of a liquid with crusts, the liquid sublayer is solved first using assumed values of its average. temperature and thickness of radius, $T_{t}$ and $\ell$ or $R_{t}$ and appropriate boundary temperatures. For any surface at which a crust exists, the boundary temperature is assumed to he the solidification temperature. Conduction in a crust is again governed by Equations (73) through (76), and the temperature profile is again quadratic within the crust. We require continuity of the heat flux at the interface with the liquid and set the volumetric source in the crust equal to that in the liquid,

$$
\begin{gathered}
S_{z}=\left(q_{\mathrm{T}}-q_{B}\right) / \ell, \\
S_{\mathrm{r}}=2 q_{\mathrm{r}} / R_{\mathrm{l}},
\end{gathered}
$$

This leads to crust thicknesses and average temperatures of

$$
\delta_{B}=2 k\left(T_{S}-T_{B}\right) /\left[\left(q_{B C}^{2}+2 k\left(T_{D}-T_{B}\right) S_{2}\right)^{1 / 2}-q_{B}\right]
$$

$$
\begin{array}{r}
\delta_{T}=2 k\left(T_{S}-T_{T}\right) /\left[\left(q_{R \ell}^{2}+2 k\left(T_{S}-T_{T}\right) S_{2}\right)^{1 / 2}-q_{T \ell}\right] \\
\delta_{R}=2 k\left(T_{S}-T_{R}\right) /\left[\left(q_{R \ell}^{2}+k\left(T_{S}-T_{R}\right) S_{r}\right)^{1 / 2}-q_{R \ell}\right] \\
\bar{T}_{B}=\left(2 T_{S}+T_{B} / 3+q_{B \ell} \delta_{B} / 6 k\right.
\end{array}
$$

$$
\begin{gathered}
\bar{T}_{T}=\left(2 T_{S}+T_{T}\right) / 3 \cdots q_{T e} \delta_{T} / 6 k \\
\bar{T}_{R}=\left(T_{S}+T_{R}\right) / 2
\end{gathered}
$$

for those trusts which are present. Here $T_{0}$ is the solidification temperature, $T_{x}$ is the average temperature of crust $x$, and $q_{x l}$ is the heat flux at its interface with the liquid, where " $x$ " may be $B, T$, or $R$. In some cases, one or more of Equations (82), (83), and (84) may have no real solution. For this to happen the effective source (divergence of the heat flux) must be negative, which may oscur if a layer is being heated by an adjacent layer. The solution is th repeat the calculations with the source made less negative by increasing the assumed liquid temperature and/or dimensions.

In general, neither the total layer thickness (or radius) nor the overall average temperature thus determined will be correct for the layer. This requires an iteration on the thickness and temperature of the liquid sublayer. A two-variable form of Newton's iteration has been found effective for this. A "bound and bisect" backup has been included for reliability.

\subsubsection{Bubble Phenomena}

Gases that rise through the debris pool as bubbles influence the heat transfer in the pool, as described in Section 2.3.2. The bubble properties and behavior also affect mixing between the layers and the aerosol and fission product release.

Owing to the importance of the bubble properties in the interlayer mixing models developed by $\mathrm{Green}^{13}$ and now implemented in CORCON-Mod3, the bubble behavior models have been improved in CORCON-Mod3. The new models are described in this section.

Bubble rise velocities are computed for three regimes based on bubble geometry and size. Regime 1 applies to small spherical bubbles with internal gas circulation. Regime 2 applies to ellipsoidal bubbles with internal gas circulation. Regime 3 applies to spherical cap bubbles.

The equation for bubble rise velocity $\mathrm{U}_{b}$ used in Regime 1 is derived using classical hydrodynamic analysis: ${ }^{50}$

$$
\mathrm{U}_{\mathrm{b}}=\frac{g \mathrm{~d}_{\mathrm{b}}^{2} \rho_{1}}{12 \mu_{1}}
$$


where $g$ is the acceleration due to gravity, $d_{b}$ is the bubble diameter, $\rho_{1}$ is the density of the surrounding liquid, and $\mu_{1}$ is the viscosity of the surrounding liquid.

The equation used for bubble rise velocity in Regime 2 is ${ }^{51}$

$$
\mathrm{U}_{b} *\left[\frac{2.14 \sigma_{l}}{\rho_{t} d_{c}}+0.505 \mathrm{gd}\right]^{1 / 2}
$$

where $\sigma_{1}$ is the surface tension of the liauid, and $d$ is the equivalent diameter of the bubble.

The equation used for bubble rise velocity in Regime 3 is the Davies-Taylor formula ${ }^{32}$

$$
U_{b}=\sqrt{8 r_{0}}
$$

where $r_{\text {g }}$ is the radius of an equivalent spherical bubble with the same volume us the spherical cap bubble.

CORCON-Mod3 determines the appropriate regime by first comparing the rise velocities for regimes 1 and 2 . If the regime 1 velocity is greater than the regime 2 velocity, the bubble is not spherical, and is, instead, in either regime 2 or 3. (If the converse is true, the bubble is spherical and the regime 1 value is appropriate.) This logic can be used because there is an inflection in the bubble rise velocity curve at the transition from spherical to ellipsoidal bubbles. Next the regime 2 and 3 velocities are compared. If the regime 2 value is greater than the regime 3 value, the bubble must be a spherical cap, otherwise the bubble is an ellipsoid. The regime 2 value is used for ellipsoidal bubbles, while the regime 3 value is used for spherical cap bubbles.

The volume of the debris pool is inflated by the volume of the gas bubbles, a phenomenon known as "level swell." CORCON-Mod2 used a form of the drift-flux correlation $^{53}$ to determine the level swell of the melt. Brockmann ${ }^{54}$ found that this correlation overpredicted the level swell for steel melts, and he developed a correlation that appears to be accurate for a wide range of material properties. Brockmann's correlation is

$$
\alpha=0.128 \mathrm{M}^{-0.0207}\left(\mathrm{j}_{\mathrm{E}}^{*}\right)^{0.584}
$$

where $\alpha$ is the volume fraction, $\mathrm{j}_{\mathrm{B}}^{*}$ is a dimensionless superficial gas velocity, given by

$$
j_{k}^{*}=\frac{j_{g}}{\left[\frac{\sigma_{t} g}{\rho_{t}}\right]^{1 / 4}}
$$

and $M$ is the Morton number, given by

$$
M=\frac{g \mu_{i}^{i}\left(\rho_{l}-\rho_{l}\right)}{\rho_{l}^{2} \sigma_{l}^{3}} .
$$

The volume fruction is limited to a maximum of 0.42 , as suggested by Blottner. ${ }^{22}$

Accurate prediction of bubble size is critical to the calculation of interlayer mixing (see Section 2.3.6). The bubble model used in CORCON-Mod3 treats three bubble formation mechanisms. In this formulation bubble size depends on the superficial gas velocity through the layer.

At low gas velocities, bubbles form at the surface and then depart when their buoyancy overcomes surface tension. A simple force balance yields the equation for the bubble diameter $d_{4}:{ }^{33}$

$$
d_{b}=0.0105 \theta \sqrt{\frac{\sigma_{l}}{B\left(\rho_{l}-\rho_{l}\right)}},
$$

where $\theta$ is the contact angle (in degrees) between the melt and the surface; in CORCON-Mod3, the contact angle is assumed to be 120 degrees.

At higher gas fluxes, bubbles grow to larger sizes before departing from the surface. The bubble size correlation implemented in CORCON-Mod3 is based on the Davidson-Schulerss equations for the bubble volume, where both high and low viscosity liquids are considered. For low viscosity liquids,

$$
\mathrm{V}_{\mathrm{bL}}=\frac{x}{6} \mathrm{~d}_{\mathrm{b}}^{3}=4.1369 \times 10^{-5}\left[\frac{\mathrm{j}_{\mathrm{g}}^{1.2}}{\mathrm{~g}^{0.6}}\right]
$$

while for high viscosity liquids,

$$
\mathrm{V}_{\mathrm{bH}}=\frac{\pi}{6} \mathrm{~d}_{\mathrm{b}}^{3}=0.01094\left[\frac{\mathrm{j}_{\mathrm{g}} \mu_{\mathrm{s}}}{\rho_{1} \mathrm{~g}}\right]^{3 / 4}
$$

where $j_{g}$ is the superficial gas velocity in $\mathrm{m} / \mathrm{sec}$ and $g$ is the acceleration of gravity $\left(9.8 \mathrm{~m} / \mathrm{s}^{2}\right)$. The code uses the 
maximum of the bubble sizes predicted using these two correlations.

At very high gas fluxes, a stable gas film may form. For a gas film, the equation for bubble radius implemented in CORCON-Mod3 is identical to the one used in CORCON-Mod2:

$$
r_{b}=3.97 \sqrt{\frac{\sigma_{t}}{g\left(\rho_{t}-\rho_{2}\right)}} .
$$

where the leading coefficient is based on the experiments of Hosler and Westwater. ${ }^{36}$ Literature values for this coefficient range from 2.2 to 4.2.36,4 The lower values are inconsistent with bubble sizes observed in meltconcrete experiments at Sandia National Laboratories and elsowhere.

CORCON-Mod3 calculates the bubble size for each of the three regimes. The Davidson-Shuler equation is used in most cases. The low gas velocity equation provides a lower bound to the bubble size, while the gas film equation provides an upper bound.

The bubble size is recalculated at each layer interface. The effects of chemistry and changes in temperature and pressure are accounted for, but bubble coalescence is not modeled. The average of the radii of the bubbles entering and leaving the layer is used to calculate a single terminal velocity for the layer.

The gas flux at any elevation is calculated using the local cross-sectional area and the total flow of gas from lower in the pool. A local gas volume fraction is then calculated, and the elevations of layer interfaces are determined from the integral of the non-gas volume

$$
\frac{m_{L}}{\rho_{L}}=\int_{L_{\text {sot }}}^{2_{\infty}}(1-\alpha(z)) A(z) d z
$$

where $m_{L}$ is the mass of the non-gas in the layer, $\rho_{L}$ is the density of the non-gas in the layer, $\alpha(z)$ is the local gas volume fraction at elevation $\mathrm{z}, \mathrm{A}(\mathrm{z})$ is the debris pool area at elevation $z_{\text {, and }} z_{\mathrm{BOT}}$ and $z_{\mathrm{TOP}}$ are the elevations of the bottom and the top of the layer, respectively.

\subsubsection{Interlayer Mixing}

Though CORCON-Mod2 included mixture layers in its layer structure, it lacked mechanistic models for mixing between the layers of the debris pool. We have developed and implemented an interlayer mixing model in CORCON-Mod3 based on the experimental and analytical work of Greene. ${ }^{13,57,58}$ The mechanistic models and their implementation into CORCON-Mod3 are described below.

Two mixture layers are possible, a layer of "light" oxide with suspended metal drops or a layer of metal with suspended "heavy" oxide drops. Mixture layers can be created in one of two ways. First, a mixture layer can be created by the entrainment of one layer, the denser of the two, into another. A mixture layer can also be created when an overlying layer becomes more dense than the layer beneath it. The latter case was handled in CORCON-Mod2 by assuming that a "layer flip" occurred; in other words, the denser oxide layer was assumed to migrate instantaneously through the metal layer and combine with the overlying oxide layer.

When a mixture layer is formed by an unstable density arrangement, mixing is assumed to occur instantaneously; that is, during a single CORCON time step. The adjacent layers are assumed to merge to form the mixture layer.

When a mixture layer is formed by entrainment, the entrained drops are assumed to be carried to the top of the overlying layer before floating free of the entraining bubble. As a result, the overlying layer immediately becomes a mixture layer, and it is redefined by the code.

CORCON-Mod3 calculates also the creation of single-phase layers due to droplet settling (deentrainment). Therefore, mixture layers may eventually stratify into distinct metal and oxide layers if density differences become greater or the gas flow through the melt decreases.

To illustrate the treatment of interlayer mixing in the code, consider the following example: molten core debris is initially stratified with an oxidic phase on the bottom (HOX layer), and a metallic phase on top (MET layer), and is rapidly ablating concrete. The gas flow rate is assumed to be sufficient to begin entraining the oxide into the metal. Once entrainment begins, droplets of the HOX layer are carried to the top of the MET layer, so the MET layer is redefined as an HMX layer (HMX implying suspended heavy oxide in a less-dense metal layer). There will be a net loss of oxide from the HOX layer until the rate of entrainment equals the rate of droplet settling out of the HMX layer. Complete entrainment of the HOX layer may occur as the HOX density decreases due to the addition of lower density concrete oxides (from ablation). If the density of the suspended oxide in the HMX layer subsequently becomes less than the density of the metals, the HMX layer is redefined as the LMX layer (or is combined with the 
LMX layer if one is present). Metals in the LMX layer will then begin to settle out of the mixture Complete stratification may occur as the gas flow rate continues to decrease, resulting in a debris configuration with a metal layer (MET) on the bottom and a less dense oxide layer (LOX) on the top. Note that the beginning and ending debris configurations in this example are the same as what was generally observed using CORCON-Mod2. The evolution of the final configuration is, however, more realistic.

The above example represents just one of many scenarios that may oscur depending on the relative densities of the oxide and metal phases, and the gas flow through the melt. (Another example is provided in the BWR sample problem shown in Section 6.0.) The comments provided in subroutines ORIENT and ADDL.YR are sufficiently detailed for the user to understand the various cases treated by the code.

As the densities of the metallic and oxidic phases and gas flows change, the onentation of the layers may also change. Coding has been included to handle all possible changes in the layer orientation.

In the entrainment calculation, two criteria are used to determine whether entrainment is possible $;^{13}$ these are

$$
\frac{\rho_{2}}{\rho_{1}}<3 \text { and } \omega>\frac{V_{b, o}}{V_{b, p e n}}
$$

where $\rho_{1}$ is the density of the material in the upper layer, and $\rho_{2}$ is the density of the material in the lower layer. In Equation (98), $\omega$ is the dimensionless bubble volume, defined by

$$
\omega=V_{b} / V_{b, p e n}
$$

where $V_{b}$ is the bubble volume and $V_{b, p e n}$ is the bubble volume required for penetration of the interface, given by

$$
V_{b, p e n}=\left[\frac{3.9 \sigma_{12}}{g\left(\rho_{1}-\rho_{k}\right)}\right]^{3 / 2}
$$

in which $\sigma_{12}$ is the surface tension of the liquid-liquid interface, $g$ is the acceleration due to gravity, $\rho_{\ell}$ is the density of the liquid surrounding the bubble, and $\rho_{k}$ is the density of the gas in the bubble. $V_{b, 0}$ in Equation (98) is the minimum bubble volume for entrainment, given by

$$
V_{b, 0}=\left[\frac{7.8 \sigma_{12}}{g\left(3 \rho_{1}-\rho_{2}-2 \rho_{k}\right)}\right]^{3 / 2}
$$

If either criterion defined by Equation (98) is not satisfied, then entrainment cannot occur. In practice, the second criterion of Equation (98) is the more restrictive of the two.

If both conditions for entrainment are satisfied, then the entrained drop volume per bubble is calculated using the correlation developed by Greene: ${ }^{13}$

$$
\frac{V_{0}}{V_{0, \max }}=\frac{1}{806} \xi^{0.152} \operatorname{Re}_{1}^{0.119} \operatorname{Re}_{2}^{0.390}
$$

In Equation (102), $V_{8}$ is the entrained drop volume per bubble, and $V_{e, \max }$ is the theoretical maximum volume that could be entrained and is given by

$$
V_{e, \max }=\frac{V_{b}\left(\rho_{1}-\rho_{g}\right)-\left(\sigma_{12} / g\right)\left(12 \pi^{2} V_{b}\right)^{1 / n}}{\left(\rho_{2}-\rho_{1}\right)}
$$

$\mathrm{Re}_{1}$ are $\mathrm{Re}_{2}$ in Equation (102) are the Reynolds numbers (based on bubble diameter) for bubbles rising in the lower and upper fluids, respectively, and $\xi$ is the normalized excess bubble volume above that required for entrainment:

$$
\xi=\left(V_{b}-V_{b, o}\right) / V_{b, o}
$$

Note that, as defined in Equation (103), $V_{e, \max }$ goes to zero at the following value for $V_{b}$ :

$$
V_{b, \min }=\left[\frac{4.91 \sigma_{12}}{g\left(\rho_{1}-\rho_{8}\right)}\right]^{3 / 2}
$$

This value for $V_{b}$ is more limiting than that given by Equation (100). Therefore, it is used along with Equation (101) to determine whether entrainment is possible.

Given the superficial gas velocity through the surface, $j_{g}$, and the bubble volume, $V_{b}$, the bubble flux (number of bubbles per unit area per unit time) is calculated as

$$
\Phi_{b}=j_{z} / V_{b}
$$

Since each bubble has associated with it an entrained drop of a known volume, the mass flux of entrained 
material can be calculated. Multiplying the mass flux of entrained material by the interfacial area and time step gives the mass entrained during the time step. This mass and its associated enthalpy are transferred into the mixture layer. If no mixture layer is available to accept the entrained mass, one is created to contain it.

Deentrainment is calculated by first calculating the drop settling velocity using the droplet drag coefficient correlations proposed by Greene. ${ }^{13}$ The correlations follow the drag curve for spherical drops until a critical value for the settling velocity is reached. At the critical settling velocity, Greene observed droplet oscillations with a coincident increase in the drag coefficient.

In the absence of drop oscillations, the drag coefficient is determined from the following set of correlations by Beard and Pruppacher: ${ }^{59}$

$$
\frac{C_{d}}{C_{d, S}}=1+0.102 \operatorname{Re}^{0.955}
$$

for $0.2<\operatorname{Re}<2.0$

$$
\frac{C_{d}}{C_{d, s}}=1+0.115 \operatorname{Re}^{0.802}
$$

for $2.0<\operatorname{Re}<21.0$

$$
\frac{C_{d}}{C_{d, s}}=1+0.1879 R^{0.632}
$$

for $21.0<\operatorname{Re}<200.0$. In these equations, $\mathrm{C}_{d, \mathrm{~s}}$ is the drag coefficient for Stokes flow:

$$
C_{d, s}=24 / R e_{d}
$$

and $\mathrm{Re}_{\mathrm{d}}$ is the Reynolds number for the droplet based on the droplet diameter.

Greene developed the following criterion for the onset of droplet oscillations:

$$
\mathrm{We}_{\text {cril }} \operatorname{Re}_{\text {cril }}^{0.65}=165
$$

In this equation, $\mathrm{We}_{\text {cril }}$ and $\mathrm{Re}_{\text {crit }}$ are the Weber and Reynolds numbers, respectively, at which drop oscillations were first observed. Coincident with the oscillations was a dramatic increase in the droplet drag coefficient. The observed drag coefficients are correlated by

$$
\frac{C_{d}}{C_{d, c r i l}}=\left[\frac{R e_{d}}{R e_{c r i t}}\right]^{1.12}
$$

where $C_{d, c r i l}$ is the drag coefficient at the onset of droplet oscillations.

A consistent droplet settling velocity $v_{d}$ and drag coefficient are determined by iteration. Given $v_{d}$, the volume fraction $\alpha_{d}$ of drops in the mixture layer, and the density of the drop material $\rho_{\mathrm{d}}$, the mass flux of settling drops is calculated using the equation

$$
\Phi_{\mathrm{d}}=v_{\mathrm{d}} \alpha_{\mathrm{d}} \rho_{\mathrm{d}}
$$

Multiplying the mass flux by the interfacial area and the time step yields the mass of drops settling out of the mixture during the time step. This mass and its associated enthalpy are transferred out of the mixture layer and into the layer below it. If no separated layer exists to accept the deentrained mass, one is created at the start of the next timestep.

In CORCON-Mod3, the user has the option of selecting whether to begin a calculation with a stratified or a fully mixed debris pool, and the user can select whether the code will perform the entrainment and deentrainment calculations. This allows the user the flexibility to begin a calculation in a fully mixed configuration and then allow the code to calculate entrainment and deentrainment, or the user can force the debris pool to remain mixed by bypassing the mixing calculation. Similarly, the user can begin a calculation with a stratified debris pool and then allow the code to calculate entrainment and deentrainment, or the user can force the pool to remain stratified by bypassing the mixing calculation.

Average thermophysical properties (thermal conductivity, viscosity, surface tension, etc.) are calculated using volume fraction or mass fraction weighing of the individual metal and oxide phase properties. Separate liquidus and solidus temperatures are calculated for the metallic and oxidic phases. The mixture layer solidus temperature is assumed to be the lower of the metal and oxide phase solidus temperatures, while the mixture liquidus temperature is chosen as the maximum of the metal and oxide phase solidus temperatures. Crust formation occurs only when the local temperature drops below the solidus temperature. Between the solidus and liquidus temperatures, the mixture is assumed to form a 
slurry The solids fraction for the mixture is determined by mass-weighing the solids fraction of the metal and oxide phases, where the solids fraction of each phase is based on the appropriate solidus and liquidus temperatures for that phase.

\subsubsection{Pool Surface Heat Transfer}

W.: anticipate that CORCON-Mod3 will be coupled to integral system codes stch as MELCOR ${ }^{8}$ and CONTAIN. ${ }^{7}$ To simplify such efforts, the pool surface has been treated as a major computational interface in CORCON-Mod3, with limited information passed in a well-defined way between above- and below-surface modules. Otherwise, these modules are quite independent; in particular, the need for simultaneous solution of above-surface and below-surface heat transfer relations has heen avoided. The treatment in CORCON-Mod3 is identical to that in CORCON-Mod2.

Each half of the problem (above- and below-surface) defines an upward heat flow, $Q_{n}$ as a function of the surface temperature, $T$. An energy balance at the surface of the pool requires finding that $T$, for which these heat flows are equal; this generally involves solution of a nonlinear--and perhaps very complicated-equation. This may be viewed somewhat differently: for each half of the problem, the boundary condition at the pool surface is the heat flow vs temperature characteristic of the other half, and the object is to find a simultaneous solution. Each "half-problem" is solved with a boundary condition representing the response of the other half, linearized about the most recently calculated surface temperature. The calculation is described in mrire, detail below.

First, the end-of-timestep response of the pool, linearized about its start-of-timestep value, $T^{n}$,

$$
Q_{1}=\left.Q^{0}\right|_{\text {Pool }}+\left.\frac{d Q_{s}}{d T_{s}}\right|_{\text {Pol }}\left(T_{s}-T_{s}^{n}\right) \text {, }
$$

is calculated in ENRCN1 and passed to the above-pool module, ATMSUR. This response includes contributions from the implicit terms in the pool energy equation, involving the change in layer temperatures with change in end-of-timestep heat loss from the surface. In ATMSUR, it is used as a boundary condition for the full nonlinear problem involving atmosphere and surroundings, resulting in a provisional end-of-timestep surface temperature, $\tilde{T}^{n+1}$. This, together with the linearization of above-surface response about $\tilde{T}_{a}^{n+1}$,

$$
Q_{3}=\left.Q_{s}^{0}\right|_{\text {sur }}+\left.\frac{d Q_{a}}{d T_{a}}\right|_{\text {sur }}\left(T_{s}-\tilde{T}^{n+1}\right) \text {. }
$$

is passed back to the below-surface modules. The linearization ultimately serves as a boundary condition for another nonlinear calculation which results in the final end-of-timestep value $T_{0}^{n+1}$. This procedure has advantages with respect to the energy-conservation equations, as will be discussed in Section 2.3.12.

In the version of ATMSUR included in CORCON-Mod3, heat loss from the pool surface includes convective heat transfer to the atmosphere and radiative heat transfer to the surroundings. Thermal radiation is the dominant mechanism. If desired, the radiative effects of aerosols in the atmosphere may be included in the calculation, with an atmospheric opacity determined from calculated aerosol concentrations. Once the optical thickness of the atmosphere is known, the one-dimensional diffusion equation is applied for infinite, parallel, optically gray plates giving

$$
\mathrm{q}_{\mathrm{rad}}=\frac{\sigma_{\mathrm{B}}\left(\mathrm{T}_{\mathrm{s}}^{4}-\mathrm{T}_{\mathrm{sur}}^{4}\right)}{1 / \varepsilon_{\mathrm{s}}+1 / \varepsilon_{\mathrm{suz}}-1+0.75 \mathrm{KL}}
$$

where $T_{s}$ is the surface temperature, $T_{\text {sur }}$ is the surroundings temperature, $\mathrm{K}$ is the extinction coefficient, $\mathrm{L}$ is the average path length for radiation in the cavity atmosphere, and $\varepsilon_{\text {s }}$ and $\varepsilon_{\text {sur }}$ are emissivities of the surface and surroundings, respectively.

Note that Equation (114) reduces to the transparent atmosphere equation as either the extinction coefficient or the average path length approaches zero.

Convection produces additional heat transfer from the pool surface. Unless the atmosphere is truly transparent, however, convection and radiation are strongly coupled; the radiation tends to increase thermal stability and reduce convection. ${ }^{\text {"n }}$ In most cases, however, we have found the convection contribution to be small.

Therefore, we have included only a very simple convection model in CORCON-Mod3. The convective heat transfer from the pool surface to the atmosphere is given by

$$
\mathrm{q}_{\text {oov }}=\mathrm{h}_{\mathrm{a}}\left(\mathrm{T}_{\mathrm{s}}-\mathrm{T}_{\mathrm{a}}\right)
$$

where $T_{a}$ is the bulk temperature of the atmosphere. The heat transfer coefficient, $h_{a}$, is assumed to be constant at $10 \mathrm{~W} / \mathrm{m}^{2} \mathrm{~K}$. 
The total heat flux from the pool surface is given by

$$
q_{1}=q_{\text {rad }}+q_{\text {oonv }}
$$

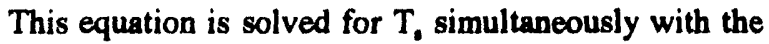
linearized pool response, Equation (112), to determine the provisional end-of-timestep surface temperature $\tilde{\mathrm{T}}_{0}^{n+1}$.

\subsubsection{Concrete Decomposition and Ablation}

The response of concrete exposed to high heat fluxes is complex. Concrete is an inhomogeneous material which undergoes changes in composition as it is heated. The most important of these changes are the vaporization of interstitial and adsorbed water at about $400 \mathrm{~K}$, the decomposition of calcium and magnesium hydroxides near $700 \mathrm{~K}$, and the decomposition of calcium and magnesium carbonates between about 1000 and $1100 \mathrm{~K}$. The carbon dioxide, water vapor, and liquid water produced within the solid concrete flow through the pores of the remaining matrix in response to pressure gradients. Finally, the remaining oxide matrix melts, at a temperature which ranges from about $1350 \mathrm{~K}$ to $1900 \mathrm{~K}$ for representative concretes. In the context of molten-core/concrete interactions, the molten and semi-molten materials are removed from the surface into the pool, as the surface recedes.

We have retained in CORCON-Mod3 the simplified model for the concrete response that was used in CORCON-Mod2. This model is based on a steady-state, one-dimensional energy balance, and is described below.

If a steady temperature profile exists in the concrete, a simple heat balance at the concrete surface yields

$$
q=\rho_{c} \Delta H_{a b l} d x_{a} / d t
$$

where $q$ is the net heat flux to the concrete, $\rho_{c}$ is the density of concrete, $\Delta \mathrm{H}_{\mathrm{abl}}$ is the ablation enthalpy of concrete, and $x_{a}$ is the position of the concrete surface.

The heat flux q must, of course, be reconciled with the interfacial heat transfer model of Section 2.3.2.2. In general, this procedure will involve an iteration to determine the temperature of the pool side of the interfacial film (gas or slag), allowing for the fact that the thermal resistance of that film may depend on the gas generation which results from the ablation. If this temperature is below the ablation temperature, the concrete is treated as an adiabatic boundary with $q$ (and $d x_{a} / d t$ ) set equal to zero.
We emphasize that the pseudo-steady temperature profile, which is used to justify Equation (117), does not appear in the equation. The sensible heat and chemical energy (and changes in these quantities) associated with the temperature profile are ignored. This is not a problem in cases where the pool contains a high temperature liquid melt: studies by ACUREX, using a more complex ablation code, have shown that the ablation process reaches a quasi-steady state within about 1 minute. ${ }^{61}$ At very late times, or early times with initially solid debris, the inaccuracies could be greater.

The quasi-steady model is also used to calculate the generation of decomposition gases; that is, the mass generation rate of each gas is taken as its partial density in the concrete times the ablation rate. Gas released in advance of the ablation front is thus ignored; this assumption neglects the initial burst of gases associated with establishing a steady profile in the concrete by a hot molten pool. The model is also in error at early times for solid debris, because no gas is generated before ablation begins, as well as at late times if ablation ceases. The ablation enthalpy for concrete in Equation (117) is calculated internally, and consists of both sensible and chemical energies. The sensible energy is computed as described in Section 2.4.1. It includes the energy necessary to raise gaseous decomposition products to the concrete ablation temperature to account for the so-called "transpiration cooling" effect. The chemical energy is included using experimentally determined heats of decomposition for three reactions: evaporation of free water $(11 \mathrm{kcal} / \mathrm{mole})$, release of chemically bound water from hydroxides $(25 \mathrm{kcal} / \mathrm{mole})$, and release of $\mathrm{CO}_{2}$ from carbonates $(40 \mathrm{kcal} / \mathrm{mole})$. The enthalpy of limes ne aggregate/common sand concrete (including decon. position products where appropriate) is illustrated in Figure 2.3 as a function of temperature.

The ablation temperature of concrete is not precisely defined because ablated material may not be completely molten. In CORCON-Mod3, we consider a melting range defined by the concrete liquidus and solidus temperatures, with the ablation temperature ordinarily chosen by the user to lie between them. The choice affects the calculated heat of ablation, as may be seen from Figure 2.3. Concrete decomposition products enter the interfacial film or the pool at the ablation temperature, with the enthalpy appropriate to that temperature. Therefore, because all enthalpies are computed from the same data base, the choice of ablation temperature has no effect on overall conservation of energy. 


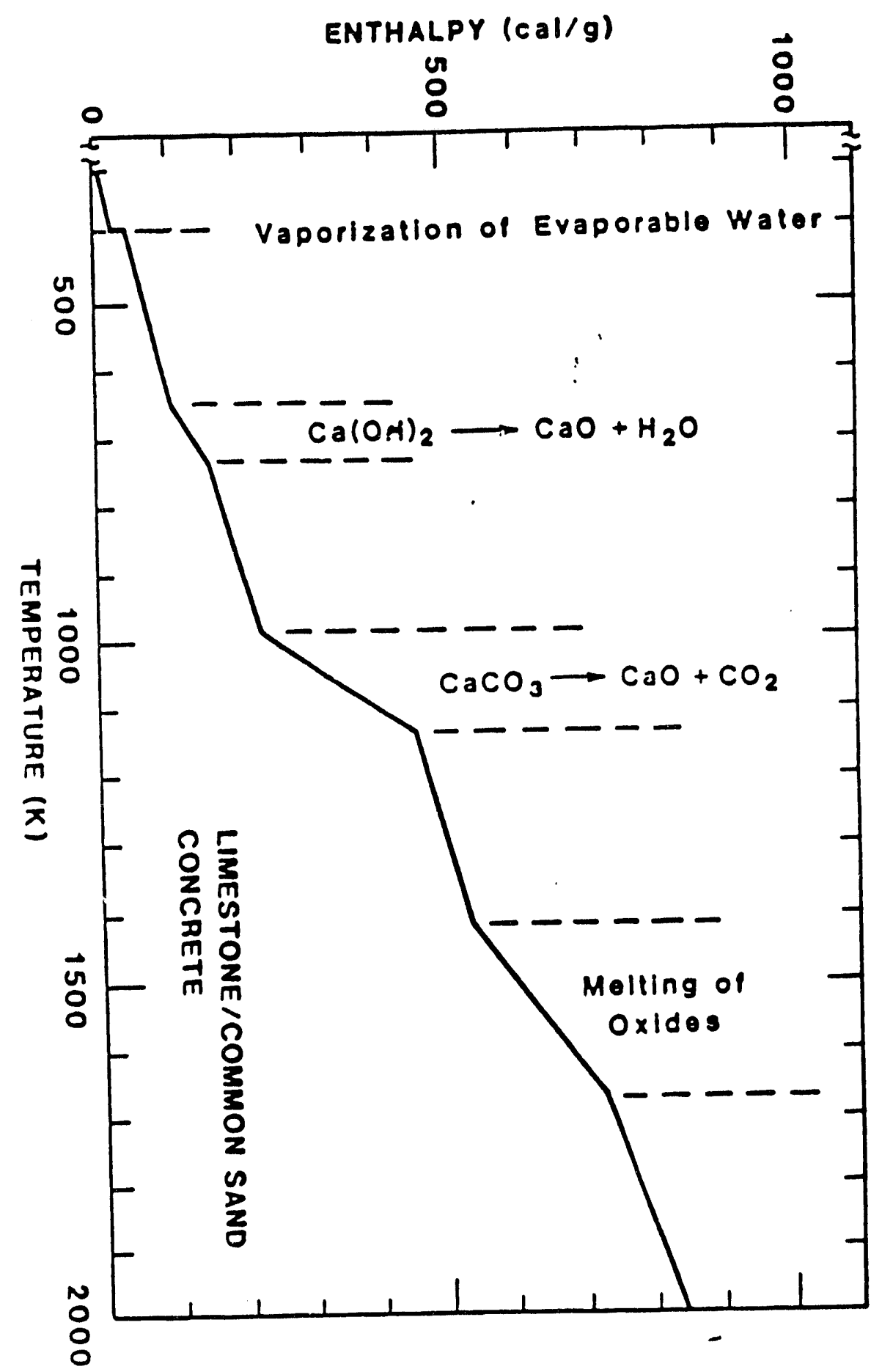


If the concrete contains reinforcing steel, the energy necessary to raise it to the concrete ablation temperatures included in the "concrete" ablation enthalpy.

\subsubsection{Time-Dependent Melt Radius}

In previous versions of CORCON, it was assumed that the melt pool, comprising the core debris, coolant, ablated concrete decomposition products, and ablated materials from the surroundings, completely fills the bottom of the reactor cavity. The assumption limits the utility of the code and may cause numerical problems if small masses of core material are required to be spread thinly over the surface concrete. For example, it is not possible, under this assumption, to simulate the transient events accompanying the initial pour of core debris onto a concrete basemat and the subsequent spreading of the molten debris across the basemat floor. CORCON-Mod3 allows the user to specify a time-dependent radius of the melt that is less than the dimension of the confining cavity. This feature extends the range of severe accident scenarios that can be simulated.

The spreading of a viscous liquid across a solid surface is a complex physical process. If the liquid begins to solidify while flowing, this compounds the complexity of the process. If a crust begins to form on the upper surface of the spreading liquid, the crust may be stable or may be broken up and mixed into the liquid. In the latter case, the resulting slurry has a high viscosity and a high surface tension. Ultimately, the shear stresses required to maintain the flow are greater than those driving the flow, and the flow stops. The molten material continues to solidify in place and may attack the substrate.

Mechanistic modeling of the spreading process described above is not attempted in implementation of the time-dependent melt radius option in CORCON-Mod3. Instead, the user enters a table of times and corresponding radii, and values of maximum and minimum allowable melt thickness. This approach allows considerable flexibility in mimicking the spreading process delineated above, but also allows the user to specify physically unreasonable melt configurations. The option, as currently implemented, will adjust the melt radius to keep the melt thickness between the maximum and minimum thicknesses specified by the user.

Alternatively, by setting the maximum and minimum melt thicknesses to be equal, a constant melt thickness can be specified, and the appropriate melt radius will be determined at each time step. A rudimentary criterion is included to determine when the melt contains too much solid material to allow the melt to continue to spread: when the total crust thickness is greater than a half of the melt thickness, spreading stops.
Each layer of the melt pool is assumed to have the same radius until the melt reaches the sidewall. Mass may be added to the melt pool, either additional core debris or concrete ablation products. With the option, it is possible to fix the radius at a particular value, and to have the melt repose on the concrete floor as a non-spreading glob. As currently implemented, the time-dependent melt radius feature is restricted to cylindrical cavities with flat floors (IGEOM $=2$ ).

Coolant may be present in the cavity when the time-dependent melt radius option is invoked. The coolant is treated correctly as long as the quantity of coolant is sufficient to cover the melt. Program execution stops when the coolant no longer covers the melt. Treating the case where the coolant does not fully cover the melt would necessitate extensive modifications to CORCON to add a radial layer structure and allow simultaneous heat transfer from the melt to a radial water layer and the surroundings. We believe that such modifications are not warranted at this time.

Additions to the energy equations to account for the exposed melt edges are described in Section 2.3.12.

\subsubsection{Chemical Reactions}

In CORCON-Mod2 it was assumed that the principal chemical reaction involved in core/concrete interactions is the oxidation of metals in the pool by concrete decomposition gases. These gases, water vapor and carbon dioxide, are reduced in the process, primarily to hydrogen and carbon monoxide. It is possible to further reduce carbon monoxide to atomic carbon. This is predicted by CORCON-Mod2 in many cases involving metallic zirconium.

In CORCON-Mod3 we treat not only the reactions of metals with gases from the concrete, but also condensed phase reactions between oxides and metals. The latter were added to the code based on the results of the SURC-4 experiment. ${ }^{5}$ In this experiment, a vigorous interaction was observed between metallic zirconium and a silicious concrete with low gas content. This interaction could only be explained by considering condensed phase chemical reactions between the zirconium and molten oxides from the concrete. The driving chemical reaction was

$$
\mathrm{Zr}+\mathrm{SiO}_{2} \rightarrow \mathrm{Si}+\mathrm{ZrO}_{2}+2.1 \mathrm{MJ} / \mathrm{kg} \mathrm{Zr}
$$

Condensed phase reactions are particularly important for core debris interactions with high silica, low gas, concretes such as the one used in the SURC-4 experiment. They are much less important for calcareous 
concretes that have a low silica content and high gas content. The user enables condensed phase chemistry using the input flag, ICHEM

To include condensed phase chemical reactions, it was necessary to expand the master species list to include uranium, aluminum, calcium, and silicon metals. These and other additions to the master species list are shown in Tahle 2.1.

CORCON-Mod3 assumes that chemical equilibrium is achieved between the reactants during each timestep. The chemical equilibrium solver minimizes the Gihbs free energy for 56 chemical species and 15 elements. These species, which are shown in Table 2.6, include all relevant condensed species (the metals, their oxides, and condensed carbon), the principal gasenus snecies of water vapur. hvdrogen, carhon dioxide, and carbon monoxide, and a variety of less-impurtant gases such as light hydrocarbons. The subroutine employed, MLTREA, has evolved from an implementation by Powers ${ }^{1 / c}$ of the method of $V$ an Zeggeren and Storey, ${ }^{62}$ It performs a simple first-order steepest descent minimization of the Gihbs function subject to constraunts on mass conservation and on non- negativity of concentrations. The version included in CORCON-Mod3 is essentially the same as that included in the third correction set for CORCON-Mod2. ${ }^{63}$ For 9 more detailed discussion of the equilibrium solver, the reader is referred to References 11 and 62.

The solution procedure has a number of significant advantages: (1) it is extremely general: (2) reactions need not be specified; (3) "onvergence dnes not require a gond initial guess (although it is much faster if one is available); and (4) the resulting FORTRAN code is relatively small for the number of species considered. The condensed phase reactants and oxidic products are

Table 2.6 Chemical species included in the CORCON chemical equilibrium solution

\begin{tabular}{|c|c|c|}
\hline Oxides & $\begin{array}{c}\text { Metals and } \\
\text { Other Elements }\end{array}$ & Gases \\
\hline $\begin{array}{l}\mathrm{FeO} \\
\mathrm{MnO} \\
\mathrm{Al}_{2} \mathrm{O}_{3} \\
\mathrm{UO}_{2} \\
\mathrm{ZrO}_{2} \\
\mathrm{Cr}_{2} \mathrm{O}_{3} \\
\mathrm{NiO} \\
\mathrm{FpMO}{ }_{2}^{*} \\
\mathrm{FpMO}_{3}{ }^{*} \\
\mathrm{Fe}_{3} \mathrm{O}_{4} \\
\mathrm{Mn}_{3} \mathrm{O}_{4} \\
\mathrm{SiO}_{2} \\
\mathrm{U}_{3} \mathrm{O}_{8} \\
\mathrm{CaO}\end{array}$ & $\begin{array}{l}\mathrm{Fe} \\
\mathrm{Cr} \\
\mathrm{Ni} \\
\mathrm{Zr} \\
\mathrm{FpM}^{*} \\
\mathrm{Mn} \\
\mathrm{C}(\mathrm{c}) \\
\mathrm{Al} \\
\mathrm{U} \\
\mathrm{Si} \\
\mathrm{UAl}_{3} \\
\mathrm{UAl}_{2} \\
\mathrm{Ca} \\
\mathrm{X}^{-m}\end{array}$ & $\begin{array}{l}\mathrm{C}(\mathrm{g}) \\
\mathrm{CH}_{4} \\
\mathrm{CO} \\
\mathrm{CO}_{2} \\
\mathrm{C}_{2} \mathrm{H}_{2} \\
\mathrm{C}_{2} \mathrm{H}_{4} \\
\mathrm{C}_{2} \mathrm{H}_{6} \\
\mathrm{H} \\
\mathrm{H}_{2} \\
\mathrm{H}_{2} \mathrm{O} \\
\mathrm{N} \\
\mathrm{NH}_{3} \\
\mathrm{~N}_{2} \\
\mathrm{O} \\
\mathrm{O}_{2} \\
\mathrm{OH} \\
\mathrm{CHO} \\
\mathrm{CH}_{2} \mathrm{O} \\
\mathrm{CrO}_{3}(\mathrm{~g}) \\
\mathrm{FpMO}_{2}(\mathrm{~g})^{*} \\
\mathrm{FpMO}_{3}(\mathrm{~g})^{*} \\
\mathrm{Al}_{2} \mathrm{O}_{2}(\mathrm{~g}) \\
\mathrm{Al}_{2} \mathrm{O}(\mathrm{g}) \\
\mathrm{AlO} \mathrm{g}) \\
\mathrm{OAlH} \mathrm{g}) \\
\mathrm{AlOH}(\mathrm{g}) \\
\mathrm{OAlOH}_{(\mathrm{O})} \\
\mathrm{AlO}_{2}(\mathrm{~g})\end{array}$ \\
\hline
\end{tabular}

"Pseudo-species representing condensed phase and gas phase fission product groups

"Inert oxide species treated as element " $X$ " in the chemical equilibrium calculation 
treated as ideal solutions in MLTREA; that is, entropy-of-mixing terms are included in their chemical potentials, and the activity of each species is equal to the mole fraction in the phase.

It should be noted that non-ideal solution chemistry has been included in the vaporization release model in VANESA. (See the discussion in Section 2.3.14.) This information could be made available to the MLTREA subroutine, but this has not yet been done. Although non-ideal solution chemistry is important for calculation of vaporization release from the core debris, we currently do not believe it to be important for the calculation of flammable gas production or energy generation, which are the primary results from the MLTREA calculation.

The IC.HEM flag. which is used to enable the condensed phase chemistry calculation, is also used to disable the production of condensed carhnn $(\mathrm{C}(\mathrm{c}))$ during the reaction of carbon dioxide with reactive metals such as zirconium or aluminum. This reaction, which is often referred to as "coking" or "carburization," is predicted by CORCON-Mod2, but has not been ohserved io any significant extent in previous melt-concrete experiments.

To disable the coking reaction, the chemical potentials of $\mathrm{C}(\mathrm{c})$ is artifically set to a large value (currently $10^{15}$ $\mathrm{cal} / \mathrm{g}$-mole). With this change in place, the code was found to predict substantial production of acetylene $\left(\mathrm{C}_{2} \mathrm{H}_{2}\right)$. Since significant acetylene production also has not been observed in any past experiments, we have suppressed the formation of acetylene by setting the chemical potential of $\mathrm{C}_{2} \mathrm{H}_{2}$ to a large value (also $10^{15} \mathrm{cal} / \mathrm{g}$-mole) whenever the coking reaction is disabled.

As with earlier versions of CORCON, CORCON-Mod3 contains coding to calculate the reduction of oxides at the prol surface by the oxygen-poor atmosphere above the melt. This feature was implemented and tested during development of CORCON-Mod1, but was bypassed in released versions of the code because of the incomplete treatment of the atmosphere. We have retained it, still bypassed, in CORCON-Mod3.

The thermodynamic-properties package (Section 2.4.1) employs the standard thermochemical reference point of separated elements in their standard states. With this reference point, heats of formation of all species are automatically included, and all heats of reaction are implicitly contained in the enthalpy data. In fact, they are calculated only for edit purposes.

\subsubsection{Mass and Energy Transfer}

Processes involving mass transfer are of considerable importance in the modeling of molten core/concrete interactions. These include injection of concrete decomposition products (condensed and gaseous) into the pool and further additions of core materials, structural materials, or coolant entering from above. It is convenient to include chemical reactions in the same calculational structure, because these reactions affect the nature of transferred masses.

These transport processes modify both the mass inventories and the energy contents of the various pool layers. The corresponding terms in the mass and energy equations are evaluated in subroutine MHTRAN. The structure of this routine closely mirrors our picture of the physical processes it models, as described in the fnllowing paragraphs.

The masses and enthalpies of all pool layers are updated for mass transfer and associated heat transfer in two passes. (Note that a calculation will not begin until there is some dehris present in the pool.) The first pass, upward through the pool, follows the rising gases and rising condensed-phase materials from concrete decomposition or concrete/melt/gas reactions. The direction of motion is determined by the density relative to the local layer material and/or the driving forces for entrainment. The compositions and enthalpies of these rising materials are followed and modified for chemical reactions. The materials are thermally equilibrated with any layers they pass through, and their energy is ultimately added to the layer where they remain. Any heat of reaction remains with the layer where the reaction occurred.

The second calculational pass, the downward pass, is similar to the first: it follows any material entering the pool from above in addition to sinking reaction products, concrete ablation products, and droplets settling out of mixture layers. Entering metallic species are added to the first layer of the core debris containing metals. If no layer with metals is present, a metallic layer is created. Addition of oxides and coolant is treated in a similar fashion. As with other material movements in CORCON, mass added to the cavity is assumed to equilibrate thermally with each layer through which it passes or eventually resides. Figures 2.4 through 2.6 show this in more explicit detail. In these figures, $Q$ denotes thermal equilibration, $\mathrm{O} / \mathrm{M} / \mathrm{G}$ refers to the oxide/metal/gas oxidation reaction, and $M X$ denotes interlayer mixing. The total heat capacity of a layer is assumed to be much greater than that of materials passing through it so that thermal equilibration takes place at the 


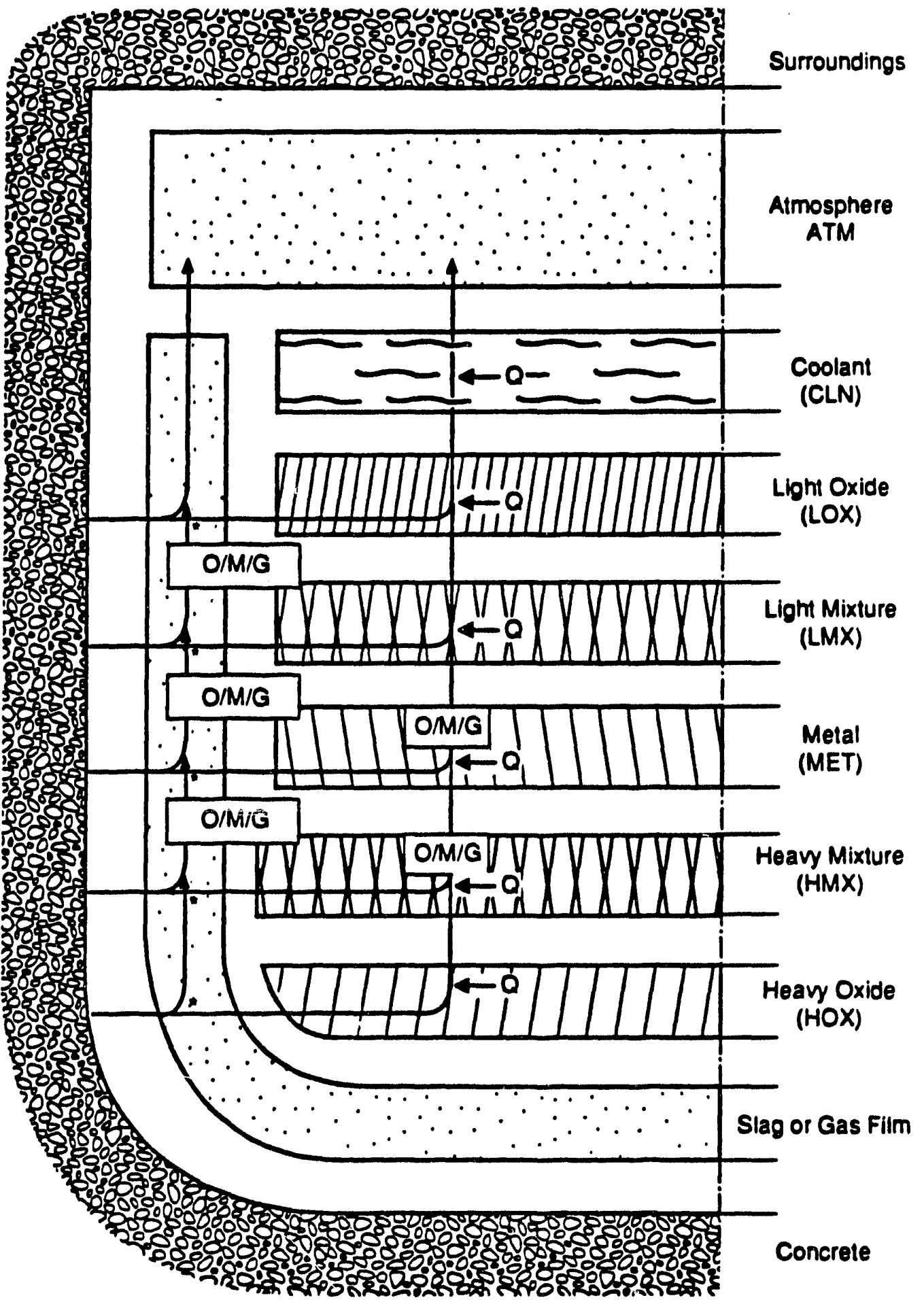

Figure 2.4 Path of gas through pool 


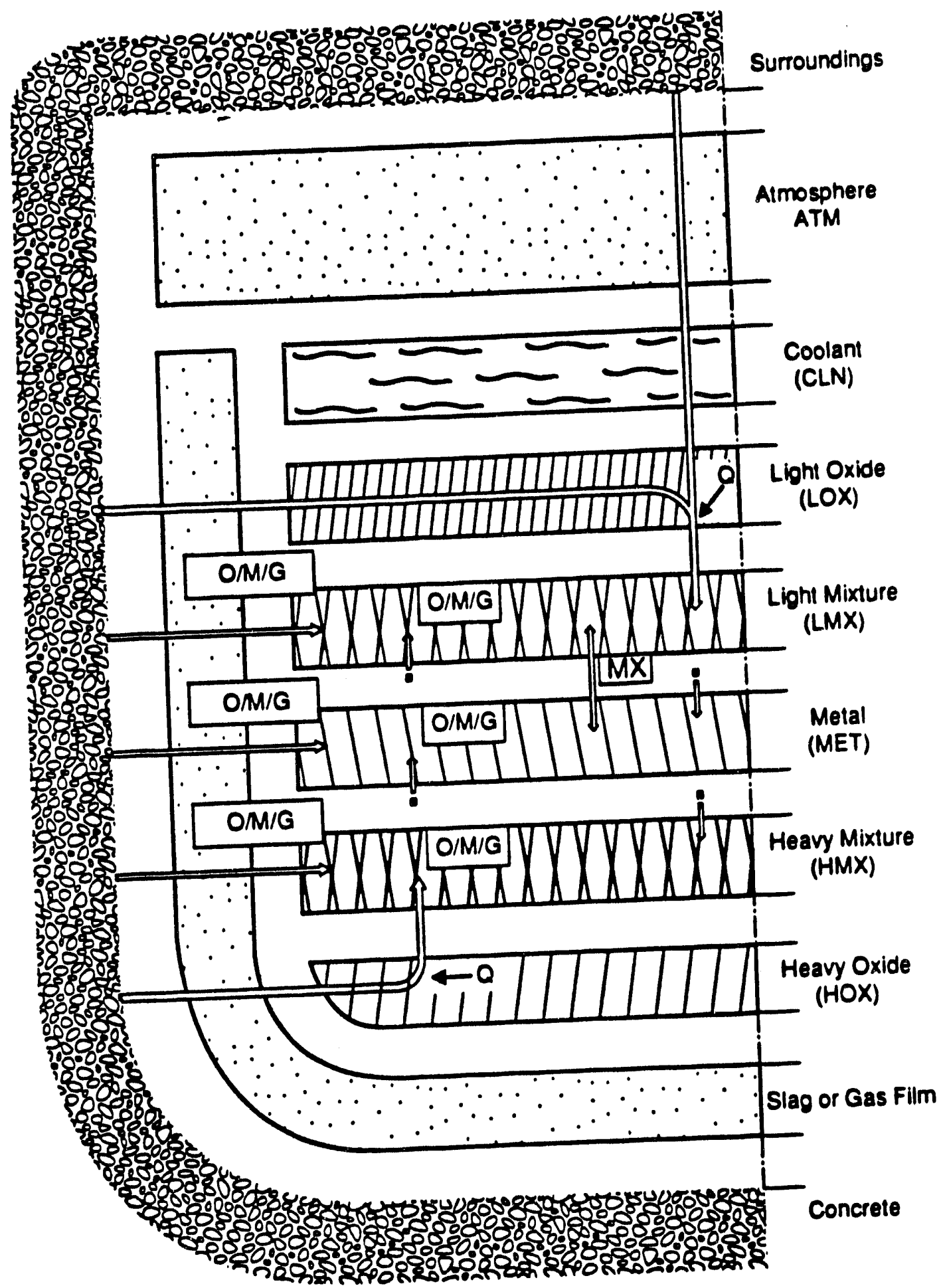

Figure 2.5 Path of metal through pool 


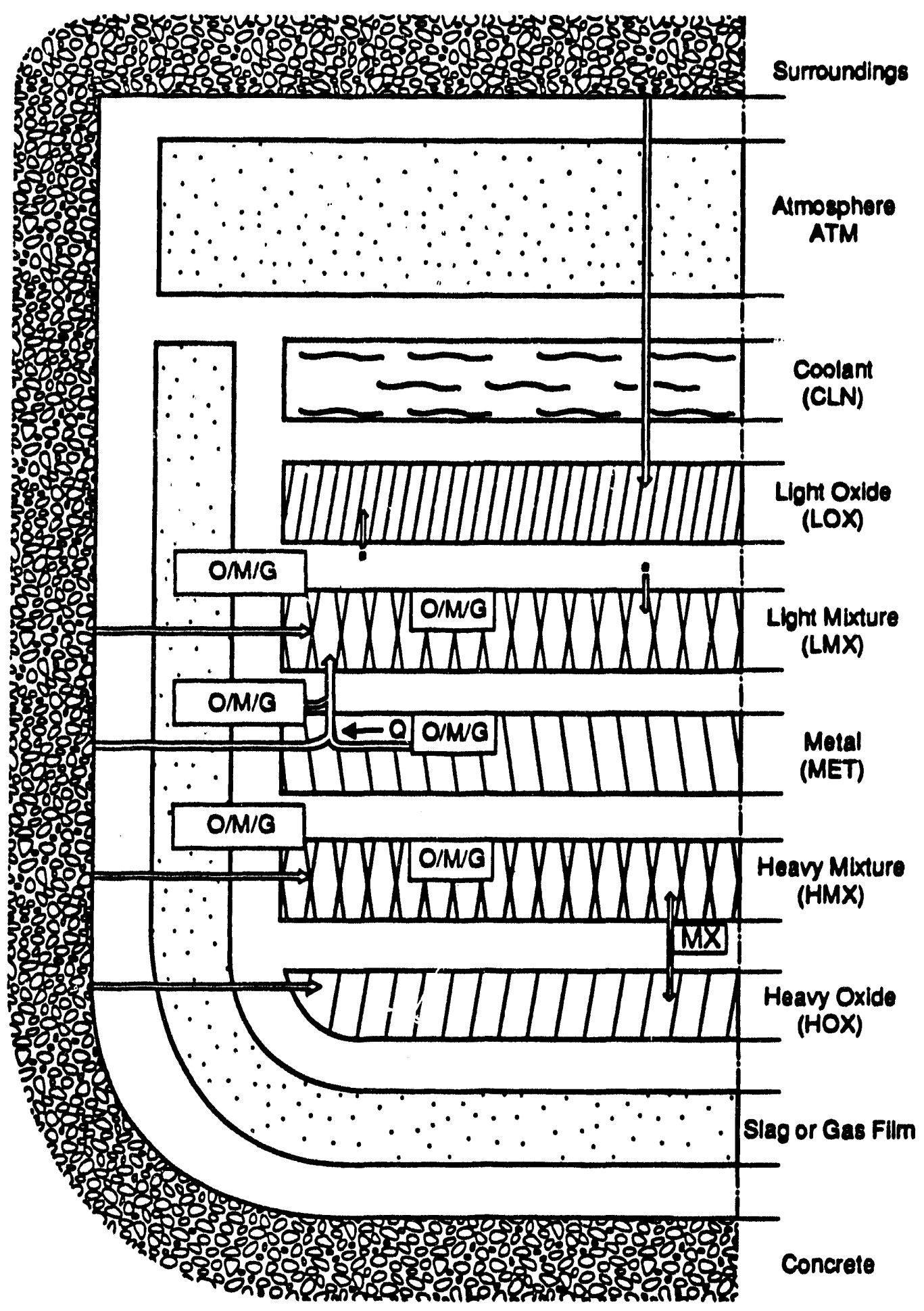

Figure 2.6 Path of oxdde through pool 
start-of-timestep layer temperature. The associated change in layer enthalpy is given by

$$
\Delta H_{L}=H\left(m_{\text {in }}, T_{l_{n}}\right)-H\left(m_{\text {and }}, T_{\text {oun }}\right)
$$

where $H$ is enthalpy, $T$ is temperature, $m$ is mass (including composition), and the subscripts "L", "in", and "out" refer to the layer, to material entering it, and to material leaving it, respectively.

Because the enthalpy package employs the standard thermochemical reference point of separated elements in their standard states, this equation will also hold including the effects of chemical reactions. If, for example, the composition of the gas which leaves the layer differs from that of the gas which entered, the entire energy effest is accounted for through the different compositions associated with $m_{\text {ou }}$ and $m_{\mathrm{ln}}$.

\subsubsection{Energy Conservation}

The energy equation to be solved for each layer of the pool is given by

$$
\begin{aligned}
& \mathrm{H}_{\mathrm{i}}^{\mathrm{n}+1}=\mathrm{H}_{\mathrm{i}}^{\mathrm{n}}+\Delta \mathrm{H}_{\text {onder 1 }}-\Delta \mathrm{H}_{\text {bowe I }} \\
& +\Delta \mathrm{H}_{\text {reac I }}+\Delta \mathrm{H}_{\text {course I }} \\
& -Q_{a b \mid 1}+Q_{B 1}-Q_{T 1}-Q_{R}
\end{aligned}
$$

where

$$
\begin{aligned}
& H_{i}^{\mathfrak{a}}=\text { the total enthalpy of layer } \mathrm{i} \text { at time } \\
& \Delta H_{\text {coter I }}=\text { the enthalpy of materials entering } \\
& \text { during the time-step } \\
& \Delta \mathrm{H}_{\text {leave }}=\text { the enthalpy of materials leaving } \\
& \text { during the time-step } \\
& \Delta \mathrm{H}_{\text {roact I }}=\text { the enthalpy gain from chemical } \\
& \text { reactions } \\
& \Delta \mathrm{H}_{\text {coume i }}=\text { the enthalpy gain from decay heat } \\
& \mathbf{Q}_{\mathrm{ab11}}=\text { the heat loss to ablate concrete } \\
& \mathbf{Q}_{\mathrm{Bi}}=\text { the heat transferred from the bottom }
\end{aligned}
$$

$$
\begin{aligned}
Q_{R J}= & \text { the heat transferred to the exposed } \\
& \text { melt edges if the time-dependent melt } \\
& \text { radius option is used. }
\end{aligned}
$$

As discussed in Section 2.3.11, the "entering," "leaving," and "reaction" terms are naturally associated. The "reaction" term is included implicitly in the other two for the thermochemical reference point employed. These terms are calculated in subroutine MHTRAN as discrete changes, involving all of the material which moves during a time-step. Residence times are not considered. In other words, all materials which result from ablation are assumed to be completely relocated during the same timestep.

Energy generation from radionuclide decay power changes slowly. Therefore, the corresponding term in the energy equation is evaluated explicitly (in the numerical methods sense) as a beginning-of-timestep power multiplied by $\Delta t$. The remaining three terms in Equation (119) involve heat flows which are driven by temperature differences. The heat loss to concrete is also evaluated explicitly, but the remaining terms are treated using a linearized-implicit algorithm. These terms involve the axial heat flows to the upper and lower surfaces of the layer (with the exception of the bottom layer for which the lower surface is adjacent to concrete), and are taken as weighted averages of the values at time $\mathbf{n}$ and the linearly-projected values at time $n+1$. This results in the equation

$$
\begin{aligned}
H_{i}^{n+1}= & H_{i}^{n+1(\operatorname{axp} u s i t)}+n \Delta t\left[\left(\tilde{Q}_{B i}^{n+1}-Q_{B i}^{n}\right)\right. \\
& \left.-\left(\tilde{Q}_{T i}^{n+1}-Q_{T i}^{n}\right)-\left(\tilde{Q}_{R}^{n+1}-Q_{R i}^{n}\right)\right]
\end{aligned}
$$

where the tilde " " denotes the linearized projection described below, and, of course, $\mathrm{H}_{1}^{\mathrm{n}+1 \text { (axplieit) }}$ includes the terms $\left(Q_{B}^{n},-Q_{T}^{n}\right) \Delta t$. The implicitness factor $\cap$ is programmed as a variable, but is set equal to 1 .

The following discussion of the terms in the energy balance is divided in two sections. The discussion in the first section applies for cases in which the time-dependent melt radius option is not invoked. The second section applied if the user chooses to invoke the time-dependent melt radius option.

\section{Melt Radius Option Not Invoked}

When the time-dependent melt radius option is not invoked, $Q_{R i}=0$, and the terms in the energy equation are determined as in CORCON-Mod2. 
We calculate the projected end-of-timestep heat fluxes from

$$
\begin{aligned}
& \tilde{Q}_{\mathrm{T}}^{\mathrm{n}+1}-Q_{\mathrm{Ti}}^{\mathrm{n}}=A_{1}\left[\left.\frac{\partial \mathrm{q}_{\mathrm{T}}}{\partial \mathrm{T}_{\mathrm{L}}}\right|_{\mathrm{i}} \Delta \mathrm{T}_{1}+\left.\frac{\partial \mathrm{q}_{\mathrm{T}}}{\partial \mathrm{T}_{\mathrm{T}}}\right|_{i} \Delta \mathrm{T}_{1}\right] \\
& =\tilde{Q}_{B i+1}^{n+1}-Q_{B i+1}^{n} \\
& =A_{1}\left[\left.\frac{\partial q_{B}}{\partial T_{B}}\right|_{i+1} \tilde{\Delta} T_{1}+\left.\frac{\partial q_{B}}{\partial T_{L}}\right|_{i+1} \tilde{\Delta} T_{i+1}\right] \text {. }
\end{aligned}
$$

if $i$ is not the top layer in the pool, or from

$$
\begin{aligned}
\tilde{Q}_{T 1}^{n+1}-Q_{T i}^{n} & =A_{1}\left[\left.\frac{\partial q_{T}}{\partial T_{L}}\right|_{i} \Delta T_{1}+\left.\frac{\partial q_{T}}{\partial T_{T}}\right|_{i}\left(\tilde{T}^{n+1}-T_{0}^{n}\right)\right] \\
& =Q_{1}^{n}+\left.\frac{d Q_{0}}{d T_{0}}\right|_{\text {sur }}\left(\tilde{T}_{0}^{n+1}-T_{0}^{n}\right)
\end{aligned}
$$

if it is. Here $q_{r(B)}$ is the upward heat flux at the top (bottom) of layer $i, T_{1}$ is the temperature of layer $i, T_{1}$ is the temperature of the interface, $A_{1}$ is its area, and $Q_{\text {. }}^{n}$ and $\left(\mathrm{dQ}_{0} / \mathrm{dT}_{2}\right)_{\mathrm{aur}}$ define the linearization of above-pool heat transfer described in Section 2.3.7. The subscripts $B, T$, and $L$ refer to the bottom and top of a layer, and to the layer average, respectively. It is important to note that $d q_{T i} / d_{T} T_{T}$ may not equal $-d q_{T i} / d T_{1}$. An example of this occurs for a primarily molten layer with a thin crust. An increase in the average temperature increases the liquid temperature and the heat flux to the crusted surface, while an increase in the surface temperature merely decreases the crust thickness with little change in heat flux (under the quasi-steady assumptions of CORCON-Mod3).

The temperatures of the interfaces (other than the top surface) are eliminated, and the change in the temperature of layer $i$ during the timestep approximated in terms of the change in enthalpy by

$$
\tilde{\Delta} \mathrm{T}_{1} \approx \mathbf{X}_{1}=\left(\mathbf{H}_{1}^{\mathrm{n}+1}-\hat{\mathrm{H}}_{1}\right) / \hat{\mathrm{C}}_{1}
$$

Here $\hat{H}_{i}$ is the total enthalpy at temperature $T_{i}$ of the contents of the layer at time $n+1$, and $C_{1}$ is the corresponding heat capacity. When coolant is present, Equation (123) is valid only so long as the coolant remains subcooled. When boiling occurs, $T_{i}^{n+1}$ must be the saturation temperature $T_{\text {oal }}$, and the final enthalpy of the coolant will determine what fraction of it has vaporized during the time-step. This is handled initially by treating $H_{c \mid a}^{n+1}$ and $T_{c \mid n}^{n+1}$ as independent variables.
Equations (120) through (123) result in a set of linear equations for the layer enthalpies at time $n+1$. As coded in subroutine ENRCN1, the equations are written in terms of the X's (which may be thought of as enthalpy changes in temperature units) in the form

$$
\begin{aligned}
\sum_{j} A_{v} X_{j} & =b_{1}^{(1)}+b_{i}^{(2)}\left(\tilde{T}_{1}^{n+1}-T_{1}^{n}\right) \\
& +b_{1}^{(3)} \Delta T_{c i n}
\end{aligned}
$$

They are solved as

$$
X_{1}=X_{i}^{(1)}+X_{i}^{(2)}\left(\tilde{T}_{i}^{n+1}-T_{i}^{n}\right)+X_{i}^{(3)} \tilde{\Delta} T_{\text {oln }}
$$

with $\left(T^{n+1}-T_{a}^{n}\right)$ and $\Delta T_{\text {cln }}$ left undetermined.

If no coolant is present, so that all the $X_{4}^{(3)}$ are 0 , the heat flow at the top surface, including the effects of all implicit terms in the energy equation, now has the form

$$
Q_{1}^{n+1}=Q_{1}^{n}+\left.\frac{d Q_{1}}{d T_{1}}\right|_{\text {pool }}\left(\tilde{T}_{n}^{n+1}-T_{1}^{n}\right) \text {. }
$$

This is the linearized pool response mentioned in Section 2.3.7. Specifically, the derivative is

$\left.\frac{d Q_{0}}{d T_{1}}\right|_{\text {pool }}=\left.\frac{d Q_{T}}{d T_{T}}\right|_{\text {top } i}+\left.\frac{d Q_{T}}{d T_{L}}\right|_{\text {top } i} X_{\text {top i }}^{(2)}$

This equation is solved simultaneously with the above-pool thermal response in subroutine ATMSUR to determine $\mathrm{T}:{ }^{n+1}$. The final evaluation of layer enthalpies and the corresponding layer temperatures is then performed in subroutine ENRCN2. This allows the implicit nature of the equations to be maintained across the pool surface even though above- and below-surface heat transfer are not evaluated simultaneously. It is the stabilizing effect of the implicit algorithm which makes possible the relatively long timesteps routinely employed in the code.

If coolant is present but remains subcooled $\mathrm{H}_{c}^{\mathrm{n}} \mathrm{I}_{\mathrm{n}}$ and $\Delta T_{\text {cin }}$ are related through Equation (123), and $\Delta T_{\text {oin }}$ may be eliminated from both Equations (125) and (126) through the relationship

$$
\tilde{\Delta} T_{\mathrm{cln}}=\frac{X_{\mathrm{cln}}^{(1)}+X_{\mathrm{cln}}^{(2)}\left(\tilde{T}_{0}^{n+1}-T_{\cdot}^{n}\right)}{1-X_{\mathrm{cln}}^{(3)}}
$$


This possibility is tested, using the above-pool thermal response from the beginning of the timestep

$$
Q_{1}^{n+1}=Q_{1}^{n}+\frac{d Q_{0}}{d T_{0}} \mid \operatorname{sur}\left(T_{0}^{n+1}-T_{0}^{n}\right)
$$

to close the set of equations. If this results in a $T_{c i n}^{+1}$ below saturation, the elimination of $\Delta T_{\text {ctm }}$ is accepted, and the solution proceeds as for the no-coolant case. If, on the other hand, this calculation results in a $T_{c i n}^{+1}$ above saturation, we assume that sufficient boiling occurred during the time-step to hold the temperature to saturation and set $T_{0}^{n+1}+$ equal to $T_{\text {at. }}$. In this case, we use the above-pool thermal response from the start of the timestep, Equation (129), to complete the solution in subroutine ENRCN1. The resulting enthalpy of the coolant corresponds to a liquid/vapor mixture at the saturation temperature. The partition of mass between the two phases is evaluated, and the vapor component is added to the gases which left the pool during the timestep. As before, the calculation is completed through ATMSUR and ENRCN2, but with the derivative $d Q_{s} / \mathrm{dT}_{s}$ set very large (negative). This procedure assures that ATMSUR will determine a value for $T_{a}^{n+1}$ very near to $T_{\text {out. }}$.

A special case arises when the coolant layer is totally depleted during a timestep, and the calculation just described results in a layer enthalpy greater than that for saturated vapor. In this case, we assume that all vapor was generated at saturation and decrement the mass and energy of the coolant layer accordingly, but we do not update the enthalpies of other layers at this time. The entire calculation is repeated, with the coolant layer now absent. Because of the detailed balance of heat-flow terms in Equation (120), energy is conserved exactly. Although the total enthalpy of the coolant is not zero at the start of the recalculation, its final enthalpy must be zero. Inspection of the equation will show that the result of the implicit calculation is to force the net heat delivered to the coolant layer during the timestep to be exactly sufficient to vaporize it at its saturation temperature.

The major approximation in this treatment of the coolant is the use of saturation conditions at the beginning of the timestep rather than at the end. This could result in an apparently super-heated liquid coolant or in one which is apparently subcooled but boiling. In practice, no significant problems have been observed.

\section{Melt Radius Option Invoked}

Consider now the case when a time-dependent melt radius is used. We must account for heat transfer from the melt edges to either the atmosphere or the coolant. First, we consider the case where coolant is absent.

The interface temperatures are determined in routine INTEMP. Temperatures of horizontal interfaces within the melt are determined as in the previous section. The temperature of the uppermost interface, between the melt and the atmosphere, is also determined as before. Since the heat flux to the atmosphere is known, this flux can be used to determine the temperatures of the exposed layer edges as follows. The linearized atmosphere response $q$ is

$$
q_{e}=q_{s}+\frac{d q_{6}}{d T_{e}}\left(T_{R i}-T_{s}\right)
$$

where $T_{R i}$ is the temperature of the edge of layer $i$ and $T$, is the provisional surface temperature (assumed known). q. must match the heat flux at each layer edge,

$$
\begin{aligned}
& q_{R 1}\left(T_{R i}^{*}+\delta T_{R l}, T_{L i}\right) \\
& =\alpha\left\{q_{1}+\frac{d q_{0}}{d T_{1}}\left(T_{R i}^{*}+\delta T_{R i}-T_{0}\right)\right\},
\end{aligned}
$$

where $T_{R i}^{*}$ is the current temperature of the edge of layer $\mathrm{i}, \delta \mathrm{T}_{\mathrm{Ri}}$ is the change in temperature required to produce equality of the fluxes, $T_{L i}$ is the fixed temperature of layer $i$, and $\alpha$ is a parameter. The value of $\alpha=0$ corresponds to an adiabatic edge, while $\alpha=1$ matches the fluxes. Then linearizing, with

$$
\begin{aligned}
& q_{R i}^{*}=q_{R i}\left(T_{R i}^{*} T_{L i}\right) \\
& q_{a}^{*}=q_{1}+d q_{0} / d T_{0}\left(T_{R i}^{*}-T_{0}\right), \\
& q_{R i}^{*}+\frac{\partial q_{R i}}{\partial T_{R i}} \partial T_{R i}=\alpha q_{a}^{*}+\alpha \frac{d q_{1}}{d T_{0}} \delta T_{R i} .
\end{aligned}
$$

Solving for the temperature correction $\delta \mathrm{T}_{\mathrm{Ri}}$,

$$
\delta T_{R i}=\frac{\alpha q_{i}^{*}-q_{R i}^{*}}{\frac{\partial q_{R i}}{\partial T_{R I}}-\alpha \frac{d q_{s}}{d T_{s}}}
$$


The new edge temperature of layer $i$ is then

$$
\min \left\{T_{R i}^{*}+\delta T_{R}, T_{L i}\right\}
$$

this constraint is imposed so that the radial heat transfer is always outward (i.e., positive).

The procedure described above is implemented at the end of the main interface temperature iteration loop in INTEMP. Following convergence of the interface temperatures, new values of the average surface temperature $T_{\text {, }}$ and the constant flux $\mathrm{q}_{\mathrm{f}}$ are required. The new values are given by

$$
T_{A}=\frac{1}{A}\left(A_{r} T_{T}+\sum_{i=1}^{k e t h-1} A_{R T} T_{R i}\right)
$$

anci

$$
q_{1}=\frac{1}{A}\left(A_{r} q_{T}+\sum_{1=1}^{i=1} A_{R 1} q_{R i}\right)
$$

where $T_{T}$ is the temperature of the horizontal interface between the melt and the atmosphere, $A_{T}$ is the area of the interface, and $q_{T}$ is the flux from the uppermost melt layer to the atmosphere through the top interface. $A_{R J}$ is the area of the edge of layer $i$, and $A$ is the total melt area exposed to the atmosphere.

Tho iayer temperatures are updated in routine ENRCN1. To account for the energy loss from the melt to the atmosphere, the energy equation for each layer becomes

$$
\begin{aligned}
H_{1}^{n+1}= & H_{1}^{n+1} \text { (explicit) } \\
& +\Omega \Delta t\left\{\left(\tilde{Q}_{B i}^{n+1}-Q_{B i}^{n}\right)-\left(\tilde{Q}_{T i}^{n+1}-Q_{T i}^{n}\right)\right. \\
& \left.-\left(\tilde{Q}_{R i}^{n+1}-Q_{R i}^{n}\right)\right\},
\end{aligned}
$$

where the last term on the right-hand side of the equation accounts for heat transfer from the melt edge, $\mathrm{H}_{4}{ }^{\mathrm{n}+1}$ is the enthalpy of layer $i$ at the time step $n+1, H_{i}^{n+1}$ (explicit) is the explicit part of the update, $\Omega$ is the implicitness factor (set equal to 1), and $\Delta t$ is the length of the time step. $Q_{B i}, Q_{T i}$, and $Q_{R i}$ are the bottom, top, and radial heat transfer, respectively, of layer $i$ at the appropriate timestep indicated by the superscript. The term, $Q_{R i}^{n+1}$ $Q_{R,}^{n}$, is given by
$\check{Q}_{R I}^{n+1}-Q_{R I}^{n}=A_{R I}\left\{\frac{\partial q_{R i}}{\partial T_{R j}}\left(T_{\bullet}^{n+1}-T_{\bullet}^{n}\right)+\frac{\partial q_{R i}}{\partial T_{L I}} \Delta T_{L}\right\}$

Equation (135) is then solved in the same way as Equation (120).

Once the layer temperatures $T_{L}$ at the new time have been determined, the new provisional surface temperature $T_{0}^{n+1}$, the new provisional heat flux $q_{1}^{n+!}$ and derivative $\mathrm{dq}_{2} / \mathrm{dT}$, and the surface emissivity are updated using area averages over the exposed surface of the melt.

Consider now the case in which the time-dependent melt radius option is invoked and coolant is present. The interface between the melt and the coolant now includes contributions from all the exposed layers. The temperatures of the layer edges are determined in the following way. The temperatures of the horizontal interfaces within the melt are determined as in the case with no time-dependent melt radius. At the melt-coolant interface, we have the energy conservation equation

$$
\begin{aligned}
& A q_{0}\left(T_{B_{C}}^{*}, \delta T_{B B^{\prime}}, T_{L e}, T_{T_{\varepsilon}^{*}}^{m}+\delta T_{T_{c}}\right) \\
& =A_{T} q_{T 1}\left(T_{B 1}^{*}+\delta T_{B 1}, T_{11}, T_{T i}^{*}+\delta T_{T i}\right) \\
& +\sum_{\mid=1}^{|c| s \mid n-1} A_{R \mid} q_{R \mid}\left(T_{R i}^{*}+\delta T_{R I}, T_{L i}\right)
\end{aligned}
$$

where $T_{x y}$ is the current temperature of the bottom $(x=B)$, layer $(x=L)$, top $(x=T)$, or edge $(x=R)$ of layer $y$, which may be coolant $(y=c)$, the top layer of the melt $(y=t)$, or the layer $i(y=i), A_{T}$ is the area of the top horizontal interface of the melt, $A_{k J}$ is the exposed area of the edge of layer $i$, and $A$ is the total exposed area of the melt. Equation (138) is solved by first setting $\delta \mathrm{T}_{\mathrm{Bc}}=$ $\delta T_{T_{1}}$ and $T_{B e}^{*}=T_{T_{1}}^{*}$ and initially setting $\delta T_{R I}=0$. Then linearizing,

$$
\begin{aligned}
& A\left\{\mathrm{q}_{c}^{*}+\frac{\partial \mathrm{q}_{\mathrm{c}}}{\partial \mathrm{T}_{\mathrm{Bc}}} \delta \mathrm{T}_{\mathrm{Ti}}+\frac{\partial \mathrm{q}_{\mathrm{c}}}{\partial \mathrm{T}_{\mathrm{Tc}}} \delta \mathrm{T}_{\mathrm{Tc}}\right\} \\
& =A_{\mathrm{T}}\left\{\mathrm{q}_{\mathrm{Ti}}^{*}+\frac{\partial \mathrm{q}_{\mathrm{Ti}}}{\partial \mathrm{T}_{\mathrm{Bt}}} \delta \mathrm{T}_{\mathrm{Bi}}+\frac{\partial \mathrm{q}_{\mathrm{Ti}}}{\partial \mathrm{T}_{\mathrm{Ti}}} \delta \mathrm{T}_{\mathrm{Ti}}\right\} \\
& +\sum_{i=1}^{i \operatorname{lin}-1} \mathrm{~A}_{\mathrm{Ri}} \mathrm{q}_{\mathrm{Ri}}^{*},
\end{aligned}
$$

where $q_{c}^{*}=q_{c}\left(T_{B c}^{*}, T_{L c}, T_{T c}^{*}\right)$, etc. 
The coolant overlying the melt is treated as a layer, and the appropriate heat fluxes are determined as described in Section 2.3.3. The quantities $\mathrm{q}_{\mathrm{c}}^{*}$ and $\partial \mathrm{q}_{\mathrm{c}} / \partial \mathrm{T}_{\mathrm{Bc}}$ are then used to determine the edge temperature of each melt layer by matching heat fluxes as

$$
q_{R i}^{*}+\frac{\partial q_{R 1}}{\partial T_{R I}} \delta T_{R}=\alpha\left\{q_{0}^{*}+\frac{\partial q_{0}}{\partial T_{R_{0}}} \delta T_{R 1}\right\},
$$

where $\alpha=0$ implies an adiabatic edge, and $\alpha=1$ matches the heat fluxes. Thus

$$
\delta T_{R 1}=\left(\alpha q_{0}^{*}-q_{R i}^{*}\right) /\left\{\frac{\partial q_{R 1}}{\partial T_{R t}}-\alpha \frac{\partial q_{0}}{\partial T_{B c}}\right\} \text {, }
$$

and the new edge temperature for layer $i$ is

$$
\min \left\{T_{R i}^{*}+\delta T_{R,}, T_{L i}\right\}
$$

where the minimum constraint is imposed so that the heat transfer is radially outward (i.e., positive). The parameter $\alpha$ is programmed as a variable but is currently set equal to 0.0 in the code.

The layer temperatures are updated in routine ENRCN1. The energy equation for each melt layer is Equation (137), as in the case with no coolant. Whes coolant is present, however,

$\tilde{Q}_{R}^{n+1}-Q_{R}^{n}=A_{R U}\left\{\frac{\partial q_{R S}}{\partial T_{R}} \Delta T_{R}+\frac{\partial q_{R U}}{\partial T_{L}} \Delta T_{L}\right\}$

where $\Delta T_{R j}$ is the change in the edge temperature over the timestep, and $\Delta T_{L}$ is the change in the layer temperature. By analogy with the expression for $\Delta T_{1}$, the change in the temperature of the horizontal interface $I$, the expression for $\Delta T_{R I}$ is

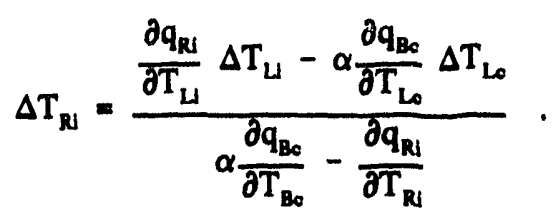

The energy equation for the coolant layer is then

$$
\begin{aligned}
& \mathbf{H}_{c}^{\mathbf{n + 1}}=\mathbf{H}_{c}^{\mathbf{A + 1}} \text { (explicit) } \\
& +a \Delta t\left\{\left(Q_{B C}^{n+1}-Q_{B C}^{D}\right)\right. \\
& -\left(Q_{T C}^{++1}-Q_{T c}^{R}\right) \\
& \left.+\sum_{l=1}^{\sum_{1}-1}\left(Q_{x}^{n+1}-Q_{x i}^{l}\right)\right\} \text {, }
\end{aligned}
$$

where the summation on the right-hand side accounts for the heat transfer from the exposed edges of the melt layers. Equation (146) with Equation (137) for the non-coolent layers are then solved in the same way as Equation (120).

As noted in Section 2.3.9, the time-dependent melt radius is limited to cases where the coolant completely covers the melt. When the coolant is insufficient to cover the melt, the code reports an error and execution stops.

\subsubsection{Cavity Shape Change}

The shape of the cross-section of the cavity is defined for computational purposes by a series of "body points." These are the intersections of a fixed series of rays with the cavity surface as shown in Figure 2.7. Given the cavity geometry at the start of a timestep, the shape change procedure provides a now cavity shape at the end of the timestep. The normal recession rate is used at each body point, as calculated by the concrete ablation model. The methods used in CORCON-Mod3 are the same as those developed for CORCON-Mod2. The shape change procedure evolved from the CASCET model written for CORCON-Modl by ACUREX/Aerotherm Corporation, ${ }^{61}$ under contract to Sandia. We first assume that the concrete recession follows the local normal at each body point and then project the resulting surface points beck onto the rays. The projection is performed by passing a circle through each normally receded point and its two nearest neighbors, and defining the new body point as the intersection of this circle with the corresponding ray as shown in Figure 2.8. A solution will exist so long as no body point is allowed to cross the neighboring ray during a timestep. In fact, there are two solutions, and we pick the one which is nearer to the normally receded point.

With one exception, the rays emanate from an origin on the vertical axis of symmetry of the cavity. We have retained the so-called "tangent ray" from CASCET. This is a single ray, parallel to the axis, which defines the edge of the flat bottom of the cavity. Because the 


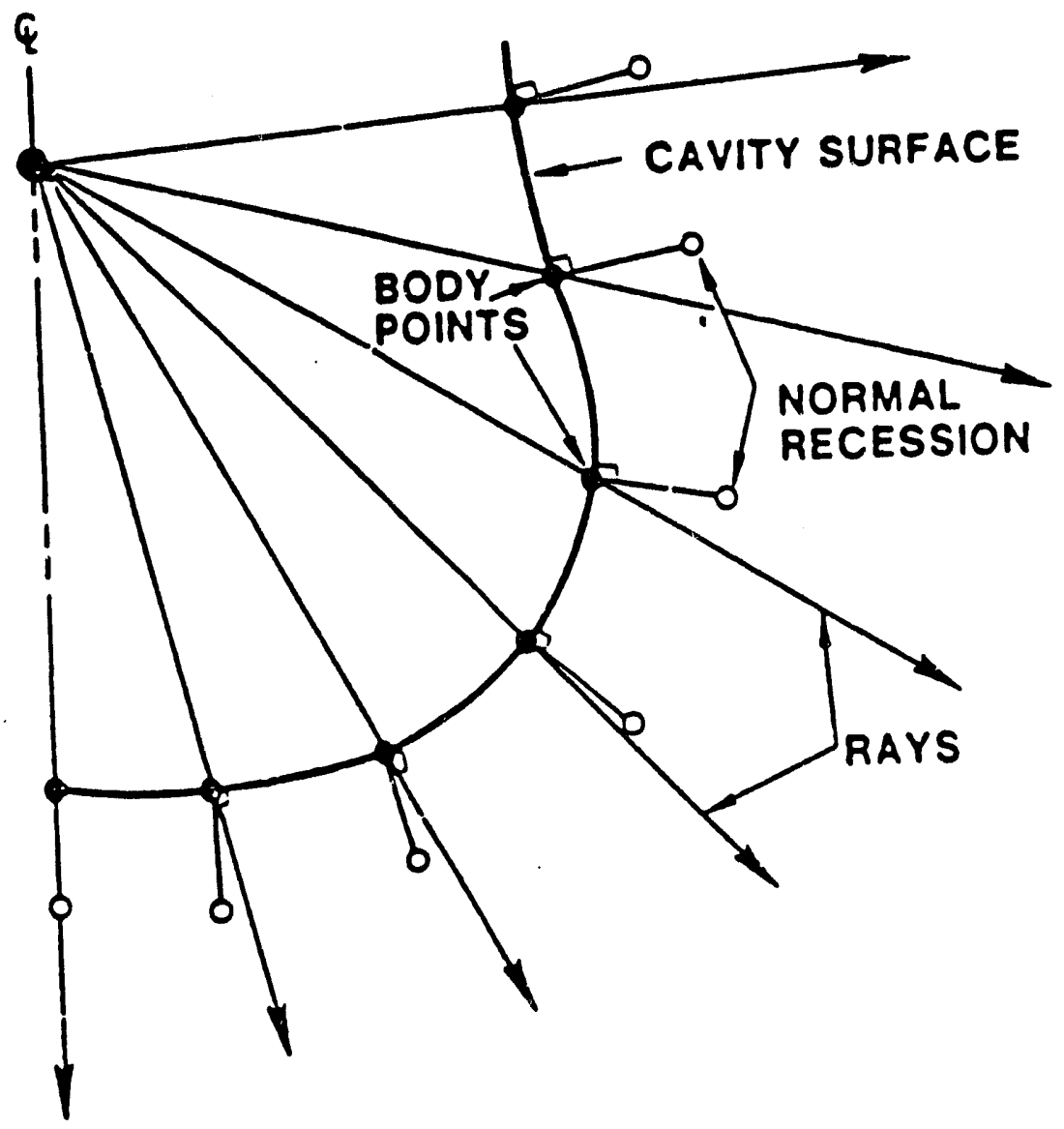

Figure 2.7 Normal surface recession 


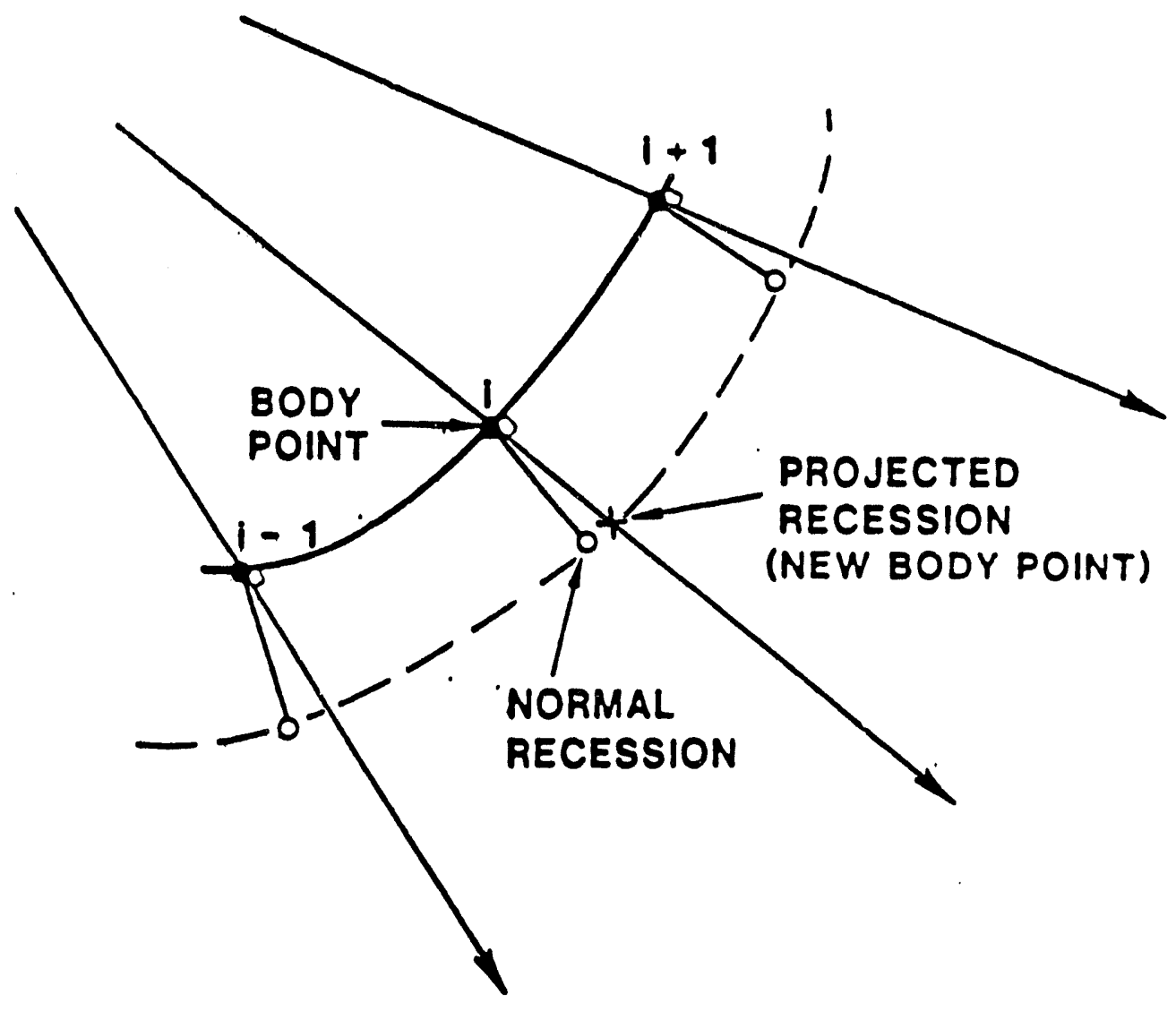

Figure 2.8 Circle intersection projection method 
corresponding body point moves vertically, the bottom is constraned to remain flat as is required by the ablation model. When the cavity surface passes through a point where the tangent ray crosses an ordinary ray, the isdering of the body points is changed appropriately.

In these calculations, the "normal" at each body point is chosen as the bisector of the angle formed by lines from the bridy point to its nearest neighbors on each side. Another modification was made to prevent numerical sharpening of corners projecting into the cavity. In this case, shown in Figure 2.9, if the normal ablation during a timestep is $\Delta s=\dot{s} \Delta t$, the point defining the comer moves $\Delta s / \cos (\gamma / 2)$. (Projection back onto the ray system is not included in this Figure, In CORCON-Mod3. the correction is made for rexession of all hody points for which $\gamma>0$.

\subsubsection{Aerosol Generation and Radionuclide Release}

As gases from decomposition of concrete pass through the melt and leave the pool, aerosols are generated by mechanisms such as vaporization/condensation and bubble bursting. These aerosols were observed in early experimental work, and were hypothesized to be an important mechanism for radionuclide release from the core debris. The VANESA model ${ }^{10}$ was developed to predict aerosol generation and radionuclide release during core-concrete interactions. VANESA has now been integrated into CORCON-Mod3.

The VANESA model is called from CORCON-Mod3 as part of the upward pass in subroutine MHTRAN (see discussion in Section 2.3.11). VANESA requires information on: the composition, temperature, and material properties of the core debris; the flow rate and composition of gases sparging the melt; the depth and temperature of any overlying coolant; and the ambient pressure. This information is provided by the remainder of CORCON and is passed to VANESA after first being converted to the form required by VANESA. The following conversions are made in subroutine CVFAC:

- CORCON's debris composition (including fission products) is converted to a VANESA composition by storing CORCON's oxide, metal, and fission product masses in the VANESA composition array $\mathrm{XM}$.

- Average metal and oxide debris temperatures are determined for VANESA by mass-weighting the temperatures of the metals and oxides in the CORCON layers.
- The metal and oxide material properties used in VANESA are calculated by mass-weighting the properties of the metals and oxides in the CORCON layers. The same is true for the hubble properties used by VANESA. All material and bubhle properties are converted to c.g.s units for use in VANESA.

- The gas flow rate and composition used in VANESA are determined from the gases exiting the surface of the melt. In other words, VANESA uses the results from the chemical equilibrium solution performed in the CORCON calls to MLTRF.A from REACT. (Note that subroutine SRG, which is used in the stand-alone version of VANESA has been eliminated in the integration of VANESA into CORCON.) CORCON gas species that are not tracked hy VANESA are included in the total flow rate, hut are not included in the VANESA gas composition. These gases are effertively treated as inert in the vaporization release calculation performed in VANESA.

- The corlant depth and temperature, and ambient pressure are passed to VANESA after first converting the depth and pressure to units required by VANESA (i.e., centimeters and atmospheres).

It should be noted that because VANESA is called during the upward pass in MHTRAN, the debris composition passed to VANESA does not include any debris added by user-specified mass addition. Since this mass is added as part of the downward pass in MHTRAN, it is added after the call to VANESA. Consequently, the CORCON and VANESA debris compositions printed in the output will be slightly different whenever the mass is being added to the cure debris.

The implementation of the VANESA model included in CORCON-Mod3 calculates the following features of radionuclide release and aerosol generation:

- The rate and total mass of aerosol generated.

- The concentration of aerosols in the evolved gases.

- The composition of the aerosol, including the contributions of non-radioactive materials, as well as those of radionuclides.

- The mean size and size distribution of the aerosols.

- The material density of the aerosol.

- The effects of coolant pools overlying the core debris on the production and nature of the aerosols. 


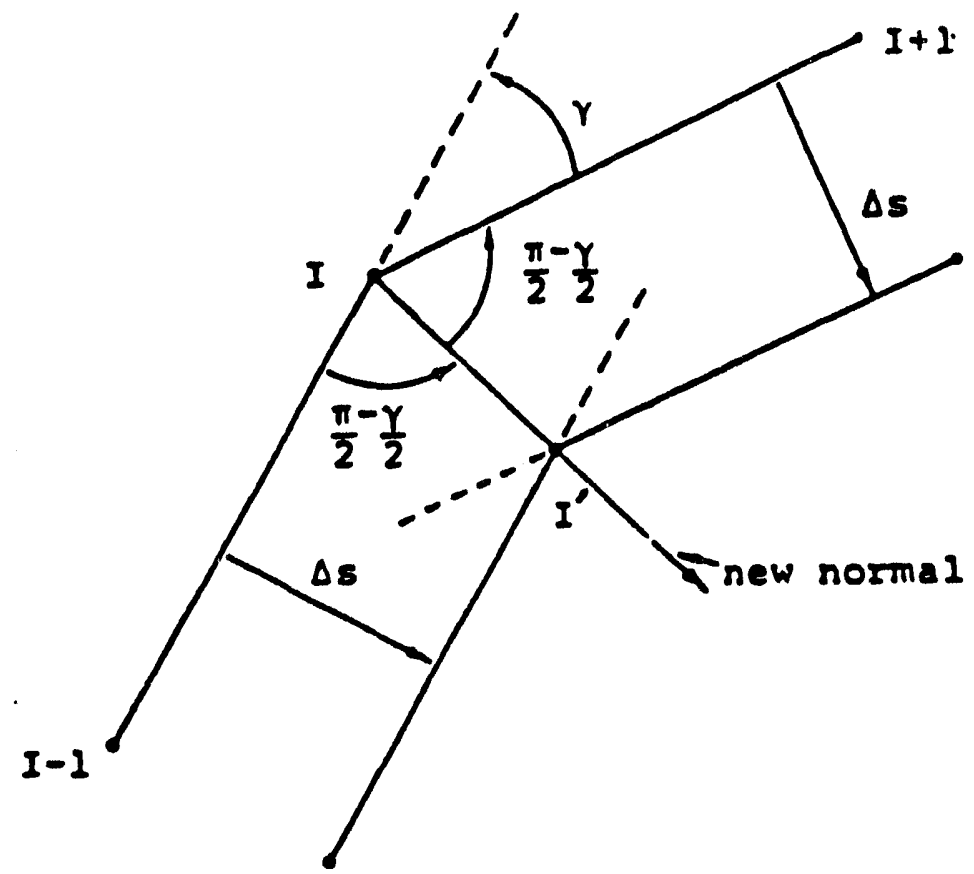

$\Delta s=\dot{s} \Delta t$

Figure 2.9 Enhanced recession for inside corner point 
After completing the above calculations, VANESA passes the calculated results for the mass and composition of the released aerosols back to the rest of CORCON-Mod3. CORCON-Mod3 uses these results to adjust the mass, composition, and enthalpy of each layer. The fission product content of each layer is also adjusted in order to correct the decay power generation for fission product release during the timestep. It should be noted that changes to the gas composition resulting from the vaporization reactions treated by VANESA (see discussion below) are not passed back to CORCON. As a result, the VANESA gas composition reported in the output is slightly different from the gas composition reported by the rest of CORCON.

The VANESA models for aerosol generation, radionuclide release, and water pool scrubbing are briefly described below. The reader is referred to Reference 10 for a more detailed discussion of the models.

\section{Model for Vaporization Release}

Vaporization is the most important of the release mechanisms. This is especially true during the early phases of the interaction when the temperatures are the highest and the oxygen potential of the core debris is the lowest. The VANESA model includes both a thermochemical analysis of vaporization and an analysis of the kinetic factors which may prevent the equilibrium limit from being reached.

Vapor formation processes can be quite complex. Consider, for example, the formation of vapor from a condensed phase species $\mathrm{MO}_{\mathrm{x}}$. Vapor formation may occur by unary vaporization, which is described by the following simple stoichiometry:

$$
\left[\mathrm{MO}_{\mathrm{x}}\right] \rightleftarrows \mathrm{MO}_{\mathrm{x}}(\mathrm{g})
$$

Vaporization may also result from reactions between the condensed phase and the adjacent gas phase. For example, the following reaction stoichiometries may occur:

$$
\begin{aligned}
{\left[\mathrm{MO}_{\mathrm{x}}\right]+\mathrm{H}_{2} \mathrm{O} } & \rightarrow \mathrm{MO}_{\mathrm{x}+1}(\mathrm{~g})+\mathrm{H}_{2} \\
{\left[\mathrm{MO}_{\mathrm{x}}\right]+\mathrm{xH}_{2} } & \rightarrow \mathrm{M}(\mathrm{g})+\mathrm{xH}_{2} \mathrm{O}
\end{aligned}
$$

A general reaction stoichiometry for vaporization into a steam/hydrogen atmosphere can be written as

$$
\left[\mathrm{MO}_{x}\right]+(w-x) \mathrm{H}_{2} \mathrm{O} \rightarrow \mathrm{MO}_{w} \mathrm{H}_{y}+(w-x-y / 2) \mathrm{H}_{2} .
$$

For this reaction, the equilibrium pressure of the vapor species $\mathrm{MO}_{\mathrm{w}} \mathrm{H}_{\mathrm{y}}$ is given by:

$$
\frac{-\Delta G(\mathrm{rxn})}{\mathrm{R}_{\mathrm{o}} \mathrm{T}}=\ln \left\{\frac{\mathrm{P}^{*}\left(\mathrm{MO}_{w} \mathrm{H}_{\mathrm{y}}\right)\left[\mathrm{P}^{*}\left(\mathrm{H}_{2}\right)\right]^{\left(w-x-\frac{y}{2}\right)}}{\mathrm{X}^{*}\left(\mathrm{MO}_{\mathrm{x}}\right)\left[\mathrm{P}^{*}\left(\mathrm{H}_{2} \mathrm{O}\right)\right]^{(w-x)}}\right\}
$$

where $P^{*}(i)$ and $X^{*}(i)$ are given by:

$$
{ }^{*}(i)=P(i) \phi(i) \text { and } X^{*}(j)=x(j) \gamma(j)
$$

where $P(i)$ and $\phi(i)$ are the partial pressure and fugacity coefficient for vapor species $i, x(j)$ and $\gamma(j)$ are the mole fraction and activity coefficient for condensed phase species $j$, and $\Delta G(r \times n)$ is given by

$$
\begin{aligned}
\Delta G(r x n)= & \Delta G_{r}\left(M_{w} H_{y}\right)-\Delta G_{f}\left(M_{x}\right) \\
& +(w-x-y / 2) \Delta G_{r}\left(H_{2}\right) \\
& -(w-x) \Delta G_{r}\left(H_{2} O^{\prime}\right)
\end{aligned}
$$

and $\Delta G_{f}(i)$ is the free energy of formation for species $i$.

Expressions of this type must be written for each of the vapor species involved in the vaporization process. The event of vaporization of the condensed phase species, $\mathrm{MO}_{\mathrm{x}}$, is then determined by the partial pressures of all vapor species composed of element $M$. As is shown in the above equation, vaporization release is a strong function of temperature and composition of evolved gases. Because the rising bubbles provide the surface area available for vaporization, release depends also on the gas flow rate through the melt.

The current implementation of VANESA assumes that the fugacity coefficients for all vapor phase species are equal to 1.0. In other words, the vapor phase is assumed to be ideal. Non-idealities are, however, considered for the condensed phase. The code calculates the activity coefficients of metallic species using a subregular solution model. For oxidic species, an associated solution model is used.

In the subregular solution model, the activity coefficient of the metal phase constituents is determined from

$$
\begin{aligned}
\ln \gamma_{i} & =\frac{1}{R_{0} T}\left\{G^{x s}+\frac{\partial G^{x s}}{\partial x_{i}}\right. \\
& \left.-\sum_{k=1}^{N} x_{k} \frac{\partial G^{x s}}{\partial x_{k}}\right\}
\end{aligned}
$$




$$
G^{x}=\sum_{i=1}^{N-1} \sum_{j=i+1}^{N} \frac{x_{i} x_{j}}{x_{i}+x_{j}}\left[W_{i j} x_{i}+h_{i j} x_{j}\right]
$$

where $W_{i j}$ and $h_{i j}$ are the interaction parameters that describe the chemical interaction between metallic constituents $i$ and $j$. The interaction coefficients are assumed to be linear functions of temperature; therefore two parameter values are needed to specify each $i, j$ coefficient. Tabulated values for these parameters are included in BLOCK DATA INTCOF. The tabular entry was simplified by making use of the following relationships between $W_{i j}$ and $h_{i j}$ :

$$
W_{i j}(T)=h_{j i}(T) \text { and } h_{i j}(T)=W_{j i}(T)
$$

Thus, it was only necessary to enter values into the upper (or lower) diagonal of each array.

The associated solutions model for the oxide phase has not yet been completed. It takes into account a richer chemistry with more constituents than are currently treated in VANESA. The chemical equilibrium of the oxide phase is solved and the associated solution model applied to this richer chemistry. Effective activity coefficients for the species treated in VANESA are then calculated and returned to VANESA. Although the data necessary to use the model are not yet completely available, the model has been formulated and the framework for its solution is included in VANESA. Until the implementation is complete, CORCON-Mod3 should be run with the user flag IDEAL set equal to 1 . The model is briefly described below.

The associated solution model adopted for the oxidic phase is based on a model suggested originally by Bottinga and Richert. ${ }^{63}$ In this model, the chemical potentials of species in the system are derived by assuming regular solution interactions between pairs of species, and recognizing that the molar volumes of the constituents can be very different. Given this basis, the following equation is derived:

$$
\begin{aligned}
\mu_{k}= & \mu_{k}+R_{o} T\left\{\ln \psi_{k}+\sum_{i=1}^{N} \psi_{i}\left[1-\frac{v_{k}}{v_{i}}\right]\right. \\
& +v_{k} \sum_{i=1}^{N} \sum_{j=1}^{N}\left\{A_{k j}-\frac{A_{i j i}}{2}\right\} \psi_{i} \psi_{j}
\end{aligned}
$$

where $\mu_{k}^{\circ}$ is standard state of the pure liquid species $k$, $\psi_{k}$ is the volume fraction of species $k$ in the mixture, $v_{k}$ is the molar volume of species $k$ in the liquid phase, and
$A_{k j}$ is the interaction coefficient for species $k-j$ interactions. The volume fraction of species $k$ is determined from

$$
\psi_{k}=\frac{N_{k} v_{k}}{\sum_{i=1}^{N} N_{i} v_{i}}
$$

where $N_{i}$ is the number of moles of $i$ in the mixture. Equation (151) can be written in terms of the activity coefficient:

$$
\begin{aligned}
\ln \gamma_{k} & =\ln \left(\frac{v_{k} \sum_{j=1}^{N} N_{j}}{\sum_{i=1}^{N} N_{i} v_{i}}\right) \\
& +\sum_{i=1}^{N} \psi_{i}\left[1-\frac{v_{k}}{v_{i}}\right] \\
& +\frac{v_{k}}{R_{0} T} \sum_{i=1}^{N} \sum_{j=1}^{N}\left[A_{k j}-\frac{A_{i j}}{2}\right] \psi_{i} \psi_{j}
\end{aligned}
$$

Tables of the oxide phase interaction coefficients, $A_{i j}$, will be included in a later update.

The VANESA model also considers kinetic limitations to the vaporization release. The model treats the following three processes:

- Transport of the volatile constituent of the condensed phase to the free surface.

- Conversion of the condensed phase constituents to the vapor phase

- Gas phase mass transport of vapor species away from the free surface

The following equation for the molar flux is solved for each vapor species:

$$
\frac{1}{A} \frac{d N_{j}}{d t}=K_{e f f, j}\left(P_{e q j}-P_{j}\right)
$$

where the $K_{e f r j}$ is the effective rate constant for formation of vapor species $\mathrm{j}, \mathrm{P}_{\text {eqj }}$ is the equilibrium partial pressure of $j$ over the condensed phase, and $P_{j}$ is the partial pressure of $j$ in the bulk gas phase. The effective rate constant, $\mathrm{K}_{\mathrm{ef} \mathrm{j}}$, is given by 


$$
K_{e f f j}=\frac{1}{\frac{P_{e q, j}}{K_{m, i} \rho_{\text {moler }} x_{i}}+\frac{1}{K_{v, j j}}+\frac{R_{0} T}{K_{e j}}}
$$

where $K_{m, 1}$ is the rate constant for condensed phase mass transport, $p_{\text {molar }}$ is the molar density of the condensed phase, $x$, is the mole fraction of constituent $i$ in the bulk condensed phase, $K_{v, j}$ is the vaporization rate constant, and $K_{w}$ is the gas phase mass transport coefficient of vapor species $\mathrm{j}$. In this equation, the subscripts $i$ and $j$ refer to the condensed and vapor phases, respectively.

The rute constant for condensed phase mass transport is calculated in the VANESA model using

$$
K_{m, i}=\frac{S h D_{i}}{d}
$$

where Sh is the Sherwood number, $D_{i}$ is the condensed phase diffusion coefficient, and $d$ is the diameter of the rising bubbles. The condensed phase diffusion coefficient is determined using the Scheibel modification of the Wilke-Chang ${ }^{65}$ correlation:

$$
D_{1}=8.2 \times 10^{-10} \mathrm{~T}\left[\frac{1+\left(\frac{3 v_{L}}{v_{1}}\right)^{2 / 3}}{\mu_{L} v_{i}^{1 / 3}}\right]
$$

where $v_{L}$ is the molar volume of the condensed phase, $v_{i}$ is the molar volume of $i$, and $\mu_{L}$ is the viscosity of the condensed phase. The Sherwood number is determined from one of several different correlations depending on the bubble geometry (e.g., size, shape, etc.). For a discussion of these correlations the reader is referred to Reference 10.

The vaporization rate constant is calculated using a form of the Hertz-Knudsen equation. ${ }^{\infty 6}$ In this model, $K_{v, l j}$ is determined from

$$
K_{v, i j}=\frac{\alpha_{1}}{\sqrt{2 \pi M_{i} R_{0} T}}
$$

where $\alpha_{i}$ is the condensation coefficient (currently set equal to 1.0 in the code), $M_{1}$ is the molecular weight of species $i$, and $T$ is the vapor phase temperature (assumed equal to the temperature of the melt).

The gas phase mass transport coefficient is calculated from

$$
K_{\mathrm{Rj}}=\frac{2 D_{j}}{d_{c}}
$$

where $D_{j}$ is the gas phase diffusion coefficient for vapor species $j$, and $d_{c}$ is the equivalent spherical bubble diameter. The gas phase diffusion coefficient is calculated using the equation proposed by Singh and Singh: ${ }^{67}$

$$
D_{j}=\frac{0.00279 T^{1.62}\left(\frac{M_{j}+M_{l}}{M_{j} M_{l}}\right)^{1 / 2}}{P\left(v_{i}^{1 / 3}+v_{l}^{1 / 2}\right)^{2}}
$$

where $M_{1}$ and $M_{8}$ are the molecular weights of species $j$ and the gas phase mixture, $v_{j}$ and $v_{k}$ are the molar volumes of species, $\mathrm{J}$ and the gas mixture, and $\mathrm{P}$ and $\mathrm{T}$ are the pressure and temperature of the gas phase.

\section{Model for Mechanical Release}

Mechanical generation of aerosols by entrainment and bubble bursting is important late in a reactor accident, when the core debris temperature has fallen such that aerosol generation by vaporization is minimal. Both droplet entrainment and bubble bursting are modeled in the code.

Droplet entrainment is modeled using a correlation developed by Kataoka and Ishii: ${ }^{60}$

$$
E^{*}=0.002\left(j_{k}^{*}\right)^{3}\left[N\left(\mu_{t}\right)\right]^{1 / 2}\left\{\frac{\rho_{1}-\rho_{B}}{\rho_{k}}\right\}
$$

where $E^{*}$ is the dimensionless entrainment flux

$$
E^{*}=\frac{\rho_{1} j_{1}}{\rho_{2} j_{2}}
$$

$\mathrm{j}_{\mathrm{k}}^{*}$ is the dimensionless gas velocity

$$
\mathrm{j}_{\mathrm{k}}^{*}=\frac{\mathrm{j}_{\mathrm{s}}}{\left[\frac{\sigma_{1} \mathrm{~g}\left(\rho_{1}-\rho_{\mathrm{s}}\right)}{\rho_{\mathrm{l}}^{2}}\right]^{1 / 4}}
$$




$$
N\left(\mu_{2}\right)=\frac{\mu_{8}}{\left.\frac{\rho_{8} \sigma_{1}^{3 / 2}}{g^{1 / 2}\left(\rho_{1}-\rho_{8}\right)^{1 / 2}}\right]^{1 / 2}}
$$

The VANESA model calculates aerosol generation by bubble bursting using the following correlation by Axbel: ${ }^{\circ}$

$$
E=\frac{3 K_{1}}{2 \pi \rho_{2} d_{b}}\left\{\frac{\text { NUMER }}{\text { DENOM }}\right\}^{1 / 2}
$$

where NUMER is

$$
\begin{aligned}
\text { NUMER } & =\left[1-\frac{d_{b}^{2}}{2 K_{2}}+\frac{9}{16} \frac{d_{b}^{4}}{K_{2}^{2}}\right]^{1 / 2} \\
& +\left[\frac{d_{b}^{2}}{4 K_{2}}-1\right]
\end{aligned}
$$

\section{DENOM is}

$$
\begin{aligned}
\text { DENOM } & =1+\frac{3}{4} \frac{d_{b}^{2}}{K_{2}} \\
& -\left[1-\frac{d_{b}^{2}}{2 K_{2}}+\frac{9}{16} \frac{d_{b}^{4}}{K_{2}^{2}}\right]^{1 / 2}
\end{aligned}
$$

the dimensionless entrainment volume, $\mathrm{E}$, is

$$
E=\frac{\rho_{1} V_{a}}{\rho_{2} V_{b}}
$$

where $V_{a}$ and $V_{b}$ are the volume of the entrained serosol and bubble, $K_{1}$ and $K_{2}$ are

$$
\begin{aligned}
& K_{1}=\frac{1.15 \pi \sigma_{1}}{c^{2}} \\
& K_{2}=\frac{6 \sigma_{1}}{g\left(\rho_{1}-\rho_{8}\right)}
\end{aligned}
$$

$d_{b}$ is the diameter of the bubble, and $c$ is the speed of sound in the gas.

If the user chooses to bypass the VANESA calculation, but still wishes to consider the effects of aerosol generation on radiant heat loss from the upper debris surface, the empirical correlation used in CORCON-Mod2 is used. This correlation is given by: ${ }^{11 d}$

$$
[A]=\left(33+240 j_{g}\right) \exp (-19000 \mathrm{~K} / \mathrm{T})
$$

where $[A]$ is the concentration $\left(g / \mathrm{m}^{3}\right.$ STP) of aerosol in the evolved gas, $T$ is the temperature of the melt (K), and $j_{8}$ is the superficial gas velocity through the melt ( $\mathrm{m} / \mathrm{s}$ STP). Note that both the superficial velocity and the concentration in this expression have been reduced to standard temperature and pressure (STP).

The temperature $T$ in Equation (170) is assumed to be the temperature of the top layer of the melt. The gas flow (in bubbles) through the pool is converted to a superficial volocity at STP, and the aerosol concentration is adjusted to the temperature and pressure directly above the pool, with the temperature taken as that of the top layer. If coolant is present, we assume that it is effective in scrubbing aerosols from the evolved gases, and set the concentration in the atmosphere equal to zero.

The empirical aerosol concentration is calculated solely for the purpose of determining the radiative properties of the atmosphere; no composition is calculated for it, and its mass is not charged against the pool inventory.

\subsubsection{Aerosol Removal By Overlying Water Pools}

The VANESA model also treats the decontamination of aerosol-laden gases passing through an overlying water pool. Aerosol removal is assumed to be due to the following mechanisms: sedimentation of aerosol particles within gas bubbles, impaction and adherence of aerosol particles on the bubble walls, and diffusion of aerosol particles to the bubble walls. If the water pool is subcooled, a fourth mechanism is assumed to be active: collapse of bubbles due to steam condensation in the subcooled pool. This model assumes that either the steam condenses completely immediately after the bubble forms in the subcooled water pool or that steam condensation is just complete when the bubble reaches the water-atmosphere interface.

The VANESA model for aerosol removal is based on the model formulated by Fuchs. ${ }^{\top}$ The aerosol removal rate is given by:

$$
\begin{aligned}
\frac{d m\left(d_{p}, x\right)}{d t}= & -\left[\alpha_{s}\left(d_{p}\right)+\alpha_{1}\left(d_{p}\right)\right. \\
& \left.+\alpha_{D}\left(d_{p}\right)\right] m\left(d_{p}, x\right)
\end{aligned}
$$

where $m\left(d_{p}, x\right)$ is the mass of aerosol having diameter $d_{p}$ at elevation $x$ above the debris surface; and $\alpha_{s}\left(d_{p}\right)$, 
$\alpha_{r}\left(d_{p}\right)$, and $\alpha_{D}\left(d_{p}\right)$ are the coefficients for removal by sedimentation, impaction, and diffusion.

The sedimentation coefficient is given by Fuchs: ${ }^{x}$

$$
\alpha_{s}\left(d_{p}\right)=\frac{1.5 \mathrm{~J}\left(d_{p}\right)}{d_{b} U_{\text {rite }}}
$$

where $J\left(d_{p}\right)$ is given by

$$
J\left(d_{p}\right)=\frac{g \rho_{p} d_{p}^{2} C}{18 \mu_{g}}
$$

$D_{b}$ is the diameter of the bubbles, $U_{\text {ree }}$ is the rise velocity of the bubbles in the water pool, $\rho_{p}$ is the material density of the aerosol particles, $\mu_{g}$ is the viscosity of the gas, $\mathrm{C}$ is the Cunningham slip correction factor

$$
C=1+\frac{2 \lambda}{d_{p}}\left[1.257+0.4 \exp \left(\frac{-0.55 d_{p}}{\lambda}\right)\right]
$$

where $\lambda$ is given by

$$
\lambda=\frac{1}{\sqrt{2} \pi d_{2}^{2} N_{A}(P / 82.06 T)}
$$

$d_{a}$ is the diameter of a gas molecule, $P$ is the local pressure, and $N_{A}$ is Avogadro's number. The equation for the sedimentation coefficient neglects the effects of water condensation on the aerosol particles. This would increase the effective particle diameter, and thereby increase aerosol removal by sedimentation. Note, however, that this effect would be compensated to some extent by the reduction in the material density of the aerosol.

The coefficient for particle diffusion is also given by Fuchs: ${ }^{\text {To }}$

$$
\alpha_{D}\left(d_{p}\right)=1.8\left[\frac{8 \theta}{U_{\text {rise }} d_{b}^{3}}\right]^{1 / 2}
$$

where $\theta$ is given by

$$
\theta=\frac{k T C}{3 \pi \mu_{z} d_{p}}
$$

and $k$ is Boltzmann's constant. The equation for the particle diffusion coefficient neglects the effect of steam production on the bubble surface. A flux of steam from the surface would, of course, reduce diffusion of particles to the surface.

The impaction coefficient describes loss of aerosol from the bubbles due to impaction on the bubble wall. This occurs because the particles cannot stay within the internally circulating gas flow. This mechanism is affected by many different processes; consequently, many of the correlations in the literature are quite complex. The VANESA model uses the relatively simple correlation shown below:

$$
\alpha_{l}\left(d_{p}\right)=\frac{8 U_{\text {rime }} \tau}{d_{b}^{2}}\left[\frac{U_{\text {rel }}}{U_{\text {rlec }}}\right]^{2}
$$

where $\tau$ is given by

$$
\tau=\frac{\rho_{p} d_{p}^{2} C}{18 \mu_{s}}
$$

and $U_{\text {rel }}$ is the relative velocity of the particles. The ratio $U_{\text {rel }} / U_{\text {rime }}$ is a parameter that depends on many variables such as bubble shape, internal circulation velocity, etc. The user may supply a value through input or may select the default value of 1.5. Values from 1.5 to 4.2 are reasonable. (The upper end of this range corresponds to spherical cap bubbles. For spherical bubbles, the geometrical basis for the impaction model is probably invalid.) Figure 2.12 illustrates the sensitivity of the pool decontamination factor (DF) to variations in this ratio.

As the above equations illustrate, the aerosol removal is sensitive to many factors including: particle size, bubble size, bubble rise velocity, and pool depth. The sensitivities of the calculated pool DF to these variables are shown in Figures 2.10 through 2.13.

The figures show a significant dependence of the pool DF on the size of the aerosol particles. Small particles $(<1 \mu \mathrm{m})$ are trapped efficiently because they diffuse quickly to the bubble wall. Large particles ( $>1 \mu \mathrm{m}$ ) are trapped efficiently because of efficient sedimentation and impaction. It is important, therefore, that the code use accurate models for calculating the particle diameter of the aerosols. These models are described below.

Aerosol formation and growth processes are extremely complex. Important processes include: homogeneous 


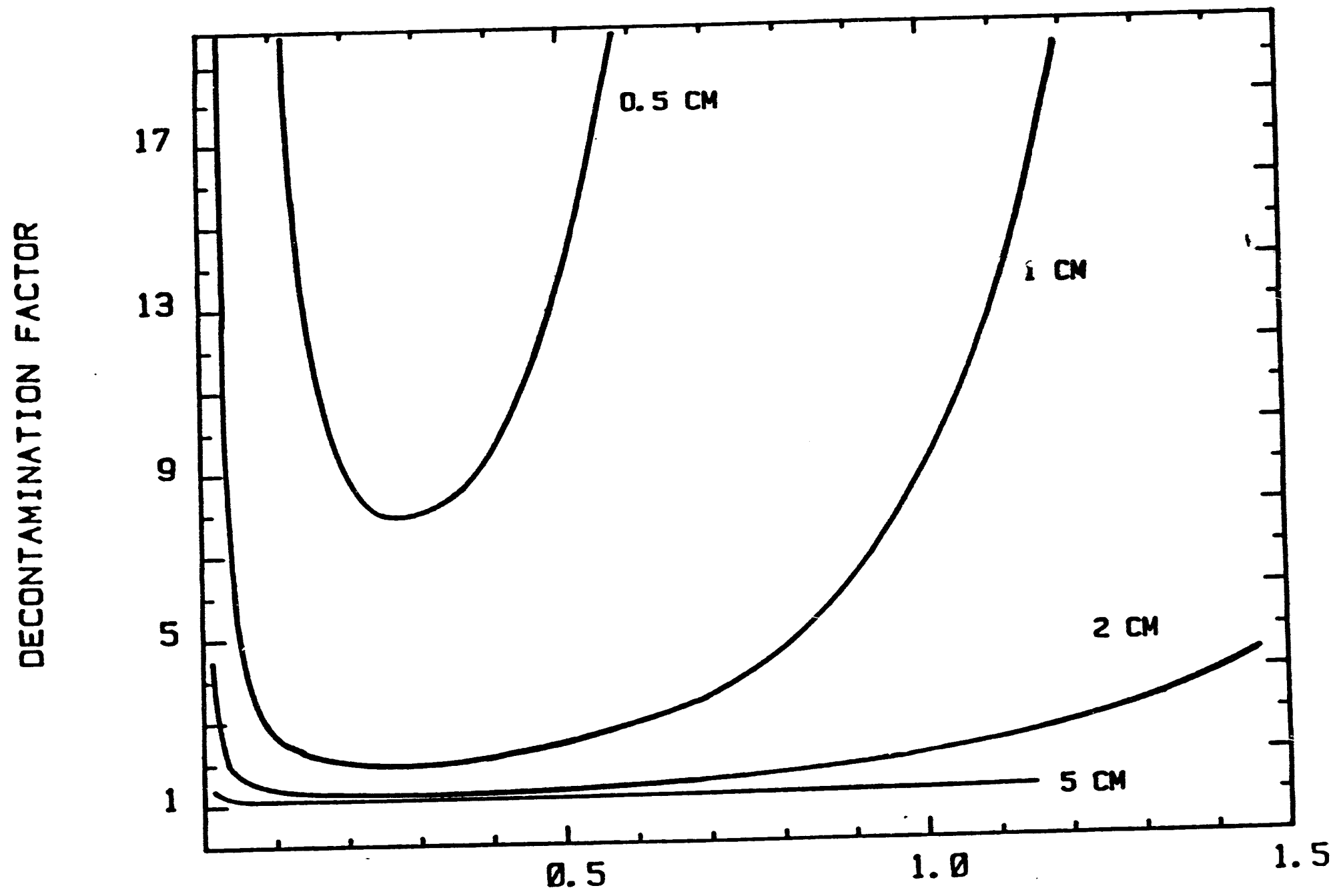

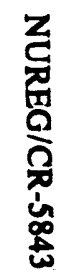




\section{Model Descriptions}

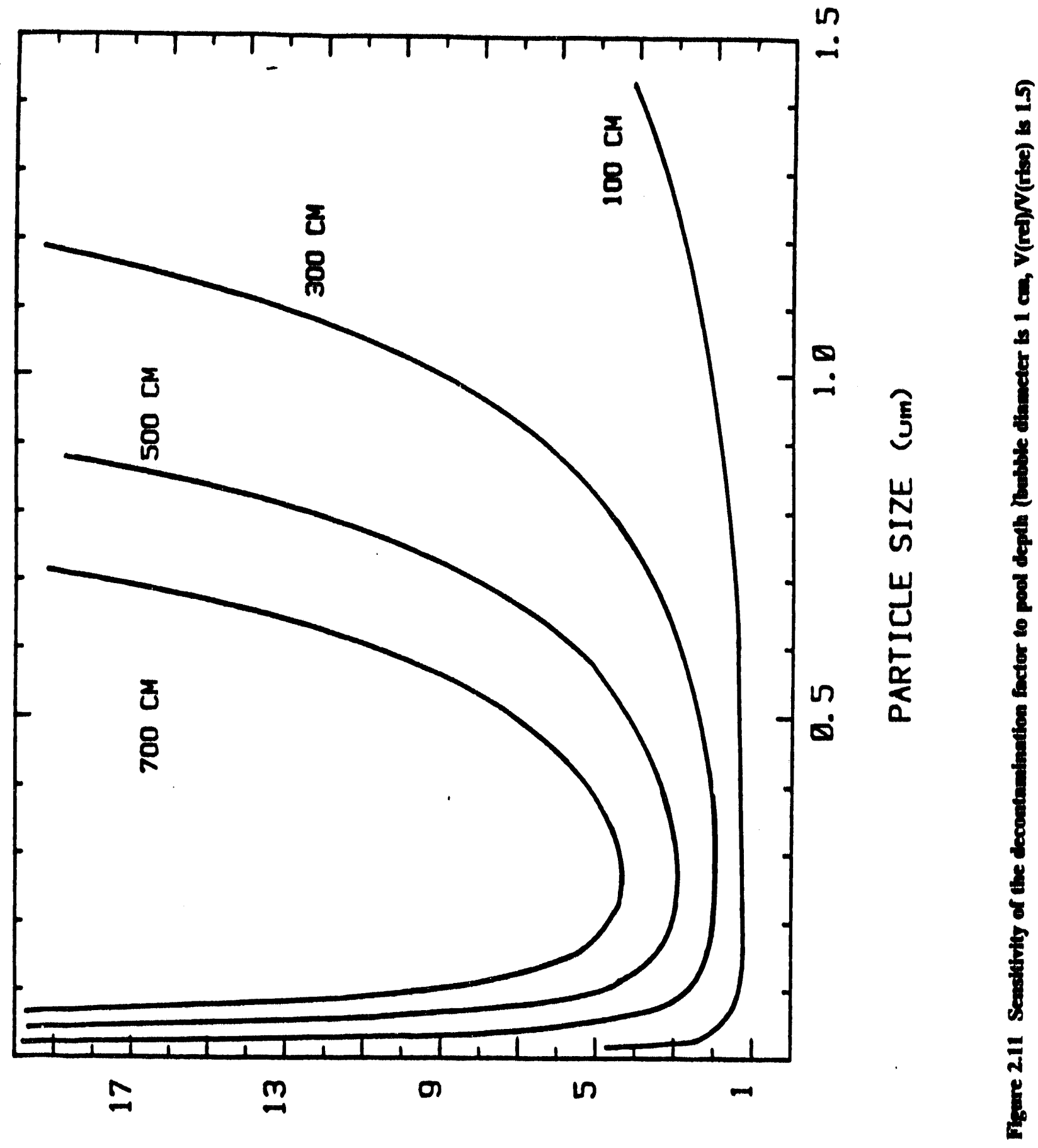

YOLJVA NOI $Y N I W Y \perp N O J 30$ 


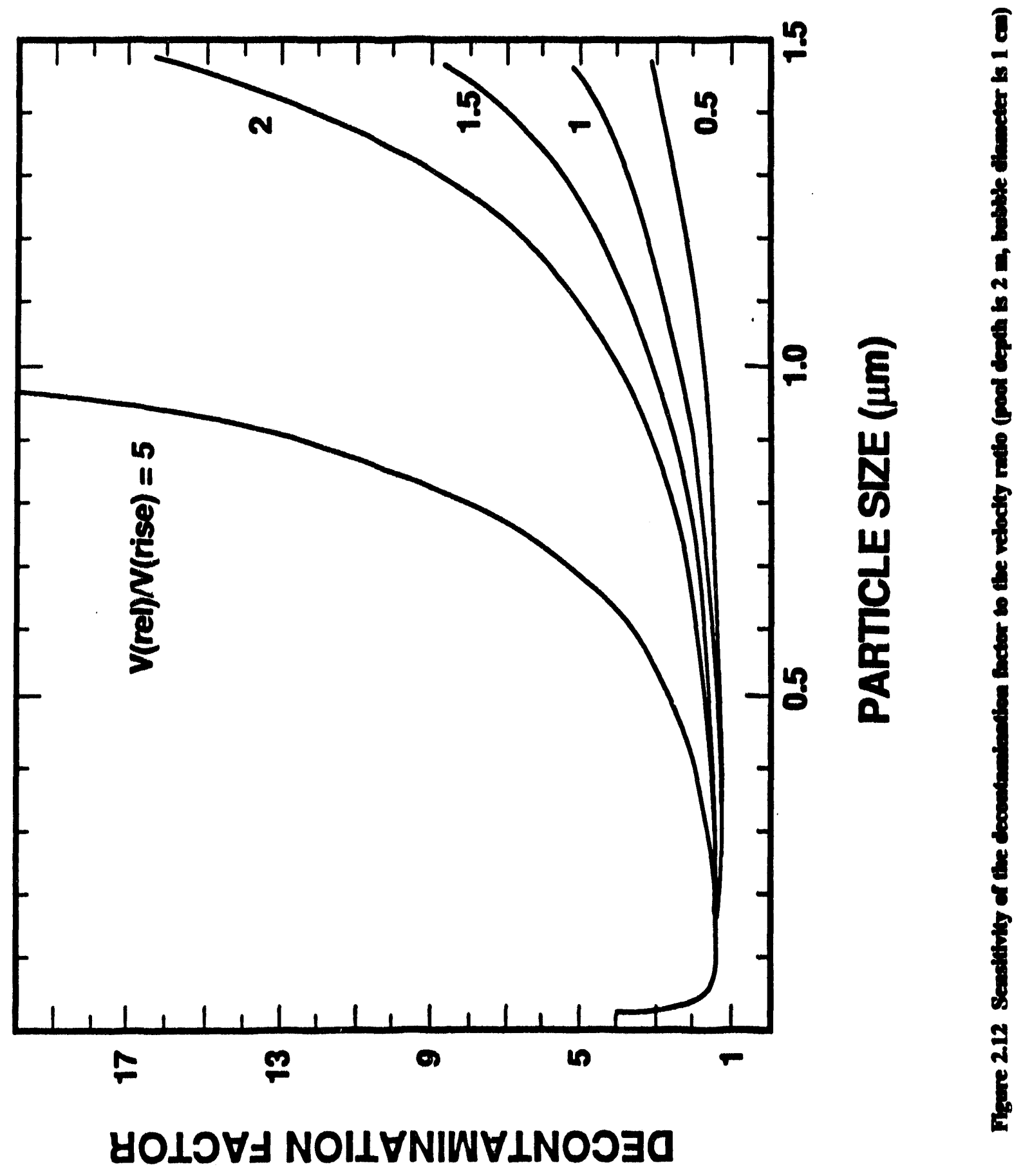




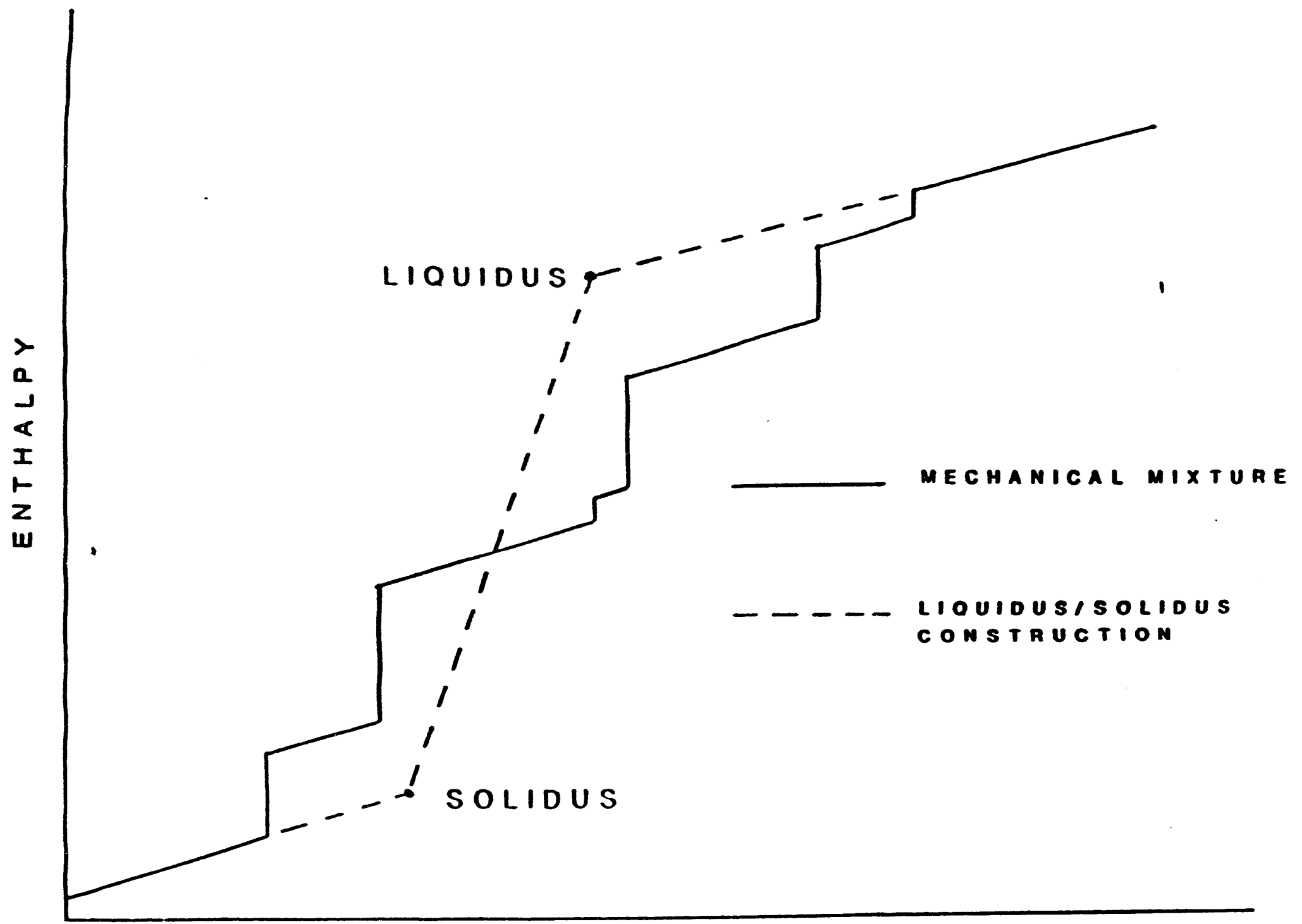

TEMPERATURE

Figure 2.13 Two-phase construction for mbxture 
nucleation in the vapor phase, condensation of vapor on nucleated particles and other surfaces, and coagulation of particles in the vapor stream. The VANESA manual ${ }^{10}$ provides an excellent summary of the models proposed to analyze these processes.

Given the complexity of the required models and the uncertainty associated with these models, the developers of the VANESA model decided to adopt an empirical approach to the calculation of the particle size. This model begins with the assumptions that a.) particle size is dictated by condensation of vapors on nucleated and coagulated particles, and $b$.) the temperature of the gas stream is low enough that essentially all of the vapor will condense. Given these assumptions, the mean particle size is

$$
d_{p}=\left[\frac{6 \mathrm{~A}}{\pi \rho_{p} n(t)}\right]^{1 / 3}
$$

where $A$ is the initial mass concentration of the condensing vapors ( $\left.\mathrm{g} / \mathrm{m}^{3} \mathrm{gas}\right), \rho_{\mathrm{p}}$ is the material density of the particles $\left(\mathrm{g} / \mathrm{cm}^{3}\right)$, and $n(t)$ is the number concentration of the particles (particles $/ \mathrm{cm}^{3}$ ). Based on experimental results which indicate a mass-weighted mean particle size of $1.2 \mu \mathrm{m}$ at vapor concentration of 50 to $150 \mathrm{~g} / \mathrm{cm}^{3}$, the number density of the aerosol particles is estimated to be $10^{8}$ particles $/ \mathrm{cm}^{3}$. Thus, the mean particle size in micrometers is calculated by

$$
d_{p}=0.266\left[\frac{A}{\rho_{p}}\right]^{1 / 3}
$$

For calculations, such as pool decontamination, that require a particle size distribution, a log normal distribution is assumed with a geometric standard deviation of 2.3. (The user may specify alternate values for the particle size and/or geometric standard deviation if desired.)

The aerosol scrubbing model divides the supplied aerosol size distribution into size classes containing equal fractions of the total mass. The upper limit to the particle size in each class is determined from

$$
\frac{1}{N}=0.5\left[1+\operatorname{erf}\left[\frac{\ln \left(D_{i+1} / \mu\right)}{\sqrt{2} \ln (\sigma)}\right]\right)
$$

where $\mathbf{N}$ is the number of size segments, $D_{i+1}$ is the upper limit to the size in class $i, \mu$ is the mean particle size, and $\sigma$ is the geometric standard deviation. Within each class, the characteristic size is determined such that half the mass lies above the characteristic size and half lies below.

The code solves for the aerosol removal as a function of position in the pool, and integrates the position-dependent removal to determine the total aerosol removed from the gas stream. Since particle removal is size dependent, the code tracks the changes in the size distribution with position in the water pool.

\subsection{Material Properties}

Calculation of the physical processes described in the preceding sections requires values for a wide range of thermodynamic and transport properties. These properties are treated as functions of composition and of temperature. However, the dependence is not always explicitly included in the CORCON-Mod3 model; for example, the values used for surface tensions are independent of temperature.

In most cases, properties are required for the mixtures of species which make up the components of the CORCON system, although the calculation of chemical equilibrium requires the chemical properties of the individual species. With a few exceptions, such as the viscosity of oxide mixtures with large silica contents, mixture properties are calculated from those of the constituent species.

The property models described below are the same as those used in CORCON-Mod2 with a few exceptions. A user option for specifying the phase diagrams of the metallic and oxidic phases is now provided in CORCON-Mod3. Also, properties used by the

CORCON-Mod3 implementation of the VANESA model have been included.

\subsubsection{Thermodynamic Properties}

The thermodynamic properties calculated are density, specific heat, enthalpy, and chemical potential.

Density - $\rho\left(\mathrm{kg} / \mathrm{m}^{3}\right)$

Condensed phases:

Mixture densities are computed from

$$
\rho_{m}=10^{3} m_{m} / \Sigma\left(m_{i} v_{i} / M_{i}\right)
$$

where the molar volumes of the individual species are given by 
Model Descriptions

$$
v_{i}=v_{i}^{0}\left[1+c_{i}(T-1673 \mathrm{k})\right] .
$$

Values for $v_{i}^{0}$ and $c_{i}$ applicable to the liquid phase are stored in subroutine DENSTY for all condensed species. ${ }^{7-73}$ The temperature range of the data from which this relationship was generated varies considerably for the different species. For many of the oxides, it is from 1200 to $1800^{\circ} \mathrm{C}$, while for others it covers the entire range from melting to boiling. However, Equation (184) is applied at all temperatures. This disregards the change in density which accompanies freezing.

Separate tables of densities and molecular weights are stored (in BLOCK DATA XNDAR) and used in the VANESA model. The tables include values for hoth condensed phase species and gas phase species. For the species considered by VANESA, the molar volume is calculated from these values by

$$
v_{i}=\frac{M_{1}}{\rho_{1}}
$$

where $M_{i}$ is the molecular weight of species $i$. The molecular weight of a mixture is calculated by molar weighing of the species molecular volumes.

\section{Gaseous phase:}

Densities are computed from the ideal gas relationship

$$
\rho_{m}=10^{3} \mathrm{pM}_{m} / R_{n} \mathrm{~T}
$$

where $M_{m}$ is the mixture molecular weight:

$$
M_{m}=\sum_{i} x_{i} M_{i}
$$

\section{Specific Heat $-c_{p}(J / k g ~ K)$}

The specific heat of any species, condensed or gaseous, is represented in the form

$$
\begin{aligned}
c_{p_{1}} & =A_{i}+10^{-3} B_{i} T+10^{-6} C_{i} T^{2} \\
& +10^{5} D_{i} / T^{2} .
\end{aligned}
$$

Within the calculation, units are $\mathrm{cal} / \mathrm{mol} \mathrm{K}$, reflecting the original data sources; a conversion to S.I. (J/kg K) is made before return from the calculational package. Values of the constants $A_{i}, B_{i}, C_{i}$, and $D_{i}$ are tabulated in subroutine CONFND, with one or more temperature ranges for each species. A single range is used for all gaseous species, and the fits are valid from 298.15 to $6000 \mathrm{~K}$. The fits for condensed species include both the liquid and one or more solid phases: in some cases, two ranges are defined for a single phase. Thus, each species requires from two to five sets of coefficients. The upper limit of validity is above $2500 \mathrm{~K}$ in most cases, although it is as low as $2000 \mathrm{~K}$ in others. ${ }^{n-n}$

In any case, the data for the highest temperature range included are used for all temperatures above the minimum for this range. Mixture specific heats are computed by mass averaging as

$$
c_{p_{e}}=4186.8 \sum \sum_{i}\left(m_{1} c_{p_{1}}\right) / m_{m} .
$$

where the constant provides the conversion to S.I. units. Specific heats are computed in subroutine CPENTH.

$$
\text { Enthalpy - h }(\mathrm{J} / \mathrm{kg})
$$

Species enthalpies are computed from integrals of the corresponding specific heats. The conventional thermochemical reference point of separated elements in their standard states $\left(25^{\circ} \mathrm{C}, 1 \mathrm{~atm}\right)$ is employed; i.e., the enthalpies correspond to the JANAF tables. The result may be expressed in the form

$$
\begin{aligned}
h_{i}(T)= & h_{i}\left(T^{\prime}\right)+\int_{T^{\prime}}^{T} d T^{\prime} c_{p_{i}}\left(T^{\prime}\right) \\
= & K_{h_{i}}^{k}+A_{i}^{k}\left(T-T_{i}^{k}\right) \\
& +10^{-3} B_{i}^{k}\left(T^{2}-T_{i}^{k}\right) / 2 \\
& +10^{-6} C_{i}^{k}\left(T^{3}-T_{i}^{k 3}\right) / 3 \\
& -10^{5} D_{i}^{k}\left(1 / T-1 / T_{i}^{k}\right)
\end{aligned}
$$

for $T$ in the kth temperature range for species i (i.e., $T_{i}^{k}$ $\left.<\mathrm{T}<\mathrm{T}_{1}^{k+1}\right)$. The $\left\{\mathrm{T}_{1}^{k}\right\}$ are the break points in the specific heat fits. The first is $298.15 \mathrm{~K}$ and the last is treated as infinite. Thus, the first integration constant, $K_{\mathrm{hi}}^{1}$, is the standard enthalpy of formation of species $i$. Any others required are computed internally, subject to the condition that the discontinuity in enthalpy at $T_{1}^{k}$ be the appropriate heat of transition, $\Delta \mathrm{H}_{\mathrm{i}, \mathrm{TR}}^{\mathrm{k}}$, if a phase change occurs at $T_{1}$, and zero otherwise. Subroutine CONFND contains tables of these heats of formation and of transition. The integration constants are evaluated internally during an initial call to CONFND from routine SETUP in order to assure full-word accuracy on machines of different word length. This is done to avoid the possibility of negative discontinuities in $h(T)$, which 
could cause numerical problems. At this time, the discontinuities in enthalpy are also replaced by smooth transitions over an arbitrary temperature range of $10 \mathrm{~K}$. As with specific heat, the enthalpy of a mixture is computed by mass averaging:

$$
h_{m}(T)=4186.8 \sum_{i}\left[m_{1} h_{1}(T)\right] / m_{m}
$$

Mass averaging of specific heats and enthalpies is really appropriate only for mechanical mixtures in which each species is unaffected by the others. Because of mutual solubilities, actual mixtures of metallic or oxidic species form one or more phases. The principal effect is a change in melting behavior; rather than the individual species moriing independently at their own melt points, the entire mixture melts over a range of temperatures.

We account for this effect--at least partially--in CORCON-Mod3. The melting range, defined by the liquidus and solidus temperatures, is prescribed by external models for mixtures of condensed species. Specific heat and enthalpy are calculated in three ranges:

1. For $T<T^{\prime}$, the calculation is as described above with the exception that extrapolated solid properties are used for any species which would ordinarily be liquid. This is simply accomplished by ignoring the presence of the sets of coefficients and integration constants for temperature ranges in which the species is liquid, so that the last set describing a solid continues to be used above the melting point.

2. For $T^{\ell}<T$, extrapolated liquid properties are used for any species which would ordinarily be solid by ignoring the presence of data for the solid.

3. For $T^{\prime}<T<T^{\prime}$, linear interpolation in enthalpy is used between the liquidus and solidus. The constant slope is returned as the specific heat. The effect of this construction is indicated in Figure 2.13.

Enthalpies are computed in subroutine CPENTH.

Chemical Potential - g (J/kg K)

Chemical potentials for the species are required in the calculation of chemical equilibrium and are computed from the molar Gibbs function

$$
g_{i}=h_{i}-T s_{i}
$$

where $s$ is the entropy and may be computed at temperature $T$ and at the reference pressure of one atmosphere from

$$
\begin{aligned}
S_{i}(T)= & S_{1}\left(T^{\prime}\right)+\int_{T^{\prime}}^{T} d T^{\prime} c_{p_{1}}\left(T^{\prime}\right) / T^{\prime} \\
= & K_{i j}^{k}+A_{i}^{k} \ln \left(T / T_{1}^{k}\right)+10^{-3} B_{1}^{k}\left(T-T_{1}^{k}\right) \\
& +10^{-6} C_{i}^{k}\left(T^{2}-T_{1}^{12}\right) / 2 \\
& -10^{5} D_{1}^{k}\left(1 / T^{2}-1 / T_{1}^{12}\right) / 2
\end{aligned}
$$

for $T$ in the $k$ th temperature range for species $i$ (i.e., $T_{i}$ $\left.<T<T_{l}^{k+1}\right)$. The first integration constant, $K_{s i}^{!}$, is the standard entropy of formation of species $i$ and, as with the enthalpy, any others required are computed internally, subject to the condition that the discontinuity in enthalpy at $T_{1}^{k}$ be the appropriate entropy transition of $\Delta H_{1, T R}^{k} / T_{1}^{k}$, if a phase change occurs at $T_{1}^{k}$ and zero otherwise. Again, subroutine CONFND contains the necessary data tables and calculates integration constants during the initial call from SETUP. The chemical potentials, evaluated in subroutine CHEMPO, are used only as internal variables in the chemical equilibrium subroutine MLTREA, which employs chemists' units of cal/mole. Therefore, no conversion to S.I. is made. MLTREA itself converts the standard chemical potentials for pure gases at one atmosphere pressure to mixture conditions using the ideal gas result

$$
g_{i}(p, T)=g_{i}^{0}(p, T)+R_{0} T \ln \left(p_{i}\right)
$$

where $p_{1}$ is the partial pressure of gaseous species $i$. No pressure correction is made for condensed species.

\section{Eree Energy Function - fef (cal/gmole K)}

The VANESA model tabulates and uses free energy data in the form of free energy functions. The free energy functions are related to the gibbs function and enthalpy by

$$
f e f_{1}=-\frac{g_{1}(T)-h_{1}(298.15)}{T}
$$

where $h_{4}(298.15)$ is the enthalpy of species $i$ at the reference temperature, $298.15 \mathrm{~K}$. The data for the species free energy functions were fit to an equation of the following form

$$
\begin{aligned}
f e f & =a_{1,1}+a_{1,2} x+a_{1,3} x^{2}+a_{1,4} x^{3} \\
& +a_{1,5} \ln (x)+a_{1,6} / x+a_{1,7} x \ln (x)
\end{aligned}
$$


where $x=T / 10000$. The seven coefficients for each VANESA chemical species are tabulated in BLOCK DATA BARRAY.

\subsubsection{Transport Properties}

The transport properties computed in CORCON-Mod3 are the dynamic viscosity, the thermal conductivity, the surface tension, and the emissivity. Detailed models are included for condensed-phase species and mixtures only. Gas-phase viscosity and thermal conductivity (required for calculation of heat-transfer coefficients at the melt/concrete interface) are treated as constants using representative values defined in subroutine GFLMPR.

\section{Dynamic Viscosity - $\mu(\mathrm{kg} / \mathrm{m} / \mathrm{s})$}

\section{Oxidic phase:}

The viscosity of molten oxides is quite complex, particularly when significant amounts of silica $\left(\mathrm{SiO}_{2}\right)$ are present. For low-silica mixtures, the viscosity is computed from the Kendell-Monroe expression. ${ }^{2,78}$

$$
\mu_{\mathrm{m}}=\left[\underset{i}{\sum \mathrm{X}_{i} \mu_{1}^{1 / 3}}\right]^{3}
$$

The viscosities of the species are determined using an Andrade form ${ }^{n}$

$$
\mu_{i}=\mu_{i}^{\circ} \exp \left[\alpha_{i} / T\right]
$$

where the constants $\mu_{i}^{\circ}$ and $\alpha_{i}$ are determined by empirical correlation.

Values of these coefficients are included (in subroutine VISCTY) for only a limited number of species. The values for $\mathrm{FeO}, \mathrm{Al}_{2} \mathrm{O}_{3}$, and $\mathrm{UO}_{2}$ are based on published data; ${ }^{2,75}$ that for $\mathrm{CaO}$ is based on our own unpublished theoretical estimate. The values for $\mathrm{ZrO}_{2}$ and $\mathrm{Cr}_{2} \mathrm{O}_{3}$ are based on analogy with $\mathrm{UO}_{2}$ and $\mathrm{FeO}$ respectively. These are important contributors to the viscosity because they may be dominant species in either the fuel-oxide layer or the light-oxide (slag) layer. Only these species are considered in the Kendell-Monroe calculation, and the resulting composition is renormalized.

For mixtures with a higher silica content, the viscosity can be greatly increased by the formation of strongly bonded chains of $\mathrm{SiO}_{4}$ tetrahedra. The viscosity is calculated from a model proposed by Shaw. ${ }^{79}$ This was originally generated as a fit to the correlation developed by Bottinga and Weill based on geologic data, ${ }^{\infty 0}$ and was shown to give good agreement with it within the original data base. Shaw's model has an extremely simple form, for which good extrapolation properties are built in:

$$
\mu_{\mathrm{m}}=\exp \left[\mathrm{s}\left(10^{4} / \mathrm{T}-1.50\right)-6.40\right]
$$

Here the viscosity, $\mu$, is in poise ( 1.0 poise $=$ $0.1 \mathrm{~kg} / \mathrm{m}-\mathrm{s}$ ) and $\mathrm{s}$ is a function of mixture composition given by

$$
s=\left(\sum_{i} n_{i} x_{i} s_{1}^{o} / \sum_{i} n_{i} x_{i}\right) x_{\text {SiO }_{2}}
$$

Data are included in subroutine VISCTY for $\mathrm{TiO}_{2}, \mathrm{FeO}$, $\mathrm{MgO}, \mathrm{CaO}, \mathrm{Li}_{2} \mathrm{O}, \mathrm{Na}_{2} \mathrm{O}, \mathrm{K}_{2} \mathrm{O}, \mathrm{Fe}_{2} \mathrm{O}_{3}$, and $\mathrm{Al}_{2} \mathrm{O}_{3}$; these values are taken from Shaw's paper. Also included are data for $\mathrm{UO}_{2}$ and $\mathrm{ZrO}_{2}$ based on an assumed analogy with $\mathrm{TiO}_{2}$, and for $\mathrm{Cr}_{2} \mathrm{O}_{3}$ based on an assumed analogy with $\mathrm{Fe}_{2} \mathrm{O}_{3}$. Again, the composition in terms of these species is renormalized for use in Equation (199).

The Shaw model is restricted to mixtures with relatively high silica contents; it is matched to the low-silica Kendell-Monroe form by simply accepting the greater of the two values calculated from Equations (197) and (199). The transition, where the two values are equal, is typically at a composition of 20 to 30 percent silica.

The maximum viscosity is limited to that for basalt, which is computed from

$$
\mu(\text { basalt })=1.94 \times 10^{-5} \exp [20950 / \mathrm{T}]
$$

where $\mu$ (basalt) is the viscosity in $\mathrm{kg} / \mathrm{m}-\mathrm{s}$ and $\mathrm{T}$ is in Kelvins.

\section{Metallic Phase:}

We assume that the viscosity of the metallic phase can be represented by the viscosity of iron (the major constituent) as given by the expression ${ }^{2}$

$$
\mu_{\mathrm{m}}=1.076 \times 10^{-3} \exp (3313 / \mathrm{T})
$$

Coolant:

The coolant viscosity is computed from ${ }^{n}$

$$
\begin{aligned}
\mu_{\text {coolanx }} & =2.414 \times 10^{-5} 10^{\alpha} \\
\alpha & =247.8 /(\mathrm{T}-140) .
\end{aligned}
$$


Two-Phase, Solid-Liquid Slurry:

CORCON-Mod3 contains a model for the enhancement of viscosity by suspended solids, in the form

$$
\mu_{1,1}=\mu_{m}\left[\frac{1+\phi / 2}{(1-\phi)^{4}}\right]
$$

where $\mu_{\mathrm{al}}$ is the slurry viscosity, $\mu_{\mathrm{m}}$ is the viscosity of the pure liquid mixture, and $\phi$ is the volume fraction of solids.

This form was suggested by Kunitz ${ }^{n}$ for slurries containing less than 50 percent solids by volume. In CORCON-Mod3, the expression is assumed to apply up to 50 percent solids. The solids fraction is computed by linear interpolation between the mixture liquidus, $T^{\prime}$, and the mixture solidus, $T^{\prime}$, as

$$
\phi(T)=\frac{T^{\prime}-T}{T^{1}-T^{\prime}}
$$

This equation is applied whenever the temperature is between the liquidus and solidus points.

Recent experiments ${ }^{\circ}$ suggest that the slurry viscosity predicted using the above equation may be significantly smaller than the true viscosity of prototypic mixtures of fuel and concrete oxides. However, these are only preliminary results, so we have made no code modifications in this area.

\section{Thermal Conductivity - $k(\mathrm{~W} / \mathrm{m} \mathrm{K})$}

Values for thermal conductivity $2,73,75,7,82$ for condensed-phase species are stored in subroutine THKOND. No temperature dependence is included. Mixture values are computed from the species values by mole-fraction averaging.

It should be mentioned that CORCON uses the same thermal conductivity for both the solid and liquid phases - literature values for the solid phase thermal conductivity are tabulated in the code. While this is a fairly reasonable assumption for the oxide phase, it may be in error for the metal phase. For example, aluminum at $900 \mathrm{~K}$ ( $33 \mathrm{~K}$ below the melting point) has a thermal conductivity of $210 \mathrm{~W} / \mathrm{m} \mathrm{K}$, while at $1000 \mathrm{~K}$, the thermal conductivity is only $93 \mathrm{~W} / \mathrm{m} \mathrm{K.} .^{83}$ Most other metals have thermal conductivities in the solid phase that are approximately twice their thermal conductivities in molten phase. To help correct for this potential problem, we have provided the user with the option of specifying a multiplier to be applied in the calculation of the metal phase thermal conductivity. It should be noted, however, that this modified thermal conductivity would still be applied to both the solid and liquid phases.

\section{Surface Tension - $\sigma(\mathrm{N} / \mathrm{m})$}

Values of surface tension ${ }^{7,74,76,78}$ for condensed-phase species are stored in subroutine SIGMY. No temperature dependence is included. Mixture values are computed from the species values by mole-fraction averaging.

Emissivity - c (-)

The calculation of radiative heat transfer requires emissivities for the radiating surfaces. In the CORCON-Mod3 code, only the emissivity of water (coolant) is stored as internal data. Values are input by the user for the ablating concrete surface, for the oxidic and metallic melt phases, and for the surroundings above the pool. The first is specified as a constant, while the last three may be input as functions of either surface temperature or time.

Emissivities are computed in subroutine EMISIV.

\subsubsection{Liquid-Solid Phase Transition}

In order to model solidification effects, we must be able to determine the liquidus and solidus temperatures for metallic and oxidic mixtures. Melting temperatures and latent heats of fusion are readily available for a wide range of materials. Values for all the condensed-phase species in the CORCON Master List (Table 2.1) are contained in the thermodynamic property tables described in Section 2.4.1. The situation is quite different for the mixtures encountered in melt/concrete interactions; data are lacking, and the mixtures are too complex for detailed analysis. Therefore, we have developed relatively simple procedures for estimating the liquidus and solidus temperatures of the various mixtures encountered in CORCON-Mod3. These temperatures are also used in the construction of a single melt transition in the enthalpy of such mixtures, as described in Section 2.4.1.

\footnotetext{
Roche, M. Presentation at the ACE Technical Advisory

Committee Mecting, Argonne National Laboratory, June 1991.
} 


\section{Concrete:}

The melting of concrete is strongly influenced by the presence of trace species such as alkali oxides.

However, melting ranges for typical concretes have been determined experimentally, and are included as part of the internal data in CORCON-Mod3 for the three built-in concrete varieties. For user-specified concretes, the liquidus and solidus temperatures must also be input by the user: the values for the built-in concretes, Table 2.4, should provide some guidance.

\section{Metallic Mixtures:}

For metallic mixtures (at least late in the accident when solidification is likely) the principal constituents should be $\mathrm{Cr}, \mathrm{Fe}$, and $\mathrm{Ni}$ from stainless steel. Therefore, we have constructed a simple fit to the iron-chromium-nickel ternary phase diagram, ${ }^{R A}$ with due consideration to the associated hinary phase diagrams. The liquidus and solidus temperatures are fit as

$$
\begin{aligned}
T^{\prime}=\max & \left(2130-510 \mathrm{~W}_{\mathrm{Fe}}-1140 \mathrm{~W}_{\mathrm{N} l},\right. \\
& 1809-90 \mathrm{~W}_{\mathrm{C} r}-440 \mathrm{~W}_{\mathrm{N} l} \\
& 1728-200 \mathrm{~W}_{\mathrm{Cr}}-40 \mathrm{~W}_{\mathrm{Fe}^{\prime}} \\
& \left.1793-230 \mathrm{~W}_{\mathrm{C} r}-130 \mathrm{~W}_{\mathrm{N}}\right)
\end{aligned}
$$

and

$$
\begin{aligned}
& \mathrm{T}^{\prime}=\max \left(2130-730 \mathrm{~W}_{\mathrm{Fo}}-3310 \mathrm{~W}_{\mathrm{Ni}},\right. \\
& 1809-90 \mathrm{~W}_{\mathrm{Cr}}-560 \mathrm{~W}_{\mathrm{Nl}} \\
& 1728-250 \mathrm{~W}_{\mathrm{Cr}}-100 \mathrm{~W}_{\mathrm{Fo}} \\
& 1783-310 \mathrm{~W}_{\mathrm{Cr}}-140 \mathrm{~W}_{\mathrm{N} j}
\end{aligned}
$$

where $\mathrm{W}_{\mathrm{C} \text { r }}, \mathrm{W}_{\mathrm{Fe}}$, and $\mathrm{W}_{\mathrm{Ni}}$ are the weight fractions of $\mathrm{Cr}$, $\mathrm{Fe}, \mathrm{Ni}$, respectively. These expressions agree with the original curves within a few tens of degrees. The corresponding contour maps are shown in Figure 2.14. As implemented in CORCON-Mod3, the presence of other elements is ignored, and the weight fractions of chromium, iron, and nickel are renormalized so that

$$
\mathrm{W}_{\mathrm{C}_{r}}+\mathrm{W}_{\mathrm{F}_{\mathrm{o}}}+\mathrm{W}_{\mathrm{Ni}}=1.0
$$

Other metals such as zirconium or silicon, which may be present in significant amounts at various times during the interaction, may substantially depress the melting temperature of the metal phase. We currently do not account for this effect in CORCON-Mod3. We have, however, provided the user with the option of specifying the solidus temperature for the metal phase. If this option is invoked, the liquidus temperature is set at $10 \mathrm{~K}$ above the user-defined solidus temperature.

\section{Oxidic Mixtures:}

The melting behavior of oxidic mixtures is far more complicated than that of the metals. The major constituents of concrete, $\mathrm{CaO}, \mathrm{SiO}_{2}$, and $\mathrm{Al}_{2} \mathrm{O}_{3}$, form a complicated ternary system (see, e.g., Reference 73). The fuel oxides, $\mathrm{UO}_{2}$ and $\mathrm{ZrO}_{2}$, appear to form a relatively simple system, but it is really a single-line in the far more complicated uranium-oxygen-zirconium ternary system. Because consideration of the complete phase diagram for concrete oxides plus fuel oxides seems to be out of the question, the melting behavior of oxidic mixtures is treated in an approximate manner.

The user may select from two different representations of the oxide phase diagram: one that assumes an ideal solution for the solid phase, and one that assumes eutectic interactions also occur. In both cases, the oxidic mixture is treated as a pseudo-binary system in which the fuel oxides form one "component" with concrete and steel oxides forming the other.

If the two components are assumed to form ideal solutions in both liquid and solid phases, the following equations for the liquidus and solidus temperatures are applicable:

$$
\begin{aligned}
& x_{1} \exp \left[-\frac{\Delta H^{\prime}}{R_{0}}\left(\frac{1}{T_{1}^{m}}-\frac{1}{T^{\ell}}\right)\right] \\
& +x_{2} \exp \left[-\frac{\Delta H^{2}}{R_{0}}\left(\frac{1}{T_{2}^{m}}-\frac{1}{T^{\ell}}\right]\right]=1 \\
& x_{1} \exp \left[\frac{\Delta H^{\prime}}{R_{0}}\left[\frac{1}{T_{1}^{m}}-\frac{1}{T^{*}}\right)\right] \\
& +x_{2} \exp \left[\frac{\Delta H^{2}}{R_{0}}\left(\frac{1}{T_{2}^{m}}-\frac{1}{T^{\natural}}\right]\right]=1
\end{aligned}
$$

where $x_{1}$ is the mole-fraction of component $i, T_{i}^{m}$ is its melting temperature, and $\Delta \mathrm{H}_{4}$ is its heat of fusion.

This formalism may be applied to a mixture of mixtures by treating $T_{1}^{m}$ as $T_{1}^{l}$, the liquidus temperature of mixture 

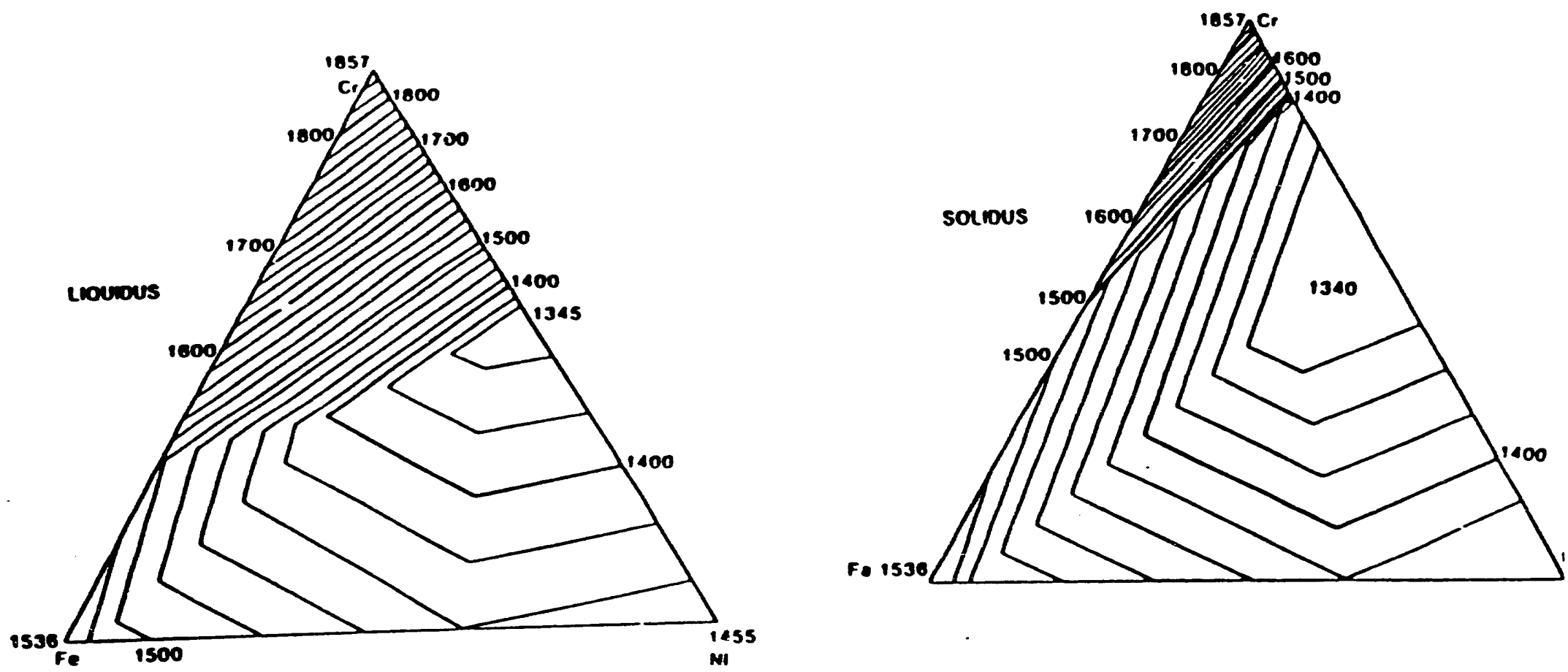

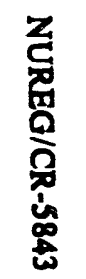

Figure 2.14 Uhrides and solidus cemperature his for Cr-Fe-NI system 
$i$, in the liquidus equation, and as $T$, the solidus temperature of mixture $i$, in the solidus Equation (207).

The melt properties for the fuel oxides $\left(\mathrm{UO}_{2}+\mathrm{ZrO}_{2}\right)$ are taken $\mathrm{as}^{24}$

$$
\begin{aligned}
\Delta H= & 20800 x+17700(1-x) \\
& -5050 \times(1-x)
\end{aligned}
$$

and

$$
\begin{aligned}
T^{\top}= & T^{1}=\Delta H /(20800 x / 2950 \\
& +17700(1-x) / 3123)
\end{aligned}
$$

where $\Delta \mathrm{H}$ is the component latent heat in cal/mole, $\mathrm{T}$ and $\mathrm{T}^{1}$ are in $\mathrm{K}$, and $\mathrm{x}$ is the mole fraction of $\mathrm{ZrO}_{2}$ in the $\mathrm{UO}_{2}+\mathrm{ZrO}_{2}$ mixture.

For the second mixture, concrete oxides + steel oxides, we use concrete properties with $T$ and $T^{\prime}$ from either built in data (for the three default concretes) or from user input data (for a nonstandard concrete). The mixture latent heat, $\Delta H$, is internally calculated from built-in enthalpy data as

$$
\Delta H=h^{\top}(\bar{T})-h^{\mathbf{i}}(\overline{\mathrm{T}}),
$$

where

$$
\bar{T}=\left(T^{*}+T^{\prime}\right) / 2
$$

and

$$
\overline{\mathbf{h}}^{\ell, s}(\overline{\mathrm{T}})=\sum_{i} \mathrm{~m}_{\mathrm{i}} \overline{\mathrm{h}}_{\mathrm{i}}^{\ell, n}(\overline{\mathrm{T}}) / \mathrm{m}
$$

Here $\overline{h_{i}, \rho}(\bar{T})$ are the enthalpies of the liquid and solid phases of the constituent species, extrapolated, if necessary, as described in Section 2.4.1.

An example of the resulting phase diagram, for $\mathrm{UO}_{2}+$ $35 \mathrm{~mol} / \mathrm{o} \mathrm{ZrO}_{2}$ and Limestone Aggregate/Common Sand Concrete is shown in Figure 2.15).

Recent experiments ${ }^{f}$ have shown that the phase diagram calculated assuming ideal solution interactions may be significantly in error for the LWR debris compositions of interest. These experiments clearly show that eutectic interactions occur in the oxide mixture. As a result, the solidus temperature of the oxide phase decreases much more rapidly with the addition of concrete oxides than shown in the figure. The experiments show that at only about 10 or 20 mole percent concrete, the solidus temperature of the mixture has fallen to near the concrete solidus temperature.

As one of the user flexibility options in CORCON-Mod3, the user may choose the ideal solution phase diagram (the default selection) or select an alternate parametric representation that accounts (at least approximately) for the observed melting behavior. In the latter, the liquidus temperature is assumed to be the same as for the ideal solution model. The solidus temperature, which is the more critical value, is calculated assuming that the solidus temperature decreases linearly with concrete addition to a user-specified concrete mole fraction. At higher concrete concentrations the solidus temperature is fixed at the concrete solidus. (The resulting phase diagram is shown in Figure 2.16.) While extremely simple, this parametric model provides a fairly accurate representation of the experiment data.

Roche, M. Presentation at the ACE Technical Advisory

Committee Meeting, Argonne National Laboratory, June 1991. 


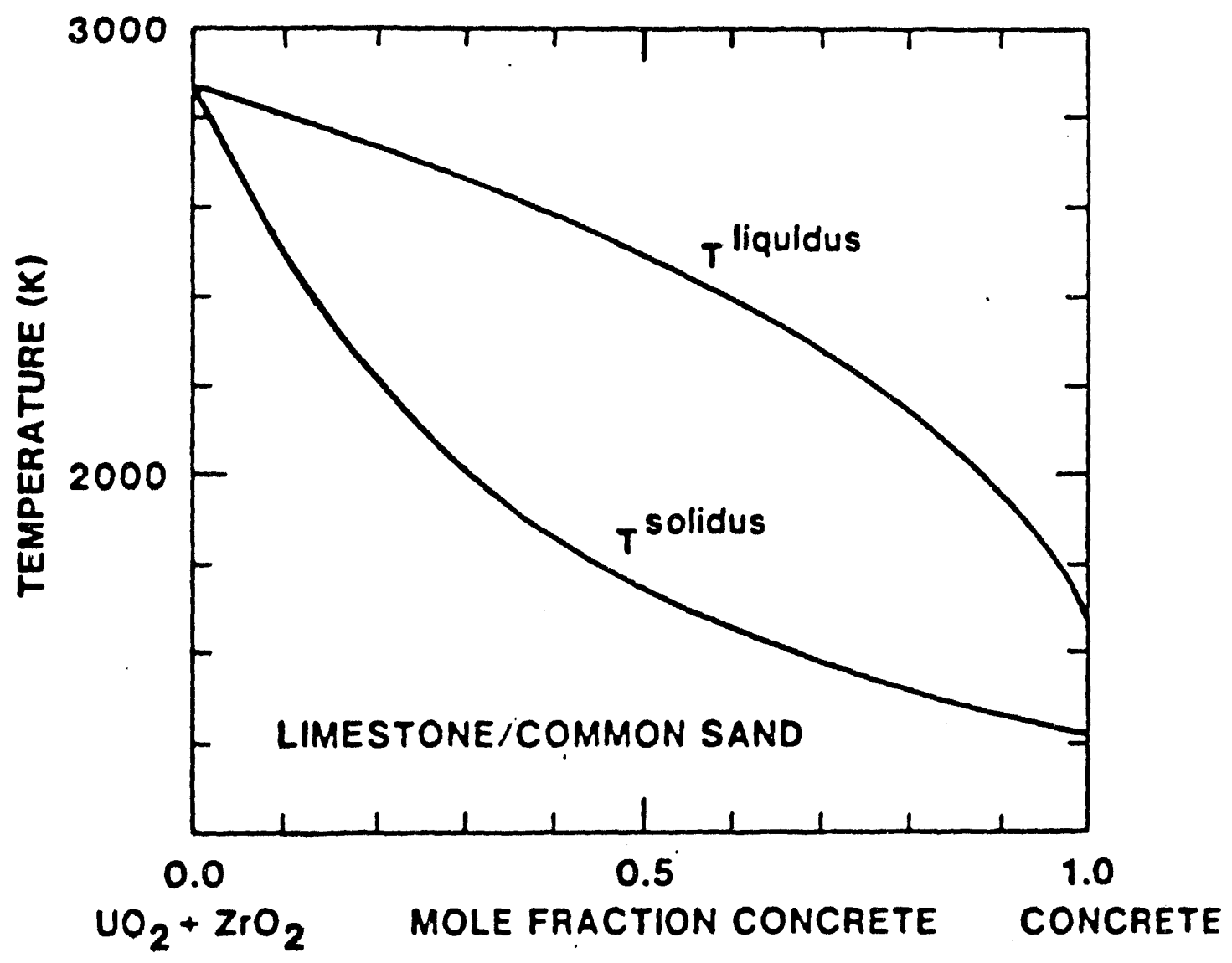

Figure 2.15 Example liquidus and solidus temperatures for oxidic mixtures 


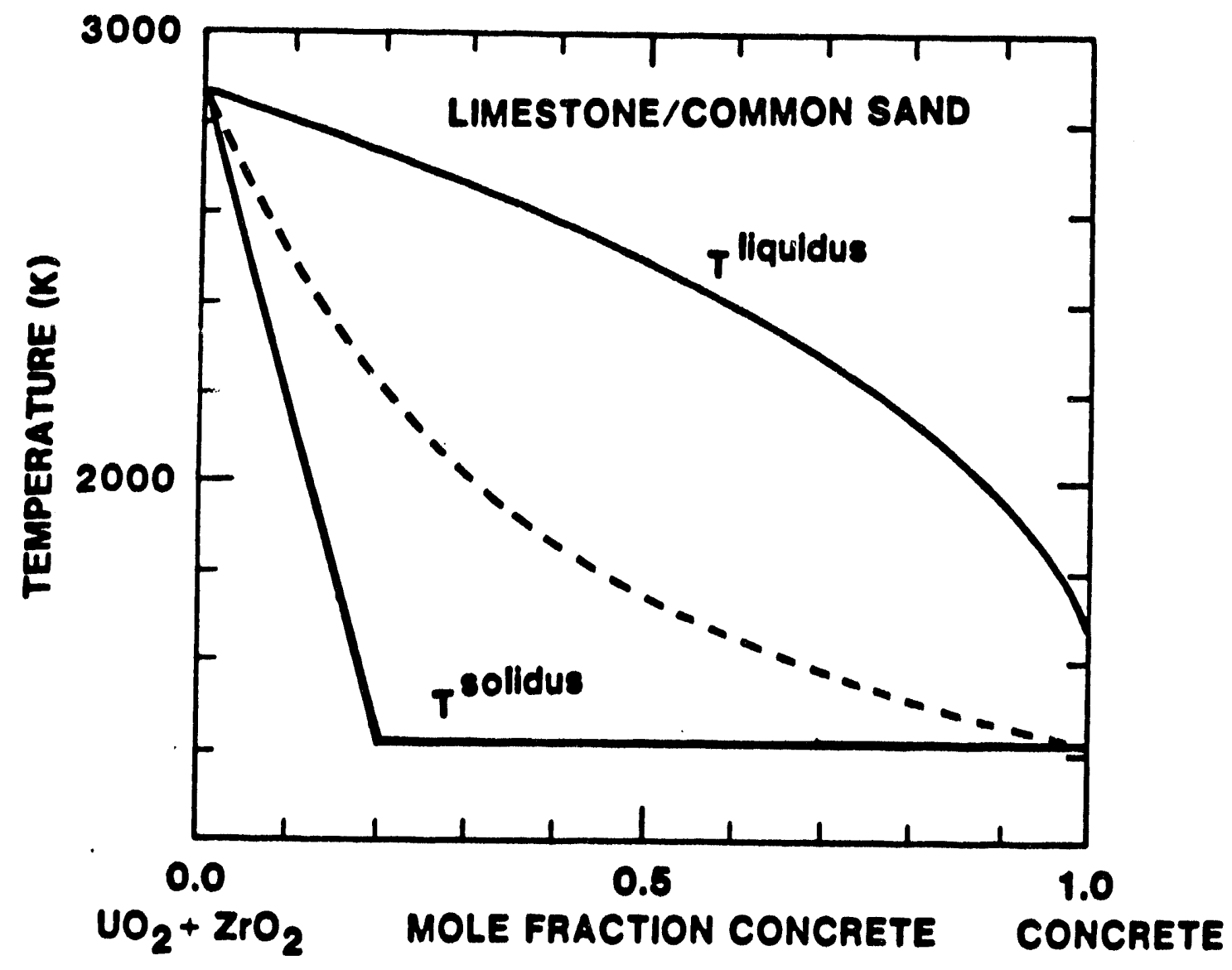

Figure 2.16 Alternate phase diagram for the oxide phase 


\subsubsection{Coolant Saturation Line}

If a coolant is present, CORCON-Mod3 considers the possibility that it may vaporize. The calculational procedure, described in Section 2.12, requires knowledge of the saturation line for the coolant--that is, the temperature at which it boils at any given pressure. The data in the Steam Tables" were fit by a nonlinear optimization program as

$$
P_{\text {ate }}(T)=1.292 \times 10^{10} \exp \left[\frac{-3880.4}{T-43.15}\right]
$$

in S.I. units (pressure $\mathrm{p}$ in $\mathrm{Pa}$, temperature $\mathrm{T}$ in $\mathrm{K}$ ). This simple expression reproduces the original data within 0.5 percent for $T<573 \mathrm{~K}$, corresponding to $\mathrm{p}_{\mathrm{es}}$ $<8.5 \mathrm{MPa}$ (which is far in excess of containment failure pressures), and is in error by only 5 percent at the critical point. This accuracy seems more than adequate for a code such as CORCON-Mod3. In addition to its extremely compact and efficient form, Equation (213) has the virtue of an exact analytic inverse:

$$
T_{\text {mat }}(p)=43.15-\frac{3800.4}{\ln \left(p / 1.292 \times 10^{10}\right)} .
$$




\subsection{Assumptions and Limitations}

The CORCON-Mod3 code has been designed to be used for the analysis of both reactor accident sequences and melt/concrete interaction experiments. The code input, described in Section 5.0, is sufficiently flexible to allow a user to describe problems of either type. Accident calculations provide information for assessment of the risks of operation of LWR's. Calculations of experiments are useful both for planning and for interpretation of results. The comparison of experiment and calculation is valuable for guiding further efforts in both areas.

The user, however, should be aware of a number of assumptions, approximations, and simplifications employed in the modeling which may affect the accuracy (or at least the interpretation) of CORCON-Mod3 calculations. It is also worth noting that a number of sensitivity parameters are available through input to allow the user to readily explore the extent of importance of some of the approximations noted below. These parameters are listed and briefly discussed in Table 4.1. Among the more important of these are:

1. The atmosphere and surroundings above the pool surface serve only to provide boundary conditions for heat and mass transfer from the pool, as CORCON does not include calculational procedures to update the temperature, pressure, or composition of the atmosphere or the temperature of the surroundings. (However, if these quantities are updated by other code modules coupled to CORCON, the current values will be used.) The calculation of radiative heat loss from the pool surface is based on a onedimensional model, and the convective loss is calculated using a constant heat transfer coefficient.

2. The calculated concrete response is based on onedimensional steady-state ablation, with no consideration given to conduction into the concrete or to decomposition in advance of the ablation front. This assumption is probably not a source of serious error in the analysis of reactor accidents, at least for the sequences with long-term interactions between core materials and concrete. The heat fluxes involved are sufficiently large that quasi-steady ablation is approached within the first few minutes of interaction if the pool is molten; the process continues for a period of hours to days, sustained by decay heat from the fission products in the melt. The steady-state ablation assumption makes it difficult to apply the code to transient interactions that occur in the first few minutes following reactor vessel failure. The code may also be inaccurate for analysis of very long-term interactions where the debris temperature may be close to the concrete ablation temperature.

3. The solidification model is preliminary. It assumes that a crust forms on any surface whose temperature falls below the solidification temperature. The mechanical stability of the crusts is not considered. We believe that both the mechanical strength of the crust and the loads imposed on it by concrete decomposition gases are important in determining the true solidification behavior of the core debris.

The code assumes also that the crust has the same properties as the bulk liquid phase. This may not be true if the liquid phase composition is changing with time. Consider for example the formation of an oxide crust early in the interaction (before significant concrete ablation). This crust material will have a solidus temperature near that of the fuel oxide mixture. As concrete is incorporated into the molten phase, the molten phase solidus temperature will decrease. The code assumes that the same change in the solidus occurs for the crust. Clearly, this is not correct.

4. If the gas-film model is used, it is used for radial heat transfer even after the melt solidifies, even though the assumptions on which the model is based are no longer valid. In particular, no radial gap develops around a layer of the melt which has completely solidified. Thus, radial ablation continues with the "solid" layer continuing to conform to the changing shape of the cavity rather than behaving as a rigid penetrator. As coded, the model also assumes that the frozen material remains gas-permeable. Because the total amount of concrete eroded is largely determined by energy considerations (the available decay energy), the effect of these modeling assumptions is primarily on the calculated shape of the cavity.

5. There is no treatment of chemical reactions between the melt and the atmosphere, or of reactions in the atmosphere. We do not consider these to be significant limitations. Melt-atmosphere interactions are probably insignificant due to the limited surface area and relatively low temperature of the surface. Reactions in the atmosphere do not affect the progress of the core-concrete interaction and so can be 
neglected in a ccunputer model such as CORCON.

Modeling of atmosphere reactions would, however, be useful when comparing code predictions to experiment results, since the gas stream in the experiments is sampled at some distance from the melt surface.

6. The code assumes ideal chemistry when calculating bulk phase chemical renctions.

7. The code uses flat plate pool boiling correlations to model heat transfer to an overlying coolant pool. Recent experiments show that heat transfer is greatly enhanced during the initial pour of coolant. This enhanced heat transfer may lead to rapid cooling of the melt surface, and a transition to nucleate boiling. The code predicts lower early heat fluxes than in the experiments, and long-term heat transfer by film boiling. Interestingly, the code predicts well the longer term (steady state) coolant heat fluxes measured in the experiments.

8. The time-dependent melt radius model allows the user to mimic the spreading of a melt across a horizontal floor, but it is not a mechanistic model of spreading.

9. The code uses the same Fe-Cr-Ni phase diagram for the metal phase that was in CORCON-Mod2. This treatment neglects important metallic components such as $\mathrm{Zr}, \mathrm{Si}$, or $\mathrm{Al}$ that may be present in the melt at various times during core-concrete interaction. In general, these constituents will reduce the melting range of the metal phase relative to the range predicted by the code. Through inpul the user can modify the phase diagram for the metal phase by specifying a constant solidus temperature. The liquidus temperature is then assumed to be $10 \mathrm{~K}$ greater than the specified solidus. 


\subsection{User Information}

In this chapter we describe a typical calculational cycle of the CORCON-Mod3 computer code. We discuss the input parameters and describe the output. Finally, we provide general programming information for the code.

\subsection{A Typical Calculational Cycle in CORCON-Mod3}

We have designed the calculational cycle in CORCON to reflect our view of the interrelationships of the various physical and chemical phenomena described in Chapter 2. The description of a typical calculational cycle in CORCON will aid user in understanding how the phenomena are modeled in the code.

At the start of a timestep, CORCON has a complete "snapshot" of the problem, that is, it has current values of all the relevant computational variables, including the cavity geometry; the physical, chemical, and thermal state of the melt pool; and the transport properties for the pool. For the first timestep, the "snapshot" comes from the initialization procedure. For later timesteps, the "snapshot" comes from the results of the preceding timestep.

The variables describing the state of the melt pool are advanced to their values at the end of the timestep by the calculational procedure described below. The basic logic is also given in Figure 4.1, which is a flow churt of the main program in CORCON-Mod3.

1. Calculate a timestep. This may be either constant or variable, as specified by the user.

2. Calculate the internal mass transport, melt/gas and condensed phase chemical reactions, and the associated energy terms.

Injection rates of concrete decomposition products (already known) are assumed to remain constant over the timestep, and the calculation proceeds in two passes.

The first pass follows rising gases and condensed phase materials (e.g., concrete slag or entrained material), layer by layer. If the material should remain in the current layer, its mass and energy are added to that of the layer; otherwise, it is equilibrated thermally with this layer and passed on to the next. Gas passing through a metal-containing layer is equilibrated chemically with the metal, and the oxidic reaction products are added to the rising oxides, with any heat of reaction remaining in the layer. When the surface of the pool is reached, the rising gases are passed to an interface routine which disposes of them and initializes any downward mass flows, such as added coolant or additional core material falling into the pool. It is in the first pass that the VANESA subroutine is called. The VANESA model calculates radionuclide release and aerosol generation, which are included in the upward flow of material exiting the pool.

In the second pass, falling condensed-phase materials are followed downward layer by layer, being equilibrated in each layer, until they are added to the appropriato layer.

3. Finish the explicit time-advancement of the layer energy equations.

This step includes the addition of decay heat and subtraction of heat lost to the concrete. It also accounts for interlayer heat transfer and loss from the pool surface besed on rates at the start of the timestep. If coolant is present and is boiling, the calculation is completed at the step; otherwise, the matrix equations for an implicit calculation of the interlayer and surface energy terms is set up. A linearized representation of the pool thermal response, surface heat flux vs surface temperature, is constructed.

4. Determine a provisional end-of-timestep value for the temperature of the surface of the pool by matching heat fluxes to and from this surface. Construct a linearized representation of the above-pool thermal response, as surface heat flux vs surface temperature.

In matching the heat fluxes, the linearized pool response and the "exact" relationships for the above-pool heat transfer are used. If desired, the change of temperature in the atmosphere and surroundings during the timestep are included here and made consistent with the heat flux. The simple routine provided could easily become part of the interface to a more general model.

5. Complete the solution of the implicit interlayer heat transfer equations, using the provisional end-of-timestep surface temperature, to determine the final layer enthalpies. Reconcile these with the known layer masses and compositions to determine the new layer temperatures.

6. Calculate the new cavity shape, using the ablation rates at the start of the timestep. These ablation rates, are treated as constant over the timestep. 


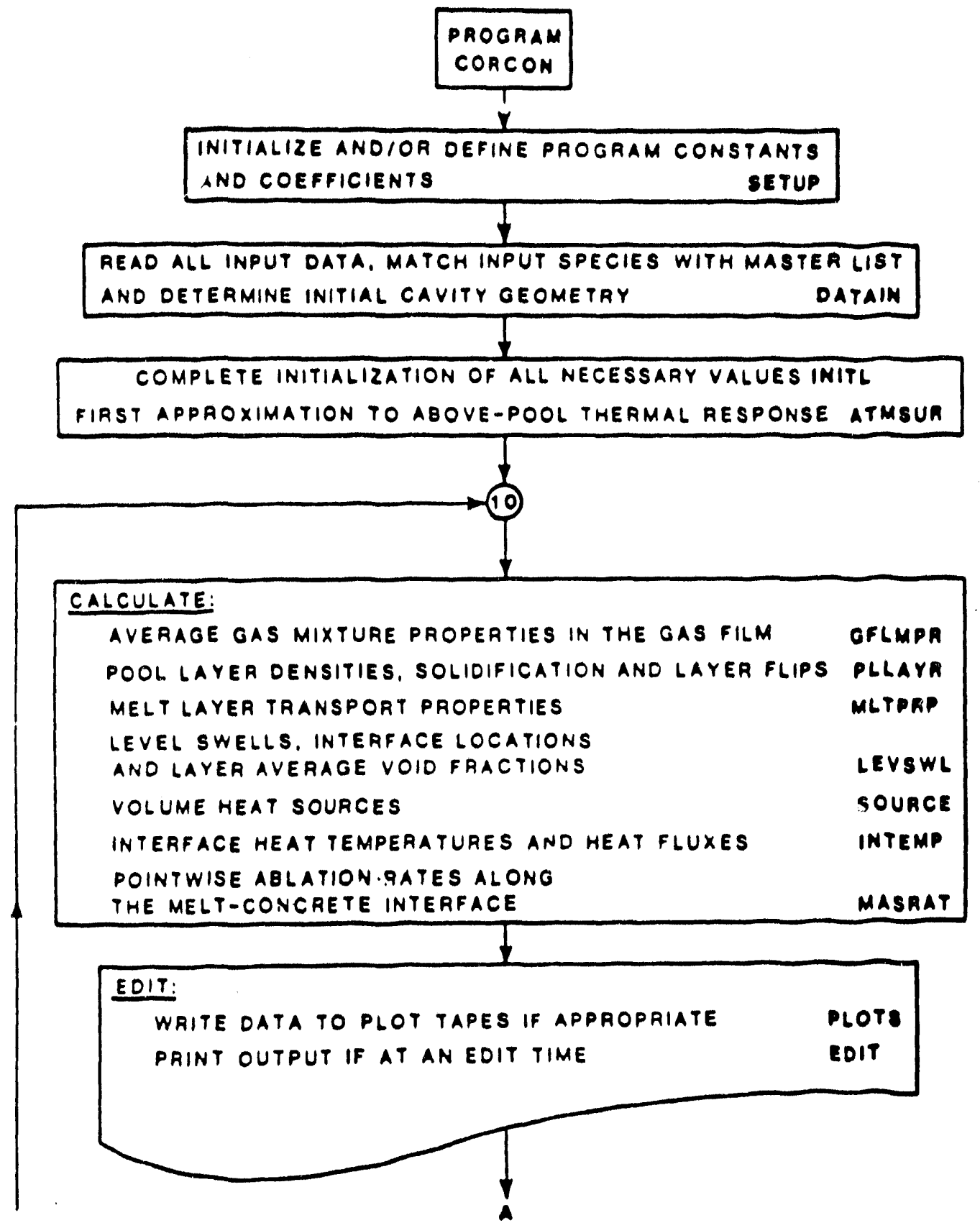

Figure 4.1 Flow diagram for CORCON-Mod3 


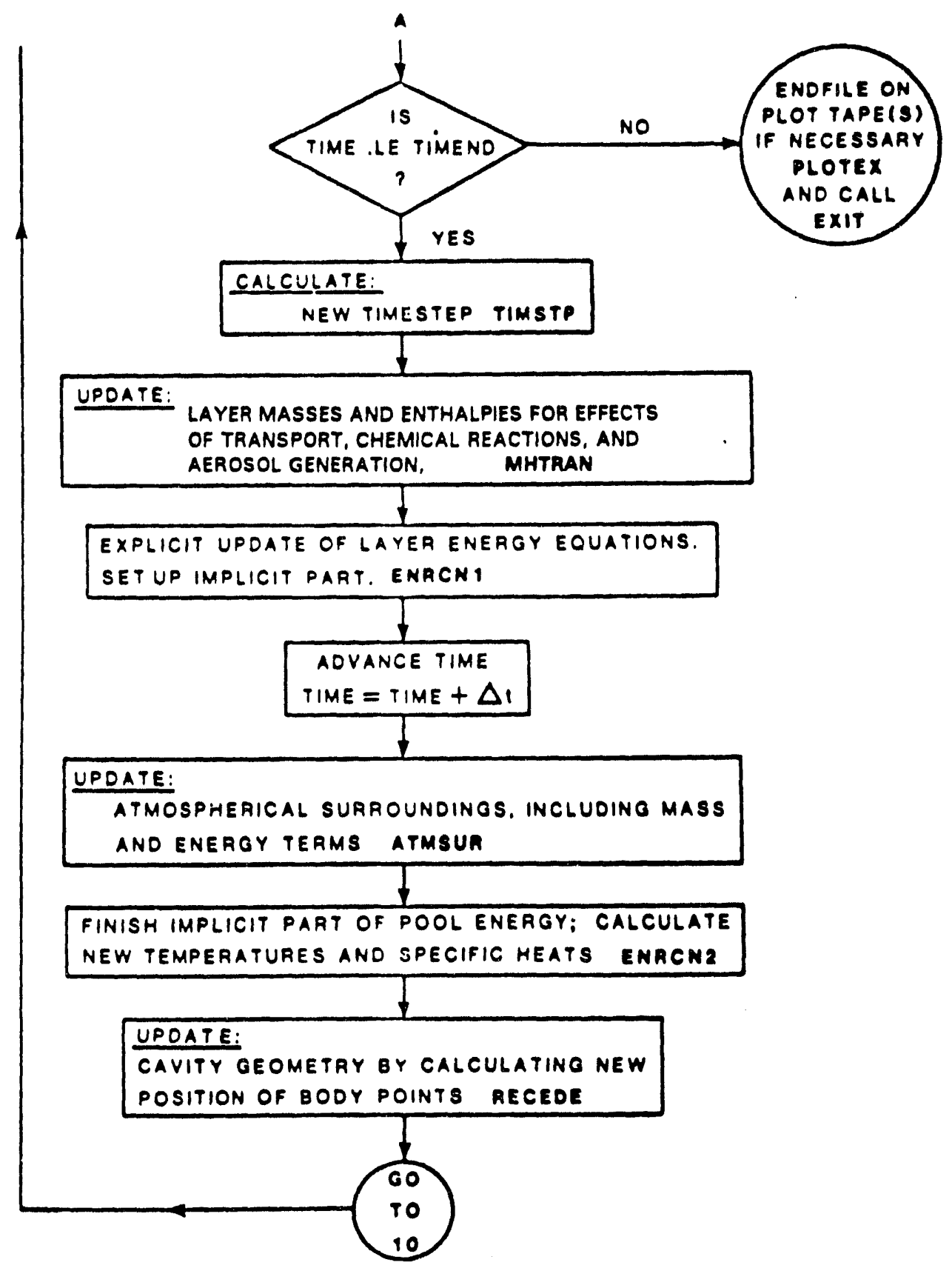

Figure 4.1 Flow diagram for CORCON-Mod3 (continued) 
7. Calculate the new layer densities. Determine if the previous layer ordering is still appropriate. If it is not, reorient the layer configuration. Layer flips occur in this step as well as creation of mixture layers.

8. Calculate new layer transport properties (viscosity, thermal conductivity, etc.) from the new compositions and temperatures.

9. Calculate bubble-rise velocities and (from known gas-flow rates) pointwise void fractions in the pnol. From the void fractions, the known volume of condensed phases in each layer and the cavity shape determine the elevation of each layer interface.

10. Evaluate decay or externally-imposer heat sources.

11. Evaluate beat transfer between layers and the resulting interlayer heat fluxes.

The linearized above-pool response which was calculated in Step 4 is used in this calculation. The final temperature of the pool surface determined here will differ slightly from the provisional value calculated in Step 4.

12. Calculate pointwise ablation rates along the melt-concrete interface.

The thermal resistance of the gas film, if it exists, is calculated based on the local conditions of gas flow and the rate at which gas is entering the film. This rate is made consistent with the local heat flux and ablation rate.

At this point, CORCON once again has a complete "snapshot" of the problem, for the end of the timestep. At this point in the calculational cycle, all the calculated variables have been evaluated at the same time level, allowing the generation of a consistent printed edit of the state of the system, if this is desired.

In the main program, the above steps are executed in the order $7-12$, edit if required, check for end of problem, $1-6$, and repeat. This order is used because steps 7-11 are also required as part of the initialization.

\subsection{Description of the Input Parameters}

CORCON-Mod3 expects input to be provided in a file called "ccmod3.in" (Unit 5). The data cards needed for input by the code are described in Table 6.1. Note that the input is compatible with the inputs used for CORCON-Mod2, in the sense that any input deck used for CORCON-Mod2 will also be accepted by CORCONMod3. There are, of course, numerous data cards that have been added to the input for the many additional features in CORCON-Mod3. Also, some models that were inactive in CORCON-Mod2 have been activated in CORCON-Mod3 (e.g., time-dependent mass addition). Finally, the reader should note that the user may now include comments within the input deck to explain a particular entry, or to provide a short description of the calculation. Comment lines are assumed hy the code if a " $\$$ " appears in the first column.

A given problem will not require all the data cards and fields described. In fact, some fields and cards are not yet operational and these are indicated by $* *[\ldots]^{* *}$ in the table. This has been done to reserve space for further upgrades of the code, and to provide the adventurous code modifier with information conceming partially implemented features.

Data cards are initially read in subroutine DATAIN, and are echoed to the output file, and to a scratch file called "echo. fil" (Unit 7). The cards are subsequently read from the scratch file by routines CONPRP, DATAIN, DCYINT, INCOOL, INGEOM, INPCON, INPGAS, MELTRD, and BCLTOV which interpret the actual data fields.

\subsubsection{Discussion of Selected Input Quantities}

Most of the input for CORCON is fairly self-explanatory, especially to the user who is familiar with the type of problems it solves. However, experience has shown that some portions of the input require further discussion.

\section{Edit and Timestep Control}

The parameter TPRIN (card 3) provides the user with detailed printout after time has passed TPRIN. This is of interest primarily in the diagnosis of code problems; the additional information is printed in terms of FORTRAN variables and requires detailed knowledge of the internal working of the code for its interpretation.

For routine calculations, TPRIN should be set greater than the problem end time TIMEND.

For both the variable timestep control (card group 3A) and the variable edit control (card group 3B), the last specification is assumed to apply to the end of the problem if TIMEND is greater than the last TEND or TED, respectively. Each group is terminated by a blank card rather than by a specified count. This facilitates the insertion of additional control intervals at significant or 
Table 4.1 Input instructions for CORCON-Mod3

Card/

Group" Field Format Variable Name Description

SECTION 1. PROBLEM IDENTIFICATION, COMPUTATIONAL OPTIONS AND CONTROL

PARAMETERS

PROBLEM TITLE

$1 \quad 1-80 \quad$ A80 $\quad$ ITITL $\quad 80$ Column run identification

\section{COMPUTATIONAL OPTIONS INDICES}

$\begin{array}{llll}2 & 1.5 & 15 & \text { ILYR }\end{array}$

Index specifying number of initial melt layers:

0 - 1 oxidic layer \& 1 metallic layer

1 - 1 metallic layer

2 - 1 oxidic layer

3 -- 1 heterogenous mixture layer

**[4 - 2 oxidic layers \& 1 metallic layer] $]^{* *}$

$>10$ - Mixing models enabled; Initial layer configuration set by ones digit (e.g., ILYR $=10$ indicates two layers with mixing on)

6-10 I5 ICOOL

Coolant index:

0 -. no coolant

1 -- coolant

11-15 I5 IGEOM

Cavity geometry index:

1 - Cylinder with hemispherical base

2 -- Cylinder with flat base

**[3 -- Cylinder with spherical-segment base]**

4 - Arbitrary shape

16-20 I5 ICON

Concrete composition index:

0 -. Nonstandard concrete

1 -- Basaltic aggregate concrete

2 - Limestone aggregate; common sand concrete

3 - Limestone aggregate concrete

21-25 I5 ICHEM

Coking and condensed phase chemistry (CPC) index:

0 - Coking off \& CPC off

: - Coking off \& CPC on

10 - Coking on \& CPC off

11 - Coking on \& CPC on

26-30 I5 IFP Decay Heat (power) generation index:

0 -- Decay power computed internally

2 -- Power deposited into oxidic and metallic layers input versus time

31-35 I5 ISUR

Index specifying surface temperature history of atmosphere surroundings:

$* *[0$ - Surface temperature history computed internally]

1 -- Surface temperature input versus time

36-40 I5 **[IABL]

Index specifying mass addition to pool due to ablation of surroundings

0 -- No mass addition

**[1 - Mass addition rates computed internally]** 
Table 4.1 Input instructions for CORCON-MOD3 (continued)

\begin{tabular}{|c|c|c|c|c|}
\hline $\begin{array}{l}\text { Card/ } \\
\text { Group" }\end{array}$ & Field & Format & Variable Name & Description \\
\hline & $41-45$ & 15 & ISPLSH & $\begin{array}{ccl}\text { Melt/coolant splashout index: } \\
0 & - & \text { No splashout from pool } \\
* *[1 & - & \text { Metallic and oxidic phase splashout mass flow rates } \\
& & \text { input versus time }]^{* *}\end{array}$ \\
\hline & $46-50$ & I5 & IPINC & $\begin{array}{l}\text { Print increment index: } \\
>0 \quad-- \text { Print output every IPINC time steps } \\
\leq 0-\text { Print controlled by Card Group 3B }\end{array}$ \\
\hline & $51-55$ & I5 & IFILM & 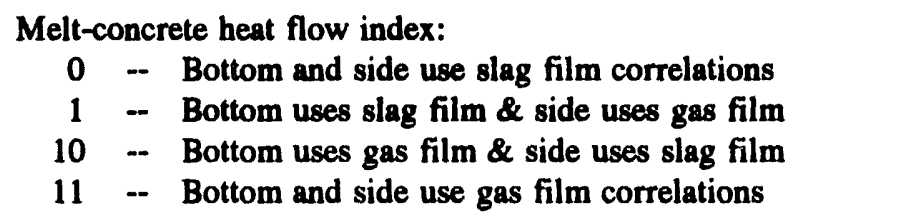 \\
\hline & $56-60$ & 15 & IRSTRT & 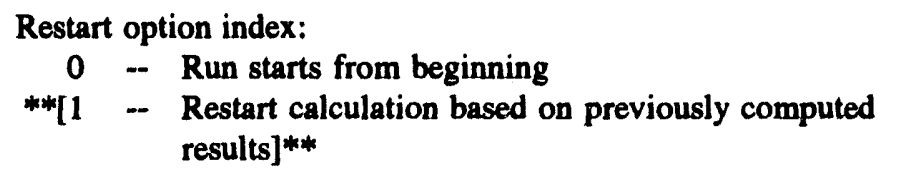 \\
\hline & $61-65$ & I5 & IMOV & $\begin{array}{l}\text { Cavity shape plot index: } \\
0 \quad-\quad \text { No plots desired } \\
1 \quad-\quad \text { Plots are desired }\end{array}$ \\
\hline & $66-70$ & I5 & IPG & $\begin{array}{l}\text { Index specifying plots of prescribed variables versus time: } \\
\begin{array}{rll}0 & - & \text { No plots desired } \\
1 & - & \text { Plots desired }\end{array}\end{array}$ \\
\hline & 71-75 & 15 & ISRABL & 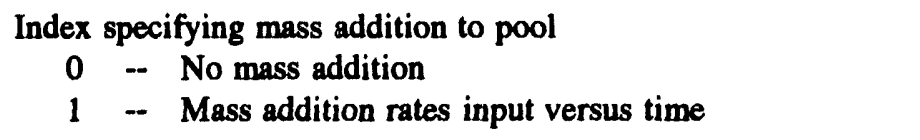 \\
\hline & $76-80$ & I5 & IAOPAC & 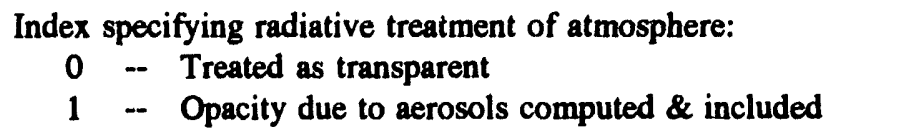 \\
\hline \multirow[t]{5}{*}{$2 A$} & 1 & A1 & '\&' & The ampersand specifies the inclusion of this card. \\
\hline & $2-5$ & 15 & ITIMR & $\begin{array}{l}\text { Time dependent melt radius (TDMR) index: } \\
0 \quad-\quad \text { TDMR option not used } \\
1 \quad-- \text { TDMR option in use (IGEOM = } 2 \text { only) }\end{array}$ \\
\hline & $6-10$ & I5 & IFVAN & 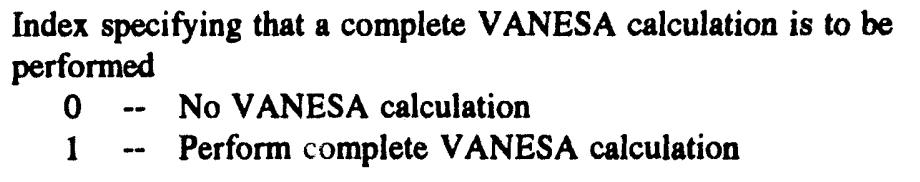 \\
\hline & $11-15$ & 15 & IVANFP & $\begin{array}{l}\text { Index specifying form of fission product information used by } \\
\text { VANESA: } \\
0 \text { - Fission product composition from CORCON } \\
1 \text { - }\end{array}$ \\
\hline & $16-20$ & 15 & IUSER & $\begin{array}{l}\text { Index specifying inclusion of additional user flexibility input: } \\
0 \text {-- No user flexibility input } \\
1 \text {-- User flexibility input to be provided }\end{array}$ \\
\hline \multicolumn{2}{|c|}{ NUREG/CR-5843 } & & & 76 \\
\hline
\end{tabular}


Table 4.1 Input instructions for CORCON-MOD3 (continued)

\begin{tabular}{|c|c|c|c|c|}
\hline $\begin{array}{l}\text { Card/ } \\
\text { Groupt }\end{array}$ & Field & Format & Variable Name & Description \\
\hline \multicolumn{5}{|c|}{ CONTROL PARAMETERS } \\
\hline \multirow[t]{5}{*}{3} & $1-10$ & E10.0 & DELTIM & $\begin{array}{l}\text { Time-step control: } \\
>0.0-- \text { DELTM is the time step (s) } \\
\leq 0.0-\text { Time step controlled by Card } 3 A\end{array}$ \\
\hline & $11-20$ & E10.0 & TIMEO & Initial time at which calculations are to begin (s) \\
\hline & $21-30$ & E10.0 & TIMEND & Final time at which calculations are to cease (s) \\
\hline & $31-40$ & E10.0 & DPRIN & $\begin{array}{l}\text { **[Diagnostic print interval - print diagnostic messages every } \\
\text { DPRIN seconds starting at TIME }=\text { TPRIN (s) }]^{* *}\end{array}$ \\
\hline & $41-50$ & E10.0 & TPRIN & Time at which diagnostic output is to begin (s) \\
\hline \multicolumn{5}{|c|}{ Variable time-step control. Include Card 3A only if DELTIM $\leq 0.0$} \\
\hline \multirow[t]{3}{*}{$3 \mathrm{~A}$} & $1-10$ & E10.0 & DTMN & Minimum time step for interval (s) \\
\hline & $11-20$ & E10.0 & DTMX & Maximum time step for interval (s) \\
\hline & $21-30$ & E10.0 & TEND & End time for interval (s) \\
\hline \multicolumn{5}{|c|}{ Repeat Card 3A for a maximum of 10 intervals, terminate with DTMN $\leq 0.0$} \\
\hline \multicolumn{5}{|c|}{ Variable edit control. Include Card/Group 3B only if IPINC $\leq 0.0$} \\
\hline \multirow[t]{3}{*}{ 3B } & $1-10$ & E10.0 & DED & Time between edits for interval (s) \\
\hline & $11-20$ & E10.0 & TED & End time for interval (s) \\
\hline & & \multicolumn{3}{|c|}{ Repeat Card 3B for a maximum of 10 intervals, terminate with DED $\leq 0.0$} \\
\hline & SECT & N 2. PR & BLEM INITIAL & NDITIONS \\
\hline & & CONCR & E CRUCIBLE IN & IAL GEOMETRY \\
\hline \multirow[t]{4}{*}{4} & $1-5$ & I5 & NRAYS & Number of rays (maximum of 100 ) \\
\hline & 6-15 & F10.0 & RO* & R-coordinate of center of ray system (m) \\
\hline & $16-25$ & F10.0 & zo & $\begin{array}{l}\text { Z-coordinate of center of ray system (m) } \\
\text { Measured positive downward. } \\
\text { Reference is arbitrary. }\end{array}$ \\
\hline & *Only & rmitted v8 & is 0.0 & \\
\hline \multicolumn{5}{|c|}{ Cylinder with hemispherical base -- see Section 4.1.1 (Figure 4.1). Include card 5 only if IGEOM $=1}$. \\
\hline \multirow[t]{4}{*}{5} & $1-10$ & F10.0 & RS & Radius of hemispherical base (m) \\
\hline & $11-20$ & F10.0 & $\mathrm{HC}$ & Height of cylindrical top section (m) \\
\hline & $21-30$ & F10.0 & RW & External radius of concrete crucible (m) \\
\hline & $31-40$ & F10.0 & HBC & $\begin{array}{l}\text { Height from external base of crucible to base of cylindrical } \\
\text { section (m) }\end{array}$ \\
\hline
\end{tabular}


- surmation

Table 4.1 Input instructions for CORCON-MOD3 (continued)

$-$

C.ard

Group Field Format Variable Name Description

( vlinder with flat base -- see Section 4 J.1 (Figure 4.2). Include card 6 only if IGEOM $=2$.

$\begin{array}{lll}1-10 & \text { F10.0 } & \text { ZT } \\ 11-20 & \text { F10.0 } & \text { RAD } \\ 21-30 & \text { F10.0 } & \text { HIT } \\ 31-40 & \text { F10.0 } & \text { RADC } \\ 41-50 & \text { F10.0 } & \text { RW } \\ 51-60 & \text { F10.0 } & \text { HBB } \\ 61-65 & 15 & \text { NBOT } \\ 66-70 & 15 & \text { NCORN }\end{array}$

Z-coordinate of cylinder top edge (m)

Radius of cylinder (m)

Height of cylinder (m)

Radius of corner (m)

External radius of concrete crucible (m)

Height from external base of crucible to base of cavity (flat bottom) (m)

Number of ray points equally spaced along flat bottom of cavity

Number of ray points equally spaced around corner (not including tangent points)

**[Cylinder with spherical-segment base -- NOT OPERATIONAL. No input for IGEOM $=3.]^{* *}$

Arbitrary shape -- see Section 4.1.1 (Figure 4.3). Include cards 7, 8 and 9 only if IGEOM $=4$.

$7 \quad 1-5 \quad 15 \quad$ NBOT

6.15 F10.0 RTANG

16-25 F10.0 RW

26-35 F10.0 HTOTL

$\begin{array}{llll}8 & 1-10 & F 10.0 & R(1)\end{array}$

$11-20 \quad F 10.0 \quad Z(1)$

$9 \quad 1-10 \quad$ F10.0 $\quad R(I)$

$11-20 \quad F 10.0 \quad Z(I)$
Number of ray points equally spaced along flat bottom of cavity

R-coordinate of tangent point (m)

External radius of concrete crucible (m)

Height from external base to top of crucible (m)

R-coordinate of body point \#1 (m)

Z-coordinate of body point $\# 2$ (m)

R-coordinate of body point I (m) for

$(I=$ NBOT +2 , NRAYS $)$

Z-coordinate of body point I (m) for

$(I=$ NBOT +2 , NRAYS $)$

(There will be (NRAYS - NBOT - 1) cards in Card Group 9)

\section{CONCRETE COMPOSITION AND PROPERTIES}

10

$\begin{array}{ccc}1-10 & \text { E10.0 } & \text { TIC } \\ 11-20 & \text { E10.0 } & \text { TW } \\ 21-30 & \text { E10.0 } & \text { EW } \\ 31-40 & \text { E10.0 } & \text { RBR }\end{array}$

Initial temperature of concrete (K)

Temperature of concrete surface (K)

(Ablation temperature)

Emissivity of concrete surface (-)

Mass fraction of reinforcing steel in the concrete (mass fraction: $k_{\text {g_FE/ }}$ kg_concrete) 
Table 4.1 Input instructions for CORCON-MOD3 (continued)

\begin{tabular}{|c|c|c|c|c|}
\hline $\begin{array}{l}\text { Card/ } \\
\text { Group\# }\end{array}$ & Field & Format & Variable Name & Description \\
\hline \multicolumn{5}{|c|}{ Include card/group 11, 12 and 13 only if ICON $=0}$. \\
\hline 11 & $1-5$ & I5 & NINP & $\begin{array}{l}\text { Number of species in concrete mixture; } \\
\text { only species available in the Master Species List may be } \\
\text { included. }\end{array}$ \\
\hline \multirow[t]{3}{*}{12} & $1-8$ & A8 & NAMSP & Name of concrete species (left justified) \\
\hline & $11-20$ & E10.0 & SM & Mass fraction of concrete species $\left(\mathrm{kg} / \mathrm{kg}_{\text {__CONC}}\right.$ ) \\
\hline & & \multicolumn{3}{|c|}{ (There will be NINP cards in group 12.) } \\
\hline \multirow[t]{4}{*}{13} & $1-10$ & E10.0 & RHOC & Density of concrete $(\mathrm{kg} / \mathrm{m} 3)$ \\
\hline & $11-20$ & E10.0 & TSOLCT & Concrete solidus temperature (K) \\
\hline & $21-30$ & E10.0 & TLIQCT & Concrete liquidus temperature (K) \\
\hline & & \multicolumn{3}{|c|}{ Include card/group 13A and 13B only if RBR $<0.0$} \\
\hline $13 A$ & $1-5$ & I5 & NRBRSP & Number of metallic species in rebar \\
\hline 13B & $1-8$ & A8 & NAMSP & Name of rebar species \\
\hline & $11-20$ & E10.0 & SM & Mass fraction of rebar species (kg/kg rebar) \\
\hline
\end{tabular}

CORE MELT CONSTITUENTS

INITIAL MASSES, COMPOSITIONS, AND TEMPERATURES

14 I5 NOSI Number of melt oxidic epecies to be input; only apecies available in the Mester Species List may be included

6-10 I5 NMSI Number of melt metallic species to be input; only species available in the Master Species Liat may bo included

11-20 E10.0 TOI Initial oxidic molt comperature (K)

21-30 E10.0 TMI initial motallic molt temperature (K)

15 1.8 A8 NAMSP Name of oxidic species (left justified)

11-20 E10.0 SM Mass of oxidic species (kg)

(There will be NOSI cards in card/group 15.)

$\begin{array}{lllll}16 & 1-8 & \text { A8 } & \text { NAMSP } & \text { Name of motallic species (left justified) }\end{array}$

11-20 E10.0 SM Mass of metallic species (kg)

(There will be NMSI cards in card/group 16.) 
Table 4.1 Input instructions for CORCON-MOD3 (continued)

\begin{tabular}{|c|c|c|c|c|}
\hline $\begin{array}{l}\text { Card/ } \\
\text { Group" }\end{array}$ & Field & Format & Variable Name & Description \\
\hline & & \multicolumn{3}{|c|}{$\begin{array}{l}\text { INTACT CORE SIZE AND POWER, AND NUMBER OF RETENTION FRACTIONS TO } \\
\text { BE MODIFIED }\end{array}$} \\
\hline \multicolumn{5}{|c|}{ Include card/groups 17 and 18 only if IFP $=0}$. \\
\hline \multirow[t]{3}{*}{17} & $1-10$ & E10.0 & XMTU & Core size (metric tons of uranium) \\
\hline & $11-20$ & E10.0 & XMWTH & Core operating power (MW thermal) \\
\hline & $21-25$ & 15 & NUM & $\begin{array}{l}\text { Number of radioactive species in the intact core inventory } \\
\text { for which the retention factor will be modified }(N U M \leq 27)\end{array}$ \\
\hline \multirow[t]{4}{*}{18} & $1-4$ & A4 & IFP1 & $\begin{array}{l}\text { Name of radioactive species whose retention factor is to be } \\
\text { modified (left justified) }\end{array}$ \\
\hline & $11-20$ & E10.0 & RET1 & Retention factor of radioactive species IFP1 \\
\hline & & \multicolumn{3}{|c|}{ (There will be NUM cards in card/group 18.) } \\
\hline & & \multicolumn{3}{|c|}{ COOLANT INITIAL MASS, COMPOSITION, AND TEMPERATURE } \\
\hline \multicolumn{5}{|c|}{ Include card/groups $18 \mathrm{~A}$ and $18 \mathrm{~B}$ only if $\mathrm{ICOOI}=1}$. \\
\hline 18A & $1-10$ & E10.0 & TCI & Initial coolant temperature $(\mathrm{K})$ \\
\hline \multirow[t]{3}{*}{ 18B } & $1-8$ & A8 & NAMSP & Name of coolant species (left justified) (H2O or H2OCLN) \\
\hline & $11-20$ & E10.0 & FMCI & Mass of coolant species 0 \\
\hline & & \multicolumn{3}{|c|}{ ATMOSPHERE INITIAL VOLUME, PRESSURE, TEMPERATURE, AND COMPOSITION } \\
\hline \multirow[t]{4}{*}{19} & $1-10$ & E10.0 & VA & Initial gas volume $\left(\mathrm{m}^{* * 3}\right)$ \\
\hline & $11-20$ & E10.0 & PA & $\begin{array}{l}\text { Initial gas pressure }\left(\mathrm{N} / \mathrm{m}^{* * 2}\right) \\
>0 \text {-- Constant for problem } \\
\leq 0 \text { - Gas pressure specified by card/groups } 20 \mathrm{~A} \& \\
\quad 20 \mathrm{~B}\end{array}$ \\
\hline & 21-30 & E10.0 & TA & Initial gas temperature (K) \\
\hline & $31-35$ & I5 & NGSINP & $\begin{array}{l}\text { Number of gaseous species in the atmosphere; } \\
\text { only species available in the Master Species List may be } \\
\text { included (NGSINP } \leq 18 \text { ). }\end{array}$ \\
\hline \multirow[t]{3}{*}{20} & $1-8$ & A8 & NAMSP & Name of gaseous species (left justified) \\
\hline & $11-20$ & E10.0 & SM & Mole fraction of gaseous species (-) \\
\hline & & \multicolumn{3}{|c|}{ (There will be NGSINP cards in group 20.) } \\
\hline
\end{tabular}

20A

$1-5 \quad 15$

NATMPR

Number of points in table of pressure of gas atmosphere versus time (1 $\leq$ NATMPR $\leq 10)$ 
Table 4.1 Input instructions for CORCON-MOD3 (continued)

\begin{tabular}{cccll}
\hline $\begin{array}{c}\text { Card/ } \\
\text { Group\# }\end{array}$ & Field & Format & Variable Name & Description \\
\hline 20B & $1-10$ & E10.0 & TPA(1) & $\begin{array}{l}\text { Table of gas pressure (PA) versus time (s); alternating values } \\
\text { of time, TPA (J) and pressure, }\end{array}$ \\
& $11-20$ & E10.0 & PAT(1) & PAT(J) for J = 1, NATMPR. \\
& $21-30$ & E10.0 & TPA(2) & If NATMPR =1, pressure is constant at PAT (1). \\
$31-40$ & E10.0 & PAT(2) & \\
& - & - & & \\
& - & - & TPA(NATMPR) & $\begin{array}{l}\text { Up to four pairs per card. May require up to } 2 \text { cards to } \\
\text { complete the table. }\end{array}$ \\
& E10.0 & E10.0 & PAT(NATMPR) &
\end{tabular}

\section{SECTION 3. MELT INTERNAL HEAT GENERATION}

DECAY POWER FOR OXIDIC AND METALLIC PHASES

Include card/group 21 only if IFP $=2$.

$21 \quad 1-5 \quad 15 \quad$ NDECO

6-10 $15 \quad$ NDECM

Include card/group 22 only if NDECO $>0$.

22

$\begin{array}{ccl}1-10 & \text { E10.0 } & \text { TIO(1) } \\ 11-20 & \text { E10.0 } & \text { PIO(1) } \\ 21-30 & \text { E10.0 } & \text { TIO(2) } \\ 31-40 & \text { E10.0 } & \text { PIO(2) } \\ \dot{-} & \dot{ } & \\ & \text { E10.0 } & \text { TIO(NDECO) } \\ & \text { E10.0 } & \text { PIO(NDECO) }\end{array}$

Include card/group 23 only if NDECM $>0$.

23

$\begin{array}{ccl}1-10 & \text { E10.0 } & \operatorname{TIM}(1) \\ 11-20 & \text { E10.0 } & \operatorname{PIM(1)} \\ 21-30 & \text { E10.0 } & \operatorname{TIM(2)} \\ 31-40 & \text { E10.0 } & \operatorname{PIM(2)} \\ \dot{\cdot} & \dot{-} & \\ \cdot & \dot{1} & \\ & \text { E10.0 } & \text { TIM(NDECM) } \\ & \text { E10.0 } & \text { PIM(NDECM) }\end{array}$

Number of points in table of oxidic phase power versus time $(0 \leq$ NDECO $\leq 30)$

Number of points in table of metallic phase power versus time $(0 \leq \mathrm{NDECM} \leq 30)$

Table of oxidic phase power $(W)$ versus time (s); alternating values of time, TIO(J) and power, PIO(J) for $\mathrm{J}=1$, NDECO.

If $\mathrm{NDECO}=1$, power is constant at PIO(1).

Up to four pairs per card. May require up to 8 cards to complete table.

Table of metallic phase power (W) versus time (s); alternating values of time, $\operatorname{TIM}(\mathrm{J})$ and power, $\operatorname{PIM}(\mathrm{J})$ for $\mathrm{J}=1, \mathrm{NDECM}$.

If $\operatorname{NDECM}=1$, power is constant at PIM(1).

Up to four pairs per card. May require up to 8 cards to complete table. 
Table 4.1 Input instructions for CORCON-MOD3 (continued)

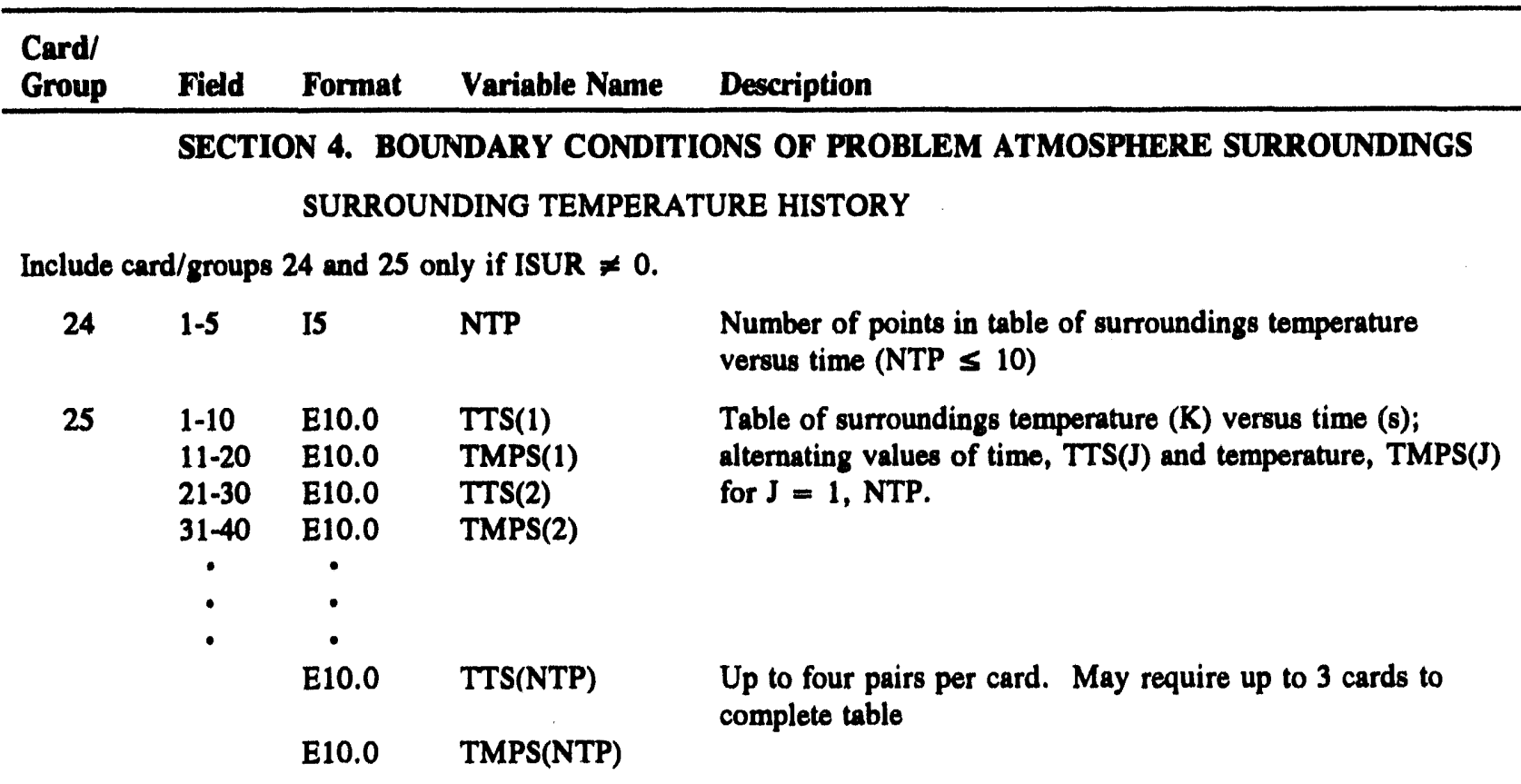

\section{RATES OF SPECIES MASS ADDITION TO POOL}

Include card/groups $26,27,28$ and 29 only if ISRABL $\neq 0$.

\begin{tabular}{|c|c|c|c|c|}
\hline 26 & $1-5$ & 15 & NSPG & $\begin{array}{l}\text { Number of species in mass addition table; } \\
\text { only species available in the Master Species List may be } \\
\text { included (NSPG } \leq 20 \text { ). }\end{array}$ \\
\hline & $6-10$ & I5 & IFPOPT & $\begin{array}{l}\text { Option flag for input of fission product mass addition } \\
0 \text {-- fission product composition calculated internally } \\
1 \text {-- fission product composition specified by user }\end{array}$ \\
\hline 27 & $1-8$ & A8 & NAMSP & Names of species in mass addition table (left justified) \\
\hline
\end{tabular}
$\begin{array}{llll}28 A & 1-5 & 15 & N M P(1)\end{array}$

$\begin{array}{crll}\text { 6-10 } & 15 & \text { NMP(2) } \\ \dot{*} & \dot{-} & \\ - & \dot{15} & \text { NMP(NSPG) }\end{array}$
Number of points in table of mass flow rate versus time for first species as defined in card/group 27.
Number of points in table of mass flow rate versus time for second species as defined in card/group 27.
Number of points in table of mass flow rate versus time for last species as defined in card/group 27. (NMP(I) $\leq 10$, $\mathrm{I}=1, \mathrm{NSPG}$ )


Table 4.1 Input instructions for CORCON-MOD3 (continued)

\begin{tabular}{|c|c|c|c|c|}
\hline $\begin{array}{l}\text { Card/ } \\
\text { Groupt }\end{array}$ & Field & Format & Variable Name & Description \\
\hline \multirow[t]{2}{*}{ 28B } & $\begin{array}{l}1-10 \\
11-20 \\
21-30 \\
31-40 \\
: \\
: \\
:\end{array}$ & $\begin{array}{c}\text { E10.0 } \\
\text { E10.0 } \\
\text { E10.0 } \\
\text { E10.0 } \\
\dot{:} \\
\text { E10.0 } \\
\text { E10.0 }\end{array}$ & $\begin{array}{l}\operatorname{TMS}(1,1) \\
\operatorname{FMS}(1,1) \\
\operatorname{TMS}(2,1) \\
\operatorname{FMS}(2,1) \\
\vdots \\
: \\
\operatorname{TMS}(\operatorname{NMP}(1), 1) \\
\operatorname{FMS}(\operatorname{NMP}(1), 1)\end{array}$ & $\begin{array}{l}\text { Tables of mass flow rate (kg/s) of species I versus } \\
\text { time (s) for each species as definod in card/group } 27 \text {; } \\
\text { alternating values of time, TMS(I,J) and rate, FMS(I,J) for (I } \\
=1, N M P(J)) \text { and }(J=1, N S P G) \text {. }\end{array}$ \\
\hline & $\begin{array}{l}1-10 \\
11-20 \\
: \\
: \\
:\end{array}$ & $\begin{array}{c}\text { E10.0 } \\
\text { E10.0 } \\
\vdots \\
: \\
\text { E10.0 } \\
\text { E10.0 }\end{array}$ & $\begin{array}{l}\text { TMS }(1,2) \\
\text { FMS }(1,2) \\
\vdots \\
\cdot \\
\text { TMS(NMP(NSPG } \\
\text { FMS(NMP(NSPG }\end{array}$ & $\begin{array}{l}\text { Start a new card 28B for each now species. } \\
\text { May require more than one card for each species. }\end{array}$ \\
\hline \multirow[t]{3}{*}{$29 \mathrm{~A}$} & $1-5$ & I5 & NOTS & $\begin{array}{l}\text { Number of points in table of temperature versus time for } \\
\text { added oxide phase. }\end{array}$ \\
\hline & $6-10$ & 15 & NMTS & $\begin{array}{l}\text { Number of points in table of temperature versus time for } \\
\text { added metallic phase. }\end{array}$ \\
\hline & $11-15$ & I5 & NCTS & $\begin{array}{l}\text { Number of points in table of temperature versus time for } \\
\text { added coolant phase. }\end{array}$ \\
\hline $29 B$ & $\begin{array}{l}1-10 \\
11-20 \\
21-30 \\
31-40 \\
\dot{:} \\
\dot{:}\end{array}$ & $\begin{array}{c}\text { E10.0 } \\
\text { E10.0 } \\
\text { E10.0 } \\
\text { E10.0 } \\
: \\
: \\
\text { E10.0 } \\
\text { E10.0 }\end{array}$ & $\begin{array}{l}\text { TIOTS(1) } \\
\text { TOTS(1) } \\
\text { TIOTS(2) } \\
\text { TOTS(2) } \\
: \\
\text { - } \\
\text { TIOTS(NOTS) } \\
\text { TOTS(NOTS) }\end{array}$ & $\begin{array}{l}\text { Tables of time (8) versus temperature (K) for added } \\
\text { oxide phase; } \\
\text { altermating values of time, TIOTS(I) and temperature, } \\
\text { TOTS(I) for (I }=1 \text {, NOTS) }\end{array}$ \\
\hline $29 \mathrm{C}$ & $\begin{array}{l}1-10 \\
11-20 \\
21-30 \\
31-40 \\
: \\
: \\
:\end{array}$ & $\begin{array}{c}\text { E10.0 } \\
\text { E10.0 } \\
\text { E10.0 } \\
\text { E10.0 } \\
: \\
\text { E10.0 } \\
\text { E10.0 }\end{array}$ & $\begin{array}{l}\text { TIMTS(1) } \\
\text { TMTS(1) } \\
\text { TIMTS(2) } \\
\text { TMTS(2) } \\
: \\
: \\
\text { TIMTS(NYTS) } \\
\text { TMTS(NOTS) }\end{array}$ & $\begin{array}{l}\text { Tables of time (s) versus temperature (K) for added } \\
\text { metal phese; } \\
\text { alternating values of time, TIMTS(I) and temperature, } \\
\text { TMTS(I) for (I = 1, NOTS) }\end{array}$ \\
\hline
\end{tabular}


Table 4.1 Input instructions for CORCON-MOD3 (continued)

\begin{tabular}{|c|c|c|c|c|}
\hline $\begin{array}{l}\text { Card/ } \\
\text { Groupt }\end{array}$ & Field & Format & Variable Name & Description \\
\hline \multirow[t]{2}{*}{ 29D } & $\begin{array}{l}1-10 \\
11-20 \\
21-30 \\
31-40 \\
\dot{:} \\
\dot{ } \\
:\end{array}$ & $\begin{array}{c}\text { E10.0 } \\
\text { E10.0 } \\
\text { E10.0 } \\
\text { E10.0 } \\
\dot{~} \\
\dot{\text { E}} \\
\text { E10.0 } \\
\text { E10.0 }\end{array}$ & $\begin{array}{l}\text { TICTS(1) } \\
\text { TCTS(1) } \\
\text { TICTS(2) } \\
\text { TCTS(2) } \\
: \\
: \\
\text { TICTS(NOTS) } \\
\text { TCTS(NOTS) }\end{array}$ & $\begin{array}{l}\text { Tables of time (s) versus temperature (K) for added } \\
\text { coolant phase; } \\
\text { alternating values of time, TICTS(I) and temperature, } \\
\text { TCTS(I) for (I = 1, NOTS) }\end{array}$ \\
\hline & & \multicolumn{3}{|c|}{ EMISSIVITIES FOR RADIATION HEAT TRANSFER COMPUTATIONS } \\
\hline \multirow[t]{3}{*}{30} & $1-4$ & A4 & IREO & $\begin{array}{l}\text { Variable name (either TIME or TEMP) for table of oxidic } \\
\text { phase emissivity versus IREO. }\end{array}$ \\
\hline & $5-8$ & A4 & IREM & $\begin{array}{l}\text { Variable name (either TIME or TEMP) for table of metallic } \\
\text { phase emissivity versus IREM. }\end{array}$ \\
\hline & $9-12$ & A4 & IRES & $\begin{array}{l}\text { Variable name (either TIME or TEMP) for table of } \\
\text { surroundings emissivity versus IRES. }\end{array}$ \\
\hline \multirow[t]{3}{*}{31} & $1-5$ & I5 & NEO & $\begin{array}{l}\text { Number of values in table of oxidic phase emissivities } \\
\text { versus IREO }(1 \leq \text { NEO } \leq 5) \text {. }\end{array}$ \\
\hline & $6-10$ & 15 & NEM & $\begin{array}{l}\text { Number of values in table of metallic phase emissivities } \\
\text { versus IREM }(1 \leq \text { NEM } \leq 5) \text {. }\end{array}$ \\
\hline & $11-15$ & 15 & NS & $\begin{array}{l}\text { Number of values in table of surroundings emissivities versus } \\
\text { NS }(1 \leq \text { NS } \leq 5) \text {. }\end{array}$ \\
\hline 32 & $\begin{array}{l}1-10 \\
11-20 \\
21-30 \\
31-40 \\
: \\
: \\
:\end{array}$ & $\begin{array}{l}\text { E10.0 } \\
\text { E10.0 } \\
\text { E10.0 } \\
\text { E10.0 } \\
\dot{~} \\
\dot{ } \\
\text { E10.0 } \\
\text { E10.0 }\end{array}$ & $\begin{array}{l}\text { TORT(1) } \\
\text { EO(1) } \\
\text { TORT(2) } \\
\text { EO(2) } \\
: \\
\cdot \\
\text { TORT(NEO) } \\
\text { EO(NEO) }\end{array}$ & $\begin{array}{l}\text { Table of oxidic phase emissivity }(-) \text { versus time (s) or } \\
\text { temperature (K); } \\
\text { alternating values of emissivity, EO(I) and time or } \\
\text { temperature, TORT(I) for } I=1, N E O \text {. } \\
\text { If NEO = } 1 \text {, emissivity is constant at } E O(1) \text {. } \\
\text { May require up to } 2 \text { cards to complete table. }\end{array}$ \\
\hline 33 & $\begin{array}{l}1-10 \\
11-20 \\
21-30 \\
31-40 \\
: \\
: \\
: \\
:\end{array}$ & $\begin{array}{l}\text { E10.0 } \\
\text { E10.0 } \\
\text { E10.0 } \\
\text { E10.0 } \\
\cdot \\
\cdot \\
\cdot \\
\text { E10.0 } \\
\text { E10.0 }\end{array}$ & $\begin{array}{l}\text { TORT2(1) } \\
\text { EMM(1) } \\
\text { TORT2(2) } \\
\text { EMM(2) } \\
: \\
: \\
\text { TORT2(NEM) } \\
\text { EMM(NEM) }\end{array}$ & $\begin{array}{l}\text { Table of metallic phase emissivity }(-) \text { versus time (s) } \\
\text { or temperature }(\mathrm{K}) \text {; } \\
\text { alternating values of emissivity, EMM(I) and time or } \\
\text { temperature, TORT2(I) for } \mathrm{I}=1, \mathrm{NEM} \text {. } \\
\text { If NEM }=1 \text {, emissivity is constant at } \operatorname{EMM(1)} \text {. } \\
\text { May require up to } 2 \text { cards to complete table. }\end{array}$ \\
\hline
\end{tabular}


Table 4.1 Input instructions for CORCON-MOD3 (continued)

\begin{tabular}{|c|c|c|c|c|}
\hline $\begin{array}{l}\text { Card/ } \\
\text { Group\# }\end{array}$ & Field & Format & Variable Name & Description \\
\hline 34 & $\begin{array}{l}1-10 \\
11-20 \\
21-30 \\
31-40 \\
: \\
\dot{.} \\
\dot{ }\end{array}$ & $\begin{array}{c}\text { E10.0 } \\
\text { E10.0 } \\
\text { E10.0 } \\
\text { E10.0 } \\
\dot{~} \\
\dot{\text { E10.0 }} \\
\text { E10.0 }\end{array}$ & $\begin{array}{l}\text { TORT6(1) } \\
\text { ES(1) } \\
\text { TORT6(2) } \\
\text { ES(2) } \\
\dot{:} \\
\dot{\text { TORT6(NS) }} \\
\text { ES(NS) }\end{array}$ & $\begin{array}{l}\text { Table of surroundings emissivity }(-) \text { versus time (s) or } \\
\text { temperature (K); } \\
\text { altemating values of emissivity, ES(I) and time or } \\
\text { temperature, TORT6(I) for } I=1, N S \text {. } \\
\text { If NS = } 1 \text {, emissivity is constant at ES(1). } \\
\text { May require up to } 2 \text { cards to complete table. }\end{array}$ \\
\hline \multicolumn{5}{|c|}{ Include card/group $34 \mathrm{~A}$ only if IAOPAC $\neq 0$} \\
\hline 34A & $1-10$ & $\begin{array}{l}\text { E10.0 } \\
\text { SPLASH }\end{array}$ & $\begin{array}{l}\text { RADLEN } \\
\text { UT OF POOL CO }\end{array}$ & $\begin{array}{l}\text { Characteristic path length (m) for atmospheric opacity } \\
\text { calculation. } \\
\text { STITUENTS }\end{array}$ \\
\hline **[Includ & SECTI & N 5. TI & Q-DEPENDENT & ULT RADIUS \\
\hline \multicolumn{5}{|c|}{ Include card/groups 38 and 39 only if IGEOM = 2 and ITIMR $=1}$. \\
\hline 38 & $1-5$ & 15 & NORAD & $\begin{array}{l}\text { Number of points in table of melt radius versus time } \\
(0 \leq \text { NORAD } \leq 12)\end{array}$ \\
\hline & $6-15$ & F10.0 & HTMIN & $\begin{array}{l}\text { Minimum allowable melt thickness }(\mathrm{cm}) \\
\text { (HTMIN } \geq 0.1 \mathrm{~cm})\end{array}$ \\
\hline & $16-25$ & F10.0 & HTMAX & $\begin{array}{l}\text { Maximum allowable melt thickness (cm). } \\
\text { (HTMAX } \leq 10^{5} \mathrm{~cm} \text { ) }\end{array}$ \\
\hline
\end{tabular}


Table 4.1 Input instructions for CORCON-MOD3 (continued)

\begin{tabular}{|c|c|c|c|c|}
\hline $\begin{array}{l}\text { Card/ } \\
\text { Group" }\end{array}$ & Field & Format & Variable Name & Description \\
\hline 39 & $\begin{array}{c}1-10 \\
11-20 \\
21-30 \\
31-40 \\
. \\
. \\
. \\
.\end{array}$ & $\begin{array}{c}\text { F10.0 } \\
\text { F10.0 } \\
\text { F10.0 } \\
\text { F10.0 } \\
\text { : } \\
\text { : } \\
\text { F10.0 } \\
\text { F10.0 }\end{array}$ & $\begin{array}{l}\text { TIMRAD(1) } \\
\text { RADTIM(1) } \\
\text { TIMRAD(2) } \\
\text { RADTIM(2) } \\
: \\
\text { TIMRAD(NORA } \\
\text { D) } \\
\text { RADTIM(NORA } \\
\text { D) }\end{array}$ & $\begin{array}{l}\text { Table of melt radius }(\mathrm{m}) \text { versus time }(\mathrm{s}) \text {; } \\
\text { alternating values of time, TIMRAD(I) and melt radius, } \\
\text { RADTIM(I) for } I=1 \text {, NORAD }\end{array}$ \\
\hline
\end{tabular}

\section{SECTION 6. USER FLEXIBILITY}

Include card/group $40 A$ and 64 only if IUSER $=1$

\begin{tabular}{|c|c|c|c|c|}
\hline \multirow[t]{15}{*}{$40 \mathrm{~A}$} & 1.5 & 15 & IBHTB & $\begin{array}{l}\text { Index specifying modification of the bulk heat transfer } \\
\text { coefficient for the bottom surface }\end{array}$ \\
\hline & $6-10$ & 15 & IBHTS & $\begin{array}{l}\text { Index specifying modification of the bulk heat transfer } \\
\text { coefficient for the side surface }\end{array}$ \\
\hline & $11-15$ & I5 & IIHT & $\begin{array}{l}\text { Index specifying modification of the interlayer heat transfer } \\
\text { coefficient }\end{array}$ \\
\hline & $16-20$ & 15 & ICHT & $\begin{array}{l}\text { Index specifying modification of the coolant beat flux } \\
\text { calculation }\end{array}$ \\
\hline & $21-25$ & 15 & ITMFB & $\begin{array}{l}\text { Index indicating modification of the minimum film boiling } \\
\text { temperature }\end{array}$ \\
\hline & $26-30$ & 15 & IEON & Index for modification of the entrainment onset criteria \\
\hline & $31-35$ & 15 & IERT & Index for modification of the entrainment rate calculation \\
\hline & $36-40$ & 15 & IDRT & Index for modification of the droplet settling calculation \\
\hline & $41-45$ & 15 & ICDC & $\begin{array}{l}\text { Index for modification of the condensed phase diffusion } \\
\text { coefficient }\end{array}$ \\
\hline & $46-50$ & I5 & IVDC & Index for modification of the vapor phase diffusion coefficient \\
\hline & $51-55$ & 15 & ICNCD & Index for modification of the condensation rate coefficient \\
\hline & $56-60$ & 15 & IMU & Index for specification of the mean aerosol particle size \\
\hline & $61-65$ & 15 & IBR & Index for modification of the bubble size models \\
\hline & $66-70$ & 15 & IKM & Index for modification of the metal phase lhermal conductivity \\
\hline & $71-75$ & I5 & IKO & Index for modification of the oxide phase thermal conductivity \\
\hline
\end{tabular}




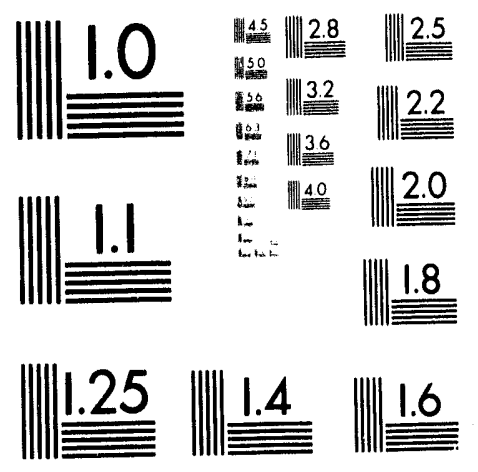



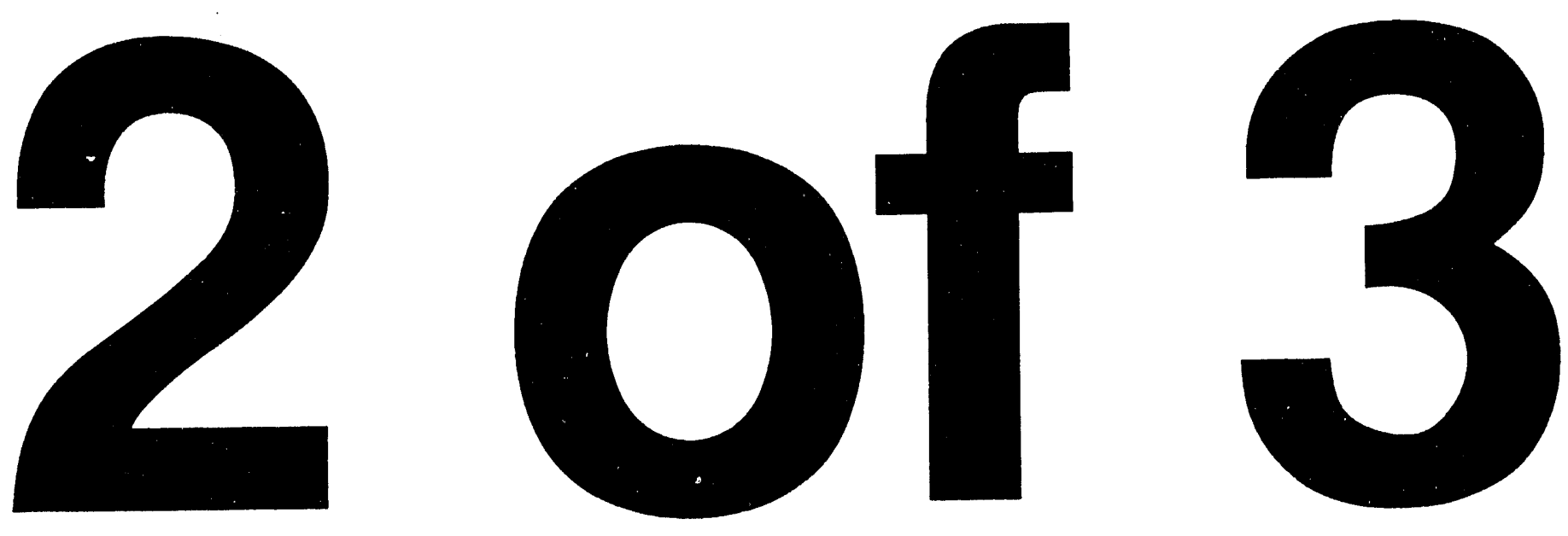
Table 4.1 Input instructions for CORCON-MOD3 (continued)

\begin{tabular}{|c|c|c|c|c|}
\hline $\begin{array}{l}\text { Card/ } \\
\text { Group\# }\end{array}$ & Field & Format & Variable Name & Description \\
\hline \multirow[t]{10}{*}{ 40B } & $1-5$ & 15 & IVSM & Index for modification of the metal phase viscosity \\
\hline & $6-10$ & 15 & IVSO & Index for modification of the oxide phase viscosity \\
\hline & $11-15$ & 15 & ISTM & Index for modification of the metal phase surface tension \\
\hline & $16-20$ & 15 & ISTO & Index for modification of the oxide phase surface tension \\
\hline & $21-25$ & 15 & IRHOM & Index for modification of the oxide phese density \\
\hline & $26-30$ & 15 & IRHOO & Index for modification of the metal phase density \\
\hline & $31-35$ & 15 & IPHM & Index for modification of the metal phase diagram \\
\hline & $36-40$ & 15 & IPHO & Index for modification of the oxide phase diagram \\
\hline & $41-45$ & I5 & IZIP & Index for modification of the cherrical equilibrium cutoff \\
\hline & & & \multicolumn{2}{|c|}{ Include card/group $41 \mathrm{~A}$ only if IBHTB $=1$. } \\
\hline \multirow[t]{4}{*}{$41 \mathrm{~A}$} & $1-10$ & E10.0 & AHTB & Coefficients A,B, and C in: \\
\hline & $11-20$ & E10.0 & BHTB & $N u=A e^{B} P_{r}^{C}$ \\
\hline & $21-30$ & E10.0 & CHTB & \\
\hline & & & Include card/grou & 41B only if IBHTB $<0$. \\
\hline \multirow[t]{2}{*}{$41 B$} & $1-10$ & E10.0 & $\mathbf{X B M}$ & $\begin{array}{l}\text { Multiplier applied to bulk convective heat transfer coefficient } \\
\text { for the bottom surface of the molt }\end{array}$ \\
\hline & & & Include card/grou & $42 \mathrm{~A}$ only if IBHTS $=1$. \\
\hline \multirow[t]{4}{*}{$42 \mathrm{~A}$} & $1-10$ & E10.0 & AHTS & Coefficients $A, B$, and $C$ in: \\
\hline & $11-20$ & E10.0 & BHTS & $\mathrm{Nu}=A \mathrm{Re}^{\mathrm{a}} \mathrm{Pr}$ \\
\hline & $21-30$ & E10.0 & CHTS & \\
\hline & & & Include card/grou & 42B only if IBHTS $<0$. \\
\hline \multirow[t]{2}{*}{ 42B } & $1-10$ & E10.0 & XSM & $\begin{array}{l}\text { Multiplier applied to bulk convective heat transfer coefficient } \\
\text { for the radial (side) surface of the melt }\end{array}$ \\
\hline & & & Include card/grou & $43 \mathrm{~A}$ only if IIHT $=1$ \\
\hline \multirow[t]{4}{*}{$43 \mathrm{~A}$} & $1-10$ & E10.0 & $\mathbf{A I}$ & Coofficients A, B, and C in: \\
\hline & $11-20$ & E10.0 & BI & $N u=A R^{B} P_{r} c$ \\
\hline & $21-30$ & E10.0 & CI & \\
\hline & & & Include card/grou & 43B only if IIHT $<1$. \\
\hline \multirow[t]{2}{*}{ 43B } & $1-10$ & E10.0 & XIM & Multiplier applied to interlayer heat transfer coefficient \\
\hline & & & Include card/grou & $44 A$ only if ICHT $=1$. \\
\hline 44A1 & $1-5$ & 15 & NCHT & $\begin{array}{l}\text { Number of entries in table for coolant heat flux ve. } \Delta T_{\text {max }} \\
\text { (NCHT } \leq 20 \text { ) }\end{array}$ \\
\hline
\end{tabular}


User Information

Table 4.1 Input instructions for CORCON-MOD3 (continued)

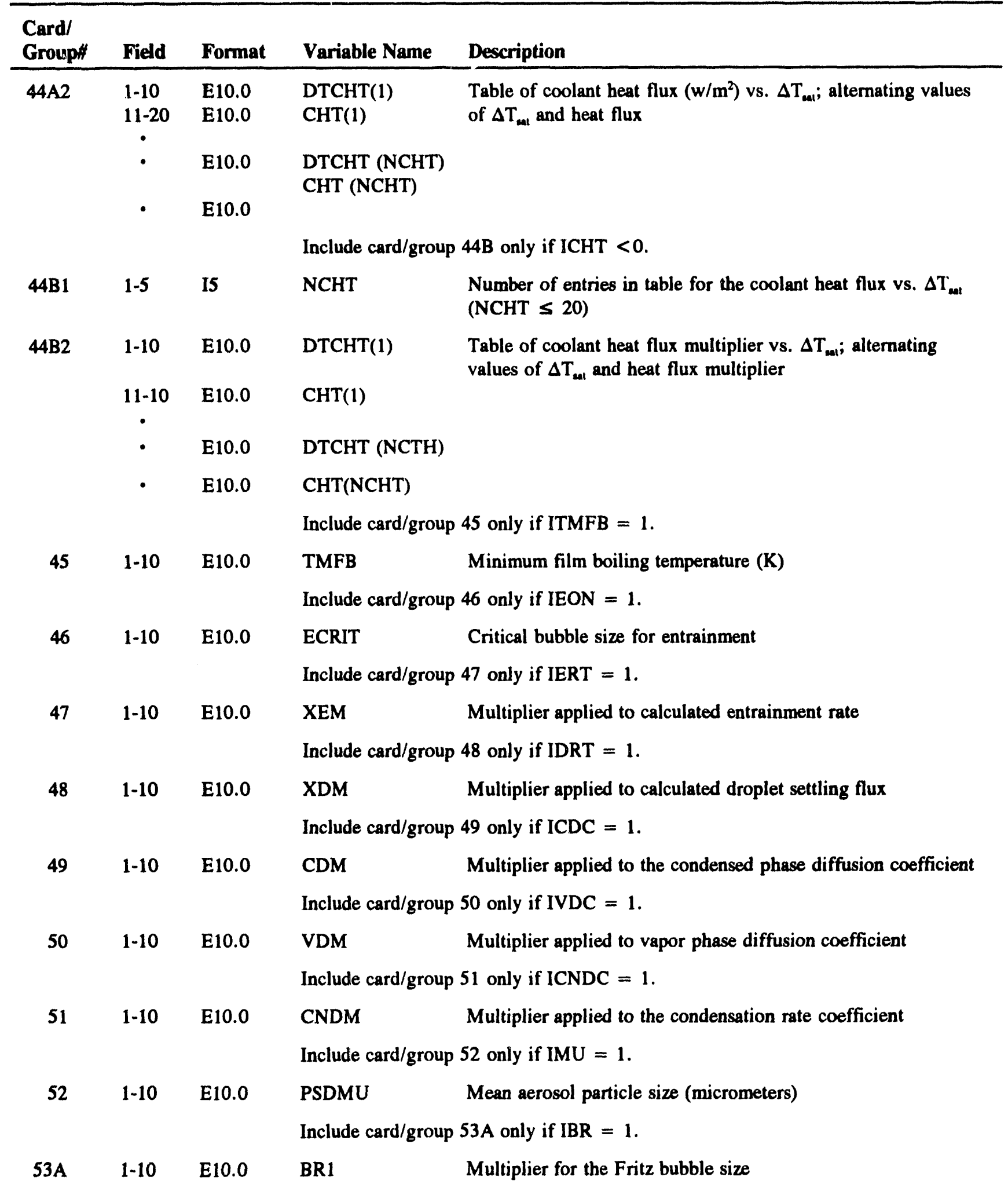


Table 4.1 Input instructions for CORCON-MOD3 (continued)

\begin{tabular}{|c|c|c|c|c|}
\hline $\begin{array}{l}\text { Card/ } \\
\text { Group\# }\end{array}$ & Field & Format & Variable Name & Description \\
\hline & $11-20$ & E10.0 & BR2 & Multiplier for the Davidson-Schuler bubble size \\
\hline & $21-30$ & E10.0 & BR3 & Multiplier for the gas film bubble size \\
\hline & & & Include card/group & 53B only if IBR $<0$. \\
\hline \multirow[t]{2}{*}{ 53B } & $1-10$ & E10.0 & BRFIX & Fixed bubble radius (m) \\
\hline & & & Include card/group & 54 only if IKM $=1$. \\
\hline $54 \mathrm{~A}$ & $1-5$ & I5 & NKMM & $\begin{array}{l}\text { Number of entries in the table of thermal conductivity } \\
\text { multiplier vs. temperature (NKMM } \leq 10)\end{array}$ \\
\hline \multirow[t]{4}{*}{ 54B } & $\begin{array}{l}1-10 \\
11-20 \\
\end{array}$ & $\begin{array}{l}\text { E10.0 } \\
\text { E10.0 }\end{array}$ & $\begin{array}{l}\text { TKMM(1) } \\
\text { XKMM(1) }\end{array}$ & $\begin{array}{l}\text { Table of thermal conductivity multiplier vs. temperature }(\mathrm{K}) \text {; } \\
\text { alternating values of temperature and thermal conductivity } \\
\text { multiplier }\end{array}$ \\
\hline & • & E10.0 & $\begin{array}{l}\text { TKMM } \\
\text { (NKMM) }\end{array}$ & \\
\hline & • & $\mathrm{E} 10.0$ & $\begin{array}{l}\text { XKMM } \\
\text { (NKMM) }\end{array}$ & \\
\hline & & & Include card/group & 55 only if IKO $=1$ \\
\hline $55 \mathrm{~A}$ & $1-5$ & I5 & NKOM & $\begin{array}{l}\text { Number of entries in table of thermal conductivity multiplier } \\
\text { vs. temperature (NKOM } \leq 10 \text { ) }\end{array}$ \\
\hline \multirow[t]{5}{*}{ 55B } & $1-10$ & E10.0 & TKOM(1) & Table of thermal conductivity multiplier vs. temperature $(\mathrm{K})$; \\
\hline & $\begin{array}{l}11-20 \\
\cdot\end{array}$ & E10.0 & XKOM(1) & $\begin{array}{l}\text { alternating values of temperature and thermal conductivity } \\
\text { multiplier }\end{array}$ \\
\hline & • & E10.0 & TKOM (NKOM) & \\
\hline & • & E10.0 & XKOM (NKOM) & \\
\hline & & & Include card/group & 56 only if IVSM $=1$ \\
\hline $56 \mathrm{~A}$ & $1-5$ & 15 & NVSMM & $\begin{array}{l}\text { Number of entries in the table of viscosity multiplier vs. } \\
\text { temperature (NVSMM } \leq 10 \text { ) }\end{array}$ \\
\hline \multirow[t]{5}{*}{ 56B } & $1-10$ & E10.0 & TVSMM(1) & Table of viscosity multipliers vs. temperature (K); alternating \\
\hline & $\begin{array}{c}11-20 \\
.\end{array}$ & & VSMM(1) & sity multiplier \\
\hline & • & E10.0 & $\begin{array}{l}\text { TVSMM } \\
\text { (NVSMM) }\end{array}$ & \\
\hline & • & E10.0 & $\begin{array}{l}\text { VSMM } \\
\text { (NVSMM) }\end{array}$ & \\
\hline & & & Include card/grol & only if IVSO $=1$ \\
\hline
\end{tabular}


Table 4.1 Input instructions for CORCON-MOD3 (continued)

\begin{tabular}{|c|c|c|c|c|}
\hline $\begin{array}{l}\text { Card/ } \\
\text { Group\# }\end{array}$ & Field & Format & Variable Name & Description \\
\hline $57 \mathrm{~A}$ & $1-5$ & I5 & NVSMO & $\begin{array}{l}\text { Number of entries in table of viscosity multiplier vs. } \\
\text { temperature (NVSMO } \leq 10 \text { ) }\end{array}$ \\
\hline \multirow[t]{2}{*}{ 57B } & $\begin{array}{c}1-10 \\
11-20 \\
: \\
:\end{array}$ & $\begin{array}{l}\text { E10.0 } \\
\text { E10.0 } \\
\text { E10.0 } \\
\text { E10.0 }\end{array}$ & $\begin{array}{l}\text { TVSMO(1) } \\
\text { VSMO(1) } \\
\text { TVSMO } \\
\text { (NVSMO) } \\
\text { VSMO } \\
\text { (NVSMO) }\end{array}$ & $\begin{array}{l}\text { Table of viscosity multiplier vs. temperature }(\mathrm{K}) \text {; alternating } \\
\text { values of temperature and viscosity multiplier }\end{array}$ \\
\hline & & & \multicolumn{2}{|c|}{ Include card/group 58 only if ISTM $=1}$. \\
\hline \multirow[t]{2}{*}{58} & $1-10$ & E10.0 & STMM & Multiplier applied to the metal phase surface tension \\
\hline & & & \multicolumn{2}{|c|}{ Include card/group 59 only if ISTO $=1}$. \\
\hline \multirow[t]{2}{*}{59} & $1-10$ & $\mathrm{E} 10.0$ & STMO & Multiplier applied to the oxide phase surface tension \\
\hline & & & \multicolumn{2}{|c|}{ Include card/group 60 only if IRHOM $=1}$. \\
\hline \multirow[t]{2}{*}{60} & $1-10$ & E10.0 & RHOMM & Multiplier applied to the metal phase density \\
\hline & & & \multicolumn{2}{|c|}{ Include card/group 61 only if IRHOO $=1}$. \\
\hline \multirow[t]{2}{*}{61} & $1-10$ & E10.0 & RHOOM & Multiplier applied to the oxide phase density \\
\hline & & & \multicolumn{2}{|c|}{ Include card/group 62 only if IPHM $=1}$. \\
\hline \multirow[t]{2}{*}{62} & $1-10$ & E10.0 & TSOLMT & Constant value for the metal phase solidus temperature (K) \\
\hline & & & \multicolumn{2}{|c|}{ Include card/group 63 only if IPHO $=1}$. \\
\hline \multirow[t]{2}{*}{63} & $1-10$ & E10.0 & XEUT & $\begin{array}{l}\text { Mole fraction of concrete oxides at which the oxide phase } \\
\text { solidus temperature has decreased to the concrete solidus }\end{array}$ \\
\hline & & & \multicolumn{2}{|c|}{ Include card/group 64 only if IZIP $=1}$. \\
\hline 64 & $1-10$ & E10.0 & ZIP & $\begin{array}{l}\text { Value of the ZILCH parameter in MLTREA = cutoff for } \\
\text { inclusion in the chemistry solution (default: } 1 . E-5 \text { moles) }\end{array}$ \\
\hline
\end{tabular}


Table 4.1 Input instructions for CORCON-MOD3 (continued)

\begin{tabular}{|c|c|c|c|c|}
\hline $\begin{array}{l}\text { Card/ } \\
\text { Group\# }\end{array}$ & Field & Format & Variable Name & Description \\
\hline \multicolumn{5}{|c|}{ SECTION 7. VANESA INPUT } \\
\hline \multicolumn{5}{|c|}{ VANESA INPUT OPTIONS } \\
\hline 65 & $1-5$ & 15 & IBUB & $\begin{array}{l}\text { Bubble diameter option index } \\
0 \text {-- Fixed bubble diameter is user specified } \\
1 \text {-- Bubble diameter is calculated internally }\end{array}$ \\
\hline & $6-10$ & 15 & KATIS & $\begin{array}{l}\text { Mechanical release option index } \\
0 \text {-- Fixed release } \\
1 \text {-- Azbel, Ishii \& Kataoka correlations are calculated } \\
\text { internally. }\end{array}$ \\
\hline & $11-15$ & 15 & INOPL & $\begin{array}{l}\text { INOPL option } \\
0 \text {-- No pool calculation } \\
1 \text {-- Pool calculation activated }\end{array}$ \\
\hline & $16-20$ & 15 & IDEAL & $\begin{array}{l}\text { Non-ideal chemistry index } \\
0 \text {-- Calculate activity coefficient for use in VANESA } \\
1 \text {-- Assume ideal chemistry }\end{array}$ \\
\hline \multicolumn{5}{|c|}{ BCL COMPOSITION } \\
\hline \multicolumn{5}{|c|}{ Include card/groups $66 \mathrm{~A}, 66 \mathrm{~B}$ and $66 \mathrm{C}$ only if IVANFP $=1$} \\
\hline $66 \mathrm{~A}$ & $\begin{array}{l}1-10 \\
11-20 \\
21-30 \\
31-40 \\
41-50 \\
51-60 \\
61-70 \\
71-80\end{array}$ & $\begin{array}{l}\text { E10.0 } \\
\text { E10.0 } \\
\text { E10.0 } \\
\text { E10.0 } \\
\text { E10.0 } \\
\text { E10.0 } \\
\text { E10.0 } \\
\text { E10.0 }\end{array}$ & $\begin{array}{l}\text { CES } \\
\text { IOD } \\
\text { XEN } \\
\text { KRY } \\
\text { TE } \\
\text { BA } \\
\text { SN } \\
\text { RU }\end{array}$ & $\begin{array}{l}\text { Mass of Cesium melt constituent }(\mathbf{k g}) \text {. } \\
\text { Mass of Iodine melt constituent }(\mathbf{k g}) \text {. } \\
\text { Mass of Xenon melt constituent }(\mathbf{k g}) \text {. } \\
\text { Mass of Krypton melt constituent }(\mathbf{k g}) \text {. } \\
\text { Mass of Tellurium melt constituent }(\mathbf{k g}) \text {. } \\
\text { Mass of Barium melt constituent }(\mathbf{k g}) \text {. } \\
\text { Mass of Tin melt constituent }(\mathbf{k g}) \text {. } \\
\text { Mass of Ruthenium melt constituent }(\mathbf{k g}) \text {. }\end{array}$ \\
\hline $66 \mathrm{~B}$ & $\begin{array}{l}1-10 \\
11-20 \\
21-30 \\
31-40 \\
41-50 \\
51-60 \\
61-70 \\
71-80\end{array}$ & $\begin{array}{l}\text { E10.0 } \\
\text { E10.0 } \\
\text { E10.0 } \\
\text { E10.0 } \\
\text { E10.0 } \\
\text { E10.0 } \\
\text { E10.0 }\end{array}$ & $\begin{array}{l}\text { MO } \\
\text { SR } \\
\text { RB } \\
\text { Y } \\
\text { TC } \\
\text { RH } \\
\text { PD } \\
\text { LA }\end{array}$ & $\begin{array}{l}\text { Mass of Molybdenum melt constituent }(\mathbf{k g}) \text {. } \\
\text { Mass of Strontium melt constituent }(\mathbf{k g}) \text {. } \\
\text { Mass of Rubidium melt constituent }(\mathbf{k g}) \text {. } \\
\text { Mass of Yttrium melt constituent }(\mathbf{k g}) \text {. } \\
\text { Mass of Technetium melt constituent }(\mathbf{k g}) \text {. } \\
\text { Mass of Rhodium melt constituent }(\mathbf{k g}) \text {. } \\
\text { Mass of Palladium melt constituent }(\mathbf{k g}) \text {. } \\
\text { Mass of Lanthanum melt constituent }(\mathbf{k g}) \text {. }\end{array}$ \\
\hline $66 \mathrm{C}$ & $\begin{array}{l}1-10 \\
11-20 \\
21-30 \\
31-40 \\
41-50 \\
51-60 \\
61-70 \\
71-80\end{array}$ & $\begin{array}{l}\text { E10.0 } \\
\text { E10.0 } \\
\text { E10.0 } \\
\text { E10.0 } \\
\text { E10.0 } \\
\text { E10.0 } \\
\text { E10.0 } \\
\text { E10.0 }\end{array}$ & $\begin{array}{l}\text { CE } \\
\text { PR } \\
\text { ND } \\
\text { SM } \\
\text { PU } \\
\text { AG } \\
\text { SB } \\
\text { NB }\end{array}$ & $\begin{array}{l}\text { Mass of Cerium melt constituent }(\mathbf{k g}) \text {. } \\
\text { Mass of Praseodymium melt constituent }(\mathbf{k g}) \text {. } \\
\text { Mass of Neodymium melt constituent }(\mathbf{k g}) \text {. } \\
\text { Mass of Samarium melt constituent }(\mathbf{k g}) \text {. } \\
\text { Mass of Plutonium melt constituent }(\mathbf{k g}) \text {. } \\
\text { Mass of Silver melt constituent }(\mathbf{k g}) \text {. } \\
\text { Mass of Antinomy melt constituent }(\mathbf{k g}) \text {. } \\
\text { Mass of Niobium melt constituent }(\mathbf{k g}) \text {. }\end{array}$ \\
\hline
\end{tabular}


Table 4.1 Input instructions for CORCON-MOD3 (continued)

\begin{tabular}{|c|c|c|c|c|}
\hline $\begin{array}{l}\text { Card/ } \\
\text { Group\# }\end{array}$ & Field & Format & Variable Name & Description \\
\hline \multirow[t]{6}{*}{67} & $1-10$ & I10 & $\mathrm{I} 1=\mathrm{NOSC}$ & $\begin{array}{l}\text { Number of size segments used to describe the aerosol size } \\
\text { distribution }(4 \leq \text { NOSC } \leq 50) \text { (If II }<0 \text {, use default: } \\
\text { NOSC }=20 .)\end{array}$ \\
\hline & $11-20$ & F10.0 & $\mathrm{F} 1=\mathrm{GSD}$ & $\begin{array}{l}\text { Geometric standard deviation of size distribution of aerosols } \\
\text { entering water pool (If } F 1<0 \text {, use default: } G S D=2.3 \text { ) }\end{array}$ \\
\hline & $21-30$ & I10 & $\mathrm{I} 2=\mathrm{IDMF}$ & $\begin{array}{l}\text { Switch that controls the diffusion mechanism for aerosol } \\
\text { entrapment by the water pool. } \\
0 \text {-- Diffusion mechanism inactive } \\
\neq 0 \text {-- Diffusion mechanism active }\end{array}$ \\
\hline & $31-40$ & 110 & $13=I M P F$ & $\begin{array}{l}\text { Switch that controls the impaction mechanism for aerosol } \\
\text { entrapment by the water pool. } \\
0 \text { - Impaction mechanism inactive } \\
\neq 0 \text {-- Impaction mechanism active }\end{array}$ \\
\hline & $41-50$ & F10.0 & $\mathrm{F} 2=\mathrm{BSIZI}$ & $\begin{array}{l}\text { Diameter of gas bubbles at base of the water pool }(\mathrm{cm}) \\
\text { (If } \mathrm{F} 2<0 \text {, use default: BSIZI }=1.0 \mathrm{~cm} \text { ) }\end{array}$ \\
\hline & $51-60$ & F10.0 & F3 = VROVR & $\begin{array}{l}\mathrm{V}(\mathrm{rel}) / \mathrm{V}(\text { rise), the ratio of gas velocity within the bubble to } \\
\text { the rise velocity of the bubble. } \\
\text { (If } \mathrm{F} 3<0 \text {, use default: VROVR }=1.0 \text { ) }\end{array}$ \\
\hline
\end{tabular}


interesting points in the development of the calculation, without providing unneeded detail during the remainder of the problem. The actual timesteps employed may differ from those specified, if necessary, to produce edits at the requested times.

Edits are produced at times which are integral multiples of the edit interval DEDIT(I), rather than a DEDIT(I)

from the previous edit. This minimizes the effect of the insertion of additional edits, which is valuable if one must compare the outputs from several calculations at the same times. To clarify this, consider as an example a calculation performed with edit controlled by an input card such as

$$
1200.0 \quad 21600.0
$$

This will produce printed output every $1200.0 \mathrm{~s}$ (20 minutes), at times of $1200.0 \mathrm{~s}, 2400.0 \mathrm{~s}, 3600.0 \mathrm{~s}$, etc. Suppose that some interesting phenomenon is observed at about $3000 \mathrm{~s}$, and that more frequent output is desirable to study it. The set of input cards

$\begin{array}{rr}1200.0 & 3000.0 \\ 60.0 & 3300.0 \\ 1200.0 & 21600.0\end{array}$

will produce additional printed output at $3000.0 \mathrm{~s}, 3060.0$ s, .., $3300.0 \mathrm{~s}$. However, the later edits will still be written at times of $3600.0 \mathrm{~s}, 4800.0 \mathrm{~s}$, etc., rather than being shifted to $4500.0 \mathrm{~s}, 5700.0 \mathrm{~s}$, etc.

If variable timestep control is specified (DELTIM $<0$ ) but no intervals are specified, then the calculation will be terminated following input.

If variable edit control is specified (IPINC < 0 ) but no intervals are specified, then the calculation will be run but no printed output will be generated. This might be desired by a user who is interested only in plots of the output data.

\section{Tables}

All input tables are automatically linearly interpolated during execution. This is true for tables given as a function of time and those specified as a function of temperature.

\section{Initial Cavity Geometry}

The definitions of most of the variables used to describe the initial cavity geometry (cards 5 to 9) should be apparent from Figures 4.2 through 4.4 , or from the definitions of the variables in Section 5.2. However, the variables NBOT and ITANG warrant further discussion.

The cavity-shape description introduces a "tangent ray" parallel to the cavity axis through the tangent point between the flat bottom of the cavity and the curved sides. This ray is used in the shape-change calculation to maintain the flat bottom of the cavity as required by the models.

For the flat-bottomed cylinder, IGEOM $=2$, or the arbitrary cavity shape, IGEOM $=4$, there is already a normal ray through this tangent point, which is numbered NBOT. The tangent ray, numbered ITANG =NBOT + 1 , defines an additional body point which initially coincides with that defined by the ray NBOT, but which diverges from it as the surface recedes. The number of the rays is changed as the calculation proceeds, to reflect their actual ordering along the cavity surface. (Note that "normal" points can cross over the tangent ray.)

In the case of a hemispherically based cavity, IGEOM = 1 , a flat bottom is assumed to exist with a radius that is one percent of the sphere radius RS, and only a single point is defined with the number ITANG $=$ NBOT $=2$.

\section{The Size and Power of the Intact Core}

The size and power of the intact core (card 17) is used to determine the concentration of fission products in the fuel if IFP $=0$. This concentration, as modified by retention factors for each element included, together with the $\mathrm{UO}_{2}$ mass in the pool, is used to evaluate the initial fission-product inventory of the melt. If the initial mass exceeds the size of the intact core, a warning is issued.

\subsubsection{Recommended Values and Default Values for Input Quantities}

Recommended values for some of the input quantities are described below. Except for the default concrete compositions listed in Table 2.2, default values are not used in CORCON. That is, if a required input value is not defined, then in general code execution is terminated with an appropriate warning or error message.

\section{Timestep Values}

The timestep for CORCON is controlled by user input (card 3 and, optionally, group 3A). In selected values for the timestep, the user should remember that the CORCON model is explicit in time for the calculation of ablation rates and convective mass and energy flows. Therefore, if the timesteps employed are too large, 


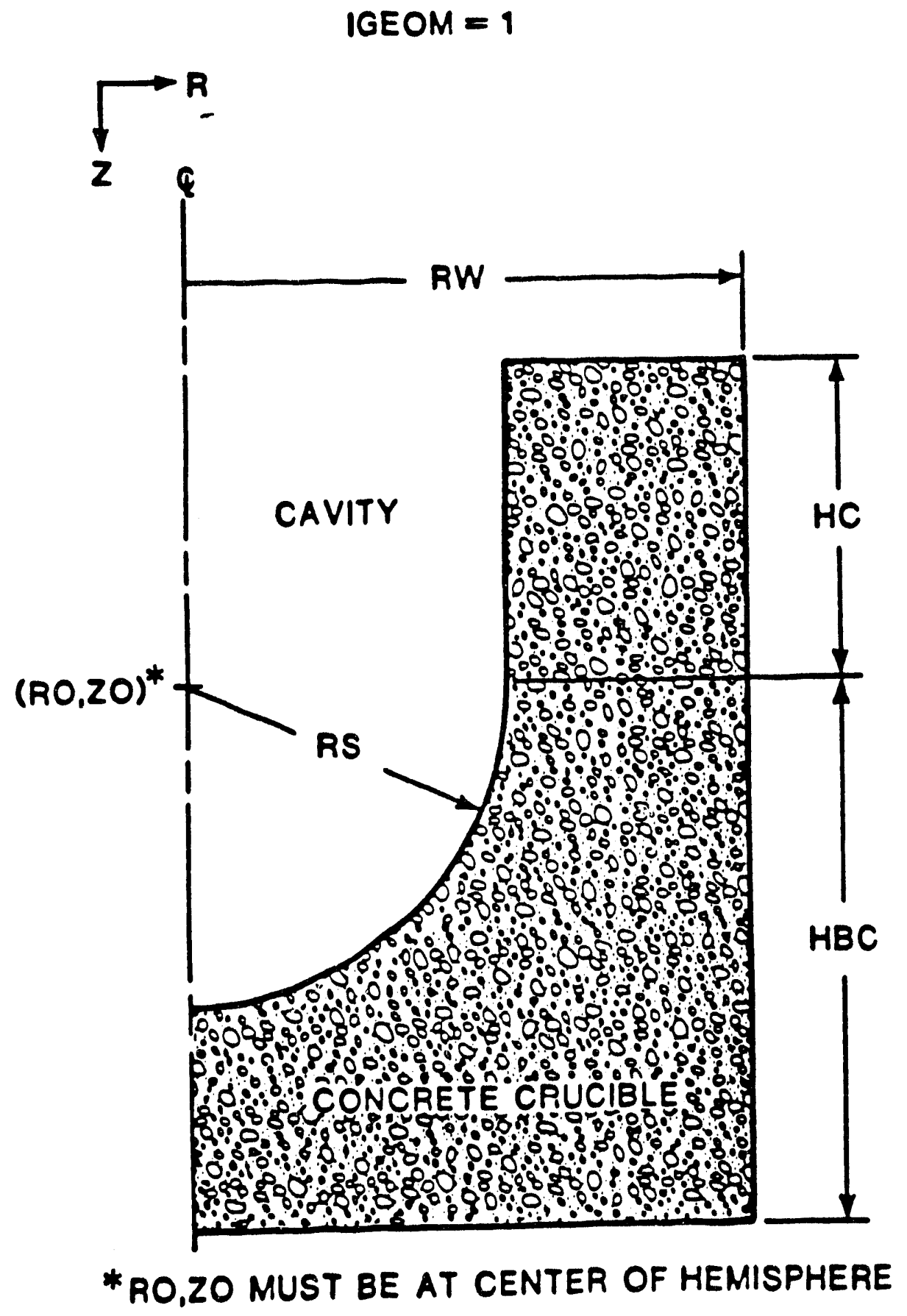

Figure 4.2 Initial cavity geometry - cylinder with hemispherical base 


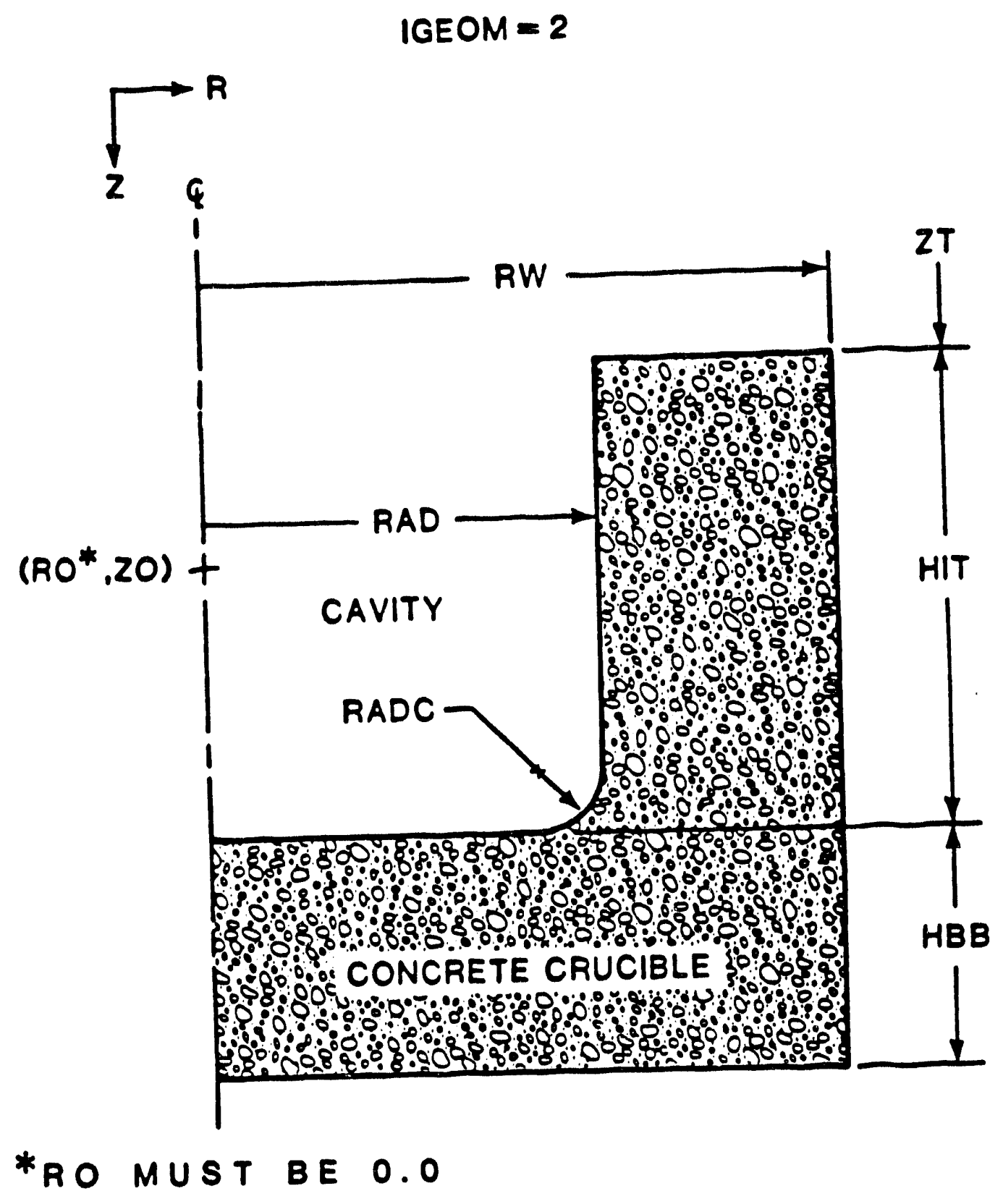

Figure 4.3 Initial cavity geometry - arbitrary shape 


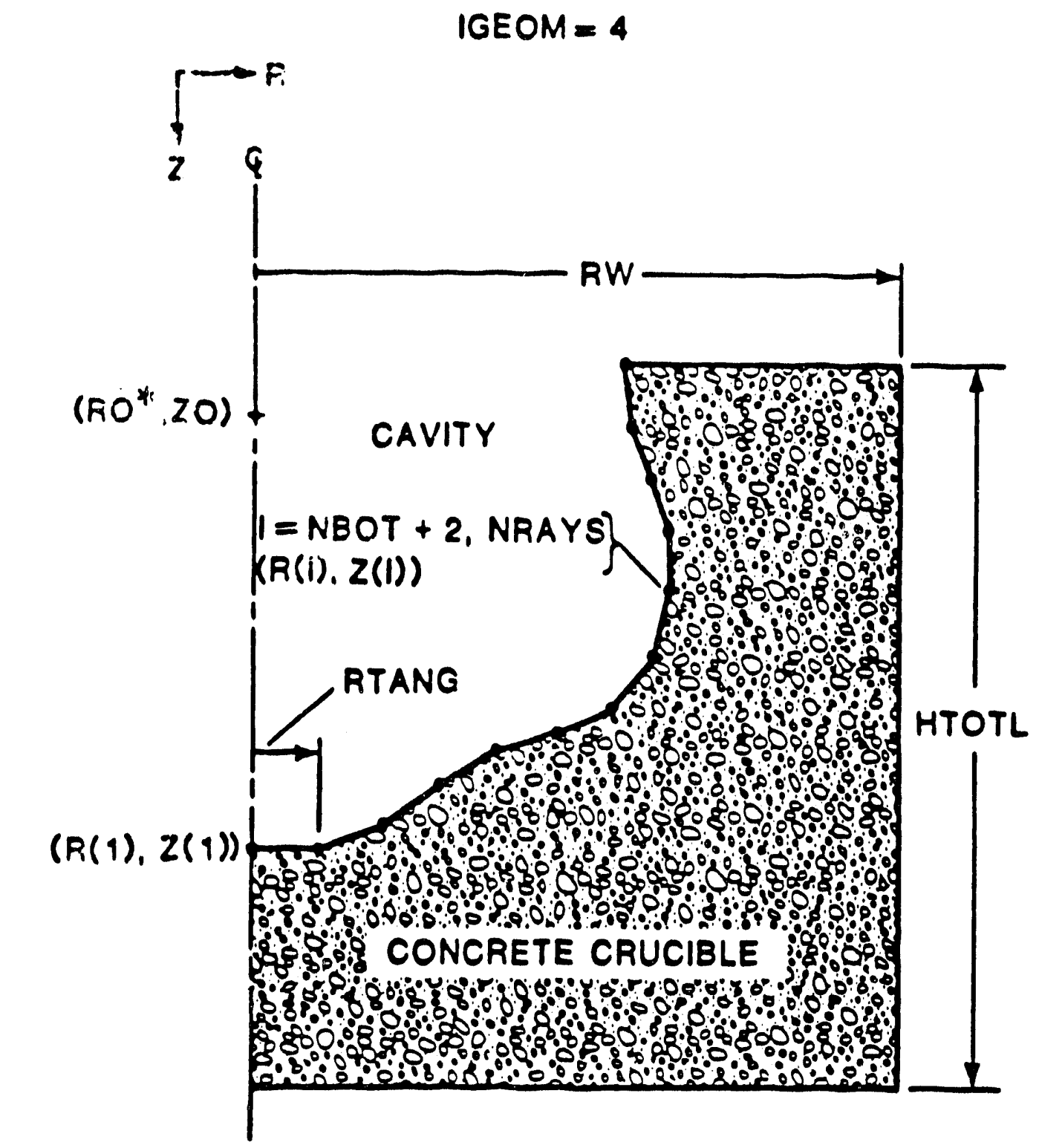

* RO MUST BE 0.0

Figure 4.4 Initial cavity geometry - cylinder with flat base 
unphysical results may be generated or the calculation may fail completely

Based on experience during the development and testing of CORCON-Mod3 and on experience with previous versions of CORCON, we recommend timestep value:, in the range of 10 to $60 \mathrm{~s}$. Short timesteps should be tised whenever conditions are changing rapidly such as during periods of high heat transfer (rapid concrete ablation), vigorous exothermic chemical reactions, or rapid interlayer mixing. Conditions such as these are most likely to occur at early times. At later times, it may be possible to increase the timestep without incurring numerical problems. Timestep control is accomplished using card group $3 \mathrm{~A}$.

\section{Concrete Ablation Temperature}

The ablation temperature for concrete, IW, is not clearly defined and must be specified by the user. The user is limited to values between the liquidus and solidus temperatures for the concrete selected. Based on past experience in simulating experiments with CORCONMod3, we currently recommend that the ablation temperature be selected at or near the liquidus temperature for the concrete. This selection implies that concrete is not displaced from the interface until it is almost completely molten. The liquidus and solidus temperatures for the default concretcs are listed in Table 2.3 .

\section{Initial Problem Time}

When using the internally calculated decay heat generation, the initial time, TIMEO, corresponds to the time following reactor shutdown (SCRAM) that the melt is deposited into the reactor cavity. If a specific time is not known, a value of TIMEO in the range of two to five hours would be reasonable.

\section{Cavity Coordinate System}

In the specification of the ray system (card 4$)$, the origin must lie on the axis of symmetry ( $R O=0.0)$. Based on sensitivity calculations, we recommend that $\mathrm{ZO} \mathrm{L}$ c chosen to maximize the number of rays whose intersection with the cavity surface is close to perpendicular, which avoids small-angle intersections. In any case, the origin must lie above the bottom of the cavity. The ray spacing should be chosen so that the separation between body points (i.e., intersections of rays with the cavity surface) is initially in the range of three to $30 \mathrm{~cm}$. Although up to 100 rays may be specified, most applications will require fewer than 60 rays. (Given the uncertainty in modeling heat transfer to the concrete, there is no real benefit to using a greater number of rays to achicve better definition of the cavity shape.)

\subsection{Description of the Output}

\subsubsection{Numerical Output}

The output from CORCON-Mod3 is stored in the file, CCMod3.OUT. This file contains several major sections.

The first section is an echo of the input data; it is an uninterpreted reproduction of the records comprising the input stream. This provides a compact, permanent record of the data used in a particular calculation, and is useful for finding input errors.

The second section contains the input data as interpreted in CORCON. It contains a description of the concrete cavity, the composition of the concrete (either default or user-specified), the fission product inventory for the melt and the initial conditions for the melt, the identity and quantity of the ccolant (if any), the composition of the atmosphere above the melt pool, the type of boundary conditions, the composition and mass flow rate of material entering the melt pool, and the radiative boundary conditions for the melt pool. Error messages are printed in this section if errors are detected. If errors are detected, code execution continues to the end of this section, and then terminates. This can reduce the number of passes needed to find all the errors in an input deck.

The third output section presents a temporal snapshot of the state of the core-concrete interaction. This is generated at the start of the problem, TIMEO, and at subsequent times as specified in the input. The information is presented in several sections, each starting on a new page of output, with a running header to identify the version of the code in use, the problem title, the problem time, and the timestep number. The sections are a general summary, and summaries of gas generation, cavity geometry, heat transfer, chemical reactions, melt pool geometry, melt pool composition, melt layer properties for the melt pool, derosol generation and radionuclide release, and aerosol removal in an overlying water pool. The latter two sections are printed only if the user has chosen to perform the VANESA calculation. These secions are described in greater detail below.

Various informational messages, such as those describing changes in melt layer orientation or warnings concerning the number of iterations required in various routines, are printed between the snapshot edits. A fourth section of 
output may be generated by use of the TPRIN variable on Card 3. This section contains extensive diagnostic information, and is intended primarily for debugging the code; it is labeled in terms of FORTRAN variable names and assumes that the user has detailed knowledge of the code. Under ordinary circumstances, this output should bo suppressed by specifying TPRIN greater than the problem end time, TIMEND.

\section{General Summary}

The general summary section of the snapshot edit contuins the pool configuration, the maximum depth and radius of the cavity, an approximate energy budget, and quantities needed to check the accuracy of the numerics of the code.

The pool configuration is given as the number of layers in the pool, the configuration of the layers, and the presence of coolant.

The maximum depth and radius of the cavity are given in meters, with the locations of the points of maximum depth and maximum radius, and the remaining axial and radial concrete thicknesses.

The energy budget is a summary of the rates of gain or loss of energy (in watts) from the pool through various mechanisms. The budget is not exact: some rates are current and some are averages over the preceding timestep. In some cases, approximations have been made in the calculation of the rates given in the energy budget which are not used in the internal CORCON calculations. For these reasons, the printed energy budget may not balance exactly, although the error is usually small (on the order of a few percent). However, large discrepancies may appear following a major discontinuity in the physical phenomena, such as problem initiation, depletion of the coolant, or layer flip. The energy budget is for debris-containing layers only; it does not include the coolant or the concrete.

Detailed checks on conservation of mass and energy in the entire pool (including coolant, if present) are made and printed in this section. The printed values are the discrepancies between the sums of layer contents and the values required by conservation within the pool boundaries, and are presented as relative errors in mass and enthalpy. Normally each value should be near machine round-off for single-precision arithmetic.

\section{Gas Generation Summary}

The gas generation summary section of the snapshot edit contains generation rates and cumulative releases for all the condensible and noncondensible gas species in the problem. These are presented as masses and moles, and include the gases in bubbles, the gas film, and vaporized coolant.

\section{Cavity Geometry Summary}

The cavity geometry summary section of the snapshot edit presents the locations of the body points defining the cavity shape, the distance of each body point from the bottom center along the concrete surface, the local slope of the wall, and the angle of the ray which defines the point. Also, the cumulative volume and surface area below each point, the average void fraction at the elevation of the point are presented for each point. The locations of interfaces between pool layers are also given.

\section{Heat Transfer Summary}

The heat transfer summary section of the snapshot edit presents, for each body point, the local normal concrete ablation rate, the film conditions, the temperature of the pool/film interface or of the pool/concrete interface, the film thickness, film-wise mass flow rate, the average velocity of the gas in the film (if a gas film is present), the Reynolds number, the film flow regime, the contributions of radiation and convection to the heat flux, and the heat transfer coefficient. If no gas film is present, the film thickness, film-wise mass flow rate, the average velocity of the gas in the film, and the Reynolds number will all be zero or very small numbers.

\section{Chemical Reactions Summary}

The chemical reactions summary section of the snapshot edit presents the results of the chemical reactions in each layer during the timestep. For a metal layer, the results of the metal/gas or metal/gas/concrete oxide reactions are printed. For a heterogeneous layer, the results of the bulk metal/oxide/gas reactions, are printed. For the film, the results of the film metal/gas reactions during the timestep are printed. The quantities of the reactants and products are printed in moles for each of the oxide, metal, and gas species involved in the reactions.

\section{Melt Pool Composition Summary}

The melt pool composition summary section of the snapshot edit presents the mass of each species present in each occupied layer of the pool and the total mass of each species present in the pool. 


\section{Melt Pool Geometry Summary}

The melt pool geometry summary section of the snapshot edit presents the radius and thickness of the melt pool. This section is printed only if the time-dependent melt radius option is used.

\section{Melt Layer Properties Summary}

The melt layer properties summary section of the snapshot edit presents the values of thermophysical and transport properties for each layer in the melt pool. Also, quantities related to gas bubbles, such as the bubble radius and velocity, are presented, and heat transfer coefficients, crust model quantities, and axial interface temperatures and layer edge temperatures are presented. Important terms in the layer energy equation are given.

All quantities are evaluated at the current time, with the exception of the heat of reaction entry, which is an average over the preceding timestep.

\section{Interlayer Mixing Summary}

The interlayer mixing summary is provided if a mixture layer exists; it is not necessary for the mixing option to be active. The summary lists properties for each phase in a mixture layer. This information supplements the layer property information provided in the preceding edit summary. Also included in this summary are the masses of oxide or metal entrained and de-entrained during the timestep.

\section{Aerosol and Radionuclide Release Summary}

The aerosol and radionuclide release summary is provided if the user has chosen to perform the VANESA calculation. This output summary provides the aerosol release rate, aerosol concentration in the gas stream, aerosol composition, aerosol material density, aerosol particle size, and the temperatures and gas composition used in the VANESA calculation.

\section{Aerosol Removal in an Overlying Water Pool Summary}

This section is provided only if the user has chosen to perform the VANESA analysis and an overlying water pool is present. The output in this section includes particle size-dependent decontamination factors and mass distribution. Also printed are the overall decontamination factor and total mass of aerosol emerging from the water pool.

\subsubsection{Graphical Output}

Output files for generating graphical depictions of the data are obtained by setting IPG to a non-zero value to obtain a set of files for plotting such things as gas generation rates and melt temperatures as functions of time, or setting IMOV to a non-zero value to obtain a file of cavity shape data for making movies of the evolution of the cavity shape.

The quantities written to the various output files for plotting are described below. Except as noted, each quantity is written at each edit time.

\section{Unit 30 (movie.dat)}

The quantities written to Unit 30 are the number of rays, the outer radius ot the concrete crucible $[\mathrm{m}]$, and the axial coordinate of the bottom of the crucible [m] (on the first line of the file). Then the time [s], the rz-coordinates of each body point, the radius of the top surface of the melt pool or coolant, and the axial location of this surface (all in meters) are written. This information can be used to generate plots of the temporal cavity shape, but doing so requires that the user develop his/her own post-processor.

\section{Unit 31 (ccmod3.plt)}

The quantities written to Unit 31 include the time [s] and then, for each layer, the layer temperature $[K]$, the layer mass $[\mathrm{kg}]$, the layer density $[\mathrm{kg} / \mathrm{m}$ ], the layer void faction [-], the temperature of the lower layer surface [K], and the heat transfer from the lower surface [W]. Also written are the coordinates $[\mathrm{m}]$ of the locations of maximum radius and maximum axial depth of the melt pool, the maximum axial and radial erosion distances, the axial erosion rate at the first body point (i.e., at the cavity centerline), the cumulative gas generated [g-mole] by species, and the rate of gas generation [ $\mathrm{g}$-moles/s] by species.

If a VANESA calculation is performed, Unit 31 is also used to store time-dependent information on radionuclide release and aerosol generation. The quantities written to Unit 31 are the aerosol generation rate $[\mathrm{g} / \mathrm{s}]$, and the aerosol concentration at ambient conditions $\left[\mathrm{g} / \mathrm{m}^{3}\right]$.

The quantities written to this file may be read by any FORTRAN program that includes the appropriate unformatted read statements. An example of the required read statements for each time point fellows:

real time(npts), tlay (npts,6), amlay (npts,6)

real sdot(npts),dz(npts),dr(npts) 
real outgas(npts,4), doutgs(npts,4), q(npts,6), auum(6), ner(npts,3) double precition Xmas

read(ntp2) times(jpt)

do $10 \mathrm{i}=1,6$

read(ntp2) thay $(\mathrm{pt} t, \mathrm{i})$, amlay $(\mathrm{jpt}, \mathrm{i}), \times 1, \times 2, \times 3, \times 4$

10 continue

$\operatorname{read}(\mathrm{ntp} 2) \mathrm{z1} 1, \mathrm{r1}, \mathrm{r2}, \mathrm{z2}$

read(ntp2) adot(jpt)

$\operatorname{read}(n t p 2) d z(j p t, d r(j p t)$

read(ntp2) (outgan(jpt, $), i=1,4$ )

read(ntp2) (doutge(jpt,i), $i=1,4$ )

read(ntp2) ( $q(p t, i), i=1,5)$

read(ntp2) aer(jpt, 1), ner(jpt, 2), xmas

$\operatorname{aer}(\mathrm{pl}, 3)=\mathrm{xmas}$

\subsection{Description of the Warning and Error Messages}

CORCON-Mod3 performs a number of error checks during execution. First the input data are examined for obvious errors or inconsistences. Then various intermediate results of the transient calculation are tested to determine if they are physically realistic within the limits of the models in CORCON-Mod3 and if they have converged numerically.

When an error condition is detected, an appropriate message is issued. Subsequent action depends on the severity of the error: execution may

- continue with a waming, or

- terminate at the end of the timestep, or

- terminate immediately.

The messages that the user may encounter are listed in Table 4.2, together with the action taken by the code.
Most of the error messages are self-explanatory. In a few cases, additional comments have been added to clarify the meaning of the message.

Examination of the code listing will reveal several other locations where an error or waming message could be printed and execution terminated by a call to FAIL. In general they correspond to errors which, short of machine error, cannot exist in CORCON- Mod3. These messages might appear if incorrect modifications were made to the code, and are included for that reason.

\subsection{General Programming Information}

CORCON-Mod3 is coded in FORTRAN which conforms to ANSI Standard X3.9-1978 ("FORTRAN 77"). ${ }^{5}$ The only changes in language from CORCON-Modl are the use of type CHARACTER for alpha-numeric data, and the use of BLOCK IF structures in the newer routines. The code contains approximately 23600 source lines, including 6200 lines of COMMENTS.

In cases where constants are required to have full machine accuracy, they are evaluated by execution of a short routine called SETUP.

The code reads data from a file named "ccmod3.in," which must exist at the start of the run. (On UNIX systems, the file name should be in lower case.) ASCII output is written to a file named "ccmod3.out," which will be created with "unknown" FORTRAN status. On some systems, this will cause an existing file with the name "ccmod3.out" to be overwritten. Additional binary files, "movie.dat" and "ccmod3.plt," may be created and written for post-execution printing and plotting. 
Table 4.2 CORCON-generated warning and error messages for each subroutine

ABLATE

*** * ABLATE *** INTERPOLATION FOR MASS ADDITION

0 AT 0 OFF LOW END OF TABLE $0 *$

[Waming only]

"* * ABLATE * * * INTERPOLATION FOR MASS ADDITION

0 AT ( ) OFF HIGH END OF TABLE $0 "$

[Warning only]

ADDLYR

*** ADDLYR * * * CONVERGENCE FAILURE.

L,HTOT(L), TLAY(L) $=0,(0,0 "$

[Warning only]

Convergence failure for layer temperature in call to TMPFND.

ATMPRO

*** * ATMPRO *** INTERPOLATION FOR ATMOSPHERIC

PRESSURE AT () OFF LOW END OF TABLE ()"

[Warning only]

** * ATMPRO * * * INTERPOLATION FOR ATMOSPHERIC

PRESSURE AT () OFF HIGH END OF TABLE ()"

[Warning only]

\section{ATMSUR}

"* * * ATMSUR * * * INTERPOLATION FOR SURROUNDINGS

TEMPERATURE AT () LOW END OF TABLE ()"

[Warning only]

"* * * ATMSUR * * * INTERPOLATION FOR SURROUNDINGS

TEMPERATURE AT () OFF HIGH END OF TABLE 0"

[Warning only]

"* * * ATMSUR * * * CONVERGENCE FAILURE"

[Immediate termination]

Failure to match the above-pool heat transfer to the pool surface. 
Table 4.2 CORCON-generated warning and error messages for each subroutine (continued)

\section{CHEKCV}

"** * CHEKCV * * * NO MORE COOLANT."

[Immediate termination]

** * * LEVSWLICHEKCV * * * NOT ENOUGH COOLANT TO COVER THE MELT AT () SECONDS."

[Immediate termination]

*** ENRCNIICHEKCV *** NOT ENOUGH COOLANT

TO COVER THE MELT AT () SECONDS."

[Immediate termination]

\section{CONFND}

"*** CONFND *** INSERT $=0$ EXCEEDS DIMENSIONS"

[Immediate termination]

Can only occur if data tables have been changed.

**** CONFND $* * *$ IOPT $=()$ OR IN $=()$ NOT IN MASTER LIST $*$

[Immediate termination]

Can only occur if data tables have been changed.

\section{CONPRP}

** * * CONPRP * * * CONCRETE INPUT SPECIES () NOT IN MASTER LIST"

[Deferred termination]

"*** CONPRP * * * REBAR INPUT SPECIES () NOT IN MASTER LIST ${ }^{n}$

[Deferred termiriation]

CPENTH

*** * CPENTH *** IOPT $=()$ IS IN ERROR"

[Immediate termination]

CREATE

"*** PLLAYR *** CONVERGENCE FAILURE.

L,HTOT(L),TLAY(L) = () () ()"

[Immediate termination] 
Table 4.2 CORCON-generated warning and error messages for each subroutine (continued)

\section{CYLIND}

"* * * CYLIND * * * NRAYS $=0$ LESS THAN NBOT + NCORN +3"

[Deferred termination]

Too few rays or two many bottom and corner points in cavity definition.

\section{DIMACH}

"D1MACH -- I OUT OF BOUNDS"

[Immediate termination]

Incorrect index in calling statement: 1 .LE. I .LE. 5. Can only occur if the code has been modified incorrectly.

DATAIN

*** * DATAIN *** NO DATA ON UNIT $5 "$

[Immediate termination]

"* * DATAIN * * * TOO MANY TIMESTEP INTERVALS"

[Deferred termination]

"*** DATATIN * ** NO TIMESTEP INPUT"

[Deferred termination]

** * DATAIN * * * TOO MANY EDIT INTERVALS"

[Deferred termination]

$" * *$ * DATAIN *** INVALID IFP $=0 "$

[Immediate termination]

$* * * *$ DATAIN $* * *$ IREO $=()$ IREM $=()$ IRES $=(0)$

[Immediate termination]

Improper type for emissivity tables. Should be "TIME" or "TEMP".

DCSEVL

"DCSEVL NUMBER OF TERMS LE 0"

[Immediate termination]

"DCSEVL NUMBER OF TERMS GT 1000"

[Immediate termination]

"DCSEVL X OUTSIDE $(-1,+1)^{n}$

[Immediate termination] 
Table 4.2 CORCON-generated warning and error messages for each subroutine (continued)

DCYINT

"*** DCYINT ***, PROBABLE ERROR. POOL CONTAINS () PERCENT OF CORE"

[Warning only]

Input has specified a mass of UO2 greater than the entire core.

DHGEN

**** DHGEN *** INTERPOLATION FOR SOURCE POWER IN OXIDE AT () OFF LOW END OF TABLE ()"

[Waming only]

**** DHGEN *** INTERPOLATION FOR SOURCE POWER IN OXIDE AT () OFF HIGH END OF TABLE ()"

[Warning only]

*** * DHGEN *** INTERPOLATION FOR SOURCE POWER IN METAL AT () OFF LOW END OF TABLE ()"

[Warning only]

"*** DHGEN * * * INTERPOLATION FOR SOURCE POWER IN METAL AT () OFF HIGH END OF TABLE ()"

[Waming only]

EDIT

- -.---

EMISIV

"*** EMISIV * **, INTERPOLATION FOR SURROUNDINGS

EMISSIVITY AT () OFF LOW END OF TABLE ()"

[Warning only]

"* * * EMISIV * * * INTERPOLATION FOR SURROUNDINGS

EMISSIVITY AT () OFF HIGH END OF TABLE ()"

[Warning only]

**** EMISIV *** INTERPOLATION FOR OXIDE

EMISSIVITY AT () OFF LOW END OF TABLE ()"

[Warning only]

** * EMISIV * * * INTERPOLATION FOR OXIDE

EMISSIVITY AT () OFF HIGH END OF TABLE ()"

[Warning only] 
Table 4.2 CORCON-generated warning and error messages for each subroutine (continued)

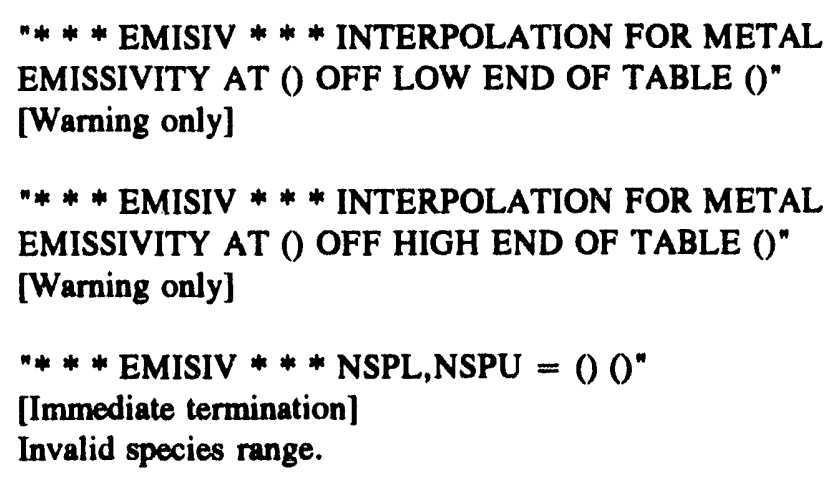

FILM

"* * * FILM * * * CONVERGENCE FAILURE. JBODY,LYR,EMDO,EMD $=()(0)(0)$

[Immediate termination]

Failure to determine consistent film properties (heat transfer coefficient and gas generation). 
Table 4.2 CORCON-generated warning and error messages for each subroutine (continued)

HTRCLN

"* * * HTRCLN * * * CONVERGENCE FAILURE"

[Immediate termination]

Failure to determine radiative contribution to the boiling heat transfer.

*** HTRCLN ***NCL $=(0)$

[Immediate termination]

Invalid coolant. Can only occur if the code has been modified incorrectly.

HTRLAY

"*** HTRLAY *** MATRIX AMTRX IS SINGULAR AS DETECTED BY SAXB"

[Immediate termination]

**** HTRLAY *** AXIAL. CONVERGENCE FAILURE. L,KOUNT,LOOPL,LOOPT $=0(0)()^{n}$

[Warning only]

Failure to determine state of layer consistent with boundary conditions. Has only been observed for vanishingly thin liquid sublayers, and usually in early iterations of INTEMP (so that the result does not affect the end-of-timestep results). Code continues, using conductive heat transfer for layer.

*** * HTRLAY*** AXIAL SOLID CORE/LIQUID SURFACE

WITH L,TS,TB,TL,TT $=0()()(0)$

[Warning only]

Solid layer is being melted from the top and bottom. Has only been observed in early iterations of INTEMP (so that the result does not affect the end-of-timestep results). Code continues, using convective heat transfer to the solidus temperature.

*** * HTRLAY*** RADIAL CONVERGENCE FAILURE. L,KOUNT,LOOPL,LOOPT $=0000$

[Waming only]

Failure to determine state of layer consistent with boundary conditions.

**** HTRLAY***, RADIAL SOLID CORE/LIQUID SURFACE

WITH L,TS,TLTR $=0()(0)$ ()

[Immediate termination]

Solid layer being melted from outside.

INCOOL

"*** INCOOL *** COOLANT () NOT IN MASTER LIST"

[Deferred termination] 
Table 4.2 CORCON-generated warning and error messages for each subroutine (continued)

INGEOM

"* * * INGEOM *** NRAYS $=0$, GREATER THAN LIMIT OF 100"

[Immediate termination]

"*** INGEOM *** RO = (). MUST BE 0.0"

[Immediate termination]

INITL

**** INITL *** INVALID ILYR $=0$ "

[Deferred termination]

INPCON

"*** INPCON *** OXIDE () NOT IN MASTER LIST"

[Deferred termination]

"** * INPCON * * * METAL () NOT IN MASTER LIST"

[Deferred termination]

INPGAS

"* * * INPGAS * * * GAS () NOT IN MASTER LIST"

[Deferred termination]

** * * INPGAS * * * INTERPOLATION FOR ATMOSPHERIC

PRESSURE AT () OFF LOW END OF TABLE ()"

[Warning only]

**** INPGAS *** INTERPOLATION FOR ATMOSPHERIC

PRESSURE AT () OFF HIGH END OF TABLE ()

[Warning only]

\section{INTEMP}

**** INTEMP * ** MATRIX AMTRX IS SINGULAR AS DETECTED BY SAXB"

[Immediate termination]

The equations for determining interface temperatures are singular. 
Table 4.2 CORCON-generated warning and error messages for each subroutine (continued)

\section{"* * * INTEMP * * * CONVERGENCE FAILURE"}

[Warning only]

Newton iteration to determine interface temperatures failed to converge in 20 iterations.

LEVSWL

"*** LEVSWL *** FIRST OCCUPIED LAYER .GT. ()"

[Immediate termination]

Can only occur if the code has been modified incorrectly.

** * LEVSWL * * * CAVITY TOO SMALL FOR MELT"

[Immediate termination]

Need to specify a larger cavity (remember level swell).

MASRAT

"*** MASRAT * * * FIRST OCCUPIED LAYER .GT. 0"

[Immediate termination]

*** * MASRAT *** CONVERGENCE FAILURE"

[Immediate termination]

Failure to match film heat transfer results to pool heat transfer at the bottom of the pool.

MASSEX

"*** MASSEX ***ICALL $=0$ "

[Immediate termination]

Invalid call to MASSEX. Can only occur if the code has been modified incorrectly.

MELTRD

*** MELTRD *** NORAD $=()^{*}$

[Immediate termination]

The number of entries in the time-radius table is greater than the maximum allowed number (MAXRAD).

"*** MELTRD *** RADTIM() $=$ () IS NEGATIVE. $"$

[Immediate termination]

"*** MELTRD *** TIMRAD() = () IS NEGATIVE."

[Immediate termination] 
Table 4.2 CORCON-generated warning and error messages for each subroutine (continued)

"*** MELTRD *** TIMRAD() > TIMRAD()"

[Immediate termination]

The entries in the time table must be increasing with time.

*** * MELTRD * * * RADTIM() > RADTIM()"

[Immediate termination]

The entries in the radius table must be increasing with time.

"** * MELTRD * * * TOO FEW ENTRIES IN TIME-RADIUS

TABLE. EXECUTION TERMINATED."

[Immediate termination]

\section{MHTRAN}

"* * * MHTRAN * * * MASS IN LAYER ()"

[Immediate termination]

Can only occur if the code has been modified incorrectly.

\section{MLTPRP}

\section{"*** MLTPRP * * * MASS IN LAYER ()"}

[Immediate termination]

Can only occur if the code has been modified incorrectly.

\section{MLTREA}

"*** MLTREA *** REDOING SOLUTION FROM NEW STARTING GUESS"

The equations for determining the next correction to the equilibrium composition are singular or failed to converge. A new starting guess will usually avoid the problem region.

**** MLTREA *** MATRIX AK IS SINGULAR IN FIRST CALL TO SAXB"

[Immediate termination]

The equations for determining the next correction to the equilibrium composition are singular despite a new starting guess.

\section{"*** MLTREA *** APPARENT CONVERGENCE FAILURE}

RELATIVE ERROR IN TOTAL MASS $=()^{n}$

[Warning only]

Failure of the chemical equilibrium routine. "Apparent" because error is often insignificant, although the code fails to recognize this. Check to verify that no serious violation of mass conservation has occurred. 
Table 4.2 CORCON-generated warning and error messages for each subroutine (continued)

PLLAYR

"** * PLLAYR * * * MASS IN LAYER O"

[Immediate termination]

Can only occur if the code has been modified incorrectly.

PRTGAS

"INVALID IRE = 0 IN PRTGAS"

[Immediate termination]

Can only occur if the code has been modified incorrectly.

\section{REACT}

** * REACT * * * IMPROPER REACTION NUMBER $=0$ "

[Immediate termination]

Can only occur if the code has been modified incorrectly.

SOLLIQ

*** SOLLIQ *** NSPL, NSPU $=()(0)$

[Immediate termination]

Invalid value for NSPL or NSPU. Can only occur if the code has been modified incorrectly.

SPHCYL

"* * SPHCYL * * * IGEOM = 3 NOT OPERATIONAL"

[Immediate termination]

SURFEB

*** * SURFEB *** CONVERGENCE FAILURE.

ICOUNT,TTA,QP,DQPDTA,QW,DQWDTA $=0(0)(0)(0)$

[Immediate termination]

Failure to match film heat transfer results to the pool heat transfer. 
Table 4.2 CORCON-generated warning and error messages for each subroutine (continued)

\section{SVLAAR}

"* * SVLAAR *** CONVERGENCE FAILURE"

[Immediate termination]

Failure to determine liquidus and solidus temperatures for oxidic mixture.

\section{TMPFND}

"*** TMPFND *** MASS IN LAYER ()"

[Immediate termination]

Can only occur if the code has been modified incorrectly.

VISCTY

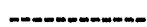

"*** VISCTY ***NSPL, NSPU $=0$ ()"

[Immediate termination]

Invalid value for NSPL or NSPU. Can only occur if the code has been modified incorrectly. 


\subsection{Coding Information}

\subsection{Descriptions of Subroutines}

Subroutines developed specifically for CORCON are listed in Table 5.1 with brief descriptions of the functions they perform. Also included are routines such as SAXB which are used by CORCON but were not developed specifically for CORCON.

Also provided is a variety of coding information that may assist the user in understanding the function of the various subroutines in the code and the overall program flow. Table 5.2 is a list of subroutines called by each routine. Table 5.3 is a list of program routines which call each subroutine.

\subsection{Use of Common}

Most of the internal data in CORCON-Mod3 are contained in and communicated through named COMMON blocks. To assist the user in understanding the flow of information between subroutines, we provide Tables 5.4, 5.5, and 5.6. Table 5.4 lists the COMMON blocks contained in each program segment. Table 5.5 lists the program segments containing each COMMON block. A list of the program variables in each COMMON block is given in Table 5.6; the variables are defined in the next section.

\subsection{Descriptions of Principal Variables}

Table 5.7 provides a dictionary of the principal FORTRAN variables used in CORCON-Mod3. For arrays shown in the table, the different array elements may correspond to different layers, to different chemical species, etc. This is indicated by the index used, as follows:
Interface $L$ is the interface between layer $L$ and layer L-1. 
Table 5.1 List of CORCON subroutines

ABLATE - Computes mass flow rate of each species in surroundings material as it ablates and falls into pool

ACTMET - Computes the activity coefficients for metal phase constituents

ACTOXD - Computes the activity coefficients for oxide phase constituents

ADDLYR - Combines two layers into a third layer

AERPTL - Determines aerosol particle size, mass concentration, and evolution rate.

ANGAVL - Finds body angle at each ray (body) point

ARBINP - Initializes an arbitrary cavity shape

ATMPRO - Determines bulk properties of the gas mixture (atmosphere) above the molten pool

ATMSUR - Updates atmosphere and surroundings, or serves as an interface to a containment response code

BARRAY - Contains parametric values for fits to the free-energy functions

BCLTOV - Groups fission product elements and converts masses from g-mol to $\mathbf{k g}$

BLKDTA - Contains data tables, including master species list, master molecular weight list, stoichiometric coefficients and phase table for MLTREA, and various pointers and global constants

BUBBLE - Determines bubble sizes and velocities where gas enters the pool and within pool layers

CHEKCV - Computes the critical volume of coolant needed to cover the melt when the time-dependent melt radius option is used

CHEKHT - Checks for valid melt thickness when the time-dependent melt radius option is used

CHEMPO - Computes enthalpy, entropy, and Gibbs free energy of each species required for the melt/gas chemical reaction calculation in MLTREA

COMBIN - Adds one mixture to another to produce a single mixture mass, composition, and enthalpy

COMBN2 - Conbines the mass and enthalpy of two layers to form a new layer.

CONFND - Initializes data tables and finds coefficients for specific heat, enthalpy, and entropy for a species as necessary for calculations in CHEMPO or CPENTH

CONPRP - Initializes concrete composition and properties

CORCON - Controls program flow

CPENTH - Computes specific heat and enthalpy of condensed melt phases for both single- and two-phase (liquid-solid) mixtures

CREATE - Creates separated layers if a mixture layer has lost a phase due to deentrainment

CVFAC - Translates CORCON variables into VANESA variables 
Table 5.1 List of CORCON subroutines (continued)

CYLIND - Initializes a flat-base cylindrical cavity shape

DATAIN - Handles the input of data for the program

DCOEFF - Calculates drag coefficients for stable droplets in the melt

DCYINT - Sets up the initial intact core inventory of radioactive species and associated retention factors, and initializes decay product pseudo-species

DCYPOW - Evaluates the decay power of each radioactive element as a function of time

DENSTY - Computes the density of the condensed phases in the pool (the metallic and oxidic melt mixtures and coolant

DENTRN - Calculates the deentrainment (settling) of suspended liquid droplets

DF - Calculates the aerosol mass gradient in the pool

DHGEN - Computes the decay heat generated within each pool layer

EDIT - Manages the printed output of the code

EMISIV - Determines the emissivities of the condensed phases of the condensed phases of the pool from input tables of emissivity vs time or temperature

ENRCN1 - Performs the explicit update of the layer energy equations, including boiling of the coolant, and sets up the implicit terms

ENRCN2 - Finishes the update of the layer energy equations by adding the implicit terms, and calculates new layer temperatures

ENTRN - Calculates bubble-drive entrainment of a heavy fluid by a less dense fluid

EXPRNT - Controls the generation of extra debug printed output

FAIL - Performs error handling when called by a subroutine which detects abnormal data or an invalid computation

FDZ - Calculates $\exp (\mathrm{z})$ for SRPP

FIIRAD - Finds the index of the body point closest to the edge of the melt

FILM - Advances the state of the gas film from one point to another and integrates results

FPUPD - Updates the fission product composition of each group given the initial composition and changes in composition due to mass addition or fission product release

GEOM - Determines the volume, surface area, and stream length of the concrete cavity as a function of body point

GFLMPR - Computes the average gas mixture properties in the gas film at each body point 
Table 5.1 List of CORCON subroutines (continued)

HCBBOT - Calculates the bubble-enhanced liquid-liquid heat transfer coefficient at the bottom of a layer

HCBINJ - Calculates the heat transfer coefficient for bubble injection at the bottom of the pool

HCBSID - Calculates the bubble-enhanced heat transfer coefficient at the side of the pool

HCBTOP - Calculates the bubble-enhanced liquid-liquid heat transfer coefficient at the top of a layer

HSPCYL - Initializes a cylindrical cavity with a hemispherical bottom

HTRCLN - Evaluates heat transfer in the coolant layer, including boiling

HTRLAY - Evaluates heat transfer in a layer, including conduction in solid regions

HTRLIQ - Computes heat transfer coefficients in a liquid layer or sub-layer, including bubble-enhanced convection, natural convection, and conduction

HTRN - Computes gas film heat transfer coefficients in all regimes

INCOOL - Inputs the initial mass, temperature, and identity of the coolant

INGEOM - Inputs and defines the initial geometry of the concrete crucible

INITL - Initializes necessary variables before entering the main computational loop

INPCON - Inputs initial masses of core constituents and their temperatures

INPGAS - Inputs and defines the initial composition and state of the atmosphere

INTEMP - Computes temperatures and heat flows at layer interfaces

INVERF - Computes the inverse of the error function

LEVSWL - Calculates pool level swell, layer interface locations, and layer average void fractions

LININT - Performs linear interpolation or extrapolation from tables

LOSSES - Calculates aerosol losses by vaporization

MASLOS - Computes mass loss from the melt due to volatilization of fission products

MASRAT - Determines concrete ablation rates (mass loss rates) at body points

MASSEX - Serves as an interface for mass exchange with atmosphere and surroundings

MECLOS - Computes aerosol losses by mechanical processes

MELTRD - Input data for the time-dependent melt radius

MHTRAN - Updates layer species masses and evaluates effects of mass transport and chemical reactions on layer enthalpies 
MLTPRP - Determines transport properties of metallic and oxide phases of the melt

MLTREA - Solves the problem of chemical equilibrium iteratively by a constrained first-order steepest descent technique, to minimize the free energy

ORIENT - Changes the layer configuration if density differences warrant a change

PAGEHD - Prints a page header with code version identification and the problem title

PHSPRP - Determines layer phase properties for aerosol calculations

PLLAYR - Calculates layer densities and determines need for reorientation of layers

PLOTS - Writes data files for a post-processor plotting program

POOLV - Integrates aerosol losses in the pool and determines aerosol size distribution

PRTGAS - Prints results from the metal/gas/oxide reactions

QMELT - Evaluates the local heat flux on the pool side of the gas film

REACT - Sets up necessary reactants for MLTREA and distributes the products returned

RECEDE - Computes the concrete normal recession and relocation of body points during a timestep

RFBS - Module of the equation solver SAXB

RLUD - Module of the equation solver SAXB

SATP - Evaluates saturation pressure as a function of temperature

SATT - Evaluates saturation temperature as a function of pressure (an ENTRY in SATP)

SAXB - Solves a system of real linear algebraic equations $\mathrm{AX}=\mathrm{B}$ (a Sandia Mathematical Library routine)

SETTL - Calculates the drag coefficients for stable and unstable drops in deentrainment

SETUP - Performs code set up requiring execution (as opposed to DATA statements) including the evaluation of machine round off

SIGMY - Computes surface tensions of the condensed phases of the pool

SOLLIQ - Sets up the components of pseudo-mixtures of oxides or metals and calls either SSMELT or SVLAAR to calculate the mixture solidus and liquidus temperatures

SOURCE - Evaluates the decay heat source in the pool

SPHCYL - Initializes a cylindrical cavity with a spherical-segment bottom

SRPP - Calculates the equilibrium partial pressures of vapor species 
Table 5.1 List of CORCON subroutines (continued)

SSMELT - Calculates the liquidus and solidus temperatures of a metallic phase based on stainless steel (Cr-Fe-Ni ternary)

SUBSIZ - Sections the aerosol size distribution and calculates the size range and characteristic size

SURFEB - Performs a surface energy balance at a point on the cavity surface to determine mass flux of ablated concrete at a point

SVLAAR - Performs a Schroeder-von Laar pseudo-binary construction of the liquidus and solidus temperatures of an oxidic phase

THKOND - Determines the thermal conductivity of the condensed phases in the pool

TIMSTP - Determines the new timestep

TMPFND - Determines the mixture temperature, given the composition and enthalpy

TMPSET - Initializes temperatures for newly-created layers

TRADII - Determines the time-dependent melt radius from a linear interpolation within the input table

UCOEFF - Calculates the droplet drag coefficient for an unstable drop

UDU - Module of equation solver SAXB

UNDEFN - Sets layer temperatures and property values to zero when a layer disappears following an orientation change

VANESA - Controls the aerosol calculations

VANFP - Calculaies the fission product content of the melt for use in aerosol calculations

VBUBL - Calculates bubble properties for use in aerosol calculations

VCFAC - Prepares the VANESA aerosol calculations for use in CORCON

VIS2PH - Computes the Kunitz two-phase viscosity multiplier for suspended solids in the melt

VISCTY - Computes the viscosity of the metallic and oxidic phases of the melt

VOUTP - Prints VANESA aerosol calculation output to the output file

VSCRIT - Computes the critical superficial gas velocities into the pool for use in determining bubble sizes

XNDAR - Initializes species molecular weights, and densities for condensed species 


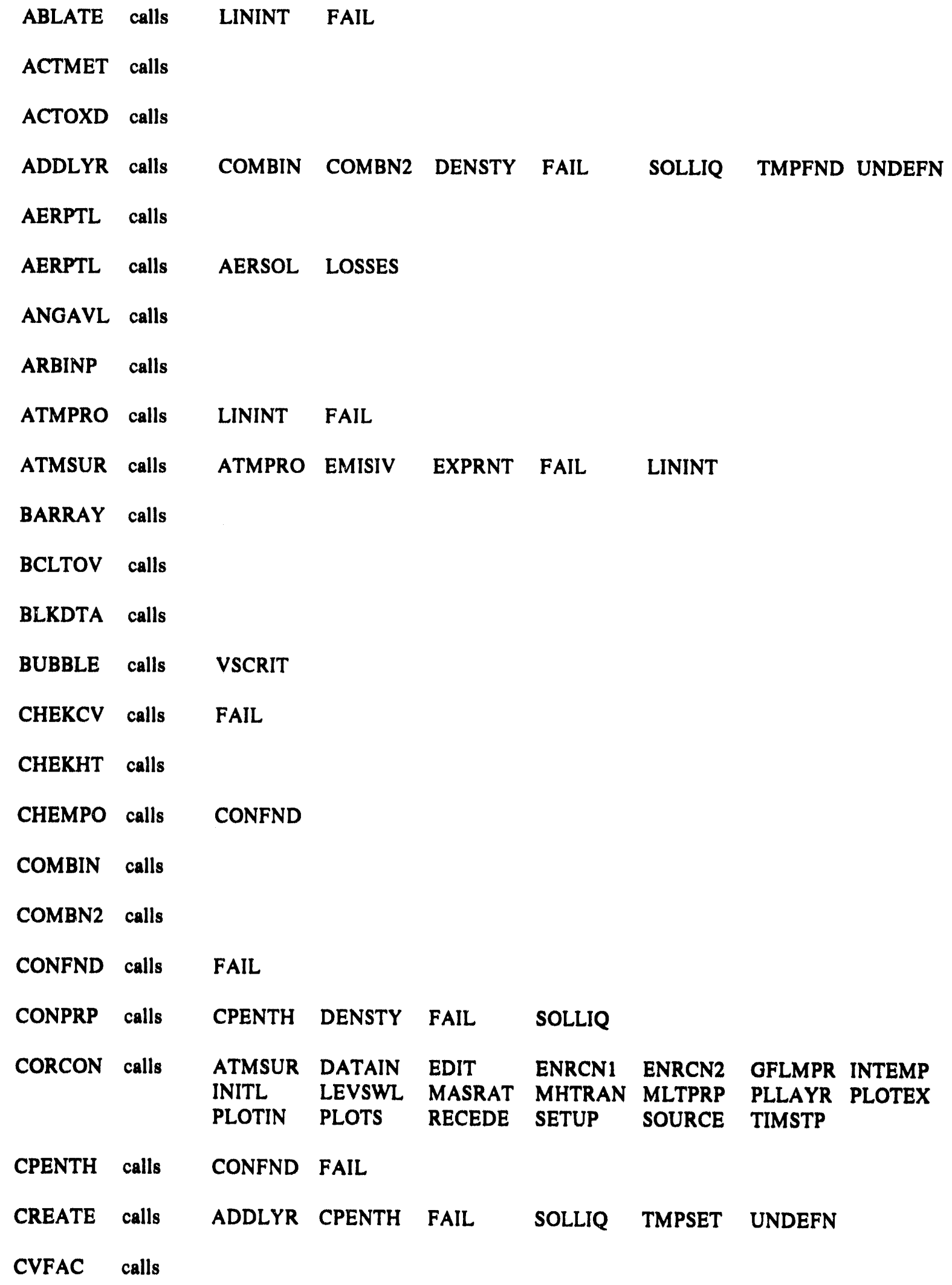


Table 5.2 Subroutines called by each program routine (continued)

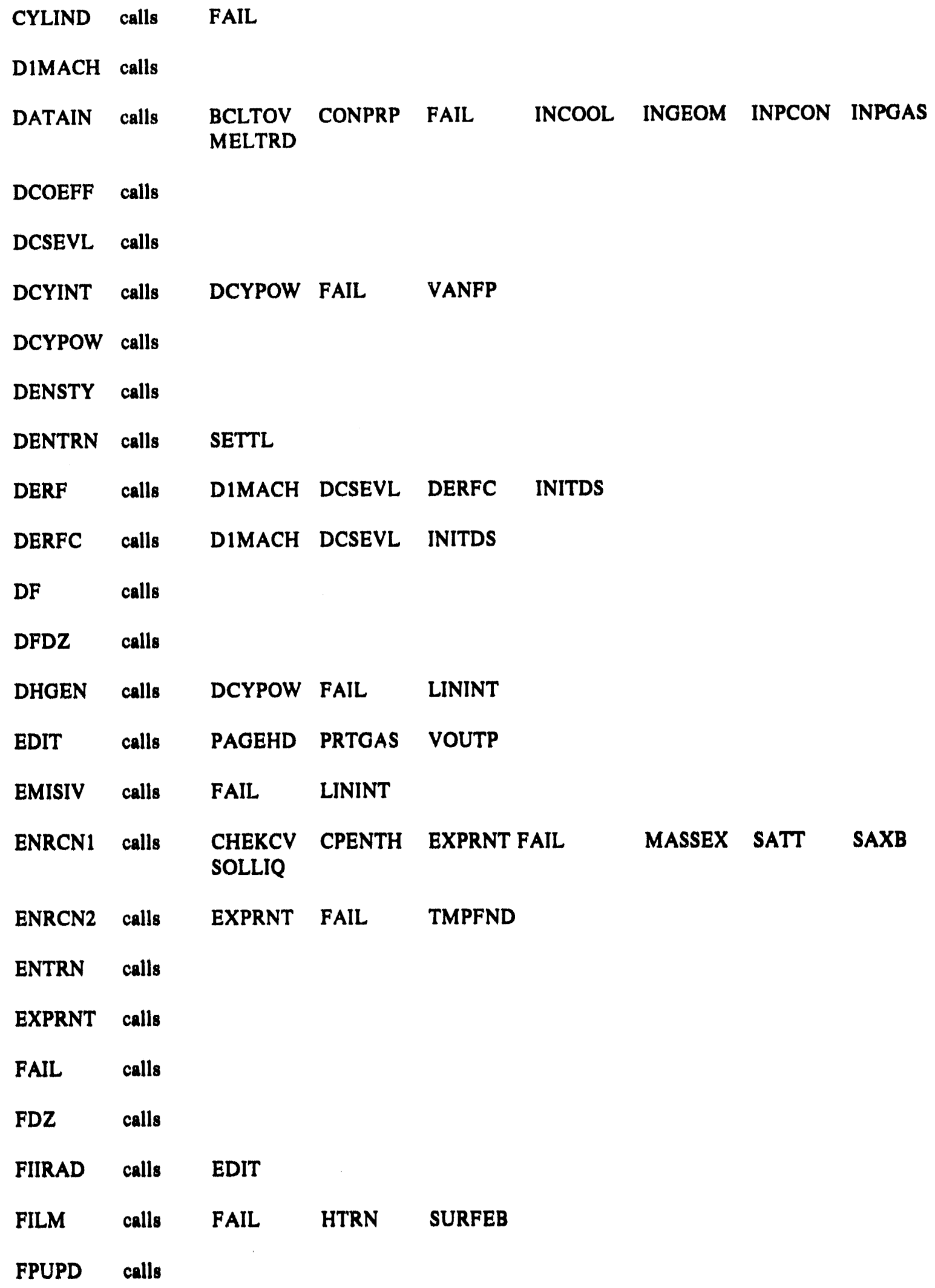




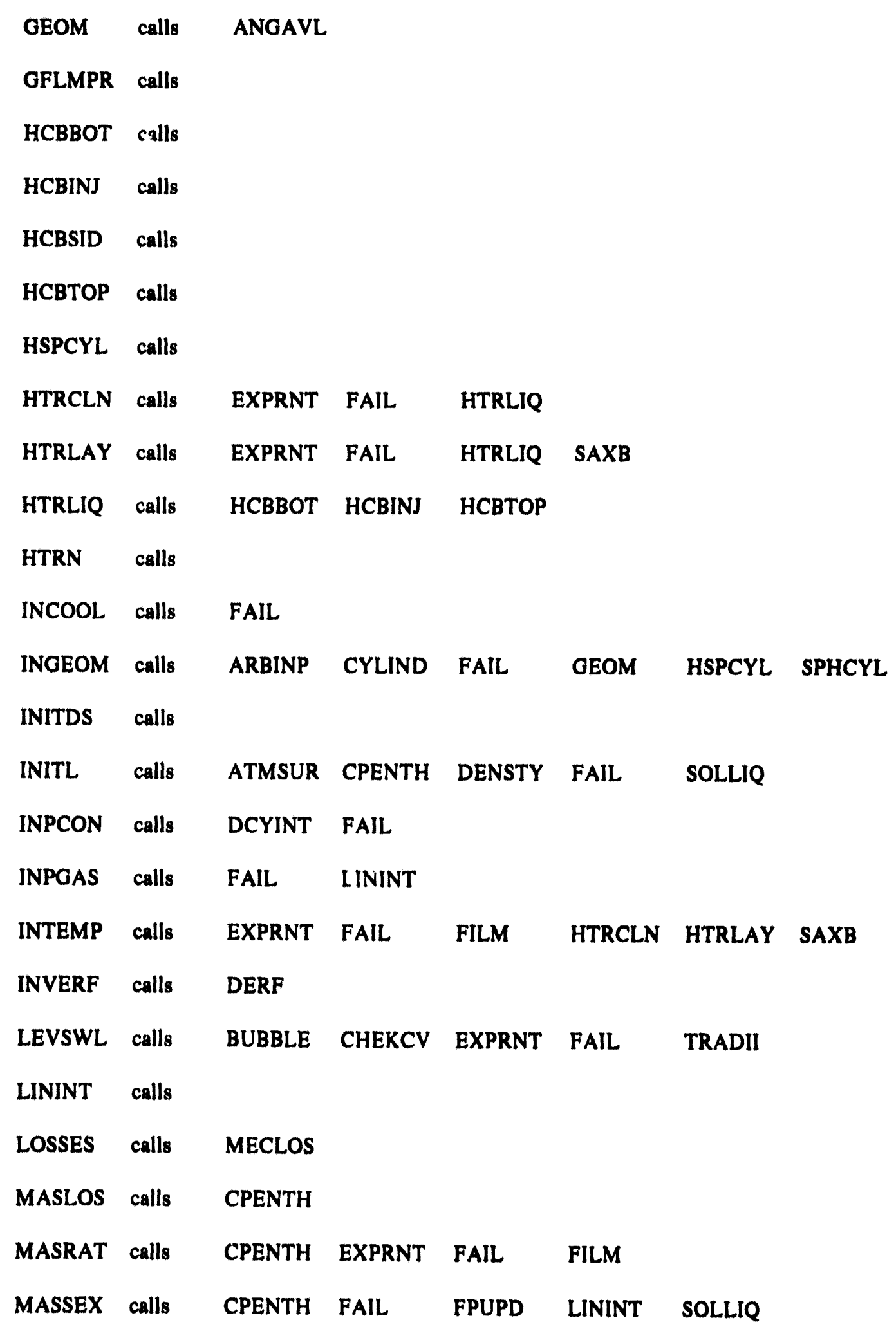


Table 5.2 Subroutines called by each program routine (continued)

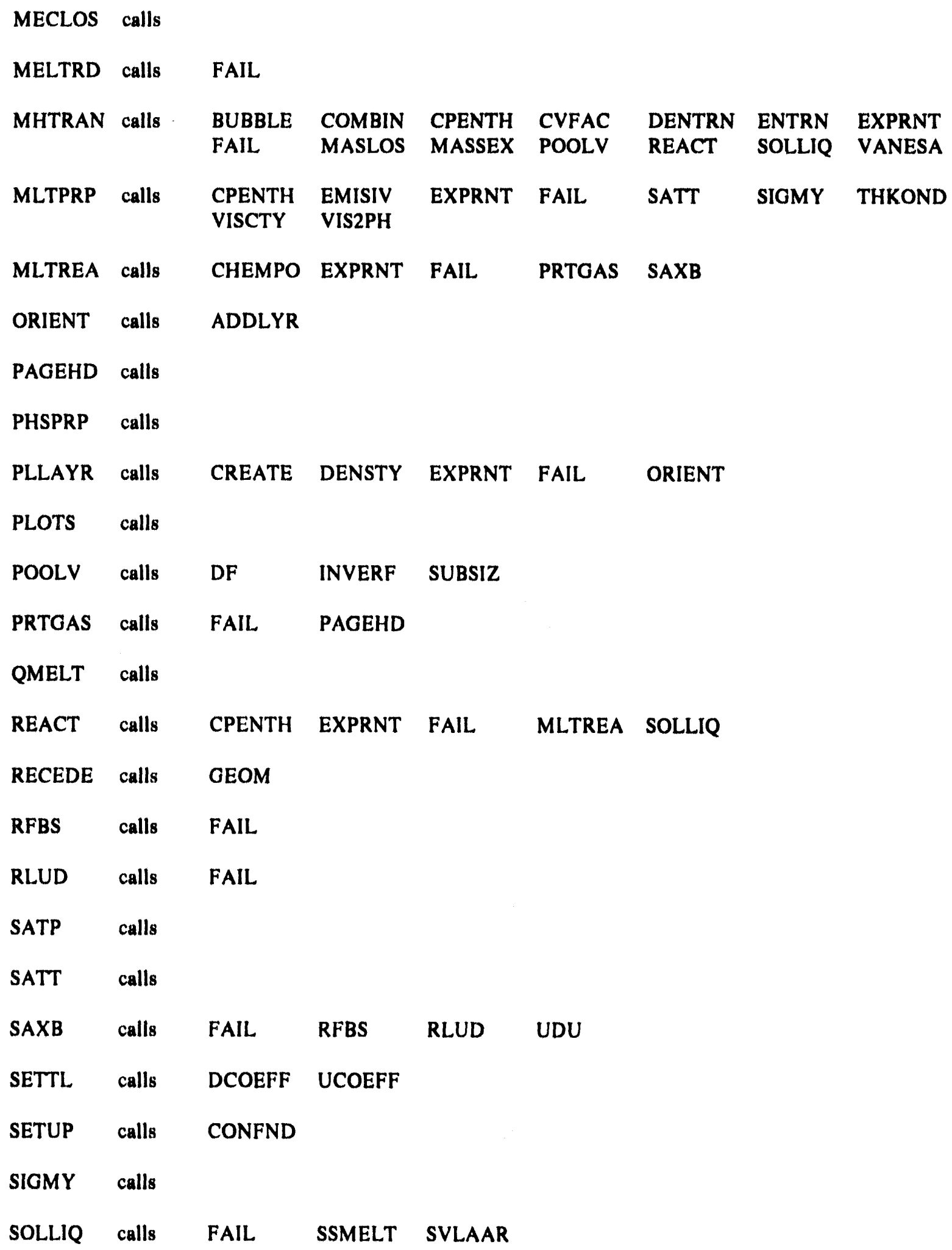


Coding Information

Table 5.2 Subroutines called hy each program routine (continued)

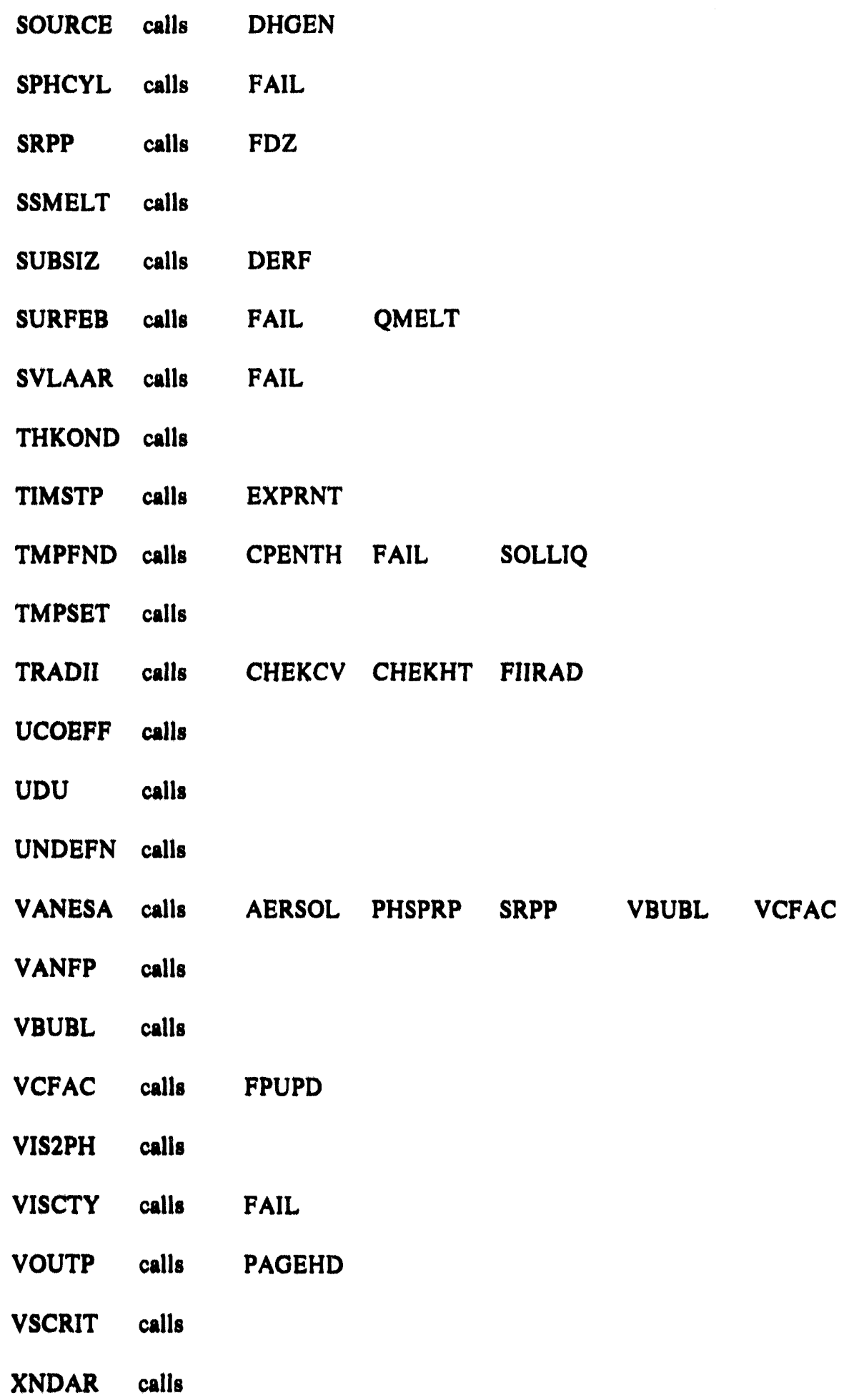


Table 5.3 Program routines which call each routine

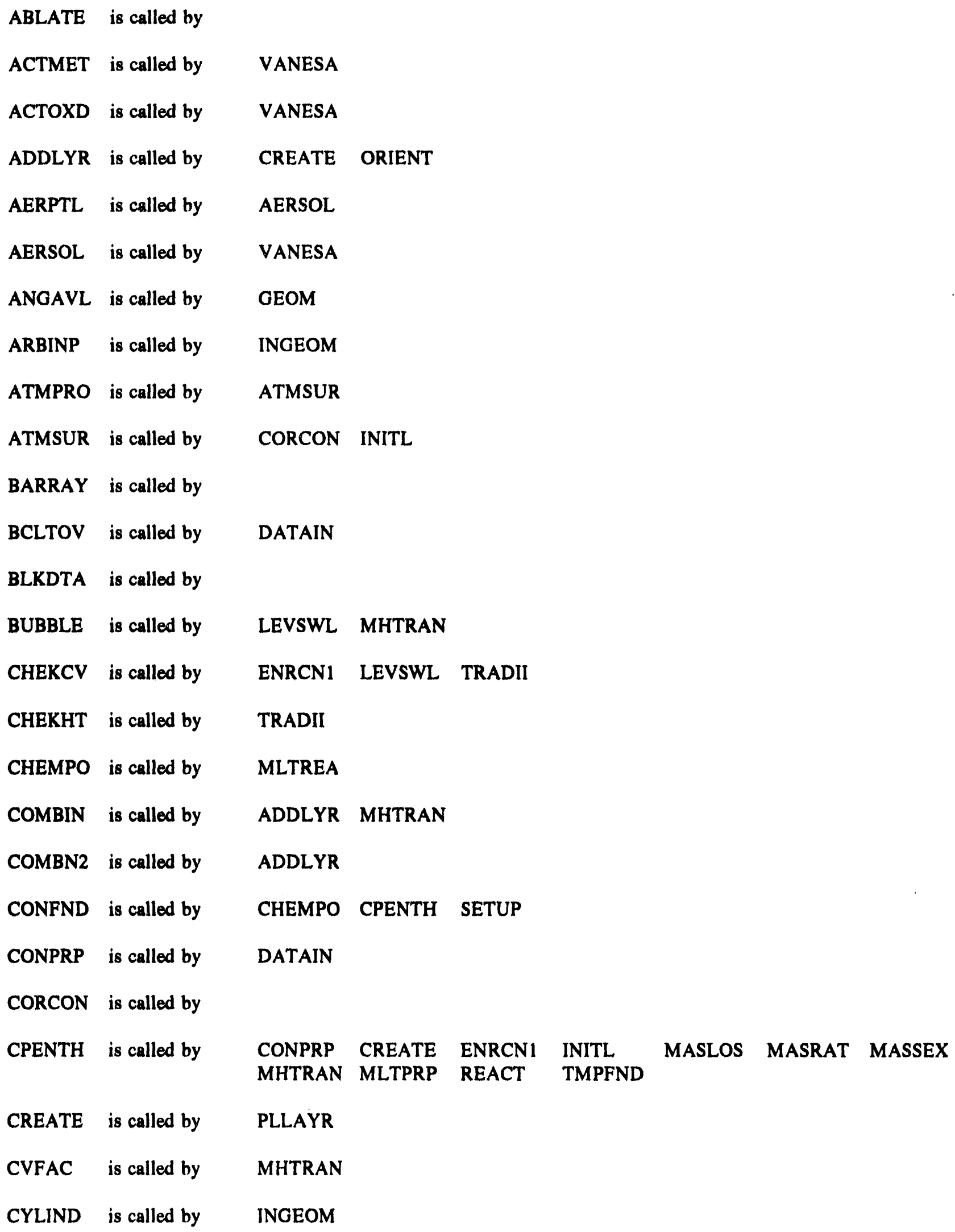


Tahle 5.3 Program routines which call each routine (continued)

\begin{tabular}{|c|c|c|c|c|c|c|c|c|}
\hline D1MACH & is called by & DERF & DERFC & & & & & \\
\hline DATAIN & is called by & CORCON & & & & & & \\
\hline DCOEFF & is called by & SETTL & & & & & & \\
\hline DCSEVL & is called by & DERF & DERFC & & & & & \\
\hline DCYINT & is called by & INPCON & & & & & & \\
\hline DCYPOW & is called by & DCYINT & DHGEN & & & & & \\
\hline DENSTY & is called by & ADDLYR & CONPRP & INITL & PLLAYR & & & \\
\hline DENTRN & is called by & MHTRAN & & & & & & \\
\hline DERF & is called by & INVERF & SUBSIZ & & & & & \\
\hline DERFC & is called by & DERF & & & & & & \\
\hline DF & is called by & POOLV & & & & & & \\
\hline DFDZ & is called by & & & & & & & \\
\hline DHGEN & is called by & SOURCE & & & & & & \\
\hline EDIT & is called by & CORCON & FIIRAD & & & & & \\
\hline EMISIV & is called by & ATMSUR & MLTPRP & & & & & \\
\hline ENRCN1 & is called by & CORCON & & & & & & \\
\hline ENRCN2 & is called by & CORCON & & & & & & \\
\hline ENTRN & is called by & MHTRAN & & & & & & \\
\hline EXPRNT & is called by & $\begin{array}{l}\text { ATMSUR } \\
\text { MASRAT }\end{array}$ & $\begin{array}{l}\text { ENRCN1 } \\
\text { MHTRAN }\end{array}$ & $\begin{array}{l}\text { ENRCN2 } \\
\text { MLTPRP }\end{array}$ & $\begin{array}{l}\text { HTRCLN } \\
\text { PLLAYR }\end{array}$ & $\begin{array}{l}\text { HTRLAY } \\
\text { REACT }\end{array}$ & $\begin{array}{l}\text { INTEMP } \\
\text { TIMSTP }\end{array}$ & LEVSWL \\
\hline FAIL & is called by & $\begin{array}{l}\text { ABLATE } \\
\text { CPENTH } \\
\text { ENRCN1 } \\
\text { INITL } \\
\text { MELTRD } \\
\text { RFBS } \\
\text { TMPFND }\end{array}$ & $\begin{array}{l}\text { ADDLYR } \\
\text { CREATE } \\
\text { ENRCN2 } \\
\text { INPCON } \\
\text { MHTRAN } \\
\text { RLUD } \\
\text { VISCTY }\end{array}$ & $\begin{array}{l}\text { ATMPRO } \\
\text { CYLIND } \\
\text { FILM } \\
\text { INPGAS } \\
\text { MLTPRP } \\
\text { SAXB }\end{array}$ & $\begin{array}{l}\text { ATMSUR } \\
\text { DATAIN } \\
\text { HTRCLN } \\
\text { INTEMP } \\
\text { MLTREA } \\
\text { SOLLIQ }\end{array}$ & $\begin{array}{l}\text { CHEKCV } \\
\text { DCYINT } \\
\text { HTRLAY } \\
\text { LEVSWL } \\
\text { PLLAYR } \\
\text { SPHCYL }\end{array}$ & $\begin{array}{l}\text { CONFND } \\
\text { DHGEN } \\
\text { INCOOL } \\
\text { MASRAT } \\
\text { PRTGAS } \\
\text { SURFEB }\end{array}$ & $\begin{array}{l}\text { CONPRP } \\
\text { EMISIV } \\
\text { INGEOM } \\
\text { MASSEX } \\
\text { REACT } \\
\text { SVLAAR }\end{array}$ \\
\hline
\end{tabular}

FDZ is called by SRPP

FIIRAD is called by TRADII

FILM is called by INTEMP MASRAT 
Table 5.3 Program routines which call each routine (continued)

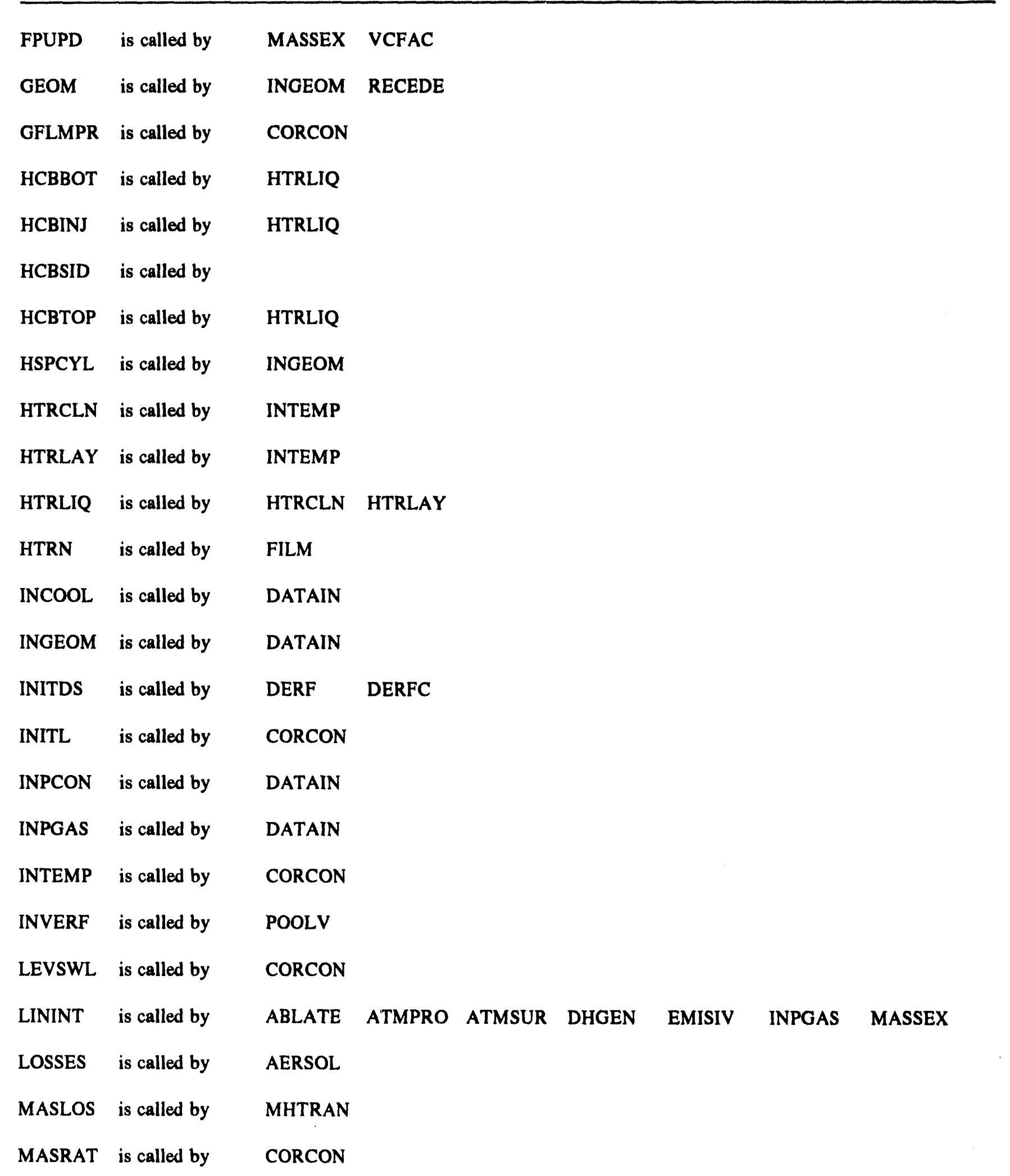


Table 5.3 Program routines which call each routine (continued)

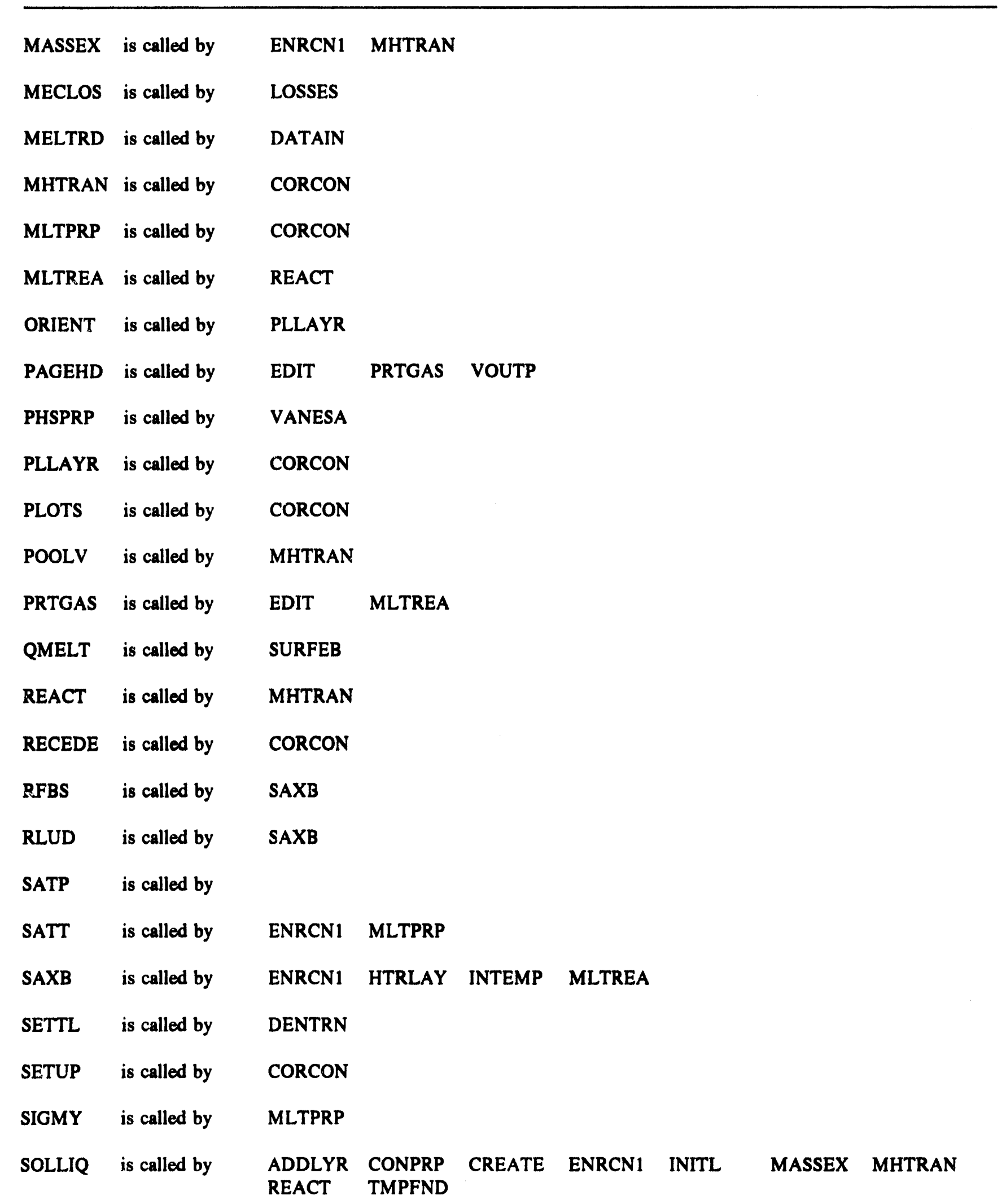


Table 5.3 Program routines which call each routine (continued)

\begin{tabular}{|c|c|c|c|c|}
\hline SOURCE & is called by & CORCON & & \\
\hline SPHCYL & is called by & INGEOM & & \\
\hline SRPP & is called by & VANESA & & \\
\hline SSMELT & is called by & SOLLIQ & & \\
\hline SUBSIZ & is called by & POOLV & & \\
\hline SURFEB & is called by & FiLM & & \\
\hline SVLAAR & is called by & SOLLIQ & & \\
\hline THKOND & is called by & MLTPRP & & \\
\hline TIMSTP & is called by & CORCON & & \\
\hline TMPFND & is called by & ADDLYR & CREATE & ENRCN2 \\
\hline TMPSET & is called by & ADDLYR & CREATE & \\
\hline TRADII & is called by & LEVSWL & & \\
\hline UCOEFF & is called by & SETTL & & \\
\hline UDU & is called by & SAXB & & \\
\hline UNDEFN & is called by & ADDLYR & CREATE & \\
\hline VANESA & is called by & MHTRAN & & \\
\hline VANFP & is called by & DCYINT & & \\
\hline VBUBL & is called by & VANESA & & \\
\hline VCFAC & is called by & VANESA & & \\
\hline VIS2PH & is called by & MLTPRP & & \\
\hline VISCTY & is called by & MLTPRP & & \\
\hline VOUTP & is called by & EDIT & & \\
\hline VSCRIT & is called by & BUBBLE & & \\
\hline XNDAR & is called by & & & \\
\hline
\end{tabular}




\begin{tabular}{|c|c|c|c|c|c|c|c|c|}
\hline ABLATE & contains & A42 & TWO & MASTER & & & & \\
\hline ACTMET & contains & ACTIV & & & & & & \\
\hline ACTOXD & contains & ACTIV & & & & & & \\
\hline ADDLYR & contains & $\begin{array}{l}\text { A45 } \\
\text { TWO }\end{array}$ & BO & B1 & B2 & B8 & MASTER & $\mathbf{M X}$ \\
\hline AERPTL & contains & $\mathrm{BCL}$ & CNDCON & CTOV & VAN & VANPRP & VPL & XND \\
\hline AERSOL & contains & BCL & CTOV & VAN & VANPRP & XND & & \\
\hline ANGAVL & contains & A3 & ONE & TWNEIT & CNSTNT & & & \\
\hline ARBINP & contains & A3 & ONE & TWNEIT & & & & \\
\hline ATMPRO & contains & A21 & TWO & ATMDAT & & & & \\
\hline ATMSUR & contains & $\begin{array}{l}\text { HEATEX } \\
\text { A20 }\end{array}$ & $\begin{array}{l}\text { ATMDAT } \\
\text { A23 }\end{array}$ & $\begin{array}{l}\text { BO } \\
\text { VAN }\end{array}$ & $\begin{array}{l}\text { B1 } \\
\text { CTOV }\end{array}$ & TWO & CNSTNT & A4 \\
\hline BCLTOV & contains & COMP & XND & BCL & A31 & & & \\
\hline BUBBLE & contains & A32 & B0 & B1 & B3 & TWNTWO & CNSTNT & \\
\hline CHEKCV & contains & $\begin{array}{l}\text { A3 } \\
\text { MRCI }\end{array}$ & BO & B1 & CNSTNT & ONE & TWO & MRCR \\
\hline CHEKHT & contains & B1 & CNSTNT & MRCR & MRCI & & & \\
\hline СНEMPO & contains & PFDAT & & & & & & \\
\hline COMBIN & contains & & & & & & & \\
\hline COMBN2 & contains & & & & & & & \\
\hline CONFND & contains & MASTER & & & & & & \\
\hline CONPRP & contains & CNDCON & A32 & B3 & MASTER & SPECNM & & \\
\hline CORCON & contuins & B4 & ONE & TWO & & & & \\
\hline CPENTH & contains & MASTER & & & & & & \\
\hline CREATE & contains & A45 & B0 & B1 & B2 & MASTER & MX & TWO \\
\hline CVFAC & contains & $\begin{array}{l}\text { ARGLST } \\
\text { MASTER } \\
\text { MX }\end{array}$ & $\begin{array}{l}\text { TWO } \\
\text { BCL } \\
\text { VAN }\end{array}$ & $\begin{array}{l}\text { BO } \\
\text { CTOV } \\
\text { VANPRP }\end{array}$ & $\begin{array}{l}\text { BI } \\
\text { CTOP }\end{array}$ & $\begin{array}{l}\text { B2 } \\
\text { VTOC }\end{array}$ & $\begin{array}{l}\text { B6 } \\
\text { CNDCON }\end{array}$ & B6A \\
\hline CYLIND & contains & A3 & ONE & FORTN & TWNEIT & CNSTNT & & \\
\hline \multicolumn{4}{|c|}{ NUREG/CR-5843 } & & \\
\hline
\end{tabular}


Table 5.4 COMMON blocks contained by each program routine (continued)

\begin{tabular}{|c|c|c|c|c|c|c|c|c|}
\hline DIMACH & contains & & & & & & & \\
\hline DATAIN & contains & $\begin{array}{l}\text { A43 } \\
\text { A23 } \\
\text { SPECNM } \\
\text { VAN }\end{array}$ & $\begin{array}{l}\text { B6 } \\
\text { A42 } \\
\text { TITLE } \\
\text { BCL }\end{array}$ & $\begin{array}{l}\text { A4 } \\
\text { B1 } \\
\text { CNDCON } \\
\text { CDF }\end{array}$ & $\begin{array}{l}\text { A9 } \\
\text { ONE } \\
\text { CTOV } \\
\text { VPL }\end{array}$ & $\begin{array}{l}\text { A17 } \\
\text { TWO } \\
\text { CTOP } \\
\text { MRCR }\end{array}$ & $\begin{array}{l}\text { A19 } \\
\text { THREE } \\
\text { COMP } \\
\text { MRCI }\end{array}$ & $\begin{array}{l}\text { A20 } \\
\text { XND }\end{array}$ \\
\hline DCOEFF & contains & & & & & & & \\
\hline DCSEVL & contains & & & & & & & \\
\hline DCYINT & contains & B6A & B6B & A31 & B6 & TWO & MASTER & SPECNM \\
\hline DCYPOW & contains & B6 & & & & & & \\
\hline DENSTY & contains & MASTER & & & & & & \\
\hline DENTRN & contains & BO & B1 & CNSTNT & MX & TWO & & \\
\hline DERF & contains & & & & & & & \\
\hline DERFC & contains & & & & & & & \\
\hline DF & contains & $\mathrm{CDF}$ & & & & & & \\
\hline DFDZ & contains & & & & & & & \\
\hline DHGEN & contains & A4 & A17 & BO & B1 & B2 & B6 & MASTER \\
\hline EDIT & contains & $\begin{array}{l}\text { A3 } \\
\text { B1 } \\
\text { TWO } \\
\text { CONSRV }\end{array}$ & $\begin{array}{l}\text { A4 } \\
\text { B2 } \\
\text { THREE } \\
\text { SPECNM }\end{array}$ & $\begin{array}{l}\text { A32 } \\
\text { B5 } \\
\text { FOUR } \\
\text { MRCR }\end{array}$ & $\begin{array}{l}\text { A39 } \\
\text { B8 } \\
\text { TWNTWO } \\
\text { MRCI }\end{array}$ & $\begin{array}{l}\text { A44 } \\
\text { B9 } \\
\text { GASEDT } \\
\text { MX }\end{array}$ & $\begin{array}{l}\text { A45 } \\
\text { ONE } \\
\text { MASTER }\end{array}$ & $\begin{array}{l}\text { BO } \\
\text { CNSTNT }\end{array}$ \\
\hline EMISIV & contains & TWO & A19 & A20 & B9 & MASTER & & \\
\hline ENRCN1 & contains & $\begin{array}{l}\text { HEATEX } \\
\text { B9 } \\
\text { MRCR }\end{array}$ & $\begin{array}{l}\text { A4 } \\
\text { TWO } \\
\text { MRCI }\end{array}$ & $\begin{array}{l}\text { BO } \\
\text { MASTER }\end{array}$ & $\begin{array}{l}\text { B1 } \\
\text { IMP }\end{array}$ & $\begin{array}{l}\text { B2 } \\
\text { CONSRV }\end{array}$ & $\begin{array}{l}\text { B5 } \\
\text { MX }\end{array}$ & B7 \\
\hline ENRCN2 & contains & HEATEX & B0 & B1 & IMP & CONSRV & & \\
\hline ENTRN & contains & BO & B1 & CNSTNT & $\mathbf{M X}$ & & & \\
\hline EXPRNT & contains & ONE & TWO & & & & & \\
\hline FAIL & contains & ONE & TWO & B4 & & & & \\
\hline FDZ & contains & & & & & & & \\
\hline FIIRAD & contains & A3 & ONE & TWNEIT & MRCR & MRCI & & \\
\hline
\end{tabular}


Table 5.4 COMMON blocks contained by each program routine (continued)

\begin{tabular}{|c|c|c|c|c|c|c|c|c|}
\hline FILM & contains & ARGLST & A4 & A32 & B1 & CNSTNT & & \\
\hline FPUPD & contains & MASTER & B6 & B6A & B6B & & & \\
\hline GEOM & contains & A3 & ONE & FOUR & FORTN & TWNEIT & CNSTNT & \\
\hline GFLMPR & contains & A32 & A39 & ONE & & & & \\
\hline НСВВОТ & contains & B1 & CNSTNT & & & & & \\
\hline HCBINJ & contains & B1 & CNSTNT & & & & & \\
\hline HCBSID & contains & B1 & CNSTNT & & & & & \\
\hline HCBTOP & contains & BO & B1 & CNSTNT & & & & \\
\hline HSPCYL & contains & A3 & ONE & FOUR & FORTN & TWNEIT & CNSTNT & \\
\hline HTRCLN & contains & $\begin{array}{l}\text { BO } \\
\text { MRCI }\end{array}$ & B1 & B5 & B8 & B9 & CNSTNT & MRCR \\
\hline HTRLAY & contains & B1 & B5 & B8 & TWO & CNSTNT & MRCR & MRCI \\
\hline HTRLIQ & contains & BO & B1 & CNSTNT & & & & \\
\hline HTRN & contains & $\begin{array}{l}\text { MX } \\
\text { B1 }\end{array}$ & $\begin{array}{l}\text { A4 } \\
\text { CNSTNT }\end{array}$ & A32 & BO & B5 & B8 & A39 \\
\hline INCOOL & contains & B9 & & & & & & \\
\hline INGEOM & contains & ONE & FORTN & CNSTNT & & & & \\
\hline INITDS & contains & & & & & & & \\
\hline INITL & contains & $\begin{array}{l}\text { HEATEX } \\
\text { BO } \\
\text { B9 } \\
\text { MX }\end{array}$ & $\begin{array}{l}\text { A3 } \\
\text { B1 } \\
\text { ONE } \\
\text { MRCR }\end{array}$ & $\begin{array}{l}\text { A4 } \\
\text { B2 } \\
\text { TWNTWO } \\
\text { MRCI }\end{array}$ & $\begin{array}{l}\text { A10 } \\
\text { B3 } \\
\text { GASEDT }\end{array}$ & $\begin{array}{l}\text { A31 } \\
\text { B5 } \\
\text { CNSTNT }\end{array}$ & $\begin{array}{l}\text { A32 } \\
\text { B8 } \\
\text { CONSRV }\end{array}$ & $\begin{array}{l}\text { A45 } \\
\text { MASTER }\end{array}$ \\
\hline INPCON & contains & A4 & A10 & A31 & B6 & MASTER & SPECNM & \\
\hline INPGAS & contains & A10 & A21 & A31 & MASTER & SPECNM & TWO & \\
\hline INTEMP & contains & $\begin{array}{l}\text { HEATEX } \\
\text { B8 }\end{array}$ & $\begin{array}{l}\text { ARGLST } \\
\text { TWO }\end{array}$ & $\begin{array}{l}\text { A3 } \\
\text { CNSTNT }\end{array}$ & $\begin{array}{l}\text { A32 } \\
\text { A4 }\end{array}$ & $\begin{array}{l}\text { B0 } \\
\text { MRCR }\end{array}$ & $\begin{array}{l}\text { B1 } \\
\text { MRCI }\end{array}$ & B5 \\
\hline INVERF & contains & & & & & & & \\
\hline
\end{tabular}


Table 5.4 COMMON blocks contained by each program routine (continued)

\begin{tabular}{|c|c|c|c|c|c|c|c|c|}
\hline LEVSWL & contains & $\begin{array}{l}\text { A3 } \\
\text { TWNTWO } \\
\text { MRCI }\end{array}$ & ${ }^{A 32}$ & $\begin{array}{l}\text { A44 } \\
\text { TWNEIT }\end{array}$ & $\begin{array}{l}\text { BO } \\
\text { CNSTNT }\end{array}$ & $\begin{array}{l}\text { B1 } \\
\text { A4 }\end{array}$ & $\begin{array}{l}\text { B3 } \\
\text { TWO }\end{array}$ & $\begin{array}{l}\text { ONE } \\
\text { MRCR }\end{array}$ \\
\hline LININT & contains & & & & & & & \\
\hline LOSSES & contains & BCL & CTOV & & & & & \\
\hline MASLOS & contains & $\begin{array}{l}\text { B0 } \\
\text { CTOV }\end{array}$ & $\begin{array}{l}\text { B1 } \\
\text { VTOC }\end{array}$ & B2 & B6 & TWO & CONSRV & MASTER \\
\hline MASRAT & contains & $\begin{array}{l}\text { ARGLST } \\
\text { B3 } \\
\text { MRCR }\end{array}$ & $\begin{array}{l}\text { A3 } \\
\text { B5 } \\
\text { MRCI }\end{array}$ & $\begin{array}{l}\text { A32 } \\
\text { TWO }\end{array}$ & $\begin{array}{l}\text { CNSTNT } \\
\text { TWNTWO }\end{array}$ & $\begin{array}{l}\text { MASTER } \\
\text { TWNEIT }\end{array}$ & $\begin{array}{l}\text { BO } \\
\text { ONE }\end{array}$ & B1 \\
\hline MASSEX & contains & $\begin{array}{l}\text { B6A } \\
\text { CONSRV } \\
\text { A4 }\end{array}$ & $\begin{array}{l}\text { B0 } \\
\text { A31 }\end{array}$ & $\begin{array}{l}\text { B1 } \\
\text { B6 }\end{array}$ & $\begin{array}{l}\text { B2 } \\
\text { A42 }\end{array}$ & $\begin{array}{l}\text { B7 } \\
\text { A43 }\end{array}$ & $\begin{array}{l}\text { B9 } \\
\text { TWO }\end{array}$ & MASTER \\
\hline MECLOS & contains & BCL. & CTOV & VAN & VANPRP & XND & & \\
\hline MELTRD & contains & $\begin{array}{l}\text { A3 } \\
\text { TWO }\end{array}$ & $\begin{array}{l}\text { A4 } \\
\text { MRCR }\end{array}$ & $\begin{array}{l}\text { BO } \\
\text { MRCI }\end{array}$ & B1 & CNSTNT & ONE & TWNEIT \\
\hline MHTRAN & contains & $\begin{array}{l}\text { A4 } \\
\text { B8 } \\
\text { VAN }\end{array}$ & $\begin{array}{l}\text { A32 } \\
\text { MASTER }\end{array}$ & $\begin{array}{l}\text { BO } \\
\text { CONSRV }\end{array}$ & $\begin{array}{l}\text { B1 } \\
\text { TWO }\end{array}$ & $\begin{array}{l}\text { B2 } \\
\text { MX }\end{array}$ & $\begin{array}{l}\text { B3 } \\
\text { CTOV }\end{array}$ & B7 \\
\hline MLTPRP & contains & BO & B1 & B2 & B8 & MASTER & B9 & $\mathbf{M X}$ \\
\hline MLTREA & contains & A4 & PFDAT & RNDOFF & & & & \\
\hline ORIENT & contains & A4 & BO & B1 & $\mathbf{M X}$ & MASTER & & \\
\hline PAGEHD & contains & ONE & TWO & TITLE & & & & \\
\hline PHSPRP & contains & BCL & VANPRP & XND & & & & \\
\hline PLLAYR & contains & $\begin{array}{l}\text { A45 } \\
\text { MASTER }\end{array}$ & $\begin{array}{l}\text { BO } \\
\text { MX }\end{array}$ & $\begin{array}{l}\text { B1 } \\
\text { A4 }\end{array}$ & B2 & B8 & B9 & TWO \\
\hline PLOTS & contains & $\begin{array}{l}\text { A32 } \\
\text { ONE }\end{array}$ & $\begin{array}{l}\text { B8 } \\
\text { TWO }\end{array}$ & $\begin{array}{l}\text { A3 } \\
\text { CNSTNT }\end{array}$ & $\begin{array}{l}\text { A4 } \\
\text { MASTER }\end{array}$ & $\begin{array}{l}\text { B0 } \\
\text { MRCR }\end{array}$ & $\begin{array}{l}\text { B1 } \\
\text { MRCI }\end{array}$ & B2 \\
\hline POOLV & contains & CDF & POOL & CTOP & VPL & & & \\
\hline PRTGAS & contains & A4 & A45 & PFDAT & MASTER & SPECNM & & \\
\hline QMELT & contains & B1 & B5 & B8 & & & & \\
\hline
\end{tabular}


Coding Information

Table 5.4 COMMON hlocks contained by each program routine (continued)

\begin{tabular}{|c|c|c|c|c|c|c|c|c|}
\hline REACT & contains & $\begin{array}{l}\text { A4 } \\
\text { GASEDT }\end{array}$ & $\begin{array}{l}\text { A45 } \\
\text { ONE }\end{array}$ & $\begin{array}{l}\text { TWO } \\
\text { PFDAT }\end{array}$ & $\begin{array}{l}\text { BO } \\
\text { MASTER }\end{array}$ & $\begin{array}{l}\text { BI } \\
\text { CONSRV }\end{array}$ & $\begin{array}{l}\text { B2 } \\
\text { MX }\end{array}$ & B7 \\
\hline RECEDE & contains & A3 & ONE & TWO & FOUR & TWNEIT & & \\
\hline RFBS & contains & & & & & & & \\
\hline RLUD & contains & & & & & & & \\
\hline SATP & contains & & & & & & & \\
\hline SAXB & contains & & & & & & & \\
\hline SETTL & contains & CNSTNT & & & & & & \\
\hline SETUP & contains & CNSTNT & RNDOFF & & & & & \\
\hline SIGMY & contains & & & & & & & \\
\hline SOLLIQ & contains & A32 & B9 & MASTER & & & & \\
\hline SOURCE & contains & BO & B1 & TWO & & & & \\
\hline SPHCYL & contains & & & & & & & \\
\hline SRPP & contains & B & & & & & & \\
\hline SSMELT & contains & & & & & & & \\
\hline SUBSIZ & contains & & & & & & & \\
\hline SURFEB & contains & A32 & & & & & & \\
\hline SVLAAR & contains & & & & & & & \\
\hline THKOND & contains & & & & & & & \\
\hline TIMSTP & contains & ONE & TWO & THREE & FOUR & TWNEIT & A3 & \\
\hline TMPFND & contains & BO & BI & B2 & B9 & TWO & MASTER & MX \\
\hline TMPSET & contains & B1 & B8 & & & & & \\
\hline TRADII & contains & $\begin{array}{l}\text { A3 } \\
\text { MRCR }\end{array}$ & $\begin{array}{l}\text { A4 } \\
\text { MRCI }\end{array}$ & BO & B1 & B8 & CNSTNT & ONE \\
\hline UCOEFF & contains & & & & & & & \\
\hline UDU & contains & RNDOFF & & & & & & \\
\hline
\end{tabular}


Coding Information

Table 5.4 COMMON blocks contained by each program routine (continued)

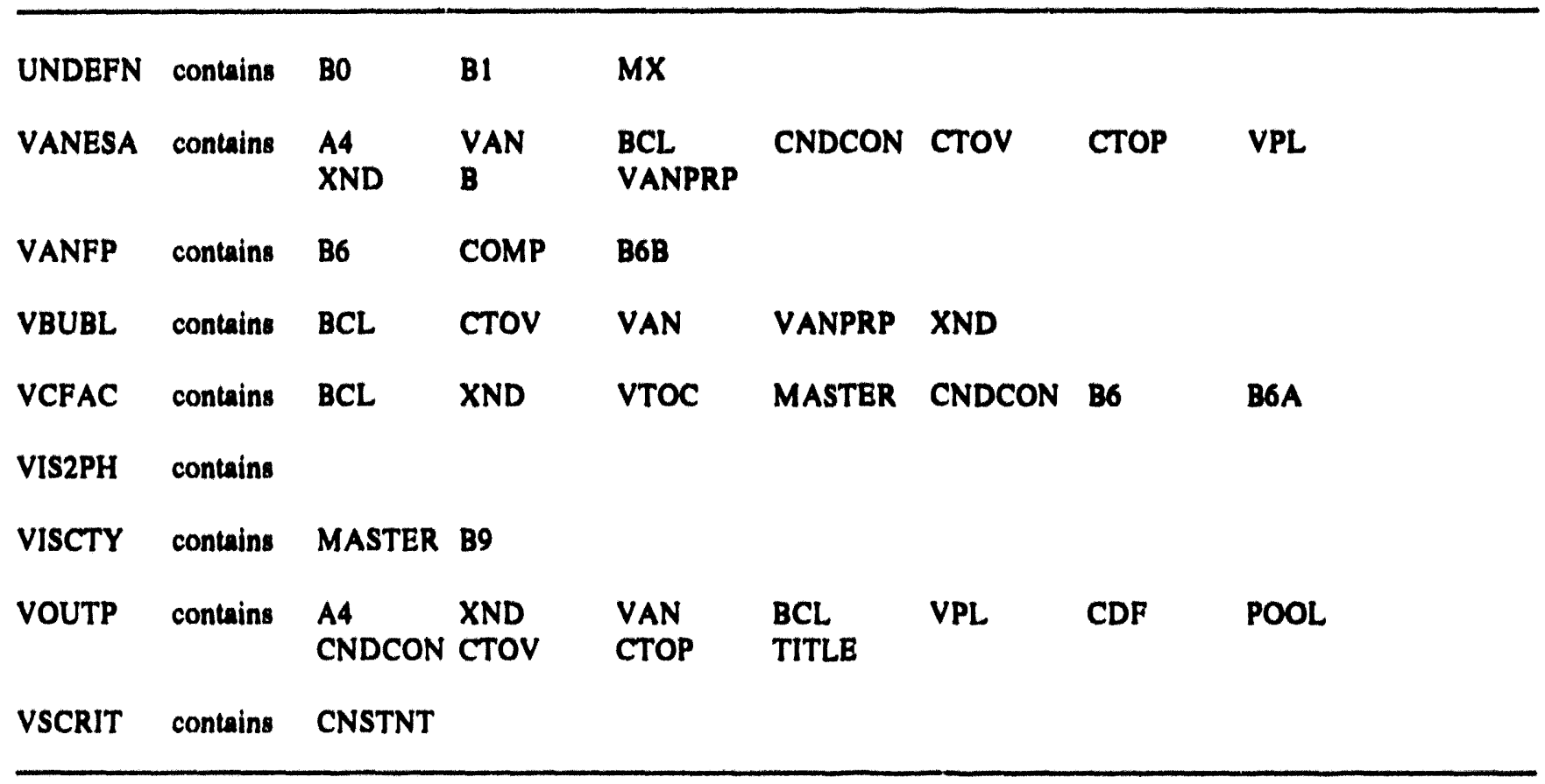

133

NUREG/CR-5843 
Tuhle 5.5 Program routines contuining anch COMMON block

\begin{tabular}{|c|c|c|c|c|c|c|c|}
\hline A10 & is contained in & INITL & INPCON & INPOAS & & & \\
\hline A17 & is contained in & DATAIN & DHOEN & & & & \\
\hline$A 19$ & is contained in & DATAIN & EMISIV & & & & \\
\hline A20 & is contuined in & ATMSUR & DATAIN & EMISIV & & & \\
\hline A21 & is contuined in & ATMPRO & INPOAS & & & & \\
\hline A23 & is contained in & ATMSUR & DATAIN & & & & \\
\hline A3 & is contained in & $\begin{array}{l}\text { ANGAVL } \\
\text { OEOM } \\
\text { MELTRD }\end{array}$ & $\begin{array}{l}\text { ARBINP } \\
\text { HSPCYL } \\
\text { PLOTS }\end{array}$ & $\begin{array}{l}\text { CHEKCV } \\
\text { INITL } \\
\text { RECEDE }\end{array}$ & $\begin{array}{l}\text { CYLIND } \\
\text { INTEMP } \\
\text { TIMSTP }\end{array}$ & $\begin{array}{l}\text { EDIT } \\
\text { LEVSWL } \\
\text { TRADII }\end{array}$ & $\begin{array}{l}\text { FIIRAD } \\
\text { MASRAT }\end{array}$ \\
\hline A31 & is contuined in & BCLTOV & DCYINT & INITL & INPCON & INPOAS & MASSEX \\
\hline A32 & is contuined in & $\begin{array}{l}\text { BUBBLE } \\
\text { INITL } \\
\text { SOLLIQ }\end{array}$ & $\begin{array}{l}\text { CONPRP } \\
\text { INTEMP } \\
\text { SURFEB }\end{array}$ & $\begin{array}{l}\text { EDIT } \\
\text { LEVSWL }\end{array}$ & $\begin{array}{l}\text { FILM } \\
\text { MASRAT }\end{array}$ & $\begin{array}{l}\text { OFLMPR } \\
\text { MHTRAN }\end{array}$ & $\begin{array}{l}\text { HTRN } \\
\text { PLOTS }\end{array}$ \\
\hline A39 & is contuined in & EDIT & OFLMPR & HTRN & & & \\
\hline A4 & is contuined in & $\begin{array}{l}\text { ATMSUR } \\
\text { HTRN } \\
\text { MELTRD } \\
\text { PRTOAS }\end{array}$ & $\begin{array}{l}\text { DATAIN } \\
\text { INIL } \\
\text { MHTRAN } \\
\text { REACT }\end{array}$ & $\begin{array}{l}\text { DHOEN } \\
\text { INPCON } \\
\text { MLTREA } \\
\text { TRADII }\end{array}$ & $\begin{array}{l}\text { EDIT } \\
\text { INTEMP } \\
\text { ORIBNT } \\
\text { VANESA }\end{array}$ & $\begin{array}{l}\text { ENRCN1 } \\
\text { LEVSWL } \\
\text { PLLAYR } \\
\text { VOUTP }\end{array}$ & $\begin{array}{l}\text { PILM } \\
\text { MASSEX } \\
\text { PLOTS }\end{array}$ \\
\hline$A 42$ & is contuined in & ABLATE & DATAIN & MASSEX & & & \\
\hline$A 43$ & is contained in & DATAIN & MASSEX & & & & \\
\hline 144 & is contained in & EDIT & LEVSWL & & & & \\
\hline A45 & is contained in & $\begin{array}{l}\text { ADDLYR } \\
\text { REACT }\end{array}$ & CREATE & EDIT & INITL & PLLAYR & PRTOAS \\
\hline$A 9$ & is contained in & DATAIN & & & & & \\
\hline ACTIV & is contained in & ACTMET & ACTOXD & INTCOF & VANESA & & \\
\hline AROLST & is contuined in & CVFAC & FILM & INTEMP & MASRAT & & \\
\hline ATMDAT & is contained in & ATMPRO & ATMSUR & & & & \\
\hline B & is contuined in & BARRAY & SRPP & VANESA & & & \\
\hline
\end{tabular}


Tahle 5.5 Program routines contuining each COMMON block (continued)

BO

is contuined in

$\begin{array}{ll}\text { ADDLYR } & \text { ATMSUR } \\ \text { CVFAC } & \text { DENTRN } \\ \text { ENTRN } & \text { HCBTOP } \\ \text { INTEMP } & \text { LEVSWL } \\ \text { MHTRAN } & \text { MLTPRP } \\ \text { SOURCE } & \text { TMPFND }\end{array}$

BLKDTA DHOEN

HTRCLN

MASLOS

ORIENT

TRADII

BUBBLE

EDIT

HTRLIQ

MASRAT

PLLAYR

UNDEFN

CHEKCV

ENRCNI

HTRN

MASSEX

PLOTS

CHEKCV

DHOEN

HCBP,OT

HTRLIQ

MASRAT

PLLAYR

TMPSET

CHEKHT

EDIT

HCBINJ

HTRN

MASSEX

PLOTS

TRADII

TMPFND

CVFAC

MASSEX

TMPFND

DHOEN

MHTRAN

EDIT

MLTPRP

ENRCN1

PLLAYR

CREATE

ENRCNI

HCBSID

INITL

MELTRD

QMELT

UNDEFN

REACT

B3

is contuined in

BUBBLE

CONPRP

INITL

LEVSW

MASRA

MHTRAN

B4

B5

is contained in

BLKDTA

CORCON

FAIL

is contained in

EDIT

INTEMP

ENRCNI

HTRCLN

MASRAT

QMELT

B6

is contained in

BLKDTA

CVFAC

DATAIN

INPCON

MASLOS

DCYINT

MASSEX

DCYPOW

VANPP

BOA is contuined in CVFAC DCYINT FPUPD MASSEX VCFAC

B6B is contained in DCYINT FPUPD VANFP

B7 is contained in ENRCNI MASSEX MHTRAN REACT

B8 is contained in

ADDLYR EDIT

INTEMP

TMPSET

MHTRAN

HTRCLN

HTRLAY

MLTPRP

PLLAYR

HTRN

PLOTS

INITL

TRADII

B9 is contained in

BLKDTA

INITL

VISCTY

EDIT

MASSEX

EMISIV

ENRCN

HTRCLN SOLLIQ

INCOOL

MLTPRP PLLAYR

SOLLIQ

TMPFND

BCL is contained in

AERPTL

BCLTOV

CVFAC

DATAIN

LOSSES

VOUTP

MECLOS

CDF is contained in

DATAIN

DF

POOLV

voUTP

COMP

is contained in

BCLTOV

DATAIN

VANFP 
Taks 5.5 Program mutines containing each COMMON block (continued)

\begin{tabular}{|c|c|c|c|c|c|c|c|}
\hline CONSRV & is contained in & $\begin{array}{l}\text { EDIT } \\
\text { MHTRAN }\end{array}$ & $\begin{array}{l}\text { ENRCN1 } \\
\text { REACT }\end{array}$ & ENRCN2 & INITL & MASLOS & MASSEX \\
\hline CNDCON & is contained in & $\begin{array}{l}\text { AERPTL } \\
\text { VOUTP }\end{array}$ & CONPRP & CVFAC & DATAIN & VANESA & VCFAC \\
\hline CNSTNT & is contained in & $\begin{array}{l}\text { ANGAVL } \\
\text { CYLIND } \\
\text { HCBBOT } \\
\text { HTRLAY } \\
\text { LEVSWL } \\
\text { TRADII }\end{array}$ & $\begin{array}{l}\text { ATMSUR } \\
\text { DENTRN } \\
\text { HCBINJ } \\
\text { HTRLIQ } \\
\text { MASRAT } \\
\text { VSCRIT }\end{array}$ & $\begin{array}{l}\text { BLKDTA } \\
\text { EDIT } \\
\text { HCBSID } \\
\text { HTRN } \\
\text { MELTRD }\end{array}$ & $\begin{array}{l}\text { BUBBLE } \\
\text { ENTRN } \\
\text { HCBTOP } \\
\text { INOEOM } \\
\text { PLOTS }\end{array}$ & $\begin{array}{l}\text { CHEKCV } \\
\text { FILM } \\
\text { HSPCYL } \\
\text { INITL } \\
\text { SETTL }\end{array}$ & $\begin{array}{l}\text { CHEKHT } \\
\text { GEOM } \\
\text { HTRCLN } \\
\text { INTEMP } \\
\text { SETUP }\end{array}$ \\
\hline CTOP & is contained in & DATAIN & POOLV & VANESA & VOUTP & & \\
\hline CTOV & is contained in & $\begin{array}{l}\text { AERPTL } \\
\text { MASLOS }\end{array}$ & $\begin{array}{l}\text { AEROSOL } \\
\text { MECLOS }\end{array}$ & $\begin{array}{l}\text { ATMSUR } \\
\text { MHTRAN }\end{array}$ & $\begin{array}{l}\text { CVFAC } \\
\text { VANESA }\end{array}$ & $\begin{array}{l}\text { DATAIN } \\
\text { VBUBL }\end{array}$ & $\begin{array}{l}\text { LOSSES } \\
\text { VOUTP }\end{array}$ \\
\hline FORTN & is contained in & CYLIND & GEOM & HSPCYL & INOEOM & & \\
\hline FOUR & is contained in & EDIT & GEOM & HSPCYL & RECEDE & TIMSTP & \\
\hline GASEDT & is contained in & EDIT & INITL & REACT & & & \\
\hline HEATEX & is contained in & ATMSUR & ENRCNI & ENRCN2 & INITL & INTEMP & \\
\hline IMP & is contuined in & ENRCNI & ENRCN2 & & & & \\
\hline MASTER & is contuined in & $\begin{array}{l}\text { ABLATE } \\
\text { CREATE } \\
\text { EMISIV } \\
\text { MASLOS } \\
\text { PLLAYR } \\
\text { VCFAC }\end{array}$ & $\begin{array}{l}\text { ADDLYR } \\
\text { CVFAC } \\
\text { ENRCNI } \\
\text { MASRAT } \\
\text { PLOTS } \\
\text { VISCTY }\end{array}$ & $\begin{array}{l}\text { BLKDTA } \\
\text { DCYINT } \\
\text { FPUPD } \\
\text { MASSEX } \\
\text { PRTGAS }\end{array}$ & $\begin{array}{l}\text { CONFND } \\
\text { DENSTY } \\
\text { INITL } \\
\text { MHTRAN } \\
\text { REACT }\end{array}$ & $\begin{array}{l}\text { CONPRP } \\
\text { DHOEN } \\
\text { INPCON } \\
\text { MLTPRP } \\
\text { SOLLIQ }\end{array}$ & $\begin{array}{l}\text { CPENTH } \\
\text { EDIT } \\
\text { INPGAS } \\
\text { ORIENT } \\
\text { TMPFND }\end{array}$ \\
\hline $\mathrm{MRCl}$ & is contained in & $\begin{array}{l}\text { CHEKCV } \\
\text { HTRCLN } \\
\text { MELTRD }\end{array}$ & $\begin{array}{l}\text { CHEKHT } \\
\text { HTRLAY } \\
\text { PLOTS }\end{array}$ & $\begin{array}{l}\text { DATAIN } \\
\text { INITL } \\
\text { TRADII }\end{array}$ & $\begin{array}{l}\text { EDIT } \\
\text { INTEMP }\end{array}$ & $\begin{array}{l}\text { ENRCNI } \\
\text { LEVSWL }\end{array}$ & $\begin{array}{l}\text { FIIRAD } \\
\text { MASRAT }\end{array}$ \\
\hline MRCR & is contuined in & $\begin{array}{l}\text { CHEKCV } \\
\text { HTRCLN } \\
\text { MELTRD }\end{array}$ & $\begin{array}{l}\text { CHEKHT } \\
\text { HTRLAY } \\
\text { PLOTS }\end{array}$ & $\begin{array}{l}\text { DATAIN } \\
\text { INITL } \\
\text { TRADII }\end{array}$ & $\begin{array}{l}\text { EDIT } \\
\text { INTEMP }\end{array}$ & $\begin{array}{l}\text { ENRCNI } \\
\text { LEVSWL }\end{array}$ & $\begin{array}{l}\text { FIIRAD } \\
\text { MASRAT }\end{array}$ \\
\hline $\mathbf{M X}$ & is contained in & $\begin{array}{l}\text { ADDLYR } \\
\text { ENTRN } \\
\text { PLLAYR }\end{array}$ & $\begin{array}{l}\text { CREATE } \\
\text { HTRN } \\
\text { REACT }\end{array}$ & $\begin{array}{l}\text { CVFAC } \\
\text { INITL } \\
\text { TMPFND }\end{array}$ & $\begin{array}{l}\text { DENTRN } \\
\text { MHTRAN } \\
\text { UNDEFN }\end{array}$ & $\begin{array}{l}\text { EDIT } \\
\text { MLTPRP }\end{array}$ & $\begin{array}{l}\text { ENRCN1 } \\
\text { ORIENT }\end{array}$ \\
\hline PFDAT & is contained in & BLKDTA & CHEMPO & MLTREA & PRTGAS & REACT & \\
\hline
\end{tabular}


Table 5.5 Program routines containing each COMMON block (continued)

\begin{tabular}{|c|c|c|c|c|c|c|c|}
\hline ONE & is contained in & $\begin{array}{l}\text { ANGAVL } \\
\text { EDIT } \\
\text { HSPCYL } \\
\text { PAGEHD }\end{array}$ & $\begin{array}{l}\text { ARBINP } \\
\text { EXPRNT } \\
\text { INGEOM } \\
\text { PLOTS }\end{array}$ & $\begin{array}{l}\text { CHEKCV } \\
\text { FAIL } \\
\text { INITL } \\
\text { REACT }\end{array}$ & $\begin{array}{l}\text { CORCON } \\
\text { FIIRAD } \\
\text { LEVSWL } \\
\text { RECEDE }\end{array}$ & $\begin{array}{l}\text { CYLIND } \\
\text { GEOM } \\
\text { MASRAT } \\
\text { TIMSTP }\end{array}$ & $\begin{array}{l}\text { DATAIN } \\
\text { GFLMPR } \\
\text { MELTRD } \\
\text { TRADII }\end{array}$ \\
\hline POOL & is contained in & POOLV & VOUTP & & & & \\
\hline RNDOFF & is contained in & MLTREA & SETUP & UDU & & & \\
\hline SPECNM & is contained in & $\begin{array}{l}\text { BLKDTA } \\
\text { INPGAS }\end{array}$ & $\begin{array}{l}\text { CONPRP } \\
\text { PRTGAS }\end{array}$ & DATAIN & DCYINT & EDIT & INPCON \\
\hline THREE & is contained in & DATAIN & EDIT & TIMSTP & & & \\
\hline TITLE & is contained in & DATAIN & PAGEHD & VOUTP & & & \\
\hline TWNEIT & is contained in & $\begin{array}{l}\text { ANOAVL } \\
\text { LEVSWL }\end{array}$ & $\begin{array}{l}\text { ARBINP } \\
\text { MASRAT }\end{array}$ & $\begin{array}{l}\text { CYLIND } \\
\text { MELTRD }\end{array}$ & $\begin{array}{l}\text { FIIRAD } \\
\text { RECEDE }\end{array}$ & $\begin{array}{l}\text { GEOM } \\
\text { TIMSTP }\end{array}$ & HSPCYL \\
\hline TWNTWO & is contained in & BUBBLE & EDIT & INITL & LEVSWL & MASRAT & \\
\hline TWO & is contained in & $\begin{array}{l}\text { ADDLYR } \\
\text { CVFAC } \\
\text { ENRCN1 } \\
\text { LEVSWL } \\
\text { PAOEHD } \\
\text { TIMSTP }\end{array}$ & $\begin{array}{l}\text { ATMPRO } \\
\text { DATAIN } \\
\text { EXPRNT } \\
\text { MASLOS } \\
\text { PLLAYR } \\
\text { TMPFND }\end{array}$ & $\begin{array}{l}\text { ATMSUR } \\
\text { DCYINT } \\
\text { FAIL } \\
\text { MASRAT } \\
\text { PLOTS }\end{array}$ & $\begin{array}{l}\text { CHEKCV } \\
\text { DENTRN } \\
\text { HTRLAY } \\
\text { MASSEX } \\
\text { REACT }\end{array}$ & $\begin{array}{l}\text { CORCON } \\
\text { EDIT } \\
\text { INPGAS } \\
\text { MELTRD } \\
\text { RECEDE }\end{array}$ & $\begin{array}{l}\text { CREATE } \\
\text { EMISIV } \\
\text { INTEMP } \\
\text { MHTRAN } \\
\text { SOURCE }\end{array}$ \\
\hline VAN & is contained in & $\begin{array}{l}\text { AERPTL } \\
\text { MHTRAN }\end{array}$ & $\begin{array}{l}\text { AERSOL } \\
\text { VANESA }\end{array}$ & $\begin{array}{l}\text { ATMSUR } \\
\text { VBUBL }\end{array}$ & $\begin{array}{l}\text { CVFAC } \\
\text { VOUTP }\end{array}$ & DATAIN & MECLOS \\
\hline VANPRP & is contained in & $\begin{array}{c}\text { AERPTL } \\
\text { VBUBL }\end{array}$ & AERSOL & CVFAC & MECLOS & PHSPRP & VANESA \\
\hline VPL & is contained in & AERPRL & DATAIN & POOLV & VANESA & VOUTP & \\
\hline VTOC & is contained in & CVFAC & MASLOS & VCFAC & & & \\
\hline XND & is contained in & $\begin{array}{l}\text { AERPTL } \\
\text { VANESA }\end{array}$ & $\begin{array}{l}\text { AERSOL. } \\
\text { VBUBL }\end{array}$ & $\begin{array}{l}\text { BCLTOV } \\
\text { VCFAC }\end{array}$ & $\begin{array}{l}\text { DATAIN } \\
\text { VOUTP }\end{array}$ & $\begin{array}{l}\text { MECLOS } \\
\text { XNDAR }\end{array}$ & PHSPRP \\
\hline
\end{tabular}




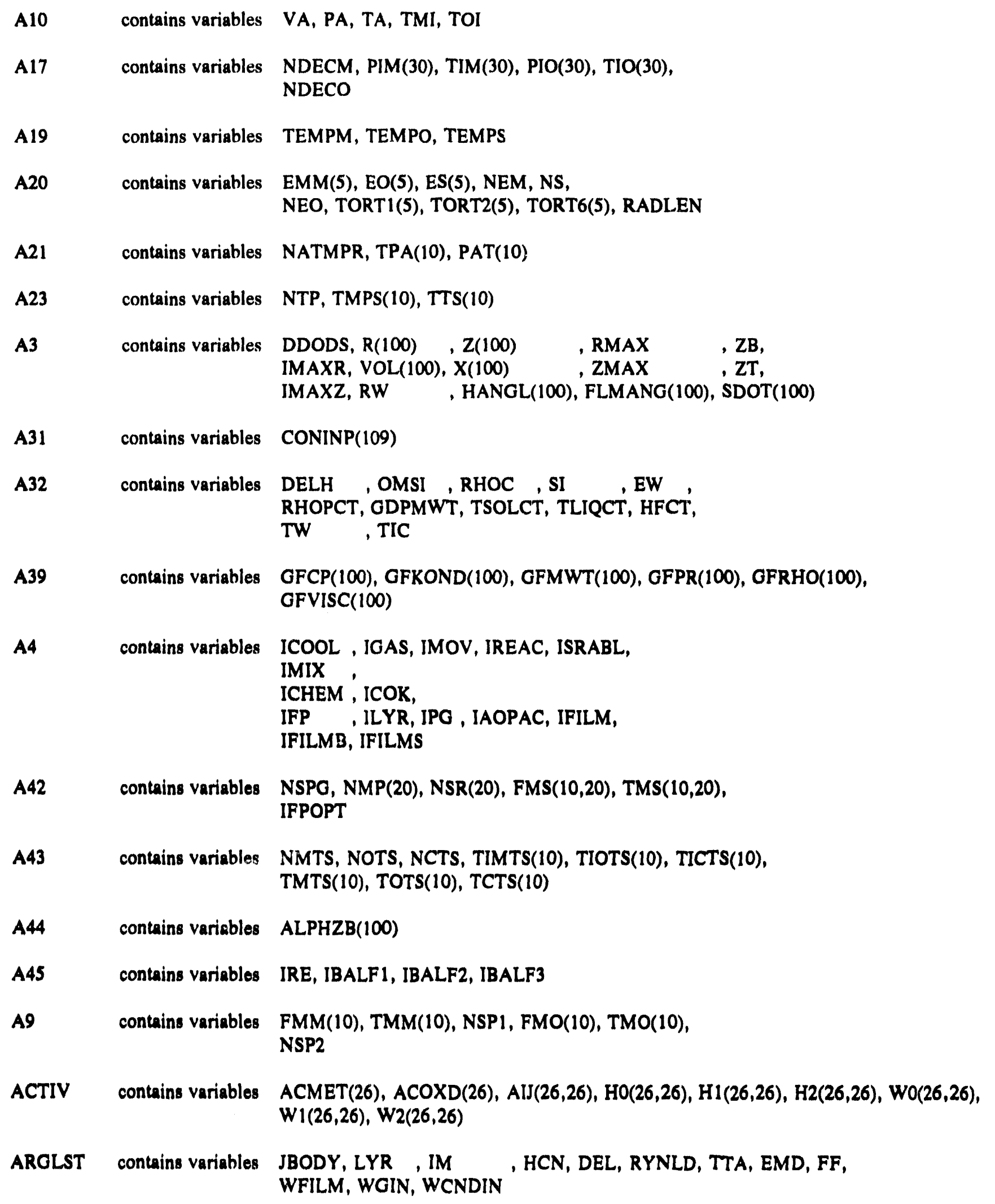


Table 5.6 Variables contained in each COMMON block (continued)

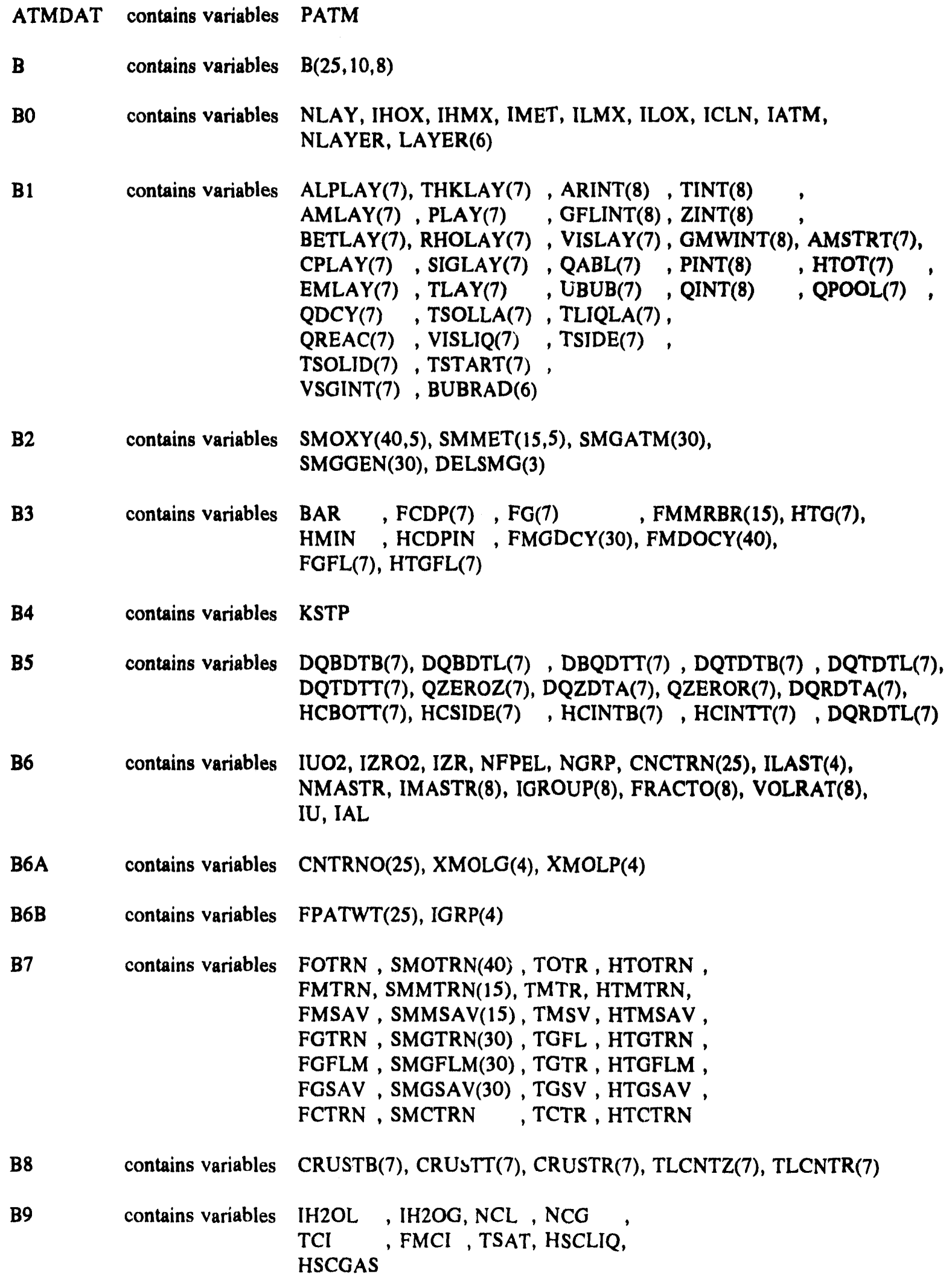

CNTRNO(25), XMOLG(4), XMOLP(4)

FPATWT(25), IGRP(4)

FOTRN , SMOTRN(40), TOTR, HTOTRN , FMTRN, SMMTRN(15), TMTR, HTMTRN, FMSAV , SMMSAV(15), TMSV, HTMSAV , FGTRN , SMGTRN(30), TGFL , HTGTRN , FGFLM , SMGFLM(30), TGTR , HTGFLM , FGSAV , SMGSAV(30), TGSV , HTGSAV , FCTRN , SMCTRN , TCTR , HTCTRN 


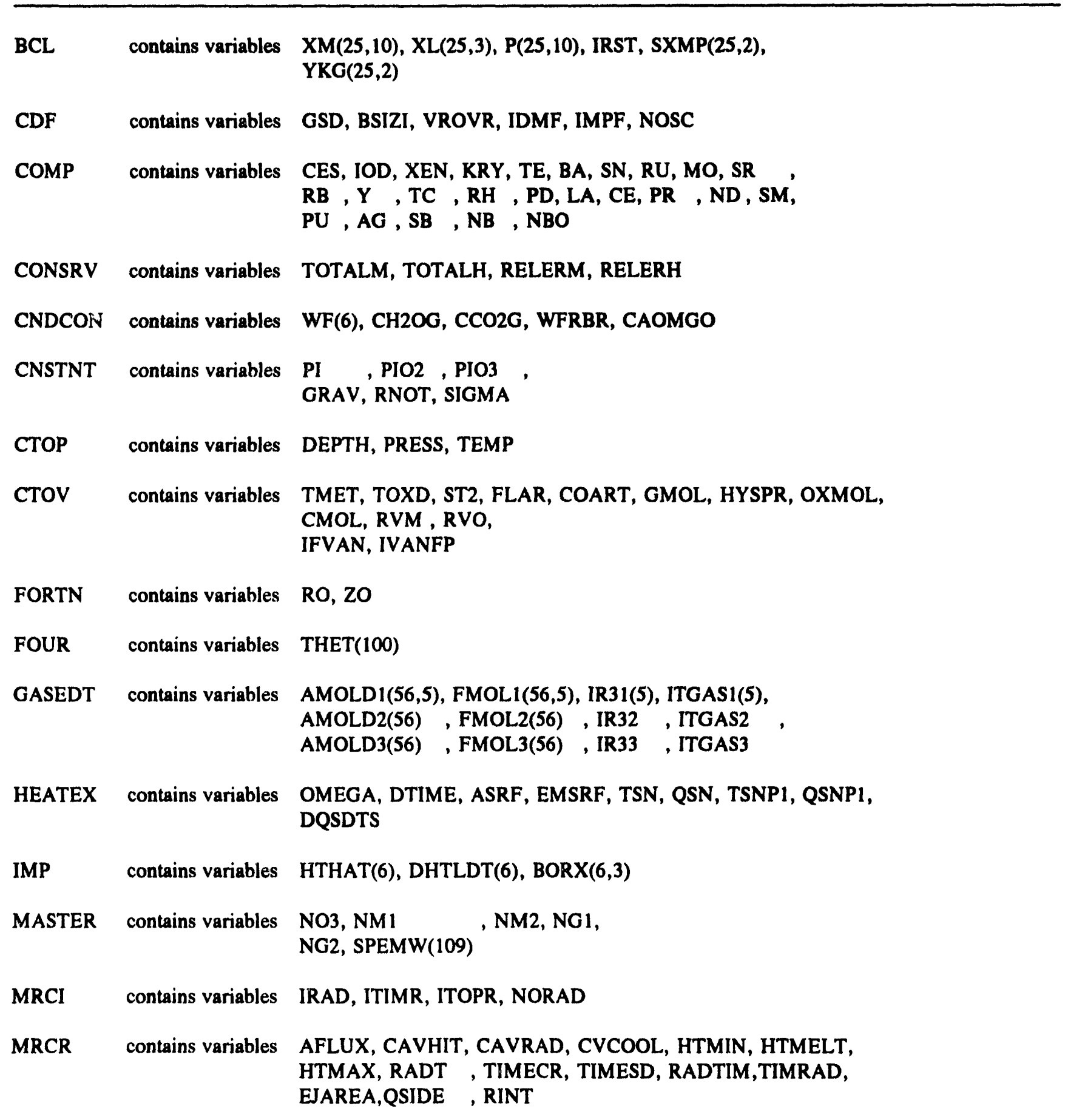


Table 5.6 Variables contained in each COMMON block (continued)

\begin{tabular}{|c|c|c|c|}
\hline $\mathbf{M X}$ & contains variables & $\begin{array}{lll}\text { FOENT } & , \text { FMENT } & , \text { FODNT } \\
\text { ENTOMX(5), ENTM(5) } & , \text { ENTMMX(5), } \\
\text { RHOM(5) } & , \text { RHOO(5) } & , \operatorname{VOLM}(5) \\
\text { AMO(5) } & , \text { RDROP(5), TSOLM(5) } \\
\text { TLIQO(5) } & \text {, SIGM(5) }, \operatorname{SIGO(5)} \\
\text { THKM(5) } & , \text { THKO(5) } & , \operatorname{HTM}(5) \\
\text { CPO(5) } & & \end{array}$ & $\begin{array}{l}\text {, FMDNT , ENTO(5) } \\
\text { DENTO(5), } \operatorname{ENTM(5)~} \\
\text {, VOLO(5), AMM(5) } \\
\text {, TSOLO(5), TLIQM(5), } \\
\text {, VISM(5) , VISO(5) } \\
\text {, HTO(5) },\end{array}$ \\
\hline PFDAT & contains variables & $\begin{array}{l}\text { NOP1 , NMP1 , NMP2, NGP1 } \\
\text { NUMSPE , IPNT(56), SG(56), NUMELE } \\
\text { ANE(56,15) }\end{array}$ & \\
\hline ONE & contains variables & IFLOR, IGEOM, IRSTRT, IT, NRAYS & \\
\hline POOL & contains variables & $\begin{array}{lll}\text { DSSG(MNOSC), APM(MNOSC), } & \text { RSIZ(M) } \\
\text { DNGSD } & , \text { ENM }\end{array}$ & NOSC), DLCC, DNMS, \\
\hline RNDOFF & contains variables & URO & \\
\hline SPECNM & contains variables & ISPNAM(109) & \\
\hline THREE & contains variables & $\begin{array}{l}\text { IDELT, NDELT, TIMDT(10), DTMIN(10 } \\
\text { IEDIT , NEDIT , TIMED(10), DEDIT(10) }\end{array}$ & 0), DTMAX(10), \\
\hline TITLE & contains variables & ITITL & \\
\hline TWNEIT & contains variables & ITANG, NBOT, RTANG & \\
\hline TWNTWO & contains variables & $\begin{array}{l}\text { BUBMLI(100), DELL(100), GFLMFL(10 } \\
\operatorname{HCON}(100), \operatorname{IMOD}(100), \operatorname{REYNLD}(10\end{array}$ & $\begin{array}{l}\text { 00), } \\
\text { D), TEMPA(100) }\end{array}$ \\
\hline TWO & contains variables & $\begin{array}{l}\text { DELTIM, IPINC , TIME, TIMEO, TPRIS } \\
\text { DPRIN , TEDIT, TIMEND }\end{array}$ & \\
\hline VAN & contains variables & $\begin{array}{l}\text { VAPOR, BURST, AER1 , AER2, GOXD } \\
\text { KATIS , DC , BUBD, PTBB, PTDIA }\end{array}$ & $\begin{array}{l}\text {, IBUB, BUBDM, BUBDO, } \\
\text { INOPL }\end{array}$ \\
\hline VANPRP & contains variables & $\begin{array}{lll}\text { BUBNM, BUBNO, DMETP , DOXP , } \\
\text { PMETP , POXP , PVAR , QMETP , } \\
\text { BNM , BNO , RTM , RTO } \\
\text { STM , STO , VISMET, VISOXD, }\end{array}$ & $\begin{array}{l}\text { G(25,10), GMET, } \\
\text { QOXP , SMETP, SOXP, } \\
\text { VMET , VOXD }\end{array}$ \\
\hline VPL & contains variables & SIZ, XMAS, DEN & \\
\hline VTOC & contains variables & XMLS(14), XOLS(33), ICVSPE(49), ICVF & $\mathrm{FP}(25)$ \\
\hline XND & contains variables & $X N(25,10), \operatorname{DARR}(25,2)$ & \\
\hline
\end{tabular}


Table 5.7 Dictionary of principal variables in CORCON

\begin{tabular}{|c|c|c|c|}
\hline $\begin{array}{l}\text { FORTRAN } \\
\text { Symbol }\end{array}$ & $\begin{array}{c}\text { Algebraic } \\
\text { Symbol }\end{array}$ & Description & Units \\
\hline ACMET(I) & $\gamma_{\mathrm{i}}$ & The activity coefficient for metal phase constituents & -- \\
\hline $\operatorname{ACOXD}(\mathrm{I})$ & $\gamma_{\mathrm{i}}$ & The activity coefficient for oxide phase constituents & \\
\hline AER1 & -- & $\begin{array}{l}\text { The concentration of aerosol particles created by } \\
\text { vaporization and bubble bursting }\end{array}$ & $g / c c$ \\
\hline AER2 & -- & $\begin{array}{l}\text { The concentration of aerosol particles created by } \\
\text { vaporization and bubble bursting, for gas at standard } \\
\text { conditions }\end{array}$ & g/cc \\
\hline AFLUX & -- & $\begin{array}{l}\text { The fraction of the top melt surface heat flux used in } \\
\text { setting the melt edge temperatures for a time-dependent } \\
\text { melt radius }\end{array}$ & -- \\
\hline AG & $\mathbf{A g}$ & Quantity of silver ( $\mathrm{Ag}$ ) in the fission product inventory & g-mol \\
\hline ALPHZB (J) & $\alpha(Z)$ & Local axial void fraction at $Z(J)$ & -- \\
\hline ALPLAY (L) & $\alpha_{\mathrm{L}}$ & Average void fraction & -- \\
\hline $\mathbf{A M}(\mathrm{I})$ & -- & Amount of species & g-mol \\
\hline AMLAY (L) & $\mathbf{m}_{\mathrm{L}}$ & Mass & kg \\
\hline AMM (L) & -- & $\begin{array}{l}\text { Mass of metal phase in new layer produced by the } \\
\text { combination of two cld layers }\end{array}$ & kg \\
\hline $\operatorname{AMO}(L)$ & - & $\begin{array}{l}\text { Mass of oxide phase in new layer produced by the } \\
\text { combination of two old layers }\end{array}$ & $\mathbf{k g}$ \\
\hline AMOLD1 $(1, L)$ & -- & Amount of species in layer at start of timestep & g-mol \\
\hline AMOLD2 (I) & -- & Amount of species in atmosphere at start of timestep & g-mol \\
\hline AMOLD3 (I) & -- & Amount of species in gas film at start of timestep & $\mathbf{g}-\mathbf{m o l}$ \\
\hline AMSTRT (L) & $\mathbf{m}_{\mathrm{L}}^{\circ}$ & Mass at start of timestep & $\mathbf{k g}$ \\
\hline ANE $(\mathbf{I}, \mathbf{K})$ & -- & Stoichiometric coefficients & -- \\
\hline $\operatorname{APM}(\mathbf{N})$ & -- & Aerosol mass in distribution range $N$ to $N+1$ & 8 \\
\hline ARINT (L) & A & Interface area & $\mathbf{m}^{2}$ \\
\hline ASRF & $\mathbf{A}_{\boldsymbol{s}}$ & Melt surface area exposed to atmosphere or coolant & $\mathbf{m}^{2}$ \\
\hline $\mathrm{B}(\mathrm{I}, \mathbf{N}, \mathbf{K})$ & -- & $\begin{array}{l}\text { Array containing parametric values for fits to the free- } \\
\text { energy functions }\end{array}$ & -- \\
\hline BA & $\mathrm{Ba}$ & Quantity of barium $(\mathrm{Ba})$ in the fission product inventory & 8-mol \\
\hline BAR & -- & Weight fraction of rebar in the concrete & -- \\
\hline BETLAY (L) & $\beta_{L}$ & Volumetric coefficient of expansion & $\mathbf{K}^{\cdot 1}$ \\
\hline
\end{tabular}


Table 5.7 Dictionary of principal variables in CORCON (continued)

\begin{tabular}{|c|c|c|c|}
\hline $\begin{array}{l}\text { FORTRAN } \\
\text { Symbol }\end{array}$ & $\begin{array}{l}\text { Algebraic } \\
\text { Symbol }\end{array}$ & Description & Units \\
\hline BNM & - & $\begin{array}{l}\text { Morton number for the metal phase in aerosol } \\
\text { calculations }\end{array}$ & -- \\
\hline BNO & -- & $\begin{array}{l}\text { Morton number for the oxide phase in aerosol } \\
\text { calculations }\end{array}$ & -- \\
\hline BSIZI & - & Initial bubble size in aerosol calculations & $\mathbf{c m}$ \\
\hline BUBD & -- & $\begin{array}{l}\text { Fixed input bubble diameter, used in aerosol } \\
\text { calculations }\end{array}$ & $\mathrm{cm}$ \\
\hline BUBDM & -- & $\begin{array}{l}\text { Bubble diameter for the metal phase, used in aerosol } \\
\text { calculations }\end{array}$ & $\mathbf{c m}$ \\
\hline BUBDO & -- & $\begin{array}{l}\text { Bubble diameter for the oxide phase, used in aerosol } \\
\text { calculations }\end{array}$ & $\mathrm{cm}$ \\
\hline BUBMLI (J) & -- & Molar flux of gas entering bubbles & $\mathrm{g}-\mathrm{mol} / \mathrm{m}^{2}-\mathrm{g}$ \\
\hline BUBNM & -- & $\begin{array}{l}\text { Number of bubbles passing through the metal phase, in } \\
\text { aerosol calculations }\end{array}$ & -- \\
\hline BUBNO & - & $\begin{array}{l}\text { Number of bubbles passing through the oxide phase, in } \\
\text { aerosol calculations }\end{array}$ & -- \\
\hline BUBRAD (L) & $\mathbf{r}_{\mathbf{h}}$ & Average bubble radius & $\mathbf{m}$ \\
\hline BURST & -- & $\begin{array}{l}\text { The concentration of aerosol particles generated by } \\
\text { bubble bursting }\end{array}$ & -- \\
\hline CAOMGO & -- & Mass fraction of $\mathrm{CaO}$ in $\mathrm{CaO}$ and $\mathrm{MgO}$ & -- \\
\hline CAVHIT & -- & $\begin{array}{l}\text { Axial location of the original cavity floor (time- } \\
\text { dependent melt radius) }\end{array}$ & $\mathbf{m}$ \\
\hline CAVRAD & -- & $\begin{array}{l}\text { Radius of the cavity, until the melt reaches the cavity } \\
\text { wall (time-dependent melt radius) }\end{array}$ & $\mathbf{m}$ \\
\hline $\mathrm{CCO} 2 \mathrm{G}$ & -- & Mass fraction of original concrete $\mathrm{CO}$ & -- \\
\hline $\mathrm{CE}$ & Ce & Quantity of cerium (Ce) in the fission product inventory & 8-mol \\
\hline CES & Cs & Quantity of cesium (Cs) in the fission product inventory & 8-mol \\
\hline CH2OG & -- & $\begin{array}{l}\text { Mass fraction of chemically bound and evaporable water } \\
\text { in the concrete }\end{array}$ & -- \\
\hline CMOL & -- & Total quantity of carbon, used in aerosol calculations & $y-\mathrm{rnol}$ \\
\hline CNCTRN (I) & -- & Fission product quantity & $\mathbf{g}-\mathbf{m o l}$ \\
\hline CNTRNO (I) & - & Original fission product quantity & g-mol \\
\hline COART & -- & Mass rate of addition of concrete to the melt & $8 / \mathbf{s}$ \\
\hline
\end{tabular}


Table 5.7 Dictionary of principal variables in CORCON (continued)

\begin{tabular}{|c|c|c|c|}
\hline $\begin{array}{l}\text { FORTRAN } \\
\text { Symbol }\end{array}$ & $\begin{array}{l}\text { Algebraic } \\
\text { Symbol }\end{array}$ & Description & Units \\
\hline CHPOT (I) & $\mu_{i}^{\circ}$ & Standard state chemical potential & $\mathrm{cal} / \mathrm{g}-\mathbf{m o l}$ \\
\hline CONINP (I) & $\mathbf{m}$ & Initial mass of condensed species & kg \\
\hline CPLAY (L) & $c_{p_{L}}$ & Specific heat & $J / \mathbf{k g}-\mathbf{K}$ \\
\hline CRUSTx (L) & $\delta_{\mathbf{x}_{\mathrm{L}}}$ & $\begin{array}{l}\text { Crust thickness of face } \times(=B \text {, bottom; }=T \text {, top; }= \\
R \text {, radial) }\end{array}$ & $\mathbf{m}$ \\
\hline CVCOOL & -- & $\begin{array}{l}\text { Critical volume of coolant required to cover the melt } \\
\text { (time-dependent melt radius) }\end{array}$ & $\mathbf{m}^{3}$ \\
\hline DARR $(I, K)$ & -- & Array containing the densities for the condensed species & $\mathbf{k g} / \mathbf{m}^{3}$ \\
\hline DDODS & -- & $\begin{array}{l}\text { Ratio of normal recision to normal distance to adjacent } \\
\text { ray }\end{array}$ & -- \\
\hline DEDIT (N) & -- & Time between printed edits & $\mathbf{s}$ \\
\hline DELH & & Heat of ablation of concrete & $\mathbf{J} / \mathbf{k g}$ \\
\hline DELL (J) & $\delta$ & Gas film thickness & $\mathbf{m}$ \\
\hline DELSMG (I) & -- & Mass of gaseous species generated during timestep & kg \\
\hline DELTIM & $\Delta t$ & Timestep & $s$ \\
\hline DEN & -- & Aerosol density & g/cc \\
\hline DEPTH & -- & Depth of coolant, used in aerosol calculations & $\mathbf{c m}$ \\
\hline DHPOW & -- & Decay heat power & $\mathbf{w}$ \\
\hline DHTLDT (L) & -- & Total heat capacity of new mass at old temperature & $\mathrm{J} / \mathrm{kg}-\mathrm{K}$ \\
\hline DLCC & -- & Linear correlation coefficient in the aerosol model & -- \\
\hline DMETP & -- & $\begin{array}{l}\text { Mass density of the metallic phase in aerosol } \\
\text { calculations }\end{array}$ & g/cc \\
\hline DNGSD & -- & $\begin{array}{l}\text { Geometric standard deviation of the aerosol particle size } \\
\text { distribution }\end{array}$ & -- \\
\hline DNMS & -- & $\begin{array}{l}\text { Mean particle size for the aerosol particle size } \\
\text { distribution }\end{array}$ & $\mu \mathrm{m}$ \\
\hline DOXP & -- & $\begin{array}{l}\text { Mass density of the metallic phase in aerosol } \\
\text { calculations }\end{array}$ & g/cc \\
\hline DPRIN & -- & Time interval for diagnostic prints & $\mathbf{s}$ \\
\hline DQBDTx (L) & $d q_{B} / d T_{x}$ & $\begin{array}{l}\text { Rate of change of upward heat flux at the bottom } \\
\text { surface with respect to temperature } \times(=B \text {, bottom; } \\
=L \text {, layer; }=T \text {, top })\end{array}$ & $W / m^{2}-K$ \\
\hline DQRDTA (L) & $d q_{R} / d T_{A}$ & $\begin{array}{l}\text { Rate of change of outward heat flux to the radial surface } \\
\text { with respect to the surface temperature }\end{array}$ & $\mathrm{W} / \mathbf{m}^{2}-\mathrm{K}$ \\
\hline
\end{tabular}


Table 5.7 Dictionary of principal variables in CORCON (continued)

\begin{tabular}{|c|c|c|c|}
\hline $\begin{array}{l}\text { FORTRAN } \\
\text { Symbol }\end{array}$ & $\begin{array}{l}\text { Algebraic } \\
\text { Symbol }\end{array}$ & Description & Units \\
\hline DQRDTL (L) & $\mathrm{dq}_{\mathrm{R}} / \mathrm{dT}_{\mathrm{L}}$ & $\begin{array}{l}\text { Rate of change of outward heat flux to the radial surface } \\
\text { with respect to the layer temperature }\end{array}$ & $W / m^{2}-K$ \\
\hline DQSDTS & $\mathrm{dq} / \mathrm{dT}$, & $\begin{array}{l}\text { Rate of change of the heat flux to/from the pool surface } \\
\text { with respect to the surface temperature }\end{array}$ & $W / \mathbf{m}^{2}-K$ \\
\hline DQTDTx (L) & $d q_{t} / d T_{x}$ & $\begin{array}{l}\text { Rate of change of upward heat flux at the bottom } \\
\text { surface with respect to temperature } \times(=\mathrm{B} \text {, bottom; } \\
=\mathrm{L} \text {, layer; = T, top) }\end{array}$ & $W / m^{2}-K$ \\
\hline DQZDTA (L) & -- & $\begin{array}{l}\text { Rate of change of heat flux to the axial surface with } \\
\text { respect to surface temperature }\end{array}$ & $W / m^{2}-K$ \\
\hline DSSG (N) & - & Aerosol particle size ranges & $\mu \mathbf{m}$ \\
\hline DTIME & - & Timestep & $\mathbf{s}$ \\
\hline DTMAX (N) & -- & Maximum timestep & $\mathbf{s}$ \\
\hline DTMIN (N) & -- & Minimum timestep & $\mathbf{s}$ \\
\hline GSD & -- & $\begin{array}{l}\text { Error in geometric standard deviation for aerosol } \\
\text { particle size distribution }\end{array}$ & -- \\
\hline EJAREA & $A_{R}$ & $\begin{array}{l}\text { Area of the edge of each layer exposed to atmosphere or } \\
\text { coolant }\end{array}$ & $\mathbf{m}^{2}$ \\
\hline EMD & -- & Energy loss to the concrete ablation products & W \\
\hline EMLAY (L) & $\epsilon_{\mathrm{L}}$ & Emissivity & -- \\
\hline EMM $(\mathbf{M}$ or $\mathbf{N})$ & $\epsilon_{M}$ & Emissivity of metallic phase & -- \\
\hline EMSRF & $\epsilon_{\text {our }}$ & Emissivity of melt surface exposed to atmosphere & -- \\
\hline EMSUR & $\epsilon_{\text {sur }}$ & Emissivity of the above-pool surroundings & -- \\
\hline ENM & -- & $\begin{array}{l}\text { Error in the natural logarithm of the aerosol particle size } \\
\text { distribution }\end{array}$ & -- \\
\hline $\mathrm{EO}(\mathrm{M}$ or $\mathrm{N})$ & $\epsilon_{\mathrm{o}}$ & Emissivity of oxidic phase & -- \\
\hline ES $(M$ or $N)$ & $\epsilon_{\text {aur }}$ & Emissivity of atmosphere surroundings & -- \\
\hline EW & $\epsilon_{w}$ & Emissivity of ablating concrete surface & -- \\
\hline FCTRN & -- & Mass of coolant added during timestep & kg \\
\hline FG (L) & -- & Gas flow rate & $\mathrm{kg} / \mathrm{s}$ \\
\hline FGFLM & -- & Mass of gas transported in film during timestep & kg \\
\hline FGSAV & -- & $\begin{array}{l}\text { Mass of gas transformed by oxide/atmosphere reaction } \\
\text { during timestep }\end{array}$ & kg \\
\hline FGTRN & - & Mass of gas transported as bubbles during timestep & kg \\
\hline FLAR & -- & $\begin{array}{l}\text { Surface area of the top melt layer, used in aerosol } \\
\text { calculations }\end{array}$ & $\mathrm{cm}^{2}$ \\
\hline
\end{tabular}


Table 5.7 Dictionary of principal variables in CORCON (continued)

\begin{tabular}{|c|c|c|c|}
\hline $\begin{array}{l}\text { FORTRAN } \\
\text { Symbol }\end{array}$ & $\begin{array}{l}\text { Algebraic } \\
\text { Symbol }\end{array}$ & Description & Units \\
\hline FLMANG (J) & - & Average slope of the gas film over a Taylor cell radius & ned \\
\hline FMCI & - & Initial mass of coolant & $\mathbf{k g}$ \\
\hline FMM (N) & - & Metallic phase splashout rate & $\mathbf{k g} / \mathbf{s}$ \\
\hline FMMRBR (I) & -- & Mass fraction composition of rebar & - \\
\hline FMO (N) & - & Oxidic phase splashout rate & $\mathrm{kg} / \mathrm{s}$ \\
\hline FMS $(\mathbf{N}, \mathbf{l})$ & - & Specified rate of additions to pool & $\mathbf{k g} / \mathbf{s}$ \\
\hline FMSAV & -- & $\begin{array}{l}\text { Mass of reinforcing steel ablated by the light oxide layer } \\
\text { during timestep }\end{array}$ & $\mathbf{k g}$ \\
\hline FMTRN & -- & $\begin{array}{l}\text { Mass of metal rising or sinking in the pool during the } \\
\text { timestep }\end{array}$ & $\mathbf{k g}$ \\
\hline FOTRN & - & $\begin{array}{l}\text { Mass of oxide rising or sinking in the pool during the } \\
\text { timestep }\end{array}$ & kg \\
\hline FPATWT (I) & -- & Fission product atomic weights & 8/g-mol \\
\hline $\mathbf{G}(\mathrm{I}, \mathrm{K})$ & -- & Free energy of species I involving element $K$ & $\mathrm{cal} / \mathrm{mol}$ \\
\hline GDPMWT & -- & $\begin{array}{l}\text { Molecular weight of gaseous products of concrete } \\
\text { ablation }\end{array}$ & $\mathrm{g} / \mathrm{g}-\mathrm{mol}$ \\
\hline GFCP (J) & -- & Gas film specific heat & $\mathrm{J} / \mathbf{k g}-\mathrm{K}$ \\
\hline GFKOND (J) & -- & Gas film thermal conductivity & $\mathbf{W} / \mathbf{m}-\mathbf{K}$ \\
\hline GFLINT (L) & -- & Mass of flow of gas at interface & $\mathbf{k g} / \mathbf{s}$ \\
\hline GFLMFL (J) & -- & Mass flow of gas in the film & $\mathbf{k g} / \mathbf{s}$ \\
\hline GFMWT (J) & -- & Gas film molecular weight & g/g-mol \\
\hline GFPR (J) & -- & Gas film Prandtl number & - \\
\hline GFRHO (J) & - & Gas film density & $\mathbf{k g} / \mathbf{m}$ \\
\hline GFVISC (J) & -- & Gas film dynamic viscosity & $\mathbf{k g} / \mathbf{m}-\mathbf{8}$ \\
\hline GMET & -- & $\begin{array}{l}\text { Quantity of gas sparging the through the metal, used in } \\
\text { merosol calculations }\end{array}$ & $\mathrm{g}-\mathrm{mol} / \mathrm{s}$ \\
\hline GMOL & -- & $\begin{array}{l}\text { Total quantity of gas sparging through the metal during } \\
\text { the timestep, used in aerosol calculations }\end{array}$ & $\mathrm{g}-\mathrm{mol} / \mathrm{s}$ \\
\hline GMWINT (L) & -- & Molecular weight of gas at interface & $\mathrm{g} / \mathrm{g}-\mathrm{mol}$ \\
\hline GOXD & - & $\begin{array}{l}\text { Quantity of gas sparging through the oxide, used in } \\
\text { aerosol calculations }\end{array}$ & $\mathrm{g}-\mathrm{mol} / \mathrm{s}$ \\
\hline GRAV & 8 & Gravitational acceleration & $\mathrm{m} / \mathbf{s}$ \\
\hline GSD & -- & $\begin{array}{l}\text { Geometric standard deviation of merosol particle size } \\
\text { distribution }\end{array}$ & - \\
\hline
\end{tabular}


Table 5.7 Dictionary of principal variables in CORCON (continued)

\begin{tabular}{|c|c|c|c|}
\hline $\begin{array}{l}\text { FORTRAN } \\
\text { Symbol }\end{array}$ & $\begin{array}{l}\text { Algebraic } \\
\text { Symbol }\end{array}$ & Description & Units \\
\hline HANGL (J) & -- & Negative of cavity surface inclination angle & rad \\
\hline HBB & -- & Concrete crucible base thickness, Figure 4.3 & $\mathbf{m}$ \\
\hline HBC & .. & $\begin{array}{l}\text { Concrete crucible dimension, top to center of } \\
\text { hemisphere, Figure } 4.2\end{array}$ & m \\
\hline $\mathrm{HC}$ & -- & $\begin{array}{l}\text { Concrete crucible dimension, top to start of hemisphere, } \\
\text { Figure } 4.2\end{array}$ & $\mathbf{m}$ \\
\hline HCINTB (L) & $h_{L}$ & Heat transfer coefficient, bulk to bottom & $W / \mathbf{m}^{2}-K$ \\
\hline HCINTT (L) & $h_{u}$ & Heat transfer coefficient, bulk to top & $W / \mathbf{m}^{2}-\mathbf{K}$ \\
\hline HCN & $\cdots$ & Heat transfer coefficient across gas film & $W / m^{2}-K$ \\
\hline HCON (J) & h & Gas film heat transfer coefficient & $W / m^{2}-K$ \\
\hline HCSIDE (L) & h & Heat transfer coefficient, bubble agitation & $W / m^{2}-K$ \\
\hline HEATUP & -- & $\begin{array}{l}\text { Heat to concrete decomposition products during } \\
\text { timestep; converted to rate [W] in EDIT }\end{array}$ & J \\
\hline HFCT & -- & Latent heat of fusion for concrete & $\mathrm{J} / \mathrm{kg}$ \\
\hline HIT & $\cdots$ & Concrete crucible depth, Figure 4.3 & $\mathbf{m}$ \\
\hline HTCTRN & -- & Total enthalpy of coolant added during timestep & J \\
\hline HTGFLM & -- & Total enthalpy of gas transported in film during timestep & J \\
\hline HTGSAV & -- & $\begin{array}{l}\text { Total enthalpy of gas transformed by oxide/atmosphere } \\
\text { reaction during timestep }\end{array}$ & J \\
\hline HTGTRN & -- & $\begin{array}{l}\text { Total enthalpy of gas transported as bubbles during the } \\
\text { timestep }\end{array}$ & $\mathbf{J}$ \\
\hline HTHAT (L) & -- & Total enthalpy of new layer mass at old temperature & J \\
\hline HTM (L) & -- & Enthalpy of the metal in the layer & J \\
\hline HTMAX & -- & $\begin{array}{l}\text { Maximum allowed melt thickness for the time-dependent } \\
\text { melt radius (entered in } \mathrm{cm} \text {; used internally in } \mathrm{m} \text { ) }\end{array}$ & $\mathbf{m}$ \\
\hline HTMELT & -- & Thickness of the melt layer (time-dependent melt radius) & $\mathbf{m}$ \\
\hline HTMIN & -- & $\begin{array}{l}\text { Minimum allowed melt thickness for the time-dependent } \\
\text { melt radius (entered in } \mathrm{cm} \text {; used internally in } \mathrm{m} \text { ) }\end{array}$ & $\mathbf{m}$ \\
\hline HTMSAV & -- & $\begin{array}{l}\text { Total enthalpy of reinforcing steel ablated by the light } \\
\text { oxide layer during the timestep }\end{array}$ & J \\
\hline HTMTRN & -- & $\begin{array}{l}\text { Total enthalpy of metal rising or sinking in pool during } \\
\text { timestep }\end{array}$ & J \\
\hline HTO (L) & -- & Enthalpy of the oxide in the layer & $\mathbf{J}$ \\
\hline
\end{tabular}


Table 5.7 Dictionary of principal variables in CORCON (continued)

\begin{tabular}{|c|c|c|c|}
\hline $\begin{array}{l}\text { FORTRAN } \\
\text { Symbol }\end{array}$ & $\begin{array}{l}\text { Algebraic } \\
\text { Symbol }\end{array}$ & Dewcription & Units \\
\hline HTOT (L) & -- & Total enthalpy & J \\
\hline HTOTRN & $-\bullet$ & $\begin{array}{l}\text { Total enthalpy of oxide rising or sinking in pool during } \\
\text { timestep }\end{array}$ & J \\
\hline HYSPR & - & Hydrogen/stenm pressure ratio & -. \\
\hline IABL & -- & Surroundings ablation index & $\cdots$ \\
\hline IAOPAC & - & Aerosol opacity index & -- \\
\hline IATM & - & Index of top surfece of the pool, including coolant & -- \\
\hline ICHEM & -- & Flag for condensed phase chemistry and coking options & -- \\
\hline ICLN & -- & Index of the coolant layer & $-\cdot$ \\
\hline ICOK & -- & Switch for coking reaction & -- \\
\hline ICON & -- & Concrete composition index & -- \\
\hline ICOOL & -- & Coolant layer flag & $\cdots$ \\
\hline IDMF & -- & Diffusion mechanism flag for aerosol calculations & -. \\
\hline IFILMB & -. & Flag for gas film on pool bottom & $\cdots$ \\
\hline IFILMS & -- & Flag for gas film on pool sido & $\cdots$ \\
\hline IFLOR & -- & Index for cavity top ray & -. \\
\hline IFP & -. & Decay heat power index & -. \\
\hline IFVAN & -- & Switch for using the VANESA aerosol model & - \\
\hline IGAS & -- & Gas phase index & -- \\
\hline IGEOM & - & Cavity geometry index & - \\
\hline IHMX & -- & Index for heavy mixed layer & -- \\
\hline IHOX & -- & Index for heavy oxide layer & -- \\
\hline ILMX & - & Index for light mixed layer & $\cdots$ \\
\hline ILOX & -- & Index for light oxide layer & -- \\
\hline ILYR & -- & Melt layer configuration index & -- \\
\hline IMASTR (N) & -- & Master list of fission product groups & -- \\
\hline IMAXR & -- & Body point at maximum cavity radius & -- \\
\hline IMAXZ & -- & Body point at maximum cavity depth & -- \\
\hline IMET & -- & Index for metal layer & - \\
\hline IMIX & -- & Flag for calculating entrainment and deentrainment & -- \\
\hline IMOD (J) & -- & Heat transfer model number & - \\
\hline IMOV & -- & Cavity shape plot index & -- \\
\hline
\end{tabular}


Table 3.7 Dictionary of princlpal variables in CORCON (continued)

\begin{tabular}{|c|c|c|c|}
\hline $\begin{array}{l}\text { PORTRAN } \\
\text { Symbol }\end{array}$ & $\begin{array}{l}\text { Algebralc } \\
\text { Symbol }\end{array}$ & Description & Unite \\
\hline IMPF & .. & Impaction mechanism flag for werowol calculations & -. \\
\hline IOD & 1 & Quantity of iodine (1) in the fission product inventory & $8 \cdot \mathrm{mol}$ \\
\hline IPO & .- & Time plot index & -. \\
\hline IPINC & -. & Print increment, number of timestops & 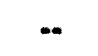 \\
\hline IPNT (I) & - & Position of Ith species in the CORCON mater liat & -• \\
\hline IR31 (L) & $\cdots$ & $\begin{array}{l}\text { Number of iterations required in MLTREA for } \\
\text { convergence }\end{array}$ & -. \\
\hline IRAD & .. & Flag for calls to TRADII (time-dopendent melt radius) & -- \\
\hline IRE & .- & Identifying number of chemical reaction & -. \\
\hline IRSTRT & .. & Restart option index & -. \\
\hline ISPNAM & - & CHARACTER $* 8$ name of muter-liat species & $\cdots$ \\
\hline ISRABL & -. & Atmosphere surroundings ablation index & -. \\
\hline IT & -. & Iteration (timestop) number & .. \\
\hline ITANO & -. & $\begin{array}{l}\text { Number of the body point constrained to move vertically } \\
\text { at the limit of a flat bottom }\end{array}$ & -- \\
\hline ITIMR & .- & Flag for time-dependent melt radius option & -. \\
\hline ITITL & -. & CHARACTER $* 80$ run identification & -. \\
\hline ITOPR & -. & Index of the top interfuce of the melt & $\cdots$ \\
\hline IUSER & .. & Flag enabling user flexibility options & - \\
\hline JBODY & -. & Index of body point & -- \\
\hline KATIS & -. & $\begin{array}{l}\text { Flag to signal use of Kataoka and Ishii, and Azbel } \\
\text { models for mechanical release of aerosols }\end{array}$ & -- \\
\hline KRY & -- & $\begin{array}{l}\text { Quantity of lanthanum (La), ytterbium }(\mathrm{Y}) \text {, } \\
\text { praseodymium }(\mathrm{Pr}) \text {, samarium }(\mathrm{Sm}) \text { and neodymium } \\
(\mathrm{Nd}) \text { in the fission product inventory }\end{array}$ & $8 \cdot \mathrm{mol}$ \\
\hline LA & La & $\begin{array}{l}\text { Quantity of lanthanum (La) in the fission product } \\
\text { inventory }\end{array}$ & $8-\mathrm{mol}$ \\
\hline LAYER (L) & $\cdots$ & Numbers (1-7) of occupied layers & $\cdots$ \\
\hline LYR & -. & Index of current melt layer & $\cdots$ \\
\hline MO & Mo & $\begin{array}{l}\text { Quantity of molybdenum (Mo) in the fission product } \\
\text { inventory }\end{array}$ & 8-mol \\
\hline NAMSP & -- & CHARACTER $* 8$ name of input species & -- \\
\hline NB & $\mathrm{Nb}$ & $\begin{array}{l}\text { Quantity of niobium }(\mathrm{Nb}) \text { in the fission product } \\
\text { inventory }\end{array}$ & g-mol \\
\hline
\end{tabular}


Table 5.7 Dictionary of principal variables in CORCON (continued)

\begin{tabular}{|c|c|c|c|}
\hline $\begin{array}{l}\text { PORTRAN } \\
\text { Symbol }\end{array}$ & $\begin{array}{l}\text { Algebralc } \\
\text { Symbol }\end{array}$ & Dewcription & Unitu \\
\hline NBO & Nbo & $\begin{array}{l}\text { Qunntity of niobium oxide (NbO) in the fission product } \\
\text { inventory }\end{array}$ & $8-\mathrm{mol}$ \\
\hline NBOT & $\cdots$ & $\begin{array}{l}\text { Number of body points on the flat bottom of cavity, } \\
\text { including the center line and tangency point }\end{array}$ & $\cdots$ \\
\hline NCL & -. & Master liat number of coolant species & - \\
\hline NCORN & $\cdots$ & $\begin{array}{l}\text { Number of body points defining a cavity comer, not } \\
\text { including tungency points }\end{array}$ & - \\
\hline ND & Nd & $\begin{array}{l}\text { Quentity of neodymium (Nd) in the fiesion product } \\
\text { inventory }\end{array}$ & 8-mol \\
\hline NDECM & -. & Number of points in metallic phase power input table & -. \\
\hline NDECO & 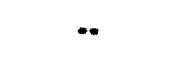 & Number of points in oxidic phase power input tuble & - \\
\hline NEM & 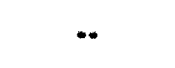 & Number of points in metullic phase emisaivity table & $\cdots$ \\
\hline NEO & -. & Number of points in metallic phase emisaivity table & $\cdots$ \\
\hline NFPEL & 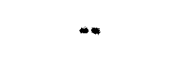 & Number of fission product elements & -- \\
\hline NOI & $\cdots$ & Location of first gas in master species list & - \\
\hline NO2 & $\cdots$ & Location of last gas in master apecies list & - \\
\hline NORP & $\because$ & Number of groups of fission product elements & 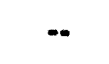 \\
\hline NINP & $-\cdot$ & Numher of concrete species input & $\cdots$ \\
\hline NLAY & $\cdots$ & $\begin{array}{l}\text { Total number of layers allowed (includes coolant and } \\
\text { atmosphere) }\end{array}$ & -- \\
\hline NLAYER & $\cdots$ & 'Total number of occupied layers & - \\
\hline NMASTR & $\cdots$ & $\begin{array}{l}\text { Maximum number of species containing a fission } \\
\text { product element }\end{array}$ & - \\
\hline NMP (I) & .. & Number of points in the input muss addition tables & $\cdots$ \\
\hline NMSI & 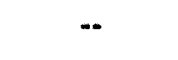 & Number of metallic species input & $\cdots$ \\
\hline NMI & $\cdots$ & Location of the first metal in the master species list & $\cdots$ \\
\hline NM2 & -. & Location of the last metal in the master species list & $\cdots$ \\
\hline NO3 & $\cdots$ & Location of the last oxide in the master species list & $\cdots$ \\
\hline NORAD & $\cdots$ & $\begin{array}{l}\text { Maximum number of entries in the table of times and } \\
\text { melt radii }\end{array}$ & - \\
\hline NOSC & $-\cdot$ & $\begin{array}{l}\text { Number of sections in the aerosol particle size } \\
\text { distribution }\end{array}$ & $\cdots$ \\
\hline NRAYS & $\cdots$ & Number of rays & $\cdots$ \\
\hline NS &.- & Number of points in surroundings emissivity table & $\cdots$ \\
\hline
\end{tabular}


Table S.7 Dictionary of principal variables in CORCON (continued)

\begin{tabular}{|c|c|c|c|}
\hline $\begin{array}{l}\text { FORTRAN } \\
\text { Symbol } \\
\end{array}$ & $\begin{array}{c}\text { Algebraic } \\
\text { Symbol }\end{array}$ & Description & Units \\
\hline NSIDE & -. & $\begin{array}{l}\text { Number of body points defining the side of the } \\
\text { cylindrical cavily }\end{array}$ & -- \\
\hline NSP1 & -. & Number of motallic species in the splachout tables & - \\
\hline NSP2 & $\cdots$ & Number of oxidic species in the splashout tables & $\cdots$ \\
\hline NSPO & - & Number of species in surroundinge & 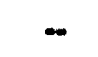 \\
\hline NSR (N) & - & Species number of epecies in fiesion product inventory & - \\
\hline NTP & $\cdots$ & Number of points in surroundinge temperature table & - \\
\hline NUMELE & $\cdots$ & Maximum number of olements & - \\
\hline NUMSPE & -. & Maximum number of chemical species & - \\
\hline OMEOA & 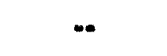 & Implicitness fraction $(-1)$ & - \\
\hline OXMOL & -- & Total quantity of oxides & g-mol \\
\hline$P(\mathbf{l}, \mathbf{K})$ & $\cdots$ & Partial pressure of apecies $K$ involving olement I & $\mathbf{P a}$ \\
\hline PA & .. & Initial gas pressure & Pa \\
\hline PAT (N) & -. & Gas (atmosphere) pressure & $\mathbf{P a}$ \\
\hline PATM & $\cdots$ & Atmospheric pressure & $\mathbf{P a}$ \\
\hline PD & Pd & $\begin{array}{l}\text { Quantity of palladium }(P d) \text { in the fission product } \\
\text { inventory }\end{array}$ & 8-mol \\
\hline PI & $\pi$ & $\mathrm{Pi}$ &.- \\
\hline PIM (N) & $\mathbf{P}_{\mathbf{m}}$ & Motallic phase input power & $\mathbf{W}$ \\
\hline PINT (L) & $\cdots$ & Interface pressure & $\mathbf{P a}$ \\
\hline $\operatorname{PIO}(\mathbf{N})$ & $\mathbf{P}$ & Oxidic phase input power & $\mathbf{W}$ \\
\hline PIO2 & $\pi / 2$ & $\mathrm{Pi} / 2$ & - \\
\hline PIO3 & $\pi / 3$ & $\mathrm{Pi} / 3$ & - \\
\hline PLAY (L) & $\mathbf{P}$ & Average pressure & $\mathbf{P a}$ \\
\hline PMETP & $\cdots$ & Volume of metallic phase & $\mathrm{cm}^{3}$ \\
\hline POXP & -- & Volume of oxidic phase & $\mathrm{cm}^{3}$ \\
\hline PR & $\operatorname{Pr}$ & $\begin{array}{l}\text { Quantity of praseodymium ( } \mathrm{Pr} \text { ) in the fission product } \\
\text { inventory }\end{array}$ & $8-\mathrm{mol}$ \\
\hline PRESS & $-\cdot$ & Ambient (atmospheric) pressure for aerosol calculations & atm \\
\hline PTBB & -- & Number of particles per bubble, for aerosol calculations & - \\
\hline PTDIA & -- & $\begin{array}{l}\text { Mean particle size for aerosol produced by mechanical } \\
\text { processes }\end{array}$ & $\mathbf{m}$ \\
\hline
\end{tabular}


Table 5.7 Dictionary of principal variables in CORCON (continued)

\begin{tabular}{|c|c|c|c|}
\hline $\begin{array}{l}\text { FORTRAN } \\
\text { Symbol }\end{array}$ & $\begin{array}{l}\text { Algebraic } \\
\text { Symbol }\end{array}$ & Description & Units \\
\hline PU & $\mathbf{P u}$ & $\begin{array}{l}\text { Quantity of plutonium }(\mathrm{Pu}) \text { in the fission product } \\
\text { inventory }\end{array}$ & 8-mol \\
\hline PVAR & -- & $\begin{array}{l}\text { Average pressure in the coolant pool for aerosol } \\
\text { calculations }\end{array}$ & atm \\
\hline QABL (L) & -- & Rate of change in enthalpy due to heat loss to concrete & $\mathbf{W}$ \\
\hline QCONV & qooson & Convective heat flux across gas film & $\mathbf{W} / \mathbf{m}^{2}$ \\
\hline QDCY (L) & -- & Internal heat source rate & $\mathbf{w}$ \\
\hline QINT (L) & -- & Interface heat flow rate (positive up) & $\mathbf{w}$ \\
\hline QMETP & -- & Molar density of metallic phase for aerosol calculations & $\mathrm{g}-\mathrm{mol} / \mathrm{cm}^{3}$ \\
\hline QOXP & - & Molar density of oxidic phase for aerosol calculations & $\mathrm{g}-\mathrm{mol} / \mathrm{cm}^{3}$ \\
\hline QPOOL (L) & -- & $\begin{array}{l}\text { Rate of change in enthalpy due to heat from adjacent } \\
\text { layers }\end{array}$ & $\mathbf{w}$ \\
\hline QRAD & $q_{\text {rad }}$ & Net radiative heat flux across the gas film & $\mathbf{W} / \mathbf{m}^{2}$ \\
\hline QREAC (L) & -- & Heat source rate from chemical reactions & w \\
\hline QSIDE (L) & $\mathbf{q}_{\mathbf{R}}$ & Radial heat flux & $\mathrm{W} / \mathrm{m}^{2}$ \\
\hline QSN & $Q^{n}$ & Surface heat flux at the start of the timestep & $\mathrm{W} / \mathrm{m}^{2}$ \\
\hline QSNP1 & $Q_{0}^{n+1}$ & Surface heat flux at the end of the timestep & $W / m^{2}$ \\
\hline QZEROR (L) & -- & $\begin{array}{l}\text { Radial heat flux to the gas film, extrapolated to a } \\
\text { surface temperature equal to the layer temperature }\end{array}$ & w \\
\hline QZEROZ (L) & -. & $\begin{array}{l}\text { Axial heat flux to the gas film, extrapolated to a surface } \\
\text { temperature equal to the layer temperature }\end{array}$ & $\mathbf{w}$ \\
\hline$R(J)$ & $\mathbf{r}$ & Radial coordinate & $\mathbf{m}$ \\
\hline RAD & -- & Concrete crucible radius, Figure 4.3 & $\mathbf{m}$ \\
\hline RADC & -- & Concrete crucible corner radius, Figure 4.3 & $\mathbf{m}$ \\
\hline RADLEN & $\mathbf{L}$ & Characteristic path length for aerosol opacity & $\mathbf{m}$ \\
\hline RADT & -- & Current melt radius (time-dependent melt radius) & $\mathbf{m}$ \\
\hline RADTIM (N) & - & $\begin{array}{l}\text { Melt radius at time TIMRAD (N) in the table of times } \\
\text { and radii (time-dependent melt radius) }\end{array}$ & $\mathbf{m}$ \\
\hline RB & $\mathbf{R b}$ & $\begin{array}{l}\text { Quantity of rubidium }(\mathrm{Rb}) \text { in the fission product } \\
\text { inventory }\end{array}$ & g-mol \\
\hline RBR & -- & Mass fraction of reinforcing steel in concrete & - \\
\hline
\end{tabular}


Table 5.7 Dictionary of principal variables in CORCON (continued)

\begin{tabular}{|c|c|c|c|}
\hline $\begin{array}{l}\text { FORTRAN } \\
\text { Symbol }\end{array}$ & $\begin{array}{l}\text { Algebraic } \\
\text { Symbol }\end{array}$ & Description & Units \\
\hline RDROP (L) & -- & Radius of entrained or deentrained drops & $\mathbf{m}$ \\
\hline RELERH & - & Relative numerical error in energy conservation & -- \\
\hline RELERM & - & Relative numerical error in mass conservation & -- \\
\hline REYNLD (J) & $\mathbf{R e}$ & Gas film Reynolds number & -- \\
\hline RH & $\mathbf{R h}$ & $\begin{array}{l}\text { Quantity of rhodium ( } R h \text { ) in the fission product } \\
\text { inventory }\end{array}$ & g-mol \\
\hline RHOC & $\rho_{\mathrm{c}}$ & Concrete density & $\mathbf{k g} / \mathbf{m}^{3}$ \\
\hline RHOLAY (L) & $\rho_{\mathrm{L}}$ & Average density & $\mathrm{kg} / \mathrm{m}^{3}$ \\
\hline RHOM (L) & -- & Density of metal phase & $\mathrm{kg} / \mathrm{m}^{3}$ \\
\hline RHOO (L) & -- & Density of oxide phase & $\mathrm{kg} / \mathrm{m} 3$ \\
\hline RHOPCT & -- & Partial density of concrete in reinforced concrete & $\mathbf{k g} / \mathbf{m}^{3}$ \\
\hline $\mathbf{R} \mathbf{I}$ & -- & Interface radius in EDIT; also used as R (J) elsewhere & $\mathbf{m}$ \\
\hline RINT (I) & -- & Interface radius & m \\
\hline RMAX & -- & Maximum radius of concrete cavity & $\mathbf{m}$ \\
\hline RNOT & $\mathbf{R}_{\text {o }}$ & Universal gas constant & $\mathrm{J} / \mathrm{g}-\mathrm{mol} \mathrm{K}$ \\
\hline RO & -- & Radial location of ray origin $(=0)$ & $\mathbf{m}$ \\
\hline RS & -- & Concrete crucible radius, Figure 4.2 & $\mathbf{m}$ \\
\hline $\operatorname{RSIZ}(\mathbf{N})$ & -- & Characteristic aerosol particle size in range $\mathbf{N}$ & $\mu \mathbf{m}$ \\
\hline RTANG & -- & Radius of flat bottom of concrete crucible & $\mathbf{m}$ \\
\hline RTM & -- & $\begin{array}{l}\text { Bubble rise time in the metal phase for aerosol } \\
\text { calculations }\end{array}$ & $\mathbf{s}$ \\
\hline RTO & -- & $\begin{array}{l}\text { Bubble rise time in the oxide phase for aerosol } \\
\text { calculations }\end{array}$ & $s$ \\
\hline RU & $\mathbf{R u}$ & $\begin{array}{l}\text { Quantity of ruthenium }(\mathrm{Ru}) \text { in the fission product } \\
\text { inventory }\end{array}$ & $\mathrm{g}-\mathrm{mol}$ \\
\hline RVM & - & $\begin{array}{l}\text { Bubble rise velocity in the metal phase for aerosol } \\
\text { calculations }\end{array}$ & $\mathrm{cm} / \mathrm{s}$ \\
\hline RVO & -- & $\begin{array}{l}\text { Bubble rise velocity in the oxide phase for aerosol } \\
\text { calculations }\end{array}$ & $\mathrm{cm} / \mathrm{s}$ \\
\hline RW & -- & Outer radius of concrete crucible, Figures $4.2-4.4$ & m \\
\hline SAREA & - & Surface area of the cavity & $\mathbf{m}^{3}$ \\
\hline SB & $\mathbf{S b}$ & $\begin{array}{l}\text { Quantity of antimony (Sb) in the fission product } \\
\text { inventory }\end{array}$ & g-mol \\
\hline
\end{tabular}


Table 5.7 Dictionary of principal variables in CORCON (continued)

\begin{tabular}{|c|c|c|c|}
\hline $\begin{array}{l}\text { FORTRAN } \\
\text { Symbol }\end{array}$ & $\begin{array}{l}\text { Algebraic } \\
\text { Symbol }\end{array}$ & Description & Units \\
\hline SCALRG & -- & Free energy divided by $R_{o} T$ for chemical system & - \\
\hline SCALRM & -- & Total moles in gas phase of chemical system & g-mol \\
\hline SDOT (J) & $\boldsymbol{s}$ & Concrete recession rate & $\mathbf{m} / \mathbf{s}$ \\
\hline SI & - & Mass fraction of concrete decomposing into gas; silicon & -- \\
\hline SIGEFF & $\sigma_{\mathrm{B}} \mathrm{F}$ & Stefan-Boltzmann constant times shape factor & $W / m^{2}-K^{4}$ \\
\hline SIGLAY (L) & $\sigma_{\mathrm{L}}$ & Surface tension & $\mathbf{N} / \mathbf{m}$ \\
\hline $\operatorname{SIGM}(L)$ & -- & Surface tension for metal phase & $\mathbf{N} / \mathbf{m}$ \\
\hline SIGMA & $\sigma_{\mathrm{B}}$ & Stefan-Boltzmann constant & $\mathrm{W} / \mathrm{m}^{2}-\mathrm{K}^{4}$ \\
\hline SIGO (L) & - & Surface tension for oxide phase & $\mathbf{N} / \mathbf{m}$ \\
\hline SIZ & -- & Aerosol particle size & $\mu \mathrm{m}$ \\
\hline SM & $\overline{--}$ & $\begin{array}{l}\text { Mass fraction of concrete species; mole fraction of } \\
\text { gaseous species; quantity of samarium }(\mathrm{Sm}) \text { in the } \\
\text { fission product inventory }\end{array}$ & $\begin{array}{c}-- \\
-- \\
\text { g-mol }\end{array}$ \\
\hline SMCTRN & -- & Mass of coolant species added during timestep & kg \\
\hline SMETP & -- & Total quantity of metal phase in aerosol calculations & g-mol \\
\hline SMGATM (I) & -- & Mass of gas in atmosphere & kg \\
\hline SMGFLM (I) & -- & $\begin{array}{l}\text { Mass of gas transported in the gas film during the } \\
\text { timestep }\end{array}$ & kg \\
\hline SMGGEN (I) & -- & Cumulative mass of gases species generated & kg \\
\hline SMGSAV (I) & -- & $\begin{array}{l}\text { Mass of gas transformed by oxide/atmosphere reaction } \\
\text { during timestep }\end{array}$ & kg \\
\hline SMGTRN (I) & -- & Mass of gas transported as bubbles during timestep & kg \\
\hline SMMET $(I, L)$ & -- & Mass of metallic species & kg \\
\hline SUMMHO & -- & $\begin{array}{l}\text { Total enthalpy of new pool mass for old temperatures } \\
\text { and melting ranges }\end{array}$ & $\mathbf{J}$ \\
\hline SMMSAV (I) & -- & $\begin{array}{l}\text { Mass of metallic products of oxide/atmosphere reaction } \\
\text { during timestep }\end{array}$ & kg \\
\hline SMMTRN (I) & -- & $\begin{array}{l}\text { Mass of metal rising or sinking in the pool during } \\
\text { timestep }\end{array}$ & kg \\
\hline SMOTRN (I) & -- & $\begin{array}{l}\text { Mass of oxide rising or sinking in the pool during the } \\
\text { timestep }\end{array}$ & $\mathbf{k g}$ \\
\hline SMOXY (I,L) & -- & Mass of oxidic species & kg \\
\hline
\end{tabular}


Table 5.7 Dictionary of principal variables in CORCON (continued)

\begin{tabular}{|c|c|c|c|}
\hline $\begin{array}{l}\text { FORTRAN } \\
\text { Symbol }\end{array}$ & $\begin{array}{c}\text { Algebraic } \\
\text { Symbol }\end{array}$ & Description & Units \\
\hline SN & Sn & Quantity of tin (Sn) in the fission product inventory & g-mol \\
\hline SOXP & - & Total quantity of oxide phase in aerosol calculations & 8-mol \\
\hline SPEMW (I) & -- & Molecular weight & 8/8-mol \\
\hline SR & Sr & $\begin{array}{l}\text { Quantity of strontium (Sr) in the fission product } \\
\text { inventory }\end{array}$ & 8-mol \\
\hline ST2 & -- & Timestep in aerosol calculations & $\mathbf{8}$ \\
\hline STM & -- & $\begin{array}{l}\text { Surface tension of the metal phase in aerosol } \\
\text { calculations }\end{array}$ & dyne/cm \\
\hline sTo & -- & $\begin{array}{l}\text { Surface tension of the oxide phase in aerosol } \\
\text { calculations }\end{array}$ & dyne/cm \\
\hline $\operatorname{SXMP}(I, K)$ & -- & Cumulative quantity of chemical species & 8-mol \\
\hline TA & $T_{.}$ & Initial gas (atmosphere) temperature & $\mathbf{K}$ \\
\hline TC & Tc & $\begin{array}{l}\text { Quantity of technetium ( } \mathrm{Tc} \text { ) in the fission product } \\
\text { inventory }\end{array}$ & 8-mol \\
\hline TCI & -- & Initial coolant temperature & $\mathbf{K}$ \\
\hline TCTR & -- & Temperature of coolant added & $\mathbf{K}$ \\
\hline TE & $\mathrm{Te}$ & $\begin{array}{l}\text { Quantity of tellurium (Te) in the fission product } \\
\text { inventory }\end{array}$ & g-mol \\
\hline TEDIT & - & Time when next edit will be generated & 8 \\
\hline TEMP & -- & $\begin{array}{l}\text { Average coolant pool temperature used in aerosol } \\
\text { calculations }\end{array}$ & $\mathbf{K}$ \\
\hline TEMPA (J) & - & Temperature of pool/film interface at $Z(J)$ & $\mathbf{K}$ \\
\hline TEMPX & $-\infty$ & $\begin{array}{l}\text { Flag for emissivity table for } \times(=M, \text { metal; }=0 \text {, } \\
\text { oxide; = S, surroundings }) ; \text { true if independent variable } \\
\text { is temperature rater than time }\end{array}$ & $\mathbf{K}$ \\
\hline TGFL & -- & Temperature of gas transported through the film & $\mathbf{K}$ \\
\hline TGSV &.- & $\begin{array}{l}\text { Temperature of gas transformed by the } \\
\text { oxide/atmosphere reaction }\end{array}$ & $\mathbf{K}$ \\
\hline TGTR & -- & Temperature of gas transported as bubbles & $\mathbf{K}$ \\
\hline THET (J) & -- & Ray angle & rad \\
\hline THKLAY (L) & $\mathbf{k}_{\mathbf{L}}$ & Thermal conductivity & $\mathbf{W} / \mathbf{m}-\mathbf{K}$ \\
\hline THKM (L) & $\mathbf{k}_{\mathrm{LM}}$ & Thermal conductivity of metal phase & $\mathbf{W} / \mathbf{m}-\mathbf{K}$ \\
\hline THKO (L) & $k_{\text {LO }}$ & Thermal conductivity of oxide phase & $\mathbf{W} / \mathbf{m}-\mathbf{K}$ \\
\hline TIC & -- & Initial concrete temperature & $\mathbf{K}$ \\
\hline
\end{tabular}


Table 5.7 Dictionary of principal variables in CORCON (continued)

\begin{tabular}{|c|c|c|c|}
\hline $\begin{array}{l}\text { FORTRAN } \\
\text { Symbol }\end{array}$ & $\begin{array}{l}\text { Algebraic } \\
\text { Symbol }\end{array}$ & Description & Units \\
\hline $\operatorname{TIM}(\mathbf{N})$ & - & Times in metal phase input power table & $\mathbf{s}$ \\
\hline TIME & $\mathbf{t}$ & Current time & $\mathbf{s}$ \\
\hline TIMECR & - & $\begin{array}{l}\text { Time at which the layer crusts are thick enough to stop } \\
\text { melt spreading (time-dependent melt radius) }\end{array}$ & $\mathbf{s}$ \\
\hline TIMEND & - & End time for calculation & 8 \\
\hline TIMEO & - & Start time for calculation & $\mathbf{s}$ \\
\hline TIMESD & - & $\begin{array}{l}\text { Time at which the spreading melt layer contacts the side } \\
\text { of the concrete cavity }\end{array}$ & $\mathbf{8}$ \\
\hline TIMRAD (N) & - & $\begin{array}{l}\text { Time at which the melt has radius RADTIM }(\mathrm{N}) \text { in the } \\
\text { table of times and radii (time-dependent melt radius) }\end{array}$ & $\mathbf{s}$ \\
\hline TINT (L) & $T_{1}$ & Interface temperature & $\mathbf{K}$ \\
\hline TIO (N) & -- & Times in oxide phase input power table & $\mathbf{s}$ \\
\hline TLAY (L) & $T_{L}$ & Average (bulk) temperature & $\mathbf{K}$ \\
\hline TLCNTR (L) & $\overline{\mathrm{T}}_{\text {tr }}$ & Average liquid temperature, radial & $\mathbf{K}$ \\
\hline TLCNTZ (L) & $\overline{\mathrm{T}}_{\mathrm{tz}}$ & Average liquid temperature, axial & $\mathbf{K}$ \\
\hline TLIQCT & $\mathrm{T}_{\mathrm{c}}^{\ell}$ & Concrete liquidus temperature & $\mathbf{K}$ \\
\hline TLIQLA (L) & $\mathrm{T}_{\mathrm{L}}^{\prime}$ & Liquidus temperature & $\mathbf{K}$ \\
\hline TLIQM (L) & -- & Metal phase liquidus & $\mathbf{K}$ \\
\hline TLIQO (L) & -- & Oxide phase liquidus & $\mathbf{K}$ \\
\hline TMET & -- & $\begin{array}{l}\text { Mass -averaged metal phase temperature used in aerosol } \\
\text { calculations }\end{array}$ & $\mathbf{K}$ \\
\hline TMI & -- & Initial temperature of metallic phase & $\mathbf{K}$ \\
\hline TMM (N) & -- & Times for metallic phase splashout table & $\mathbf{s}$ \\
\hline TMO (N) & - & Times for metallic phase splashout table & $\mathbf{s}$ \\
\hline TMPS (N) & - & Temperature of surroundings & $\mathbf{K}$ \\
\hline TMS $(\mathbf{N}, \mathbf{I})$ & -- & Time in mass addition table & $\mathbf{8}$ \\
\hline TMSV & -- & $\begin{array}{l}\text { Temperature of metallic products of oxide/atmosphere } \\
\text { reaction }\end{array}$ & $\mathbf{K}$ \\
\hline TMTR & -- & Temperature of metal rising of sinking in the pool & $\mathbf{K}$ \\
\hline TOI & -- & Initial temperature of the oxidic phase & $\mathbf{K}$ \\
\hline TORT1 $(\mathbf{M}$ or $\mathbf{N})$ & -- & Time or temperature for oxidic phase emissivity table & $\mathrm{s}$ or $\mathrm{K}$ \\
\hline
\end{tabular}


Table 5.7 Dictionary of principal variables in CORCON (continued)

\begin{tabular}{|c|c|c|c|}
\hline $\begin{array}{l}\text { FORTRAN } \\
\text { Symbol }\end{array}$ & $\begin{array}{l}\text { Algebraic } \\
\text { Symbol }\end{array}$ & Description & Units \\
\hline TORT2 (M or $N)$ & - & Time or temperature for metallic phase emissivity table & $\mathbf{s}$ or $\mathrm{K}$ \\
\hline TORT6 (M or is) & -- & Time or temperature for surroundings emissivity table & $\mathbf{s}$ or $\mathrm{K}$ \\
\hline TOTALH & - & Total pool enthalpy required by energy conservation & $\mathbf{J}$ \\
\hline TOTALM & - & Total pool mass required by mass conservation & kg \\
\hline TOTR & - & Temperature of oxide rising or sinking in the pool & $\mathbf{K}$ \\
\hline TOXD & -- & $\begin{array}{l}\text { Mass-averaged oxide phase temperature used in aerosol } \\
\text { calculations }\end{array}$ & $\mathbf{K}$ \\
\hline TPA (N) & - & Time in gas (atmosphere) pressure table & $\mathbf{s}$ \\
\hline TPRIN & - & Time for start of diagnostic print & $\mathbf{s}$ \\
\hline \multicolumn{4}{|l|}{ TSAT } \\
\hline TSIDE (L) & -- & Side (radial) boundary temperature & $\mathbf{K}$ \\
\hline TSN & $\mathbf{T}$ & Surface temperature at the start of the timestep & $\mathbf{K}$ \\
\hline TSNP1 & $\mathbf{T}$ & Surface temperature at the end of the timestep & $\mathbf{K}$ \\
\hline TSOLCT & $\mathrm{T}_{\mathrm{c}}^{*}$ & Concrete solidus temperature & $\mathbf{K}$ \\
\hline TSOLID (L) & -- & Solidification temperature & $\mathbf{K}$ \\
\hline TSOLLA (L) & $T_{L}^{s}$ & Solidus temperature & $\mathbf{K}$ \\
\hline TSOLM (L) & -- & Metal phase solidus & $\mathbf{K}$ \\
\hline TSOLO (L) & -- & Oxide phase solidus & $\mathbf{K}$ \\
\hline TSTART (L) & -- & Temperature at the start of a timestep & $\mathbf{K}$ \\
\hline TTA & -- & Current layer temperature & $\mathbf{K}$ \\
\hline TTS $(\mathbf{N})$ & -- & Time in surroundings temperature table & $\mathbf{s}$ \\
\hline TW & $\mathrm{T}_{\mathbf{w}}$ & Concrete ablation temperature & $\mathbf{K}$ \\
\hline UBUB (L) & $\mathrm{U}_{\mathrm{z}}$ & Bubble rise velocity & $\mathbf{m} / \mathbf{s}$ \\
\hline URO & -- & Unit round off error & -- \\
\hline VA & -- & Initial gas volume & $\mathbf{m}^{3}$ \\
\hline VAPOR & -- & $\begin{array}{l}\text { The concentration of aerosol particles generated by } \\
\text { vaporization }\end{array}$ & - \\
\hline VISLAY (L) & - & Dynamic viscosity & $\mathrm{kg} / \mathrm{m}-\mathrm{s}$ \\
\hline VISLIQ (L) & $\mu_{8}$ & Dynamic viscosity of liquid & $\mathrm{kg} / \mathrm{m}-\mathrm{s}$ \\
\hline VISM (L) & - & Dynamic viscosity of the metal phase & $\mathrm{kg} / \mathrm{m}-\mathrm{s}$ \\
\hline VISMET & -- & $\begin{array}{l}\text { Mass-averaged dynamic viscosity of the metal phase, } \\
\text { used in aerosol calculations }\end{array}$ & $\mathrm{g} / \mathrm{cm}-\mathrm{s}$ \\
\hline
\end{tabular}


Table 5.7 Dictionary of principal variables in CORCON (continued)

\begin{tabular}{|c|c|c|c|}
\hline $\begin{array}{l}\text { FORTRAN } \\
\text { Symbol }\end{array}$ & $\begin{array}{l}\text { Algebraic } \\
\text { Symbol }\end{array}$ & Description & Units \\
\hline VISO (L) & -- & Dynamic viscosity of the oxide phase & $\mathrm{kg} / \mathrm{m}-\mathrm{s}$ \\
\hline VISOXD & -- & $\begin{array}{l}\text { Mass-averaged dynamic viscosity of the oxide phase, } \\
\text { used in aerosol calculations }\end{array}$ & $\mathrm{g} / \mathrm{cm}-\mathrm{s}$ \\
\hline VMET & -- & $\begin{array}{l}\text { Superficial gas velocity through the metal, used in } \\
\text { aerosol calculations }\end{array}$ & $\mathrm{cm} / \mathrm{s}$ \\
\hline VOL (J) & - & Cumulative cavity volume & $\mathbf{m}^{3}$ \\
\hline VOLM (L) & -- & Volume of the metal phase in entrainment calculations & $\mathbf{m}^{3}$ \\
\hline VOLO (L) & -- & Volume of the oxide phase in entrainment calculations & $\mathbf{m}^{3}$ \\
\hline VOLRAT (l) & -- & Exponential volatization rate of fission products & $\mathbf{s}^{-1}$ \\
\hline VOXD & -- & $\begin{array}{l}\text { Superficial gas velocity through the oxide, used in } \\
\text { aerosol calculations }\end{array}$ & $\mathrm{cm} / \mathrm{s}$ \\
\hline VROVR & -- & Velocity ratio for aerosol calculations & -- \\
\hline VSGINT (L) & $\mathrm{V}_{\mathrm{s}}$ & Superficial gas velocity & $\mathbf{m} / \mathbf{s}$ \\
\hline WF (I) & -- & Weight fraction of concrete constituents & -- \\
\hline WFRBR & -- & Weight fraction of rebar in concrete & -- \\
\hline$X(J)$ & -- & Path length in the film & m \\
\hline XEN & -- & $\begin{array}{l}\text { Quantity of ruthenium }(\mathrm{Ru}), \text { technetium }(\mathrm{Tc}) \text {, rhodium } \\
(\mathrm{Rh}) \text {, and palladium }(\mathrm{Pd}) \text { in the fission product } \\
\text { inventory }\end{array}$ & g-mol \\
\hline $\mathrm{XL}(\mathrm{I}, \mathrm{K})$ & -- & Quantity of fission product inventory species & kg \\
\hline $\mathbf{X M}(\mathbf{I}, \mathbf{K})$ & -- & Quantity of fission product inventory species & g-mol \\
\hline XMAS & -- & Aerosol generation rate & $g / s$ \\
\hline XMTU & -- & Core size, metric tonnes of uranium & $\mathbf{M g}$ \\
\hline XMWTH & -- & Core operating power (thermal) & MW \\
\hline $\mathrm{XN}(\mathrm{I}, \mathrm{K})$ & -- & $\begin{array}{l}\text { Array containing the molecular weights for species } \mathrm{K} \text { of } \\
\text { element I }\end{array}$ & $\mathrm{g} / \mathrm{g}-\mathrm{mol}$ \\
\hline $\mathbf{Y}$ & Y & $\begin{array}{l}\text { Quantity of ytterbium }(Y) \text { in the fission product } \\
\text { inventory }\end{array}$ & g-mol \\
\hline YKG $(I, N)$ & -- & Array containing the quantities of fission product in $\mathbf{~ k g}$ & kg \\
\hline$z(j)$ & z & Axial coordinate (positive down) & $\mathbf{m}$ \\
\hline ZB & -- & Axial coordinate of the bottom of the concrete crucible & $\mathbf{m}$ \\
\hline ZINT (L) & -- & Interface location & $\mathbf{m}$ \\
\hline
\end{tabular}


Table 5.7 Dictionary of principal variables in CORCON (continued)

\begin{tabular}{lclc}
\hline $\begin{array}{l}\text { FORTRAN } \\
\text { Symbol }\end{array}$ & $\begin{array}{c}\text { Algebraic } \\
\text { Symbol }\end{array}$ & \multicolumn{1}{c}{ Description } & Units \\
\hline ZMAX & -- & Axial coordinate of deepest point in the cavity & $\mathrm{m}$ \\
$\mathrm{ZO}$ & -- & Axial coordinate of ray origin & $\mathrm{m}$ \\
$\mathrm{ZT}$ & -- & $\begin{array}{l}\text { Axial coordinate of the top of the concrete crucible, } \\
\text { Figure 4.3 }\end{array}$ & $\mathrm{m}$ \\
\hline
\end{tabular}




\subsection{Sample Problems}

To illustrate the use of CORCON-Mod3, and to verify the correct functioning of the code following its installation, we have provided a set of sample problems. The input decks for these samples should provide valuable help to the new user in assembling correct input decks. The sample problems also demonstrate the capabilities of the code.

No one problem utilizes all the capabilities of CORCONMod3. The CORCON standard problem exercises the more commonly used capabilities of CORCON; the additional sample problem exercises some of the newer features in the code.

\subsection{The CORCON Standard Problem}

The CORCON standard problem describes deposition of essentially the entire core of a typical large $(3400 \mathrm{MWt})$ PWR into a reactor cavity formed of limestone aggregate/common sand concrete. Deposition takes place three hours after reactor SCRAM, at which time the zirconium cladding is assumed to be approximately 50 percent oxidized. The melt also includes a significant amount of steel, from the upper internals and the breached lower head; the steel is assumed to be $\mathbf{5}$ percent oxidized. The initial water inventory of 60 metric tonnes represents the contents of the accumulators of a typical PWR. The cavity radius is taken as $\mathbf{3 . 0}$ meters, and does not include the area of the keyway in a plant such as Zion or Indian Point. Containment pressure is assumed to rise linearly from 1.5 bars at deposition time to 3.0 bars 3 hours later. The effects of aerosol opacity are included with the characteristic path length taken as a nominal $1.0 \mathrm{~m}$.

This problem does not model any particular plant or accident sequence. It is intended merely to provide a typical problem to exercise the basic capabilities of CORCON.

The calculation assumes that the core melt is initially stratified. It remains stratified because the interlayer mixing models are not activated (ILYR $<10$ ).

Condensed phase chemistry is not calculated and the coking reaction is disabled (ICHEM $=0)$. Slag heat transfer is assumed at the bottom surface, while gas film heat transfer is assumed at the side surface (IFILM =1). A full VANESA calculation is performed (IFVAN $=1$ ), and the VANESA fission product composition is determined from the CORCON composition (IVANFP = 0 ). The effects of aerosol in the cavity atmosphere is included in the calculation of radiative heat transfer $($ IAOPAC $=1)$.
The release calculation is performed assuming ideal chemistry $($ IDEAL $=1)$.

The data cards used for this problem are shown in Table 6.1. This input follows the input description of Section 4.1.

A partial output listing for time 16200 seconds $(270 \mathrm{~min}$ ) for this calculation is shown in Table 6.2. Plots of the output are shown in Figures 6.1 through 6.9.

In the Standard Problem calculation, the melt remains stratified in a three layer configuration with the heavy oxide layer on the bottom, a metal layer in the middle, and a light oxide on the top. Unlike the calculations with earlier versions of CORCON, no layer flip is calculated during the three hours of the interaction.

Because the heavy oxide layer remains on the bottom of the melt, axial (downward) ablation is rather slow. This is true because an oxide crust exists at the interface with the concrete. The crust remains throughout the calculation and grows to a quasi-steady thickness of approximately 4 centimeters. As the crust grows, the ablation rate decreases to a nearly constant value of 2 $\mathrm{cm} / \mathrm{hr}$.

Figure 6.2 shows the maximum axial and radial ablation distances calculated by the code. Note that the hot metallic layer ablates concrete at a fairly rapid rate during the first hour of the calculation. After about one hour, the lower oxide layer has grown such that the maximum radial ablation location is adjacent to the oxide layer rather than the metal layer. This is true until 80 minutes later. At this time, the maximum radial ablation location is adjacent to the metal phase, and the maximum radial ablation distance again increases rapidly.

The calculated layer temperatures are shown in Figure 6.3. The three layer temperatures decrease during the first 100 minutes of the interaction. At approximately, 110 minutes after the start of the interaction, the overlying coolant pool boils off, and the layers begin to increase in temperature. By the end of the calculation, the layers have reached a new quasi-steady temperature levels of between 2100 and $2200 \mathrm{~K}$.

Figures 6.4 and 6.5 show the cumulative amount of gas entering the cavity atmosphere, and the rate of gas generation. As expected, the early gas generation is dominated by the steam from coolant boiling. (Note that the steam production is initially low because the coolant pool is subcooled. When the coolant is subcooled, steam is assumed to condense before reaching the pool surface.) Boiloff of the coolant is shown clearly in Figure 6.5 at 
about 295 minutes, where the steam generation rate abruptly decreases.

Figure 6.6 shows the calculated rates of energy generation and energy loss. Energy generation occurs by ratinnuclide decay and by chemical reactions. Energy losses include heat losses to the concrete and to the overlying wai. pool, atmosphere, and structures. In adt:won, energy lost from the melt in heating the incoming ablation products is included. This term is usually small, as is the case here. In cases where the ablation rate is extremely rapid, this term is larger.

Heat transfer to the coolant is initially quite high. During the first 15 minutes of the interaction, the coolant heat flux is greater than $1 \mathrm{MW} / \mathrm{m}^{2}$ over the approximately $32 \mathrm{~m}^{2}$ surface area. After 110 minutes, however, the coolant heat flux has decreased to $0.5 \mathrm{MW} / \mathrm{m}^{2}$. Boiloff of the coolant occurs at this time, and the surface heat flux drops rapidly. Late in the calculation, the heat flux due to thermal radiation is approximately $0.3 \mathrm{MW} / \mathrm{m}^{2}$.

In this sample calculation, energy generation from chemical reactions is much smaller than that from radionuclide decay. This is true because the ablation rate and consequently gas generation rates are relatively low. In calculations with higher ablation rates, the energy generation from chemical reactions can greatly exceed the contribution from radionuclide decay.

Figures 6.7 through 6.9 show the calculated aerosol concentration and aerosol generation rate for the CORCON Standard Problem. The general trend of the aerosul concentration and aerosol generation curves follows that of the gas generation and melt temperature curves. The aerosol concentration is greatest early in the calculation, with a maximum value of approximately 200 $\mathrm{g} / \mathrm{m}^{3}$ at STP, but declines rapidly to a quasi-steady level of approximately 30 to $40 \mathrm{~g} / \mathrm{m}^{3}$. Similarly, the aerosol generation rate is highest at the onset of the interaction (at $>150 \mathrm{~g} / \mathrm{s}$ ), but quickly reaches a nearly constant value of $20 \mathrm{~g} / \mathrm{s}$.

Another important safety consideration is the magnitude of radionuclide release from the core debris. The calculated release fractions for several of the important refractory radionuclides are shown below:

\begin{tabular}{|c|c|}
\hline Radionuclide & $\begin{array}{l}\text { Release } \\
\text { Fraction }\end{array}$ \\
\hline Tellurium & 0.399 \\
\hline Molybdenum & $1.30 \times 10^{6}$ \\
\hline Uranium & $5.24 \times 10^{5}$ \\
\hline Barium & $3.49 \times 10^{3}$ \\
\hline Strontium & $4.34 \times 10^{3}$ \\
\hline Cerium & $1.13 \times 10^{-3}$ \\
\hline Lanthanum & $8.53 \times 10^{5}$ \\
\hline
\end{tabular}

It should be noted that these releases are for only the first three hours of the interaction. Though the bulk of the release occurs during this period, radionuclides will continue to be released for several hours. It should also be noted that these are releases from the walt surface rather than into the cavity atmosphere. A significant fraction of the released radionuclides is trapped within the overlying water pool. Currently, this material is effectively lost from the problem once it becomes trapped. It is not returned to the core debris if the water pool evaporates completely. Also, the code does not treat decay power generation in the water pool from the trapped radionuclides.

\subsection{Additional Sample Problem}

A second sample problem is provided to illustrate some of the new features of CORCON-Modi3. This problem simulates an accident at a typical BWR plunt. The coreconcrete interaction is assumed to begin three hours after reactor shutdown. Initially, approximately one third of the core is deposited into the reactor cavity. The zirconium included in the initial pour is assumed to the 20 percent oxidized. During the next 3 hours of the calculation, the second third of the core is assumed to enter the cavity. The reactor cavity is assumed to be constructed from basaltic concrete and is assumed to have a diameter of 3.2 meters. Water is added after one hour of the interaction. The water addition rate of $20 \mathrm{~kg} / \mathrm{s}$ (approximately $300 \mathrm{gal} / \mathrm{min}$ ) is sufficicnt to fill the 5 meter deep cavity in approximately 2 hours. The pressure in the cavity atmosphere is assumed to rise from 1.5 bars to 2.5 bars during the 3 hours of the calculation.

The core melt is assumed to be wcll-rixed nitially, and the interlayer mixing models are enabled (ILYK $=13)$. Condensed phase chemistry is 
calculated and coking is disabled (ICHEM = 1). Slag film heat transfer is assumed at both the bottom and side surfaces (IFILM $=0)$. A complete VANESA calculation is performed (IFVAN $=1$ ), and the VANESA fission product composition is determined from the CORCON composition (IVANFP $=0$ ). The release calculation is performed assuming ideal chemistry (IDEAL $=1$ ). The effects of aerosols on thermal radiation is included (IAOPAC $=1$ ), and a mean path length of 3.0 meters is assumed. The optional user flexibility input is provided (IUSER $=1$ ), with the following user modifications specified:

1) the thermal conductivity for the metal phase is multiplied by 0.5 ,

2) the viscosity of the oxide phase is multiplied by 5.0,

3) the oxide phase solidus temperature is assumed to decrease to the concrete solidus at 20 mole percent concrete oxides.

The input for this sample problem is shown in Table 6.3. A partial listing of the output for time $=16200 \mathrm{~s}$ (270 $\mathrm{min}$ ) from the calculation is provided in Table 6.4. of the calculated results are provided in Figures 6.10 through 6.18 .

The melt is assumed to be well-mixed at the start of the interaction. After 10 minutes, the melt is calculated to be a dense oxide layer and a mixture of dense oxides and metal. In this sample calculation, a mixture of dense oxides and metals is calculated to form (i.e., an HMX layer). (The oxides are denser than the metals because fuel oxides are being continuously added to the melt.) Immediately, this layer begins to stratify, with the dense oxides settling out of the mixture. The evolution of the layering configuration is shown clearly in the layer temperature plot of Figure 6.12.

Figures 6.10 and 6.11 show the calculated axial ablation rate, and axial and radial ablation distances for the BWR sample problem. Because the heavy oxide layer is on the bottom of the melt during most of the calculation, the ablation rate is slow due to the existence of a oxide crust at the interface with the concrete. The spike in ablation rate seen at 9 minutes arises from the changes in layer configuration occurring at this time. The crust reaches a quasi-steady thickness of $2.5 \mathrm{~cm}$ prior to water addition. After water addition, the crust is calculated to shrink as concrete oxides mix with the fuel oxides lowering the mixture solidus temperature. By the end of the calculation the crust thickness is $0.2 \mathrm{~cm}$. Thinning of the crust is not accompanied by an increase in the ablation rate because the oxide temperature has fallen to $1865 \mathrm{~K}$.

Figure 6.12 shows the rapid decline in the melt temperature during the first hour of the calculation. At this point, water is added onto the melt. After water addition, the melt temperature decreases at a slightly faster rate. Approximately 100 minutes after water addition, energy generation from radionuclide decay and chemical reactions exceeds losses to the coolant and the concrete, and the temperature of the melt begins to increase slightly.

Figures 6.13 and 6.14 show the cumulative gas generation and the gas generation rate calculated for the BWR sample problem. Production of $\mathrm{H}_{2} \mathrm{O}$ and $\mathrm{CO}_{2}$ is suppressed. The code predicts that gas reactions with the HMX mixture produce methane $\left(\mathrm{CH}_{4}\right)$ and other hydrocarbons at the expense of steam and carbon dioxide production. The steam release seen at 270 minutes is, of course, the steam produced by boiling of the coolant.

The calculated energy generation and energy loss terms are shown in Figure 6.15. The decay power is calculated to increase from $7 \mathrm{MW}$ to $11 \mathrm{MW}$ as core debris enters the cavity. Energy generation from chemical reactions is comparable to the decay power during the first hour of the calculation. The spike in chemical energy generation at 8 to 10 minutes occurs when the core debris becomes fully mixed and all of the oxides, metals, and gases are suddenly assumed to react chemically. When water is added at 240 minutes, heat loss through the melt surface quickly increases to $20 \mathrm{MW}\left(0.65 \mathrm{MW} / \mathrm{m}^{2}\right)$, and then declines to a level that almost exactly balances the decay power.

Figures 6.16 through 6.18 show the calculated aerosol concentration and aerosol generation rate for the BWR sample problem. The high concentration and generation rates seen during the first 10 minutes of interaction arise from the relatively high melt temperatures and the gas generation rate spikes seen in Figures 6.12 and 6.14 respectively. The gas generation spike occurred during the rapid melt layer configuration changes. This could also influence the chemistry driving release. After the first 10 minutes the high hydrogen to steam ratio (i.e., low oxygen potential) in the gas stream promotes the reduction of refractory oxides to more volatile species. This enhances the release of both oxides from the concrete such as silica and fission product oxides such as $\mathrm{BaO}$ and $\mathrm{La}_{2} \mathrm{O}_{3}$. The aerosol generation rate and concentration increase to about $200 \mathrm{~g} / \mathrm{s}$ and $250 \mathrm{~g} / \mathrm{m}^{3}$ (STP). At water addition at 240 minutes, the aerosol release shows a distinct drop corresponding to the melt temperature decrease. The increasing generation rate and 
concentration during the last 1 1/2 hours of the concentration can be attributed to the slight increase in melt temperature and to the release of concrete oxides such as $\mathrm{NaO}_{2}$ and $\mathrm{KO}_{2}$, which comprise nearly $3 / 4$ of the aerosol mass at the end of the calculation.

The following are the release fractions calculated for some of the more important radionuclides:

\begin{tabular}{lll} 
Badionuclide & $\begin{array}{l}\text { Release } \\
\text { Eraction }\end{array}$ & $\begin{array}{l}\text { New Result } \\
(2.28)\end{array}$ \\
\cline { 3 - 3 } Tellurium & & 0.155 \\
Molybdenum & $6.28 \times 10^{-9}$ & $5.8 \times 10^{-9}$ \\
Uranium & $6.23 \times 10^{-5}$ & $5.65 \times 10^{-5}$ \\
Barium & 0.110 & $9.92 \times 10^{-2}$ \\
Strontium & 0.187 & $1.89 \times 10^{-1}$ \\
Cerium & 0.0513 & $4.48 \times 10^{-2}$ \\
Lanthanum & $2.26 \times 10^{-3}$ & $1.83 \times 10^{-3}$
\end{tabular}

Note that these releases are relative to the total mass of core debris in the reactor cavity (i.e., two-thirds of the total core mass). To determine release fractions relative to the total core inventory, multiply the above values by $2 / 3$. 


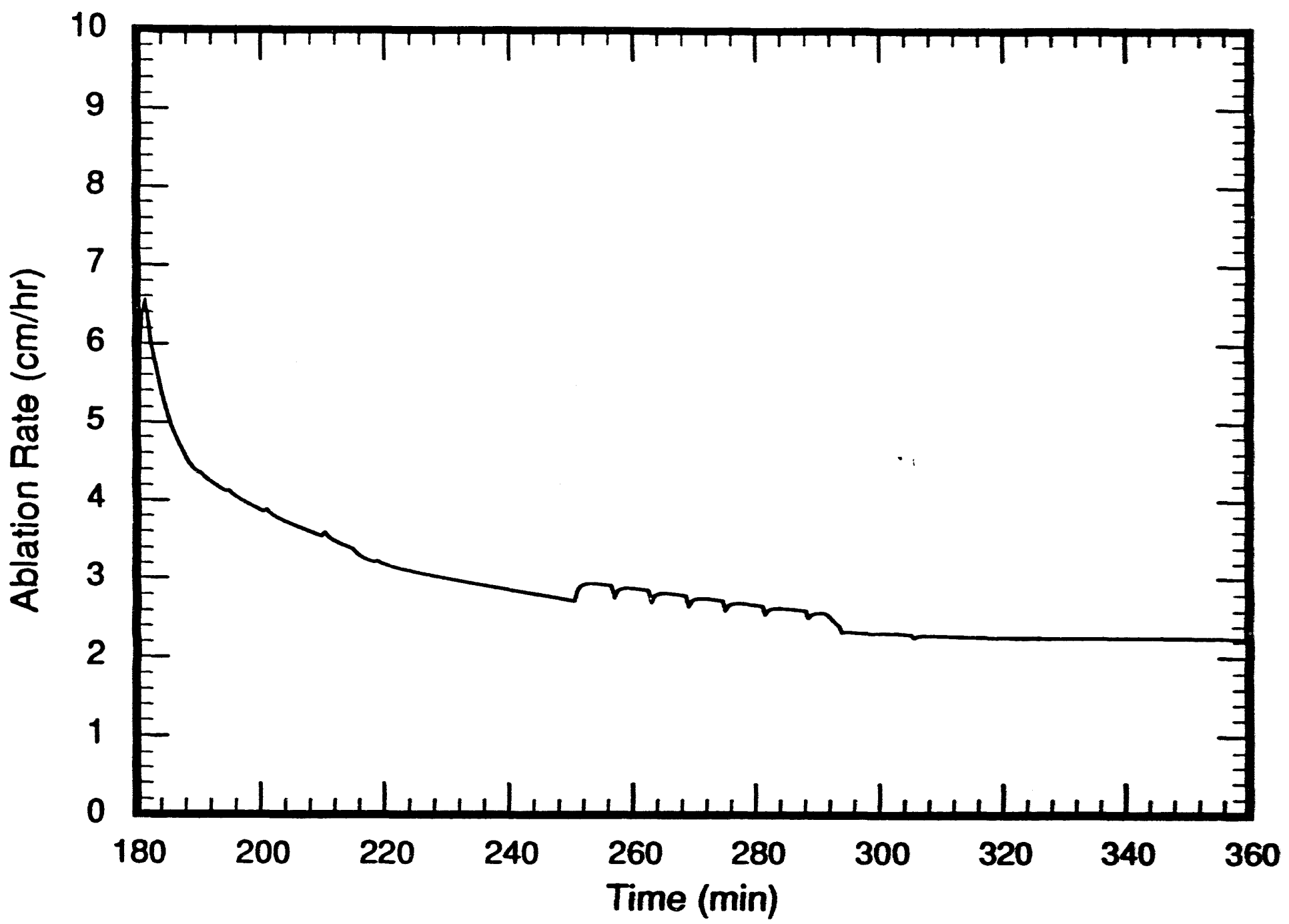

Figure 6.1 Axial ablation rate calculated for the CORCON standard problem 


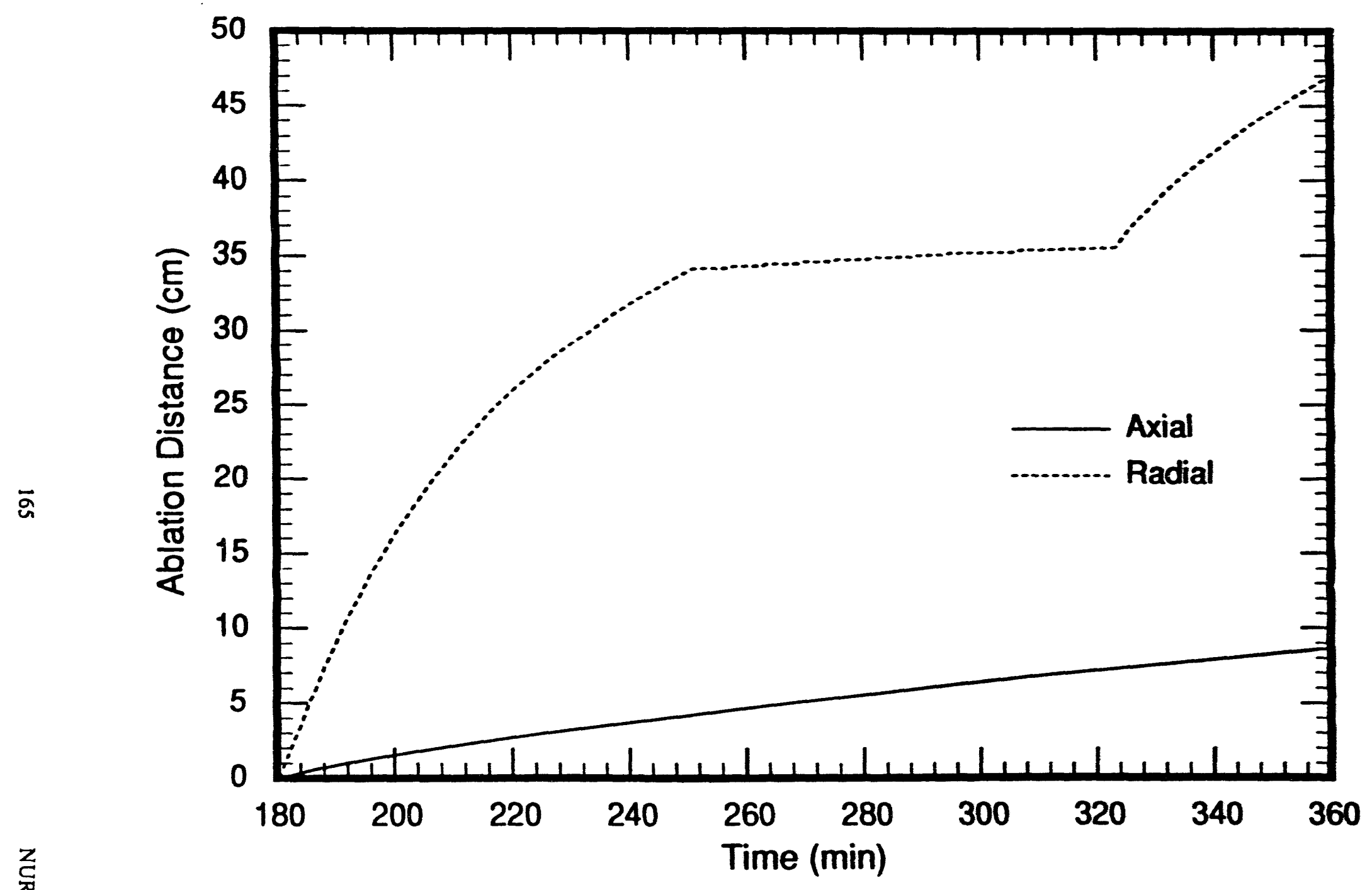

Figure 6.2 Axial and radial ablation distance calculated for the CORCON standard problem 


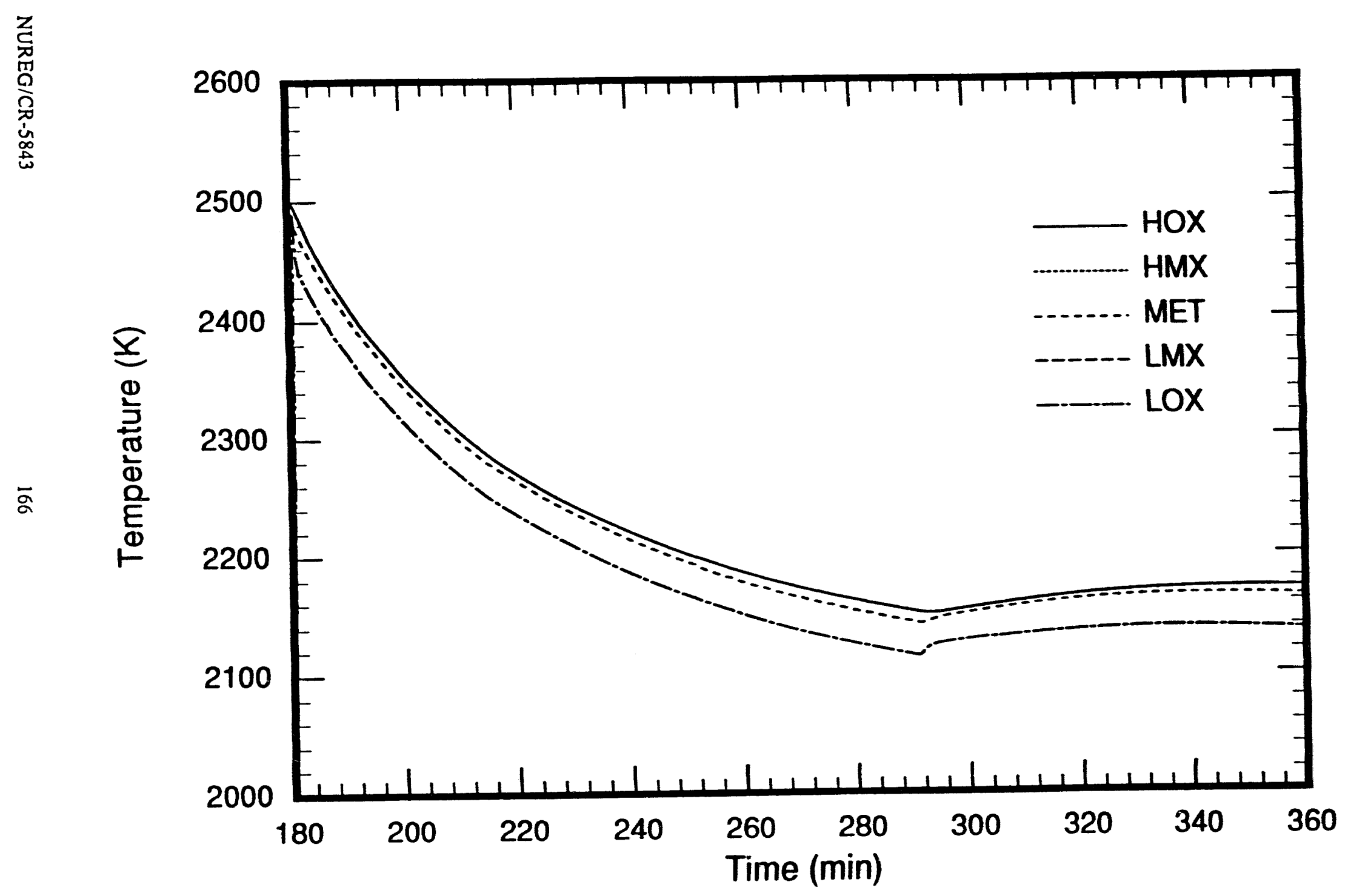

Figure 6.3 Layer temperatures calculated for the CORCON standard problem 


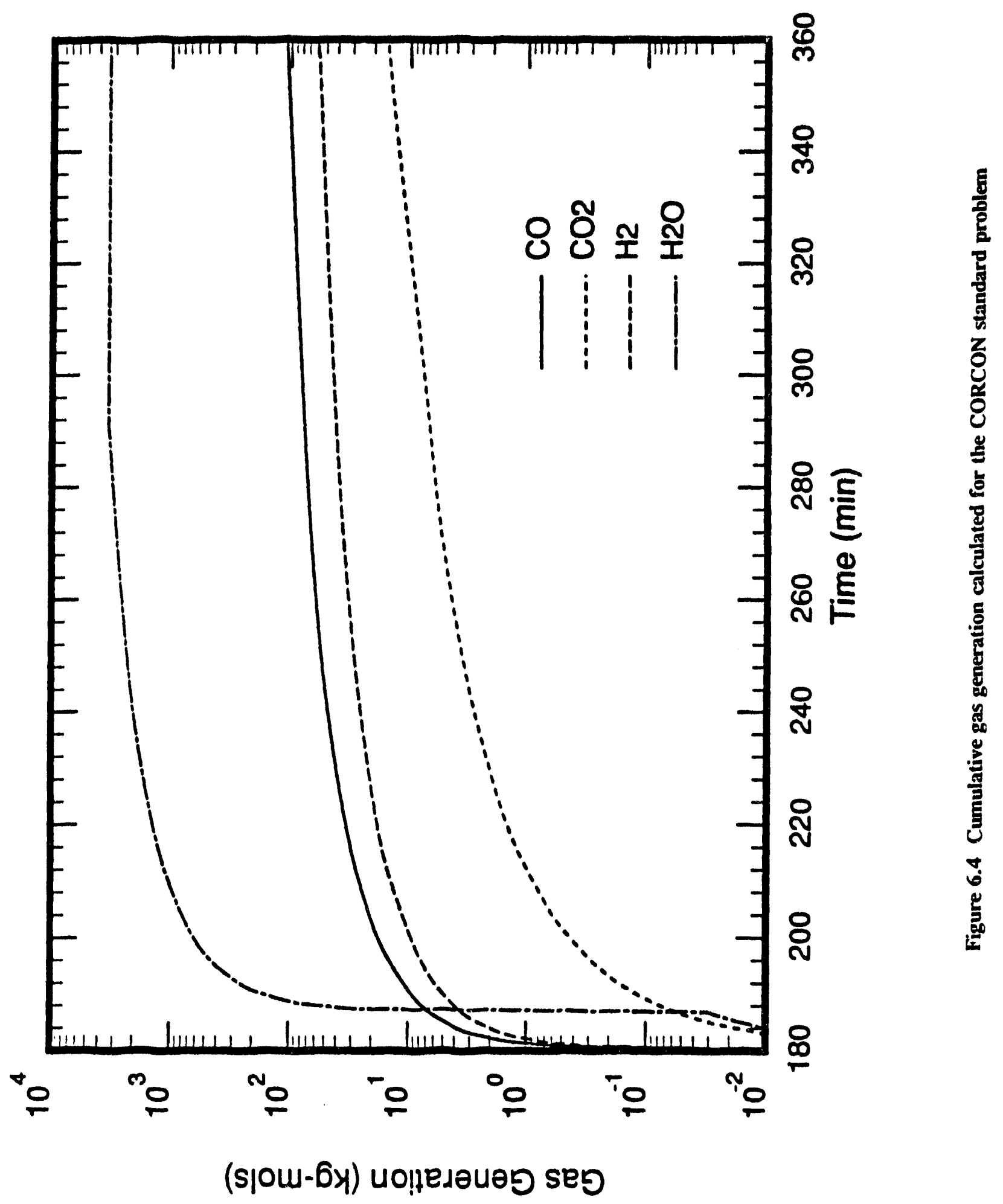




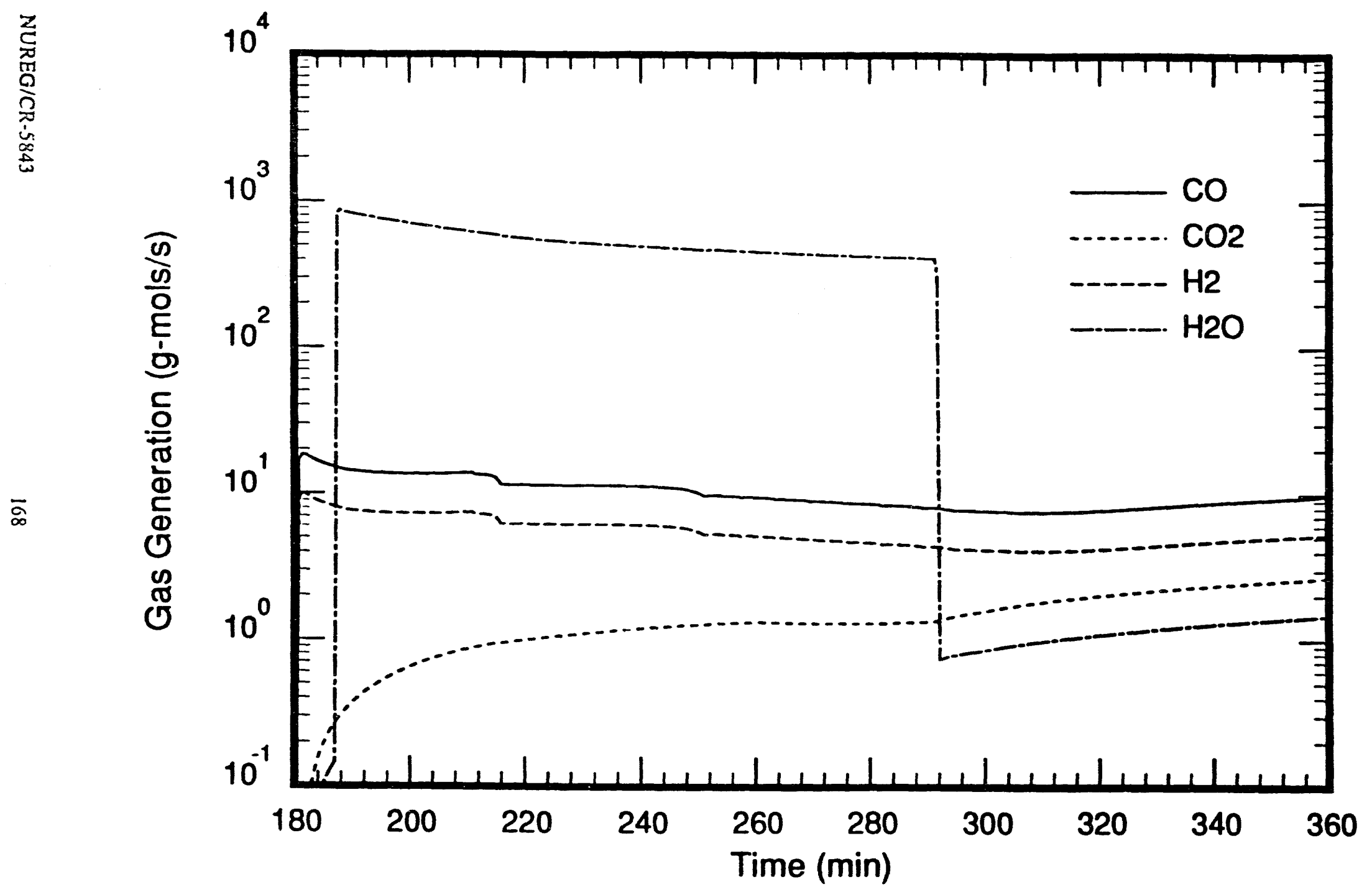




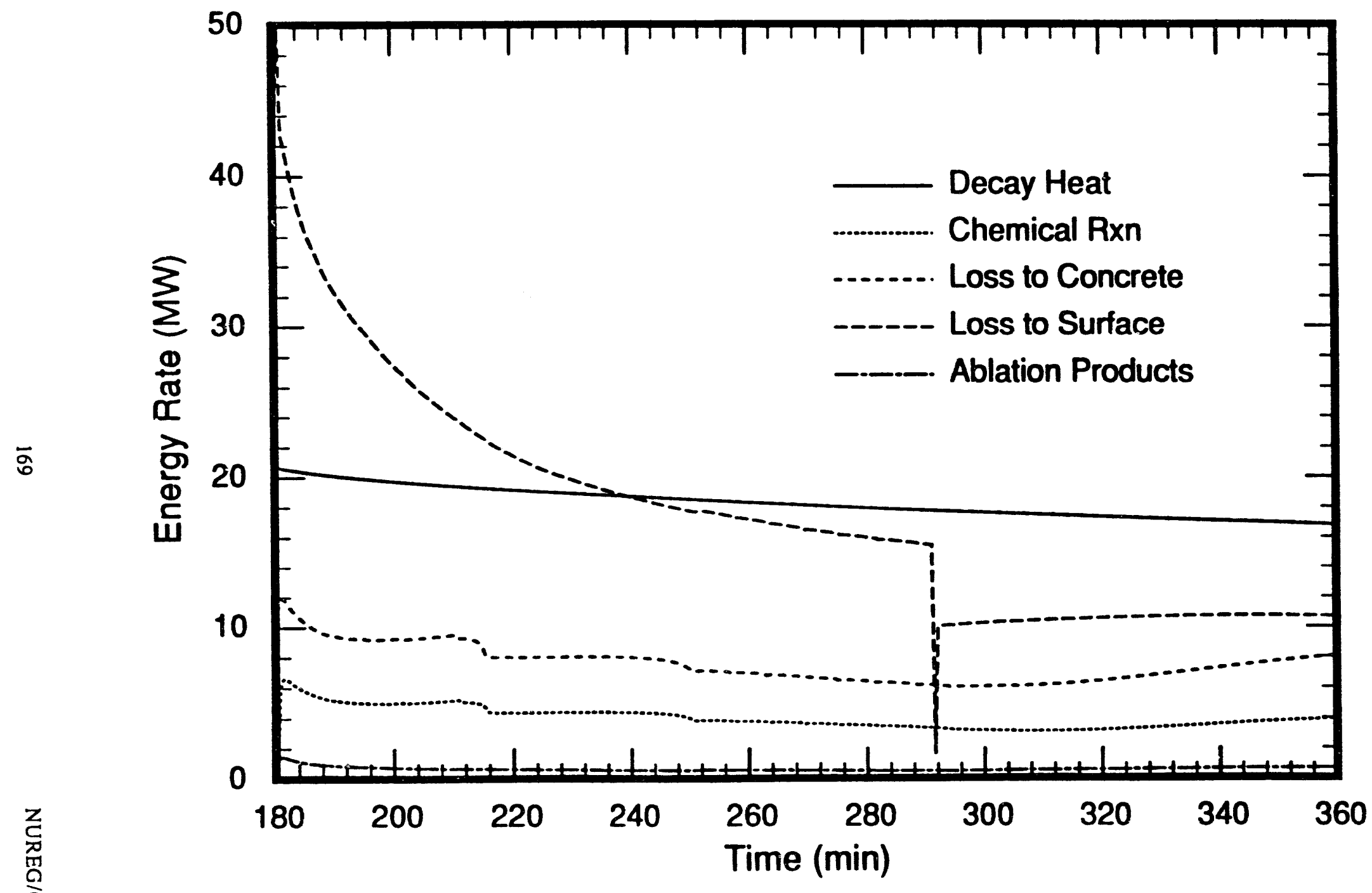

Figure 6.6 Energy terms calculated for the CORCON standard problem 


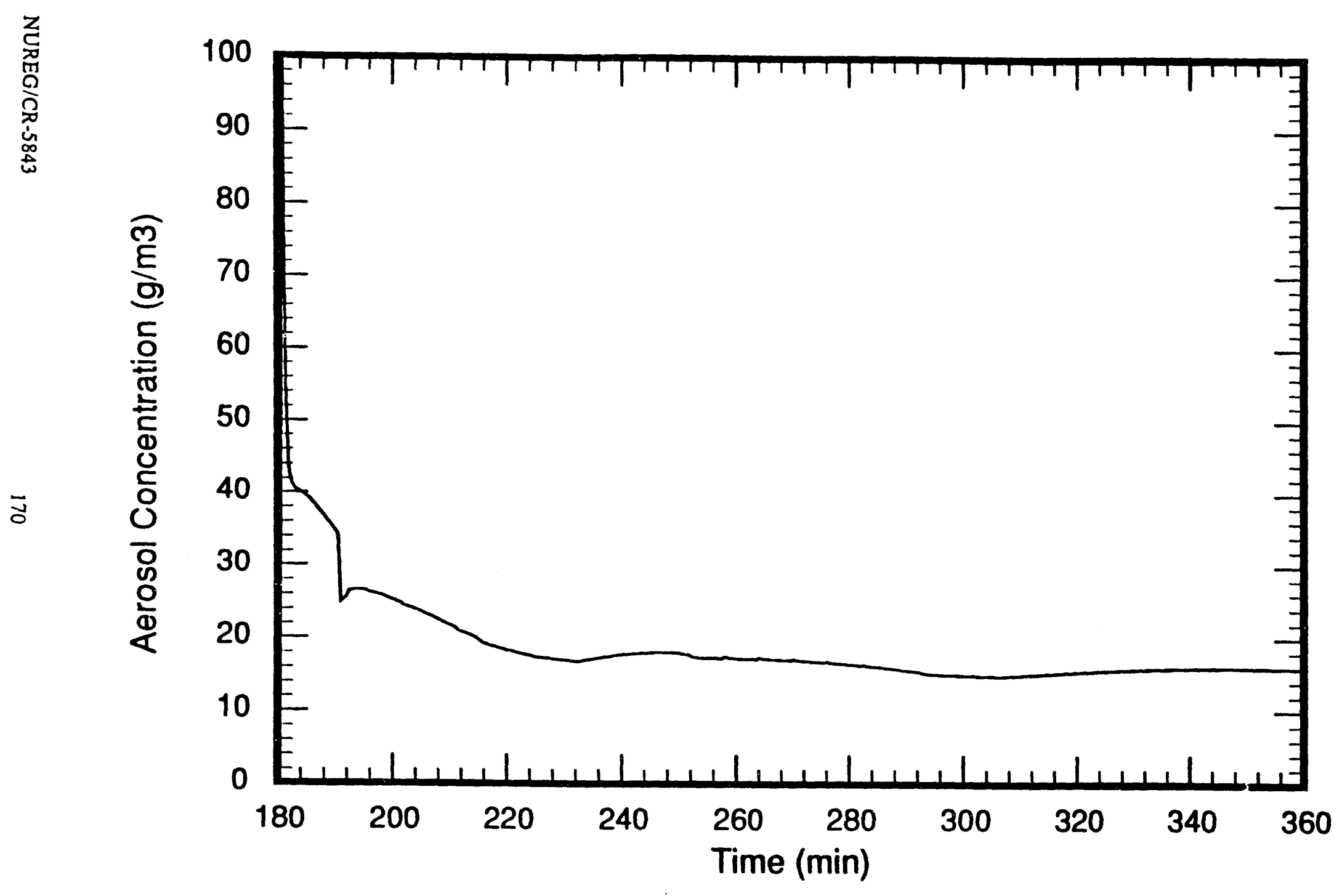

Figure 6.7 Aerosol concentration (ambient conditions) calculated for the CORCON standard problem 


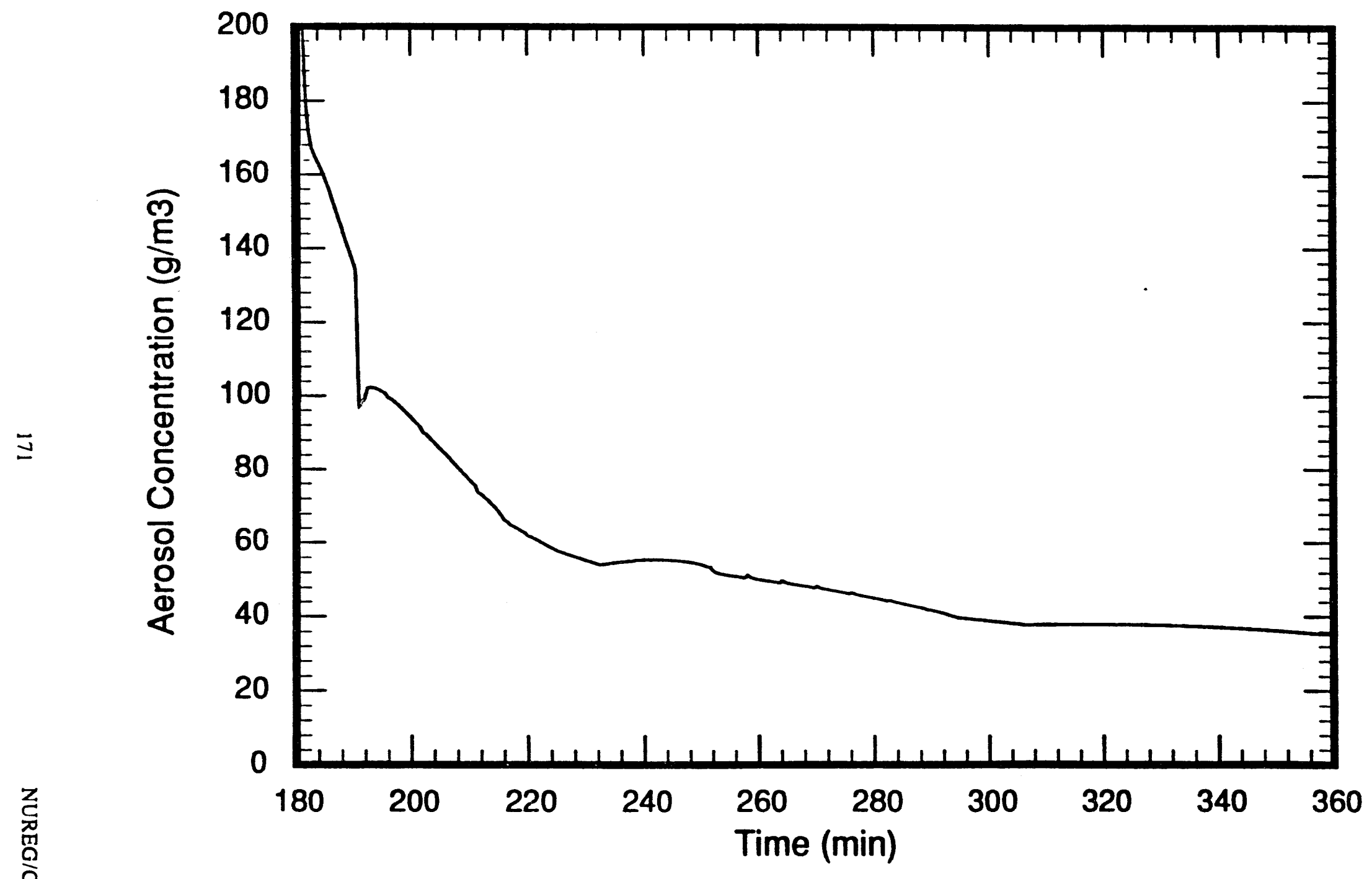

Figure 6.8 Aerosol concentration (STP) calculated for the CORCON standard problem 


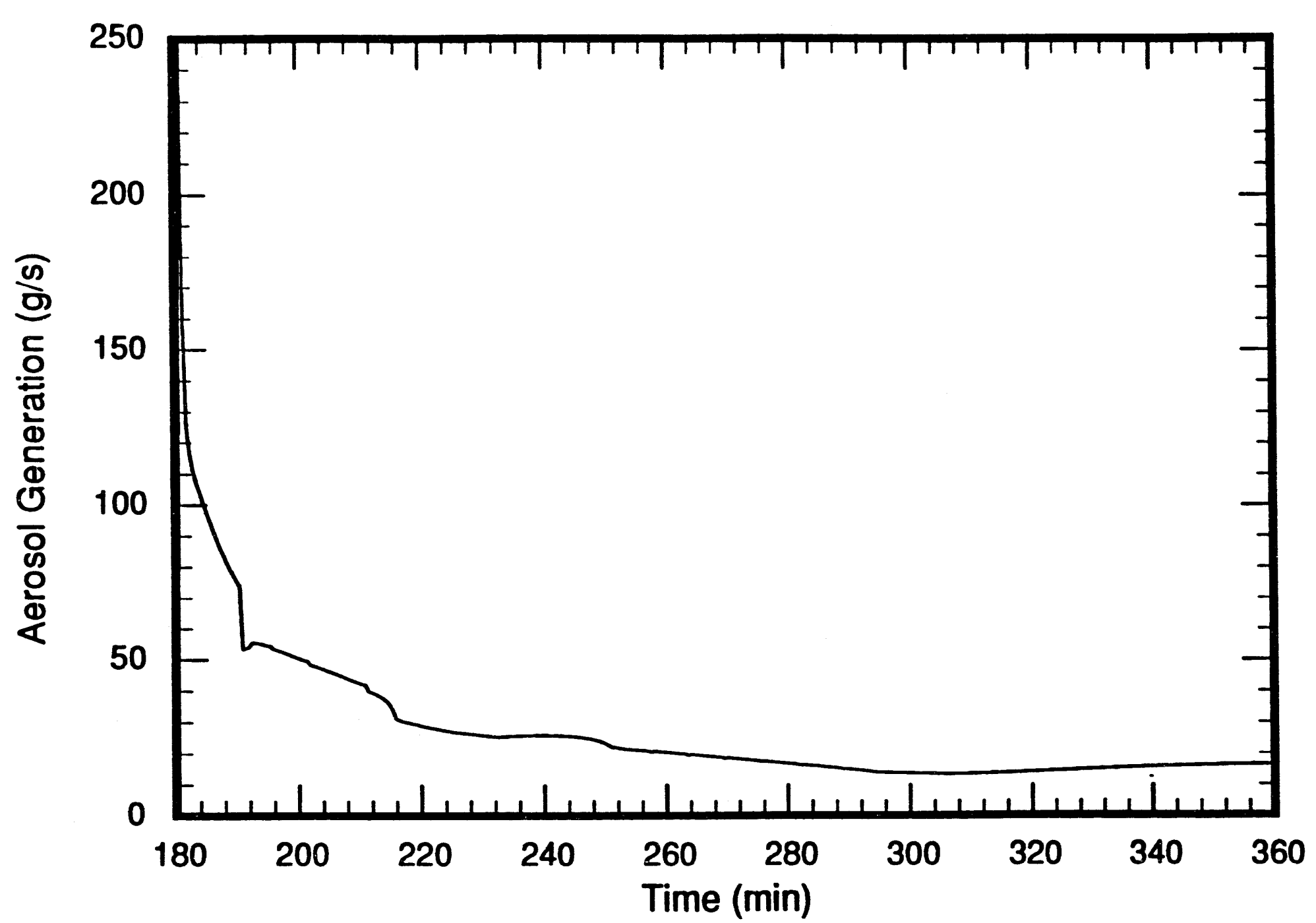

Figure 6.9 Aerosol generation rate calculated for the CORCON standard problem 


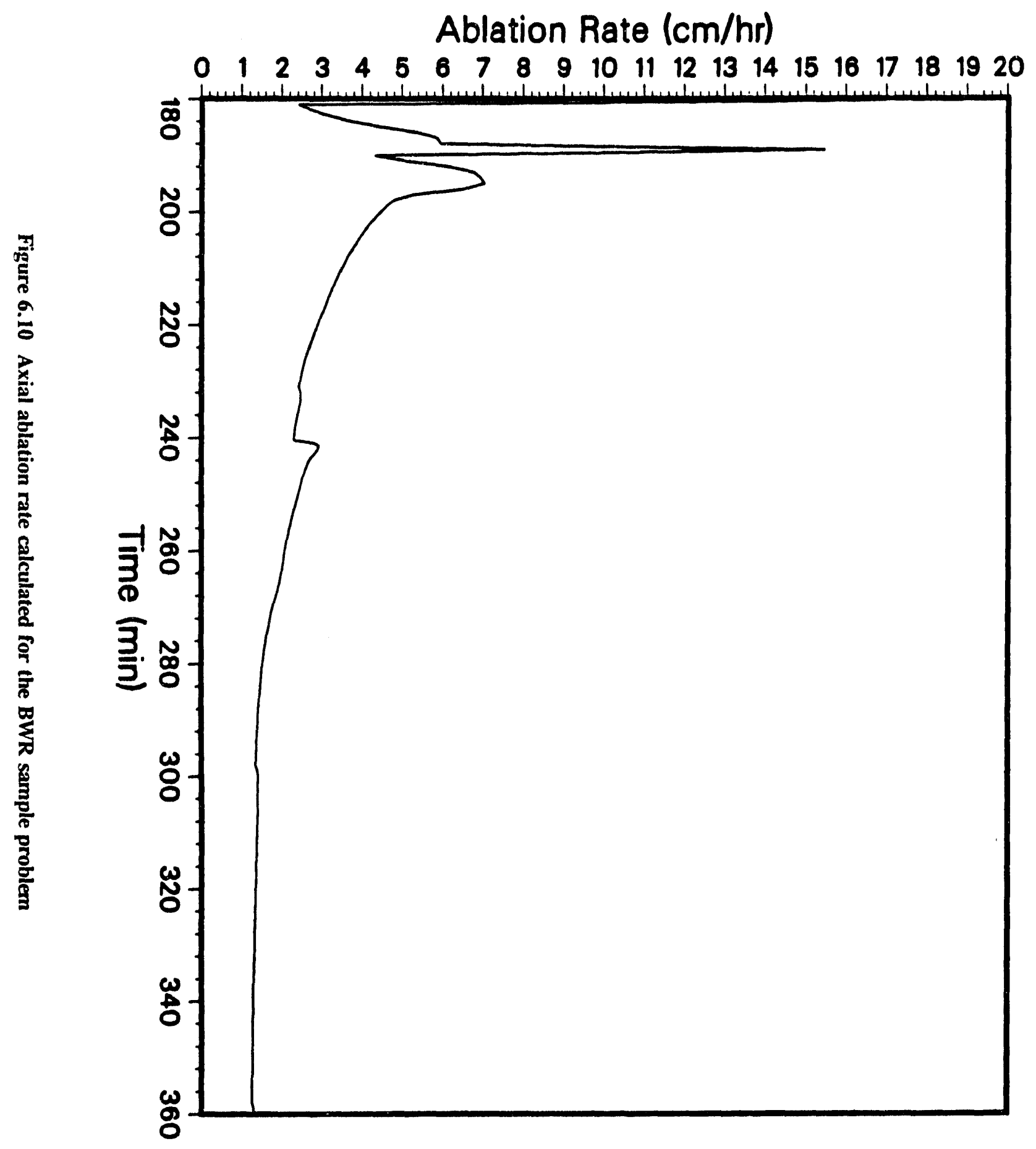




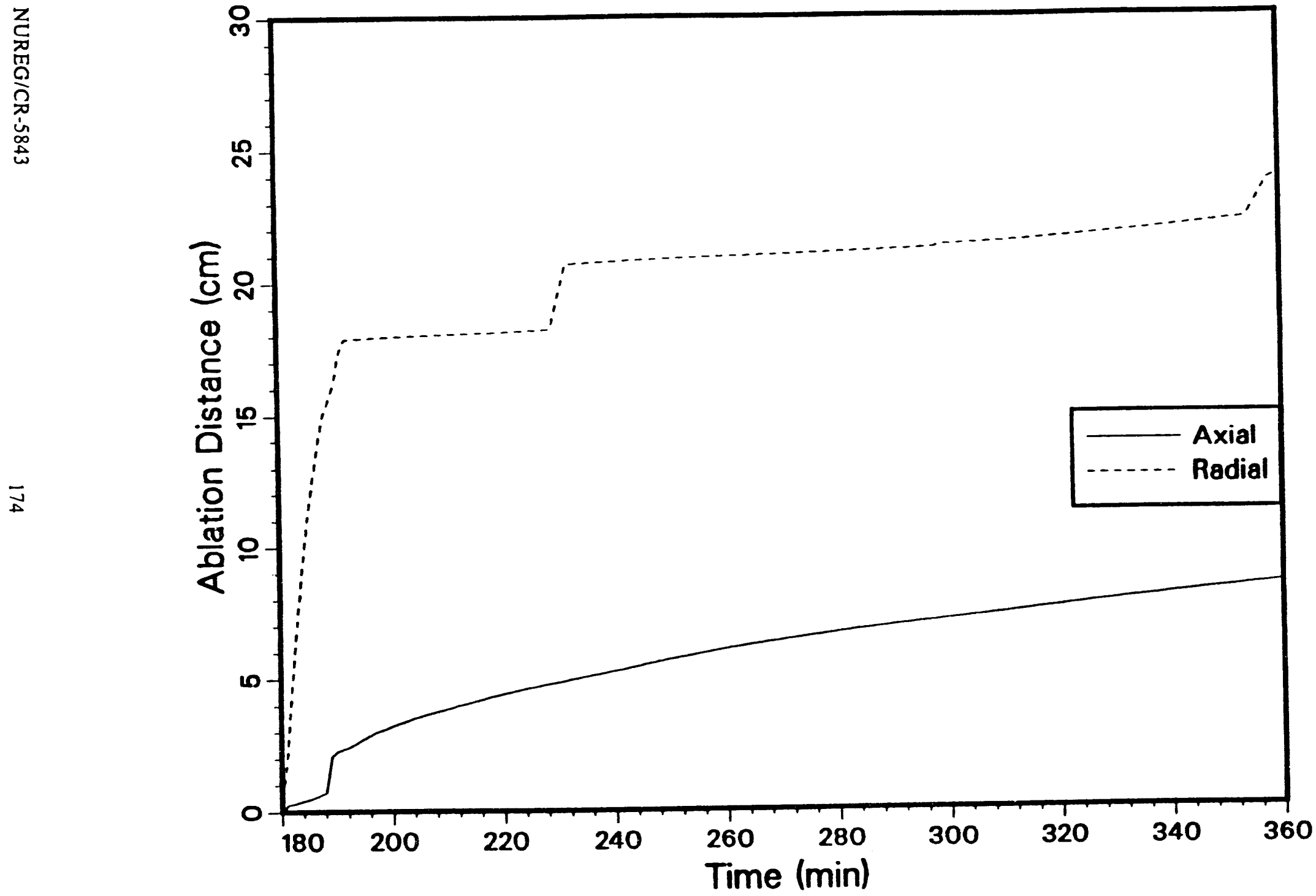

Figure 6.11 Axial and radial ablation distances calculated for the BWR sample problem 


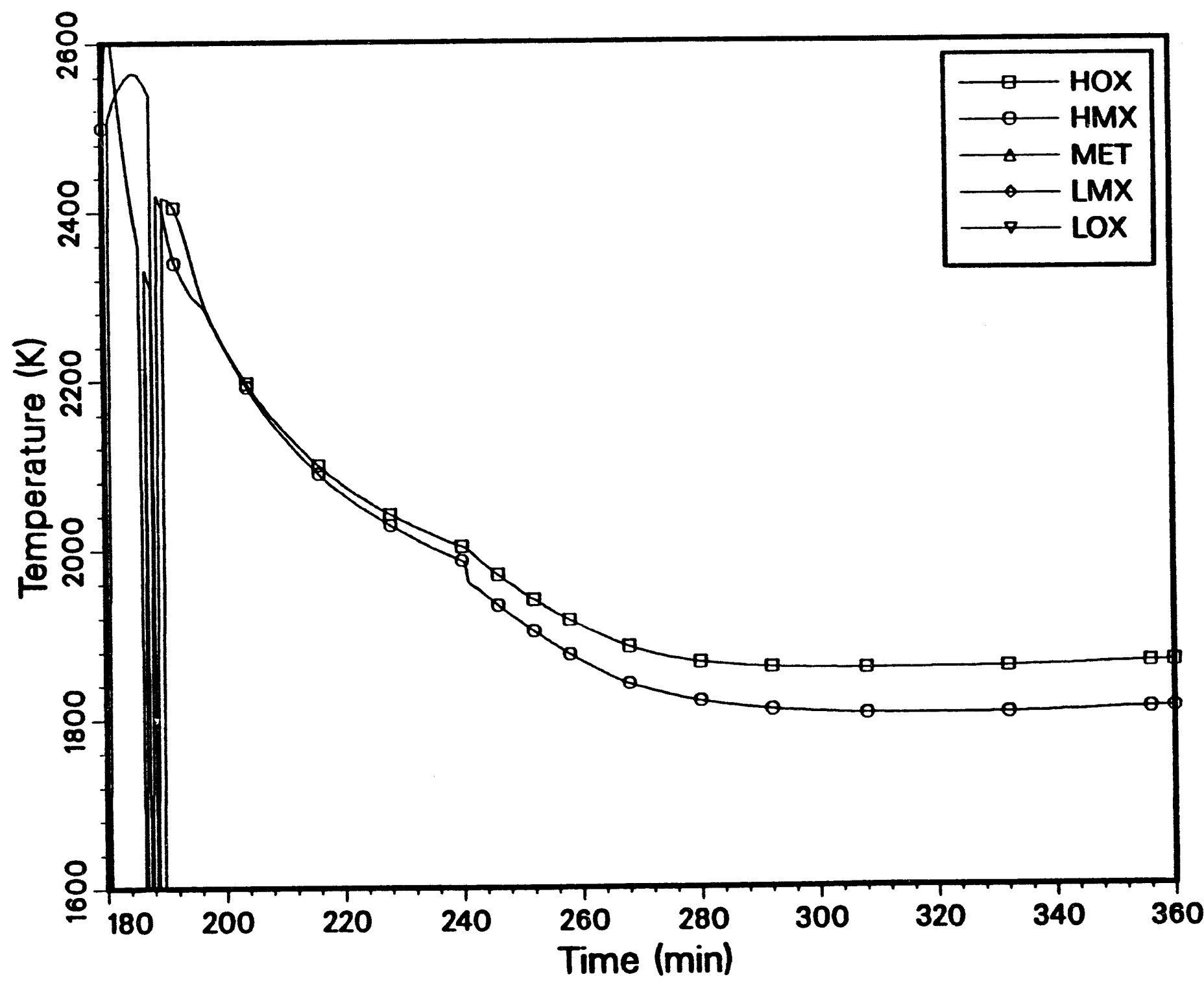

Figure 6.12 Layer temperatures calculated for the BWR sample problem 


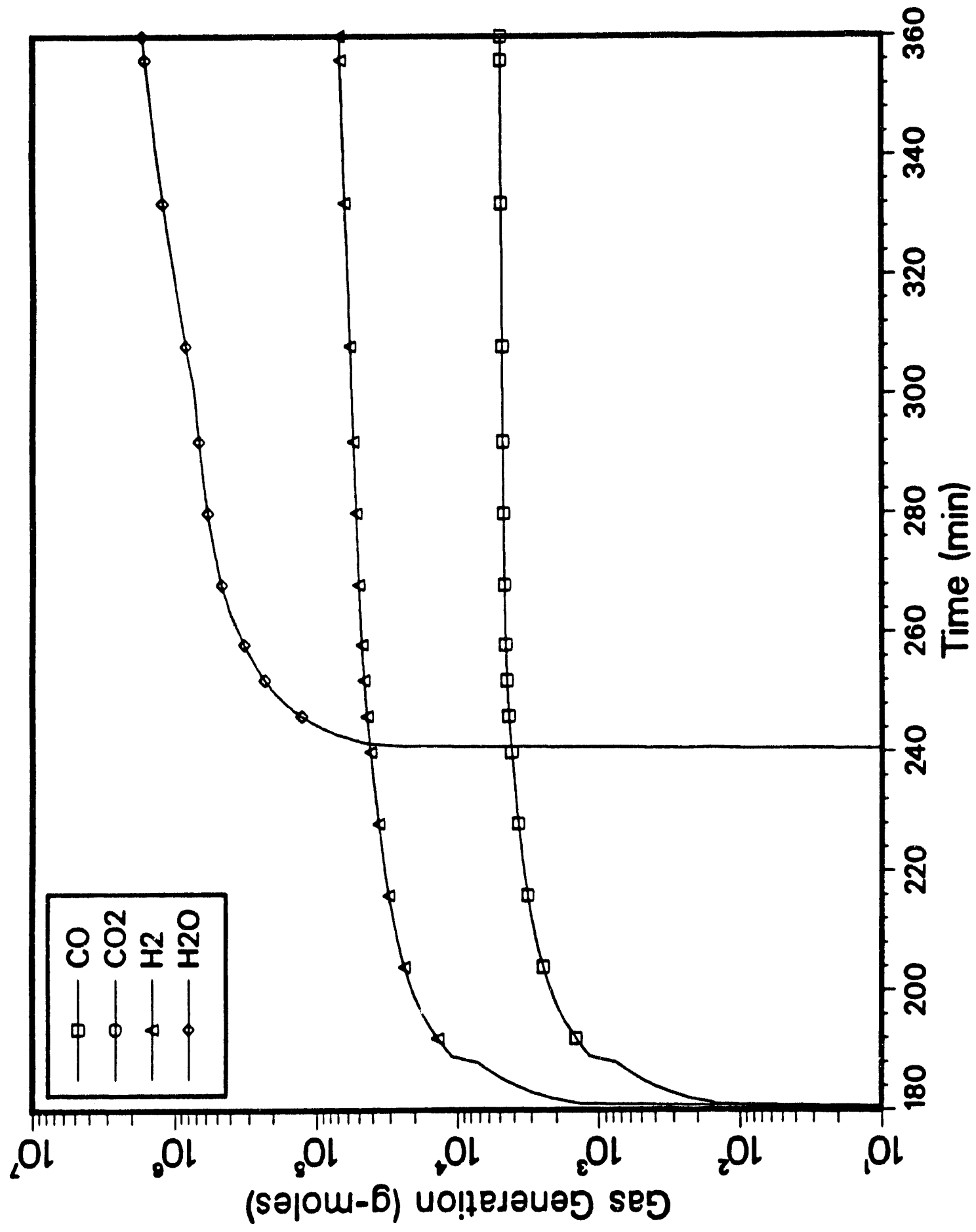




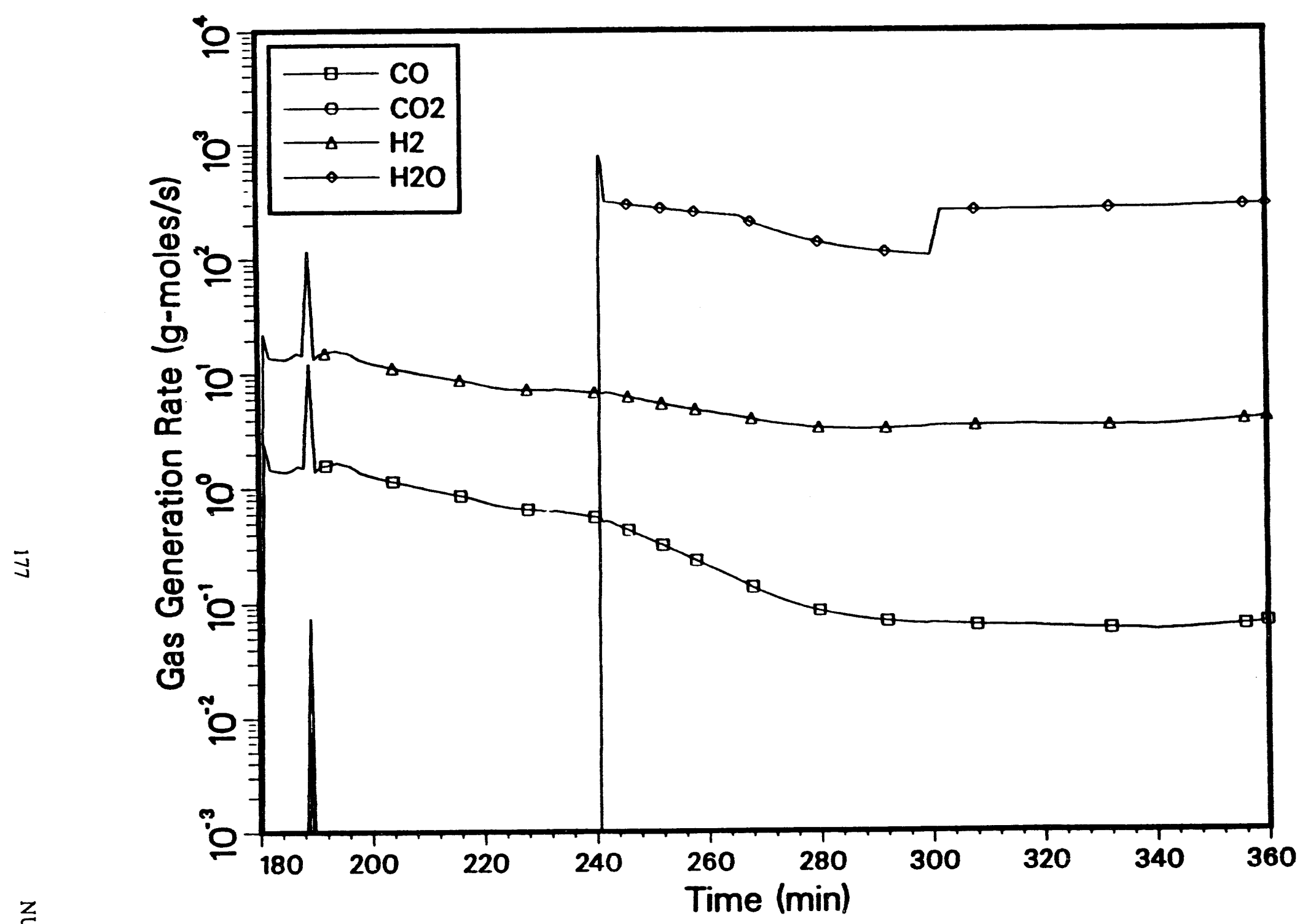

Figure 6.14 Gas generation rate calculated for the BWR sample problem 


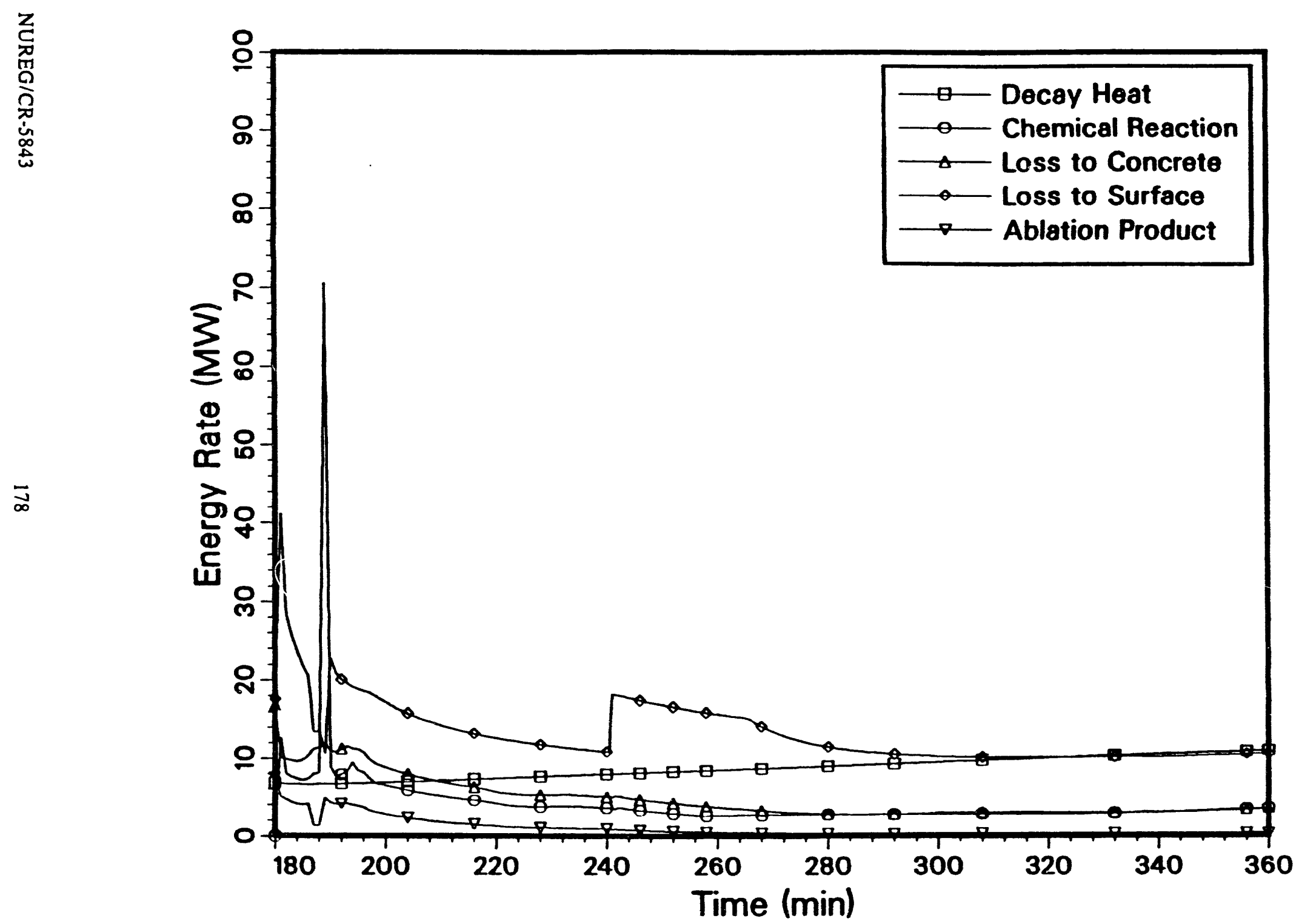

Figure 6.15 Energy terms calculated for the BWR sample problem 


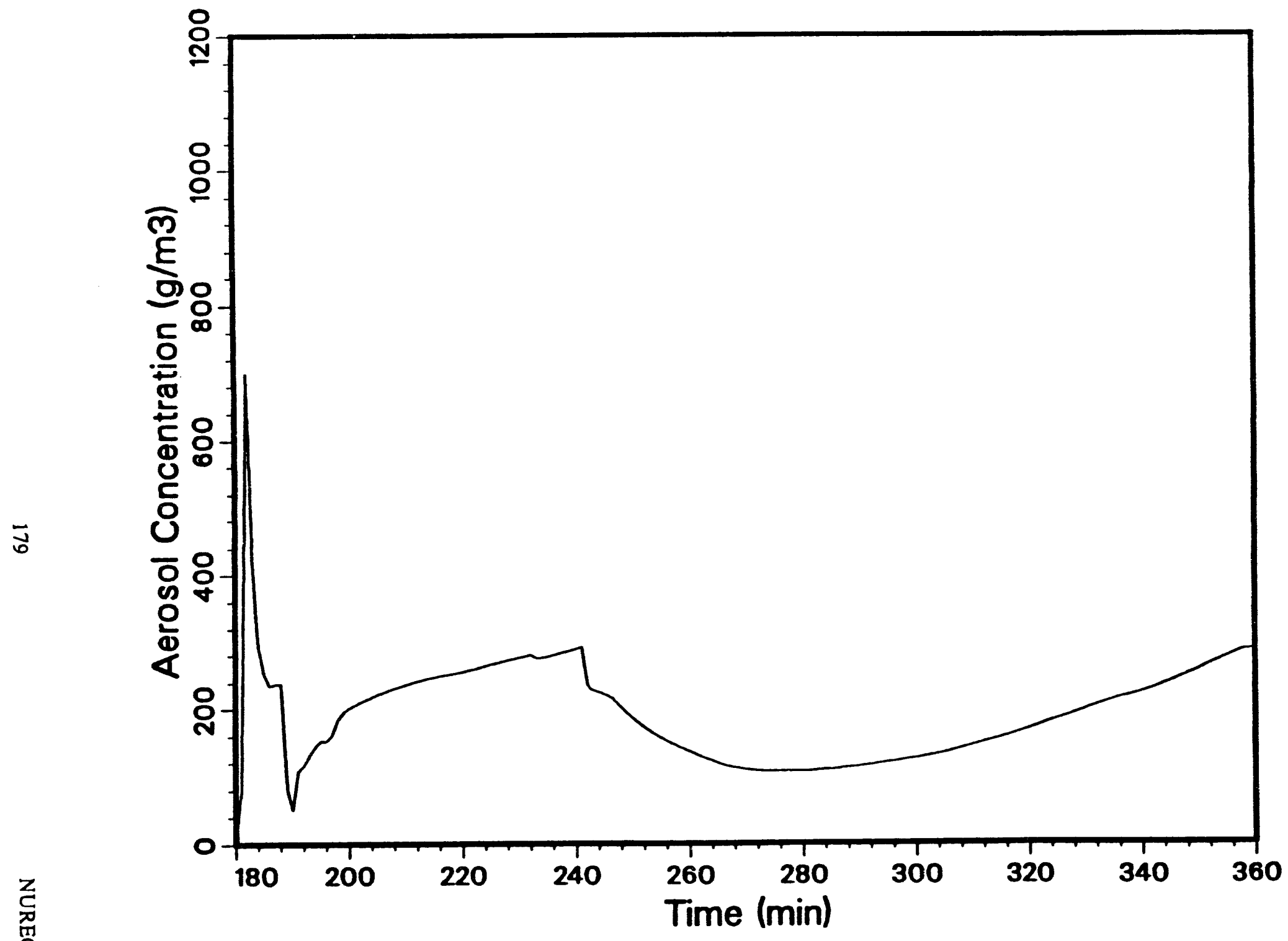

Figure 6.16 Aerosol concentration (ambient conditions) calculated for the BWR sample problem 


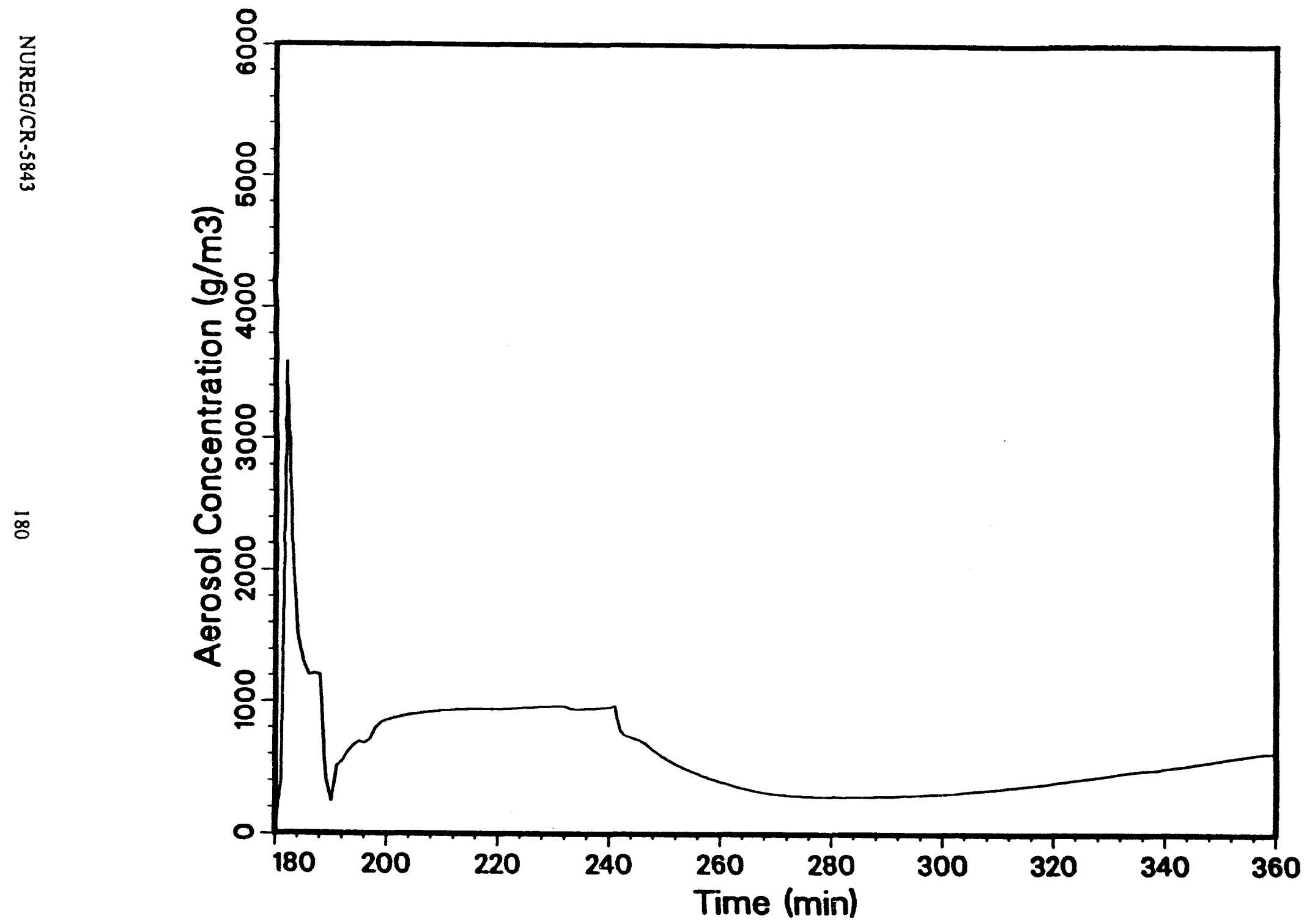

Figure 6.17 Aerosol concentration (STP) calculated for the BWR sample problem 


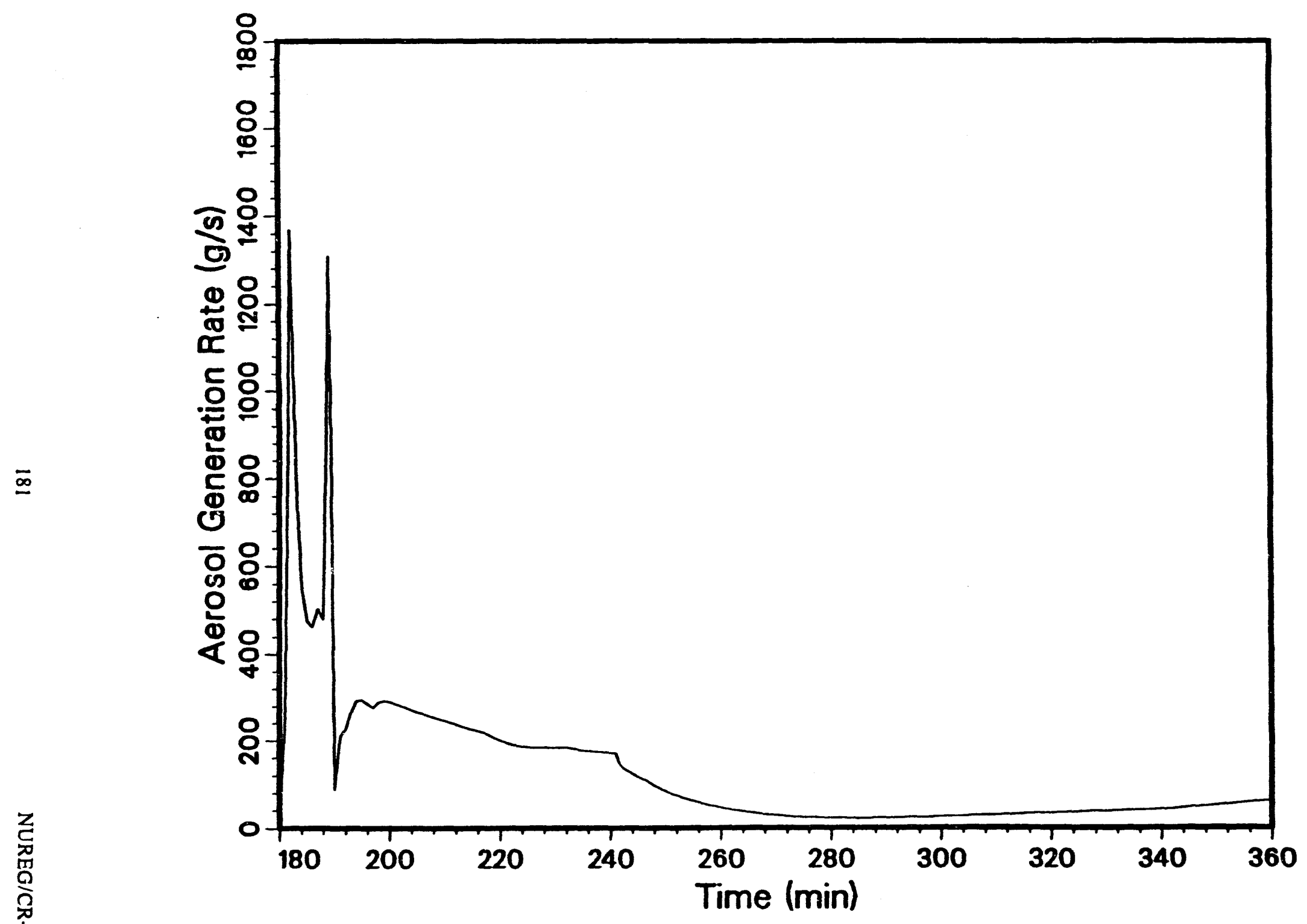

Figure 6.18 Aerosol generation rate calculated for the BWR sample problem 


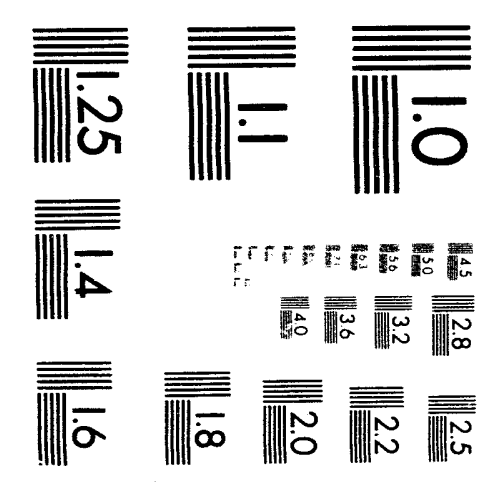



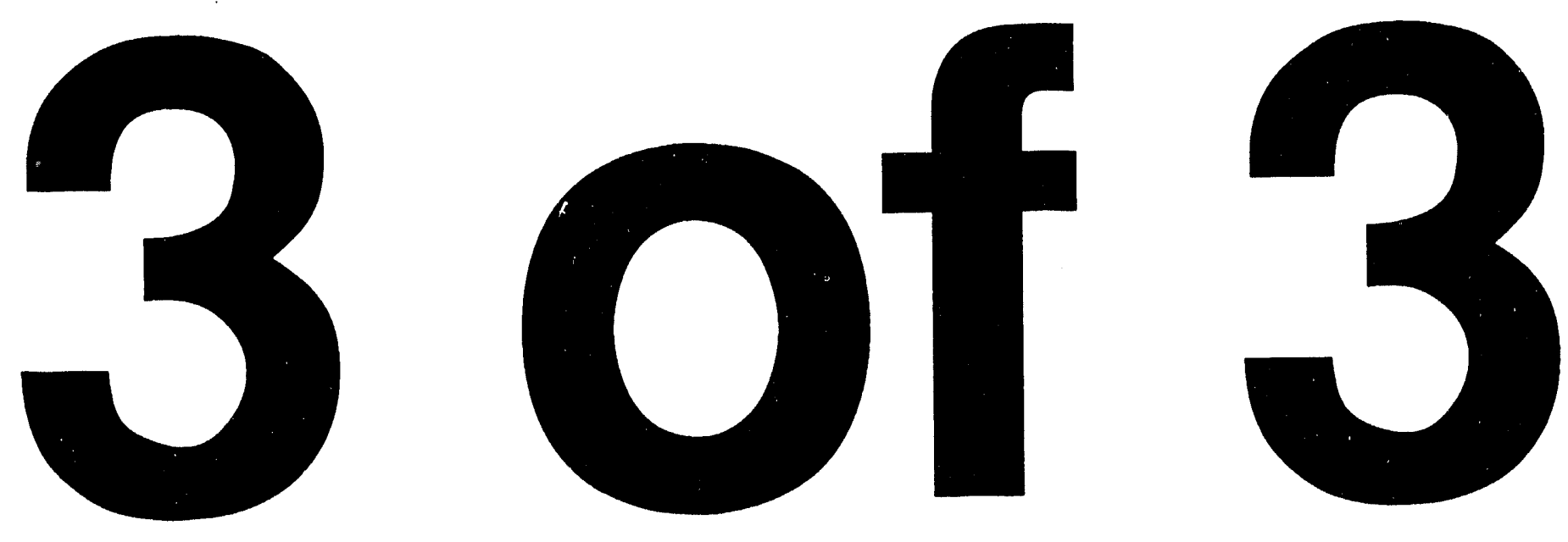


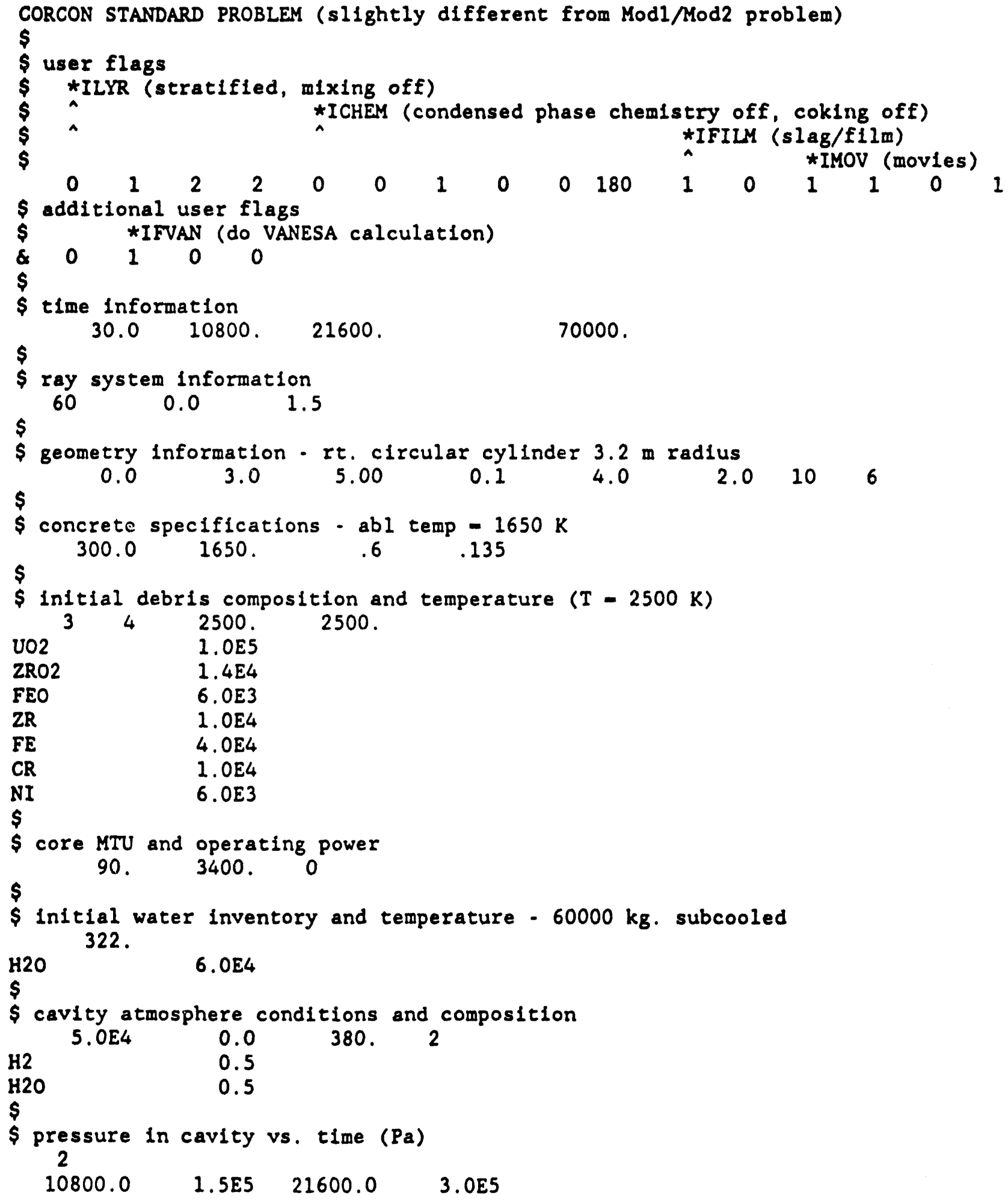


Table 6.1 Input listing for the CORCON standard problem (continued)

$\$$

$\$$ temperature of surfaces above melt

$$
2
$$

$10800.0 \quad 400.0 \quad 21600.0 \quad 800.0$

$\$$

\$ emissivities vs, time - oxides, metals, surroundings

TIMETIMETIME

$\begin{array}{lll}1 & 1 & 1\end{array}$

$$
\begin{array}{ll}
0.0 & 0.8 \\
0.0 & 0.6 \\
0.0 & 0.6
\end{array}
$$

$\$$

$\$$ mean beam length for atmospheric opacity calculation (m)

3.0

$\$$

$\$$ VANESA input

$\$$ user options

\$

$1 \quad 1 \quad 1 \quad 1$

* IDEAL-1, run with 1deal chemistry turned on

$\$$

$\$$ pool scrubbing parameters

$$
20
$$

$$
2.3
$$

1

1

1.0

1.0 
Table 6.2 Output listing for the CORCON standard problem

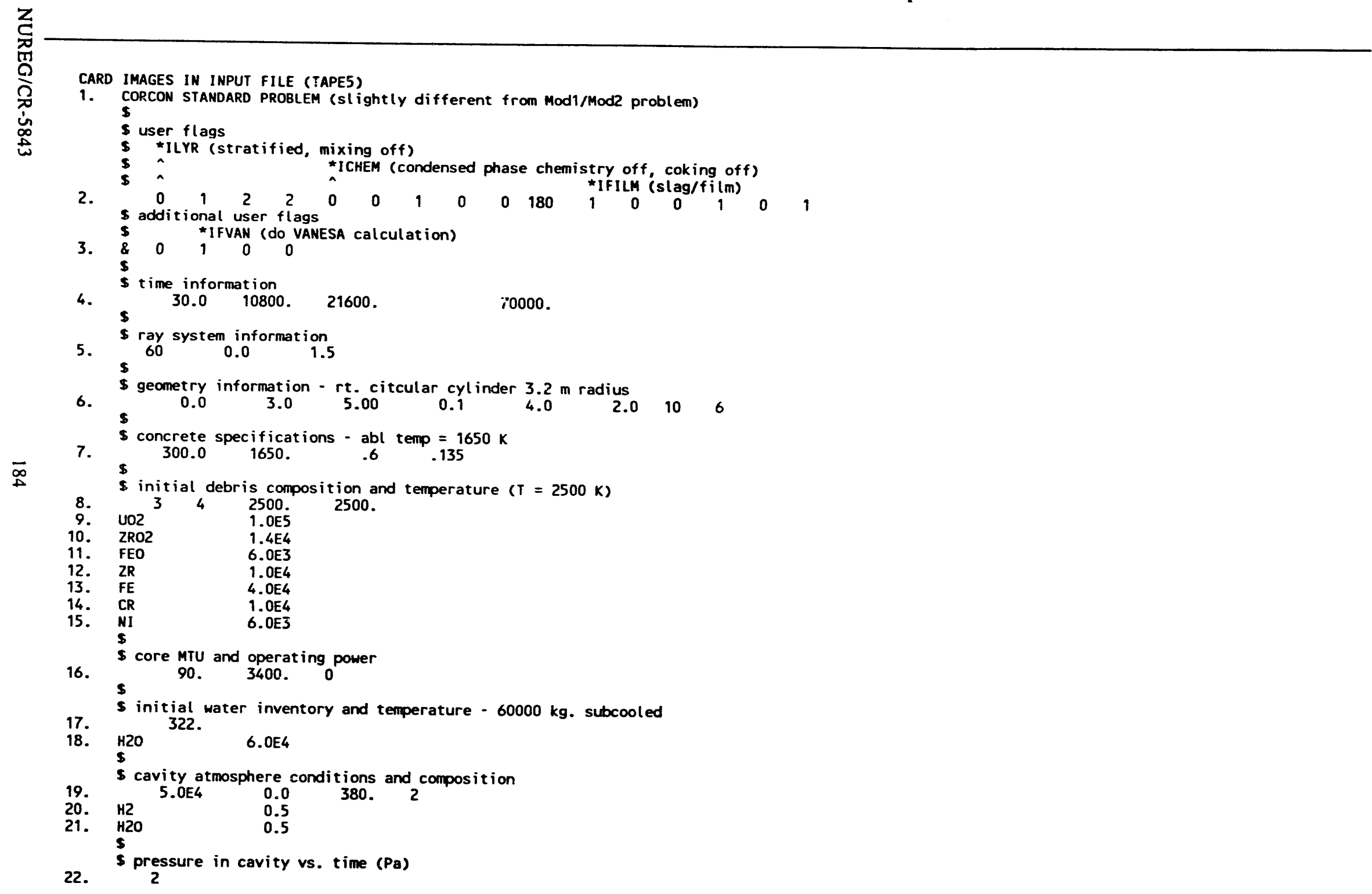


Table 6.2 Output listing for the CORCON standard problem (continued)

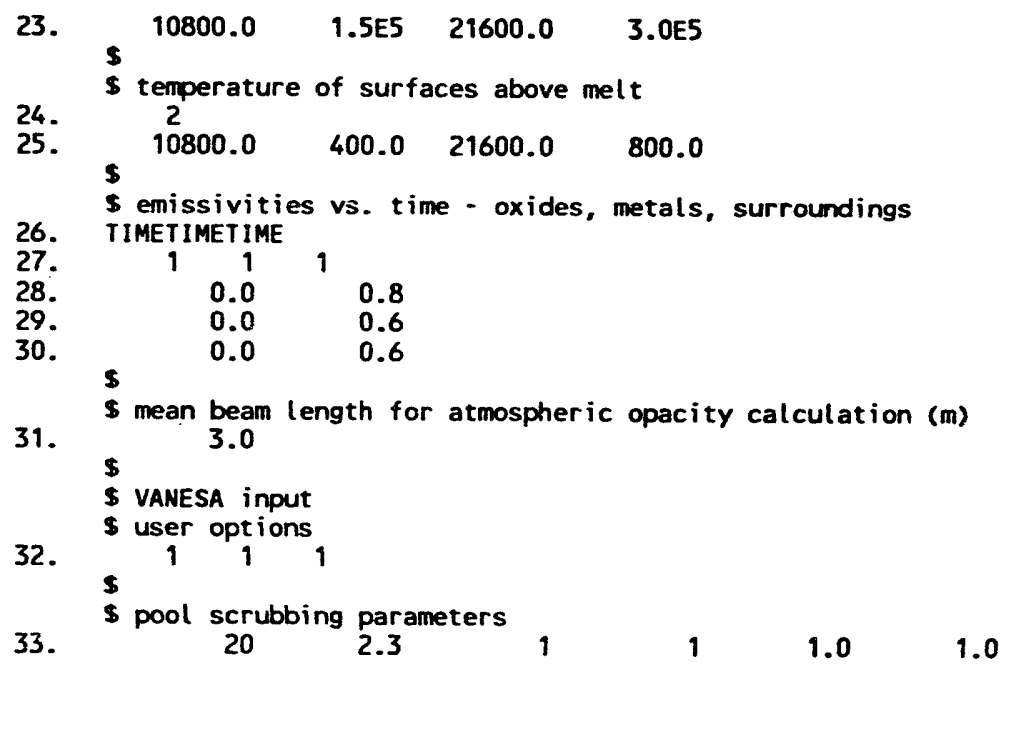


Table 6.2 Output listing for the CORCON standard problem (continued)

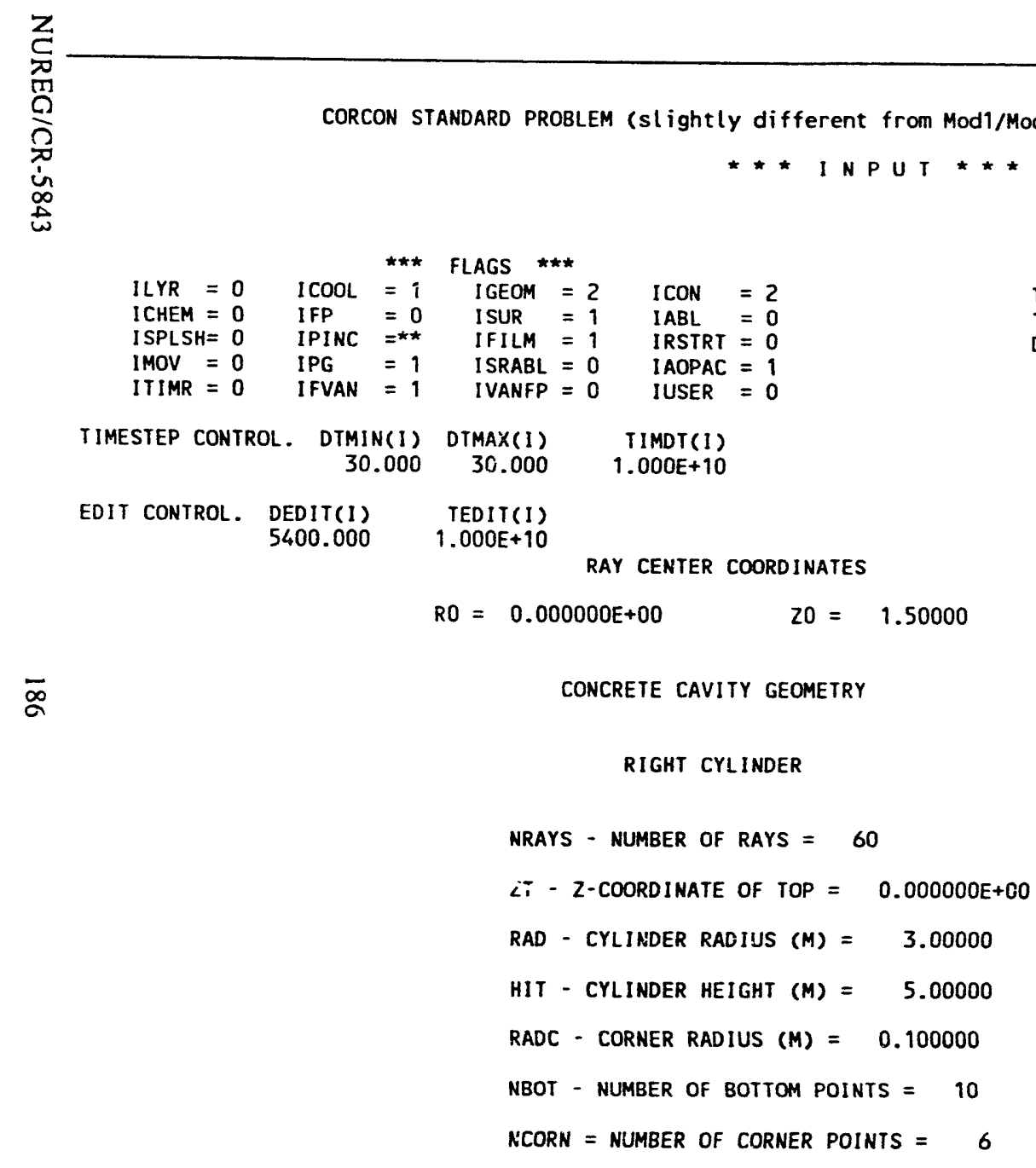


Table 6.2 Output listing for the CORCON standard problem (continued)

\section{* * * CONCRETE SPECIFICATIONS * * *}

LIMESTONE AGGREGATE - COMMON SAND CONCRETE
RHOC
RBR
$(K G / K G$
SI
TSOL
TLIO
TIC
(K)
TW
DELH
EW

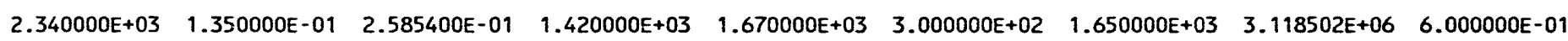
SPECIES NAME MASS FR.(KG/KG C) MOLECULAR HT.

$\begin{array}{llr}\text { S102 } & 0.3580 & 60.0843 \\ \text { TIO2 } & 0.0018 & 79.8988 \\ \text { MNO } & 0.0003 & 70.9374 \\ \text { MGO } & 0.0048 & 40.3044 \\ \text { CAO } & 0.3130 & 56.0794 \\ \text { NA2O } & 0.0008 & 61.9790 \\ \text { K20 } & 0.0122 & 94.1960 \\ \text { FE203 } & 0.0144 & 159.6922 \\ \text { AL203 } & 0.0360 & 101.9613 \\ \text { CR203 } & 0.0001 & 151.9902 \\ \text { CO2 } & 0.2115 & 44.0098 \\ \text { H2OEVAP } & 0.0270 & 18.0152 \\ \text { H2OCHEM } & 0.0200 & 18.0152\end{array}$

MELT FISSION PRODUCT INVENTORY

BASED ON $9.00000 E+01$ METRIC TON URANIUM CORE OPERATED AT $3.40000 E+03$ MW(THERMAL)

MELT CONTAINS 8.81390 +01 METRIC TONS URANIUM, CORRESPONDING TO 97.93 PERCENT OF CORE

ELEMENTS, RETAINED FRACTIONS (**DENOTES USER INPUT, OTHERS FROM WASH 1400), AND GRAM-ATOMS IN MELT

$\begin{array}{lll}\text { MO } & (0.970) & 1.8885 E+03 \\ \text { SB } & (0.850) & 6.5124 E+00 \\ \text { ZR } & (0.990) & 2.3563 E+03 \\ \text { NB } & (0.990) & 3.6129 E+01 \\ \text { LA } & (0.990) & 5.3006 E+02 \\ \text { EU } & (0.990) & 5.1556 E+01 \\ \text { I } & (0.100) & 1.0109 E+01\end{array}$

TC $(0.970) \quad 4.8318 E+02$

TE $(0.850) 1.6880 E+02$

CE $(0.990) \quad 1.2329 E+03$

PU $(0.990) \quad 2.2541 \mathrm{E}+03$

PR $(0.990) \quad 4.6084 E+02$

RB $(0.190) \quad 5.0434 E+01$
RU (0.970) $1.1976 E+03$
SR $(0.900) \quad 6.3021 E+02$
NP $(0.990) 1.2203 E+02$
AM $(0.990) \quad 1.6241 E+01$
ND $(0.990) \quad 1.4801 E+03$
CS $(0.190) \quad 2.2933 E+02$

$\begin{array}{lll}\text { RH } & (0.970) & 2.1821 E+02 \\ \text { BA } & (0.900) & 5.5260 E+02 \\ \text { CM } & (0.990) & 5.0995 E+00 \\ \text { Y } & (0.990) & 3.5403 E+02 \\ \text { SM } & (0.990) & 1.7342 E+02 \\ \text { BR } & (0.100) & 1.7078 E+00\end{array}$

FISSION PROOUCTS GROUPED AS 4 PSEUDO-SPECIES

3.96277E+03 GRAM-ATOMS OF FPM MO $\quad 4.76552 \mathrm{E}-01$ WITH ATOMIC FRACTIONS TC 1.21930E-01 
Table 6.2 Output listing for the CORCON standard problem (continued)

\section{GAS INPUT}

SPECIES NAME MOLE FR. (-) MOLECULAR WT.

$\begin{array}{lrr}H 2 & 0.5000 & 2.0158 \\ H 20 & 0.5000 & 18.0152\end{array}$

$\star \star \star$ MELT INTERNAL CONDITIONS $\star \star \star ~$

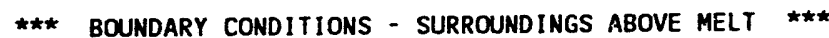

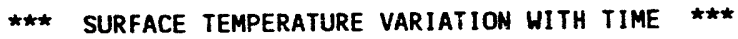

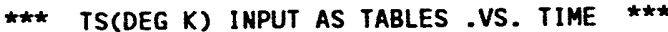

$$
\begin{array}{cc}
\text { TIME(SEC) } & \text { TS(DEG K) } \\
1.0800 E+04 & 4.0000 E+02 \\
2.1600 E+04 & 8.0000 E+02
\end{array}
$$

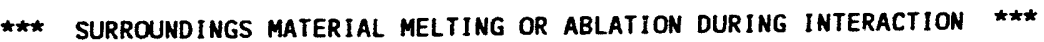

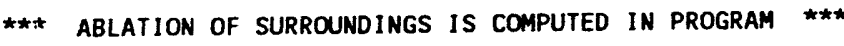

TIME $0.0000 E+00$

$$
\text { EO } 8.0000 E-01
$$

TIME $0.0000 E+00$

EM 6.0000E-01

TIME $0.0000 E+00$

ES $6.0000 E-01$

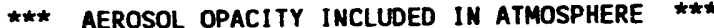

CHARACTERISTIC PATH LENGTH $(M)=3.0000 E+00$

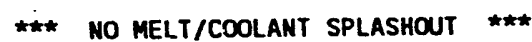

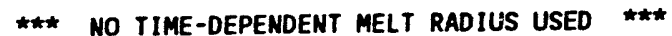


Table 6.2 Output listing for the CORCON standard problem (continued)

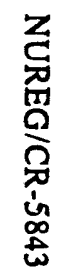

* * * VANESA INPUT * * *

BUBbLE DIAMETER IS CALCULATED

PARTICLE DIAMETER $=\quad 1.00$ MICROMETERS

PARTICLES/BUBBLE IS CALCULATED

$\begin{array}{rr}\text { INITIAL MELT COMPOSITION } \\ \text { G-MOLES } & \text { KG } \\ 716242.6 & 40000.000 \\ 192322.5 & 10000.000 \\ 83511.5 & 6000.000 \\ 0.0 & 0.000 \\ 102197.2 & 6000.000 \\ 1888.7 & 181.198 \\ 1899.3 & 191.966 \\ 0.0 & 0.000 \\ 6.5 & 0.793 \\ 168.8 & 21.538 \\ 0.0 & 0.000 \\ 0.0 & 0.000 \\ 0.0 & 0.000 \\ 0.0 & 0.000 \\ 0.0 & 0.000 \\ 370372.0 & 100000.000 \\ 113619.0 & 14000.000 \\ 134.0 & 37.755 \\ 552.8 & 84.772 \\ 630.3 & 65.310 \\ 1524.8 & 496.813 \\ 5976.6 & 1028.693 \\ 36.1 & 3.935 \\ 11.8 & 3.070 \\ 109625.1 & 10000.000\end{array}$

*** MATER POOL DECONTAMIMATION ***

INITIAL BUBBLE SIZE(CA $)=1.00$

SIZE SEGHENTS IN DISTRIBUTION $=20$

ALL SCAVENGING MECHANISHS ARE OPERATING

IMPACTION BASE ON V(REL)/VRRISE)

1.00

GEONETRIC STAMDARD DEVIATION OF INPUT SIZE DISTRIBUION $=2.30$ 
TIME $=16200.00$

CORCON STANDARD PROBLEM (slightly different from Mod1/Mod2 problem)

$\star \star \star \star$ GENERAL SUMMARY $* \star \star \star *$
IT. NO. $=180$

MELT AND COOLANT LAYERS

$\begin{array}{ll}\text { MUNBEP. OF LAYERS, } & \text { NLYR }=4 \\ \text { CONFIGURATION, } & \text { ILYR }=4 \\ \text { COOLANT PRESENT, } & \text { ICOOL }=1\end{array}$

EXTREME CAVITY DIMENSIONS, WITH LOCATIONS

$$
\text { RADIAL }
$$

MAXIMUM CAVITY RADIUS

$(\mathrm{M})=3.34555$

OUTSIDE RADIUS OF CONCRETE $(M)=4.00000$

$\begin{array}{lrr}\text { CORRESPONDING BODY POINT } & (\mathrm{H})= & 0.65445 \\ & = & 24\end{array}$

(SEE MANUAL FOR EXPLANATION AND CAVEATS)
INTERNAL (DECAY) SOURCE
$(W)=1.813 E+07$
CHEMICAL REACTION SQURCE
$(W)=3.591 E+06$

$\checkmark$ HEAT LOSS TO CONCRETE

HEATUP OF ABLATION PROOUCTS $(H)=1.243 E+06$

HEAT LOSS FROM SURFACE $\quad(W)=1.592 E+07$

(TO COOLANT)

CHANGE IN POOL ENTHALPY

$(H)=-2.126 E+06$

(SUMMATION OF M*DH/DT)

NUMERICAL CHECKS ON MASS AMD EMERGY CONSERVATION

RELATIVE ERROR IN MASS $=9.41733 E-06$

RELATIVE ERROR IN ENTHALPY $=9.41753 E-06$

CHECK ON RECESSION CALCULATION (DD/DS SHOULD BE .LE. 1)

MAXIMUM DD/DS $=0.01143$

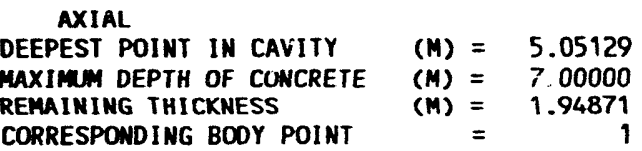

CORRESPONDING BODY POINT

1.94871

APPROXIMATE OVERALL ENERGY PUDGET FOR DEBRIS 
Table 6.2 Output listing for the CORCON standard problem (continued)

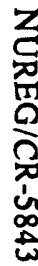

TIME $=16200.00$

CORCON VERSION 2.26

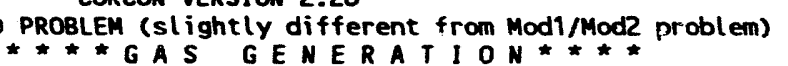

IT. NO. $=180$

GAS EXITING POOL (INCLUDES FILM AND COOLANT)

\begin{tabular}{|c|c|c|c|c|c|}
\hline \multirow{4}{*}{$\begin{array}{l}\text { SPECIES } \\
\text { C(G) } \\
\mathrm{CH} 4\end{array}$} & \multicolumn{2}{|l|}{ GEMERAT } & \multicolumn{2}{|r|}{ RELEASE } & \multirow{3}{*}{$\begin{array}{l}\text { SPECIES } \\
C(G)\end{array}$} \\
\hline & MASS $(K G / S)$ & MOLES $(1 / \$)$ & MASS (KG) & MOLES $(-)$ & \\
\hline & $2.50932 E-10$ & $2.08918 E-08$ & $5.72707 E-06$ & $4.76819 E-04$ & \\
\hline & $1.59294 E-04$ & $9.92945 E-03$ & 4.06591E-01 & $2.53444 E+01$ & $\mathrm{CH}_{4}$ \\
\hline co & $2.48936 E-01$ & $8.88729 E+00$ & $1.80765 E+03$ & $6.45349 E+04$ & co \\
\hline $\mathrm{CO} 2$ & $5.87581 E-02$ & $1.33534 \mathrm{E}+\mathrm{CO}$ & $2.26163 E+02$ & $5.13892 E+03$ & $\mathrm{co2}$ \\
\hline $\mathrm{C2} \mathrm{HZ}$ & $0.00000 E+00$ & $0.00000 E+00$ & $0.00000 E+00$ & $0.00000 E+00$ & $\mathrm{C} 2 \mathrm{H} 2$ \\
\hline $\mathrm{C}^{2} \mathrm{H}_{4}$ & $4.94777 \mathrm{E}-05$ & $1.76372 E-03$ & $9.37977 \mathrm{E}-02$ & $3.34359 E+00$ & $\mathrm{C} 2 \mathrm{H} 4$ \\
\hline $\mathrm{C2} \mathrm{H} 6$ & $1.69260 E-08$ & 5.62896E-07 & 2.69389E-05 & $8.95890 E-04$ & $\mathrm{C2} \mathrm{H} 6$ \\
\hline $\begin{array}{l}\mathrm{H} \\
\mathrm{H} 2\end{array}$ & $\begin{array}{l}2.04044 E-05 \\
0.67077 F-03\end{array}$ & $2.02445 E-02$ & $3.34079 E-01$ & SOE +02 & $\stackrel{H}{H 2}$ \\
\hline H2O & $7.62427 \mathrm{E}+00$ & $4.23213 \mathrm{E}+02$ & $5.01489 E+04$ & $\begin{array}{l}3.48209 E+04 \\
2.78370 E+06\end{array}$ & $\begin{array}{l}172 \\
\mathrm{H} 2 \mathrm{O}\end{array}$ \\
\hline N & $0.00000 E+00$ & $0.00000 E+00$ & $0.00000 E+00$ & $0.00000 E+00$ & $N$ \\
\hline NH3 & $0.00000 E+00$ & $0.00000 E+00$ & $0.00000 E+00$ & $0.00000 E+00$ & NH3 \\
\hline N2 & $0.06000 E+00$ & $0.00000 E+00$ & $0.00000 E+00$ & $0.00000 E+00$ & N2 \\
\hline 0 & $1.91931 \mathrm{E}-12$ & $1.19961 E-10$ & $4.64784 \mathrm{E}-07$ & $01 E-05$ & o \\
\hline 02 & $2.71057 \mathrm{E}-17$ & $8.47086 E-16$ & $2.96563 E-11$ & $9.26793 \mathrm{E}-10$ & 02 \\
\hline $\mathrm{OH}$ & $6.57951 E-10$ & $3.86864 E-08$ & $4.25586 E-05$ & $2.50237 \mathrm{E}-03$ & $\mathrm{OH}$ \\
\hline Сно & $2.46702 E-07$ & $8.50161 E-06$ & $2.58788 E-03$ & $8.91811 E-02$ & CHO \\
\hline $\mathrm{CH} 2 \mathrm{O}$ & $1.56753 E-0 ?$ & 5.22054E-06 & $9.59070 E-04$ & $3.19411 E-02$ & CH2O \\
\hline CRO3(G) & $4.38491 E-22$ & $4.38516 E-21$ & $1.39577 \mathrm{E}-15$ & $1.39586 E-14$ & $\operatorname{CRO3}(G)$ \\
\hline FPMO2(G) & $7.18199 E-17$ & $5.51032 E-16$ & $7.19931 E-11$ & $5.52361 E-10$ & FPMO2(G) \\
\hline FPMO3 (G) & $3.59882 E-21$ & $2.45928 E-20$ & $1.07032 E-14$ & $7.31408 E-14$ & FPMO3 (G) \\
\hline AL202(G) & $0.00000 E+00$ & $0.00000 E+00$ & $0.00000 E+00$ & $0.00000 \mathrm{E}+00$ & AL202(G) \\
\hline AL20(G) & $0.00000 E+00$ & $0.00000 E+00$ & $0.00000 E+00$ & $0.00000 E+00$ & $A L 20(G)$ \\
\hline & $D O O E+00$ & $0.00000 E+C O$ & $0.00000 E+00$ & $0.00000 E+00$ & $A L O(G)$ \\
\hline OALH(G) & $0.00000 E+00$ & $0.00000 E+00$ & $0.00000 E+00$ & $0.00000 E+00$ & OALH(G) \\
\hline ALOH(G) & $10 E+00$ & $00 E+00$ & $0.00000 E+00$ & $0.00000 E+00$ & ALOH(G) \\
\hline OALOH(G) & OOOE+OO & $0.00000 E+00$ & $0.00000 E+00$ & $0.00000 E+00$ & OALOH(G) \\
\hline ALO2(G) & $300 E+C O$ & $0.00000 E+00$ & $0.00000 E+00$ & $0.00000 E+00$ & ALO2(G) \\
\hline
\end{tabular}


Table 6.2 Output listing for the CORCON standard problem (continued)

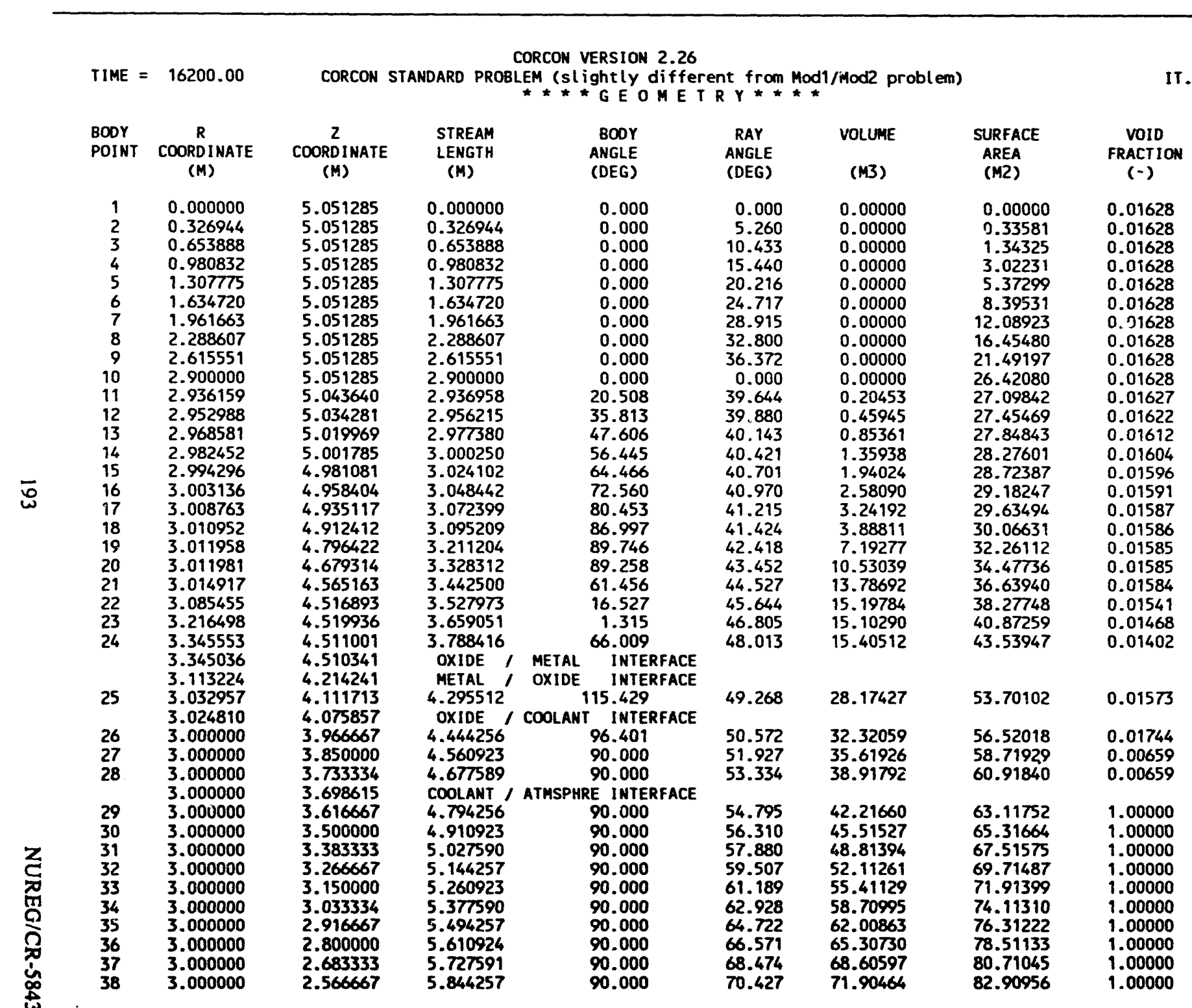


Table 6.2 Output listing for the CORCON standard problem (continued)

$\begin{array}{lllll}39 & 3.000000 & 2.450000 & 5.960924 & 90.000 \\ 40 & 3.000000 & 2.333333 & 6.077591 & 90.000 \\ 41 & 3.000000 & 2.216667 & 6.194258 & 90.000 \\ 42 & 3.000000 & 2.100000 & 6.310925 & 90.000 \\ 43 & 3.000000 & 1.983333 & 6.427591 & 90.000 \\ 44 & 3.000000 & 1.866667 & 6.544258 & 90.000 \\ 45 & 3.000000 & 1.750000 & 6.660925 & 90.000 \\ 46 & 3.000000 & 1.633333 & 6.777592 & 90.000 \\ 47 & 3.000000 & 1.516667 & 6.894258 & 90.000 \\ 48 & 3.000000 & 1.400000 & 7.010925 & 90.000 \\ 49 & 3.000000 & 1.283333 & 7.127592 & 90.000 \\ 50 & 3.000000 & 1.166667 & 7.244259 & 90.000 \\ 51 & 3.000000 & 1.050000 & 7.360926 & 90.000 \\ 52 & 3.000000 & 0.933333 & 7.477592 & 90.000 \\ 53 & 3.000000 & 0.816667 & 7.594259 & 90.000 \\ 54 & 3.000000 & 0.700000 & 7.710926 & 90.000 \\ 55 & 3.000000 & 0.583333 & 7.827592 & 90.000 \\ 56 & 3.000000 & 0.466667 & 7.944259 & 90.000 \\ 57 & 3.000000 & 0.350000 & 8.060926 & 90.000 \\ 58 & 3.000000 & 0.233334 & 8.177593 & 90.000 \\ 59 & 3.000000 & 0.116667 & 8.294260 & 90.000 \\ 60 & 3.000000 & 0.000000 & 8.410927 & 90.000\end{array}$

\subsection{9}

74.476

76.564

$78.69 \mathrm{C}$

80.848

83.032

85.236

87.455

89.682

91.909

94.131

96.340

98.531

100.697

104.931

106.991

100.006

110.973

112.891

114.755

0.000000

8.294260
8.410927

90.000

114.755
116.565

75.20332

81.50198
81.80066

85.09933

85.09933

8.39000

91.69667

94.99535

98.29401
101.59269

104.89136

108.19003

111.48870

14.78737

118.08604

121.38472

124.68338

127.98206

131.28073

134.57941

137.87807

141.17674
144.47542
85.10868

\subsection{9}

89.50691

91.70602

93.90514

98.10426

100.50249

102.70161

107.09984

109.29895

111.49806

113.69718

115.89630

18.8950

118.09541

122.49365

122.49365

124.69277

126.89188

131.29012
1.00000

1.00000

1.00000

1.00000

.00000

1.00000

1.00000

1.00000

1.00000

1.00000

1.00000

1.00000

1.00000

1.00000
1.00000

1.00000

1.00000

1.00000

1.0000

1.00000

1.00000

1.00000

1.00000
$i .00000$ 
Table 6.2 Output listing for the CORCON standard problem (continued)

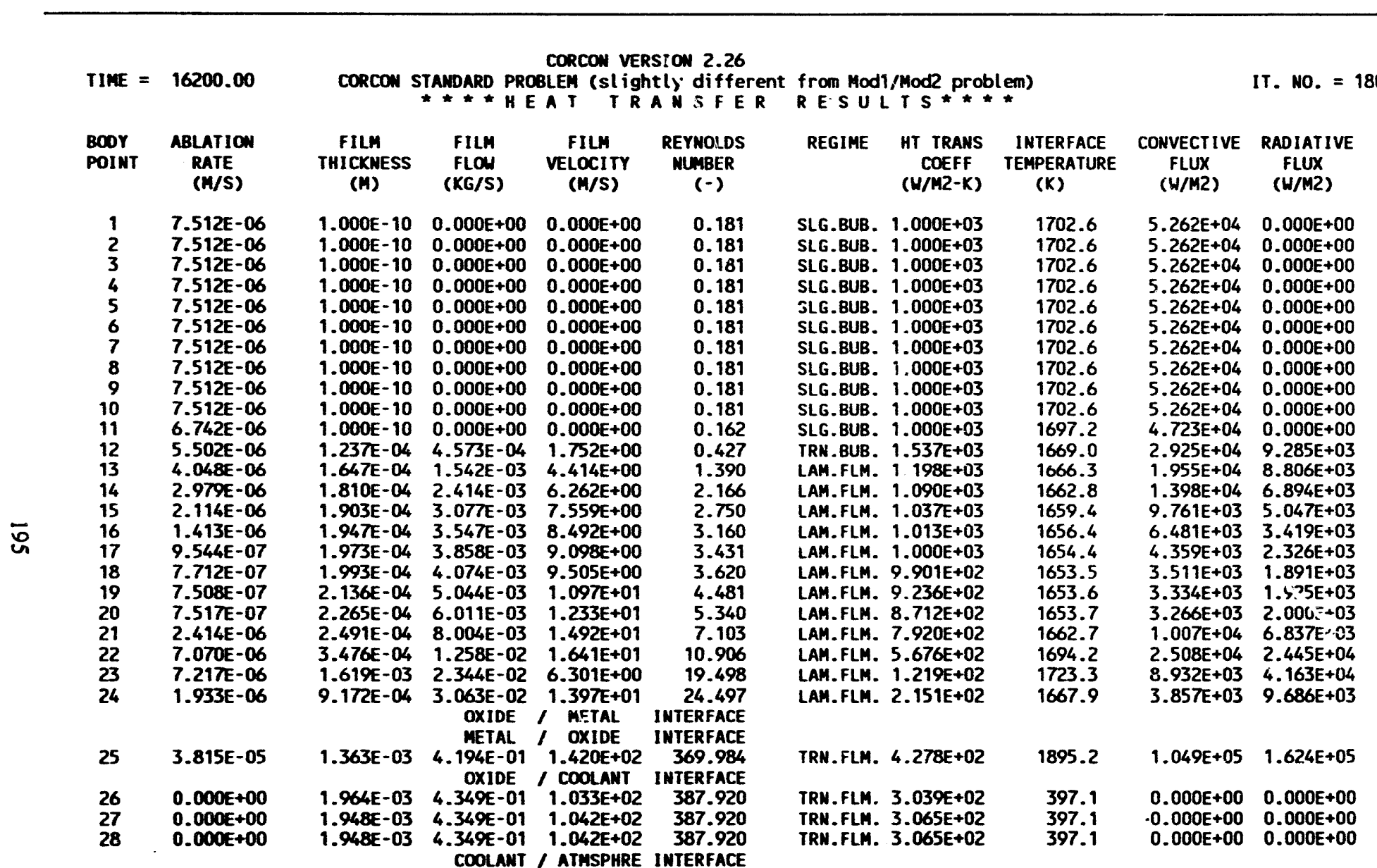


Table 6.2 Output listing for the CORCON standard problem (continued)

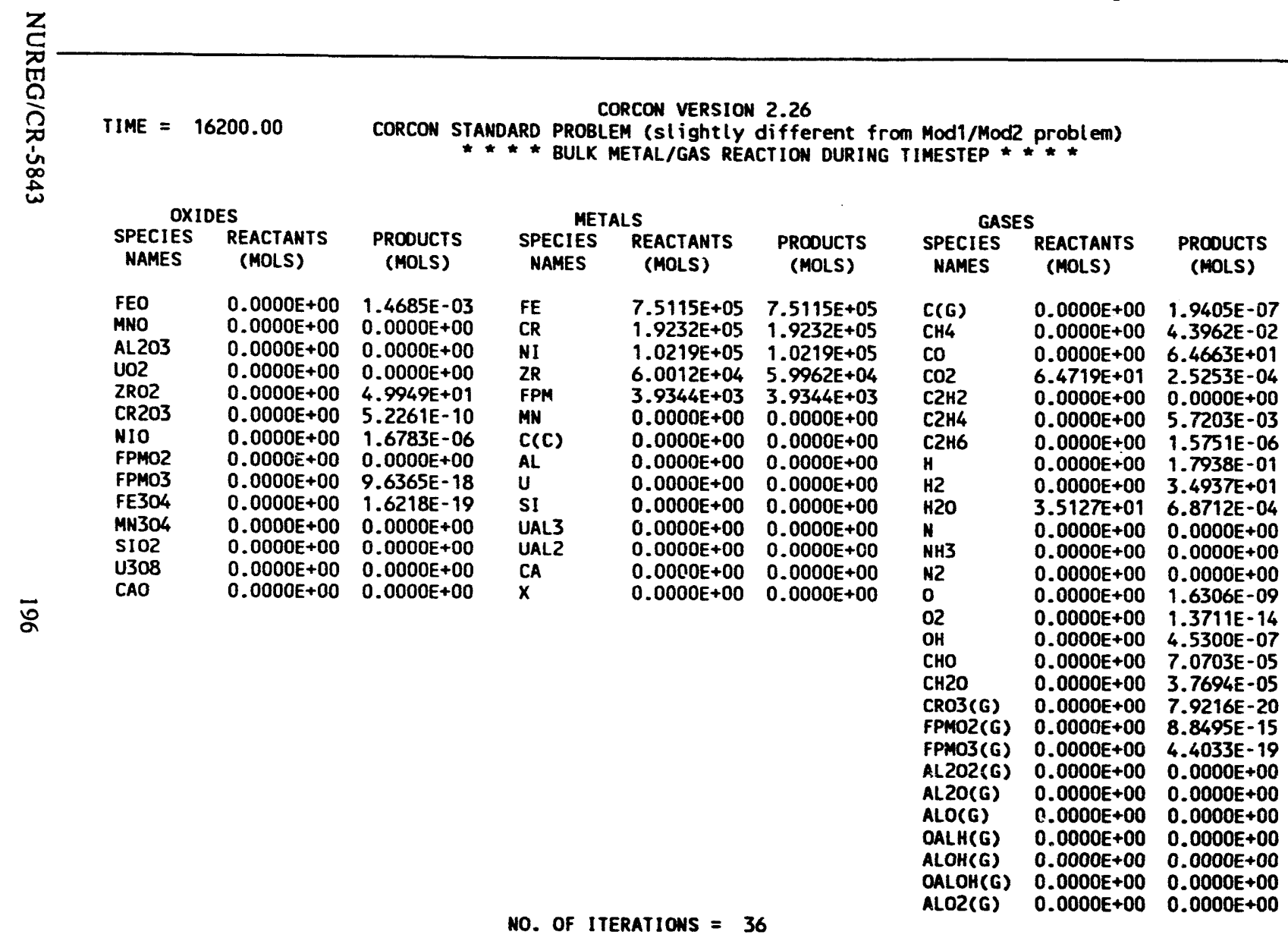


Table 6.2 Output listing for the CORCON standard problem (continued)

TIME $=16200.00$

OXIDES OXIDES SPECIES REACTAN

ACTANTS

$0.0000 \mathrm{E}+00$

$0.0000 \mathrm{E}+00 \quad 0.0000 \mathrm{E}+00$

$0.0000 E+00 \quad 0.0000 E+00$

$0.0000 E+00 \quad 0.0000 E+00$

$0.0000 \mathrm{E}+00 \quad 1.5623 \mathrm{E}+02$

$0.0000 E+00 \quad 9.1865 E-10$

$0.0000 E+00 \quad 3.6791 E-06$

$0.0000 E+00 \quad 0.0000 E+00$

$0.0000 E+00 \quad 8.9785 E-18$

$0.0000 \mathrm{E}+00 \quad 1.2365 \mathrm{E}-19$

$0.0000 \mathrm{E}+00 \quad 0.0000 \mathrm{E}+00$

$\begin{array}{lll}\text { MN3O4 } & 0.0000 E+00 & 0.0000 E+00 \\ \text { U308 } & 0.0000 E+00 & 0.0000 E+00\end{array}$

$\overline{9}$

CAO
CORCON VERSION 2.26

ANDARD PROBLEM (slightly different from Mod1/Mod2 problem)
IT. NO. $=180$

NO. OF ITERATIONS $=36$

GASES

$\begin{array}{lcc}\begin{array}{l}\text { SPECIES } \\ \text { NAMES }\end{array} & \begin{array}{c}\text { REACTANTS } \\ \text { (MOLS) }\end{array} & \begin{array}{c}\text { PRODUCTS } \\ \text { (MOLS) }\end{array} \\ \text { CCG) } & 0.0000 E+00 & 4.3271 E-07 \\ \text { CH4 } & 0.0000 E+00 & 2.5392 E-01 \\ \text { CO } & 0.0000 E+00 & 2.0196 E+02 \\ \text { CO2 } & 2.0230 E+02 & 5.7203 E-04 \\ \text { C2H2 } & 0.0000 E+00 & 0.0000 E+00 \\ \text { C2H4 } & 0.0000 E+00 & 4.7191 E-02 \\ \text { C2H6 } & 0.0000 E+00 & 1.5312 E-05 \\ H & 0.0000 E+00 & 4.2796 E-01 \\ \text { H2 } & 0.0000 E+00 & 1.0899 E+02 \\ \text { H2O } & 1.0981 E+02 & 1.5092 E-03 \\ \text { N } & 0.0000 E+00 & 0.0000 E+00 \\ \text { NH3 } & 0.0000 E+00 & 0.0000 E+00 \\ \text { N2 } & 0.0000 E+00 & 0.0000 E+00 \\ \text { O } & 0.0000 E+00 & 1.9682 E-09 \\ \text { O2 } & 0.0000 E+00 & 1.1701 E-14 \\ \text { OH } & 0.0000 E+00 & 7.0759 E-07 \\ \text { CHO } & 0.0000 E+00 & 1.8434 E-04 \\ \text { CH2O } & 0.0000 E+00 & 1.1892 E-04 \\ \text { CRO3(G) } & 0.0000 E+00 & 5.2339 E-20 \\ \text { FPMO2(G) } & 0.0000 E+00 & 7.6814 E-15 \\ \text { FPMO3(G) } & 0.0000 E+00 & 2.9745 E-19 \\ \text { AL2O2(G) } & 0.0000 E+00 & 0.0000 E+00 \\ \text { AL2O(G) } & 0.0000 E+00 & 0.0000 E+00 \\ \text { ALO(G) } & 0.0000 E+00 & 0.0000 E+00 \\ \text { OALH(G) } & 0.0000 E+00 & 0.0000 E+00 \\ \text { ALOH(G) } & 0.0000 E+00 & 0.0000 E+00 \\ \text { OALOH(G) } & 0.0000 E+00 & 0.0000 E+00 \\ \text { ALO2(G) } & 0.0000 E+00 & 0.0000 E+00\end{array}$

$\begin{array}{lll}A L 02(G) & 0.0000 E+00 & 0.0000 E+00\end{array}$ 
Table 6.2 Output listing for the CORCON standard problen: (continued)

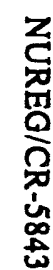

CORCOW VERSION 2.26

TIME $=16200.00 \quad$ CORCON STANDARD PROBLEM (slightly different from Mod1/Mod? problem)

IT. NO. $=180$

OXIDE METAL OXIDE COOLANT

MASS OF LAYER $1.2424 E+05 \quad 6.3804 E+04 \quad 1.3990 E+04 \quad 9.9014 E+03$

mSS OF SPECIES

\begin{tabular}{|c|c|c|}
\hline $\begin{array}{l}\text { FPALIKAET } \\
\text { FPHALOG } \\
\text { FE304 } \\
\text { FE }\end{array}$ & $\begin{array}{l}1.3383 E+03 \\
6.7653 E+00 \\
5.9750 E+03 \\
1.1275 E+00 \\
1.8040 E+01 \\
1.1764 E+03 \\
4.4579 E-01 \\
1.3185 E+01 \\
5.4122 E+01 \\
1.3528 E+02 \\
9.9997 E+4 \\
1.4000 E+04 \\
5.2406 E-01 \\
0.0000 E+00 \\
0.0000 E+00 \\
0.0000 E+00 \\
1.5109 E+03 \\
1.1119 E+01 \\
4.3336 E-01 \\
0.0000 E+00\end{array}$ & $\begin{array}{l}4.1958 E+04 \\
1.3000 E+04 \\
5.9995 E+03 \\
5.4555 E+03 \\
3.9049 E+02\end{array}$ \\
\hline
\end{tabular}

$3.8309 \mathrm{E}+03$

$1.9338 \mathrm{E}+01$

$1.6931 \mathrm{E}-01$

$3.2230 E+00$
$5.1567 E+01$

$3.3626 \mathrm{E}+03$

$1.2506 \mathrm{E}+00$

$3.8901 E+01$

$1.5471 E+02$

3.8672E+02

$0.0000 E+00$

$6.1388 \mathrm{E}+03$

$1.4996 E+00$

$2.3492 E-04$

$4.0894 \mathrm{E}-34$

$3.8946 \mathrm{E}-14$

$0.0000 E+00$

$0.0000 E+00$

$0.0000 E+00$

$1.6749 E-15$

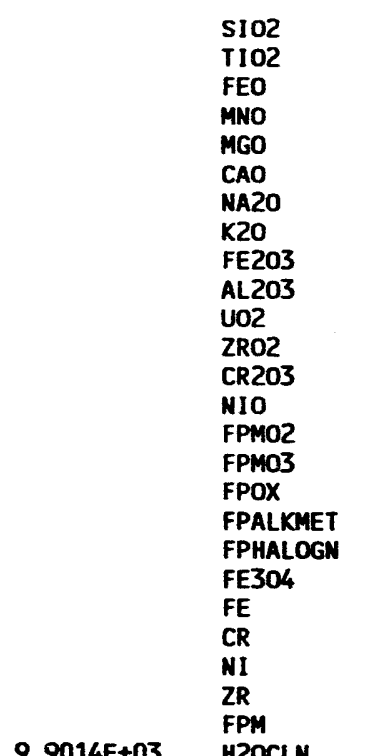

$9.9014 E+03$

HZOCLN 
Table 6.2 Output listing for the CORCON standard problem (continued)

TIME $=16200.00$

$\begin{array}{lcc} & & \text { OXIDE } \\ \text { MASS } & \text { (KG) } & 1.2424 E+05 \\ \text { DENSITY } & \text { (KG/M3) } & 8.1834 E+03 \\ \text { THEREAL EXPANSIVITY } & (1 / K) & 3.5134 E-05\end{array}$

AVEPAGE TEMPERATURE

$\begin{array}{ccc}\text { (K) } & 2.1751 E+03 & 2.1677 \mathrm{E}+03 \\ \text { (K) } & 1.7026 \mathrm{E}+03 & 2.1745 \mathrm{E}+03\end{array}$

INTERFACE TEMPERAT

SOLIDUS TEMPERATURE

LIOUIDUS TEMPERATURE

SPECIFIC ENTHALPY

(K)

$\begin{array}{ccc}\text { (K) } & 2.1751 E+03 & 2.1677 \mathrm{E}+03 \\ \text { (K) } & 1.7026 \mathrm{E}+03 & 2.1745 \mathrm{E}+03\end{array}$

(K) $\quad 1.6678 E+03$

$2.1663 \mathrm{E}+03$

$2.1663 \mathrm{E}+03$

$2.7876 \mathrm{E}+06$

$(\mathrm{K})$

TOTAL ENTHALPY

$(\mathrm{J} / \mathrm{KG}$

SPECIFIC HEAT

(J/KG K)

VISCOSITY

(KG/M S)

THERMAL CONDUCTIVITY (W/M K)

THERMAL DIFFUSIVITY (M2/S)

SURFACE TENSION

$(N / M)$

$(-)$

$-5.2026 E+11$

$5.6732 E+02$

$1.7888 E+02$

$2.8134 E+00$

6.0599E-07

$4.8896 \mathrm{E}-01$

8.0000E-01

CORCON VERSION 2.26 (stightly different from Mod1/Mod2 problem)

IT. NO. $=180$

SUPERFICIAL GAS VEL

BUBBLE RADIUS

BUBBLE VELOCITY

VOID FRACTION

$\begin{array}{ccc}\text { (M/S) } & 6.0021 E-03 & 8.2516 \mathrm{E}-03 \\ \text { (M) } & 1.7714 \mathrm{E}-03 & 1.84\end{array}$

(M/S) $\quad 4.6921 \mathrm{E}-04$

4.6921E-04
$1.5844 \mathrm{E}-02$

BOT CRUST THICKNESS (H)
HT COEFF, LIO TO BOT (H/M2

$(-)$

Z-AVE LIO TEMPERATURE (K)

HT COEFF, LIQ TO TOP (H/MZ K)

TOP CRUST THICKNESS

(M)

R-AVE LIQ TEMPERATURE (K)

HT COEFF, LIO TO SID (H/KR K)

SIDE CRUS'T THICKNESS

(M)

DECAY HEAT

HEAT TO CONCRETE

MEAT OF REACTION

(W)

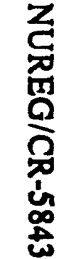

$4.8306 E+02 \quad 7.1721 E+04 \quad 2.8838 E+04 \quad 1.0386 E+03$

$2.1906 E+03 \quad 2.1677 E+03 \quad 2.1408 E+03 \quad 3.9714 E+02$

$3.0639 E+04 \quad 7.5815 E+04 \quad 1.2956 E+03 \quad 2.7328 E+04$

$\begin{array}{llll}0.0000 E+00 & 0.0000 E+00 & 0.0000 E+00 & 0.0000 E+00\end{array}$

$\begin{array}{llll}2.2325 E+03 & 2.1677 E+03 & 2.1408 E+03 & 3.9714 E+02 \\ 7.0102 E+01 & 1.3018 E+04 & 1.0882 E+03 & 1.0000 E-10\end{array}$

$1.0000 E-10$

$\begin{array}{llll}1.6045 E+07 & 2.0809 E+06 & 1.4077 E-10 & 0.0000 E+00\end{array}$

$\begin{array}{llll}1.8039 E+06 & 4.0124 E+06 & 8.6398 E+05 & 0.0000 E+00\end{array}$

$0.0000 E+00 \quad 3.5914 E+06 \quad 0.0000 E+00 \quad 0.0000 E+00$

$\begin{array}{llll}1.4246 E+07 & 1.6236 E+07 & 1.5925 E+07 & -9.5822 E+04\end{array}$ 
Table 6.2 Output listing for the CORCON standard problem (continued)

TIME $=16200.00$

\section{CORCON VERSION 2.26}

(slightly different from Mod1/ModR problem)

IT. NO. $=180$

TEMPERATURE OF METAL (K)

TEMPERATURE OF OXIDE (K)

AEROSOL - AMBIENT CONDITIONS (G/CC)

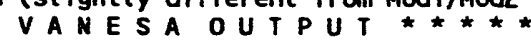

( 298.15 (K) AND 1 ATM. ) (G/CC)
(K)

GAS (G-MOLES/S)

AEROSOL RATE (GRAMS/S)

AEROSOL DENSITY (G/CMB)

PARTICLE SIZE (MICROMETERS)

BUBBLE DIAMTETER FOR THE METAL PHASE (CM)

$2.1677 E+03$
$2.1724 E+03$
$1.6011 E-05$
$4.5203 E-05$

$1.5768 \mathrm{E}+01$

$2.1587 E+00$

$5.1680 \mathrm{E}-01$

$3.7568 \mathrm{E}-01$

MECHANICAL RELEASES FRON THE AZBEL AND THE ISHII AND KATAOKA CORRELATIONS ARE CALCULATED.

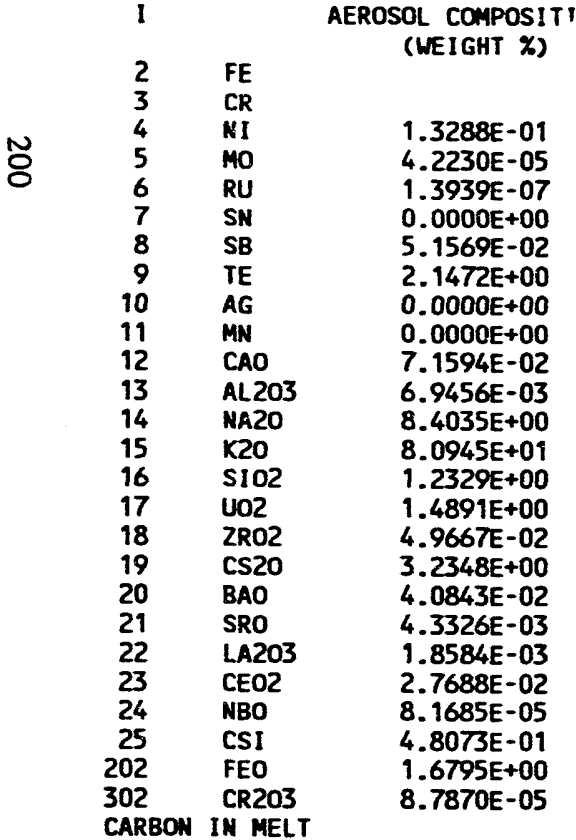

$$
\begin{gathered}
\text { (KG) } \\
4.1957 E+04 \\
1.0000 E+04 \\
5.9995 E+03 \\
1.8118 E+02 \\
1.9193 E+02 \\
0.0000 E+00 \\
3.5001 E-01 \\
1.6951 E+01 \\
0.0000 E+00 \\
0.0000 E+00 \\
4.5390 E+03 \\
5.2200 E+02 \\
1.6966 E+00 \\
5.2091 E+01 \\
5.1692 E+03 \\
9.9986 E+04 \\
2.0139 E+04 \\
1.1987 E+01 \\
8.4491 E+01 \\
6.5023 E+01 \\
4.9684 E+02 \\
1.0293 E+03 \\
3.9345 E+00 \\
9.3738 E-01 \\
5.9752 E+03 \\
2.0237 E+00 \\
0.0000 E+00 \\
5.4555 E+03
\end{gathered}
$$

$\begin{array}{ll}1.1841 E-02 & 1.1475 E-04 \\ 2.3028 E-06 & 1.5092 E-07 \\ 7.2155 E-09 & 4.6916 E-09 \\ 0.0000 E+00 & 0.0000 E+00 \\ 2.2160 E-03 & 5.5855 E-01 \\ 8.8038 E-02 & 2.1298 E-01 \\ 0.0000 E+00 & 0.0000 E+00 \\ 0.0000 E+00 & 0.0000 E+00 \\ 6.6791 E-03 & 3.1164 E-05 \\ 3.5638 E-04 & 1.3888 E-04 \\ 7.0935 E-01 & 8.5309 E-01 \\ 4.4954 E+00 & 7.0141 E-01 \\ 1.0735 E-01 & 4.2576 E-03 \\ 2.8854 E-02 & 3.2393 E-05 \\ 2.1088 E-03 & 7.2723 E-07 \\ 6.0052 E-02 & 6.8164 E-01 \\ 1.3935 E-03 & 2.8717 E-03 \\ 2.1875 E-04 & 4.2697 E-03 \\ 2.9841 E-05 & 6.4637 E-05 \\ 8.4160 E-04 & 1.0986 E-03 \\ 3.9241 E-06 & 3.6349 E-05 \\ 9.6804 E-03 & 6.9467 E-01 \\ 1.2230 E-01 & 4.1655 E-04 \\ 3.0246 E-06 & 2.1690 E-07\end{array}$

LOSS(MOLES)

RELEASE FRACTION

$.4555 E+03$ 
Table 6.2 Output listing for the CORCON standard problem (continued)

$\begin{array}{llcc}1 & & \begin{array}{c}\text { GAS COMPOSITION } \\ \text { (WEIGH } \% \text { ) }\end{array} & \begin{array}{c}\text { RELEASE RATE } \\ \text { (GRAMS/SECOND) }\end{array} \\ 1 & \text { H2O } & \begin{array}{l}4.6914 E+00 \\ 2\end{array} & 1.3327 E+01 \\ 2 & \text { H2 } & 3.0311 E+01 & 9.6350 E+00 \\ 3 & H & 1.6385 E-01 & 2.6042 E-02 \\ 4 & \text { OH } & 3.4767 E-03 & 9.3238 E-03 \\ 5 & 0 & 1.2898 E-05 & 3.2529 E-05 \\ 6 & 02 & 8.5013 E-07 & 4.2894 E-06 \\ 7 & \text { CO2 } & 1.9020 E+00 & 1.3199 E+01 \\ 8 & \text { CO } & 6.2928 E+01 & 2.7794 E+02\end{array}$

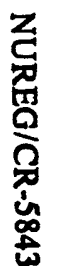


Table 6.2 Output listing for the CORCON standard problem (concluded)

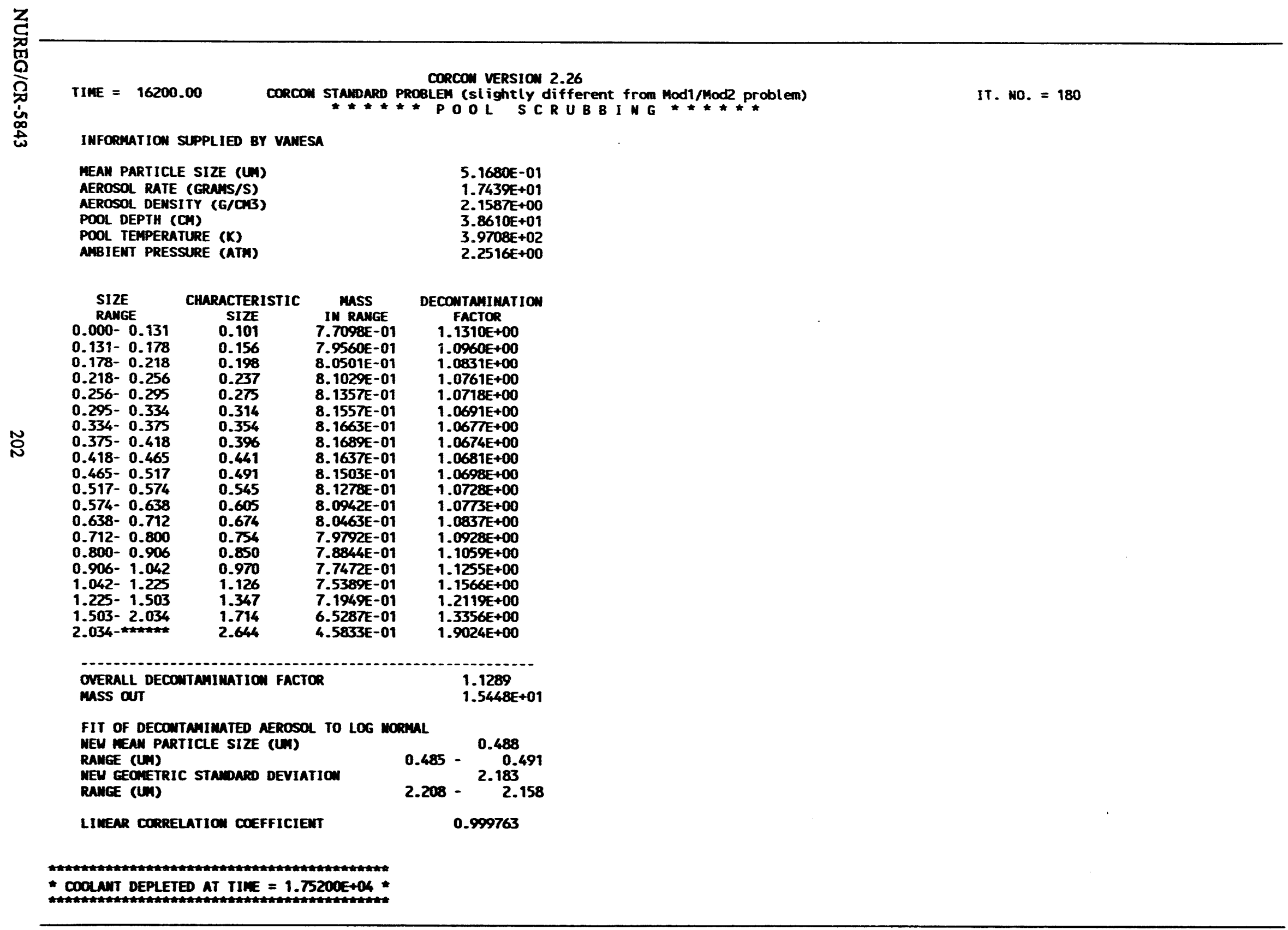

MEAM PARTICLE SIZE (UI)

AEROSOL RATE (GRAHS/S)

POOL DEPTH (CM)

POOL TEAPERATURE (K)

2.1587E+00

$3.9708 \mathrm{E}+02$

IN RAMGE

SIZE 7.9560E-01

$0.131-0.178$

$.178-0.218$

$.218-0.256$

0.156

$1.0761 E+00$

$0.275 \quad 8.1357 E-01 \quad 1.0718 E+00$

$0.314 \quad 8.1557 E-01 \quad 1.0691 E+00$

0.354 8.1603E-01 1.067F+00

8.1689E-01 1.0674E+00

0.441 8.1637E-01 1.0681E+00

$0.491 \quad 8.1503 \mathrm{E}-01-1.0698 \mathrm{O}+00$

$0.545 \quad 8.1278 E-01 \quad 1.0728 E+00$

0.605 8.0942E-01 1.0773E+00

$0.674 \quad 8.0463 E-01 \quad 1.0837 \mathrm{E}+00$

$1.0928 E+00$

$1.1059 E+00$

$1.1255 E+00$

$1.1566 \mathrm{E}+00$

$1.2119 E+00$

$\begin{array}{llll}1.503-2.034 & 1.714 & 6.5287 E-01 & 1.3356 E+00 \\ 2.034-2.644 & 4.5833 E-01 & 1.9024 E+00\end{array}$

OVERALL DECOUTAMIMATIOA FACTOR

1289

FIT OF DECONTMIMATED AEROSOL TO LOG MODMAL

ARTICLE SIZE (IN)

$2008-$

0.999763 
Table 6.3 Input listing for the BWR sumple problem

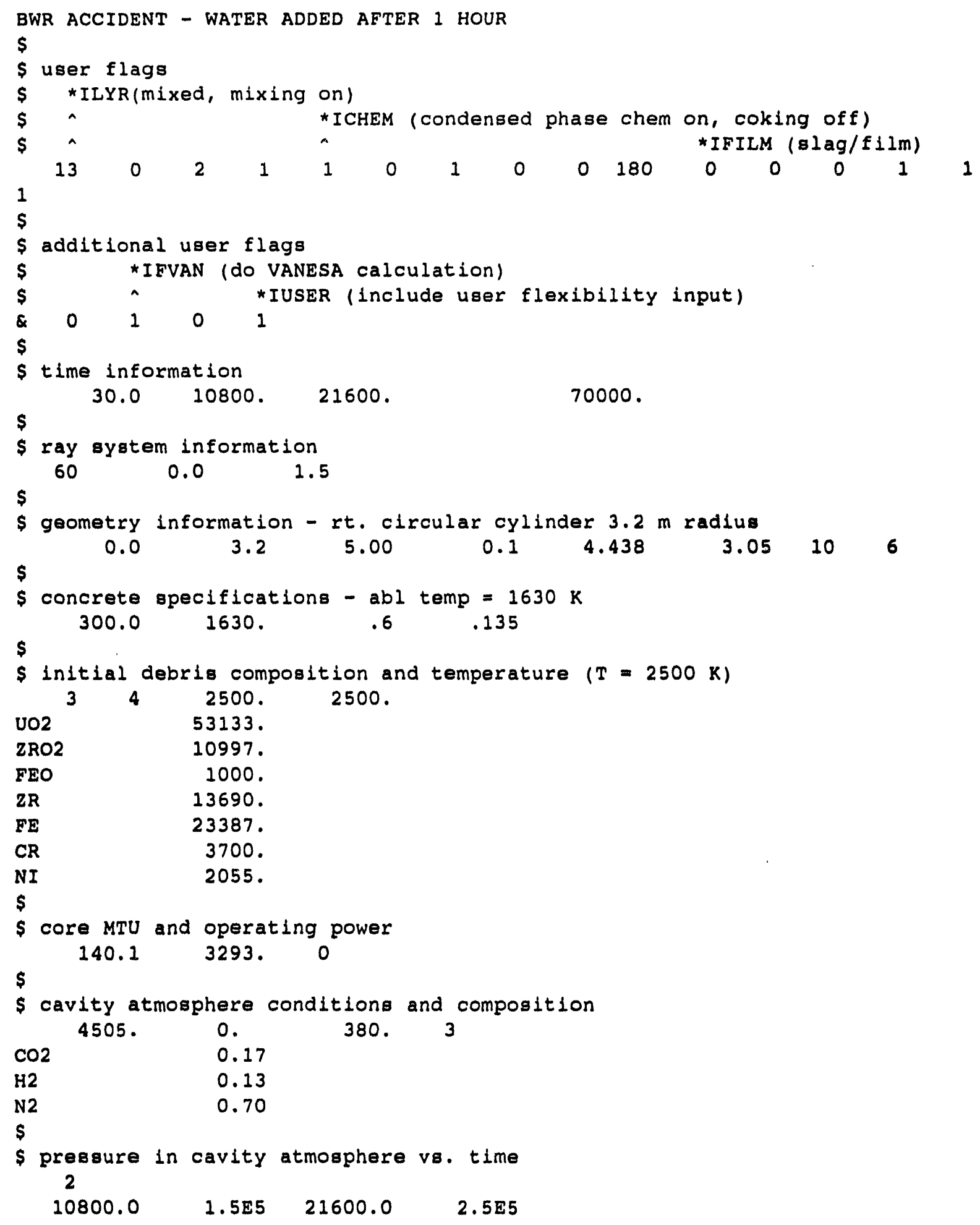


Table 6.3 Input listing for the BWR sample problem (continued)

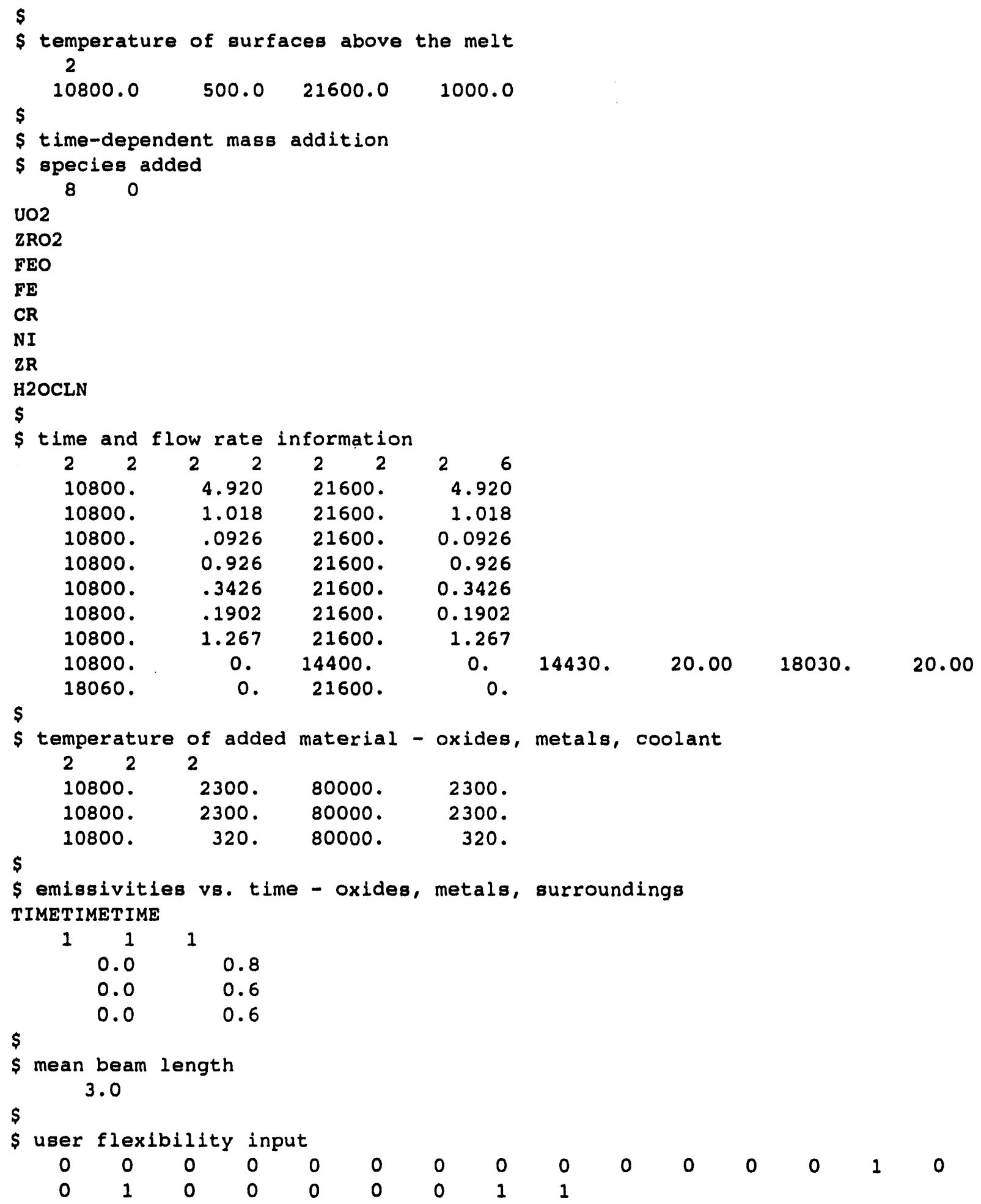


Table 6.3 Input listing for the BWR sample problem (continued)

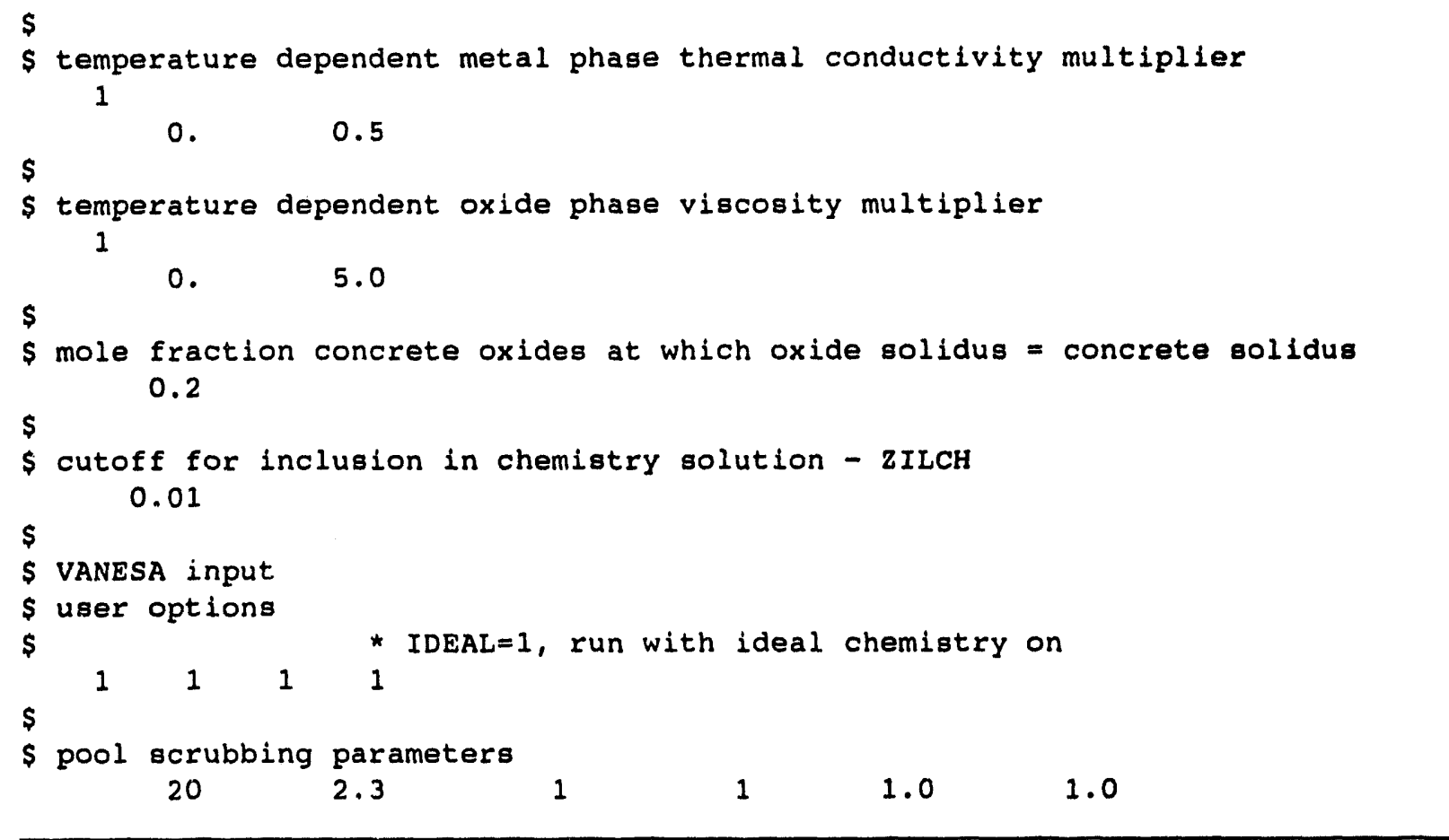


Table 6.4 Output listing for the BWR sample problem

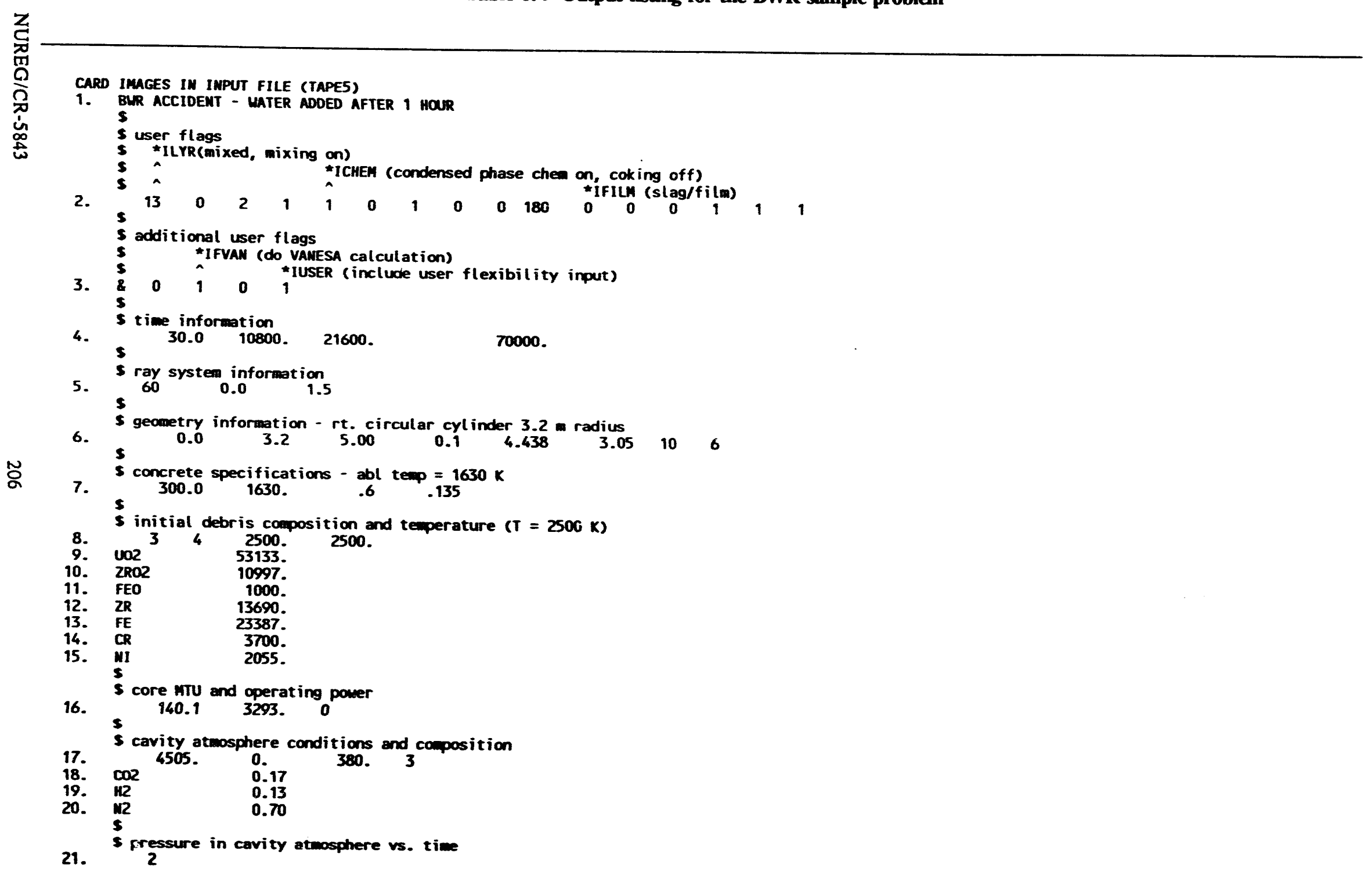




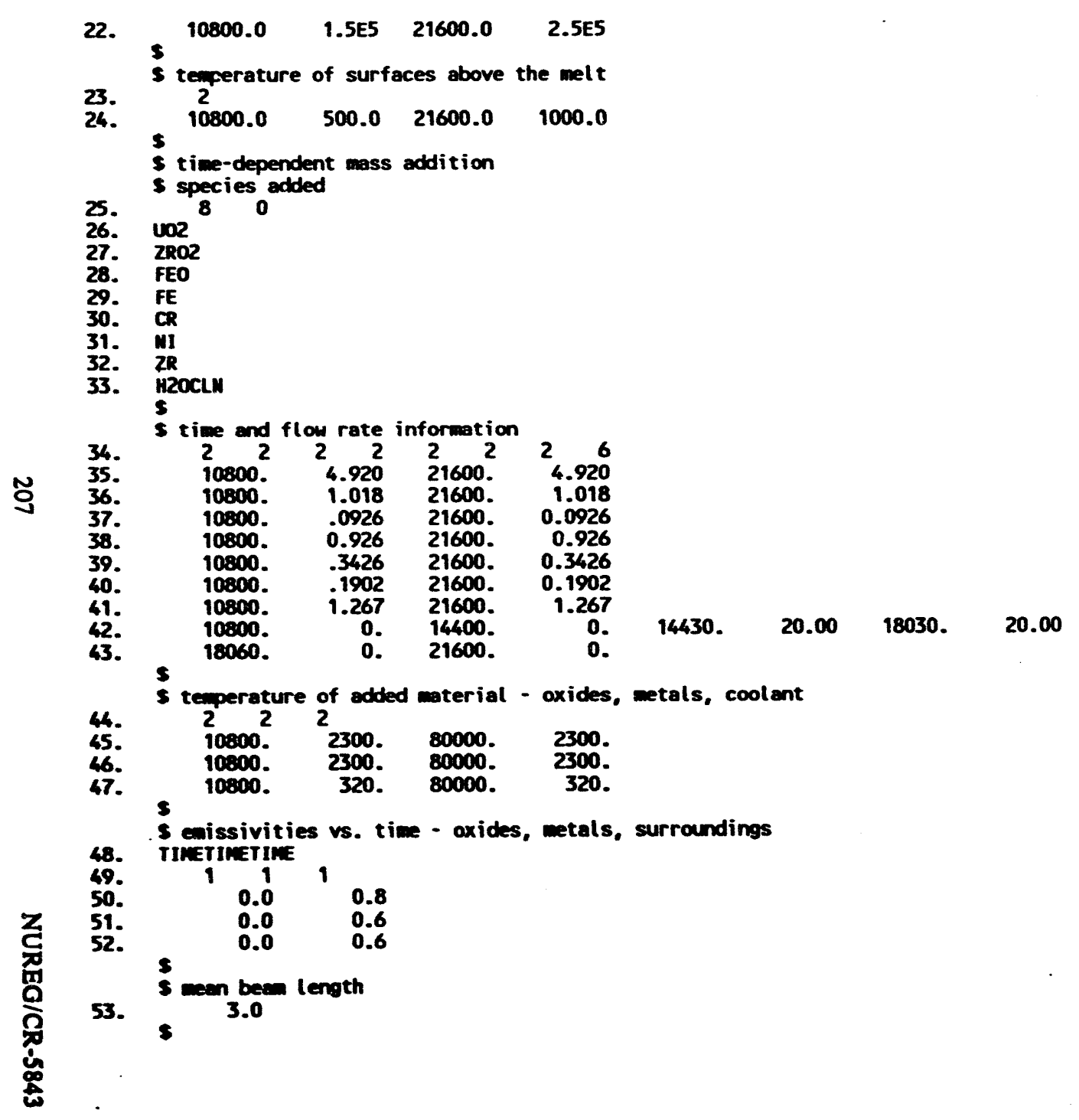


Table 6.4 Output listing for the BWR sample problem (continued)

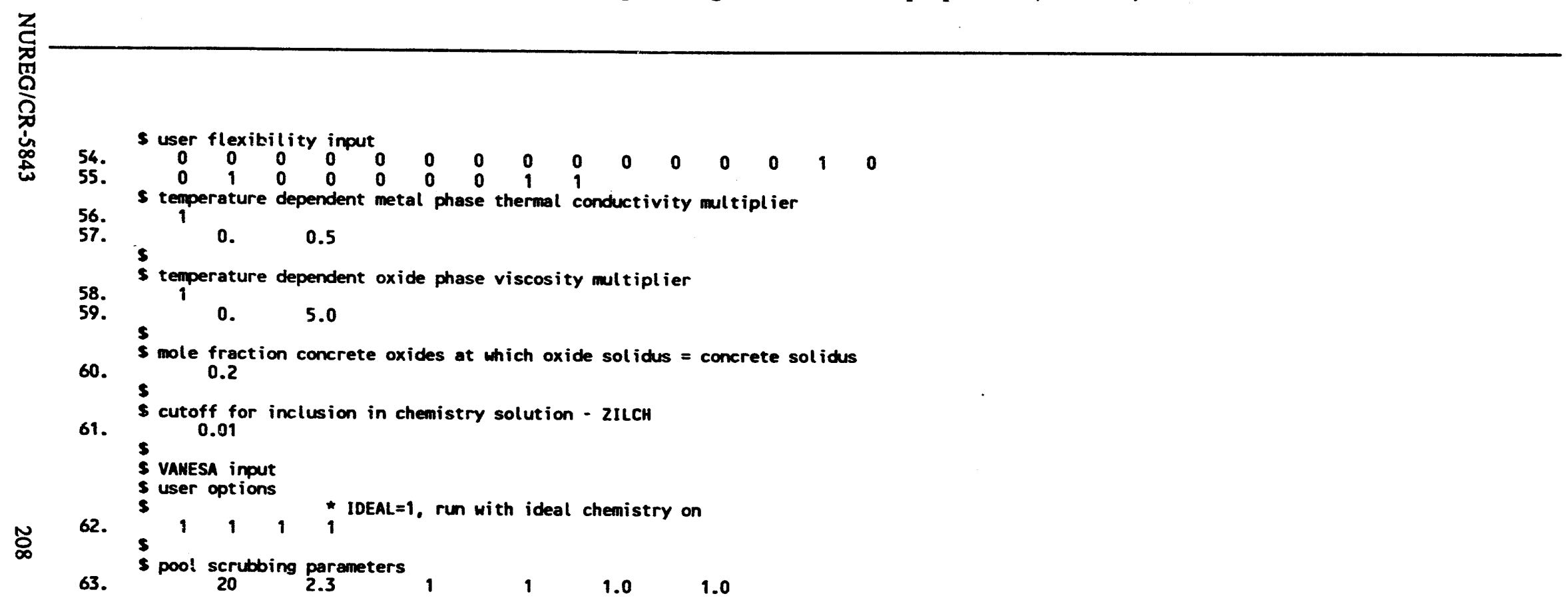


BWR ACCIDENT - HATER ADDED AFTER 1 HOUR

*** INPUT

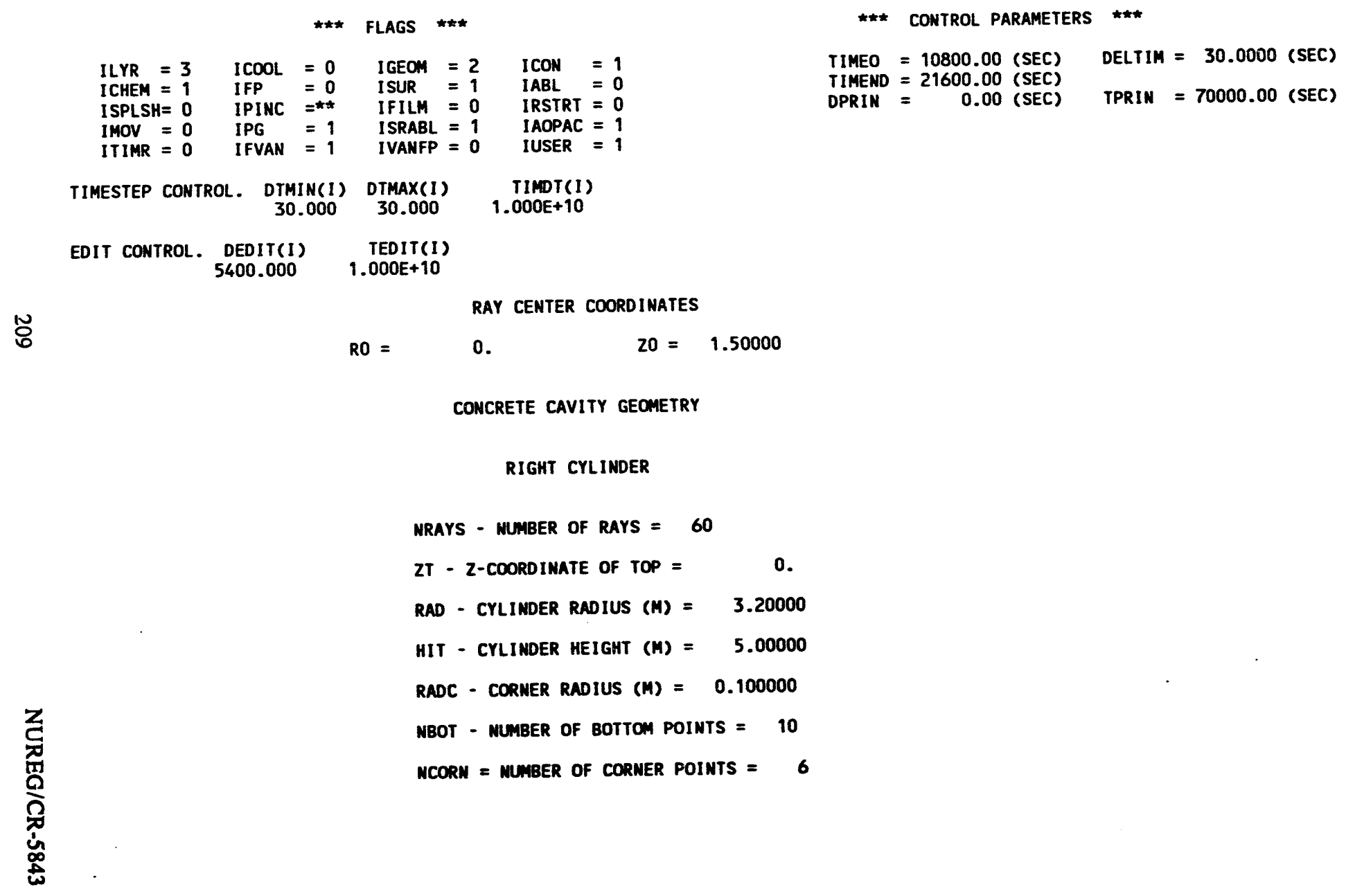


Table 6.4 Output listing for the BWR sample problem (continued)

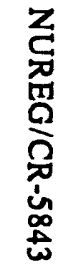

* * * CONCRETE SPECIFICATIONS * * *

BASALTIC AGGREGATE CONCRETE
RHOC

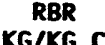
SI
TSOL
TLIO
IIC
(K)
DELH
(J/KG)
$\underset{(-)}{E W}$

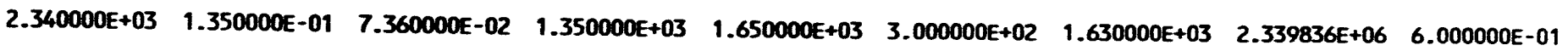
SPECIES MANE MASS FR.(KG/KG C) MOLECULAR WT.

$\begin{array}{lllr} & \text { SIO2 } & 0.5484 & 60.0843 \\ & \text { IIOZ } & 0.0105 & 79.8988 \\ & \text { MGO } & 0.0616 & 40.3044 \\ & \text { CAO } & 0.0882 & 56.0794 \\ & \text { NA20 } & 0.0180 & 61.9790 \\ & \text { K20 } & 0.0539 & 94.1960 \\ N & \text { FE203 } & 0.0626 & 159.6922 \\ 0 & \text { AL203 } & 0.0832 & 101.9613 \\ & \text { CO2 } & 0.0150 & 44.0098 \\ & \text { HZOEVAP } & 0.0386 & 18.0152 \\ & \text { HZOCHEM } & 0.0200 & 18.0152\end{array}$

MELT FISSION PROOUCT INVEMTORY

BASED ON 1.40100E+02 METRIC TON URANIUH CORE OPERATED AT $3.29300 E+03$ MH(THERMAL)

MELT CONTAIUS 4.68309E+01 METRIC TONS URANIUH, CORRESPONDING TO 33.43 PERCENT OF CORE

ELERENTS, RETAIWED FRACTIONS («DENOTES USER INPUT, OTHERS FROM WASH 1400), AND GRAM-ATOMS IN MELT

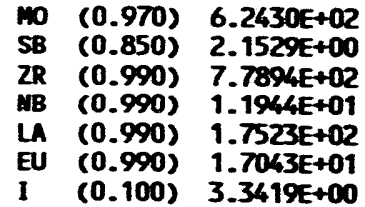

I $(0.100) \quad 3.3419 E+00$

$$
\text { M. } 46552 E-01
$$

TE $4.25953 E-02$

IC (0.970) 1.5973E+02

TE $(0.850) \quad 5.5801 E+01$

CE $(0.990)$ 4.0756E+02

PU $(0.990) 7.4516 E+02$

PR (0.990) $1.5235 \mathrm{E}+02$

RB $(0.190) 1.6673 \mathrm{E}+01$

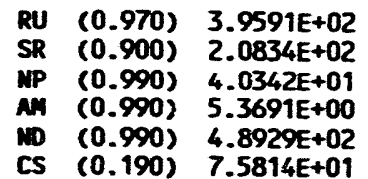

$\begin{array}{lll}\text { RH } & (0.970) & 7.2135 E+01 \\ \text { BA } & (0.900) & 1.8268 E+02\end{array}$

CM (0.990) 1.6858E+00

$Y(0.990) 1.1704 E+02$

SW (0.990) 5.7331E+01

BR $(0.100) 5.6457 E-01$

FISSION PRODUCTS GROUPED AS 4 PSELDO-SPECIES

HITH ATOAIC FRACTIOUS

RU 3.02216E-01

RH 5.50639E-02

SB $1.64339 E-03$

3.39030E+03 GRAN-ATOAS Of FPOX

SR $6.14511 E-02$

iA 5.16855E-02

\section{WITH ATOMIC FRACTIOUS}

BA 5.38829E-02

is $3.52284 E-03$

2R 2.29756E-01

PU 2.19792E-01 
9.24864E+01 GRAM-ATOMS OF FPALKMET UITH ATOMIC FRACTIONS

$$
\text { RB } 1.80272 E-01 \text { CS } 8.19728 E-01
$$

3.90643E+00 GRAM-ATOMS OF FPHALOGN MITH ATONIC FRACTIONS

$$
\text { BR } 1.44524 \mathrm{E}-01 \quad \text { I } 8.55476 \mathrm{E}-01
$$

INITIAL POUER AT START OF CORCON, 1.08000E+04 SEC AFTER SCRAM IS 6.86111 +06 MATTS

REPRESENTING 0.62 PERCENT OF OPERATING PONER OF FRACTION OF CORE IN MELT

INITIAL CORE MASSES (KG) OF CONSTITUENTS AND TEMPERATURES

$\begin{array}{lrrrrrr}\text { OXIDES } & \text { SPEMH } & \text { CONINP } & \text { METALS } & \text { SPEAW } & \text { CONINP } \\ \text { FEO } & 7.1846397 E+01 & 1.0000000 E+03 & \text { FE } & 5.5847000 E+01 & 2.3387000 E+04 \\ \text { UO2 } & 2.7002780 E+02 & 5.3133000 E+04 & \text { CR } & 5.1995998 E+01 & 3.7000000 E+03 \\ \text { ZRO2 } & 1.2321880 E+02 & 1.0997000 E+04 & \text { NI } & 5.8709999 E+01 & 2.0550000 E+03 \\ \text { FPOX } & 1.4748474 E+02 & 5.0001773 E+02 & \text { ZR } & 9.1220001 E+01 & 1.3690000 E+04 \\ \text { FPALKMET } & 1.2435149 E+02 & 1.1500827 E+01 & \text { FPM } & 9.9529694 E+01 & 1.3038666 E+02 \\ \text { FPHALOGN } & 1.2011028 E+02 & 4.7920219 E-01 & & & & \end{array}$

IMITIAL TEMPERATURE (TO) IN DEGREES $K=2.50001000+03$

INITIAL TEMPERATURE (TM) IN DEGREES $K=2.5000000 E+03$

INITIAL MASS OF OXIDES $=6.5641984 E+04$

INITIAL MASS OF ETALS $=4.2962387 \mathrm{E}+04$

$$
\text { * * reacting gas mixture * * * }
$$

** ATMOSPHERIC PRESSURE SPECIFIEd AS OF TIME

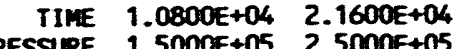

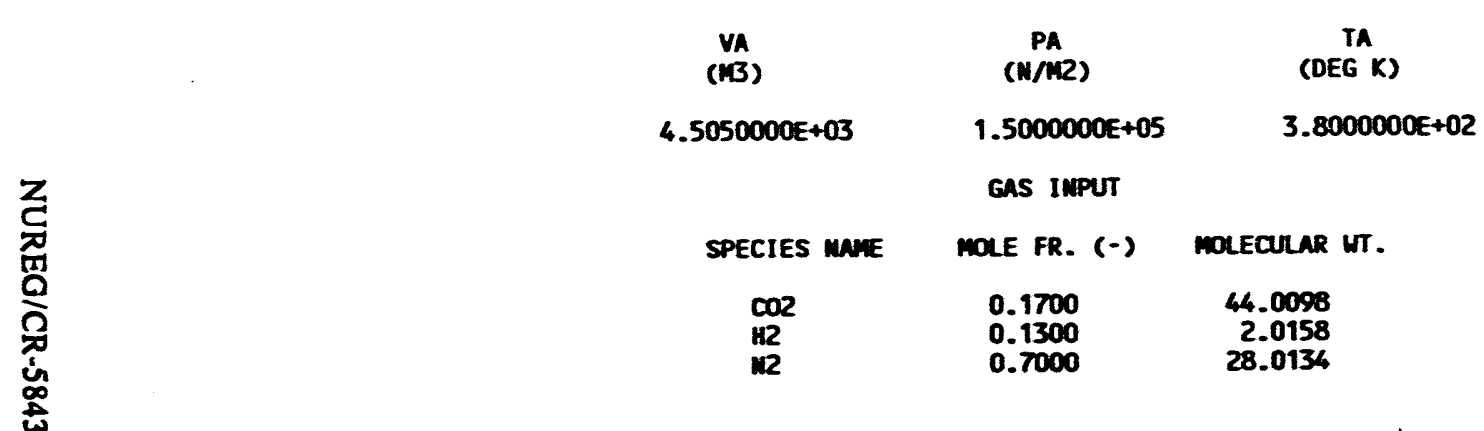


Table 6.4 Output listing for the BWR sample problem (continued)

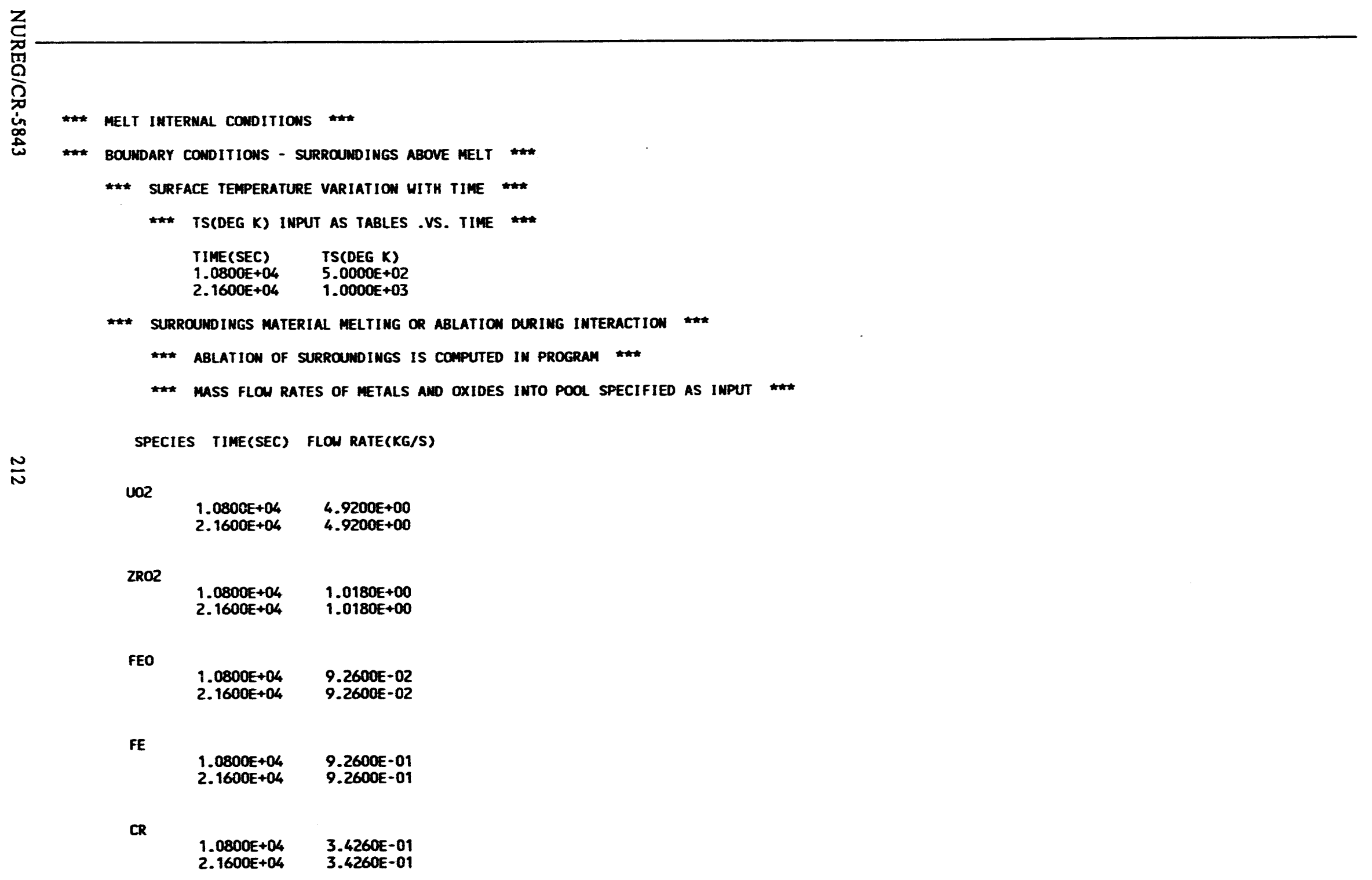


Table 6.4 Output listing for the BWR sample problem (continued)

$\begin{array}{ll}1.0800 E+04 & 1.9020 E-01 \\ 2.1600 E+04 & 1.9020 E-01\end{array}$

ZR

$\begin{array}{ll}1.0800 E+04 & 1.2670 E+00 \\ 2.1600 E+04 & 1.2670 E+00\end{array}$

HZOCLN

$\begin{array}{ll}1.0800 E+04 & 0.0000 E+00 \\ 1.4400 E+04 & 0.0000 E+00 \\ 1.4430 E+04 & 2.0000 E+01 \\ 1.8030 E+04 & 2.0000 E+01 \\ 1.8060 E+04 & 0.0000 E+00 \\ 2.1600 E+04 & 0.0000 E+00\end{array}$

$\begin{array}{ll}.8060 E+04 & 0.0000 E+00 \\ 2.1600 E+04 & 0.0000 E+00\end{array}$

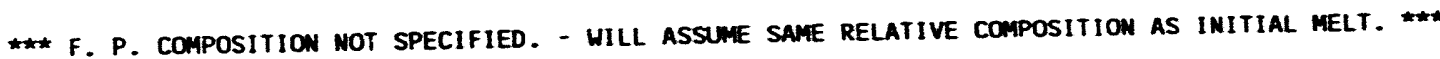

$\frac{N}{\omega}$

\# T(DEG K) IMPUT AS TABLES .VS. TIME

$\begin{array}{lc}\text { TIME(SEC) } & \text { TMET(DEG K) } \\ 1.0800 E+04 & 2.3000 E+03 \\ 8.0000 E+04 & 2.3000 E+03 \\ & \\ \text { TIME(SEC) } & \text { TOX(DEG K) } \\ 1.0800 E+04 & 2.3000 E+03 \\ 8.0000 E+04 & 2.3000 E+03 \\ & \\ \text { TIME(SEC) } & \text { TCLN(DEG K) } \\ 1.0800 E+04 & 3.2000 E+02 \\ 8.0000 E+04 & 3.2000 E+02\end{array}$

* BoumoarY conditIONS - RADIATIVE hEAT TRAMSFER **

SUPFACE EMISSIVITIES .VS. TIME OR TEMP.

TIME
EO
$8.0000 E-001$

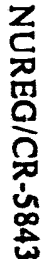

$0.0000 E+00$

EM 6.0000E-01

TIME $0.0000 E+00$

ES $6.0000 E-01$ 
*** aEROSOL OPACITY IMCLIDED III ATMOSPHERE \#\#

CHARACTERISTIC PATH LEMGTH $(\boldsymbol{H})=3.0000 \mathrm{E}+00$

* WELT/COOLANT SPLASHOUT \#*

* WO TIME-DEPENDENT MELT RADIUS USED ***

\#* PLOTS WERE WOT SPECIFIED, IMON $=0$

* aDOITIOULL USER SUPPLIED MODEL INPUTS **

*** THE USER uAS CHOSEM to mOOIFY SOME CORCON MODELS AND PARMETERS

$1 \mathrm{INH}=1$

THE USER HAS CHOSEM TO SUPPLY TERPERATURE-DEPEMDEMT MUHTIPLIERS

$\stackrel{N}{\stackrel{\Xi}{\Phi}}$

FOR THE CALCULATED METAL MIXTURE THERML COMOUCTIVITY
TEP(K)
MUTIPLIER

$0.0000 E+00$

5.0000E-01

IVSO $=1$

THE USER HAS CHOSEN TO SUPPLY TERPERATURE-DEPEADENT MULTIPLIERS

FOR THE CALCULATED OXIDE MIXTURE VISCOSITY

$$
\text { TEP(K) MUTIPLIER }
$$

O.0000E+CO S.0000E+CO

IPHO = 1

THE USER ans CHOSEM TO MODIfY THE OXIDE PHASE DIAGRAM

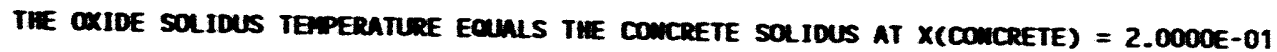

IZIP $=1$

THE USER uns chosen to SUPPLY THE VALUE FOR ZILCH USED In MITREA

ZILCH $=1.0000 E-02$ 
* * vamesa IIMPUT * * *

DUBBLE DINMETER IS CMLORATED PRRTICLE DINWETER = 1.00 MICROFETERS PARTICLES/BUBBLE IS CALCULATED IDEAL CHEAISTRY IS ASSUNED

IMITINal Melt composition

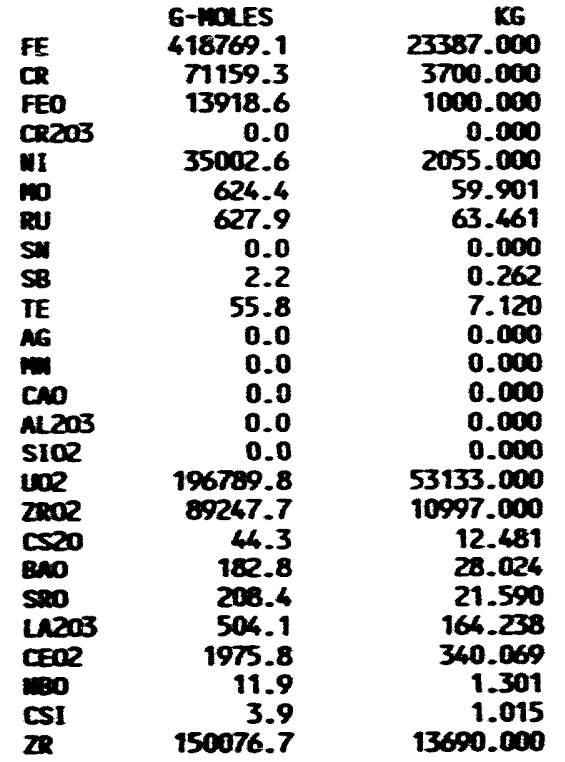

* IUTER POO DECONTMIMATIOO * 
Table 6.4 Output listing for the BWR sample problem (continued)

$\operatorname{TIVE}=10800.00$

BUR ACCIDEWT - MATER CORCON VERSION 2.28

- Mater adoed after 1 hour

IT. m. $=0$

MELT MD COOLMT LAYERS

MUMBER OF LAYERS, MLYR $=1$

COMFIGURATIOW, ILYR $=2$
WO COOLANT PRESENT, ICOOL $=0$

EXTREME CAVITY DIHEWSIOUS, MITH LOCATIOAS

RAOINL

MaXIVIM CAVITY RADIUS

(I) $=3.20000$

OUTSIDE RADIUS OF CONCRETE $(M)=4.43800$

REMIIUIMG THICKIESS $\quad$ (M) $=1.23800$

REMRESPO THICK BODY POINT

AxIAL

DEEPEST POINT IN CAVITY $\quad$ (H) $=5.00000$

MAXIMUN DEPTH OF CONCRETE (N) $=\mathbf{8 . 0 5 0 0 0}$

$\begin{array}{ll}\text { REMAIHING THICXNESS } & \text { (M) }=3.05000\end{array}$

CORRESPOWDING BCOY POINT

a APProximate OVERALL EMERG BUDET FOR DEBris

(SEE Mavil FOR EXPLAMATIO AD CAVEATS)
IMTERUAL (DECAY) SOURCE
(U) $=6.848 E+06$ CHENICAL REACTION SOURCE
$(U)=0.000 E+00$
HEAT LOSS TO CONCRETE
(u) $=1.686 E+07$
UEATUP OF ABLATION PROOUC
(W) $=0.000 E+\infty 0$
HEAT LOSS FROM SURFACE
$(W)=1.756 E+07$
(TO SURRoudimCS)

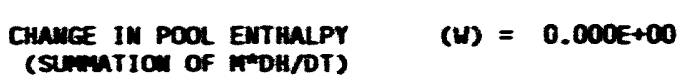

MUERICAL CHECKS ON MASS AMO EMERG COUSERVATION

RELATIVE ERRCR IN MASS = $0.00000 E+00$

RELATIVE ERROR IN EMTHALPY $=0.00000 E+00$

CHECK ON RECESSION CALCULATION CDO/DS SWOULD BE .LE. i)

maximin Doros $=0.00000$ 
Table 6.4 Output listing for the BWR sample problem (continued)

TIME $=10800.00$

* * * * G A S GE 1 HOUR

1T. No. $=0$

GAS EXITIMG POOL (INCLUDES FILM AND COOLAMT)

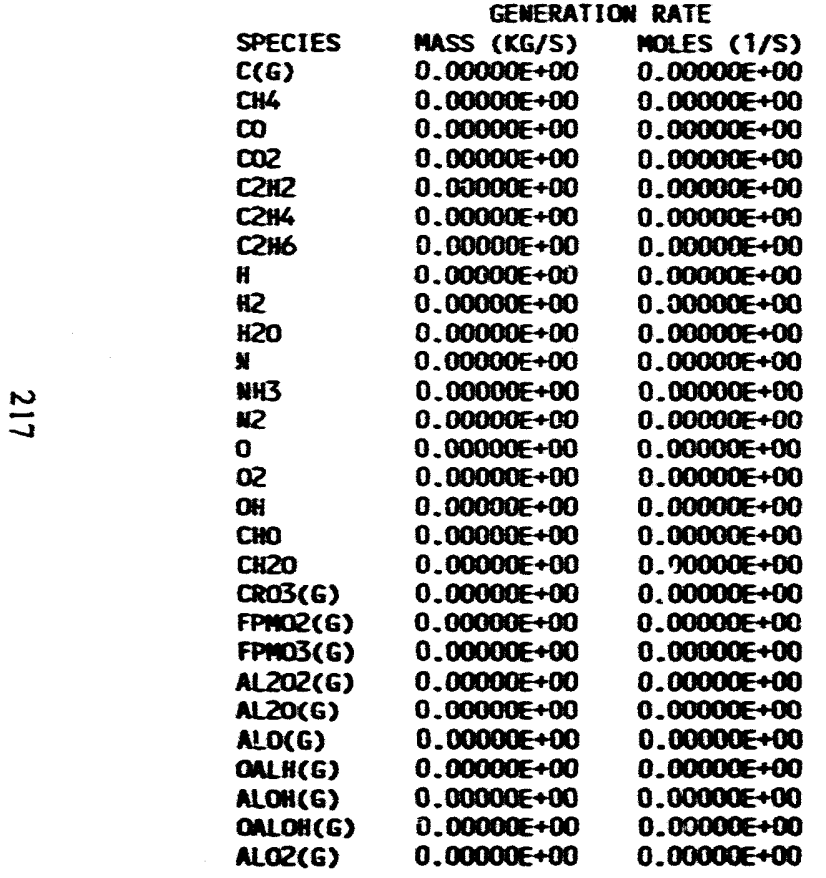

CMILLATIVE RELEASE

\begin{tabular}{|c|c|c|}
\hline $\begin{array}{l}\text { MASS (KG) } \\
0.00000 E+00 \\
0.00000 E+00 \\
0.00000 E+00 \\
0.00000 E+00 \\
0.00000 E+00 \\
0.00000 E+00 \\
0.00000 E+00 \\
0.00000 E+00 \\
0.00000 E+00 \\
0.00000 E+00 \\
0.00000 E+00 \\
0.00000 E+00 \\
0.00000 E+00 \\
0.00000 E+00 \\
0.00000 E+00 \\
0.00000 E+00 \\
0.00000 E+00 \\
0.00000 E+00 \\
0.00000 E+00 \\
0.00000 E+00 \\
0.00000 E+00 \\
0.00000 E+00 \\
0.00000 E+00 \\
0.00000 E+00 \\
0.00000 E+00 \\
0.00000 E+00 \\
0.00000 E+00 \\
0.0000\end{array}$ & 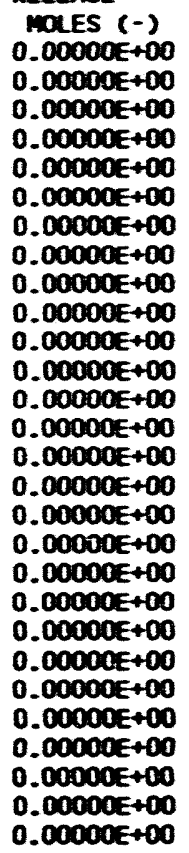 & $\begin{array}{l}\text { SPECIES } \\
\text { C(G) } \\
\text { CH4 } \\
\text { CO } \\
\text { COZ } \\
\text { C2H2 } \\
\text { C2H4 } \\
\text { C2HG } \\
H \\
H 2 \\
H 2 O \\
H \\
\text { HH3 } \\
H 2 \\
0 \\
\text { O2 } \\
\text { OH } \\
\text { CHO } \\
\text { CHZO } \\
\text { CRO3(G) } \\
\text { FPHOZ(G) } \\
\text { EPHO3(G) } \\
\text { AL2O2(G) } \\
\text { AL2O(G) } \\
\text { ALO(G) } \\
\text { OALH(G) } \\
\text { ALOH(G) } \\
\text { OALOH(G) } \\
\text { ALOZ(G) }\end{array}$ \\
\hline
\end{tabular}

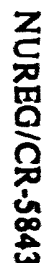


Table 6.4 Output listing for the BWR sample problem (continued)

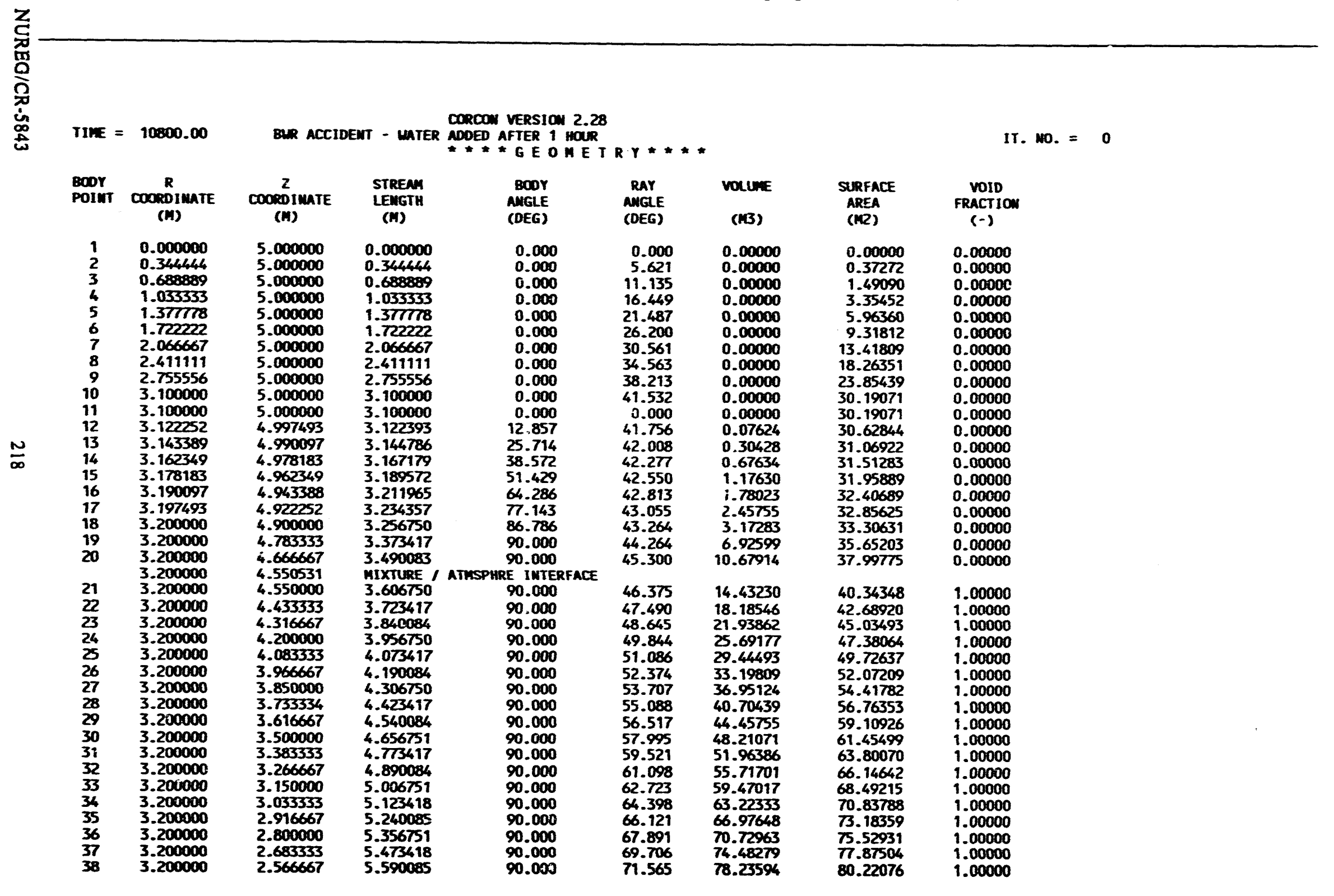


Table 6.4 Output listing for the BWR sample problem (continued)

\begin{tabular}{|c|c|c|c|c|c|c|c|c|}
\hline $\begin{array}{l}39 \\
40 \\
41 \\
42 \\
43 \\
44 \\
45 \\
46 \\
47 \\
48 \\
49 \\
50 \\
51 \\
52 \\
53 \\
54 \\
55 \\
56 \\
57 \\
58\end{array}$ & $\begin{array}{l}3.200000 \\
3.200000 \\
3.200000 \\
3.200000 \\
3.200000 \\
3.200000 \\
3.200000 \\
3.200000 \\
3.200000 \\
3.200000 \\
3.200000 \\
3.200000 \\
3.200000 \\
3.200000 \\
3.200000 \\
3.200000 \\
3.200000 \\
3.200000 \\
3.200000 \\
3.200000 \\
3.200000 \\
3.200000\end{array}$ & $\begin{array}{l}2.450000 \\
2.333333 \\
2.216667 \\
2.100000 \\
1.983333 \\
1.866667 \\
1.750000 \\
1.633333 \\
1.516667 \\
1.400000 \\
1.283333 \\
1.166667 \\
1.050000 \\
0.933333 \\
0.816667 \\
0.700000 \\
0.583333 \\
0.466667 \\
0.350000 \\
0.233334 \\
0.116667 \\
0.000000\end{array}$ & $\begin{array}{l}5.706752 \\
5.823419 \\
5.940085 \\
6.056752 \\
6.173419 \\
6.290086 \\
6.405753 \\
6.523419 \\
6.640086 \\
6.756753 \\
6.873420 \\
6.990087 \\
7.106753 \\
7.223420 \\
7.340087 \\
7.456753 \\
7.573420 \\
7.690087 \\
7.806754 \\
7.923420 \\
8.040087 \\
8.156754\end{array}$ & $\begin{array}{l}90.000 \\
90.000 \\
90.000 \\
90.000 \\
90.000 \\
90.000 \\
90.000 \\
90.000 \\
90.000 \\
90.000 \\
90.000 \\
90.000 \\
90.000 \\
90.000 \\
90.000 \\
90.000 \\
90.000 \\
90.000 \\
90.000 \\
90.000 \\
90.000 \\
90.000\end{array}$ & $\begin{array}{r}73.465 \\
75.403 \\
77.376 \\
79.380 \\
81.411 \\
83.463 \\
85.533 \\
87.614 \\
89.702 \\
91.790 \\
93.873 \\
95.947 \\
98.005 \\
100.042 \\
102.054 \\
104.036 \\
105.985 \\
107.896 \\
109.767 \\
111.595 \\
113.378 \\
115.115\end{array}$ & $\begin{array}{r}81.98910 \\
85.74225 \\
89.49541 \\
93.24856 \\
97.00172 \\
100.75487 \\
104.50803 \\
108.26118 \\
112.01434 \\
115.76749 \\
119.52065 \\
123.27380 \\
127.02695 \\
130.78011 \\
134.53326 \\
138.28641 \\
142.03957 \\
145.79272 \\
149.54588 \\
153.29903 \\
157.05219 \\
160.80534\end{array}$ & $\begin{array}{r}82.56648 \\
84.91220 \\
87.25793 \\
89.60365 \\
91.94937 \\
94.29509 \\
96.64082 \\
98.98653 \\
101.33226 \\
103.67798 \\
106.02370 \\
108.36942 \\
110.71514 \\
113.06087 \\
115.40659 \\
117.75231 \\
120.09804 \\
122.44376 \\
124.78949 \\
127.13521 \\
129.48093 \\
131.82664\end{array}$ & $\begin{array}{l}1.00000 \\
1.00000 \\
1.00000 \\
1.00000 \\
1.00000 \\
1.00000 \\
1.00000 \\
1.00000 \\
1.00000 \\
1.00000 \\
1.00000 \\
1.00000 \\
1.00000 \\
1.00000 \\
1.00000 \\
1.00000 \\
1.00000 \\
1.00000 \\
1.00000 \\
1.00000 \\
1.00000 \\
1.00000\end{array}$ \\
\hline
\end{tabular}


Table 6.4 Output listing for the BWR sample problem (continued)

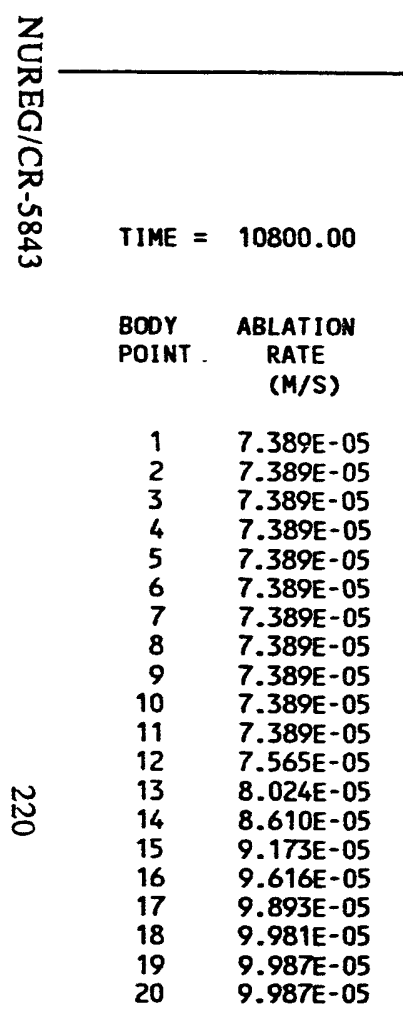

BUR ACCIDENT - WATER CORCON VERSION 2.28

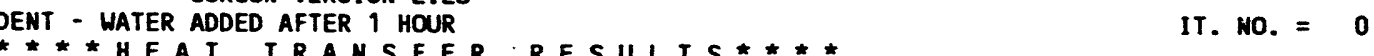

SLG.BUB. 1.000E+03 2018.4

SLG.BUB. $1.000 E+03$

SLG.BUB. $1.000 E+03$

SLG.BUB. $1.000 E+03$

2018.4

2018.4

SLG.BB.

$1.000 E+03$

SLG.BUB. 1.000E+03

SLG.BUB. $1.000 E+03$

SLG.BUB. $1.000 E+03$

SLG.BUE. $1.000 E+03$

SLG.BUB. $1.000 E+03$

SLG.BUB. $1.000 E+03$

SLG.FLM. $1.000 E+03$

SLG.FLM. $1.000 E+03$

SLG.FLM. $1,000 E+03$

SLG.FLM. $1.000 E+03$

SLG.FLM. $1.000 E+03$

$3.884 E+05$ O. $0.000 E+00$

$3.884 E+05 \quad 0.000 E+00$

$3.884 E+05 \quad 0.000 E+00$

$\begin{array}{lll}3.884 E+05 & 0.000 E+00\end{array}$

$3.884 E+05 \quad 0.000 E+00$

$3.884 E+05 \quad 0.000 E+00$

$3.884 \mathrm{E}+05 \quad 0.000 \mathrm{E}+00$

$3.884 E+05 \quad 0.000 E+00$

$\begin{array}{lll}2018.4 & 3.884 \mathrm{E}+05 & 0.000 \mathrm{E}+00\end{array}$

$3.976 E+05 \quad 0.000 E+00$

$4.217 \mathrm{E}+05 \quad 0.000 E+00$

$4.525 E+05 \quad 0.000 E+00$

$2112.1 \quad 4.821 E+05 \quad 0.000 E+00$

$2135.4 \quad 5.054 \mathrm{E}+05 \quad 0.000 \mathrm{E}+00$

$2150.0 \quad 5.200 E+05 \quad 0.000 E+00$

$2154.6 \quad 5.246 \mathrm{E}+05 \quad 0.000 \mathrm{E}+00$

$\begin{array}{lll}2154.9 & 5.249 E+05 & 0.000 E+00\end{array}$ 
Table 6.4 Output listing for the BWR sample problem (continued)

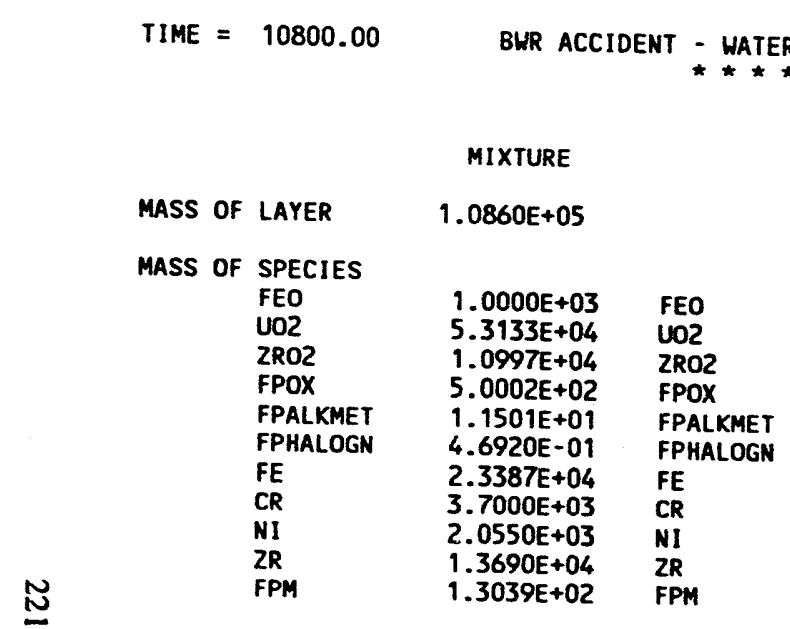

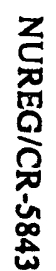

CORCON VERSION 2.28

ADDED AFTER 1 HOUR

C O MOUROSI TION

IT. NO. $=0$

$1.3039 \mathrm{E}+02$ 
Table 6.4 Output listing for the BWR sample problem (continued)

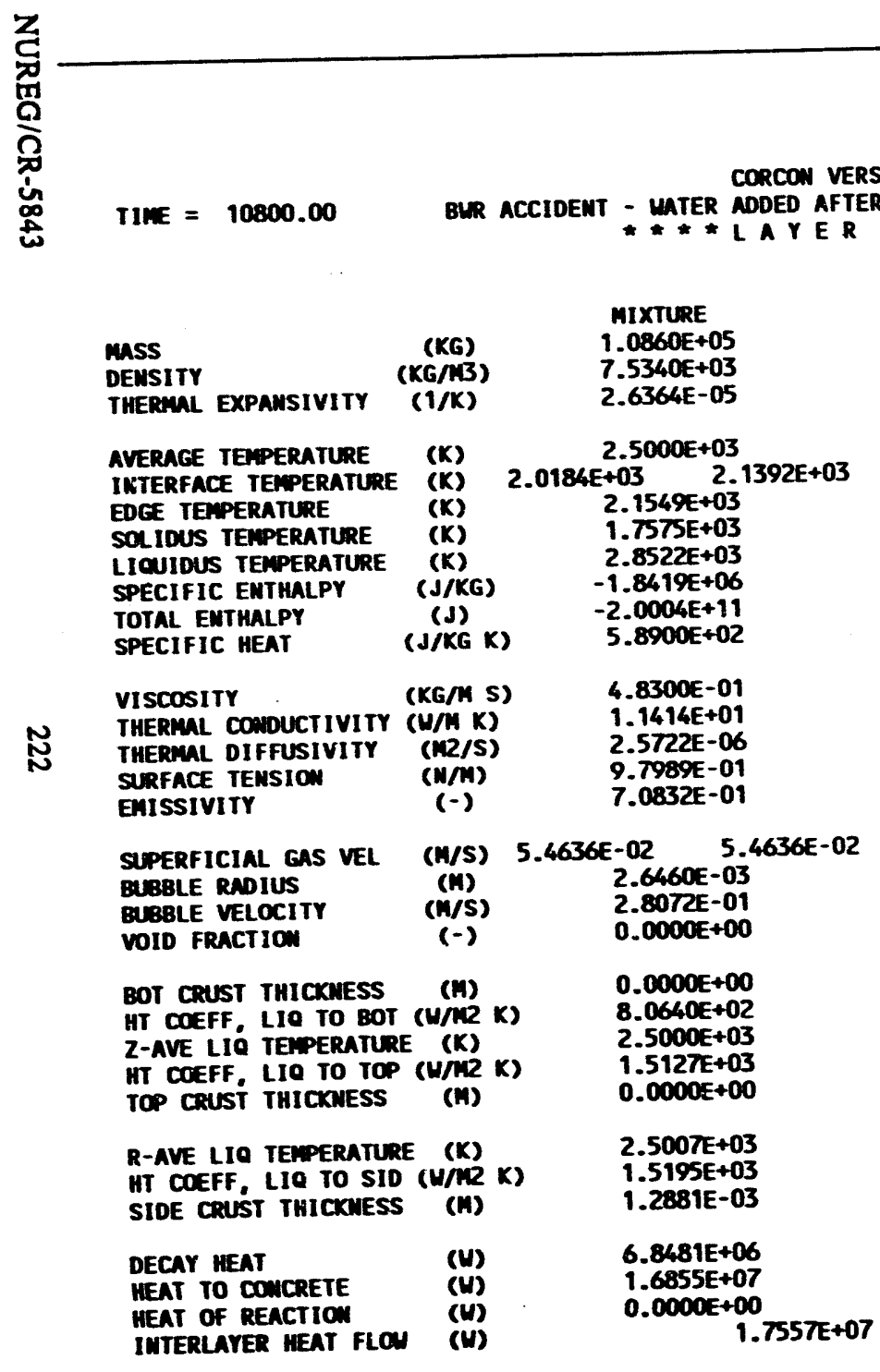


TIME $=10800.00 \quad$ BUR ACCIDENT - MATER CORCON VERSION 2.28

*** INTERLAYER MIXING****
1T. NO. $=0$

LONER MIXTURE LAYER

$\begin{array}{llll} & & & \\ & & & \\ \text { PXIDE } & \text { METAL } \\ \text { PHASE MASSES } & \text { (KG) } & 6.5642 E+04 & 4.2962 E+04 \\ \text { PHASE DENSIIIES } & \text { (KG/M) } & \mathbf{8 . 4 0 8 0 E + 0 3} & 6.5014 E+03 \\ \text { LIOUIDUS TEMPERATURE } & \text { (K) } & 2.4432 E+03 & 1.7575 E+03 \\ \text { SOLIDUS TEMPERATURE } & \text { (K) } & 2.8522 E+03 & 1.7675 E+03 \\ \text { PHASE ENTHALPIES } & \text { (J) } & -2.6854 E+11 & 6.8492 E+10 \\ \text { MASS ENTRAINED } & \text { (KG) } & 0.0000 E+00 & 0.0000 E+00 \\ \text { MASS DE-ENTRAINED } & \text { (KG) } & 0.0000 E+00 & 0.0000 E+00\end{array}$

MLTREA REQUIRED 111 ITERATIONS

MLTREA REQUIRED 51 ITERATIOUS

N

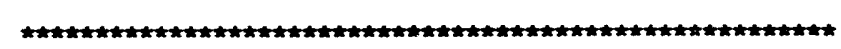

* LaYer orientation change at TIMe = 1 .08300e+04

+ LAYER HAX BECOMES A MEU MET LAYER

mLTREA REQUIRED 111 ITERATIONS

MLTREA RECUIRED 51 ITERATIONS

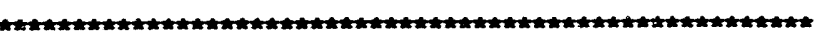

- LaYer orientation change at time = 1.08600E+04

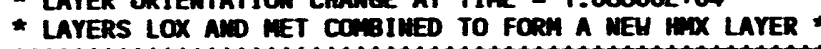

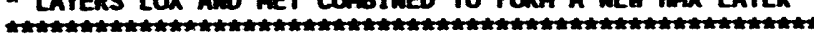

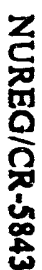


Table 6.4 Output listing for the BWR sample problem (continued)

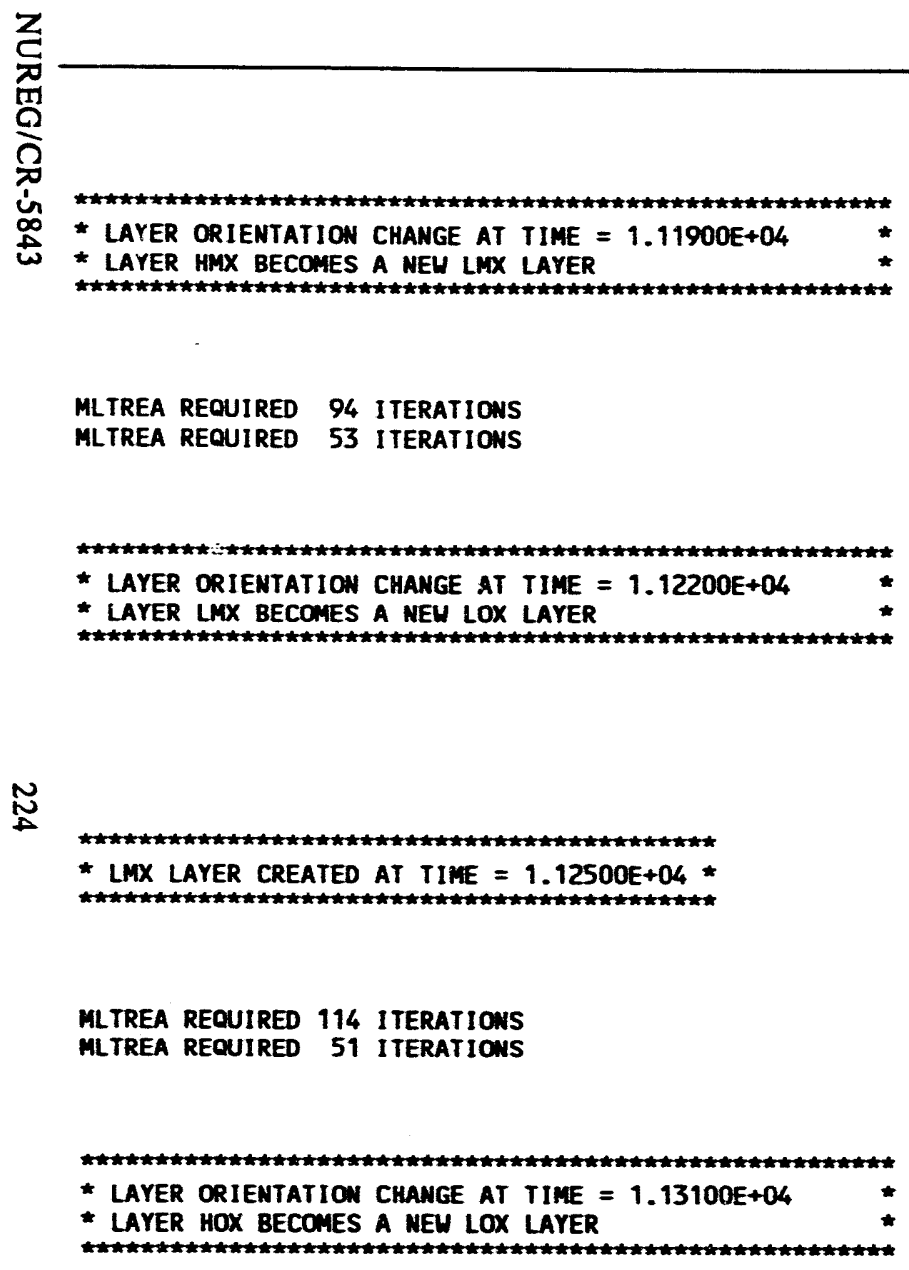

MLTREA REOUIRED 51 ITERATIONS 
- LAYER ORIENTATION CHANGE AT TIME $=1$ 13400E+04

* layer orientation change at tIME = 1.13400 e+04

* LAYERS loX and MET COMBINED TO FORM a Nen hMX LAYER *

\author{
MLTREA REQUIRED 94 ITERATIONS \\ MLTREA RECUIRED SI ITERATION \\ INITDS ETA MAY BE TOO SMALL \\ INITDS ETA MAY BE TOO SMALL \\ INITDS ETA MAY BE TOO SWALL \\ INITDS ETA MAY BE TOO SMALL \\ MLTREA REQUIRED 52 ITERATIONS \\ MLTREA REOUIRED 51 ITERATIOWS
}


meLt and COOLNT LAYERS

MMBER OF LAYERS, MLYR $=3$
CONFIGURATION, ILYR $=0$
WO COOLANT PRESEN, ICOOL $=0$

EXTREAE CAVITY DImEUSIOUS, UITH LOCATIOUS

\section{RADIAL}

MXImin CAVITY Rapius

OUTSIDE DADIUS OF COA $=3.40992$

REMINI RAIUS OF CONCRETE $(A)=4.43800$

$\begin{aligned} & \text { (H) } & =1.02808 \\ & = & 22\end{aligned}$

N

(SEE MUULL FOR EXPLAMATIO AD CAVEATS)
INTERIAL (DECAY) SOURCE
$(U)=8.780 E+06$ CHEAICAL REACTIOW SOURCE
(U) $=2.674 E+06$
$\begin{array}{ll}\text { HEAT LOSS TO CONCRETE } & \text { (H) }=3.107 E+06 \\ \text { HEATUP OF ABLATIOH PROOUCTS } & \text { (U) }=-1.212 E+06\end{array}$
HEAT LOSS FROM SURFACE
(U) $=-1.212 E+06$
(TO SURROUnoIMGS)
(W) $=1.344 E+07$

CHNGE IH POOL ENTHLLPY

$(U)=-1.372 E+10$

MHERICAL CHECKS ON MSS AD ENERG COUSERVATION

RELATIVE ERROR III MASS = $-2.26490 E-06$

RELATIVE ERROR IN ENTHLLPY $=-1.07288 E-06$
NXIAL

DEEPEST POINT IN CAVITY (H) $=5.06399$

$\begin{array}{ll}\text { MXIMUM DEPTH OF CONCRETE } & \text { (N) }=8.05000 \\ \text { REMAIMIMG THICKYESS } & \text { (H) }=2.98601\end{array}$

REMINIMG THICKMESS

$=2.98601$
CORRESPONIMG BOOY POIUT

CHECK ON RECESSION CALCULATION (DD/DS SHOULD BE .LE. 1)

mximn DO/DS $=0.02083$ 
TIIE $=16200.00$

Concon VERsion 2.20

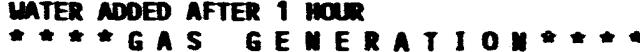

IT. $100 .=180$

GAS EXITIMG POOL (INCLUES FILH ND COOLAT)

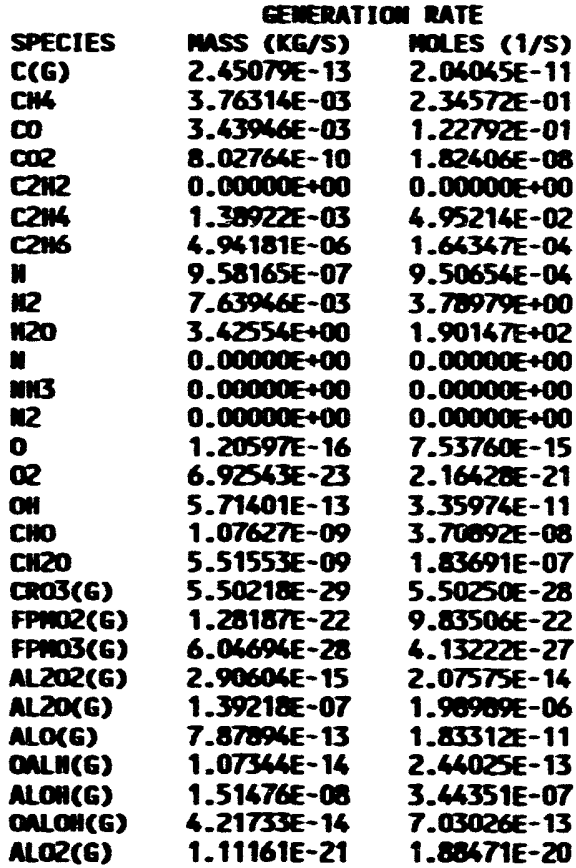

COMULTIVE RELERSE

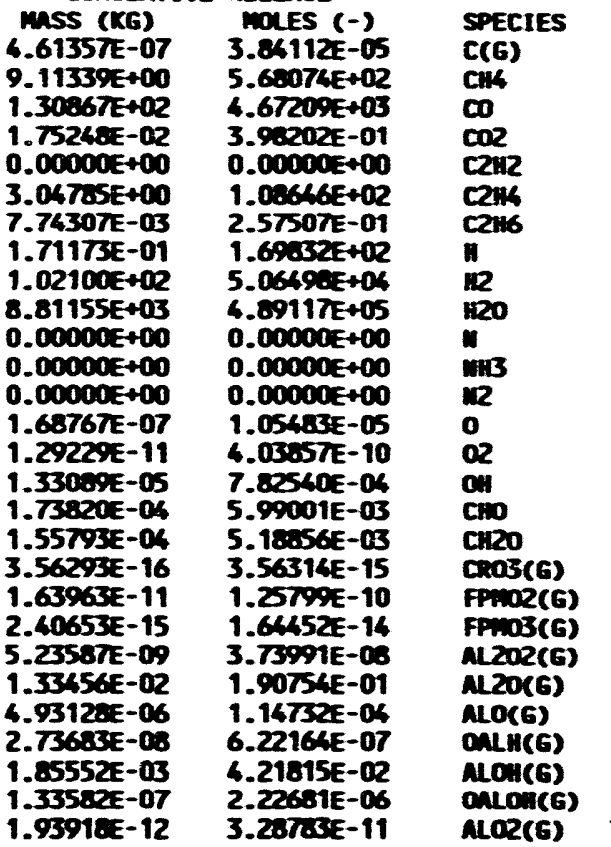


Table 6.4 Output listing for the BWR sample problem (continused)

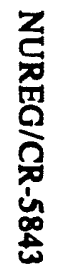

TIME = 16200.00

BOOY R
POINT Coopdimate

(H)

0.000000

0.350742

0.701483

1.402967

1.753709

2. 104451

2.455193

2.805934

3.100000

3.146289

3.161209

3.176493

3.190768

3.202973

3.218485

3.221335

3.229483

3.312683

3.387869

3.409921

3.359040

3.229580

3.221595

3.200000

3.200000

3.200000

3.200000

3.200000

3.200000

3.200000

3.200000

3.200000

3.200000

3.200000
BMR ACCIDENT - MATER ADDED AFTER 1 MOUR

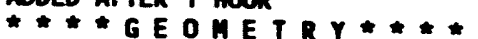

2
COORDIMATE
(I)

STRENM

LENGT

BODY
ANGLE

RAY

5.063988

5.063988

5.063988

5.063988

5.063988

5.063988

5.063988

5.063988

5.063988

5.063988

5.052261

5.041134

5.026852

4.989352

4.967273

4.967273
4.944720

4.922668

4.813581

4.729066

4.625760

4.545898

4.342703

4.304179

4.200000

4.083333

3.966667

3.850000

3.733334

3.616667

3.500000

3.419232

3.266667

3.150000

3.033333

2.916667

2.916667
2.800000

0.000000

0.350742

0.701483

1.052226

1.402967

1.753709

2.104451

2.455193

2.805934

3.100000

3.147751

3.166363

3.187282

3.209797

3.233302
3.257242

3.280647

3.302882

3.412274

3.502693

3.592498

3.698131 100.226
OX1DE / MIXTURE INTERFACE
4.033756

MIXTURE / COOLAMT INTERFACE

4.179492

4.296159

4.412826

4.412826
4.529492

4.529492
4.646159

4.762826

COOLANT

4.996160

5.112826

5.229493

5.346160

5.462827
5.579494

95.855

95.855
90.000

90.000

90.000

90.000

90.000

INTERFACE

90.000

90.000

90.000

90.000
90.000

.000

0.000

0.000

0.000

0.000

.465

3.886
6.857

9.686

.561

182

49.844

59.521
1T. 10. = 180

volume

(DEG) (MB)

AREA

(in2)

VOID

$(-)$

$\begin{array}{ll}0.000 & 0.00000 \\ 5.621 & 0.00000\end{array}$

11.1350 .00000

$\begin{array}{ll}16.449 & 0.00000 \\ 21.487 & 0.00000\end{array}$

$26.200 \quad 0.00000$

$30.561 \quad 0.00000$

$\begin{array}{ll}34.563 & 0.00000\end{array}$

$0.000 \quad 0.000000$

$41.532 \quad 0.35935$

$41.756 \quad 0.7070$

1.15758

1.71198

2.35697

3.07064

3.80313

4.52139

9.27686

11.00869

14.75801

$48.646 \quad 24.56062$

29.19392

32.96707

36.70023

40.45338

$55.088 \quad 44.20654$

$\begin{array}{ll}56.517 & 47.95969\end{array}$

47.95969
51.71285

55.46600

59.21915

62.97231

66.72547

70.47862
74.23177

0.38648

1.54591

3.67050
6.18365

9.66195

13.91322

18.93744

24.73459

30.19071

31.12774

31.49655

31.91305

32.36362

32.83557

33.31804

33.79009

34.26074

36.45766

40.30645

42.46235

49.46302

52.40676

54.75248

57.09021

59.44393

61.70965

64.13538

66.48110

68.82682

71.17254

73.51826

75.86399

78.20971
80.55543

0.00732

0.00732

0.00732

0.00732

0.00752

0.00752

0.00732

0.00732

0.00732

0.00732

0.00752

0.00728

0.00726

0.00720

0.00717

0.00714

0.00712

0.00710

0.0068

0.00671

0.00666

0.00710

0.00363

0.0029

0.0029

0.0029

0.00298

0.0029

1.00000

1.00000

1.00000

1.00000

1.00000

1.00000 
Table 6.4 Output listing for the BWR sample problem (continesed)

\begin{tabular}{|c|c|c|c|c|c|c|c|}
\hline $\begin{array}{l}37 \\
38 \\
39 \\
40 \\
41 \\
42 \\
43 \\
44 \\
45 \\
46 \\
47 \\
48 \\
49 \\
50 \\
51 \\
52 \\
53 \\
54 \\
55 \\
56 \\
57 \\
58 \\
59 \\
60\end{array}$ & $\begin{array}{l}3.200000 \\
3.200000 \\
3.200000 \\
3.200000 \\
3.20000 \\
3.200000 \\
3.200000 \\
3.200000 \\
3.200000 \\
3.200000 \\
3.200000 \\
3.200000 \\
3.200000 \\
3.200000 \\
3.200000 \\
3.200000 \\
3.200000 \\
3.200000 \\
3.200000 \\
3.200000 \\
3.200000 \\
3.20000 \\
3.200000 \\
3.200000\end{array}$ & $\begin{array}{l}2.683333 \\
2.56667 \\
2.450000 \\
2.333333 \\
2.216667 \\
2.100000 \\
1.983333 \\
1.866667 \\
1.750000 \\
1.633333 \\
1.51667 \\
1.400000 \\
1.283333 \\
1.166667 \\
1.050000 \\
0.933333 \\
0.816667 \\
0.700000 \\
0.583533 \\
0.466657 \\
0.350000 \\
0.233334 \\
0.116667 \\
0.000000\end{array}$ & $\begin{array}{l}5.696160 \\
5.812827 \\
5.929494 \\
6.046161 \\
6.162827 \\
6.279494 \\
6.396161 \\
6.512828 \\
6.629695 \\
6.746161 \\
6.862828 \\
6.979495 \\
7.096162 \\
7.212829 \\
7.329695 \\
7.466162 \\
7.562829 \\
7.679695 \\
7.796162 \\
7.9122829 \\
8.029695 \\
8.166162 \\
8.262629 \\
8.379496\end{array}$ & $\begin{array}{l}90.000 \\
90.000 \\
90.000 \\
90.000 \\
90.000 \\
90.000 \\
90.000 \\
90.000 \\
90.000 \\
90.000 \\
90.000 \\
90.000 \\
90.000 \\
90.000 \\
90.000 \\
90.000 \\
90.000 \\
90.000 \\
90.000 \\
90.000 \\
90.000 \\
90.000 \\
90.000 \\
90.000\end{array}$ & $\begin{array}{l}69.706 \\
71.565 \\
77.465 \\
75.403 \\
77.376 \\
79.380 \\
81.411 \\
83.463 \\
85.533 \\
87.614 \\
89.702 \\
91.790 \\
93.873 \\
95.947 \\
9.005 \\
100.042 \\
102.054 \\
104.036 \\
105.985 \\
107.896 \\
109.767 \\
111.595 \\
113.378 \\
115.115\end{array}$ & $\begin{array}{r}77.99493 \\
81.73808 \\
85.49124 \\
89.24639 \\
92.99755 \\
9.75070 \\
100.50386 \\
104.25701 \\
10.01017 \\
111.76332 \\
115.51648 \\
119.26963 \\
123.02279 \\
126.77594 \\
130.52910 \\
134.28226 \\
138.03562 \\
161.78656 \\
145.56172 \\
169.29688 \\
153.04803 \\
156.80118 \\
1600.55434 \\
164.30750\end{array}$ & 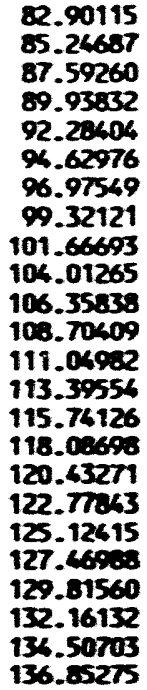 \\
\hline
\end{tabular}


Table 6.4 Outpat listing for the BWR sample problem (continued)

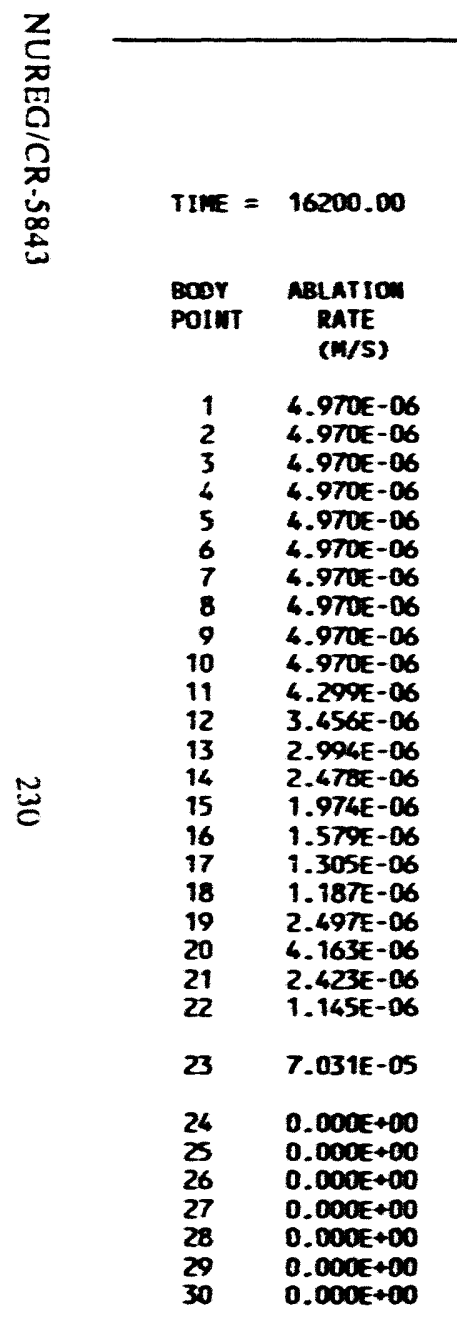

BAR ACCIDEWT - UATER CORCOD VERSIOW 2.26

- * heat transfer Results....

FILM FIL FIL REYMOLS

$\underset{(M)}{\text { FLOU }} \begin{array}{ccc}\text { VELOCITY } \\ \text { (KG/S) }\end{array}$

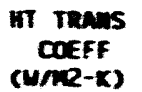

St6.008. 1.000E+03

SLG.018. 1.000E+03

SLG.018. 1.000E+03

stG.eve. 1.000E+03

SIG. $018.1 .000 E+03$

S2. 0 .01. 1.000E+03

SLG.Des. 1.000E+03

SLG.808. 1.000E+03

SL6.808. 1.000e+03

SLG.808. 1.000E+03

SLG.FLH. 1.000E+03

SLG.FL. 1.000E-03

SLG.FLA. 1.000E+0s

SLG.FL. $1.000 \mathrm{C}+03$

SLG.FLn. 1.000E+0S

SLG.FL. 1.000E+03

SLG.FLA. 1.000E+0S

SLG.FIn. 1.000E+05

SL.FLn. 1.000 EOS

SLG.FLn. 2.532E+03

SLG.FLT. 1.000E+03

SLE.FLF. 1.000E+03

SLG.FLE. 1.000E+03

SLG.FIT. 1.000E+03

StG.FIn. 1.000E+03

SLG.FLH. 1.000E+0

SLG.FLA. $1.000 \mathrm{C}+03$
(K)

1656.1

IT. $10 .=100$

CONECTIVE NOLATIV

Fux FLux

$2.612 E+04$ 0.000E+00

$2.612 E+040.000 E+00$

$2.612=+04$ 0.000E+00

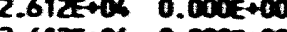

$2.612 E+04$ 0.00BE+00

$0.0012+00$

$2.612 \mathrm{E}+04 \quad 0.000 \mathrm{E}+00$

$2.612=+040.000 E+00$

$2.612 \mathrm{E}+0 \mathrm{OK}$ O.000E+00

$2.612 E+04$ O.000E+00

$2.260 E+06$ 0.000E+00

$1.816 E+040.000 E+00$

$i .574 E+04$ O.000E-00

1.30ectar 0.000E+00

$1.030 \mathrm{C}+04 \quad 0.000 \mathrm{E}+00$

6.301E+03 0.000e+00

$6.858 E+03 \quad 0.000 E+00$

$6.237 \mathrm{E}+03 \quad 0.000 \mathrm{E}+00$

$1.312 E+04$ 0.000E+00

2.180E+Q6 0.000E+00

$1.273 E+06$ 0.000E+00

6.017E+03 0.000E+00

3.695E+05 0.000E+00

$0.000 E+000$ O.000E +00

$393.6 \quad 0.000 E+00 \quad 0.000 E+00$

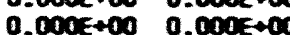

$0.008 E+\infty 0$ O.000E+CO

$0.000 E+600.000 E+\infty 0$

$0.000 E+00$ 0.000E+00

$0.000 E+00$ O.000E+00 


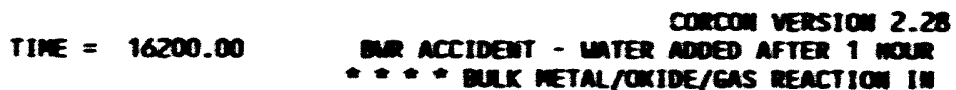

IT. 10.100

\begin{tabular}{|c|c|c|c|c|c|}
\hline \multicolumn{2}{|c|}{ ouIDES } & \multicolumn{4}{|c|}{ netals } \\
\hline $\begin{array}{l}\text { SPECIES } \\
\text { WAES }\end{array}$ & $\begin{array}{l}\text { REACTAMTS } \\
\text { (mos) }\end{array}$ & $\begin{array}{l}\text { Prooucts } \\
\text { (mols) }\end{array}$ & $\begin{array}{l}\text { SPECIES } \\
\text { mines }\end{array}$ & $\begin{array}{l}\text { DEnctamis } \\
\text { (noLS) }\end{array}$ & $\begin{array}{l}\text { Peopucts } \\
\text { (nols) }\end{array}$ \\
\hline 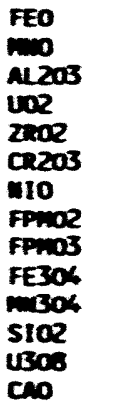 & $\begin{array}{l}1.9766 E+01 \\
0.0000 E+00 \\
2.3525 E+01 \\
6.3560 E+02 \\
7.2761 E+02 \\
1.8156 E-10 \\
3.7069 E-06 \\
2.2500 E-36 \\
1.26901-16 \\
3.2003 E-18 \\
0.0000 E+00 \\
2.5571 E+02 \\
4.8513 E-16 \\
9.7071 E+01\end{array}$ & $\begin{array}{l}2.1204 E-03 \\
0.0000 E+00 \\
1.0559 E+00 \\
6.1102 E+02 \\
1.0712 E+03 \\
2.1527 E-11 \\
1.5396 E-06 \\
0.0000 E+00 \\
3.7533 E-21 \\
1.157 E E-23 \\
0.0000 E+00 \\
2.7372 E-02 \\
3.5076 E-21 \\
9.407 \pi+01\end{array}$ & 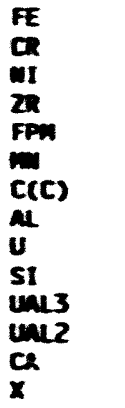 & 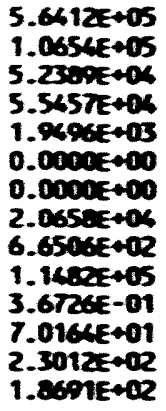 & $\begin{array}{l}5.64 \\
1.06 \\
5.23 \\
5.51 \\
1.94 \\
0.00 \\
0.00 \\
2.07 \\
6.90 \\
1.15 \\
2.80 \\
6.06 \\
2.32 \\
1.86\end{array}$ \\
\hline
\end{tabular}

w. OF ITEATIOUS $=41$

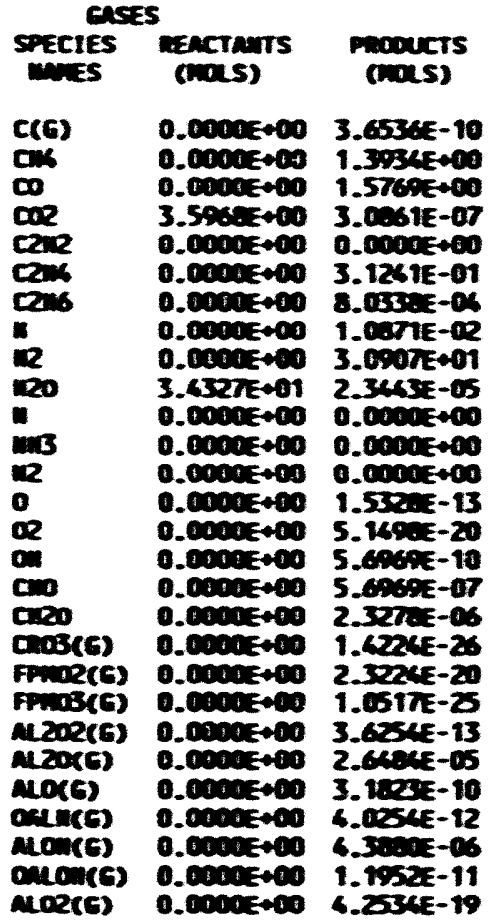


Table 6.4 Odpet listing for the BWR sumple problen (continued)

TIFE $=16200.00$

2. Coicon vession 2.20

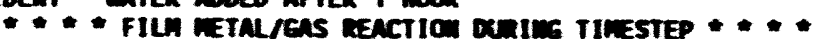

IT. $10 .=190$

\begin{tabular}{|c|c|c|c|c|c|}
\hline \multicolumn{2}{|c|}{ OXIDES } & \multicolumn{4}{|c|}{ netals } \\
\hline $\begin{array}{l}\text { PECIES } \\
\text { MANES }\end{array}$ & $\begin{array}{l}\text { REACTAMTS } \\
\text { (mOLS) }\end{array}$ & $\begin{array}{l}\text { Prooucts } \\
\text { (mols) }\end{array}$ & $\begin{array}{c}\text { SPECIES } \\
\text { WAES }\end{array}$ & $\begin{array}{l}\text { REACTAMTS } \\
\text { (nOLS) }\end{array}$ & $\begin{array}{l}\text { Pnopucts } \\
\text { (nols) }\end{array}$ \\
\hline 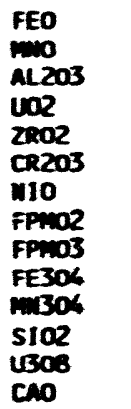 & $\begin{array}{l}0.0000 E+\infty 00 \\
0.0000 E+00 \\
0.0000 E+\infty 0 \\
0.0000 E+\infty 0 \\
0.0000 E+\infty 0 \\
0.0000 E+\infty 0 \\
0.0000 E+\infty 0 \\
0.0000 E+\infty 0 \\
0.0000 E+\infty 0 \\
0.0000 E+\infty 0 \\
0.0000 E+\infty 0 \\
0.0000 E+\infty 0 \\
0.0000 E+\infty 0 \\
0.0000 E+\infty 0\end{array}$ & $\begin{array}{l}3.4666 E-05 \\
0.0000 E+00 \\
4.0162 E-02 \\
2.0957 E+01 \\
3.4657 E+01 \\
2.3390 E-13 \\
2.5112 E-00 \\
0.0000 E+00 \\
1.6156 E-23 \\
3.8670 E-26 \\
0.0000 E+00 \\
6.6176 E-04 \\
2.1627 E-23 \\
3.5951 E+00\end{array}$ & 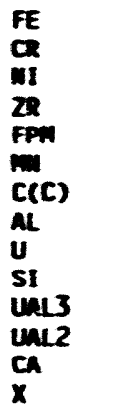 & $\begin{array}{l}5.6616 E+05 \\
1.0654 E+05 \\
5.2309 E+04 \\
5.5113 E+04 \\
1.9696 E+03 \\
0.00000+00 \\
0.0000 E+00 \\
2.0722 E+04 \\
6.9054 E+02 \\
1.1500 E+05 \\
2.0939 E-01 \\
6.0602 E+01 \\
2.3225 E+02 \\
0.0000 E+00\end{array}$ & $\begin{array}{l}5.6 \\
1.0 \\
5.22 \\
5.50 \\
1.9 \\
0.0 \\
0.0 \\
2.07 \\
6.67 \\
1.15 \\
3.7 \\
7.07 \\
2.2 \\
0.0\end{array}$ \\
\hline
\end{tabular}

wo. Of ITERATIOnS $=45$

\begin{tabular}{|c|c|c|}
\hline $\begin{array}{l}\text { PECIES } \\
\text { mes }\end{array}$ & $\begin{array}{l}\text { Renctamts } \\
\text { (mols) }\end{array}$ & \\
\hline 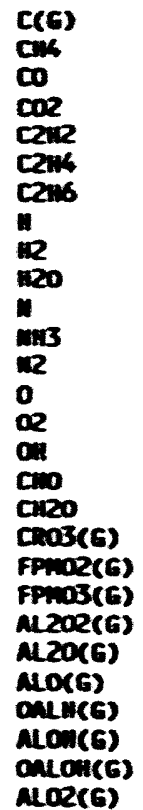 & 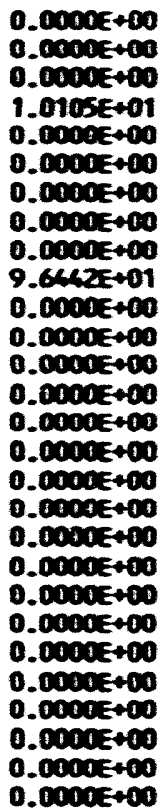 & 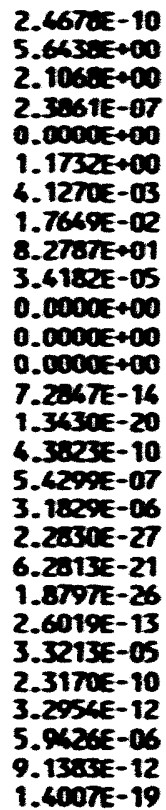 \\
\hline
\end{tabular}




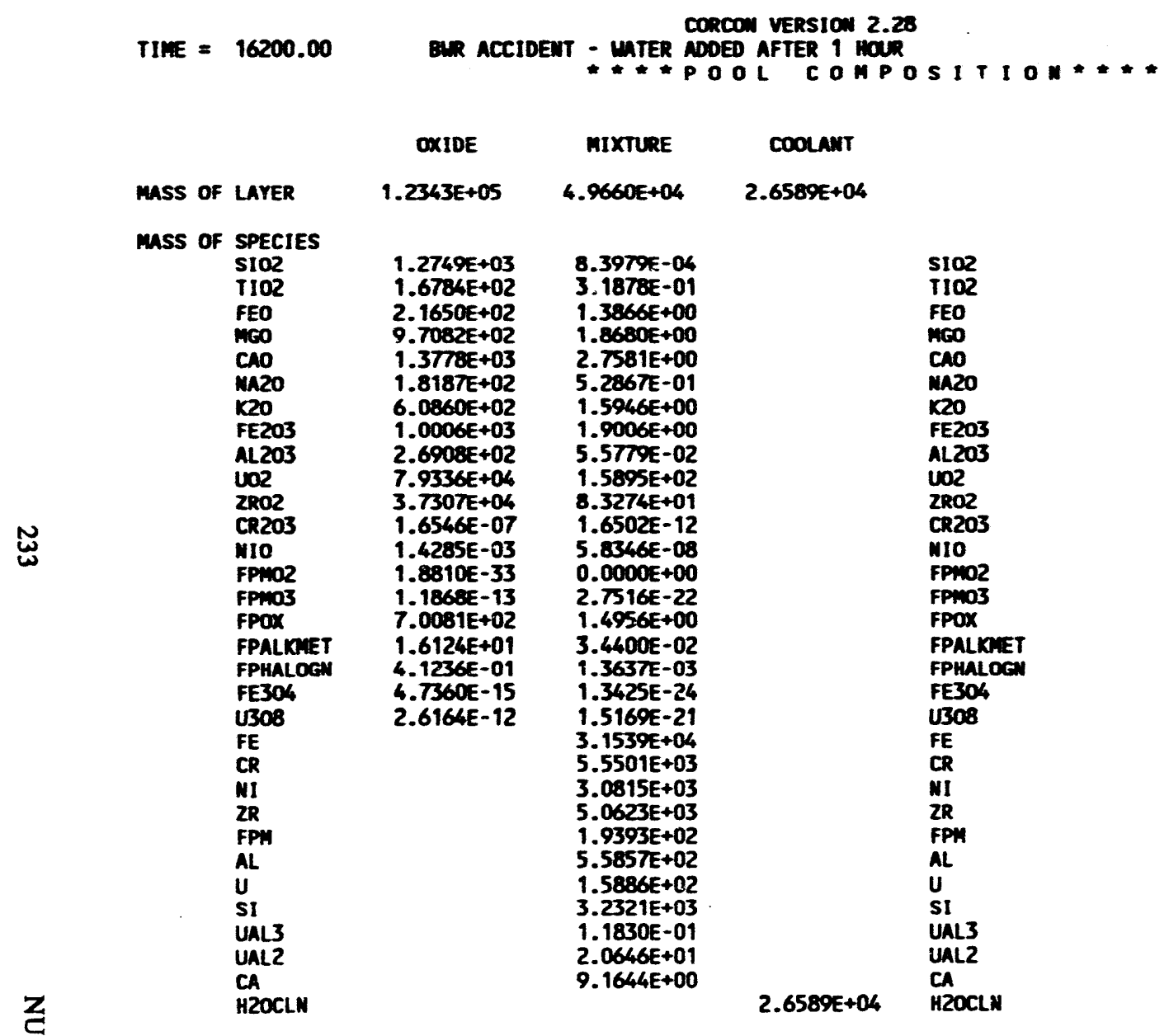


Table 6.4 Output listing for the BWR sample problem (continued)

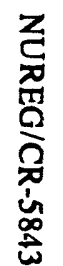

TIME $=16200.00$

CORCON VERSION 2.28

BUR ACCIDENT - WATER ADDED AFTER I HOUR

* * * Layer properties****

IT. พO. $=180$

\begin{tabular}{|c|c|c|c|c|}
\hline $\begin{array}{l}\text { MASS } \\
\text { EENSITY } \\
\text { THERMAL EXPANSIVITY }\end{array}$ & $\begin{array}{c}(\mathrm{KG}) \\
(\mathrm{KG} / \mathrm{MB}) \\
(1 / \mathrm{K})\end{array}$ & $\begin{array}{c}\text { OXIDE } \\
1.2343 E+05 \\
7.0929 E+03 \\
4.6053 E-05\end{array}$ & $\begin{array}{l}\text { MIXTURE } \\
4.9660 E+04 \\
6.02777+03 \\
2.2891 E-05\end{array}$ & $\begin{array}{c}\text { COOLANT } \\
2.6589 E+04 \\
9.3580 E+02 \\
3.6328 E-04\end{array}$ \\
\hline $\begin{array}{l}\text { AVERAGE TEMPERATURE } \\
\text { 1WTERFACE TERMERATURE } \\
\text { EDGE TEMPERAPURE } \\
\text { SOL IDUS TEMPERATURE } \\
\text { LIQUDUS TEMPERATURE } \\
\text { SPECIFIC ENTALPY } \\
\text { TOTAL ENTHALPY } \\
\text { SPECIFIC HEAT }\end{array}$ & $\begin{array}{c}(K) \\
E(K) \\
(K) \\
(K) \\
(K) \\
(J / K G) \\
(J) \\
(J / K G K)\end{array}$ & $\begin{array}{r}1.8798 E+03 \\
1.6561 E+03 \quad 1.8 \\
1.6360 E+03 \\
1.7838 E+03 \\
2.7553 E+03 \\
-5.2045+06 \\
-6.4239 E+19 \\
6.0514 E+02\end{array}$ & $\begin{array}{l}1.8358 E+03 \\
E+03 \quad 1.6 \\
1.7760 E+03 \\
1.7534 E+03 \\
2.7664 E+03 \\
1.3785 E+06 \\
6.8454 E+10 \\
7.4533 E+02\end{array}$ & $\begin{array}{c}3.9341 E+02 \\
33 E+03 \quad 4.031 \\
3.9341 E+02 \\
2.6821 E+02 \\
2.7821 E+02 \\
-1.5478 E+07 \\
-4.1153 E+11 \\
4.2651 E+03\end{array}$ \\
\hline $\begin{array}{l}\text { SCOSITY } \\
\text { ECRMA CONDUCTIVITY } \\
\text { ERMAL DIFFUSIVITY } \\
\text { RFACE TENSIOW } \\
\text { IISSIVITY }\end{array}$ & $\begin{array}{c}(K G / M S) \\
(W / M K S) \\
(M 2 / S) \\
(N / M) \\
(-)\end{array}$ & $\begin{array}{l}7.4446 E+02 \\
2.8554 E+00 \\
6.6526 E-07 \\
5.1718 E-01 \\
8.0000 E-01\end{array}$ & $\begin{array}{l}6.9844 E-03 \\
2.4069 E+01 \\
5.3574 E-06 \\
1.6444 E+00 \\
6.0086 E-01\end{array}$ & $\begin{array}{l}0.0000 E-01 \\
1.5033 E-07 \\
7.3000 E-02 \\
1.0000 E+00\end{array}$ \\
\hline
\end{tabular}

SUPERFICIAL GAS VEL (M/S) $2.1406 E-03 \quad 2.7123 E-03 \quad 2$ 5993E-03 $5.7956 E-04$ BUBBLE RADIUS BUBBLE VELOCITY

VOID FRACTIOW $($ (M)

$(-)$

$1.0211 E-02$
$3.2471 E-03$

$6.9650 E-03$

$1.0331 \mathrm{E}-0$

.

6.1839E-03

2.7339-01

BOT CRUST THICKMESS

(M)

HT COEFF, LIO TO BOT (W/M2 K)

HT COEFF HO TO TOP (WMP

TOP CRUST THICKMESS (M)

1.6761E-02

$0.0000 E+0 C$

$1.7162 E+02$

3.0615E-03

$7.8850 E+03$

$1.4951 E+04$

$0.0000 E+00$

$7.5858 \mathrm{E}+02$

$3.9341 \mathrm{E}+02$

$0.0000 E+00 \quad 4.4561 E-03$

R-AVE LIO TEMPERATURE (K)

HT COEFF, LIO TO SID (W/ME

SIDE CRUST THICKMESS

$1.8874 E+03$

$0.0000 E+00$

DECAY HEAT

HEAT TO CONCRETE

(M)

$1.8358 E+03$

$7.0885 E-02$

.1745E+03

$3.9341 \mathrm{E}+02$

$1.0000 E-10$

$0.0000 E+00$

$0.0000 E+00$

$\begin{array}{lll}7.6810 E+06 & 1.0987 E+06 & 0.0000 E+00\end{array}$

$\begin{array}{llll}\text { (W) } & 9.6937 E+05 & 2.1373 E+06 & 0.00000 E+00 \\ \text { (W) } & 0.0000 E+00 & 2.6739 E+06 & 0.0000+00\end{array}$

(H)

$8.0071 E+06 \quad 1.3443 E+07-3.0993 E+05$ 
Table 6.4 Output listing for the BWR sample problem (continued)

\section{CORCON VERSION 2.28}

TIME $=16200.00$ BIR ACCIDEMT - UATER ADDED AFTER 1 HOUR

* * * INTERIAYER MIXING****

IT. NO. $=180$

LONER MIXTURE LAYER

$\begin{array}{lcll} & & \text { OXIDE } & \text { METAL } \\ \text { PHASE MASSES } & (K G) & 2.5417 E+02 & 4.9406 E+04 \\ \text { PHASE DENSITIES } & \text { (KG/MB) } & 7.1571 E+03 & 6.0228 E+03 \\ \text { LIQUIDUS TEMPERATURE } & \text { (K) } & 1.9640 E+03 & 1.7534 E+03 \\ \text { SOLIDUS TEMPERATURE } & \text { (K) } & 2.7664 E+03 & 1.7634 E+03 \\ \text { PHASE EMTHALPIES } & \text { (J) } & -1.3325 E+09 & 6.9787 E+10 \\ \begin{array}{l}\text { MASS EMTRAIMED } \\ \text { MASS DE-EMTRAINED }\end{array} & \text { (KG) } & 0.0000 E+00 & 0.0000 E+00 \\ \text { (KG) } & 2.5508 E+02 & 0.0000 E+00\end{array}$


Table 6.4 Output listing for the BWR sample problem (continued)

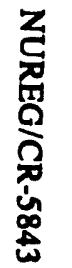

TIME $=16200.00$

BAR ACCIDEUT CORCON VERSION 2.28

- miter adoed after i moir

ANESA OUTPUT ****.*.*.

IT. 10. $=180$

TEMPERATURE OF METAL (K)

TEMPERATURE OF OXIDE (K)

$1.8369 E+03$

AEROSOL - STANDARD STATE CONDITIOWS (G/CC) $\quad \begin{aligned} & 1.0962 E-04 \\ & \mathbf{3} .0112 E-04\end{aligned}$

( 298.15 (K) AND 1 ATM.)

CAS (G-MOLES/S)

AEROSOL RATE (GRAMS/S)

AEROSOL DENSITY (G/CWB)

PARTICLE SIZE (MICROMETERS)

GUBBLE DIAMETER FOR THE METAL PHASE (CM)

BUBBLE DINMETER FOR TME OXIDE PHASE (CM)

$3.9139 E+00$

$2.8835 E+01$

$2.1824 E+00$

$9.8129 E-01$
$2.0706 E+00$

$2.0706 \mathrm{t}+00$

MECHANICAL RELEASES FROA THE AZBEL AND THE ISHII AMD KATAOU

జ

$\begin{array}{ccc}1 & \text { AEROSOL COMPOSITI } \\ \text { (WEIGHT X) }\end{array}$

CKG)
(KITIO
$3.1511 E+04$
$5.5398 E+03$
$3.0757 E+03$
$8.9914 E+01$
$9.5250 E+01$
$0.0000 E+00$
$3.8510 E-01$
$8.5732 E+00$
$0.0000 E+00$
$0.0000 E+00$
$1.3806 E+03$
$2.6913 E+02$
$1.8240 E+02$
$6.1025 E+02$
$1.2749 E+03$
$7.9339 E+04$
$3.7360 E+04$
$1.7913 E+01$
$3.5917 E+01$
$2.4404 E+01$
$2.4540 E+02$
$4.7559 E+02$
$1.9491 E+00$
$8.9518 E-01$
$2.1511 E+02$
$1.6546 E-07$
$0.0000 E+00$
$5.0242 E+03$

LOSS(MOLES)

RELEASE FRACTION

$\begin{array}{ll}1.7107 E-04 & 2.2240 E-04 \\ 1.6212 E-12 & 8.3773 E-09 \\ 4.4787 E-12 & 3.3728 E-08 \\ 0.0000 E+00 & 0.0000 E+00 \\ 8.5752 E-06 & 2.1320 E-02 \\ 5.1191 E-03 & 1.9793 E-01 \\ 0.0000 E+00 & 0.0300 E+00 \\ 0.0000 E+00 & 0.0000 E+00 \\ 1.0429 E-01 & 2.3555 E-02 \\ 3.4859 E-03 & 5.3254 E-03 \\ 2.5181 E+00 & 3.8645 E-01 \\ 2.7179 E+00 & 2.9093 E-01 \\ 7.3693 E+00 & 6.7549 E-02 \\ 3.5068 E-04 & 8.3010 E-05 \\ 2.7814 E-04 & 3.1543 E-06 \\ 2.2365 E-03 & 5.8427 E-02 \\ 4.6681 E-03 & 1.4437 E-01 \\ 9.2608 E-03 & 2.45633 E-01 \\ 1.5805 E-05 & 3.0109 E-03 \\ 1.2734 E-03 & 6.8293 E-02 \\ 1.6453 E-08 & 4.2344 E-06 \\ 1.2819 E-03 & 4.1200 E-01 \\ 4.2759 E-03 & 2.3861 E-04 \\ 3.7482 E-10 & 8.4718 E-12\end{array}$

ZIRCONIUN IN MELT

$5.0242 E+03$ 


\begin{tabular}{|c|c|c|c|}
\hline 1 & & $\begin{array}{l}\text { GAS composition } \\
\text { (WEIGHT } X \text { ) }\end{array}$ & $\begin{array}{l}\text { RELEASE RATE } \\
\text { (GRAMS/SECOWD) }\end{array}$ \\
\hline $\begin{array}{l}1 \\
2 \\
3 \\
4 \\
5 \\
6 \\
7 \\
8\end{array}$ & $\begin{array}{l}\mathrm{H} 2 \mathrm{O} \\
\mathrm{H2} \\
\mathrm{H} \\
\mathrm{OH} \\
0 \\
\mathrm{O} \\
\mathrm{CO} \\
\mathrm{CO}\end{array}$ & $\begin{array}{l}1.8230 E-04 \\
9.6818 E+01 \\
4.4239 E-02 \\
6.7414 E-09 \\
2.2706 E-12 \\
1.8782 E-18 \\
1.4430 E-06 \\
3.1373 E+00\end{array}$ & $\begin{array}{l}1.2854 E-04 \\
7.6390 E+00 \\
1.7453 E-03 \\
4.4875 E-09 \\
1.4214 E-12 \\
2.3523 E-18 \\
2.4856 E-06 \\
3.4395 E+00\end{array}$ \\
\hline
\end{tabular}


Table 6.4 Output listing for the BWR sample problem (continued)

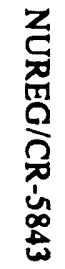

TIME $=16200.00$

BUR ACCIDENT CORCON VERSION 2.28

$$
\star \star * * * \text { POOL SCRUBBING } * * * * *
$$

IT. Mo. $=180$

INFORMATIOM SUPPLIED BY VANESA

MEAN PARTICLE SIZE (UN)

AEROSOL RATE (GRAMS/S)

AEROSOL DENSITY (G/CM3)

POOL DEPTH (CH)

POOL TEMPERATURE (K)

AMBIENT PRESSURE (ATM)

$$
\begin{aligned}
& 9.8129 E-01 \\
& 2.8835 E+01 \\
& 2.1824 E+00 \\
& 8.6848 E+01 \\
& 3.9336 E+02 \\
& 2.0500 E+00
\end{aligned}
$$

\begin{tabular}{|c|c|c|c|}
\hline $\begin{array}{l}\text { S1ZE } \\
\text { RANGE } \\
.000-0.249 \\
.249-0.337 \\
.337-0.414 \\
.414-0.487 \\
.487-0.560 \\
.560-0.634 \\
.634-0.712 \\
.712-0.795 \\
.795-0.884 \\
.884-0.981 \\
.981-1.090 \\
.090-1.212 \\
.212-1.353 \\
.353-1.519 \\
.519-1.721 \\
.721-1.978 \\
.978-2.327 \\
.327-2.853 \\
.853-3.862 \\
.862-* \star * \star \star \star\end{array}$ & $\begin{array}{c}\text { CHARACTERISTIC } \\
\text { SIZE } \\
0.192 \\
0.296 \\
0.376 \\
0.451 \\
0.523 \\
0.596 \\
0.672 \\
0.753 \\
0.838 \\
0.931 \\
1.034 \\
1.149 \\
1.280 \\
1.432 \\
1.614 \\
1.841 \\
2.137 \\
2.558 \\
3.255 \\
5.021\end{array}$ & $\begin{array}{c}\text { MASS } \\
1 \text { RANGE } \\
1.1988 E+00 \\
1.2362 E+00 \\
1.2433 E+00 \\
1.2407 E+00 \\
1.2321 E+00 \\
1.2187 E+00 \\
1.2007 E+00 \\
1.1778 E+00 \\
1.1496 E+00 \\
1.1151 E+00 \\
1.0734 E+00 \\
1.0227 E+00 \\
9.6117 E-01 \\
8.8595 E-01 \\
7.9341 E-01 \\
6.7877 E-01 \\
5.3627 E-01 \\
3.6109 E-01 \\
1.6050 E-01 \\
8.6443 E-03\end{array}$ & $\begin{array}{c}\text { DECONTAMINATIO } \\
\text { FACTOR } \\
1.2027 E+00 \\
1.1663 E+00 \\
1.1596 E+00 \\
1.1621 E+00 \\
1.1702 E+00 \\
1.1830 E+00 \\
1.2008 E+00 \\
1.2241 E+00 \\
1.2542 E+00 \\
1.2929 E+00 \\
1.3432 E+00 \\
1.4097 E+00 \\
1.5000 E+00 \\
1.6274 E+00 \\
1.8172 E+00 \\
2.1241 E+00 \\
2.6885 E+00 \\
3.9928 E+00 \\
8.9828 E+00 \\
1.6679 E+02\end{array}$ \\
\hline
\end{tabular}

$\begin{array}{ll}\text { OVERALL DECONTAMINATION FACTOR } & 1.5591 \\ \text { MASS OUT } & 1.8495 \mathrm{E}+01\end{array}$

$\begin{array}{ll}\text { OVERALL DECONTAMINATION FACTOR } & 1.5591 \\ \text { MASS OUT } & 1.8495 \mathrm{E}+01\end{array}$

FIT OF DECONTAMIMATED AEROSOL TO LOG MORMAL

FIT OF DECONTAMIMATED AEROSOL TO LOG MORMAL
RANGE (UM)
RANGE (UNA)
RANGE (UN)
$\begin{array}{cc}0.726 \\ 0.707- & 0.745 \\ 1.945- & 1.829 \\ 1.719\end{array}$
LINEAR CORRELATION COEFFICIENT
0.990469 


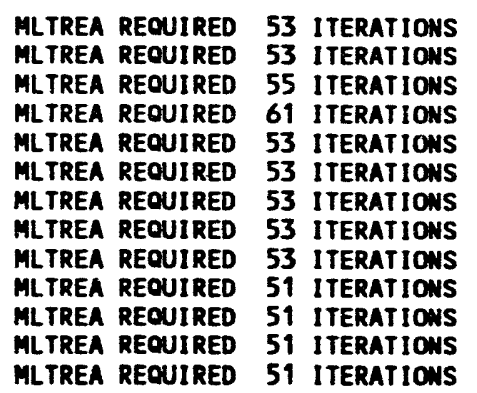


Table 6.4 Output listing for the BWR sample problem (continued)

MELT AND COOLANT LAYERS

MUMBER OF LAYERS, MLYR $=3$

CONFIGURATION, ILYR $=0$
NO COOLANT PRESENT, ICOOL $=0$

EXTREME CAVITY DIMENSIONS, MITH LOCATIONS

\begin{tabular}{|c|c|c|}
\hline $\begin{array}{l}\text { RADIAL } \\
\text { MAXIMUM CAVITY RADIUS } \\
\text { OUTSIDE RADIUS OF CONCRETE } \\
\text { REMAINING THICKNESS } \\
\text { CORRESPOWIHG BOOY POINT }\end{array}$ & $\begin{array}{l}(M)= \\
(M)= \\
(M)= \\
(M)=\end{array}$ & $=$ \\
\hline
\end{tabular}

AXIAL

DEEPEST POINT IN CAVITY (M) $=5.08486$

MAXIMUM DEPTH OF CONCRETE (M) $=\mathbf{8 . 0 5 0 0 0}$

REMAINING THICKMESS

CORRESPONDING BOOY POINT

APPROXIMATE OVERALL EMERGY BUDGET FOR DEBRIS

(SEE MAMUAL FOR EXPLANATION AND CAVEATS)
INTERHAL (DECAY) SOURCE
(W) $=1.103 E+07$ CHEMICAL REACTION SOURCE
(W) $=3.580 E+06$
HEAT LOSS TO CONCRETE
(W) $=3.499 E+06$
HEATUP OF ABLATION PRODUCTS
(W) $=-1.487 \mathrm{E}+06$
HEAT LOSS FROM SURFACE
(W) $=1.058 E+07$
(TO SURROUNDIMGS)
(W) $=-2.164 E+10$

CHANGE IN POOL ENTHALPY
(SUMHATION OF M^DH/DT)

NUMERICAL CHECKS ON MASS AND ENERGY CONSERVATION

RELATIVE ERROR IN MASS $=-4.05312 E-06$

RELATIVE ERROR IN EMTHALPY $=-1.78814$ E-07

CHECK ON RECESSION CALCULATION (DO/DS SHOULD BE .LE. 1)

MAXIMUM DD/DS $=0.00402$ 
TIME $=21600.00$

GAS EXITING POOL (INCLUDES FILM AND COOLANT)

\section{GENERATION RATE}

$\begin{array}{lll}\text { SPECIES } & \text { MASS (KG/S) } & \text { MOLES (1/S) } \\ \text { C(G) } & 9.49796 E-14 & 7.90771 E-12 \\ \text { CH4 } & 5.24064 E-03 & 3.26670 E-01 \\ \text { CO } & 1.85434 E-03 & 6.62017 E-02 \\ \text { CO2 } & 3.28865 E-10 & 7.47253 E-09 \\ \text { C2H2 } & 0.00000 E+00 & 0.00000 E+00 \\ \text { C2H4 } & 1.61999 E-03 & 5.77475 E-02 \\ \text { C2H6 } & 8.171977-06 & 2.71770 E-04 \\ \text { H } & 7.31174 E-07 & 7.25443 E-04 \\ \text { H2 } & 8.23833 E-03 & 4.08688 E+00 \\ \text { H2O } & 5.25124 E+00 & 2.91490 E+02 \\ \text { N } & 0.00000 E+00 & 0.00000 E+00 \\ \text { NH3 } & 0.00000 E+00 & 0.00000 E+00 \\ \text { N2 } & 0.00000 E+00 & 0.00000 E+00 \\ \text { O } & 4.28101 E-17 & 2.67573 E-15 \\ \text { O2 } & 1.75147 E-23 & 5.47356 E-22 \\ \text { OH } & 2.96114 E-13 & 1.74110 E-11 \\ \text { CHO } & 5.53478 E-10 & 1.90734 E-08 \\ \text { CH2O } & 3.79128 E-09 & 1.26266 E-07 \\ \text { CRO3(G) } & 1.13606 E-29 & 1.13612 E-28 \\ \text { FPMO2(G) } & 3.54648 E-23 & 2.72101 E-22 \\ \text { FPMO3(G) } & 1.32464 E-28 & 9.05205 E-28 \\ \text { AL202(G) } & 1.55346 E-15 & 1.10962 E-14 \\ \text { AL20(G) } & 1.00210 E-07 & 1.43234 E-06 \\ \text { ALO(G) } & 3.88366 E-13 & 9.03576 E-12 \\ \text { OALH(G) } & 6.32648 E-15 & 1.43820 E-13 \\ \text { ALOH(G) } & 1.15627 E-08 & 2.62855 E-07 \\ \text { OALOH(G) } & 2.38082 E-14 & 3.96881 E-13 \\ \text { ALO2(G) } & 3.51935 E-22 & 5.96699 E-21\end{array}$

CUMULATIVE RELEASE

$\begin{array}{lll}\text { MASS (KG) } & \text { MOLES (-) } & \text { SPECIES } \\ 4.61905 E-07 & 3.84568 E-05 & \text { C(G) } \\ 3.20791 E+01 & 1.99962 E+03 & \text { CH4 } \\ 1.41000 E+02 & 5.03383 E+03 & \text { CO } \\ 1.75267 E-02 & 3.98245 E-01 & \text { CO2 } \\ 0.00000 E+00 & 0.00000 E+00 & \text { C2H2 } \\ 1.04995 E+01 & 3.74275 E+02 & \text { C2H4 } \\ 4.20700 E-02 & 1.39910 E+00 & \text { C2H6 } \\ 1.74762 E-01 & 1.73392 E+02 & \text { H } \\ 1.40011 E+02 & 6.94568 E+04 & \text { H2 } \\ 3.05659 E+04 & 1.69667 E+06 & \text { H2O } \\ 0.00000 E+00 & 0.00000 E+00 & \text { H } \\ 0.00000 E+00 & 0.00000 E+00 & \text { HH3 } \\ 0.00000 E+00 & 0.00000 E+00 & \text { N2 } \\ 1.68767 E-07 & 1.05483 E-05 & 0 \\ 1.29229 E-11 & 4.03857 E-10 & \text { O2 } \\ 1.33104 E-05 & 7.82628 E-04 & \text { OH } \\ 1.76750 E-04 & 6.09098 E-03 & \text { CHO } \\ 1.74482 E-04 & 5.81101 E-03 & \text { CH2O } \\ 3.56293 E-16 & 3.56314 E-15 & \text { CRO3(G) } \\ 1.63963 E-11 & 1.25799 E-10 & \text { FPMO2(G) } \\ 2.40653 E-15 & 1.64452 E-14 & \text { FPMO3(G) } \\ 5.24340 E-09 & 3.74528 E-08 & \text { AL2O2(G) } \\ 1.38175 E-02 & 1.97499 E-01 & \text { AL20(G) } \\ 4.93327 E-06 & 1.14778 E-04 & \text { ALO(G) } \\ 2.73988 E-08 & 6.22858 E-07 & \text { OALH(G) } \\ 1.90972 E-03 & 4.34137 E-02 & \text { ALOH(G) } \\ 1.33697 E-07 & 2.22872 E-06 & \text { OALOH(G) } \\ 1.93918 E-12 & 3.28783 E-11 & \text { ALO2(G) }\end{array}$


Table 6.4 Output listing for the BWR sample problem (continued)

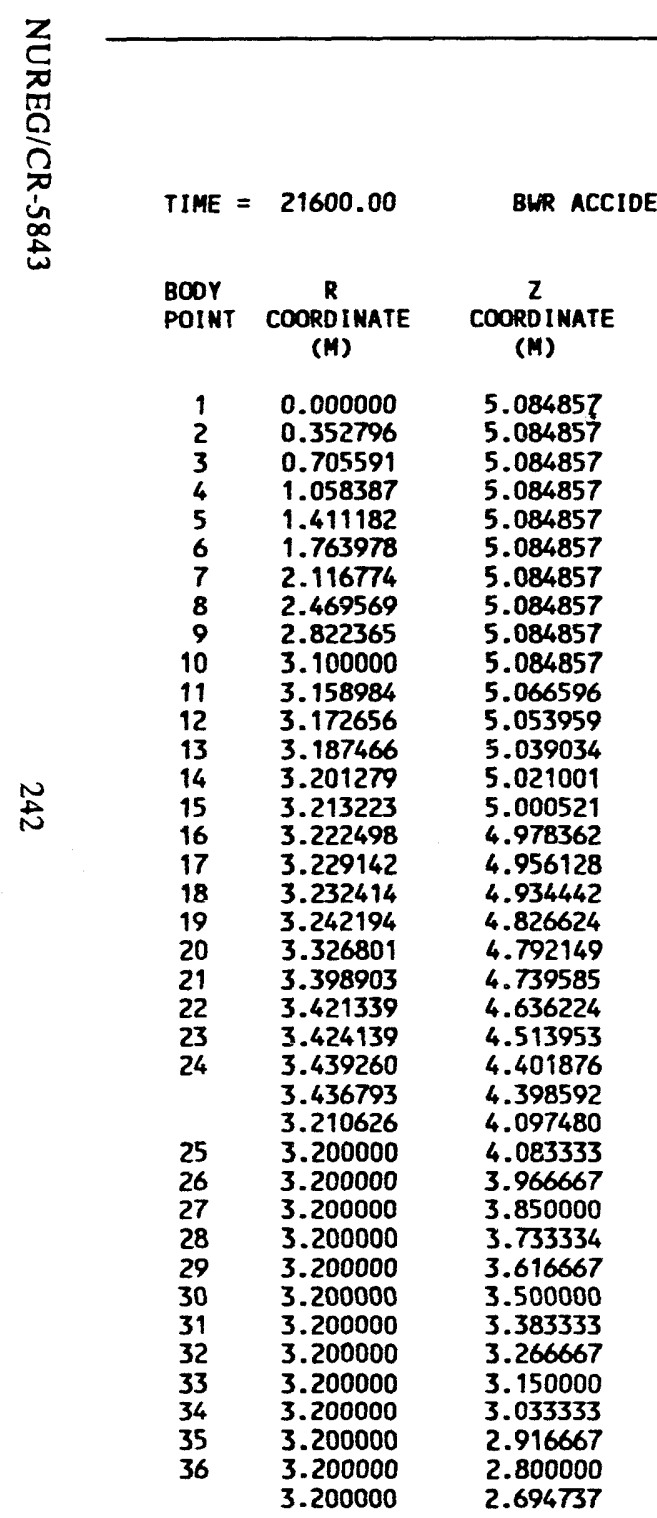

CORCON VERSION 2.28

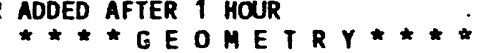

\begin{tabular}{|c|c|c|c|}
\hline $\begin{array}{l}\text { TREAM } \\
\text { ENGTH } \\
\text { (M) }\end{array}$ & $\begin{array}{l}\text { BODY } \\
\text { ANGLE } \\
\text { (DEG) }\end{array}$ & $\begin{array}{c}\text { RAY } \\
\text { ANGLE } \\
\text { (DEG) }\end{array}$ & $\begin{array}{c}\text { VOLUME } \\
\text { (M3) }\end{array}$ \\
\hline $\begin{array}{l}000000 \\
352796 \\
705591 \\
058387 \\
411182 \\
763978 \\
116774 \\
469569 \\
822365 \\
100000 \\
161746 \\
180363 \\
201390 \\
224105 \\
247813 \\
271836 \\
295041 \\
316972 \\
425232 \\
516594 \\
605822 \\
711591 \\
833894 \\
946985\end{array}$ & $\begin{array}{r}0.000 \\
0.000 \\
0.000 \\
0.000 \\
0.000 \\
0.000 \\
0.000 \\
0.000 \\
0.000 \\
0.000 \\
29.973 \\
43.983 \\
48.886 \\
56.149 \\
63.518 \\
70.326 \\
77.392 \\
83.118 \\
53.493 \\
29.131 \\
56.923 \\
83.221 \\
85.502 \\
104.613\end{array}$ & $\begin{array}{r}0.000 \\
5.621 \\
11.135 \\
16.449 \\
21.487 \\
26.200 \\
30.561 \\
34.563 \\
38.213 \\
0.000 \\
41.532 \\
41.756 \\
42.008 \\
42.277 \\
42.550 \\
42.813 \\
43.055 \\
43.264 \\
44.264 \\
45.300 \\
46.375 \\
47.490 \\
48.646 \\
49.844\end{array}$ & $\begin{array}{r}0.00000 \\
0.00000 \\
0.00000 \\
0.00000 \\
0.00000 \\
0.00000 \\
0.00000 \\
0.00000 \\
0.00000 \\
0.00000 \\
0.56187 \\
0.95974 \\
1.43392 \\
2.01201 \\
2.67383 \\
3.39468 \\
4.12153 \\
4.83264 \\
8.38247 \\
9.55094 \\
11.41847 \\
15.19463 \\
19.69472 \\
23.84123\end{array}$ \\
\hline $\begin{array}{l}.940 \times 03 \\
\text { OXIDE } \\
\text { IIXTURE }\end{array}$ & $\begin{array}{l}\text { MIXTURE INTERFAC } \\
\text { COOLANT INTERFAC }\end{array}$ & & \\
\hline $\begin{array}{l}345376 \\
462043 \\
578710 \\
.695376 \\
812043 \\
928710 \\
045377 \\
162044 \\
278710 \\
.395377 \\
512044 \\
628711\end{array}$ & $\begin{array}{r}108.455 \\
90.000 \\
90.000 \\
90.000 \\
90.000 \\
90.000 \\
90.000 \\
90.000 \\
90.000 \\
90.000 \\
90.000 \\
90.000\end{array}$ & $\begin{array}{l}51.086 \\
52.374 \\
53.707 \\
55.088 \\
56.517 \\
57.995 \\
59.521 \\
61.098 \\
62.723 \\
64.398 \\
66.121 \\
67.891\end{array}$ & $\begin{array}{l}34.87402 \\
38.62718 \\
42.38033 \\
46.13348 \\
49.88664 \\
53.63980 \\
57.39295 \\
61.14610 \\
64.89926 \\
68.65242 \\
72.40557 \\
76.15872\end{array}$ \\
\hline
\end{tabular}

IT. NO. $=360$

SURFACE VOID

AREA

$(-)$

$0.00000 \quad 0.00640$

$0.39102 \quad 0.00640$

$\begin{array}{ll}1.56407 & 0.00640\end{array}$

$\begin{array}{ll}1.56407 & 0.00640 \\ 3.51916 & 0.00640\end{array}$

$\begin{array}{ll}6.25628 & 0.00640\end{array}$

$\begin{array}{ll}6.25628 & 0.00640 \\ 9.7 / 543 & 0.00640\end{array}$

$14.07663 \quad 0.00640$

$\begin{array}{ll}14.07663 & 0.00640 \\ 19.15986 & 0.00640\end{array}$

$25.02512 \quad 0.00640$

25.025120 .00640

0.00640

$31.40483-0.00640$

31.75150 .00636

32.195280 .00633

33.0511960 .00630

33.128960 .00627

33.614650 .00625

$34.08499 \quad 0.00623$

$34.53018-0.00623$

$36.73225 \quad 0.00621$

38.617690 .00602

$40.50303 \quad 0.00587$

$42.76927 \quad 0.00583$

$45.39940 \quad 0.00582$

$47.83797 \quad 0.00579$

$56.14754 \quad 0.00630$

$58.49326 \quad 0.00260$

$60.83898 \quad 0.00260$

$63.18470 \quad 0.00260$

$65.53043 \quad 0.00260$

$67.87615 \quad 0.00260$

$\begin{array}{ll}70.22187 & 0.00260\end{array}$

$72.56759 \quad 0.00260$

$74.91331 \quad 0.00260$

$77.25904 \quad 0.00260$

$79.60476 \quad 0.00260$

$81.95048 \quad 0.00260$ 
Table 6.4 Output listing for the BWR sample problem (continued)

\begin{tabular}{|c|c|c|c|c|c|c|c|c|}
\hline $\begin{array}{l}37 \\
38 \\
39 \\
40 \\
41 \\
42 \\
43 \\
44 \\
45 \\
46 \\
47 \\
48 \\
49 \\
50 \\
51 \\
52 \\
53 \\
54 \\
55 \\
56 \\
57 \\
58 \\
59 \\
60\end{array}$ & $\begin{array}{l}3.200000 \\
3.200000 \\
3.200000 \\
3.200000 \\
3.200000 \\
3.200000 \\
3.200000 \\
3.200000 \\
3.200000 \\
3.200000 \\
3.200000 \\
3.200000 \\
3.200000 \\
3.200000 \\
3.200000 \\
3.200000 \\
3.200000 \\
3.200000 \\
3.200000 \\
3.200000 \\
3.200000 \\
3.200000 \\
3.200000 \\
3.200000\end{array}$ & $\begin{array}{l}2.6833333 \\
2.566667 \\
2.450000 \\
2.333333 \\
2.216667 \\
2.100000 \\
1.983333 \\
1.866667 \\
1.750000 \\
1.633333 \\
1.516667 \\
1.400000 \\
1.283333 \\
1.166667 \\
1.050000 \\
0.933333 \\
0.816667 \\
0.700000 \\
0.583333 \\
0.466667 \\
0.350000 \\
0.233334 \\
0.116667 \\
0.000000\end{array}$ & $\begin{array}{l}5.745378 \\
5.862044 \\
5.978711 \\
6.095378 \\
6.212045 \\
6.328712 \\
6.445378 \\
6.562045 \\
6.678712 \\
6.795379 \\
6.912045 \\
7.028712 \\
7.145379 \\
7.262046 \\
7.378713 \\
7.495379 \\
7.612046 \\
7.728713 \\
7.845379 \\
7.962046 \\
8.078712 \\
8.195379 \\
8.312046 \\
8.428713\end{array}$ & $\begin{array}{l}90.000 \\
90.000 \\
90.000 \\
90.000 \\
90.000 \\
90.000 \\
90.000 \\
90.000 \\
90.000 \\
90.000 \\
90.000 \\
90.000 \\
90.000 \\
90.000 \\
90.000 \\
90.000 \\
90.000 \\
90.000 \\
90.000 \\
90.000 \\
90.000 \\
90.000 \\
90.000 \\
90.000\end{array}$ & $\begin{array}{r}69.706 \\
71.565 \\
73.465 \\
75.403 \\
77.376 \\
79.380 \\
81.411 \\
83.463 \\
85.533 \\
87.614 \\
89.702 \\
91.790 \\
93.873 \\
95.947 \\
98.005 \\
100.042 \\
102.054 \\
104.036 \\
105.985 \\
107.896 \\
109.767 \\
111.595 \\
113.378 \\
115.115\end{array}$ & $\begin{array}{r}79.91188 \\
83.66503 \\
87.41819 \\
91.17134 \\
94.92450 \\
98.67765 \\
102.43081 \\
106.18396 \\
109.93712 \\
113.69027 \\
117.44343 \\
121.19658 \\
124.94974 \\
128.70290 \\
132.45605 \\
136.20921 \\
139.96237 \\
143.71552 \\
147.46867 \\
151.22183 \\
154.97499 \\
158.72813 \\
162.48129 \\
166.23445\end{array}$ & $\begin{array}{r}84.29620 \\
86.64192 \\
88.98765 \\
91.33337 \\
93.67909 \\
96.02481 \\
98.37054 \\
100.71626 \\
103.06198 \\
105.40770 \\
107.75343 \\
110.09914 \\
112.44487 \\
114.79059 \\
117.13631 \\
119.48203 \\
121.82776 \\
124.17348 \\
126.51920 \\
128.86493 \\
131.21065 \\
133.55637 \\
135.90208 \\
138.24780\end{array}$ & $\begin{array}{l}1.00000 \\
1.00000 \\
1.00000 \\
1.00000 \\
1.00000 \\
1.00000 \\
1.00000 \\
1.00000 \\
1.00000 \\
1.00000 \\
1.00000 \\
1.00000 \\
1.00000 \\
1.00000 \\
1.00000 \\
1.00000 \\
1.00000 \\
1.00000 \\
1.00000 \\
1.00000 \\
1.00000 \\
1.00000 \\
1.00000 \\
1.00000\end{array}$ \\
\hline
\end{tabular}




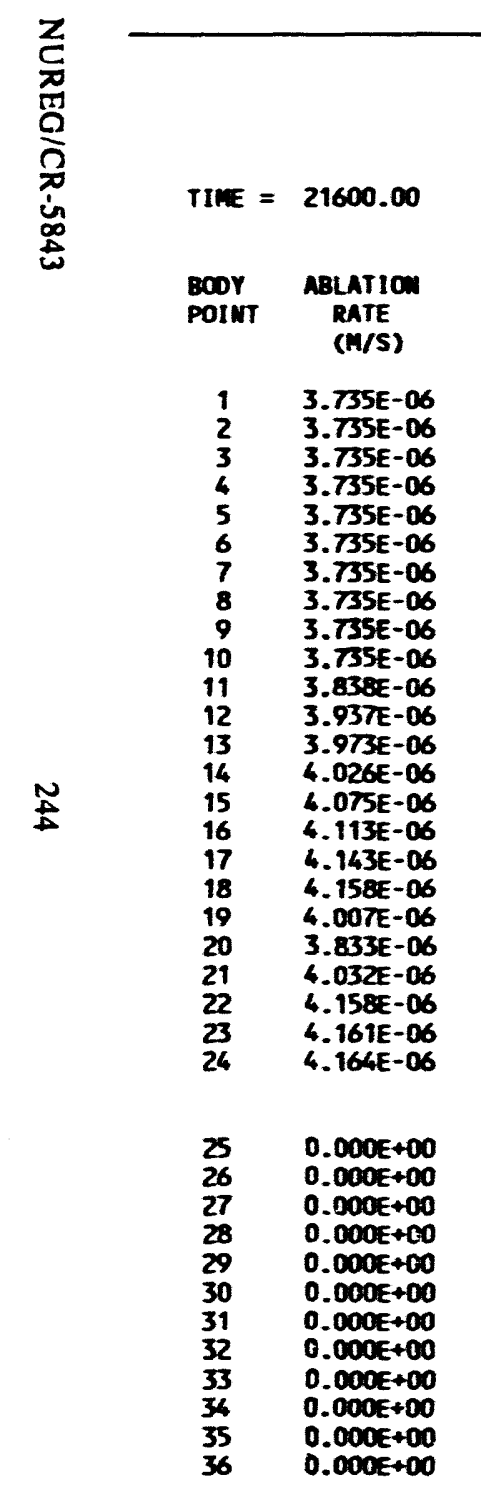
BUR ACCIDEWT - UATER CORCOW VERSIOW 2.28

\begin{tabular}{|c|c|c|c|}
\hline $\begin{array}{l}\text { FILn } \\
\text { THICKMESS } \\
\text { (H) }\end{array}$ & 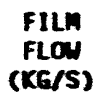 & $\begin{array}{l}\text { FILA } \\
\text { VELOCITY } \\
\text { (W/S) }\end{array}$ & $\begin{array}{c}\text { REVIOLOS } \\
\text { MEER } \\
(-)\end{array}$ \\
\hline
\end{tabular}
* * neat trainsfer results....

tr. $10 .=360$

IEGInE TITAMS
COEFF
(UNR-K)

IITERFACE

comective motative

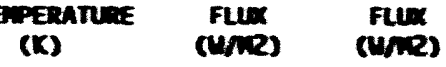

$\begin{array}{ccc}1.000 E-10 & 0.000 E+00 & 0.000 E+00\end{array}$ 0.020

S26.008. 1.000E+03 sL6.003. 1.000E+03 st6.805. 1.000E+03 st6.eve. 1.000E+03 SL6. 200 . 1.000E+03 SL6.008. 1.000E+03 st6.003. $1.000 \mathrm{E}+03$ sL6.eus. 1.000E+03 SLG.018. 1.000E+03 SLG.008. 1.000E+03 TE. EUS. 1.000E+03 SLG.FU. 1.000E-05 STG.FL. 1.000E+0B se.Fin. 1.000E+03 SLG.FU. 1.000E-03 st6.FLI. 1.000E+03 SLG.FLI. 1.000E+03 SLG.FLI. 1.00BE+03 SLG.FU. 1.000E+03 SLG.FL. 1.000E+03 SLG.FLH. 1.000E=03 SLG.FLA. 1.000E+0S STG.FL. 1.000E+03 SL6.FLA. 1.00BE+03

$\begin{array}{lll}1649.6 & 1.963 E+06 & 0.000 E+60 \\ 1649.6 & 1.963 E+04 & 0.004=000\end{array}$ $1649.6 \quad 1.963 E+04$ 0.060 $1649.6 \quad 1.963 E+06$ a.cone+en $1649.6 \quad 1.963 \mathrm{x}+04$ 0.000

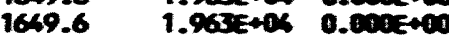
$1649.6 \quad 1.963 E+640.003 E+00$ $1649.6 \quad 1.9635+040.660+000$ 1669.6 1.963E+04 $0.003 E+60$ $1650.2 \quad 2.017 \mathrm{E}+04$ 0.600 $1650.7 \quad 2.06 \%=+06 \quad 0.000=000$ $1650.92 .000+040.000=000$ 1651.2 2.116e+0\% :.003E+os $1651.4 \quad 2.162 E+06$ 0.000E+00 $1651.62 .162 E+640.0005+60$ $1651.8 \quad 2.17 \mathrm{E}+66$ O.000E+60 1651.1 2.105en

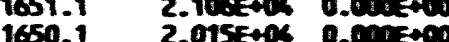
$1651.2 \quad 2.0110=0 \%$ Q.000 $1651.2 \quad 2.1190040 .010+6$ 1651.9 2. $100 \mathrm{E}+04$ Q.000 $\begin{array}{lll}1651.9 & 2.100 E+06 & 0.000 E+00 \\ 1651.9 & 2.100 E+06 & 0.000 E+10\end{array}$ 1.000E-10 $9.321 E-03 \quad 0.000 E+00$ OXIDE

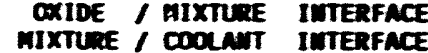
$1.000 E-10 \quad 9.061 E-02 \quad 0.000 E+00 \quad 75.766$ $\begin{array}{llll}1.000 E-10 & 9.061 E-02 & 0.000 E+00 & 75.766 \\ 1.000 E-10 & 9.061 E-02 & 0.000 E+00 & 75.766\end{array}$ $\begin{array}{cccc}1.000 E-10 & 9.061 E-02 & 0.000 E+00 & 75.766 \\ 1.000 E-10 & 9.061 E-02 & 0.000 E+00 & 75.766\end{array}$ $1.000 E-10 \quad 9.061 E-02 \quad 0.000 E+00 \quad 75.766$ $\begin{array}{llll}1.000 E-10 & 9.061 E-02 & 0.000 E+\infty 0 & 75.766\end{array}$ 1.000E-10 9.061E-02 $0.000 E+\infty 0$ T5.766 $\begin{array}{llll}1.000 E-10 & 9.061 E-02 & 0.000 E+00 & \\ 15.766\end{array}$ 1.000 - 10 9.061E-02 0.000 E +00 75.766 $1.000 E-10$ 9.061E-02 $0.000 E+00 \quad 75.766$ 1.000E-10 $9.061 E-02 \quad 0.000 E+00 \quad 75.766$ $\begin{array}{llll}1.000 E-10 & 9.061 E-02 & 0.000 E+00 & 75.766 \\ 1.000 E-10 & 9.061 E-02 & 0.000 E+00 & 75.766\end{array}$

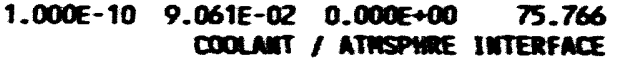

SLG.FLH. 1.000E+03 SLG.FLA. 1.000E+05 SLG.FLW. 1.000E+03 sechin. 1.000.003 sc. se.FLn. 1.000 sec.FL. 1.000 sL6.FL. 1.buetos SLG.FLn. 1.000E+0s st6.run. 1.000teds SL6.FLn. $1.000 E+03$
SLG. FLI. $1.000 E+03$

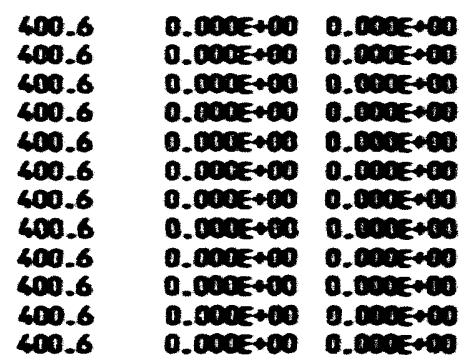


TINE $=21600.00$$$
\text { OXIDES }
$$

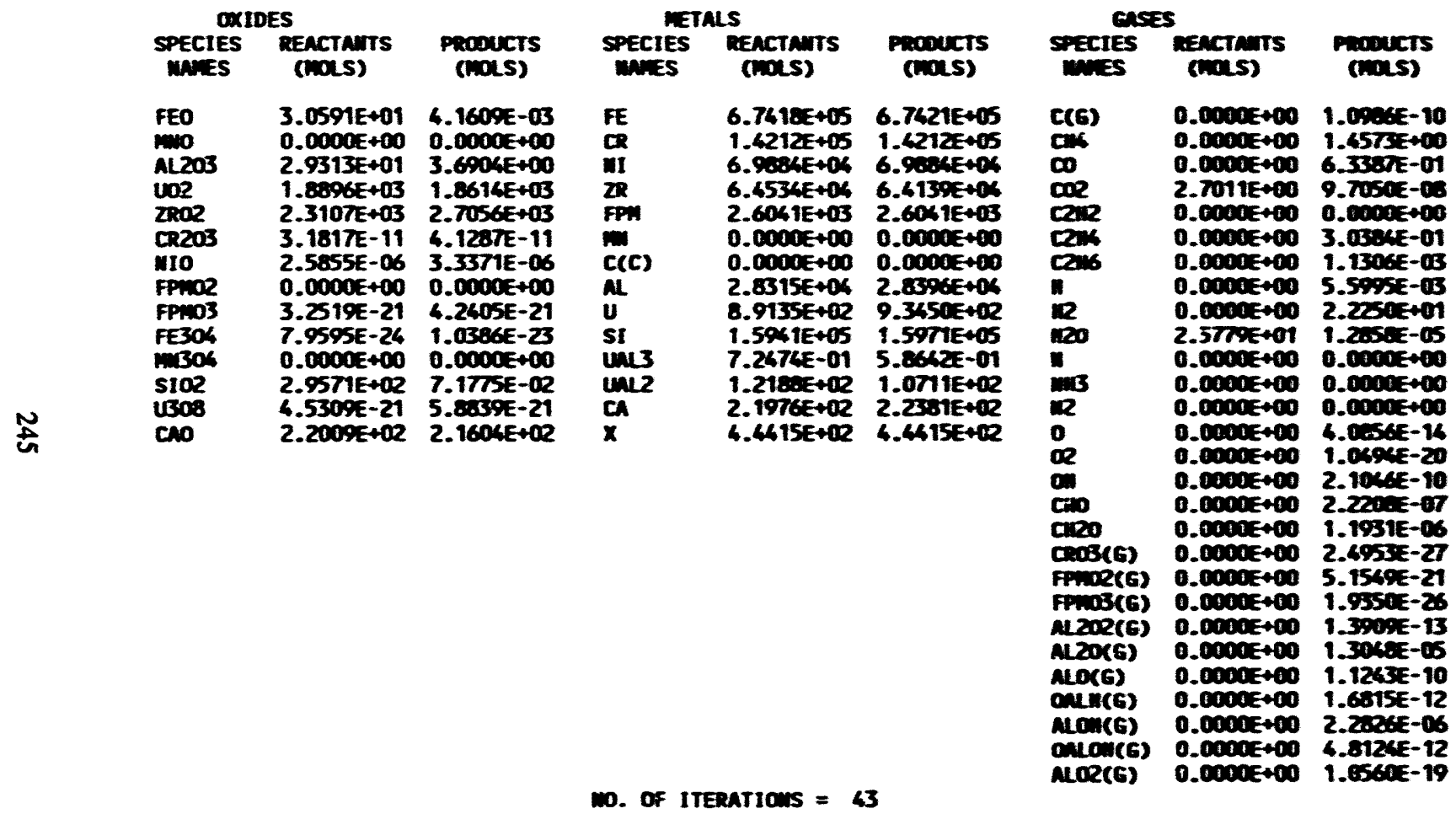

colecom vetsion 2.20

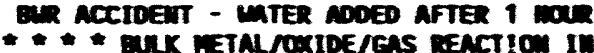

IT. $10 .=360$ 
Table 6.4 Output listing for the BWR sample problem (oontined)

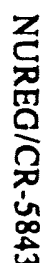

TIME $=21600.00$

BUR ACCIDENT - MATER ADOED AFTER 1 HOW

$$
\text { * * Film metal.gas reaction durimg timestep * * * }
$$

IT. $10 .=360$

OXIDES

\begin{tabular}{|c|c|c|c|c|c|}
\hline & & & & & \\
\hline $\begin{array}{l}\text { SPECIES } \\
\text { MAMES }\end{array}$ & $\begin{array}{l}\text { REACTANTS } \\
\text { (HOLS) }\end{array}$ & $\begin{array}{l}\text { PROOUCTS } \\
\text { (MOLS) }\end{array}$ & $\begin{array}{c}\text { SPECIES } \\
\text { MANES }\end{array}$ & $\begin{array}{l}\text { REACTAMTS } \\
\text { (MOLS) }\end{array}$ & $\begin{array}{l}\text { Prooucts } \\
\text { (mols) }\end{array}$ \\
\hline $\begin{array}{l}\text { FEO } \\
\text { MNO } \\
\text { AL2O3 } \\
\text { UO2 } \\
\text { ZRO2 } \\
\text { CR2O3 } \\
\text { N1O } \\
\text { FPMO2 } \\
\text { FPMO3 } \\
\text { FE304 } \\
\text { WN304 } \\
\text { S102 } \\
\text { U308 } \\
\text { CAO }\end{array}$ & $\begin{array}{l}0.0000 E+00 \\
0.0000 E+00 \\
0.0000 E+00 \\
0.0000 E+00 \\
0.0000 E+00 \\
0.0000 E+00 \\
0.0000 E+00 \\
0.0000 E+00 \\
0.0000 E+00 \\
0.0000 E+00 \\
0.0000 E+00 \\
0.0000 E+00 \\
0.0000 E+00 \\
0.0000 E+00\end{array}$ & $\begin{array}{l}3.3529 E-05 \\
0.0000 E+00 \\
6.4360 E-02 \\
2.9314 E+01 \\
4.0574 E+01 \\
2.3488 E-13 \\
2.7279 E-08 \\
0.0000 E+00 \\
9.4048 E-24 \\
1.9863 E-26 \\
0.3000 E+00 \\
8.1247 E-04 \\
1.9448 E-23 \\
3.7372 E+00\end{array}$ & $\begin{array}{l}\text { FE } \\
C R \\
\text { MI } \\
\text { ZR } \\
\text { FPM } \\
\text { WI } \\
C(C) \\
\text { AL } \\
U \\
\text { SI } \\
\text { UAL3 } \\
\text { UAL2 } \\
C A \\
X\end{array}$ & $\begin{array}{l}6.7421 E+05 \\
1.4212 E+05 \\
6.9884 E+04 \\
6.4139 E+04 \\
2.6041 E+03 \\
0.0000 E+00 \\
0.0000 E+00 \\
2.8396 E+04 \\
9.3450 E+02 \\
1.5971 E+05 \\
5.8642 E-01 \\
1.0711 E+02 \\
2.2381 E+02 \\
0.0000 E+00\end{array}$ & $\begin{array}{l}6.7621 E+05 \\
1.4212 E+05 \\
6.9884 E+04 \\
6.4098 E+04 \\
2.6041 E+03 \\
0.0000 E+00 \\
0.0000 E+00 \\
2.8366 E+04 \\
8.9029 E+02 \\
1.5971 E+05 \\
7.2514 E-01 \\
1.2188 E+02 \\
2.2007 E+02 \\
0.0000 E+00\end{array}$ \\
\hline
\end{tabular}

走

$0.0000 E+00 \quad 3.7372 E+10$

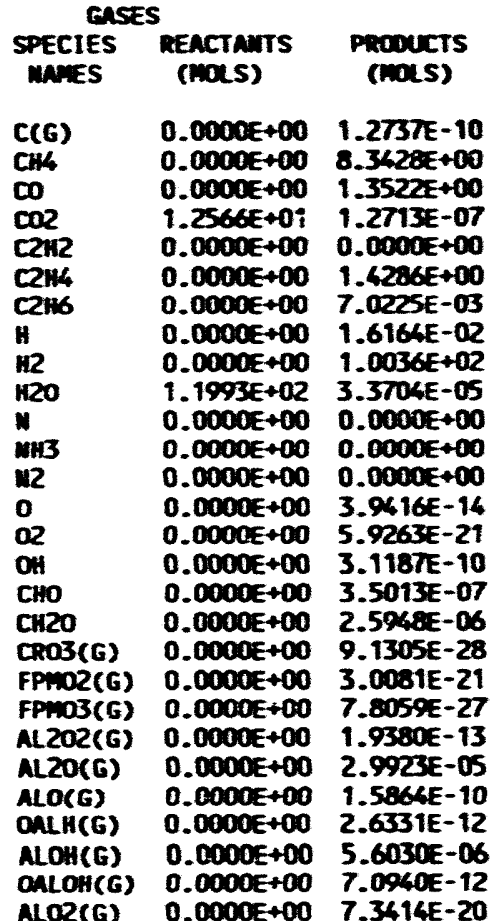


Table 6.4 Output listing for the BWR sample problem (continued)

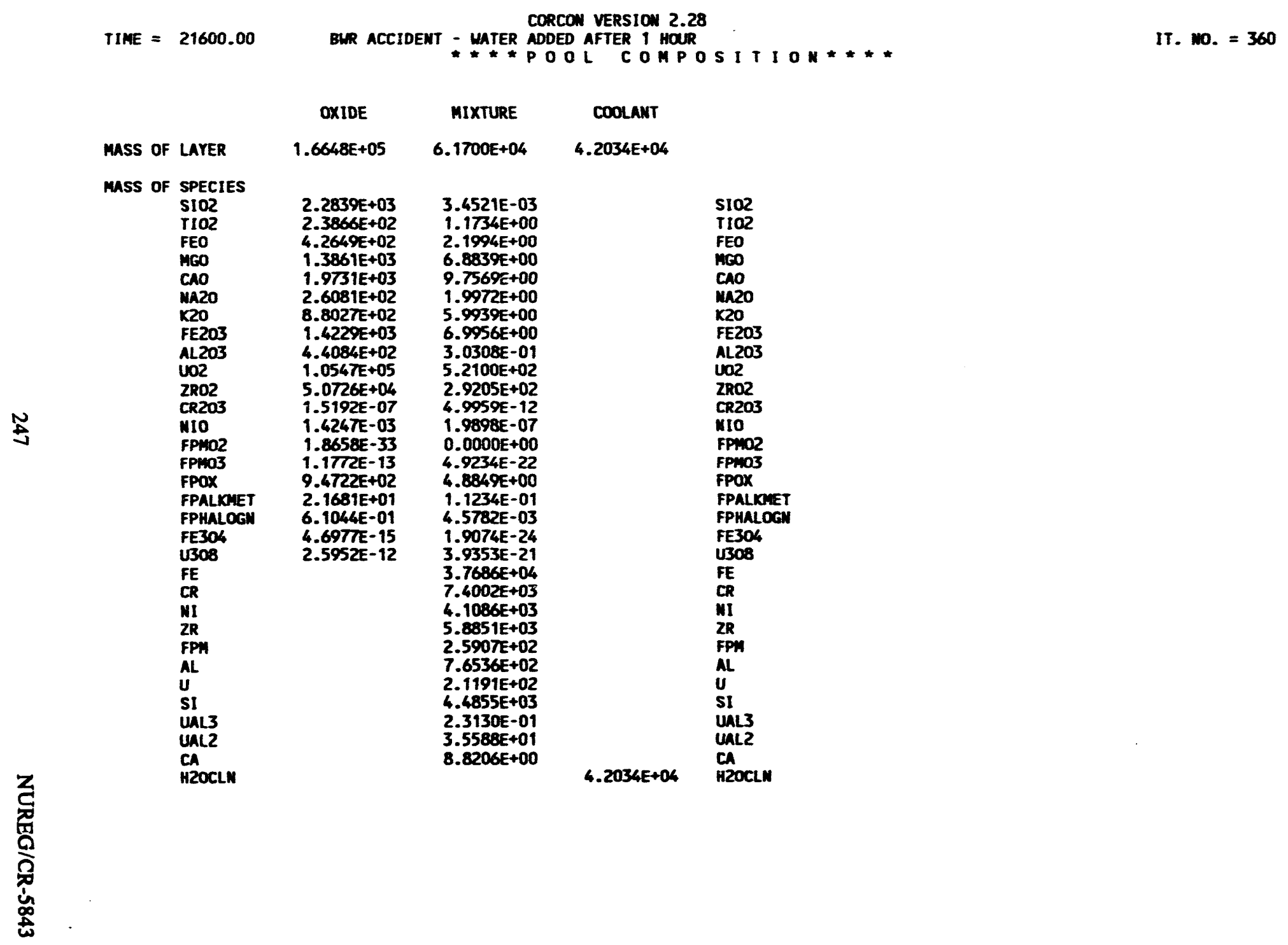




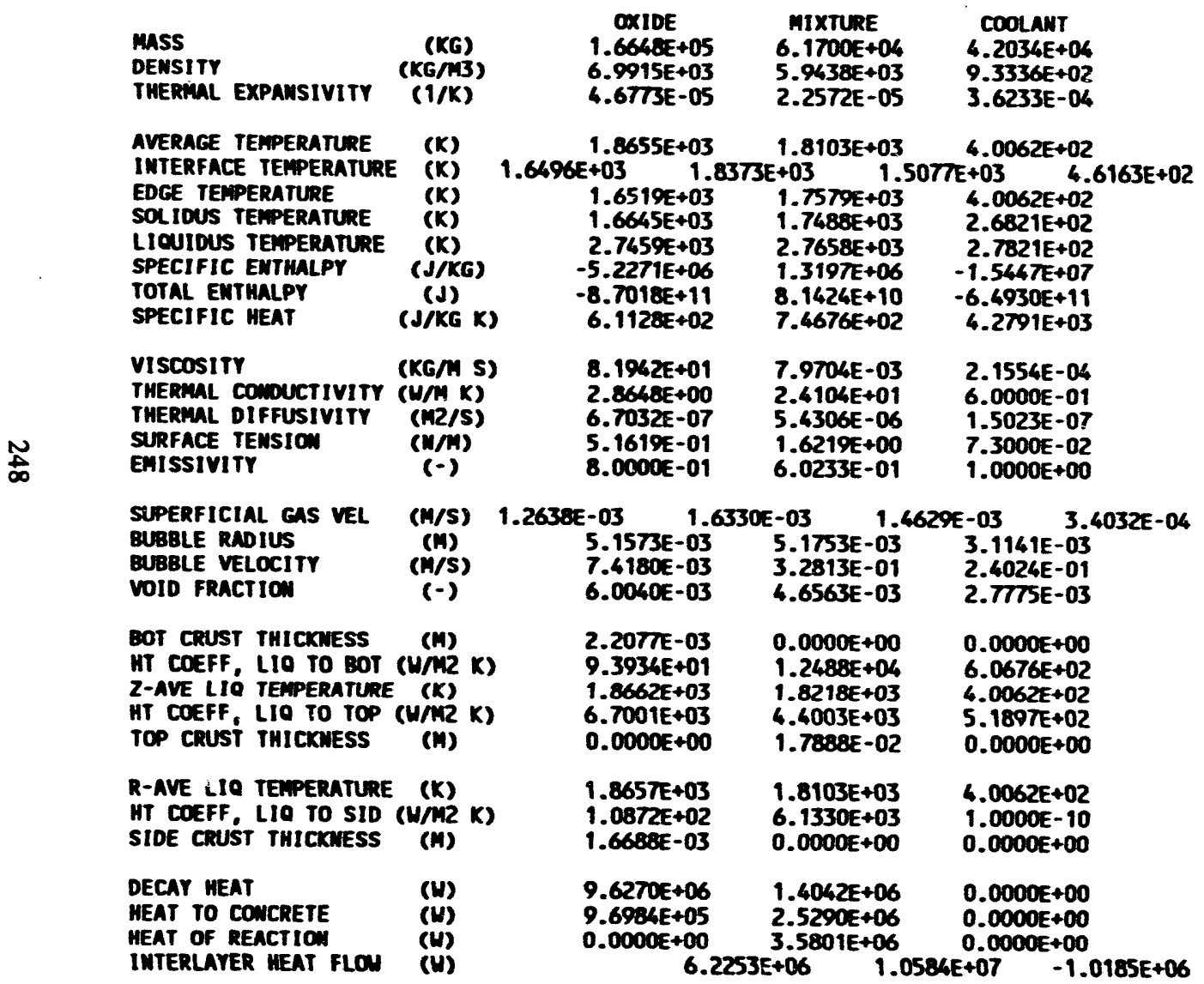


TIME $=21600.00$

BUR ACCIDENT CORCON VERSION 2.28

$$
\text { * * * I I T TERLAYER A I XING**** }
$$

IT. ผ0. $=360$

LOWER MIXTURE LAYER

$\begin{array}{llrl} & & & \\ \text { PHASE MASSES } & \text { (KG) } & 8.5336 E+02 & 6.0847 E+04 \\ \text { PHASE DENSITIES } & \text { (KG/M3) } & 7.0653 E+03 & 5.9306 E+03 \\ \text { LIOUIDUS TEMPERATURE } & \text { (K) } & 1.9657 \mathrm{~T}+03 & 1.7488 E+03 \\ \text { SOLIDUS TEMPERATURE } & \text { (K) } & 2.7658 E+03 & 1.7589 E+03 \\ \text { PHASE ENTHALPIES } & \text { (J) } & -4.5495 E+09 & 8.5968 E+10 \\ \text { MASS EMTRAINED } & \text { (KG) } & 0.0000 E+00 & 0.0000 E+00 \\ \text { MASS DE-ENTRAINED } & \text { (KG) } & 2.2460 E+02 & 0.0000 E+00\end{array}$


Table 6.4 Output listing for the BWR sample problem (continued)

\section{TEMPERATURE OF METAL (K) \\ $1.8102 E+03$ \\ TEMPERATURE OF OXIDE (K) $1.8651 E+03$ \\ AEROSOL - AMBIENT CONDITIONS (G/CC) $2.8787 \mathrm{E}-04$ \\ AEROSOL - STANDARO STATE CONDITIONS (G/CC) $6.1913 E-04$
( 298.15 (K) AND 1 ATM.) \\ GAS (G-MOLES/S) \\ AEROSOL RATE (GRAMS/S) \\ $4.1542 E+00$ \\ $6.2927 \mathrm{E}+01$ \\ $2.0860 E+00$ \\ $\begin{array}{ll}\text { PARTICLE SIZE (MICRONETERS) } & 1.3745 E+00 \\ \text { BUBBLE DINMETER FOR THE METAL PHASE (CM) } & 1.0374 E+00\end{array}$ \\ BUBBLE DIAMETER FOR THE METAL PHASE (CM)}

MECHANICAL RELEASES FRON THE AZBEL AND THE ISHII AND KATAOKA CORRELATIONS ARE CALCULATED.

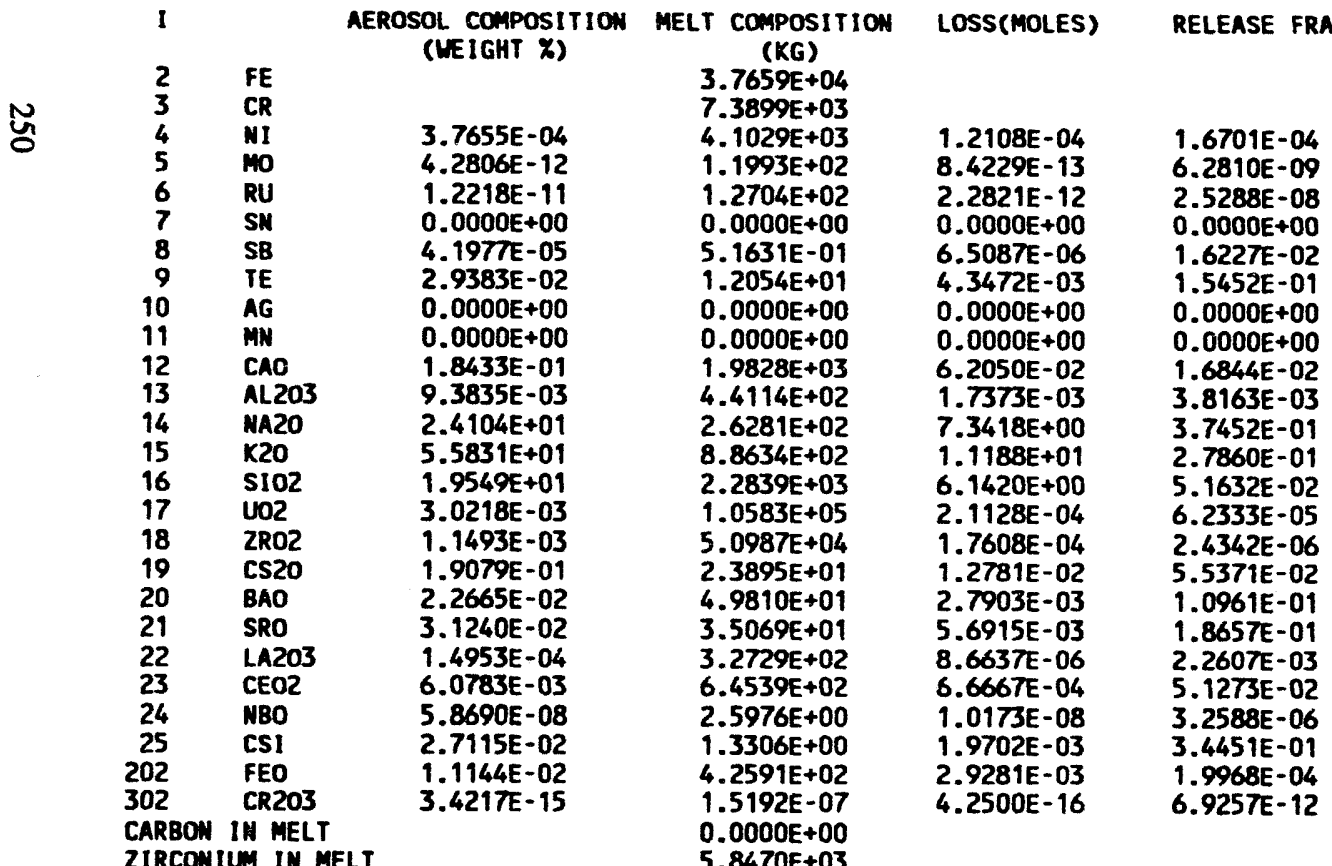


Table 6.4 Output listing for the BWR sample problem (continued)

$\begin{array}{llcc}1 & & \begin{array}{c}\text { GAS COMPOSITION } \\ \text { (WEIGHT } \text { ) }\end{array} & \begin{array}{c}\text { RELEASE RATE } \\ \text { (GRAMS/SECOND) }\end{array} \\ 1 & \text { H2O } & 2.1330 E-04 & 1.5963 E-04 \\ 2 & \text { H2 } & 9.8371 E+01 & 8.2380 E+00 \\ 3 & \text { H } & 3.5088 E-02 & 1.4693 E-03 \\ 4 & \text { OH } & 5.9564 E-09 & 4.2083 E-09 \\ 5 & \text { O } & 1.5731 E-12 & 1.0452 E-12 \\ 6 & \text { O2 } & 1.5013 E-18 & 1.9957 E-18 \\ 7 & \text { CO2 } & 8.5647 E-07 & 1.5658 E-06 \\ 8 & \text { CO } & 1.5936 E+00 & 1.8543 E+00\end{array}$


Table 6.4 Output listing for the BWR sample problem (continued)

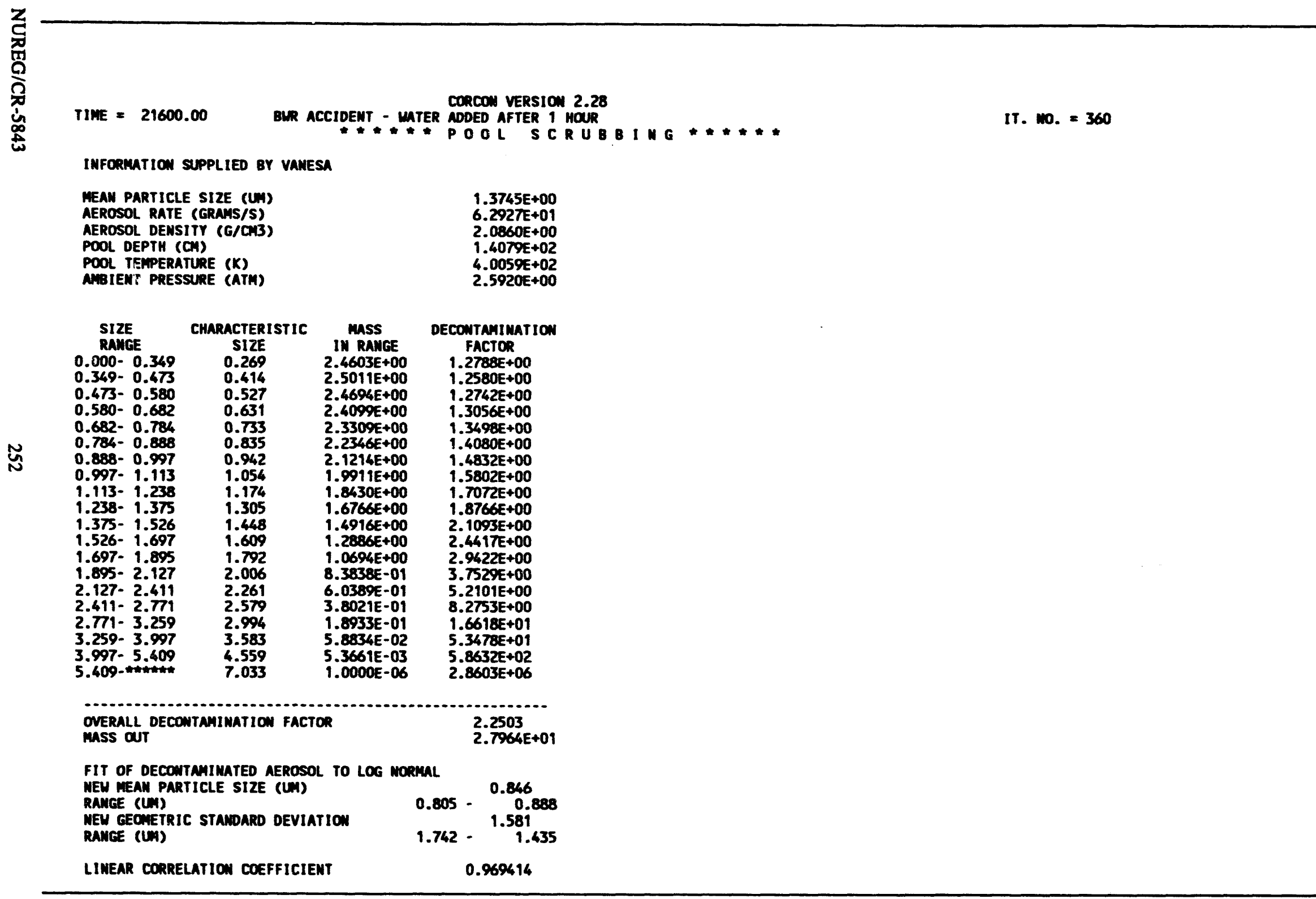

MEAN PARTICLE SIZE (UH)

RATE (GRAMS/

POOL DEPTH (CH)

TEOTER (K)

$6.2927 E+01$
$2.0860 E+00$
$1.4079 E+02$
$4.0059 E+02$

$2.5920 E+00$

\begin{tabular}{|c|c|c|c|}
\hline $\begin{array}{c}\text { SIZE } \\
\text { RANGE } \\
0.000-0.349 \\
0.349-0.473 \\
0.473-0.580 \\
0.580-0.682 \\
0.682-0.784 \\
0.784-0.888 \\
0.888-0.997 \\
0.997-1.113 \\
1.113-1.238 \\
1.238-1.375 \\
1.375-1.526 \\
1.526-1.697 \\
1.697-1.895 \\
1.895-2.127 \\
2.127-2.411 \\
2.411-2.771 \\
2.771-3.259 \\
3.259-3.9997 \\
3.997-5.409\end{array}$ & $\begin{array}{c}\text { ARACTERISTIC } \\
\text { S1ZE } \\
0.269 \\
0.414 \\
0.527 \\
0.631 \\
0.733 \\
0.835 \\
0.942 \\
1.054 \\
1.174 \\
1.305 \\
1.448 \\
1.609 \\
1.792 \\
2.006 \\
2.261 \\
2.579 \\
2.994 \\
3.583 \\
4.559\end{array}$ & $\begin{array}{c}\text { MASS } \\
\text { IN RANGE } \\
2.4603 E+00 \\
2.5011 E+00 \\
2.4694 E+00 \\
2.4099 E+00 \\
2.3309 E+00 \\
2.2346 E+00 \\
2.1214 E+00 \\
1.9911 E+00 \\
1.8430 E+00 \\
1.6766 E+00 \\
1.4916 E+00 \\
1.2886 E+00 \\
1.0694 E+00 \\
8.3838 E-01 \\
6.0389 E-01 \\
3.8021 E-01 \\
1.8933 E-01 \\
5.883-E-02\end{array}$ & $\begin{array}{r}\text { DECONTAMIMATI } \\
\text { FACTOR } \\
1.2788 E+00 \\
1.2580 E+00 \\
1.2742 E+00 \\
1.3056 E+00 \\
1.3498 E+00 \\
1.4080 E+00 \\
1.4832 E+00 \\
1.5802 E+00 \\
1.7072 E+00 \\
1.8766 E+00 \\
2.1093 E+00 \\
2.4617 E+00\end{array}$ \\
\hline
\end{tabular}

OVERALL DECONTAMIMATION FACTOR

FIT OF DECONTMIMATED AEROSOL TO LOG MOOHAL

OFEAM PARTICLE SIZE (IM)

MEY GEONETRIC STANDARD DEVIATION $-0.805 \cdot 1.581$

LINEAR CORRELATION COEFFICIENT $\quad 0.969414$ 


\subsection{References}

1. USNRC, Reactor Safety Study, NUREG-75-014, WASH-1400, 1975.

2. Murfin, W. B., A Preliminary Model for CoreConcrete Interactions, SAND77-0370, Sandia National Laboratories, Albuquerque, NM, 1977.

3. Muir, J. F., et al., CORCON-Mod1: An Improved Model for Molten-Core/Concrete Interactions, SAND80-2415, Sandia National Laboratories, Albuquerque, NM, 1981.

4. Cole, R. K., D. P. Kelly, and M. A. Ellis, CORCON-Mod2: A Computer Program for Analysis of Molten-Core Concrete Interactions, NUREG/CR-3920, SAND84-1246, Sandia National Laboratories, Albuquerque, NM, 1984.

5. ANSI, American National Standard Programming Language FORTRAN, X3.9-1978, American National Standards Institute, New York, NY, 1978.

6. Copus, E. R., R. E. Blose, J. E. Brockmann, R. D. Gomez, and D. A. Lucero, Core-Concrete Interactions Using Molten Steel with Zirconium on a Basaltic Basemat: The SURC-4 Experiment, NUREG/CR-4994, SAND87-2008, Sandia National Laboratories, Albuquerque, NM, 1989.

7. Murata, K. K., D. E. Carroll, K. E. Washington, F. Gelbard, G. D. Valdez, D. C. Williams, and K. D. Bergeron, User's Manual for CONTAIN 1.1: A Computer Code for Severe Nuclear Reactor Accident Containment Analysis, NUREG/CR-5026, SAND87-2309, Sandia National Laboratories, Albuquerque, NM, 1989.

8. Summers, R. M., et al., MELCOR 1.8.0: A Computer Code for Nuclear Reactor Severe Accident Source Term and Risk Assessment Analyses, NUREG/CR-5531, SAND90-0364, Sandia National Laboratories, Albuquerque, NM, 1991.

9. Greene, G. A. and R. A. Bari, International Standard Problem No. 24, SURC-4 Experiment on Core-Concrete Interactions, Vol. 2: Workshop Summary Report, Prepared by Brookhaven National Laboratory for the OECD Committee on the Safety of Nuclear Installations, June 1989.
10. Powers, D. A., J. E. Brockmann, and A. W. Shiver, VANESA: A Mechanistic Model of Radionuclide Release and Aerosol Generation During Core Debris Interactions With Concrete, NUREG/CR-4308, SAND85-1370, Sandia National Laboratories, Albuquerque, NM, 1986.

11. Light Water Reactor Safety Research Program Quarterly (or Semiannual) Report, Nuclear Fuel Cycle Program, Sandia National Laboratories, Albuquerque, NM.
a. January - March 1976
SAND76-0369 September 1976
b. October - December 1978 SAND70.0820 July 1979
c. April - June 1979. April 1980
d. January - March 1980 July 1980
SAND79-2057
SAND80-1304
(1 of 4)
e. January - March 1981 July 1981
SAND81-1214
(1 of 4)
f. April - September 1981
SAND81-0006 February 1992
g. Oct 1981 - March 1982 December 1982
h. April - September 1982 October 1983
SAND82-1572
SAND83-1576

12. Powers, D. A., et al., Exploratory Study of Molten Core Material/Concrete Interactions July 1975 March 1977, SAND77-2042, Sandia National Laboratories, Albuquerque, NM, 1978.

13. Greene, G. A., "Heat, Mass, and Moment' $m$ Transfer in a Multi-Fluid Bubbling Pool," Advances in Heat Transfer, Vol. 21, pp. 270 345, 1991.

14. Nelson, L. S., M. J. Eatough, and K. P. Guay, Explosive Interactions Between Molten Alumimum and Aqueous Coolants, SAND88-2959, Sandia National Laboratories, Albuquerque, NM, 1989.

15. Blose, R. E., J. E. Gronager, A. J. Suo-Antilla, and J. E. Brockmann, SWISS: Sustained Heated Metallic Melt/Concrete Interactions With Overlying Water Pools, NUREG/CR-4727, SAND85-1546, Sandia National Laboratories, Albuquerque, NM. 1987. 
16. Spencer, B. W., et al., MACE Scoping Test Data Report, MACE-TR-D03, Argonne National Laboratory, Argonne, IL, 1991.

17. Copus, E. R., et al., Simultaneous Interactions Between Molten Aluminum, Siliceous Concrete, and Overlying Water Pools: The SRL-3 Experiment, SAND92-0443, Sandia National Laboratories, Albuquerque, NM.

18. Greene, G. A., et al., "Some Observations on Simulated Molten Debris-Coolant Layer Dynamics, "International Meeting on Light Water Reactor Severe Accident Evaluation, Cambridge, MA, September 1983.

19. Fauske \& Associates, Inc., Technical Support for the Debris Coolability Requirements for Advanced Light Water Reactors in the Utility/EPRI Light Water Reactor Requirements Document, DOE/ID10278, June 1990.

20. Thompson, D. H., and J. K. Fink, ACE MCCI Test L6 Test Data Report, Vols. 1 and 2, ACETR-C26 v.1 and v.2, Argonne National Laboratory, Argonne, IL, 1991.

21. Bennett, D. E., SANDIA-ORIGEN User's Manual, NUREG/CR-0987, SAND79-0299, Sandia National Laboratories, Albuquerque, NM, 1979.

22. Blottner, F. G., Hydrodynamics and Heat Transfer Characteristics of Liquid Pools with Bubble Agitation, NUREG/CR-0944, SAND79-1132, Sandia National Laboratories, Albuquerque, NM, 1979.

23. Konsetov, V. V., "Heat Transfer during Bubbling of Gas Through Liquid, "International Journal of Heat Mass Transfer, Vol. 9, pp. 1103-1108, 1966.

24. Ginsberg, T. and G. A. Greene, "BNL Program in Support of LWR Degraded Core Accident Analysis, " Proceedings of the 10th Water Reactor Safety Research Information Meeting, Vol. 2, NUREG/CP-0041, pp. 364-395, 1983.

25. Bradley, D. R., "Modeling of Heat Transfer Between Core Debris and Concrete, " in ANS Proceedings of the 1988 National Heat Transfer Conference, Houston, TX, pp. 37-49, July 24-27, 1988.
26. Kutateladze, S. S., and I. G. Malenkov, "Boiling and Bubbling Heat Transfer Under Conditions of Free and Forced Convection, "6th International Heat Transfer Conference, Toronto, Canda, 1978.

27. Sokolov, V. N., and A. D. Salamakin, "Heat Exchange Between a Gas-Liquid System and a Heat Exchange Element, " Zhumal Prikladnoi Khimii (Journal of Applied Chemistry of the USSR), Vol. 35, No. 11, 1962.

28. Greene, G. A., and T. F. Irvine, "Heat Transfer Between Stratified Immiscible Liquid Layers Driven by Gas Bubbling Across the Interface, " in ANS Proceedings of the 1988 National Heat Transfer Conference, Houston, TX, July 24-27, 1988.

29. Farmer, M. T., J. J. Sienicki, and B. W. Spencer, "CORQUENCH: A Model for Gas SpargingEnhanced Melt-Water, Film Boiling Heat Transfer," ANS Special Session on Thermal Hydraulics of Severe Accidents, November 11-15, 1990.

30. McAdans, W. H., Heat Transmission, McGrawHill Book Co., New York, NY, 1954.

31. Kulacki, F. A., and R. J. Goldstein, "Thermal Convection in a Horizontal Fluid Layer with Uniform Volumetric Energy Source, " Journal of Fluid Mechanics, Vol. 55, No. 2, p. 271, 1975.

32. Kulacki, F. A., and M. E. Nagle, Journal of Heat Transfer, Vol. 97, p. 204, 1975.

33. Kulacki, F. A., and A. A. Emara, Trans ANS, Vol. 22, p. $44 \%, 1975$.

34. Epstein, $\mathrm{M}$., "Heat Conduction in the $\mathrm{UO}_{2}$. Cladding Composite Body with Simultaneous Solidification and Melting, "Nuclear Science and Engineering, Vol. 51, 1973.

35. Alsmeyer, H., and M. Reirnann, "On the Heat and Mass Transport Processes of a Horizontal Melting or Decomposing Layer Under a Molten Pool," Nuclear Reactor Safety Heat Transfer, Winter Annual Meeting ASME, Atlanta, GA, pp. 47-53, 1977.

36. Berenson, P. J., "Transition Boiling Heat Transfer From a Horizontal Surface, " Journal of Heat Transfer, Vol. 83, pp. 351-358, 1961. 
37. Bergles, A. E., et al., Two-Phase Flow and Heat Transfer in the Power and Process Industries, McGraw Hill, New York, NY, 1981.

38. Keenan, J. H., et al., Steam Tables, Thermodynamic Properties of Water Including Vapor, Liquid, and Solid Phases (International System of Units-S.I.), John Wiley and Sons, New York, NY, 1978.

39. Rohsenow, W. M., "A Method of Correlating Heat Transfer for Surface Boiling of Liquids," Trans. ASME, Vol. 74, pp. 979-976, 1952.

40. Zuber, N., "On Stability of Boiling Heat Transfer," Trans. ASME, Vol. 80, pp. 711-720, 1958.

41. Zuber, N., et al., "The Hydrodynamic Crisis in Pool Boiling of Saturated and Subcooled Liquids," in International Developments in Heat Transfer, Part II, ASME, New York, NY, pp. 230-235, 1961.

42. Rohsenow, W. H., "Boiling, " in Handbook of Heat Transfer, McGraw-Hill, New York, NY, pp. 1328, 1973.

43. Ivey, H. J., "Acceleration and the Critical Heat Flux in Pool Boiling," Chartered Mechanical Engineering, Vol. 9, pp. 413-427, 1962.

44. Duignan, M. R., and G. A. Greene, "Enhanced Convective and Film Boiling Heat Transfer by Surface Injection, " Diss., State University of New York at Stony Brook, NY, 1989.

45. Siviour, J. B., and A. J. Ede, "Heat Transfer in Subcooled Film Boiling, " paper B3.12, in Proceedings of the Fourth International Heat Transfer Conference, Paris-Versailles, France, 1970.

46. Dhir, V. K. and G. P. Purohit, "Subcooled Film Voiling Heat Transfer from Spheres," Nuclear Engr. and Design, Vol. 47, 1978, pp. 49-66.

47. Toda, S., and M. Mori, "Subcooled Film Boiling and the Behavior of Vapor Film on a Horizontal Wire and Sphere," 7 th International Heat Transfer Conference, Munich, Germany, pp. 173-178, 1982.

48. Bradfield, W. S., "On the Effect of Subcooling on Wall Superheat in Pool Boiling, "Journal of Heat Transfer, pp. 269-270, 1967.
49. Cole, Jr., R. K., "A Crust Formation and Refreezing Model for Molten-Fuel/Concrete Interactions Codes," Paper 12.5, in Proceedings, International Meeting on Light Water Reactor Severe Accident Evaluation, Cambridge, MA, 1983.

50. Clift, R., J. R. Grace, and M. E. Weber, Bubbles, Drops, and Particles, Academic Press, New York, NY, 1978.

51. Mendelson, H. D., Journal of the American Institute of Chemical Engineering, Vol. 13, p. 250 , 1967.

52. Davies, R. M., and G. I. Taylor, Proceedings of the Royal Society of London A, Vol. 200, p. 375, 1950.

53. Wallis, G. B., One-Dimensional, Two-Phase Flow, McGraw-Hill, New York, NY, 1969.

54. Brockmann, J. E., Validation of Models of Gas Holdup in the CORCON Code, NUREG/CR-5433, SAND89-1951, Sandia National Laboratories, Albuquerque, NM, 1989.

55. Davidson, J. F., and B. O. G. Schuler, Transactions of the Institute of Chemical Engineers, Vol. 38, pp. 144, 335, 1960.

56. Hosler, E. R., and J. W. Westwater, "Film Boiling on a Horizontal Plate," ARS Journal, pp. 553-558, April 1962.

57. Greene, G. A., J. C. Chen, and M. T. Conklin, "Onset of Entrainment Between Immiscible Liquid Layers Due to Rising Gas Bubbles," International Journal of Heat and Mass Transfer, Vol. 31, p. $1309,1988$.

58. Greene, G. A., J. C. Chen, and M. T. Conklin, "Bubble-Induced Entrainment Between Stratified Liquid Layers," International Journal of Heat and Mass Transfer, Vol. 34, p. 149, 1990.

59. Beard, K. V., and H. R. Pruppacher, "A Determination of the Terminal Velocity and Drag of Small Water Drops by Means of a Wind Tunnel, "Journal of Atmospheric Science, Vol. 26, p. 1066, 1969. 
60. Cheung, F. B., S. H. Chan, T. C. Chawla, and D. H. Cho, "Radiative Heat Transfer in a Heat Generating and Turbulent Convecting Fluid Layer," International Journal of Heat Mass Transfer, Vol. 23, pp. 1313-1323, 1980.

61. Kwong, K. C., R. A. S. Beck, and T. C. Derbidge, CORCON Program Assistance, FR-7910/AS, ACUREX Corporation/Aerotherm Aerospace Systems Division, Mt. View, CA, 1979.

62. Van Zeggeren, F. H., and S. H. Storey, The Computation of Chemical Equilibrium, Cambridge University Press, Cambridge, 1970.

63. Bottinga, Y., D. F. Weill, and P. Richert, "Thermodynamic Modeling of Silicate Melts," Thermodynamics of Minerals and Melts, R. C. Newton, A. Navrotsky, and B. J. Wood, eds., Springer-Verlag, 1981.

64. Scheibel, E. G., "Physical Chemistry in Chemical Engineering," Ind. Eng. Chem., Vol. 46, p. 2007, 1954.

65. Wilke, C. R., and P. Chang, "Correlations of Diffusion Coefficients in Dilute Solutions, " AICHE Journal, Vol. 1, p. 264, 1955.

66. Ziemniak, S. E., "A Study of Interfacial Resistance to Mass Transfer at High Evaporation Rates," Diss., Rennesselaer Polytechnic Institute, 1968.

67. Singh, P. C., and S. Singh, "Development of a New Correlation for Binary Gas Phase Diffusion Coefficients, "International Commission on Heat Mass Transfer, Vol. 10, p. 123, 1983.

68. Kataoka, I, and M. Ishii, "Mechanistic Modeling of Pool Entrainment Phenomenon," International Joumal of Heat Mass Transfer, Vol. 27, p. 1999, 1984.

69. Azbel, D. S., et al., "Acoustic Resonance Theory for the Rupture of Film Cap of a Gas Bubble at a Horizontal Gas-Liquid Interface," Two Phase Momentum, Heat and Mass Transfer in Chemical Process and Energy Engineering Systems, Vol. 1, Hemisphere Publishing Co., pp. 159-170, 1978.
70. Fuchs, N. A., The Mechanics of Aerosols, Pergamon Press, 1964.

71. Powers, D. A., and A. W. Frazier, VISRHO: A Computer Subroutine for Estimating the Viscosity and Density of Complex Silicate Melts, SAND760649, Sandia National Laboratories, Albuquerque, NM, 1977.

72. SNL, Core-Meltdown Experimental Review, NUREG-0205, SAND74-0382, Sandia National Laboratories, Albuquerque, NM, 1977.

73. Weast, R. C., Ed., Handbook of Chemistry and Physics, CRC Press, Cleveland, OH, 1976.

74. JANAF Thermochemical Tables, Physicochemical Studies, DOW Chemical USA, Midland, MI (including supplements).

75. Samsonov, G. V., ed., The Oxide Handbook, translated from Russian by C. N. Turton and T. I. Turton, IFI/Plenum Press, New York, NY, 1973.

76. Robie, R. A., B. S. Hemingway, and J. R. Fisher, Thermodynamic Properties of Minerals, Geological Survey Bulletin 1452, U. S. Department of Interior, U. S. Government Printing Office, Washington, DC, 1978.

77. Touloukian, Y. S., ed., Thermophysical Properties of Matter. The TPRC Data Series, Vols. 1-8, IFI/Plenum Press, New York, NY, 1970.

78. Kendell, J., and K. P. Monroe, "The Viscosity of Liquids, III Ideal Solution of Solids in Liquids," Journal of the American Chem. Soc., Vol. 39, No. 9, p. 1802, September 1917.

79. Shaw, H. R., "Viscosities of Magmatic Silicate Liquids: An Empirical Method of Prediction," American Journal of Science, Vol. 272, pp. 870-893, 1972.

80. Bottinga, Y., and D. F. Weill, "The Viscosity of Magmatic Silicate Liquids: A Model for Calculation," American Joumal of Science, Vol. 272, pp. 438-475, 1972. 
81. Ogino, Y., F. O. Borgmann, and M. G. Frohberg, "On the Viscosity of Liquid Iron," Japan Inst. of Metals Joum., Vol. 37, p. 1230, 1973.

82. Kunitz, M., Journal of General Physiology, Vol, 9 p. $715,1926$.

83. Parrott, J. E., and A. D. Stuckes, Thermal Conductivity of Solids, Pion Limited, London, England, 1975.
84. Nause, R. C., and M. T. Leonard, Thermophysical Property Assessment for Savannah River Site Production Reactor Materials, 89/6507, Science Applications International Corporation, Albuquerque, NM, 1989.

85. Speich, G. R., "Cr-Fe-Ni (Chromium-Iron Nickel), "Metals Handbook, Vol. 8, American Society for Metals, Metals Park, OH, p. 424, 1973. 


\section{DISTRIBUTION LIST}

External Distribution:

25 U.S. Nuclear Regulatory Commission Office of Nuclear Regulatory Research Washington, DC 20555

Attn: B. W. Sheron, NLS-007

C. G. Tinkler, Jr. NLN-344

G. R. Marino, NLS-007

A. M. Rubin, NLN-344

R. B. Foulds, NLN-344

A. Notafrancesco, NLN-344

J. A. Murphy, NLS-007

S. Basu, NLN-344

E. S. Beckjord, NLS-007

F. Eltawila, NLN-344

T. P. Speis, NLS-007

5 U.S. Nuclear Regulatory Commission Office of Nuclear Reactor Regulation Washington, DC 20555

Attn: T. C. Murley, $12 \mathrm{G} 18$

F. P. Gillespie

A. C. Thadani, 8E2 (3)

2 U.S. Department of Energy

Albuquerque, New Mexico

P.O. 5400

Albuquerque, NM 87185

Attn: C. E. Garcia, Director

2 Electric Power Research Institute

3412 Hillview Avenue

Palo Alto, CA 94303

Attn: A. Machiel

3 Brookhaven National Laboratory

$130 \mathrm{BNL}$

Upton, NY 11973

Attn: T. Pratt
3 Oak Ridge National Laboratory

P.O. Box Y

Oak Ridge, TN 37830

Attn: T. Kress

3 Argonne National Laboratory

9700 S. Cass Avenue

Argonne, IL 60439

Attn: B. Spencer

1 Nuclear Safety Oversight Commission 1133 15th Street, NW

Room 307 ;

Washington, DC 20005

Attn: Cathy Anderson

1 Battelle Columbus Laboratory

505 King Avenue

Columbus, OH 43201

Attn: P. Cybulskis

1 UCLA Nuclear Energy Laboratory 405 Hilgaard Avenue Los Angeles, CA 90024

Attn: I. Catton

1 Los Alamos National Laboratories

P.O. Box 1663

Los Alamos, NM 87545

Attn: M. Stevenson

1 University of Wisconsin

Nuclear Engineering Department

1500 Johnson Drive

Madison, WI 53706

Attn: M. L. Corradini 
1 EG\&G Idaho

Willo Creek Building, W-3

P.O. Box 1625

Idaho Falls, ID 83415

Attn: R. Hobbins

1 Battelle Pacific Northwest Laboratory P.O. Box 999

Richland, WA 99352

Attn: M. Freshley

1 Department of Energy

Scientific and Tech. Info. Center

P.O. Box 62

Oak Ridge, TN 37831

1 Fauske and Associates, Inc. 16W070 West 83rd Street

Burr Ridge, IL 60952

Attn: R. Henry

\section{IAEA}

Division of Nuclear Reactor Safety

Wagranesrstrasse 5

P.O. Box 100

A/1400 Vienna

AUSTRIA

Attn: M. Jankowski

1 Department LWR Fuel

Belgonuucleaire

Rue de Champde Mars. 25

B-1050 Brussels

BELGIUM

Attn: Mr. H. Bairiot, Chief

1 U.S. Nuclear Regulatory Commission Advising Comm. on Reactor Safeguard Washington, DC 20555

Attn: M. D. Houston, P-315
1 Atomic Energy Canada, Ltd. Chalk River, Ontario CANADA KOJ IJO

Attn: R. D. MacDonald

1 Institute of Nuclear Energy Research P.O. Box 3

Lungtan

Taiwan 325

REPUBLIC OF CHINA

Attn: Sen-I Chang

1 Department of Nuclear Safety

Finnish Center for Radiation

and Nuclear Safety

P.O. Box 268

SF-00181 Helsinki

FINLAND

Attn: Jorma V. Sandberg

1 CEN Cadarache

18108 Saint Paul Lez Durance

FRANCE

Attn: A. Meyer-Heine

1 Energy Research Inc.

P. O. Box 2034

Rockville, MD 20847-2034

Attn: Dr. Mohsen Khatib Rahbar

1 Centre d'Estudes Nuclearies (IPSNDAS)

Commisariat a l'Energie Atomique

Boite Postale No. 6

F-92265 Fontenay-aux-Roses Cedex FRANCE

Attn: Jacques Duco 
2 Gesellschaft fur Reaktorsicherheit (GRS)

Postfach 101650

Glockengrasse 2

5nO0 Koeln 1

GERMANY

Attn: Dr. Ing. Manfred Fimhaber

1 Kraftwerk Union

Hammerbacher Strasse 1214

Postfach 3220

D-8520 Erlangen 2

GERMANY

Attn: Dr. M. Peeks

2 Kernforschungszentrum Karlsruhe

Postfach 3640

75 Karlsruhe

GERMANY

Attn: S. Hagen

2 Japan Atomic Energy Research Institute Tokai-Mura, Naka-Gun

Ibaraki-Ken 319-11

JAPAN

Attn: K. Soda

1 Reactor Centrum Nederland

1755 ZG Petten

THE NETHERLANDS

Attn: Dr. K. J. Brinkman

1 I. V. Kurchatov Institute

of Atomic Energy

Nuclear Safety Department

Moscow, 123182

RUSSIA

Attn: V. Asmalov
1 Sub. Emplazamientos y Programas

Consejo de Seguridad Nuclear

Justo Dorado II

28040 Madrid

SPAIN

Attn: Jose Angel Martinez

1 Consejo de Seguridad Nuckan

SOR Angela de la Cruz No. 3

Madrid

SPAIN

Attn: Juan Bagues

1 Nucleare e della Protezione

Sanitaria (DISP)

Ente Nazionnle Energie

Alternative 7 (ENEA)

Viale Regina Margherita, 125

Casella Postale M. 2358

I-00100 Roma A.D.

ITALY

Attn: Mr. G. Petrangeli

1 Thermodynamics \& Rad. Physics

CEC Joint Research Center, Ispra I-21020 Ispra (Varese)

ITALY

Attn: Alan V. Jones

2 Statens Karnkraftinspektion

P.O. Box 27106

S-10252 Stockholm SWEDEN

Attn: W. Frid

1 E.T.S. Ingenieros Industriales

Jost Gutierrez Abascal, 2

28006 Madrid

SPAIN

Attn: Professor Agustin Alonso 
1 Department of Nuclear Power Safety

Royal Institute of Technology

S-100 11 Stockholm

SWEDEN

Attn: B. Raj Sehgal

1 UKAEA Culham Laboratory

Abingdon

Oxfordshire OX14 3DB

UNITED KINGDOM

Attn: B. D. Turland E5.157

1 Light Water Reactor Safety Program

Paul Scherrer Institute

CH-5232 Villigen PSI

SWITZERLAND

Attn: J. Peter Hosemann

1 Swiss Federal Nuclear Safety Directorate

CH-5303 Wurenlingen

SWITZERLAND

Attn: S. Chakraborty

1 Korea Atomic Energy Research Inst.

Korea Adv. Energy Research Inst.

P.O. Box 7

Daeduk-Danji

TAEJAN 305-606

Attn: Mr. Hee Dong Kim

2 UKAEA

Winfrith, Dorchester

Dorset DT2 8DH

UNITED KINGDOM

Attn: A. Nichols 102/A50

S. Kinnersly 204/A32 
Sandia Distribution:

1810 D. W. Schaeffer

6400 N. R. Ortiz

6403 W. A. von Riesemann

6404 D. A. Powers

6418 R. K. Cole

6418 S. L. Thompson

6422 M. D. Allen

6422 R. E. Blose

6422 J. E. Brockmann

6422 M. Pilch

6423 K. O. Reil

6429 R. O. Griffith

6429 K. K. Murata

6429 K. E. Washington (5)

7141 Technical Library (5)

7151 Technical Publications

7613 Document Processing for DOE/OSTI (10)

8523-2 Central Technical Files 

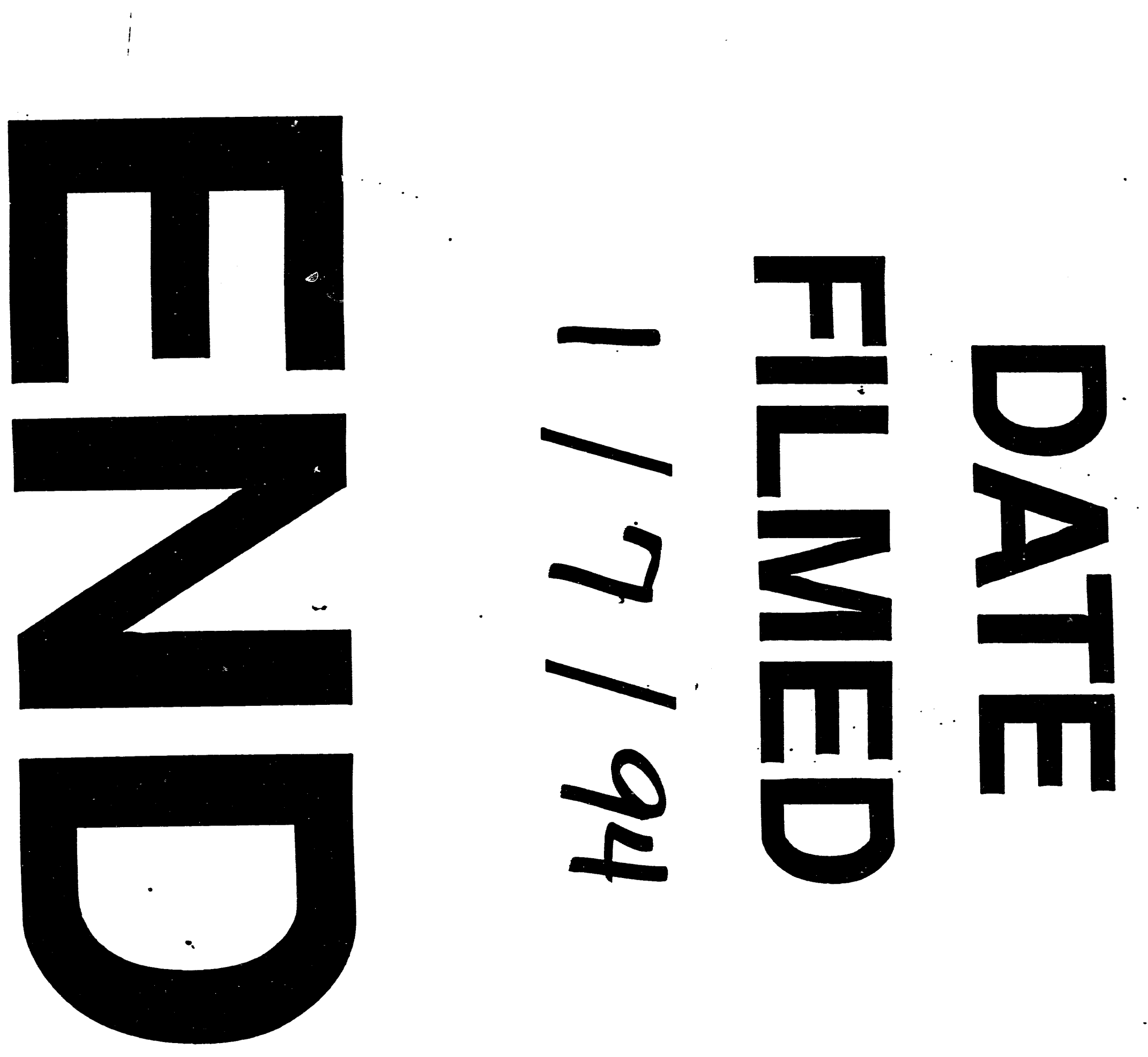
